\title{
Reduced or modified dietary fat for preventing cardiovascular disease (Review)
}

Hooper L, Summerbell CD, Thompson R, Sills D, Roberts FG, Moore HJ, Davey Smith G

Hooper L, Summerbell CD, Thompson R, Sills D, Roberts FG, Moore HJ, Davey Smith G.

Reduced or modified dietary fat for preventing cardiovascular disease.

Cochrane Database of Systematic Reviews 2012, Issue 5. Art. No.: CD002137.

DOI: 10.1002/14651858.CD002137.pub3.

www.cochranelibrary.com 
TABLE OF CONTENTS

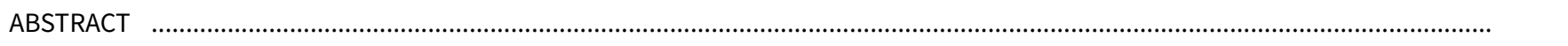

PLAIN LANGUAGE SUMMARY

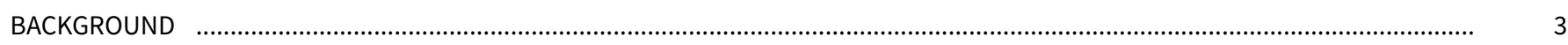

OBJECTIVES

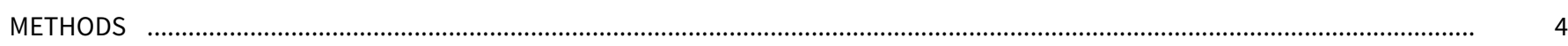

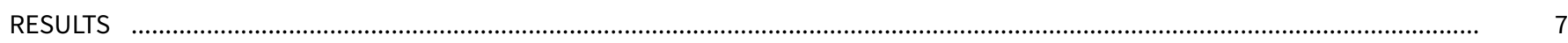

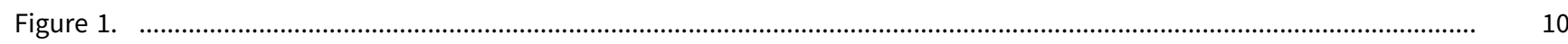

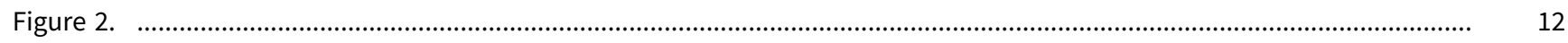

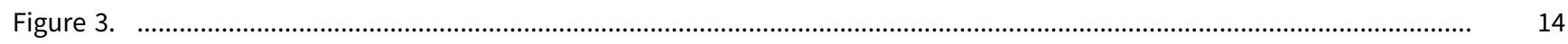

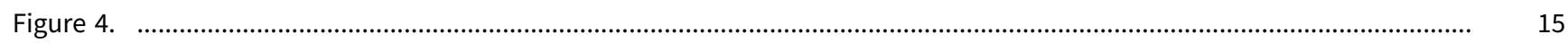

DISCUSSION

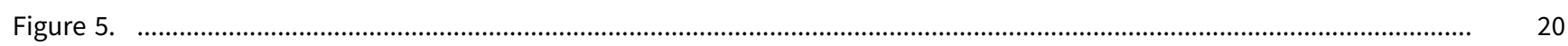

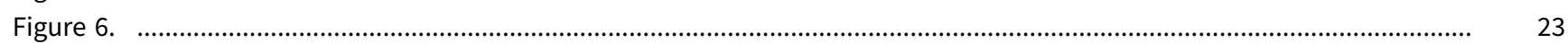

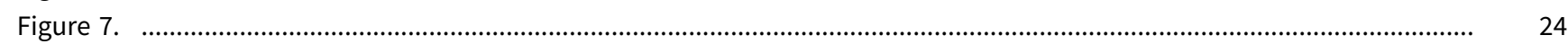

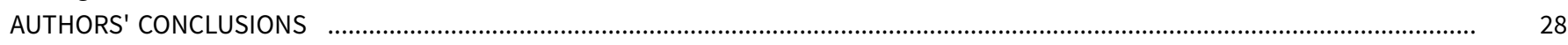

ACKNOWLEDGEMENTS

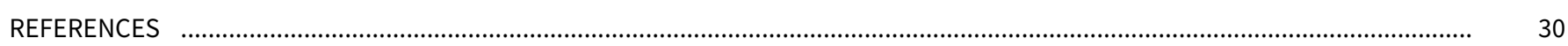

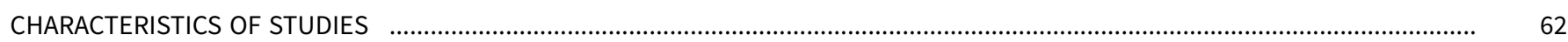

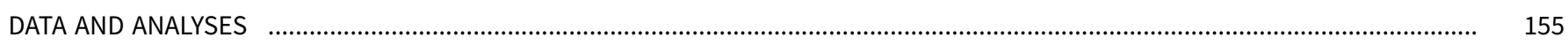

Analysis 1.1. Comparison 1 fat modification or reduction vs usual diet - primary outcomes, Outcome 1 Total mortality. .......... 156

Analysis 1.2. Comparison 1 fat modification or reduction vs usual diet - primary outcomes, Outcome 2 Cardiovascular 157 mortality.

Analysis 1.3. Comparison 1 fat modification or reduction vs usual diet - primary outcomes, Outcome 3 Combined cardiovascular events.

Analysis 2.1. Comparison 2 fat modification or reduction vs usual diet - secondary outcomes, Outcome 1 Myocardial infarctions.

Analysis 2.2. Comparison 2 fat modification or reduction vs usual diet - secondary outcomes, Outcome 2 Stroke. ................... Analysis 2.3. Comparison 2 fat modification or reduction vs usual diet - secondary outcomes, Outcome 3 Cancer deaths. ....... Analysis 2.4. Comparison 2 fat modification or reduction vs usual diet - secondary outcomes, Outcome 4 Cancer diagnoses. ... Analysis 2.5. Comparison 2 fat modification or reduction vs usual diet - secondary outcomes, Outcome 5 Diabetes diagnoses. . Analysis 2.6. Comparison 2 fat modification or reduction vs usual diet - secondary outcomes, Outcome 6 Non-fatal MI. ........... Analysis 3.1. Comparison 3 fat modification or reduction vs usual diet - tertiary outcomes, Outcome 1 Weight, kg. ................. Analysis 3.2. Comparison 3 fat modification or reduction vs usual diet - tertiary outcomes, Outcome $2 \mathrm{BMI}, \mathrm{kg} / \mathrm{m} 2$. ................ Analysis 3.3. Comparison 3 fat modification or reduction vs usual diet - tertiary outcomes, Outcome 3 LDL cholesterol, $\mathrm{mmol} /$ L.

Analysis 3.4. Comparison 3 fat modification or reduction vs usual diet - tertiary outcomes, Outcome $4 \mathrm{HDL}$ cholesterol, mmol/ Lkg.

Analysis 3.5. Comparison 3 fat modification or reduction vs usual diet - tertiary outcomes, Outcome 5 Total cholesterol, mmol/ L.

Analysis 3.6. Comparison 3 fat modification or reduction vs usual diet - tertiary outcomes, Outcome 6 Triglycerides, $\mathrm{mmol} / \mathrm{L}$. .. Analysis 3.7. Comparison 3 fat modification or reduction vs usual diet - tertiary outcomes, Outcome 7 Systolic Blood Pressure, $\mathrm{mmHg}$.

Analysis 3.8. Comparison 3 fat modification or reduction vs usual diet - tertiary outcomes, Outcome 8 Diastolic Blood Pressure, $\mathrm{mmHg}$.

Analysis 4.3. Comparison 4 fat reduction vs fat modification - primary outcomes, Outcome 3 Combined cardiovascular events.

Analysis 5.1. Comparison 5 fat reduction vs fat modification - secondary outcomes, Outcome 1 Myocardial infarction. ............

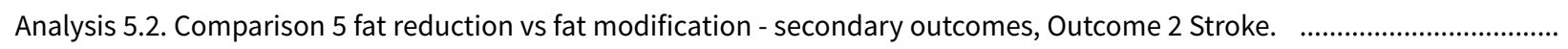
Analysis 5.4. Comparison 5 fat reduction vs fat modification - secondary outcomes, Outcome 4 Cancer diagnoses. ................. Analysis 5.6. Comparison 5 fat reduction vs fat modification - secondary outcomes, Outcome 6 Non-fatal MI. ........................

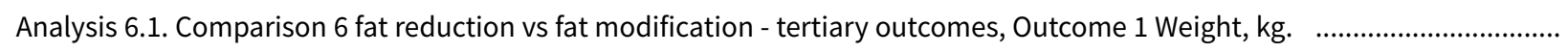

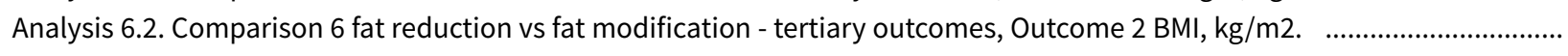
Analysis 6.3. Comparison 6 fat reduction vs fat modification - tertiary outcomes, Outcome 3 LDL cholesterol, mmol/L. ........... (1)

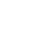

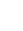

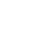

12

.

9

0

3

4

8
10

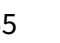

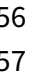


Analysis 6.4. Comparison 6 fat reduction vs fat modification - tertiary outcomes, Outcome $4 \mathrm{HDL}$ cholesterol, mmol/Lkg. $\quad \ldots . . . \quad 177$ Analysis 6.5. Comparison 6 fat reduction vs fat modification - tertiary outcomes, Outcome 5 Total cholesterol, mmol/L. .......... 178 Analysis 6.6. Comparison 6 fat reduction vs fat modification - tertiary outcomes, Outcome 6 Triglycerides, mmol/L. ............... 178 Analysis 6.7. Comparison 6 fat reduction vs fat modification - tertiary outcomes, Outcome 7 Systolic Blood Pressure, $\mathrm{mmHg}$. .. $\quad 178$ Analysis 6.8. Comparison 6 fat reduction vs fat modification - tertiary outcomes, Outcome 8 Diastolic Blood Pressure, $\mathrm{mmHg}$. . 179

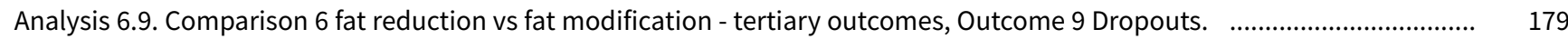

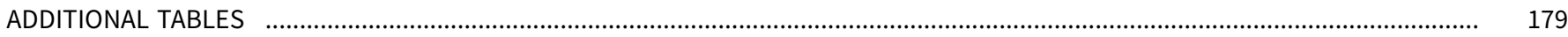

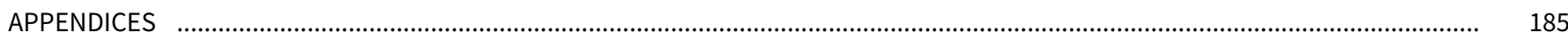

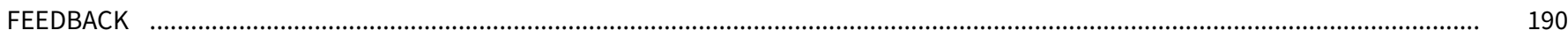

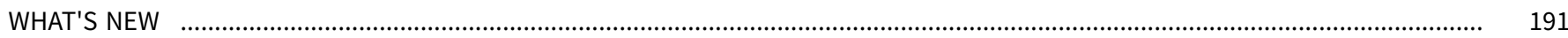

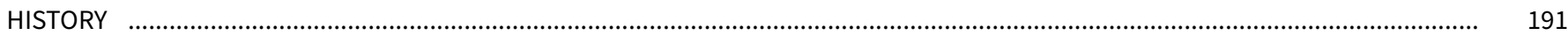

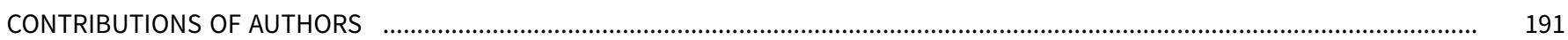

DECLARATIONS OF INTEREST

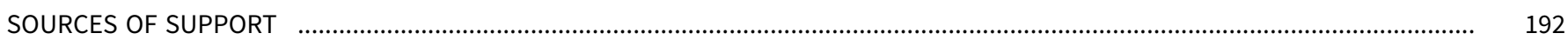

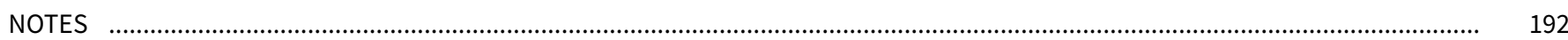

INDEX TERMS 
[Intervention Review]

\section{Reduced or modified dietary fat for preventing cardiovascular disease}

Lee Hooper ${ }^{1}$, Carolyn D Summerbell ${ }^{2}$, Rachel Thompson ${ }^{3}$, Deirdre Sills ${ }^{4}$, Felicia G Roberts 5 , Helen J Moore ${ }^{6}$, George Davey Smith ${ }^{7}$

1Norwich Medical School, University of East Anglia, Norwich, UK. ${ }^{2}$ School of Medicine, Pharmacy and Health, Wolfson Research Institute, Queen's Campus, Durham University, Stockton-on-Tees, UK. ${ }^{3}$ World Cancer Research Fund International, London, UK. ${ }^{4}$ Kings College London, London, UK. 5Ipswich Hospital, Ipswich, Suffolk, UK. 6School of Medicine and Health, Wolfson Research Institute, Queen's Campus, Durham University, Stockton-on-Tees, UK. ${ }^{7}$ MRC Integrative Epidemiology Unit, School of Social and Community Medicine, University of Bristol, Bristol, UK

Contact: Lee Hooper, Norwich Medical School, University of East Anglia, Norwich Research Park, Norwich, Norfolk, NR4 7TJ, UK. l.hooper@uea.ac.uk.

Editorial group: Cochrane Heart Group.

Publication status and date: Stable (no update expected for reasons given in 'What's new'), published in Issue 11, 2017.

Citation: Hooper L, Summerbell CD, Thompson R, Sills D, Roberts FG, Moore HJ, Davey Smith G. Reduced or modified dietary fat for preventing cardiovascular disease. Cochrane Database of Systematic Reviews 2012, Issue 5. Art. No.: CD002137. DOI: 10.1002/14651858.CD002137.pub3.

Copyright @ 2017 The Cochrane Collaboration. Published by John Wiley \& Sons, Ltd.

\section{A B S T R A C T}

\section{Background}

Reduction and modification of dietary fats have differing effects on cardiovascular risk factors (such as serum cholesterol), but their effects on important health outcomes are less clear.

\section{Objectives}

To assess the effect of reduction and/or modification of dietary fats on mortality, cardiovascular mortality, cardiovascular morbidity and individual outcomes including myocardial infarction, stroke and cancer diagnoses in randomised clinical trials of at least 6 months duration.

\section{Search methods}

For this review update, the Cochrane Central Register of Controlled Trials (CENTRAL), MEDLINE and EMBASE, were searched through to June 2010. References of Included studies and reviews were also checked.

\section{Selection criteria}

Trials fulfilled the following criteria: 1) randomised with appropriate control group, 2) intention to reduce or modify fat or cholesterol intake (excluding exclusively omega-3 fat interventions), 3) not multi factorial, 4) adult humans with or without cardiovascular disease, 5) intervention at least six months, 6) mortality or cardiovascular morbidity data available.

\section{Data collection and analysis}

Participant numbers experiencing health outcomes in each arm were extracted independently in duplicate and random effects metaanalyses, meta-regression, sub-grouping, sensitivity analyses and funnel plots were performed.

\section{Main results}

This updated review suggested that reducing saturated fat by reducing and/or modifying dietary fat reduced the risk of cardiovascular events by $14 \%$ (RR $0.86,95 \% \mathrm{Cl} 0.77$ to $0.96,24$ comparisons, 65,508 participants of whom $7 \%$ had a cardiovascular event, $1250 \%$ ). Subgrouping suggested that this reduction in cardiovascular events was seen in studies of fat modification (not reduction - which related directly to the degree of effect on serum total and LDL cholesterol and triglycerides), of at least two years duration and in studies of men 
(not of women). There were no clear effects of dietary fat changes on total mortality (RR $0.98,95 \% \mathrm{Cl} 0.93$ to $1.04,71,790$ participants) or cardiovascular mortality (RR $0.94,95 \% \mathrm{Cl} 0.85$ to $1.04,65,978$ participants). This did not alter with sub-grouping or sensitivity analysis.

Few studies compared reduced with modified fat diets, so direct comparison was not possible.

\section{Authors' conclusions}

The findings are suggestive of a small but potentially important reduction in cardiovascular risk on modification of dietary fat, but not reduction of total fat, in longer trials. Lifestyle advice to all those at risk of cardiovascular disease and to lower risk population groups, should continue to include permanent reduction of dietary saturated fat and partial replacement by unsaturates. The ideal type of unsaturated fat is unclear.

\section{PLAIN LANGUAGE SUMMARY}

\section{Cutting down or changing the fat we eat may reduce our risk of heart disease}

Modifying fat in our food (replacing some saturated (animal) fats with plant oils and unsaturated spreads) may reduce risk of heart and vascular disease, but it is not clear whether monounsaturated or polyunsaturated fats are more beneficial. There are no clear health benefits of replacing saturated fats with starchy foods (reducing the total amount of fat we eat). Heart and vascular disease includes heart attacks, angina, strokes, sudden cardiovascular death and the need for heart surgery. Modifying the fat we eat seems to protect us better if we adhere in doing so for at least two years. It is not clear whether people who are currently healthy benefit as much as those at increased risk of cardiovascular disease (people with hypertension, raised serum lipids or diabetes for example) and people who already have heart disease, but the suggestion is that they would all benefit to some extent. 


\section{B A C K G R O U N D}

In 1949 Ryle and Russell in Oxford documented a dramatic increase in coronary heart disease, and the Registrar General's Statistical Tables of 1920 to 1955 showed that there had been a 70-fold increase in coronary deaths during this 35 year period (Oliver 2000; Ryle 1949). This sudden surge in coronary heart disease sparked research into its causes. A case control study published in 1953 of 200 post-myocardial infarction patients and age-matched controls established that those with disease had higher LDL cholesterol levels (Oliver 1953).

Meanwhile in 1949 in the US Gofman had separated lipids into lipoprotein classes through ultra centrifugation, describing the low density lipoproteins (LDL) as 'atherosclerogenic' (Gofman 1949). The following year Keys proposed that the concentration of plasma cholesterol was proportional to dietary saturated fat intake (Keys 1950), and this relationship was confirmed in work by Hegsted (Hegsted 1965; Hegsted 2000) who published an equation explaining the relationship in 1965 and subsequently in 2000, suggesting that dietary saturated fat increases serum cholesterol and so increases cardiovascular risk, while polyunsaturated fats reduce both (this has since been further refined):

$\Delta$ serum cholesterol (in $\mathrm{mg} / \mathrm{dl}$ ) $=2.16^{*} \Delta$ dietary saturated fat intake (as percentage of energy) $-1.65^{*} \Delta$ dietary polyunsaturated intake (as percentage of energy) $+6.77^{*} \Delta$ dietary cholesterol intake (in units of $100 \mathrm{mg} /$ day) -0.53

The Seven Countries Study compared CHD mortality in 12000 men aged 40-59 in seven countries and found positive correlations between CHD mortality and total fat intake in 1970, then in 1986 between CHD mortality and saturated fat intake (Keys 1986, Thorogood 1996). A migrant study of Japanese men confirmed in 1974 that men in California had the diet richest in saturated fat and cholesterol, and the highest CHD rates, those in Hawail had intermediate diet and CHD rates, and those in Japan had a diet lowest in saturated fat and cholesterol, and the least CHD (Kagan 1974, Robertson 1977). However, recent systematic reviews of the observational data have not confirmed these early studies. Skeaff 2009 included 28 US and European cohorts (including 6600 coronary heart disease deaths among 280,000 participants) investigating the effects of total, saturated, monounsaturated, trans and omega- 3 fats on coronary heart disease deaths and events. They found no clear relationship between total, saturated or monounsaturated fat intake and coronary heart disease events or deaths. There was evidence that trans fats increased both coronary heart disease events and deaths, and that total polyunsaturated fats and omega- 3 fats decreased them. Siri-Tarino 2010 included 21 prospective epidemiologic studies assessing the relationship between saturated fats and coronary heart disease, stroke and cardiovascular disease, finding that saturated fat intake was not associated with risk of coronary heart disease, stroke or cardiovascular disease. Observational studies are potentially powerful at providing associations between dietary factors and cardiovascular risk, but the scale of measurement error is such that detecting such effects may be difficult. Thus intervention studies are needed to clarify cause and effect, to ensure that confounding is not either hiding or generating true relationships. Trials also directly address the issue of whether altering dietary fat in adults is helpful in reducing the risk of cardiovascular diseases in the general population and in those at high risk. It is essential that intervention trials form the basis of evidence based practice in this area.

Most intervention studies have assessed the effect of dietary interventions on risk factors for heart disease, and separate work ties the effect of altering these risk factors to changes in disease incidence and mortality. Systematic review in this area follows the same pattern, so that there are systematic reviews of the effect of dietary fat advice on serum lipid levels (Brunner 1997; Brunner 2009; Clarke 1997; Denke 1995; Kodama 2009; Mensink 1992; Shafiq 2010; Weggemans 2001; Yu-Poth 1999) suggesting that dietary changes cause changes in serum lipids and reviews on the effect of lipid level alterations on cardiovascular morbidity and mortality (Briel 2009; De Caterina 2010; Law 1994; Robinson 2009; Rubins 1995; Walsh 1995), suggesting that changes in lipids do affect cardiovascular risk. Other risk factors dealt with in a similar way are blood pressure (Bucher 1996; Law 1991; Shah 2007), body weight or fatness (Astrup 2000; Hession 2009; SIGN 1996), angiographic measurements (Marchioli 1994), antioxidant intake (Ness 1997), metabolic profile (Kodama 2009) and alcohol intake (Rimm 1996).

A problem with this two-level approach is that any single dietary alteration may have effects over a wide range of risk factors for cardiovascular disease. An example of this is the choice of substitution of saturated fats by carbohydrate, polyunsaturated fats or monounsaturated fats in the diet. This choice may alter lipid profile, and may also affect blood pressure, body weight, oxidative state, rate of cholesterol efflux from fibroblasts, insulin resistance, post-prandial triacylglycerol response, blood clotting factors, and platelet aggregation. There may also be further risk factors of which we are not yet aware. Evidence of beneficial effect on one risk factor does not rule out an opposite effect on another unstudied risk factor, and therefore an overall null (or harmful) effect of intervention. While understanding the effects of dietary advice on intermediate risk factors helps to ensure diets are truly altered by advice, and illuminates mechanisms, the best way of combining the effects on all of these risk factors is to not study risk factors, but to study the effects of dietary change on important outcomes, on cardiovascular morbidity and mortality, and on total mortality.

Substantial randomised controlled trial data on the effects of dietary fat on mortality and morbidity does exist - the first version of this systematic review included over 18,000 participants in trials of at least six months duration, reporting on over 1400 deaths, over 800 cardiovascular deaths, and over 1200 cardiovascular events (Hooper 2000, Hooper 2001). The review found no clear relationship between fat modification (reduction of saturated fats, including studies that replaced the missing calories with carbohydrates - low fat diets - and studies that replaced the missing calories with other fats - modified fat diets) and total or cardiovascular mortality, but did find that such modification reduced the rate of cardiovascular events by around $16 \%$ (rate ratio $0.84,95 \% \mathrm{Cl} 0.72$ to 0.99 compared with usual diet). Since 2000 several important new studies have been published. These include some very large studies that have modified dietary fat intake in women over several years (including the Women's Health Initiative that included over 2000 women with, and over 48,000 women without, cardiovascular disease at baseline for over eight years (WHI with CVD 2006; WHI without CVD 2006) and the Women's Healthy Eating and Living Study including over 3000 women for 11 years (WHEL 2007)) allowing us to add a substantial 
body of new research on low fat diets (many of the large studies included in the first version of the review were of modified fat diets rather than reduced fat diets), as well as information on the effects of these changes in women (as previous large studies were mainly in men). The results of $\mathrm{WHI}$ in particular have raised many questions about both the effects of fat on health and on how we best conduct research to understand the relationship (Michels 2009; Prentice 2007; Stein 2006; Yngve 2006).

Public health dietary advice on prevention of cardiovascular disease has changed a little over time, with a focus on fat modification during the 1960s and fat reduction during the 1990 s. Recent recommendations by the American Heart Association suggest that, among other dietary measures, Americans should "limit intake of saturated fat to $7 \%$ of energy, trans fat to $1 \%$ of energy, and cholesterol to $300 \mathrm{mg} /$ day by choosing lean meats and vegetable alternatives, fat-free (skim) or low-fat (1\% fat) dairy products and minimize intake of partially hydrogenated fats" (Lichtenstein 2006).

How effective are these alterations in dietary fat at reducing cardiovascular morbidity and mortality? Should we replace the energy from saturated and trans fats with carbohydrates, polyunsaturated or monounsaturated fats?

This systematic review aimed to assess the effects of reducing or modifying dietary fat on mortality, cardiovascular mortality and cardiovascular events, as well as on individual cardiovascular outcomes and total cancers, and cardiovascular risk factors. Additionally we used the whole database of studies to use meta-regression to explore effects of changes in total, saturated, polyunsaturated, monounsaturated, trans, weight and study duration on the primary outcomes. Finally we included studies that directly compare reduced with modified dietary fats.

\section{OB JECTIVES}

The aim of this systematic review was to assess the effect of change in dietary fats, which would be expected to result in lipid lowering, on mortality and cardiovascular morbidity, using all available randomised clinical trials. For this update interventions were classified as low fat, modified fat or combined low and modified fat diets and effects of each type of dietary intervention were assessed.

\section{Specific questions included:}

1. Does reducing saturated fat intake, by reducing and/or modifying dietary fat, in the longer term (at least six months) reduce mortality, cardiovascular mortality or cardiovascular morbidity (or individual health events such as myocardial infarction, stroke, diabetes or cancer)?

2. Does a long term reduced fat diet, compared with usual diet, reduce mortality, cardiovascular mortality or cardiovascular morbidity (or individual health events such as myocardial infarction, stroke, diabetes or cancer)?

3. Does a long term reduced fat diet alter classic cardiovascular risk factors (weight, body mass index, systolic or diastolic blood pressure, serum total, LDL or HDL cholesterol and triglyceride)?

4. Does a long term modified fat diet, compared with usual diet, reduce mortality, cardiovascular mortality or cardiovascular morbidity (or individual health events such as myocardial infarction, stroke, diabetes or cancer)?
5. Does a long term modified fat diet alter classic cardiovascular risk factors (weight, body mass index, systolic or diastolic blood pressure, serum total, LDL or HDL cholesterol and triglyceride)?

6. Does reducing and modifying dietary fat (as a combined intervention) in the longer term reduce mortality, cardiovascular mortality or cardiovascular morbidity (or individual health events such as myocardial infarction, stroke, diabetes or cancer)?

7. Does a long term reduced and modified fat diet (as a combined intervention) alter classic cardiovascular risk factors (weight, body mass index, systolic or diastolic blood pressure, serum total, LDL or HDL cholesterol and triglyceride)?

8. Are effects on mortality, cardiovascular mortality or cardiovascular morbidity moderated by differences in baseline cardiovascular risk, mode of intervention, control group total fat intake, control group saturated fat intake, gender, setting or decade of publication, or changes in total fat intake, saturated fat intake, polyunsaturated fat intake, monounsaturated fat intake, trans fat intake, dietary cholesterol, weight, serum LDL cholesterol, or study duration?

9. Is a long term reduced fat diet or a modified fat diet more effective in reducing mortality, cardiovascular mortality or cardiovascular morbidity (or individual effects such as myocardial infarction, stroke or cancer diagnoses)?

10.Is a long term reduced fat diet or a modified fat diet more acceptable to people trying to adhere to these diets?

11.Is a long term reduced fat diet or a modified fat diet more effective in altering classic cardiovascular risk factors (weight, body mass index, systolic or diastolic blood pressure, serum total, LDL or HDL cholesterol and triglyceride)?

\section{METHODS}

\section{Criteria for considering studies for this review Types of studies}

Randomized controlled trials only. Randomization of individuals was accepted, or of larger groups where there were at least six of these groups randomized. Studies where allocation was not truly randomised (e.g. divisions based on days of the week or first letter of the family name were excluded) or where allocation was not stated as randomised (and no further information was available from the authors) were excluded.

\section{Types of participants}

Studies of adults (18 years or older, no upper age limit) at any risk of cardiovascular disease (with or without existing cardiovascular disease) were accepted. Participants could be of either gender, but those who were acutely ill, pregnant or lactating were excluded.

\section{Types of interventions}

All randomised controlled trials of interventions stating an intention to reduce or modify dietary fat or cholesterol, such as would be expected to result in improvement of serum lipid profile, were considered. These included an intention to reduce total fat intake, modify fat intake (while maintaining total fat intake), and reduction and modification of fat intake, all compared to a usual diet type control or modification of dietary fat compared to reduced total fat. The intervention had to be dietary advice, supplementation (of fats, oils or modified or low fat foods) or a 
provided diet, and the control group usual diet, placebo or a control diet.

A low fat diet was considered to be one that aimed to reduce fat intake to $<30 \% \mathrm{E}$ from fat, and at least partially replace the energy lost with carbohydrates (simple or complex), protein or fruit and vegetables. A modified fat diet was considered to be one that aimed to include $30 \%$ or more energy from total fats, and included higher levels of mono-unsaturated or poly-unsaturated fats than a 'usual' diet.

Interventions excluded (unless they were present in addition to those above) were addition of alpha-linolenic acid, omega- 3 fats or fish oils (as the effect of these is dealt with in a separate review), high fibre diets and garlic (as pulses, fruits and vegetables may have various effects other than lipid lowering) or exploration of varying forms of carbohydrate (unless also specifically low in fat or fat modified). Also excluded were all multiple risk factor interventions other than diet or supplementation (unless the effects of diet or supplementation could be separated, as in a factorial design, so the additional intervention was consistent or randomised between the intervention and control groups), and studies that aimed for weight loss in one arm but not the other. Atkins-type diet aiming to increase protein and fat intake were excluded, as were studies where fat was reduced by means of a fat-substitute (like Olestra). Enteral and parenteral feeds were excluded, as were formula weight reducing diets.

Examples: studies that reduced or modified fats and encouraged physical activity in one arm and compared with encouraging physical activity in the control were included; studies that reduced or modified fats and encouraged physical activity in one arm and compared with no intervention in the control were excluded; studies that reduced or modified fats and encouraged fruit and vegetables in one arm and compared with no intervention in the control were included.

\section{Types of outcome measures}

\section{Primary outcomes:}

The main outcomes were total and cardiovascular mortality and combined cardiovascular events. Combined cardiovascular events included any of the following data available from a trial: cardiovascular deaths, cardiovascular morbidity (non-fatal myocardial infarction, angina, stroke, heart failure, peripheral vascular events, atrial fibrillation) and unplanned cardiovascular interventions (coronary artery bypass surgery or angioplasty).

\section{Secondary outcomes:}

Secondary outcomes included individual types of cardiovascular events, including total myocardial infarction, non-fatal myocardial infarction, stroke (fatal and non-fatal), diabetes diagnosis, cancer deaths and cancer diagnoses, and quality of life measures (including informal outcomes such as feelings of health, time off work).

\section{Tertiary outcomes:}

Tertiary outcomes were process outcomes, and included changes in saturated and total fat intakes, and classic cardiovascular risk factors (weight, body mass index, systolic or diastolic blood pressure, serum total, LDL or HDL cholesterol and triglyceride).
Trials were only included where primary outcome data (mortality or cardiovascular morbidity) could be collected (by communication with authors if necessary). Studies where it was known that no events occurred were included, and their data used to assess tertiary outcomes.

\section{Search methods for identification of studies}

\section{Electronic searches}

The initial searches were run in March to June 1998 and included The Cochrane Library, MEDLINE, EMBASE, CAB Abstracts, CVRCT Registry, SIGLE, bibliographies and experts. A comprehensive search strategy was developed to search for nutrition based randomised controlled trials with morbidity or mortality outcomes.

MEDLINE on SilverPlatter was searched for randomised controlled trials on diet and cardiovascular disease or mortality from 1966 to May 1998. An additional MEDLINE (SilverPlatter 1966 to June 1998) search strategy was run to collect papers where only lipid outcomes were mentioned (see Appendix 1 for details of both searches). These search strategies were adapted for use on The Cochrane Library (Issue 2, 1998), EMBASE (Ovid online to May 1998), the CVRCT Registry (May 1998), CAB Abstracts (Ovid online, 1973 to March 1998) and SIGLE (to January 1999). Published systematic reviews addressing diet and heart health were sought as a source of RCTs using similar strategies on MEDLINE (Silver Platter, 1966March 1998) and The Cochrane Library (Issue 1, 1998).

Cochrane Review Groups in areas related to this review include the Diabetes Group (now the Endocrine and Metabolic Disorders Group), Stroke Group, Renal Group, Hypertension Group and Peripheral Vascular Disease Group. The groups were contacted and asked to search their trial registers for relevant trials.

The review authors updated the searches in June 2010, and modified searches were run on Cochrane Central Register of Controlled Trials (CENTRAL on The Cochrane Library), Ovid MEDLINE and EMBASE. Modification was due to altered database accessibility as well as altered recommended RCT filters. The searches for the earlier review were run as a wider search for studies for several reviews (including a variety of dietary factors), the recent searches were focused on dietary fat interventions only. As databases other than these three had not provided any included studies during the first version of the review, and the numbers of titles and abstracts were not feasible to handle for the update, these were the only databases used for this update. The search strategies used for the update search are shown in Appendix 2.

No language restrictions were applied to the searches.

\section{Searching other resources}

Bibliographies of all identified systematic reviews, major nonsystematic reviews and included trials were searched for further trials for the first review and the update. Experts in the field were contacted for references to studies not yet identified by the search process. Attempts were made to obtain translations of relevant non-English articles, or contact with the author was established to enable assessment of eligibility. 


\section{Data collection and analysis}

\section{Selection of studies}

Articles were only rejected on initial screen if the reviewer could determine from the title and abstract that the article was not a report of a randomized controlled trial; the trial did not address a low or modified fat diet; the trial was exclusively in children less than 18 years old, pregnant women or the critically ill; the trial was of less than 6 months duration; or the intervention was multifactorial. When a title/abstract could not be rejected with certainty, the full text of the article was obtained for further evaluation.

The inclusion of studies was assessed independently by two assessors (LH and one of RLT, DS, FR, HM, Indra Tumur and Dorotheé Fagard) and differences between reviewers' results resolved by discussion and, when necessary, in consultation with a third reviewer (Rudolph Reimersma, see acknowledgements). Trials were categorised as "possible" (where all inclusion criteria appeared to be met or where the ascertainment, or otherwise, of outcome events was uncertain, to be resolved by writing to the author) or "excluded". Attempts were made to contact all authors of "possible" trials in order to confirm or ascertain whether inclusion criteria were met.

\section{Data extraction and management}

A data extraction form was designed for this review. Data concerning participants, interventions and outcomes, trial quality characteristics (Chalmers 1990), data on potential effect modifiers including participants baseline risk of cardiovascular disease, trial duration, intensity of intervention (dietary advice, diet provided, dietary advice plus supplementation, supplementation alone), medications used (particularly lipid lowering medication) and smoking status, numbers of events and total patient years in trial were extracted. Where provided, data on risk factors for cardiovascular disease including blood pressure, lipids and weight were collected.

Baseline risk of cardiovascular disease was defined as follows: high risk are participants with existing vascular disease including a history of myocardial infarction, stroke, peripheral vascular disease, angina, heart failure or previous coronary artery bypass grafting or angioplasty; moderate risk are participants with a familial risk, dyslipidaemia, diabetes mellitus, hypertension, chronic renal failure; low risk are other participants or mixed population groups.

Original reports of trial results were independently extracted by two reviewers (LH and one of CDS, RLT, DS, FR, HM, Indra Tumur, Dorotheé Fagard, Rudolph Reimersma), differences were resolved by discussion.

\section{Assessment of risk of bias in included studies}

Trial quality characteristics were assessed using the Cochane Collaboration's tool for assessment of risk of bias; studies already included were re-assessed using this tool (Higgins 2011). All validity data were extracted by two reviewers independently ( $\mathrm{LH}$ and one of CDS, RLT, DS, FR, HM, Indra Tumur, Dorotheé Fagard, Rudolph Reimersma), and differences resolved by discussion.

\section{Measures of treatment effect}

Primary measures of interest were the effect of intervention on:
1. total and cardiovascular mortality

2. combined cardiovascular events (including cardiovascular deaths, non-fatal myocardial infarction, stroke, angina, heart failure, peripheral vascular disease, angioplasty and coronary artery bypass grafting)

3. quality of life measures.

\section{Unit of analysis issues}

We did not include any cluster randomised trials in this review, and cross-over studies (such as the Finnish Mental Hospital study, Finnish Mental Hosp 1972) were excluded as this design would be inappropriate for assessing effects on cardiovascular events or mortality.

Where there was more than one relevant intervention arm but only one control arm the relevant intervention arms were either pooled to create a single pair-wise comparison (where the intervention arms were equivalently appropriate for this review) as described in the Cochrane Handbook (Higgins 2011). Intervention arms that were not appropriate for this review, or less appropriate than another arm, were excluded. When two arms were appropriate for different subgroups then the control group was used once with each intervention arm, but the subgroups were not pooled overall.

When assessing event data we aimed to avoid counting more than one outcome event for any one individual within any one comparison. Where we were unclear (for example, where a paper reported numbers of heart attacks, but did not report the number of people who experienced a heart attack, in each arm) we asked authors for further information.

\section{Dealing with missing data}

As the outcomes of our review were often not the planned outcomes of relevant trials (many studies with relevant methods, participants, intervention, control and duration had other primary and secondary outcomes, so mortality and morbidity were reported in many studies only as reasons for dropout, or not reported at all), we tried to contact the authors of all identified studies that were appropriate for inclusion on the basis of participants, intervention, comparison and methodology, to ask about mortality and morbidity, the review's primary and secondary outcomes. This allowed inclusion of many studies that would otherwise have had to be omitted. Studies which were otherwise relevant but where presence or absence of primary outcomes could not be established were retained in the section of studies awaiting classification.

It was often unclear where data on primary or secondary outcome events may still have been missing, and so data were not imputed for this review.

\section{Assessment of heterogeneity}

Heterogeneity was examined using the 12 test, and considered important where > 50\% (Higgins 2003; Higgins 2011).

\section{Assessment of reporting biases}

Funnel plots were drawn to examine the possibility of small study bias, including publication bias (Egger 1997), for the primary outcomes total mortality and combined cardiovascular events. 


\section{Data synthesis}

The data within the original review were in the form of rates. Treatment effect was measured as a rate ratio and meta-analysis performed as a weighted average of (In) rate ratios (as described by Hasselblad 1995). For trials with a zero in one arm of the data a small number (0.5) was added to the number of events in both groups. Meta-analysis was performed using random effects methodology (DerSimonian 1986) within S-PLUS (Higgins 1999). For the update of the review we checked that using event data gave similar results to rate data as described above - using the same studies the outcomes of the meta-analysis using MantelHaenszel random effects methods were almost identical (presence or absence of statistical significance was never different, and scale of effect size was always similar). For this reason, within the update, numbers of events in each study arm, and total number of participants randomised, were extracted, and Mantel-Haenszel random effects meta-analysis carried out in Review Manager software. Event and continuous outcome data were extracted for the latest time point available within the trial (and always at least six months from inception).

Trials where it was known that there were no events in either intervention group were included in the review for completeness, but were not included in the meta-analysis (where it was stated that no events of particular type occurred this was detailed in Characteristics of included studies). These studies inclusion or otherwise would not influence the results of the review. Where trials ran one control group and more than one included intervention group, data from the intervention group providing the comparison that best assessed the effect of altering dietary fat was used. Where the intervention groups appeared equal in this respect the intervention groups were merged (simply added for dichotomous data, and using the techniques described in the Higgins 2011 for continuous data). It was planned that if trials randomized by cluster were identified the patient numbers would be reduced to an "effective sample size" (as described by Hauck 1991), however none were identified that were both included and had cardiovascular events or deaths.

\section{Subgroup analysis and investigation of heterogeneity}

For this update dietary interventions were classified as low fat, modified fat or combined low and modified fat diets. Pre-specified analyses included:

Effects of low, modified, and combined low and modified fat diets compared with usual or standard diet on the following outcomes:

- total mortality

- cardiovascular mortality

- combined cardiovascular events

Pre-specified subgroups for primary outcomes included:

- mean follow-up time of up to and including, or over, 2 years

- initial level of cardiovascular risk (low, medium, high)

- mode of intervention (advice, supplementation or provision of diet).

Further subgroups, added into the updating of the systematic review, included:

- control group total fat intake,
- control group saturated fat intake,

- year of first publication of results

- gender, and

- setting (workplace, community, outpatient), at the request of the Cochrane Occupational Health Field.

We explored the effects of different levels of dietary fats achieved in trials (all difference between the intervention and control groups, as a percentage of energy) using meta-regression on total mortality, cardiovascular mortality and total cardiovascular events by:

- total fat intake

- saturated fat intake

- monounsaturated fat intake

- polyunsaturated fat intake

- trans fat intake

- body weight

- LDL cholesterol

The effects of low fat, modified fat and low and modified fat diets (all compared with control or usual diet), and low fat vs modified fat diets on secondary and tertiary outcomes were assessed.

Random effects meta-regression (Berkley 1995) was performed using the STATA command metareg (Sharp 1998, Sterne, Bradburn and Egger 2001, Sterne 2009).

\section{Sensitivity analysis}

Sensitivity analyses were carried out for primary outcomes, subgrouping by type of dietary fat intervention (reduced fat, modified fat or both) assessing the effect of

- running Mantel-Haenszel fixed effects relative risk metaanalyses (rather than random effects), as events could be considered to be rare (percentages of participants experiencing events was $6 \%$ for mortality, $2 \%$ for cardiovascular mortality and $7 \%$ for cardiovascular events) (Higgins 2011),

- running Peto fixed effects odds ratio meta-analysis, which may be more useful than Mantel-Haenszel when events are rare but works better when studies have similar numbers of participants in each arm (not the case with several included studies such as WHI)

- excluding the largest study (WHI with CVD 2006, WHI without CVD 2006),

- excluding studies which were not free of systematic difference in care (or unclear)

- excluding studies that were not free of dietary differences other than fat (or unclear).

\section{RE S U L T S}

\section{Description of studies}

\section{Results of the search}

The initial search strategy resulted in 16,821 potential titles and abstracts, which were scanned for relevant studies. The 2010 update search found an additional 5,191 titles and abstracts to assess for inclusion, making 22,012 titles and abstracts screened in total. Two hundred and seventy six papers were collected as full text in the first review, and a further 254 were collected in the review 
update, and all 530 were assessed for inclusion in duplicate. The papers were then amalgamated into studies. Of these a total of 48 randomised controlled trials were included in the review. For all included studies we searched for additional publications to ensure that we did not miss any relevant methodology or outcome data from that dataset. This full set of published papers (along with any additional information provided by authors) was data extracted (along with assessment of validity) in duplicate for each included trial. A further 15 studies (17 papers) were allocated to Studies awaiting classification as it could not be established whether they had collected data on mortality or morbidity, the outcomes of this review (Characteristics of studies awaiting classification).

\section{Included studies}

Forty eight studies were included in the review (Included studies) and were described in Characteristics of included studies. Some included studies included several comparison arms (e.g. the Kuopio study includes four comparisons: Sarkkinen Fat Mod 1995; Sarkkinen Red \& Mod 1995; Sarkkinen Red Fat 1995; Sarkkinen Red vs Mod1995 and the Monounsaturated fat Obesity study includes three comparisons: Due Low fat 2008; Due Mod fat 2008; Due Low vs Mod 2008); and some studies were reported in several sections for ease of analysing subgroup data (e.g. the Minnesota Coronary Study was reported separately for men (Minnesota Coron men 1989) and women (Minnesota Coron women1989); the National Diet-Heart Study was reported in its various sub-studies (NDHS Faribault 1968; NDHS Open 1st L\&M 1968; NDHS Open 1st mod 1968, NDHS Open 2nd L\&M 1968, NDHS Open 2nd Mod 1968); and the Women's Health Initiative was reported by cardiovascular risk (WHI with CVD 2006, WHI without CVD 2006). In total there were 60 comparisons included in the review.

The main study papers ranged in publication date from 1965 to 2009, and the comparisons were conducted in North America (30), Europe (26), Australia/ New Zealand (3) and the Middle East (1). Ten of the comparisons included only people at high risk of cardiovascular disease, 17 at moderate risk, and 33 at low risk. Sixteen comparisons included only men, 14 only women, and 30 both men and women.

Dietary interventions varied from trials which provided dietary advice (in varying degrees of intensity and duration, 35 studies), provided advice plus some dietary supplementation (such as oils or margarines, nine studies), to studies that provided most food eaten by participants (via institutional provision, meals provided for those living independently or study shops, 16 studies). The setting for most studies was the community (in that participants were living in the community - the actual setting for provision of advice, group work etc was usually unclear, although interventional advice was occasionally clearly in a home or community setting, and occasionally in a primary or secondary healthcare setting), but three studies took place in institutions (Minnesota Coron men 1989; Minnesota Coron women1989; NDHS Faribault 1968; Veterans Admin 1969), and no studies appeared to have been carried out in occupational settings.

Twenty five comparisons, including 61,958 participants and first published between 1965 and 2007, compared a reduced fat diet with usual or control diet (Ley 2004; BDIT Pilot Studies 1996; BRIDGES 2001; CARMEN 2000; CARMEN MS sub-study 2002; DO IT 2006; Seppelt 1996; Lean 1997; Anderson 1990; Sarkkinen Red Fat 1995; Ball 1965; Boyd 1988; Moy 2001; MSFAT 1997; Due Low fat
2008; Nutrition \& Breast Health; Ole Study 2002; Polyp Prevention 1996; Simon 1997; McKeown-Eyssen 1994; Black 1994; WHEL 2007; WHI with CVD 2006; WHI without CVD 2006; WINS 2006).

Fifteen comparisons, including 13,004 participants and published between 1965 and 2007, compared a modified fat diet with control or usual diet (Frenkiel 1986, Houtsmuller 1979, Sarkkinen Fat Mod 1995, Dullaart 1992, Rose 1965, Minnesota Coron men 1989, Minnesota Coron women1989, MRC 1968, Due Mod fat 2008, NDHS Faribault 1968, NDHS Open 1st mod 1968, NDHS Open 2nd Mod 1968, Oslo Diet-Heart 1966, Sydney Diet-Heart 1978, Veterans Admin 1969). Ten interventions, including 4,931 participants and published between 1968 and 2006, compared a reduced and modified fat diet with usual or control diet (beFIT 1997; DART 1989; Sarkkinen Red \& Mod 1995; MeDiet 2002; NDHS Open 1st L\&M 1968; NDHS Open 2nd L\&M 1968; Oxford Retinopathy 1978; PREMIER 2003; Sondergaard 2003; STARS 1992). Nine interventions, including 1,290 participants and published between 1994 and 2009, compared a low fat diet with a modified fat diet (Sarkkinen Red vs Mod1995; Due Low vs Mod 2008; McAuley 2005; Rivellese 1994; Azadbakht 2007; THIS DIET 2008; Sacks high protein 2009; Sacks low protein 2009; Strychar 2009). One intervention (144 participants) was not classifiable (Curzio 1989).

Of these comparisons 12 stated that an intended outcome was to assess mortality or cardiovascular morbidity of some sort (DART 1989; DO IT 2006; Rose 1965; Ball 1965; Minnesota Coron men 1989; Minnesota Coron women1989; MRC 1968; Oslo DietHeart 1966; Sydney Diet-Heart 1978; THIS DIET 2008; Veterans Admin 1969; WHEL 2007). A further 36 intended to monitor lipids, blood pressure, weight or other cardiovascular risk factor outcomes, seven aimed to assess effects on cancers or cancer related outcomes such as polyps, two the feasibility of dietary intake, and the remaining three studies aimed to assess bile acid kinetics or diabetic retinopathy. Of the 48 included studies, nine recruited participants with cardiovascular disease (were secondary prevention studies), 12 recruited those at increased risk of CVD (including those recruited on the basis of raised lipids, blood pressure or weight), and 25 recruited people from the general population or without specific CVD risk (primary prevention). A further two studies recruited a mix of participants (at high and lower CVD risk).

Of the 51 comparisons of a modified and/or low fat diet with a control or usual diet, four measured the total fat intake in the control arm as less than $30 \%$ of energy (30\%E), $1830 \%-34.9 \% \mathrm{E}$, $1935 \%-39.9 \% \mathrm{E}$, six had a control arm with at least $40 \% \mathrm{E}$, and it was unclear what the total fat intake in the control arm was in the remaining four comparisons. Three of the included comparisons had a saturated fat intake in the control group of less than $10 \%$ of energy (10\%E), $1910 \%-14.9 \% \mathrm{E}, 1415 \%-19.9 \% \mathrm{E}$ and one comparison $20+\% \mathrm{E}$, while saturated fat intake in the control group was unclear in a further 14 comparisons.

Of the 60 intervention arms only 21 provided data on mortality (including 71,790 participants and 4292 deaths), 16 on cardiovascular mortality $(65,978$ participants and 1407 cardiovascular deaths), and 23 on combined cardiovascular events (65,508 participants and 4887 events). In 25 of the included arms none of the participants experienced any deaths or cardiovascular events, and in three further studies it was clear that events had occurred, but it was not clear in which arm(s) the events had 
occurred (BDIT Pilot Studies 1996; Oxford Retinopathy 1978; Simon 1997), so that data could not be included in the meta-analyses.

\section{Excluded studies}

Two hundred and eighty eight trials have been excluded (Excluded studies, the full texts assessed in duplicate for inclusion), and the reasons for these exclusions were described in Characteristics of excluded studies.
One more trial was ongoing (Ongoing studies) and described in Characteristics of ongoing studies.

\section{Risk of bias in included studies}

To understand the risk of bias in the individual included studies in a visual way, see Figure 1, and the summary of studies included in the review, see Figure 2 . 
Figure 1. Methodological quality summary: review authors' judgements about each methodological quality item for each included study.

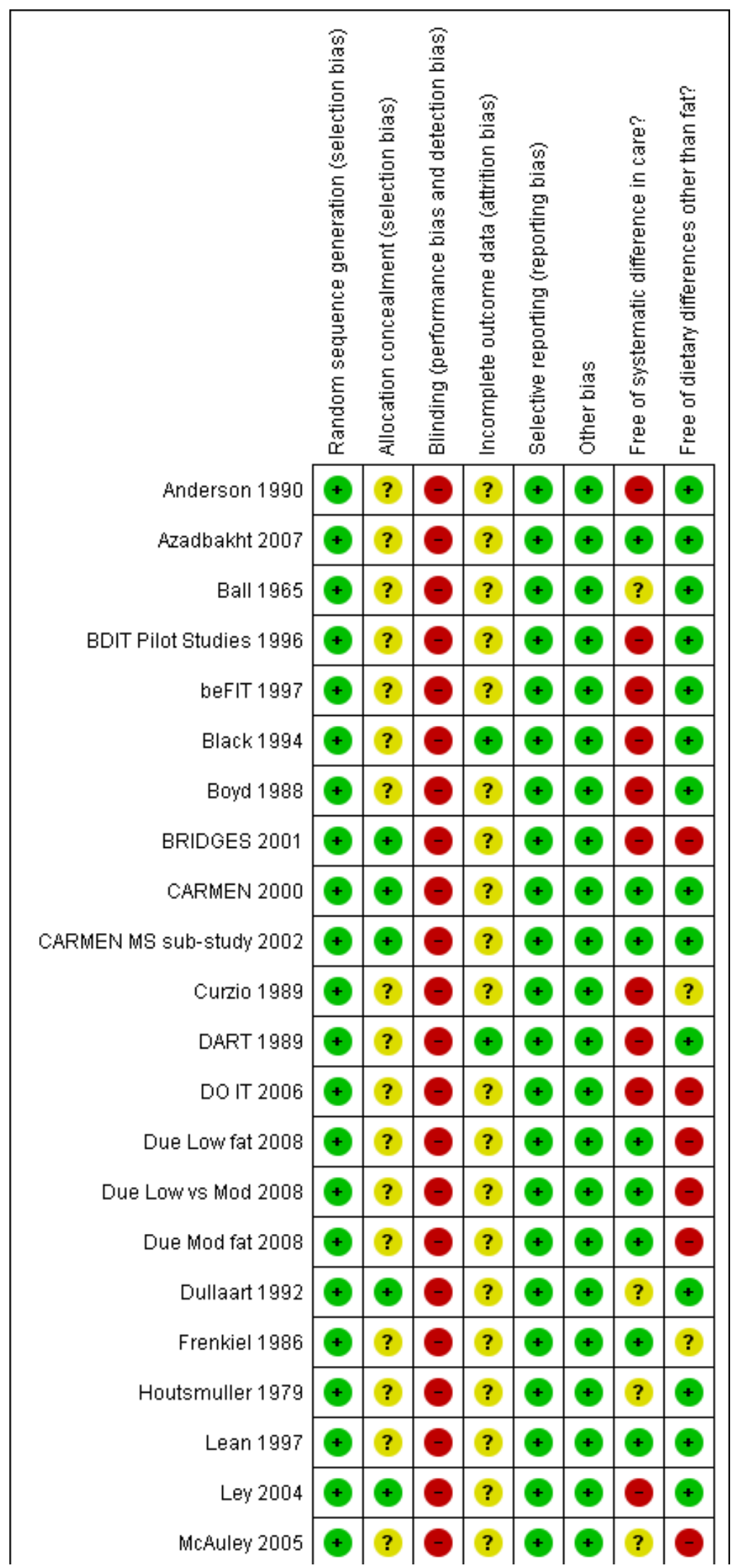


Figure 1. (Continued)

\begin{tabular}{|c|c|c|c|c|c|c|c|c|}
\hline McAuley 2005 & + & $?$ & - & $?$ & $\odot$ & $\odot$ & $?$ & $\odot$ \\
\hline McKeown-Eyssen 1994 & $\odot$ & $\odot$ & - & $?$ & $\odot$ & $\odot$ & $\odot$ & $\Theta$ \\
\hline MeDiet 2002 & $\odot$ & $?$ & $\odot$ & $?$ & $\odot$ & $\odot$ & $\odot$ & $\Theta$ \\
\hline Minnesota Coron men 1989 & $\odot$ & $?$ & $\odot$ & $\odot$ & $\odot$ & $\odot$ & $\odot$ & + \\
\hline Minnesota Coron women 1989 & $\odot$ & $?$ & $\odot$ & $\odot$ & $\odot$ & $\odot$ & $\odot$ & $\odot$ \\
\hline Moy 2001 & $\odot$ & $?$ & $\odot$ & $?$ & $\odot$ & $\odot$ & $\odot$ & $?$ \\
\hline MRC 1968 & $?$ & $?$ & $\Theta$ & $\odot$ & $\odot$ & $\odot$ & $\odot$ & + \\
\hline MSFAT 1997 & $\odot$ & $\odot$ & $\Theta$ & $?$ & $\odot$ & $\odot$ & $\odot$ & $\odot$ \\
\hline NDHS Faribault 1968 & $\odot$ & $\odot$ & $\odot$ & $\odot$ & $\odot$ & $\odot$ & $\odot$ & $\odot$ \\
\hline NDHS Open 1st L\&M 1968 & $\odot$ & $\odot$ & + & $\Theta$ & $\odot$ & $\odot$ & $\odot$ & $\odot$ \\
\hline NDHS Open 1st $\bmod 1968$ & $\odot$ & $\odot$ & $\odot$ & $\odot$ & $\odot$ & $\odot$ & $\odot$ & + \\
\hline NDHS Open 2nd L\&M 1968 & $\odot$ & + & $\odot$ & $\Theta$ & $\odot$ & $\odot$ & $\odot$ & + \\
\hline NDHS Open 2nd Mod 1968 & + & + & + & $\odot$ & + & $\odot$ & $\odot$ & + \\
\hline Nutrition \& Breast Health & $\odot$ & + & $\odot$ & $?$ & $\odot$ & $\odot$ & $\odot$ & + \\
\hline Ole Study 2002 & $\odot$ & $\odot$ & $\odot$ & $?$ & $\odot$ & $\odot$ & + & 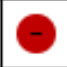 \\
\hline Oslo Diet-Heart 1966 & + & + & $\odot$ & $\odot$ & $\odot$ & $\odot$ & $\odot$ & $\odot$ \\
\hline Oxford Retinopathy 1978 & $\odot$ & + & $\Theta$ & $?$ & $\odot$ & $\odot$ & $\odot$ & $\odot$ \\
\hline Polyp Prevention 1996 & + & + & $\odot$ & $?$ & $\odot$ & $\odot$ & $\odot$ & $\theta$ \\
\hline PREMIER 2003 & $\odot$ & + & $\odot$ & $\odot$ & $\odot$ & $\odot$ & $\odot$ & $\theta$ \\
\hline Rivellese 1994 & $\odot$ & $?$ & $\odot$ & $?$ & $\odot$ & $\odot$ & $\odot$ & 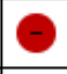 \\
\hline Rose 1965 & $\odot$ & $?$ & $\odot$ & $\odot$ & $\odot$ & $\odot$ & $\odot$ & + \\
\hline Sacks high protein 2009 & + & $\odot$ & $\odot$ & $?$ & $\odot$ & $\odot$ & $\odot$ & + \\
\hline Sacks low protein 2009 & + & + & + & $?$ & $\odot$ & $\odot$ & + & $\odot$ \\
\hline Sarkkinen Fat Mod 1995 & $\odot$ & $?$ & $\odot$ & $?$ & $\odot$ & $\odot$ & $\odot$ & $\odot$ \\
\hline Sarkkinen Red \& Mod 1995 & + & $?$ & - & $?$ & $\odot$ & $\odot$ & $\odot$ & + \\
\hline Sarkkinen Red Fat 1995 & + & $?$ & $\odot$ & $?$ & $\odot$ & $\odot$ & + & + \\
\hline Sarkkinen Red vs Mod1995 & $\odot$ & $?$ & $\Theta$ & $?$ & $\odot$ & $\odot$ & $\odot$ & + \\
\hline Seppelt 1996 & $\odot$ & $?$ & $?$ & $?$ & $\odot$ & $\odot$ & $\odot$ & + \\
\hline Simon 1997 & + & $?$ & $\odot$ & $?$ & + & $\odot$ & $\odot$ & + \\
\hline Sondergaard 2003 & $\odot$ & $\odot$ & $\odot$ & $?$ & $\odot$ & $\odot$ & $\odot$ & $\odot$ \\
\hline STARS 1992 & $\odot$ & $\odot$ & $\odot$ & $?$ & $\odot$ & $\odot$ & & \\
\hline
\end{tabular}


Figure 1. (Continued)

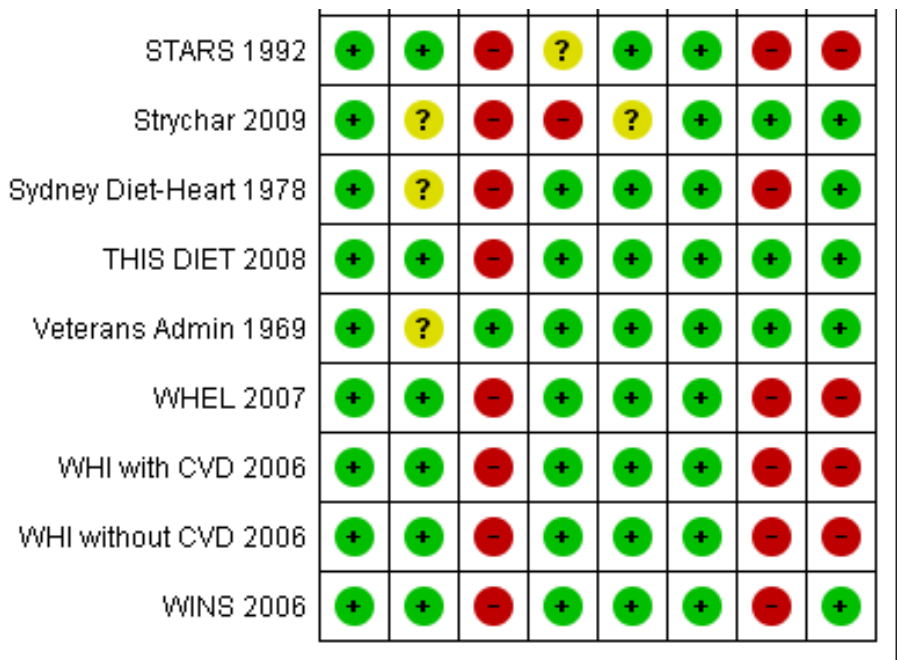

Figure 2. Methodological quality graph: review authors' judgements about each methodological quality item presented as percentages across all included studies.

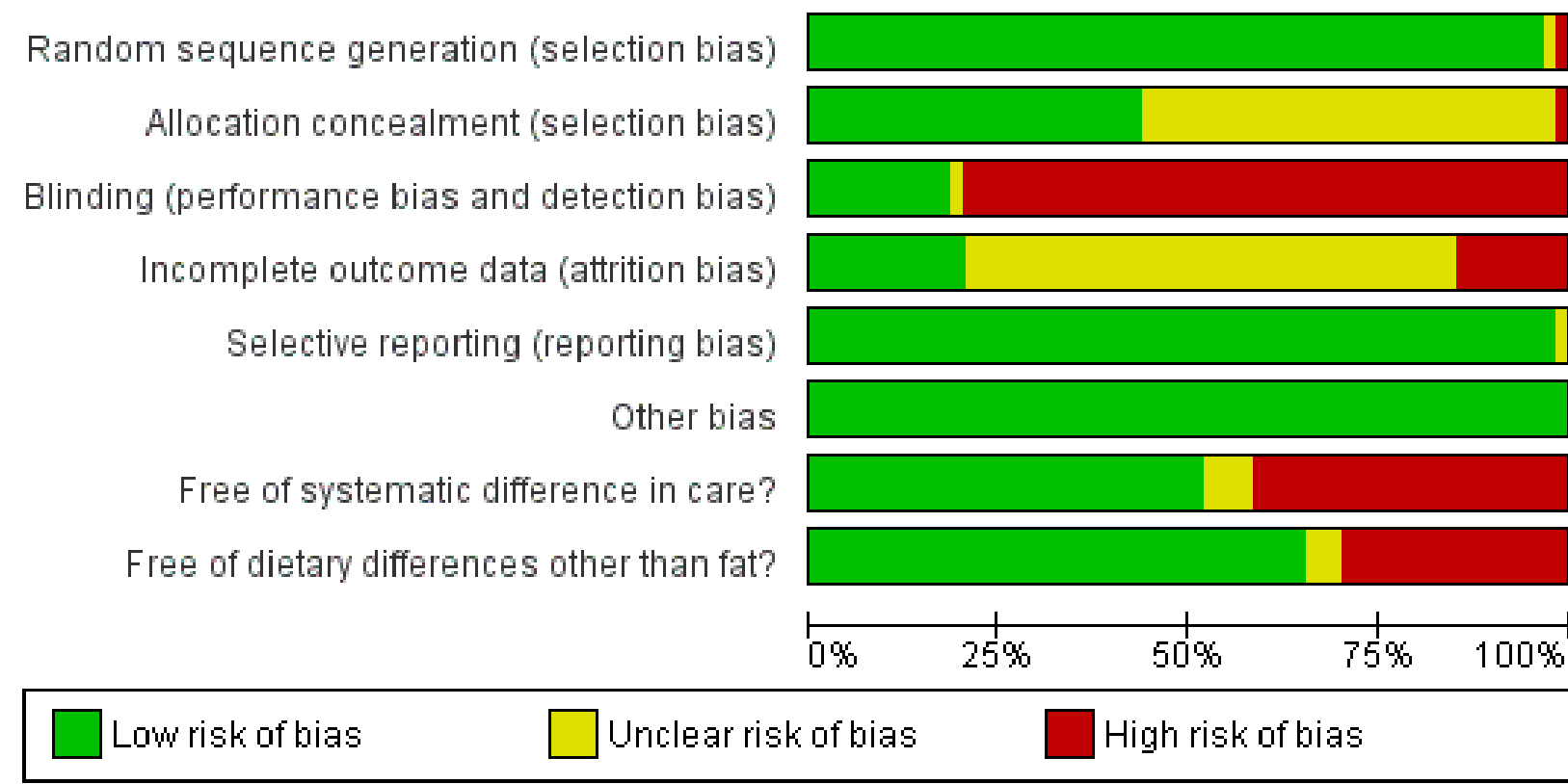

\section{Allocation}

All trials included were randomised controlled trials, those with detected pseudo random allocation (for example where participants are randomised according to birth date or alphabetically from their name) were excluded. Allocation concealment was judged well done in 26 comparisons (Ley 2004; BRIDGES 2001; CARMEN 2000; CARMEN MS sub-study 2002; Dullaart 1992; MSFAT 1997; NDHS Faribault 1968; NDHS Open 1st L\&M 1968; NDHS Open 1st mod 1968; NDHS Open 2nd L\&M 1968; NDHS Open 2nd Mod 1968; Nutrition \& Breast Health; Ole Study 2002; Oslo Diet-Heart 1966; Oxford Retinopathy 1978; Polyp Prevention 1996; PREMIER 2003; Sacks low protein 2009; Sacks high protein 2009;
STARS 1992; THIS DIET 2008; McKeown-Eyssen 1994; WHEL 2007; WHI with CVD 2006; WHI without CVD 2006; WINS 2006), not done in one (Sondergaard 2003) and unclear in the remainder.

\section{Blinding}

Blinding of participants is not easy in dietary studies, as the participants usually have to follow instructions to attain the specific dietary goals. However, it is feasible in some circumstances, including when food is provided via an institutional setting, or meals provided at a central setting and remaining meals packed to take away, through use of a trial shop, where very specific foodbased dietary advice is provided for all participants, or where the 
same dietary advice is provided to both groups but a different supplement (e.g. dietary advice to reduce fats, then provision of different oils or fats) is provided. Where participants are not blinded it is difficult to ensure that study staff, health care providers and outcome assessors are blinded. The 11 comparisons that appear to have had adequate participant and study personnel blinding were Minnesota Coron men 1989, Minnesota Coron women1989, NDHS Faribault 1968, NDHS Open 1st L\&M 1968, NDHS Open 1st mod 1968, half the participants in NDHS Open 2nd L\&M 1968, half the participants in NDHS Open 2nd Mod 1968, Sacks low protein 2009, Sacks high protein 2009, the Ole Study 2002 and Veterans Admin 1969), and blinding was inadequate or unclear in the remaining studies.

\section{Incomplete outcome data}

Assessing whether incomplete outcome data have been addressed was difficult as the primary outcomes for this review were often seen as dropouts and exclusions from the original studies. When mortality and/or cardiovascular events were noted in any one study it is still feasible that some participants left that study feeling unwell or because the diet was inconvenient (so were simply lost to follow up from the perspective of the study) and later died or experienced a cardiovascular event. However, in some cases studies checked medical records or death registers to ensure that such events were all collected (these 10 studies included DART 1989, NDHS Faribault 1968, Oslo Diet-Heart 1966, PREMIER 2003, Sydney Diet-Heart 1978, THIS DIET 2008, Veterans Admin 1969, Black 1994, WHEL 2007, WINS 2006). In the other studies it is not possible to know whether additional deaths or cardiovascular events occurred, that were not counted or ascertained within this review.

\section{Selective reporting}

Assessment of selective reporting is difficult when the outcome of interest is simply a cause of dropouts in most included studies. We tried to contact all of the trialists to ask about deaths and outcome events, but it is possible that some trialists did not reply as they felt that their data did not reflect the expected or hoped for pattern of events. All of the included studies have either reported that the participants did not experience any of our primary outcomes, published their outcome data or have provided the data they did possess. For this reason all the included studies have been graded as 'Free of selective reporting'.

\section{Other potential sources of bias}

The studies were assessed for risk of bias in relation to 'systematic difference in care' and 'dietary differences other than fat'. The 29 comparisons free of systematic differences in care between the study arms included CARMEN 2000, CARMEN MS sub-study 2002, Frenkiel 1986, Seppelt 1996, Lean 1997, Sarkkinen Fat Mod 1995, Sarkkinen Red Fat 1995, Sarkkinen Red \& Mod 1995, Sarkkinen Red vs Mod1995 Rose 1965, Minnesota Coron men 1989, Minnesota Coron women1989, MSFAT 1997, Due Low fat 2008, NDHS Faribault 1968, NDHS Open 1st L\&M 1968, NDHS Open 1st mod 1968, NDHS Open 2nd L\&M 1968, NDHS Open 2nd Mod 1968, Ole Study 2002, Oxford Retinopathy 1978, PREMIER 2003, Rivellese 1994, Sacks low protein 2009, Sacks high protein 2009, Strychar 2009, Azadbakht
2007, THIS DIET 2008, Veterans Admin 1969), while 27 comparisons clearly did have differences in care (such as differential time provided for those on the intervention to learn a new diet, and/or differential medical follow-up), and four were unclear.

Some comparisons were partially confounded by dietary changes other than those directly related to dietary fat intakes (for example, some studies encouraged intervention participants to make changes to their fat intake as well as changes to fruit and vegetable or fibre or salt intakes). The 39 comparisons that appeared free of such differences included Ley 2004, BDIT Pilot Studies 1996, beFIT 1997, CARMEN 2000, CARMEN MS sub-study 2002, DART 1989, Houtsmuller 1979, Seppelt 1996, Lean 1997, Anderson 1990, Sarkkinen Fat Mod 1995, Sarkkinen Red Fat 1995, Sarkkinen Red \& Mod 1995, Sarkkinen Red vs Mod1995, Dullaart 1992, Rose 1965, Ball 1965, Boyd 1988, Minnesota Coron men 1989, Minnesota Coron women1989, MRC 1968, MSFAT 1997, NDHS Faribault 1968, NDHS Open 1st L\&M 1968, NDHS Open 1st mod 1968, NDHS Open 2nd L\&M 1968, NDHS Open 2nd Mod 1968, Nutrition \& Breast Health, Oxford Retinopathy 1978, Sacks low protein 2009, Sacks high protein 2009, Simon 1997, Strychar 2009, Sydney DietHeart 1978, Azadbakht 2007, THIS DIET 2008, Veterans Admin 1969, Black 1994, WINS 2006. A further three comparisons were unclear, and the remainder, 18 comparisons, were confounded as to the effects of changes in fat.

\section{Effects of interventions}

\section{Reduced, modified or reduced and modified dietary fat vs. usual or control diet}

\section{Primary outcomes}

\section{Total mortality}

There was no clear effect of any dietary fat intervention compared to usual or control diet on mortality (RR $0.98,95 \% \mathrm{Cl} 0.93$ to 1.04 , $1^{2} 0 \%, 71,790$ participants, 4292 deaths, $p_{\text {effect }} 0.53$, Analysis 1.1 ). Similarly there was no effect of modified fat vs usual diet (RR 1.02, $95 \% \mathrm{Cl} 0.88$ to 1.18 , I2 34\%, 11,441 participants, 1120 deaths, $p_{\text {effect }}$ 0.81 ), reduced fat vs usual diet (RR $0.97,95 \% \mathrm{Cl} 0.90$ to $1.04, \mathrm{I}^{2}$ $0 \%, 58,130$ participants, 2936 deaths, $p_{\text {effect }} 0.42$ ), or reduced and modified fat vs usual diet (RR $0.97,95 \% \mathrm{Cl} 0.76$ to $1.23,120 \%, 2219$ participants, 236 deaths, peffect $^{0.78) \text {. }}$

Sensitivity analyses (using Mantel-Haenszel fixed effects relative risk meta-analysis, Peto odds ratio (fixed effects), removing the largest single study (WHI), removing studies with a systematic difference in care between the intervention and control arms, and removing studies with dietary differences between arms other than in dietary fat intake) did not alter the lack of statistically significant effect overall or for modified fat intake, reduced fat intake or reduced and modified fat intake compared with usual or control diet (Table 1). The funnel plot does not suggest severe small study bias, although there may be some smaller studies missing suggesting increased total mortality in the intervention group (so addition of any such studies would further lessen the likelihood of a protective effect of dietary fat modification or reduction on mortality). The funnel plot is shown in Figure 3. 
Figure 3. Funnel plot of comparison: fat modification or reduction vs usual diet - total mortality.

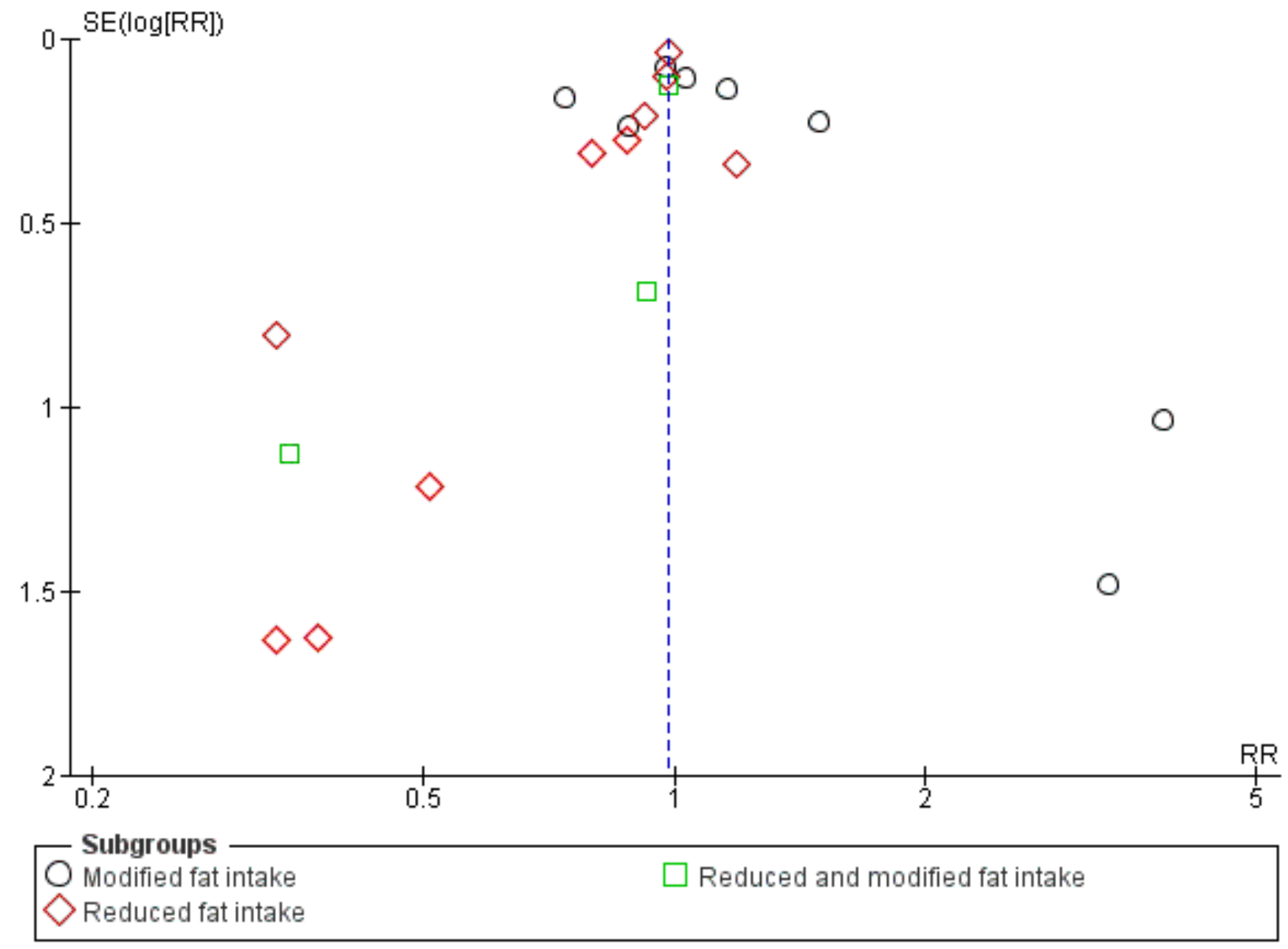

No important effects of reduced and/or modified fat diets compared to usual or control diets on mortality were seen when studies were subgrouped by duration (mean duration up to two years or over two years), cardiovascular risk (low, moderate or high cardiovascular risk), mode of intervention (dietary advice, advice plus supplementation or diet provided), total fat in the control group (less than $30 \%$ of energy from fat, 30\% - 34.9\%E, 35\% $39.9 \% \mathrm{E}$ or $40 \% \mathrm{E}$ and over from fat), saturated fat in the control group (less than $10 \%$ of energy from saturated fat, $10 \%-14.9 \% \mathrm{E}$ or $15 \%-19.9 \% \mathrm{E}$ from saturated fat), by gender (studies of men, of women and of men and women combined), by setting (community or residential institution, no studies of workplaces were identified) or by year of first publication of results (Table 2).

We explored the effects of dietary fats on total mortality, by using meta-regression of the difference between the control and intervention of duration, total fat intake, saturated fat intake, monounsaturated fat intake, polyunsaturated fat intake, trans fat intake (all by percentage of energy), weight (in $\mathrm{kg}$ ) and serum $\mathrm{LDL}$ cholesterol (in $\mathrm{mmol} / \mathrm{L}$ ) achieved in trials. The results of all meta-regressions are shown in Table 3. As only two trials reported trans fat intakes achieved by study arm meta-regression on trans fats could not be carried out, and with only six of the studies reporting serum LDL cholesterol levels achieved the power of this analysis to suggest a result was limited. Because so few studies reported LDL cholesterol, we also ran a post-hoc meta-regression by total serum cholesterol (as a surrogate for LDL cholesterol) achieved in each arm. We did not observe any clear relationships between treatment-control group differences in dietary or serum characteristics and mortality, but power for this analysis was limited.

\section{Cardiovascular mortality}

There was no clear effect of any dietary fat intervention compared to usual diet on cardiovascular mortality (RR $0.94,95 \% \mathrm{Cl} 0.85$ to 1.04, 12 0\%, 65,978 participants, 1407 cardiovascular deaths, peffect $_{\text {end }}$ 0.23 ), Analysis 1.2. Again, there was no effect within any dietary fat subgroup: modified fat diet vs usual diet RR 0.92 (95\% Cl 0.73 to $1.15,\left.\right|^{2} 45 \%, 10,788$ participants, 593 cardiovascular deaths, $p_{\text {effect }}$ $0.46)$; reduced fat vs usual diet RR $0.96(95 \% \mathrm{Cl} 0.82$ to 1.13 , I2 $0 \%, 52,971$ participants, 602 cardiovascular deaths, peffect 0.65 ); or reduced and modified fat vs usual diet RR 0.98 (95\% Cl 0.76 to 1.27, 12 0\%, 2219 participants, 212 cardiovascular deaths, $p_{\text {effect }}$ 0.88 ), Analysis 1.2. Sensitivity analyses did not alter the lack of clear effects of modified fat intake, reduced fat intake, reduced and modified fat intake or all combined compared to usual or control diets on cardiovascular mortality (Table 1).

Subgrouping (as above) did not suggest important effects of reduced and/or modified fat diets on cardiovascular mortality (Table 2). 
We explored the effects of dietary fats on cardiovascular mortality in meta-regression (Table 3). There were insufficient studies to explore the effects of trans fats. As only six of the studies reported serum LDL cholesterol levels achieved, we also ran a post-hoc meta-regression by total serum cholesterol (as a surrogate for LDL cholesterol) achieved in each arm. We did not observe any clear relationships between duration, weight, any dietary or serum characteristics and mortality, but power for this analysis was limited.

\section{Cardiovascular events}

There was a reduction in cardiovascular events for any dietary fat intervention compared with usual diet (RR $0.86,95 \% \mathrm{Cl} 0.77$ to
0.96, I2 50\%, 65,508 participants, 4887 people with cardiovascular events, peffect 0.007) Analysis 1.3. Sensitivity analyses, using Mantel-Haenszel fixed effects meta-analysis, Peto fixed effects odds ratio, and removing the largest study comparisons (WHI with CVD 2006, WHI without CVD 2006) maintained this clear effect of the intervention, while removing studies with a systematic difference in care between the intervention and control arms, or removing studies with dietary differences other than dietary fat differences both removed the statistical significance of the effect (Table 1). A funnel plot did not suggest severe small study (or publication) bias, but it is likely that a few small studies with more cardiovascular events in the intervention groups may be missing from the review (Figure 4).

Figure 4. Funnel plot of comparison: fat modification or reduction vs usual diet - combined cardiovascular events.

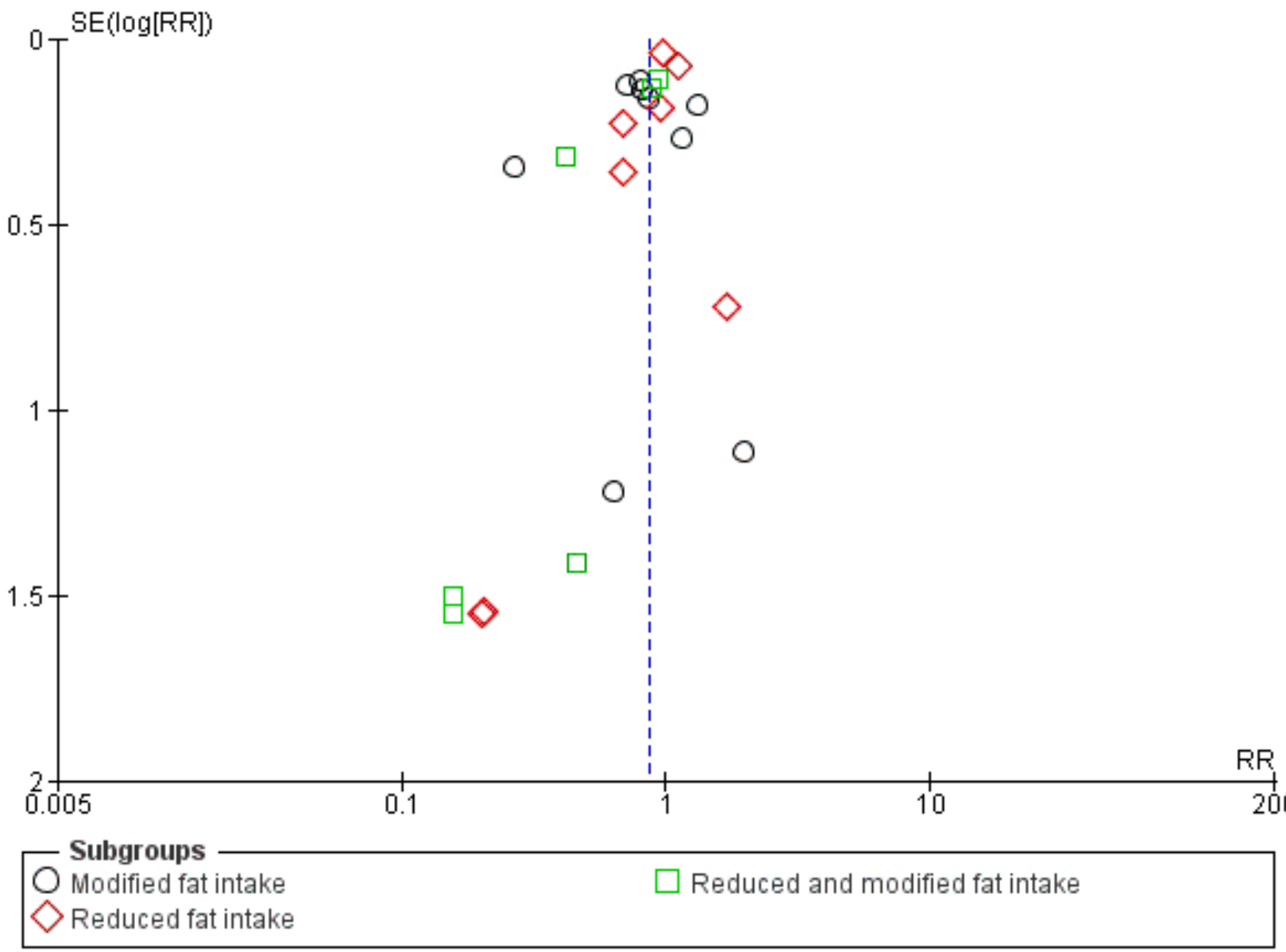

None of the subgroups of types of dietary fat change showed a clear effect of dietary fat change compared with usual diet in its own right, but the effects in both groups that included modification of fat were of marginal significance: modified fat vs usual fat RR 0.82 (95\% Cl 0.66 to $1.02,1^{2} 61 \%, 11,660$ participants, 855 people with CVD events, peffect 0.07 ); reduced and modified fat vs usual diet RR 0.77 (95\% Cl 0.57 to $1.03,\left.\right|^{2} 40 \%, 3193$ participants, 400 people with events, $\left.p_{\text {effect }} 0.08\right)$. There was no suggestion of an effect on cardiovascular events in studies that compared reduced fat vs usual intake (RR $0.97,95 \% \mathrm{Cl} 0.87$ to $1.08, \mathrm{I}^{2} 17 \%, 50,655$ participants, 3632 people with CVD events, $p_{\text {effect }} 0.55$ ).
Sensitivity analyses of the modified fat studies, using MantelHaenszel fixed effects relative risk meta-analysis suggested a strong and statistically significant effect of modified fat on cardiovascular events (RR $0.83,95 \% \mathrm{Cl} 0.73$ to $0.93, \mathrm{I}^{2} 61 \%, 11,660$ participants, 855 events, Peffect 0.002), as did the Peto odds ratio, and using fixed effects meta-analysis on the reduced and modified fat subgroup suggested risk reduction of $16 \%$ and of marginal statistical significance (RR $0.84,95 \%$ Cl 0.71 to $1.00,1240 \%, 3193$ participants, 400 events, peffect 0.05 , also reiterated by the Peto odds ratio analysis). Removing studies with systematic differences in care between intervention and control groups, or other dietary 
differences, both removed statistical significance. No sensitivity analyses of the reduced fat intake compared with control or usual diet suggested important or statistically significant effects (Table 1 ).

As multiple analyses were run for sub-grouping, only those subgroups statistically significant to $P<0.01$ are reported here (and in Table 2) as being statistically significant. Subgrouping by duration suggested no important effect in studies with duration of two years or less, but a clear and statistically significant effect in studies of more than two years duration. There was little suggestion of different effects of fat modification and/or reduction in those at low, moderate or high cardiovascular risk. Subgrouping suggested a strong effect of dietary advice plus supplementation, only marginal effect in those given dietary advice (without supplementation, but often with high levels of support and encouragement), and no effect in those provided with their food (via shops or group eating). There was no clear effect of baseline total fat intake on cardiovascular events. Many studies did not report saturated fat intakes, and sub-grouping by saturated fat intake in the control group did not result in important effects. Dietary fat intervention reduced cardiovascular events in men, but not in women or in combined studies of men and women, and studies in community settings reduced events, but those in residential institutions did not. Studies published in the 1960s, and in the 1990s, reduced cardiovascular events significantly, but not studies published in other decades (Table 2).

We explored the effects of dietary fats on cardiovascular events, by using meta-regression of the difference between the control and intervention of total fat intake, saturated fat intake, monounsaturated fat intake, polyunsaturated fat intake, trans fat intake (all by percentage of energy), weight (in $\mathrm{kg}$ ) and serum LDL cholesterol (in $\mathrm{mmol} / \mathrm{L}$ ) achieved in trials (Table 3). Metaregression of trans fats was not feasible due to lack of trials. As only seven studies reported LDL cholesterol, we also ran a post-hoc meta-regression by total serum cholesterol (as a surrogate for LDL cholesterol) achieved in each arm. As studies of shorter duration appeared to have less effect on events than longer interventions, we also performed a second post-hoc analysis, in which we carried out the meta-regression on duration alongside each dietary or serum factor. We did not observe any clear relationships between any dietary or serum characteristic and cardiovascular events (with or without co-regression on duration), but power for these analyses were limited.

\section{Secondary outcomes}

\section{Total myocardial infarction}

There was no clear effect of altering dietary fat intakes (compared to usual diet) on myocardial infarction (RR $0.93,95 \% \mathrm{Cl} 0.84$ to $1.02,126 \%, 64,891$ participants, 2068 people with fatal or non-fatal myocardial infarcts, $p_{\text {effect }} 0.13$ ) (Analysis 2.1). Neither was there any effect of any of the distinct dietary fat changes: modified fat intake RR 0.91 (95\% Cl 0.72 to 1.16, 12 45\%, 11,831 participants, 579

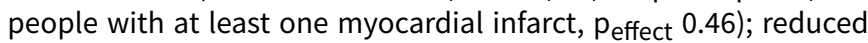
fat vs usual fat intake RR $0.97(95 \% \mathrm{Cl} 0.86$ to $1.08,120 \%, 50,522$ participants, 1203 people having a myocardial infarct, $p_{\text {effect }} 0.54$ ); and reduced and modified fat vs usual intake RR 0.90 ( $95 \% \mathrm{Cl} 0.72$ to $1.11,120 \%, 2538$ participants, 286 people with myocardial infarcts, peffect 0.32).

\section{Stroke}

There was no evidence of an effect of general dietary fat advice (RR $0.99,95 \% \mathrm{Cl} 0.89$ to $1.11, \mathrm{I}^{2} 0 \%, 59,853$ participants, 1140 people with at least one fatal or non-fatal stroke, peffect 0.87 ), or modified, reduced or both vs usual diet on stroke (Analysis 2.2). As $95 \%$ of the weight of this analysis was due to inclusion of the WHI trial (WHI with CVD 2006, WHI without CVD 2006), we also checked the effect of dietary fat advice on stroke excluding both parts of this study, and there was the suggestion of an effect of dietary change on stroke (RR $0.61,95 \% \mathrm{Cl} 0.37$ to $1.02, \mathrm{I}^{2}$ 0\%, 11,018 participants, 64 people with a stroke, peffect 0.06 ).

\section{Cancer deaths}

There was no effect of altering dietary fat intakes on cancer deaths (RR $0.98,95 \% \mathrm{Cl} 0.91$ to $1.06,120 \%, 65724$ participants, 2818 cancer deaths, $p_{\text {effect }} 0.66$ ), or of any specific type of fat changes, although there was a marginally significant effect of modified fat intake increasing cancer deaths, although this included only 91 cancer deaths (Analysis 2.3).

\section{Cancer diagnoses}

There was no evidence of an effect of altered dietary fat intake on cancer diagnoses (RR $0.96,95 \% \mathrm{Cl} 0.91$ to 1.01 , I2 1\%, 59,082 participants, 6115 people with at least one cancer diagnosis, $p_{\text {effect }}$ 0.11) (Analysis 2.4). Almost all the evidence on cancer diagnoses come from studies that reduced dietary fat, and only 138 diagnoses were made in studies where modified fat or reduced and modified fat interventions had been undertaken.

\section{Diabetes diagnoses}

Most data on diabetes diagnoses came from the WHI study, and neither the study itself, or the combined data set suggested that the intervention reduced the risk of diabetes being diagnosed (RR $0.96,95 \% \mathrm{Cl} 0.90$ to $1.02,120 \%, 49,859$ participants, 3367 diabetic diagnoses, $p_{\text {effect }} 0.20$ ) (Analysis 2.5).

\section{Non-fatal myocardial infarction}

There was no evidence of an effect of reduced and/or modified dietary fat vs usual fat diet on people diagnosed with at least one non-fatal MI, overall or within any subgroup of fat change (overall RR $0.95,95 \% \mathrm{Cl} 0.81$ to $1.12, \mathrm{I}^{2} 21 \%, 54,883$ participants, 1403 people with at least one infarct, $p_{\text {effect }} 0.55$ (Analysis 2.6).

\section{Quality of life}

Few studies considered quality of life. The Women's Health Initiative assessed quality of life at baseline using the SF-36 tool, but we were unable to find whether quality of life was compared between dietary intervention and control groups during the study. The Ole Study 2002 did not use a formal quality of life tool, but asked participants about their feelings about the diet they were on. They provided a scale of 1 (dislike extremely) to 7 (like extremely). Of the 13 control participants (eating meals 5 days per week in the study centre, with other meals in takeout containers) 6 participants were neutral about the diet (neither like nor dislike, 4 on the scale), and the remaining control participants were a little mor positive, choosing number 5 or 6 . Results were very similar in the low fat group, with 5 being neutral, 8 choosing 5 or 6 on the scale, and one choosing 3 (a little on the negative side of neutral). 


\section{Tertiary outcomes}

Note that the effects of dietary fat changes on tertiary outcomes discussed below represent a subset of all trials - we have only included assessment of effect of dietary interventions on these outcomes presented in studies that reported on occurrence of deaths and/or cardiovascular events (the review's primary or secondary outcomes). These outcomes are reported here as providing information on the potential mechanisms of any effects of dietary fat changes and also on whether participants adhered appropriately to their allocated interventions.

\section{Weight and Body Mass Index (BMI)}

Only two studies, including 99 participants, assessed the effects of modified fat intake compared to usual diet on weight, and did not find any clear effect (Analysis 3.1). One of these studies, and an additional study (116 participants in total) assessed the effect of a modified fat diet on BMI, again finding no clear effect (Analysis 3.2).

Sixteen studies (11,058 participants) compared reduced fat diets with usual diets and assessed effects on weight, finding a significant reduction in weight in those on low fat diets compared to usual diets (MD $-0.83 \mathrm{~kg}, 95 \% \mathrm{Cl}-1.37$ to $-0.30,1254 \%$, peffect 0.002 ). Similarly, the ten comparisons of reduced fat with usual intake found a reduction in $\mathrm{BMI}$ in those on the reduced fat diets (MD $-0.47 \mathrm{~kg} / \mathrm{m}^{2}, 95 \% \mathrm{Cl}-0.72$ to $-0.23, \mathrm{I}^{2} 51 \%$, peffect 0.0002 ).

No studies assessed the impact of reduced and modified fat intakes compared to usual diets on weight, and only two studies (111 participants) assessed impact on BMI, finding no clear effect (MD $-0.20 \mathrm{~kg} / \mathrm{m}^{2}, 95 \% \mathrm{Cl}-1.30$ to $0.91, \mathrm{I}^{2} 0 \%, \mathrm{p}_{\text {effect }} 0.73$ ).

In understanding the causative pathway between dietary advice or provision and mortality or morbidity it is useful to understand whether intermediate outcomes are being modified. Within this review this is complicated by the problem that some studies experienced outcomes, but did not report intermediate markers, and other studies experienced no events, but reported intermediate outcomes. For this reason we carried out a post-hoc analysis of the intermediate markers (tertiary outcomes) only for the studies that reported the primary outcomes. These are reported briefly below for weight and BMI, confirming clear and statistically significant reductions in both weight and BMI for those on reduced fat diets compared with control, but with no studies reporting these outcomes for modified or reduced and modified fat diets:

- Total mortality, modified fat intake: no studies reported weight or BMI

- Total mortality, reduced fat intake: 7 studies reported weight (MD $-0.92 \mathrm{~kg}, 95 \% \mathrm{Cl}-1.41$ to -0.43 , no heterogeneity), 3 studies reported BMI (MD $-0.48 \mathrm{~kg} / \mathrm{m}^{2}, 95 \% \mathrm{Cl}-0.65$ to -0.31 , no heterogeneity)

- Total mortality, reduced and modified fat intake: no studies reported weight or BMI

- Cardiovascular mortality, modified fat intake: no studies reported weight or BMI

- Cardiovascular mortality, reduced fat intake: 3 studies reported weight (MD $-0.55 \mathrm{~kg}, 95 \% \mathrm{Cl}-1.70$ to 0.61 , no heterogeneity), 2 studies reported $\mathrm{BMI}$ (MD $-0.44 \mathrm{~kg} / \mathrm{m}^{2}, 95 \% \mathrm{Cl}-0.60$ to -0.29 , no heterogeneity)
- Cardiovascular mortality, reduced and modified fat intake: no studies reported weight or BMI

- Cardiovascular events, modified fat intake: no studies reported weight or BMI

- Cardiovascular events, reduced fat intake: 3 studies reported weight (MD $-1.00 \mathrm{~kg}, 95 \% \mathrm{Cl}-1.60$ to -0.40 , no heterogeneity), 3 studies reported BMI (MD $-0.43 \mathrm{~kg} / \mathrm{m}^{2}, 95 \% \mathrm{Cl}-0.57$ to -0.28 , no heterogeneity)

- Cardiovascular events, reduced and modified fat intake: no studies reported weight or BMI

\section{Low density lipoprotein (LDL) cholesterol}

Two studies (116 participants) assessed the effect of a modified fat intake compared to usual or control diet on LDL cholesterol, finding no significant effect (MD $-0.20 \mathrm{mmol} / \mathrm{L}, 95 \% \mathrm{Cl}-0.47$ to 0.07 , $120 \%, p_{\text {effect }} 0.14$ ). The 14 comparisons (6971 participants) that assessed the effect of a low fat diet compared to usual diet on LDL cholesterol found a significant reduction in LDL on a low fat diet (MD $-0.10 \mathrm{mmol} / \mathrm{L}, 95 \% \mathrm{Cl}-0.14$ to $-0.05,120 \%$, $\mathrm{p}_{\text {effect }}<0.0001$ ). A reduced and modified fat intake also appeared to reduce LDL cholesterol in comparison to a usual diet (MD $-0.21 \mathrm{mmol} / \mathrm{L}, 95 \% \mathrm{Cl}-0.35$ to $-0.08,4$ comparisons, 627 participants, $12 \%$, peffect 0.002 ), see Analysis 3.3 .

Post-hoc analysis of LDL cholesterol only for the studies that reported the primary outcomes confirmed clear reductions in LDL in the reduced fat subgroup, non-statistically significant reductions in the reduced and modified fat subgroup, and no relevant studies for the modified fat subgroup:

- Total mortality, modified fat intake: no studies reported LDL

- Total mortality, reduced fat intake: four studies reported LDL (MD $-0.09 \mathrm{mmol} / \mathrm{L}, 95 \% \mathrm{Cl}-0.14$ to -0.03 , no heterogeneity)

- Total mortality, reduced and modified fat intake: two studies reported LDL (MD $-0.25 \mathrm{mmol} / \mathrm{L}, 95 \% \mathrm{Cl}-0.63$ to 0.12 , I2 56\%)

- Cardiovascular mortality, modified fat intake: no studies reported LDL

- Cardiovascular mortality, reduced fat intake: four studies reported LDL (MD $-0.09 \mathrm{mmol} / \mathrm{L}, 95 \% \mathrm{Cl}-0.14$ to -0.03 , no heterogeneity)

- Cardiovascular mortality, reduced and modified fat intake: two studies reported LDL (MD $-0.25 \mathrm{mmol} / \mathrm{L}, 95 \% \mathrm{Cl}-0.63$ to 0.12 , 12 $56 \%)$

- Cardiovascular events, modified fat intake: no studies reported LDL

- Cardiovascular events, reduced fat intake: four studies reported LDL (MD $-0.10 \mathrm{mmol} / \mathrm{L}, 95 \% \mathrm{Cl}-0.15$ to -0.04 , no heterogeneity)

- Cardiovascular events, reduced and modified fat intake: two studies reported LDL (MD $-0.25 \mathrm{mmol} / \mathrm{L}, 95 \% \mathrm{Cl}-0.63$ to 0.12 , $\mathrm{I}^{2}$ $56 \%)$

\section{High density lipoprotein (HDL) cholesterol}

None of the interventions appeared to alter HDL cholesterol: modified fat intake MD $-0.04 \mathrm{mmol} / \mathrm{L}(95 \% \mathrm{Cl}-0.18$ to $0.09,3$ comparisons, 152 participants, $\mathrm{I}^{2} 0 \%$, peffect 0.54 ); reduced fat intake MD $-0.01 \mathrm{mmol} / \mathrm{L}$ ( $95 \% \mathrm{Cl}-0.02$ to $0.01,15$ comparisons, 7082 participants, $\mathrm{I}^{2} 0 \%$, peffect 0.30 ); and reduced and modified fat intake MD $-0.01 \mathrm{mmol} / \mathrm{L}(95 \% \mathrm{Cl}-0.04$ to 0.01 , four comparisons, 2073 participants, $120 \%$, peffect 0.36 ), see Analysis 3.4 . 
Post-hoc analysis of HDL cholesterol only for the studies that reported the primary outcomes confirmed a lack of effects on HDL cholesterol in any group (with no studies reporting HDL in the modified fat subgroup):

- Total mortality, modified fat intake: no studies reported HDL

- Total mortality, reduced fat intake: four studies reported HDL (MD $0.00 \mathrm{mmol} / \mathrm{L}, 95 \% \mathrm{Cl}-0.03$ to 0.04 , no heterogeneity)

- Total mortality, reduced and modified fat intake: three studies reported HDL (MD $-0.01 \mathrm{mmol} / \mathrm{L}, 95 \% \mathrm{Cl}-0.04$ to 0.02 , no heterogeneity)

- Cardiovascular mortality, modified fat intake: no studies reported HDL

- Cardiovascular mortality, reduced fat intake: four studies reported HDL (MD $0.00 \mathrm{mmol} / \mathrm{L}, 95 \% \mathrm{Cl}-0.03$ to 0.04 , no heterogeneity)

- Cardiovascular mortality, reduced and modified fat intake: three studies reported HDL (MD $-0.01 \mathrm{mmol} / \mathrm{L}, 95 \% \mathrm{Cl}-0.04$ to 0.02 , no heterogeneity)

- Cardiovascular events, modified fat intake: no studies reported HDL

- Cardiovascular events, reduced fat intake: four studies reported LDL (MD $0.10 \mathrm{mmol} / \mathrm{L}, 95 \% \mathrm{Cl}-0.03$ to 0.04 , no heterogeneity)

- Cardiovascular events, reduced and modified fat intake: three studies reported $\mathrm{HDL}$ (MD $-0.01 \mathrm{mmol} / \mathrm{L}, 95 \% \mathrm{Cl}-0.04$ to 0.02 , no heterogeneity)

\section{Total cholesterol}

More studies reported total than LDL or HDL cholesterol, and all three dietary fat interventions found significant reductions in total cholesterol: modified fat intake MD $-0.44 \mathrm{mmol} / \mathrm{L}(95 \% \mathrm{Cl}-0.60$ to -0.28 , 8 comparisons, 2280 participants, $\left.\right|^{2} 59 \%$, peffect $<0.00001$ ); reduced fat intake MD $-0.10 \mathrm{mmol} / \mathrm{L}(95 \% \mathrm{Cl}-0.14$ to $-0.05,15$ comparisons, 7602 participants, $120 \%$, peffect $<0.0001$ ); reduced and modified fat intake MD $-0.26 \mathrm{mmol} / \mathrm{L}(95 \% \mathrm{Cl}-0.47$ to $-0.04,5$ comparisons, 2131 participants, $1251 \%$, peffect 0.02 ).

Post-hoc analysis of total cholesterol only for the studies that reported the primary outcomes confirmed clear total cholesterol reductions for most comparisons:

- Total mortality, modified fat intake: four studies reported total cholesterol (MD $-0.47 \mathrm{mmol} / \mathrm{L}, 95 \% \mathrm{Cl}-0.85$ to -0.10 , no heterogeneity)

- Total mortality, reduced fat intake: five studies reported total cholesterol (MD $-0.08 \mathrm{mmol} / \mathrm{L}, 95 \% \mathrm{Cl}-0.13$ to -0.03 , no heterogeneity)

- Total mortality, reduced and modified fat intake: three studies reported total cholesterol (MD $-0.33 \mathrm{mmol} / \mathrm{L}, 95 \% \mathrm{Cl}-0.61$ to $-0.06,1265 \%)$

- Cardiovascular mortality, modified fat intake: two studies reported total cholesterol (MD $-0.32 \mathrm{mmol} / \mathrm{L}, 95 \% \mathrm{Cl}-0.67$ to 0.04 , no heterogeneity)

- Cardiovascular mortality, reduced fat intake: four studies reported total cholesterol (MD $-0.08 \mathrm{mmol} / \mathrm{L}, 95 \% \mathrm{Cl}-0.14$ to -0.02 , no heterogeneity)

- Cardiovascular mortality, reduced and modified fat intake: three studies reported total cholesterol (MD $-0.33 \mathrm{mmol} / \mathrm{L}, 95 \% \mathrm{Cl}-0.61$ to $-0.06,1265 \%$ )
- Cardiovascular events, modified fat intake: four studies reported total cholesterol (MD $-0.44 \mathrm{mmol} / \mathrm{L}, 95 \% \mathrm{Cl}-0.54$ to -0.35 , no heterogeneity)

- Cardiovascular events, reduced fat intake: three studies reported total cholesterol (MD $-0.08 \mathrm{mmol} / \mathrm{L}, 95 \% \mathrm{Cl}-0.14$ to -0.02 , no heterogeneity)

- Cardiovascular events, reduced and modified fat intake: three studies reported total cholesterol (MD $-0.33 \mathrm{mmol} / \mathrm{L}, 95 \% \mathrm{Cl}-0.61$ to $-0.06,1265 \%$ )

\section{Triglycerides}

Modified fat intake and reduced and modified fat intake studies both reduced fasting triglycerides (modified fat intake MD $-0.11 \mathrm{mmol} / \mathrm{L}, 95 \% \mathrm{Cl}-0.22$ to $-0.00,5$ comparisons, 706 participants, $120 \%, p_{\text {effect }} 0.04$; reduced and modified fat intake MD $-0.27 \mathrm{mmol} /$ L, $95 \% \mathrm{Cl}-0.53$ to $-0.00,3$ comparisons, 218 participants, $120 \%$, Peffect 0.05 ), see Analysis 3.6. However, studies that reduced total fat intake compared to usual diet did not have any effect on fasting triglycerides (MD $-0.00 \mathrm{mmol} / \mathrm{L}, 95 \% \mathrm{Cl}-0.00$ to $0.00,13$ comparisons, 6875 participants, $\mathrm{I}^{2} \mathrm{0} \%$, $\mathrm{p}_{\text {effect }} 1.00$ ).

Post-hoc analysis of the triglycerides for the studies that reported the primary outcomes only confirmed that diets both reducing and modifying dietary fats appeared to reduce triglycerides, while there were insufficient studies to tell for modified fat and reduced fat diets:

- Total mortality, modified fat intake: one study reported triglycerides (MD $-0.10 \mathrm{~mol} / \mathrm{L}, 95 \% \mathrm{Cl}-0.26$ to 0.06 )

- Total mortality, reduced fat intake: four studies reported triglycerides (MD $-0.02 \mathrm{mmol} / \mathrm{L}, 95 \% \mathrm{Cl}-0.13$ to 0.08 , no heterogeneity)

- Total mortality, reduced and modified fat intake: two studies reported triglycerides (MD $-0.31 \mathrm{mmol} / \mathrm{L}, 95 \% \mathrm{Cl}-0.62$ to -0.01 , no heterogeneity)

- Cardiovascular mortality, modified fat intake: no studies reported triglycerides

- Cardiovascular mortality, reduced fat intake: four studies reported triglycerides (MD $-0.02 \mathrm{mmol} / \mathrm{L}, 95 \% \mathrm{Cl}-0.13$ to 0.08 , no heterogeneity)

- Cardiovascular mortality, reduced and modified fat intake: two studies reported triglycerides (MD $-0.31 \mathrm{mmol} / \mathrm{L}, 95 \% \mathrm{Cl}-0.62$ to -0.01 , no heterogeneity)

- Cardiovascular events, modified fat intake: one study reported triglycerides (MD $-0.26 \mathrm{~mol} / \mathrm{L}, 95 \% \mathrm{Cl}-0.50$ to -0.02 )

- Cardiovascular events, reduced fat intake: four studies reported triglycerides (MD $-0.50 \mathrm{mmol} / \mathrm{L}, 95 \% \mathrm{Cl}-1.05$ to 0.05 , I2 52\%)

- Cardiovascular events, reduced and modified fat intake: two studies reported triglycerides (MD $-0.31 \mathrm{mmol} / \mathrm{L}, 95 \% \mathrm{Cl}-0.62$ to -0.01 , no heterogeneity)

\section{Systolic and diastolic blood pressure (BP)}

No studies reported the effects of modified fat intake on systolic or diastolic BP, and only one reported (non-significant) effects of reduced and modified fat on systolic and diastolic BP. Six included comparisons reported effects of reduced fat diets on blood pressure, but again pooled results did not suggest clear effects (systolic BP MD $-0.56 \mathrm{mmHg}, 95 \% \mathrm{Cl}-11.22$ to $1.06,6$ comparisons, 3981 participants, $120 \%$, peffect 0.25 ; diastolic BP MD 
$-0.35 \mathrm{mmHg}, 95 \% \mathrm{Cl}-0.96$ to $0.26,6$ comparisons, 3543 participants, $120 \%$, peffect 0.26 ). See Analysis 3.7 and Analysis 3.8 for blood pressure analyses.

Post-hoc analysis of the systolic and diastolic blood pressure only for the studies that reported the primary outcomes confirmed that few studies reported blood pressure and there was no clear effect on blood pressure in those studies that did:

- Total mortality, reduced fat intake: four studies reported SBP (MD $-0.55 \mathrm{mmHg}, 95 \% \mathrm{Cl}-1.54$ to 0.43 , no heterogeneity)

- Total mortality, reduced fat intake: three studies reported dBP (MD - $0.32 \mathrm{mmHg}, 95 \% \mathrm{Cl}-0.94$ to 0.30 , no heterogeneity)

- Total mortality, reduced and modified fat intake: no studies reported systolic or diastolic BP

- Cardiovascular mortality, reduced fat intake: three studies reported SBP (MD $-0.67 \mathrm{mmHg}, 95 \% \mathrm{Cl}-2.23$ to 0.89 , no heterogeneity)

- Cardiovascular mortality, reduced fat intake: two studies reported dBP (MD $-0.43 \mathrm{mmHg}, 95 \% \mathrm{Cl}-6.20$ to 1.58 , no heterogeneity)

- Cardiovascular mortality, reduced and modified fat intake: no studies reported systolic or diastolic BP

- Cardiovascular events, reduced fat intake: four studies reported sBP (MD $-0.55 \mathrm{mmHg}, 95 \% \mathrm{Cl}-11.22$ to 1.06 , no heterogeneity)

- Cardiovascular events, reduced fat intake: three studies reported dBP (MD $-0.32 \mathrm{mmHg}, 95 \% \mathrm{Cl}-0.94$ to 0.30 , no heterogeneity)

- Cardiovascular events, reduced and modified fat intake: no studies reported systolic or diastolic BP

\section{Reduced fat vs. modified fat diet}

\section{Primary outcomes}

There were no included studies comparing reduced with modified fat diets that reported total or cardiovascular mortality. Three studies (with 912 participants) reported 28 cardiovascular events, and not suggesting any difference in the effects of a reduced or modified fat diet (RR $1.13,95 \% \mathrm{Cl} 0.41$ to 3.06 , Analysis 4.3).

\section{Secondary outcomes}

No studies compared reduced with modified fat diets and provided data on diabetes diagnoses. One small study provided data on myocardial infarction, stroke and non-fatal MI, and there were no clear effects for any outcome. A further study with two comparisons found no cancer deaths and there was no difference between low and modified fat in terms of cancer diagnoses (with only 4 diagnoses). See Analysis 5.1, Analysis 5.2, Analysis 5.3, Analysis 5.4, Analysis 5.5 and Analysis 5.6 for further details.

\section{Tertiary outcomes}

Several small studies comparing reduced with modified fat diets reported tertiary outcomes. There were no clear differences between reduced and modified fat diets for weight, BMI, total, LDL or HDL cholesterol, triglycerides, systolic or diastolic blood pressure. See Analysis 6.1, Analysis 6.2, Analysis 6.3, Analysis 6.4, Analysis 6.5, Analysis 6.6, Analysis 6.7 and Analysis 6.8 for further details.

There were insufficient studies in this section of the review to carry out sensitivity analyses or sub-grouping. One study directly addressed the acceptability of modified compared to reduced fat diets finding little difference between low and modified fat diets in terms of craving, fullness, hunger, desire to eat, wellness, distaste, cost, personal inconvenience, family inconvenience or feelings of deprivation. This study also assessed quality of life for all four arms using the SF-36, but data have not yet been reported (Sacks low protein 2009, Sacks high protein 2009). A further study reported that the modified fat diet advised was 'monotonous' in comparison to the low fat diet, although it was not clear why this was (Rivellese 1994).

In the nine studies that directly compared reduced with modified fat diets there was little difference in numbers of dropouts in those on modified fat diets (RR of dropping out $0.84,95 \% \mathrm{Cl} 0.58$ to 1.22 in reduced compared to modified fat diets, $\mathrm{P}=0.36,1353$ participants, 237 dropouts, $1244 \%)$, Analysis 6.9.

\section{DISCUSSION}

\section{Addressing the specific questions}

Does reducing saturated fat intake, by reducing and/or modifying dietary fat, in the longer term (at least 6 months) reduce mortality, cardiovascular mortality or cardiovascular morbidity (or individual health events such as myocardial infarction, stroke, diabetes or cancer)?

- This review suggested that dietary saturated fat reduction (through reduction and/or modification of dietary fat) may be protective of cardiovascular events overall, reducing them by 14\% (RR $0.86,95 \% \mathrm{Cl} 0.77$ to 0.96, 24 comparisons, 65,614 participants, with marginal $1^{2}$ of $50 \%$, Analysis 1.3$)$. This was moderate GRADE evidence (See summary of findings, Figure 5).

- Despite the reduction in total cardiovascular events, there was no clear evidence of reductions in any individual outcome (total or non-fatal myocardial infarction, stroke, cancer deaths or diagnoses, diabetes diagnoses).

- Despite a large number of participants involved in randomised controlled trials of at least six months duration there were no clear effects of dietary fat changes on total mortality (RR $0.98,95 \% \mathrm{Cl} 0.93$ to $1.04,21$ comparisons, 71,795 participants, without important heterogeneity, Analysis 1.1) or cardiovascular mortality (RR $0.94,95 \%$ Cl 0.85 to $1.04,16$ comparisons, 65,983 participants, without important heterogeneity, Analysis 1.2). 
Figure 5. Summary of findings, for reduced or modified fat diets vs usual diet (primary outcomes).

\begin{tabular}{|c|c|c|c|c|c|c|}
\hline \multicolumn{7}{|c|}{$\begin{array}{l}\text { Patient or population: patients with preventing cardiovascular disease } \\
\text { Settings: } \\
\text { Intervention: fat modification or reduction } \\
\text { Comparison: usual diet }\end{array}$} \\
\hline \multirow[t]{2}{*}{ Outcomes } & \multirow{2}{*}{\multicolumn{2}{|c|}{$\begin{array}{l}\text { Illustrative comparative risks }{ }^{\star}(95 \% \mathrm{Cl}) \\
\text { Assumed risk Corresponding risk } \\
\text { usual diet } \begin{array}{l}\text { fat modification or } \\
\text { reduction }\end{array}\end{array}$}} & \multirow{2}{*}{$\begin{array}{l}\text { Relative } \\
\text { effect } \\
(95 \% \mathrm{CI}) \\
\end{array}$} & \multirow{2}{*}{$\begin{array}{l}\text { No of Participants } \\
\text { (studies) }\end{array}$} & \multirow{2}{*}{$\begin{array}{l}\text { Quality of the } \\
\text { evidence } \\
\text { (GRADE) }\end{array}$} & \multirow[t]{2}{*}{ Comments } \\
\hline & & & & & & \\
\hline \multirow{4}{*}{$\begin{array}{l}\text { Total mortality } \\
\text { Follow-up: } 0.5 \text { to } 11 \text { years }\end{array}$} & \multicolumn{2}{|c|}{ Study population } & \multirow{4}{*}{$\begin{array}{l}\text { RR } 0.98 \\
-(0.93 \text { to } 1.04)\end{array}$} & \multirow{4}{*}{$\begin{array}{l}71790 \\
\text { (21 studies) }\end{array}$} & \multirow{4}{*}{$\begin{array}{l}\text { ニニニニ } \\
\text { high }^{1}\end{array}$} & \\
\hline & 59 per 1000 & $\begin{array}{l}58 \text { per } 1000 \\
(55 \text { to } 61)\end{array}$ & & & & \\
\hline & \multicolumn{2}{|c|}{ Medium risk population } & & & & \\
\hline & 68 per 1000 & $\begin{array}{l}67 \text { per } 1000 \\
(63 \text { to } 71)\end{array}$ & & & & \\
\hline \multirow{4}{*}{$\begin{array}{l}\text { Cardiovascular mortality } \\
\text { Follow-up: } 0.5 \text { to } 11 \text { years }\end{array}$} & \multicolumn{2}{|c|}{ Study population } & \multirow{4}{*}{$\begin{array}{l}\text { RR } 0.94 \\
-(0.85 \text { to } 1.04)\end{array}$} & \multirow{4}{*}{$\begin{array}{l}65978 \\
\text { (16 studies) }\end{array}$} & \multirow{4}{*}{$\begin{array}{l}\text { ニニニニ } \\
\text { high }^{1}\end{array}$} & \\
\hline & 20 per 1000 & $\begin{array}{l}19 \text { per } 1000 \\
(17 \text { to } 21)\end{array}$ & & & & \\
\hline & \multicolumn{2}{|c|}{ Medium risk population } & & & & \\
\hline & 45 per 1000 & $\begin{array}{l}42 \text { per } 1000 \\
(38 \text { to } 47)\end{array}$ & & & & \\
\hline \multirow{4}{*}{$\begin{array}{l}\text { Combined cardiovascular events } \\
\text { Follow-up: } 0.5 \text { to } 8.1 \text { years }\end{array}$} & \multicolumn{2}{|c|}{ Study population } & \multirow{4}{*}{$\begin{array}{l}\text { RR } 0.86 \\
-(0.77 \text { to } 0.96)\end{array}$} & \multirow{4}{*}{$\begin{array}{l}65609 \\
\text { (24 studies) }\end{array}$} & \multirow{4}{*}{$\begin{array}{l}\text { ニニニニ } \\
\text { moderate }\end{array}$} & \\
\hline & 77 per 1000 & $\begin{array}{l}66 \text { per } 1000 \\
\text { ( } 59 \text { to } 74) \\
\end{array}$ & & & & \\
\hline & \multicolumn{2}{|c|}{ Medium risk population } & & & & \\
\hline & 152 per 1000 & $\begin{array}{l}131 \text { per } 1000 \\
(117 \text { to } 146)\end{array}$ & & & & \\
\hline \multirow{4}{*}{$\begin{array}{l}\text { Combined cardiovascular events - Modified } \\
\text { fat intake } \\
\text { Follow-up: } 0.5 \text { to } 6 \text { years }\end{array}$} & \multicolumn{2}{|c|}{ Study population } & \multirow{4}{*}{$\begin{array}{l}\text { RR } 0.83 \\
-(0.67 \text { to } 1.02)\end{array}$} & \multirow{4}{*}{$\begin{array}{l}11761 \\
\text { (10 studies) }\end{array}$} & \multirow{4}{*}{$\begin{array}{l}\text { ニニニニ } \\
\text { low }^{1,4}\end{array}$} & \\
\hline & 81 per 1000 & $\begin{array}{l}67 \text { per } 1000 \\
(54 \text { to } 83)\end{array}$ & & & & \\
\hline & \multicolumn{2}{|c|}{ Medium risk population } & & & & \\
\hline & 225 per 1000 & $\begin{array}{l}187 \text { per } 1000 \\
(151 \text { to } 229)\end{array}$ & & & & \\
\hline \multirow{4}{*}{$\begin{array}{l}\text { Combined cardiovascular events - Reduced } \\
\text { fat intake } \\
\text { Follow-up: } 0.5 \text { to } 8.1 \text { years }\end{array}$} & Study populat & tion & RR 0.97 & 50655 & ニニニン & \\
\hline & 73 per 1000 & $\begin{array}{l}71 \text { per } 1000 \\
\text { (64 to } 79)\end{array}$ & & & high $^{1}$ & \\
\hline & Medium risk $\mathrm{p}$ & population & & & & \\
\hline & 115 per 1000 & $\begin{array}{l}112 \text { per } 1000 \\
(100 \text { to } 124)\end{array}$ & & & & \\
\hline Combined cardiovascular events - Reduced & Study populat & tion & RR 0.77 & 3193 & ニンニン & \\
\hline $\begin{array}{l}\text { and modified fat intake } \\
\text { Follow-up: } 0.6 \text { to } 4.3 \text { years }\end{array}$ & 146 per 1000 & $\begin{array}{l}112 \text { per } 1000 \\
(83 \text { to } 150)\end{array}$ & $-(0.57$ to 1.03$)$ & (6 studies) & $\operatorname{low}^{1,5}$ & \\
\hline & Medium risk $\mathrm{p}$ & population & & & & \\
\hline & 100 per 1000 & $\begin{array}{l}77 \text { per } 1000 \\
(57 \text { to } 103)\end{array}$ & & & & \\
\hline
\end{tabular}

${ }^{*}$ The basis for the assumed risk (e.g. the median control group risk across studies) is provided in footnotes. The corresponding risk (and its $95 \%$ confidence interval) is based on the assumed risk in the comparison group and the relative effect of the intervention (and its $95 \% \mathrm{Cl}$ ).

Cl: Confidence interval; RR: Risk ratio

GRADE Working Group grades of evidence

High quality: Further research is very unlikely to change our confidence in the estimate of effect

Moderate quality: Further research is likely to have an important impact on our confidence in the estimate of effect and may change the estimate

Low quality: Further research is very likely to have an important impact on our confidence in the estimate of effect and is likely to change the estimate.

Very low quality: We are very uncertain about the estimate.

1 Problems in this set of dietary trials include a large proportion of comparisons were not blinded, and most have differential levels of attention and support to the intervention groups, as well as dietary advice or changes additional to the fat intervention. It is also difficult to ascertain the true dietary changes achieved (as most rely on self-reported dietary assessment or records). Despite this the data set is very large and the data are remarkably consistent. As these biases tend to favour the intervention over the control arm, but the data do not show evidence of a benefit of the intervention, the risk of bias in the results is reduced. For this reason we have chosen to state that there are no limitations in design, which allows the GRADE evidence not to suggest that further trials should be conducted.

2 Problems in this set of dietary trials include that a large proportion of comparisons were not blinded, and most have differential levels of attention and support to the intervention groups, as well as dietary advice or changes additional to the fat intervention. It is also difficult to ascertain the true dietary changes achieved (as most rely on self-reported dietary assessment or records). These limitations would tend to favour a positive effect in the intervention group over the control group

${ }^{3}$ There is evidence of heterogeneity in the studies, so the inconsistency has been explored below in subgroups

${ }^{4}$ Fewer than 1000 events in total, so limited power to estimate true effect. Using fixed effects meta-analysis (both Mantel-Haenszel and Peto) alters the statistical significance of this outcome, suggesting that there is an important and statistically significant effect of a modified fat diet on cardiovascular events. While $p$ for heterogeneity was 0.009 , and I squared $61 \%$ (both suggesting important heterogeneity), there is evidence that the fixed effects analysis may deal better with studies with a low risk of events. For this reason it is unclear whether fixed or random effects methodolociv provides the best estimate of effect. The outcome has been 


\section{Figure 5. (Continued)}

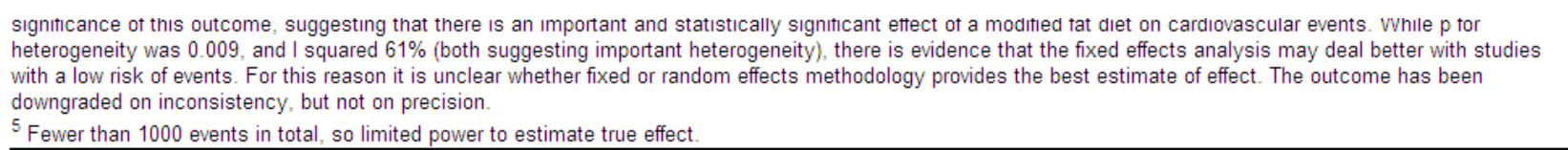

${ }^{5}$ Fewer than 1000 events in total, so limited power to estimate true effect

Does a long term reduced fat diet, compared with usual diet, reduce mortality, cardiovascular mortality or cardiovascular morbidity (or individual health events such as myocardial infarction, stroke, diabetes or cancer)?

- There were no clear effects of reduced fat diets on any of these outcomes despite the inclusion of almost 62000 participants in studies of over 6 months. This was despite clear and statistically significant reductions in weight, BMI and total and LDL cholesterol in reduced fat vs. usual diet studies.

Does a long term reduced fat diet alter classic cardiovascular risk factors (weight, body mass index, systolic or diastolic blood pressure, serum total, LDL or HDL cholesterol and triglyceride)?

- The studies included in this review represent a subset of all relevant studies assessing the effect of reduced fat diets on classic cardiovascular risk factors, so should be treated with caution. However, they do represent a good set of data from which to assess the longer term effects of such interventions in people living in the community.

- There was evidence that reduced fat diets resulted in modestly lower weight (MD $-0.83 \mathrm{~kg}, 95 \% \mathrm{Cl}-1.37$ to $-0.30,11,058$ participants, $\mathrm{I}^{2} 54 \%$ ) and body mass index (MD $-0.47 \mathrm{kgm}^{-2}$, $95 \% \mathrm{Cl}-0.72$ to $-0.23,5972$ participants, $1251 \%)$. Similary modest improvements were seen in LDL cholesterol (MD $-0.10 \mathrm{mmol} /$ $\mathrm{L}, 95 \% \mathrm{Cl}-0.14$ to $-0.05,5971$ participants, $\mathrm{I}^{2} 0 \%$ ) and total cholesterol (MD $-0.10 \mathrm{mmol} / \mathrm{L}, 95 \% \mathrm{Cl}-0.14$ to $-0.05,7602$ participants, $\mathrm{I}^{2} \mathrm{0 \%}$ ), compared with those on usual or control diets.

- Other risk factors (HDL cholesterol, triglycerides, systolic and diastolic blood pressure) did not appear to alter with reduced fat diets.

Does a long term modified fat diet, compared with usual diet, reduce mortality, cardiovascular mortality or cardiovascular morbidity (or individual health events such as myocardial infarction, stroke, diabetes or cancer)?

- There were no clear effects of modified fat diets on total or cardiovascular mortality, despite the inclusion of over 13000 participants in studies of over six months.

- There was a suggestion (but without statistical significance) that modified fat diets reduced cardiovascular events by $18 \%$ (RR $0.82,95 \% \mathrm{Cl} 0.66$ to 1.02 , peffect $0.07,11,660$ participants, |2 $61 \%$ ).

- There were no suggestions of effects on individual health outcomes (with no data found on diabetes diagnosis), except for a suggestion that modified fat diets increased the risk of cancer deaths (RR $1.46,95 \% \mathrm{Cl} 0.96$ to 2.21 , peffect $0.08,9903$ participants, $120 \%)$
Does a long term modified fat diet alter classic cardiovascular risk factors (weight, body mass index, systolic or diastolic blood pressure, serum total, LDL or HDL cholesterol and triglyceride)?

- The studies included in this review represent a subset of all relevant studies assessing the effect of modified fat diets on classic cardiovascular risk factors, so should be treated with caution. However, they do represent a good set of data from which to assess the longer term effects of such interventions in people living in the community.

- A modified fat diet appeared to reduce serum total cholesterol by around $7 \%$ (MD $-0.44 \mathrm{mmol} / \mathrm{L}, 95 \% \mathrm{Cl}-0.60$ to $-0.28,2280$ participants, $\mathrm{I}^{2} 59 \%$ ) and modestly reduce fasting serum triglycerides (MD $-0.11 \mathrm{mmol} / \mathrm{L}, 95 \% \mathrm{Cl}-0.22$ to $-0.00,701$ participants, $120 \%)$.

- There were no clear effects on serum LDL or HDL cholesterol, weight or BMI, although the numbers of participants in these comparisons were low (99 to 150 only). We found no data assessing the effect of modified fat diets on systolic or diastolic blood pressure.

- Few of the studies that compared a modified fat diet with a usual diet and experienced deaths or cardiovascular events reported intermediate outcomes.

Does reducing and modifying dietary fat (as a combined intervention) in the longer term reduce mortality, cardiovascular mortality or cardiovascular morbidity (or individual health events such as myocardial infarction, stroke, diabetes or cancer)?

- There were no clear effects of reduced and modified fat diets on total or cardiovascular mortality, despite the inclusion of almost 5000 participants in studies of over 6 months.

- There was a suggestion (with marginal statistical significance), that reduced and modified fat diets reduced cardiovascular events by $23 \%$ (RR $0.77,95 \% \mathrm{Cl} 0.57$ to 1.03 , peffect $0.08,3193$ participants, $1240 \%)$.

- There were no suggestions of effects on individual health outcomes (with no data found on diabetes diagnosis and few events for most outcomes).

Does a long term reduced and modified fat diet (as a combined intervention) alter classic cardiovascular risk factors (weight, body mass index, systolic or diastolic blood pressure, serum total, LDL or HDL cholesterol and triglyceride)?

- The studies included in this review represent a subset of all relevant studies assessing the effect of reduced and modified fat diets on classic cardiovascular risk factors, so should be treated with caution. However, they do represent a good set of data from which to assess the longer term effects of such interventions in people living in the community. 
- A reduced and modified fat diet appeared to modestly reduce serum total cholesterol (MD $-0.26 \mathrm{mmol} / \mathrm{L}, 95 \% \mathrm{Cl}-0.47$ to $-0.04,2131$ participants, $1251 \%$ ), serum LDL cholesterol (MD $-0.21 \mathrm{mmol} / \mathrm{L}, 95 \% \mathrm{Cl}-0.35$ to $-0.08,627$ participants, $120 \%$ and fasting serum triglycerides (MD $-0.27 \mathrm{mmol} / \mathrm{L}, 95 \% \mathrm{Cl}-0.53$ to $-0.00,218$ participants, $1^{2} 0 \%$ ).

- There were no clear effects on serum HDL cholesterol or BMI.

- Few of the studies that compared a reduced and modified fat diet with a usual diet and experienced deaths or cardiovascular events reported intermediate outcomes.

- We located only one study that assessed effects on systolic and diastolic blood pressure, and no studies reported weight.

Are effects on mortality, cardiovascular mortality or cardiovascular morbidity moderated by differences in baseline cardiovascular risk, mode of intervention, control group total fat intake, control group saturated fat intake, gender, setting or decade of publication, or changes in total fat intake, saturated fat intake, polyunsaturated fat intake, monounsaturated fat intake, trans fat intake, dietary cholesterol, weight, serum LDL cholesterol, or study duration?

- Lack of effects of reduced and/or modified dietary fat on total and cardiovascular mortality did not alter with any subgrouping, and no meta-regression suggested relationships with these outcomes and any continuous factor tested.

- Subgrouping suggested that effects on cardiovascular events of reduced and/or modified fat diets were due to studies with duration greater than two years (not those of two years or less), those given dietary advice plus a supplement (not diet alone or diet provided), studies of men (not of women), those in a community rather than residential setting (no studies in workplace locations were identified). There was no suggestion that baseline CVD risk or baseline total fat intakes altered the effects of dietary fat changes on cardiovascular events. There was a suggestion of an effect in studies published in the 1960s and 1990s. Overall, the data suggest that modified or modified and reduced fat diets (but not reduced fat diets), help to reduce cardiovascular events in longer studies of men.

- Meta-regression suggested no clear effects of the amount of difference between intervention and control arms in total fat intake, saturated fat intake, polyunsaturated fat intake, monounsaturated fat intake or total cholesterol on cardiovascular events. Nor were there clear effects of duration.

- There were insufficient data to assess the associations of trans fats or LDL cholesterol with cardiovascular mortality or events.

Is a long term reduced fat diet or a modified fat diet more effective in reducing mortality, cardiovascular mortality or cardiovascular morbidity (or individual effects such as myocardial infarction, stroke or cancer diagnoses)?

- There were no studies that provided data to assess the differences between reduced and modified fat diets on total or cardiovascular mortality. Only three studies assessed the comparative effects of reduced compared to modified fat diets on cardiovascular events ( 28 events in total).

- One study provided some data on myocardial infarction and stroke, and two comparisons provided information on cancer diagnoses.
Is a long term reduced fat diet or a modified fat diet more acceptable to people trying to adhere to these diets?

- One study (two comparisons) directly addressed the acceptability of modified compared to reduced fat diets - no clear differences were identified and the quality of life data have not yet been reported (Sacks high protein 2009; Sacks low protein 2009). One further study reported that the modified fat diet advised was 'monotonous' in comparison to the low fat diet (Rivellese 1994).

- In the eight studies that directly compared reduced with modified fat diets there was no difference in numbers of dropouts in either arm.

Is a long term reduced fat diet or a modified fat diet more effective in altering classic cardiovascular risk factors (weight, body mass index, systolic or diastolic blood pressure, serum total, LDL or HDL cholesterol and triglyceride)?

- Several small studies provided outcome data comparing the effects of reduced and modified fat diets on these risk factors. There were no clear differences.

- When (indirectly) comparing effects on risk factors in studies that compared reduced fat with usual diets with those in studies that compared modified fat with usual diets, effects in reducing risk factors in the modified fat studies were greater than those in the reduced fat trials for total and LDL cholesterol, and triglycerides (with similarly little effect on HDL cholesterol). Data for weight, BMI and blood pressure were scarce for modified fat trials, so data were not comparable.

- The stronger effect of modified fat diets over reduced fat diets in reducing serum lipids may relate to the apparently stronger effect of modified fat diets than reduced fat diets on cardiovascular events. It is not clear from the included studies whether this is due to the greater intrinsic effect of modified fat diets on cardiovascular events, or that modified fat diets are easier to comply with than reduced fat diets.

\section{Summary of main results}

This review suggested that dietary fat manipulation (reduction and/or modification) may be protective of cardiovascular events, reducing them by $14 \%$ ( $\mathrm{RR} 0.86,95 \% \mathrm{Cl} 0.77$ to $0.96,24$ comparisons, 65,614 participants, with marginal heterogeneity, Analysis 1.3). This was moderate GRADE evidence (See summary of findings, Figure 5). Subgrouping suggested that this reduction in cardiovascular events was due to studies of fat modification, or fat modification and reduction (not studies of fat reduction alone), seen in studies of at least two years duration, in studies of men (and not those of women), and in those with moderate or high cardiovascular risk at baseline (not general population groups).

Despite a large number of participants involved in randomised controlled trials of at least six months duration there were no clear effects of dietary fat changes on total mortality (RR 0.98, 95\% Cl 0.93 to $1.04,21$ comparisons, 71,795 participants, without important heterogeneity, Analysis 1.1) or cardiovascular mortality (RR 0.94, $95 \% \mathrm{Cl} 0.85$ to $1.04,16$ comparisons, 65,983 participants, without important heterogeneity, Analysis 1.2). This did not alter with subgrouping or sensitivity analysis, and was moderate GRADE evidence (Figure 5). 
Figure 7. Summary of findings, reduced or modified dietary fat vs usual diet, important secondary outcomes (myocardial infarction, stroke, cancer diagnosis)

\begin{tabular}{|c|c|c|c|c|c|}
\hline \multicolumn{6}{|c|}{$\begin{array}{l}\text { Patient or population: patients with preventing cardiovascular disease } \\
\text { Settings: (secondary outcomes) } \\
\text { Intervention: fat modification or reduction } \\
\text { Comparison: usual diet }\end{array}$} \\
\hline$\overline{\text { Outcomes }}$ & $\begin{array}{l}\text { Illustrative cor } \\
\text { Assumed risk } \\
\text { usual diet }\end{array}$ & $\begin{array}{l}\text { parative risks }{ }^{\star}(95 \% \mathrm{Cl}) \\
\text { Corresponding risk } \\
\text { fat modification or reduction }\end{array}$ & $\begin{array}{l}\text { Relative effect } \\
(95 \% \mathrm{CI})\end{array}$ & $\begin{array}{l}\text { No of Participants } \\
\text { (studies) }\end{array}$ & $\begin{array}{l}\text { Quality of the evidence } \\
\text { (GRADE) }\end{array}$ \\
\hline \multirow{4}{*}{$\begin{array}{l}\text { Myocardial infarctions } \\
\text { Follow-up: } 0.5 \text { to } 8.1 \text { years }\end{array}$} & \multicolumn{2}{|c|}{ Study population } & \multirow{4}{*}{$\begin{array}{l}\text { RR } 0.92 \\
-(0.84 \text { to } 1.02)\end{array}$} & \multirow{4}{*}{$\begin{array}{l}64997 \\
\text { (20 studies) }\end{array}$} & \multirow{4}{*}{$\begin{array}{l}\text { ニニニニ } \\
\text { moderate }\end{array}$} \\
\hline & 32 per 1000 & $\begin{array}{l}29 \text { per } 1000 \\
(27 \text { to } 33)\end{array}$ & & & \\
\hline & \multicolumn{2}{|c|}{ Medium risk population } & & & \\
\hline & 49 per 1000 & $\begin{array}{l}45 \text { per } 1000 \\
(41 \text { to } 50)\end{array}$ & & & \\
\hline \multirow{4}{*}{$\begin{array}{l}\text { Stroke } \\
\text { Follow-up: } 0.5 \text { to } 8.1 \text { years }\end{array}$} & \multicolumn{2}{|c|}{ Study population } & \multirow{4}{*}{$\begin{array}{l}\text { RR } 0.99 \\
-(0.89 \text { to } 1.11)\end{array}$} & \multirow{4}{*}{$\begin{array}{l}59959 \\
\text { (12 studies) }\end{array}$} & \multirow{4}{*}{$\begin{array}{l}\text { ニニニニ } \\
\text { moderate }\end{array}$} \\
\hline & 20 per 1000 & $\begin{array}{l}20 \text { per } 1000 \\
\text { (18 to } 22)\end{array}$ & & & \\
\hline & \multicolumn{2}{|c|}{ Medium risk population } & & & \\
\hline & 19 per 1000 & $\begin{array}{l}19 \text { per } 1000 \\
(17 \text { to } 21) \\
\end{array}$ & & & \\
\hline \multirow{4}{*}{$\begin{array}{l}\text { Cancer diagnoses } \\
\text { Follow-up: } 0.5 \text { to } 11 \text { years }\end{array}$} & \multicolumn{2}{|c|}{ Study population } & \multirow{4}{*}{$\begin{array}{l}\text { RR } 0.96 \\
-(0.91 \text { to } 1.01)\end{array}$} & \multirow{4}{*}{$\begin{array}{l}58847 \\
\text { (12 studies) }\end{array}$} & \multirow{4}{*}{$\begin{array}{l}\text { ニニニニ } \\
\text { moderate }\end{array}$} \\
\hline & 106 per 1000 & $\begin{array}{l}102 \text { per } 1000 \\
(96 \text { to } 107) \\
\end{array}$ & & & \\
\hline & \multicolumn{2}{|c|}{ Medium risk population } & & & \\
\hline & 51 per 1000 & $\begin{array}{l}49 \text { per } 1000 \\
(46 \text { to } 52) \\
\end{array}$ & & & \\
\hline \multirow{2}{*}{\multicolumn{6}{|c|}{$\begin{array}{l}{ }^{2} \text { The basis for the assumed risk (e.g. the medi } \\
\text { is based on the assumed risk in the compariso } \\
\text { Cl: Confidence interval; RR: Risk ratio; }\end{array}$}} \\
\hline & & & & & \\
\hline \multicolumn{6}{|c|}{$\begin{array}{l}\text { GRADE Working Group grades of evidence } \\
\text { High quality: Further research is very unlikely to change our confidence in the estimate of effect. } \\
\text { Moderate quality: Further research is likely to have an important impact on our confidence in the estimate of effect and may change the estimate. } \\
\text { Low quality: Further research is very likely to have an important impact on our confidence in the estimate of effect and is likely to change the estimate. } \\
\text { Very low quality: We are very uncertain about the estimate. }\end{array}$} \\
\hline \multicolumn{6}{|c|}{$\begin{array}{l}1 \text { Problems in this set of dietary trials include a large proportion of comparisons were not blinded, and most have differential levels of attention and support to the } \\
\text { intervention groups, as well as dietary advice or changes additional to the fat intervention. It is also difficult to ascertain the true dietary changes achieved (as most rely } \\
\text { on self-reported dietary assessment or records). Despite this the data are remarkably consistent. }\end{array}$} \\
\hline \multicolumn{6}{|c|}{$\begin{array}{l}{ }^{2} \text { Fixed effects analysis produced similar results (RR } 0.93,95 \% \mathrm{Cl} 0.86 \text { to } 1.02 \text { ). Any effects appeared to arise from modified fat }(\mathrm{RR} 0.90,95 \% \mathrm{Cl} 0.71 \text { to } 1.15 \text { ) and } \\
\text { reduced and modified fat diets (RR } 0.90,95 \% \mathrm{Cl} 0.0 .72 \text { to } 1.11 \text { ), rather than reduced fat diets (RR } 0.97,95 \% \mathrm{Cl} 0.86 \text { to } 1.08 \text { ). }\end{array}$} \\
\hline \multicolumn{6}{|c|}{$\begin{array}{l}3 \text { Fixed effects analysis produced similar results ( } R R 0.99,95 \% \mathrm{Cl} 0.88 \text { to } 1.10 \text { ). There were no clear effects in studies of reduced fat diets (RR } 1.01,95 \% \mathrm{Cl} 0.90 \text { to } \\
1.13 \text { ) and there were insufficient events to understand effects of modified ( } \mathrm{RR} 0.81,95 \% \mathrm{Cl} 0.40 \text { to } 1.66 \text { ) or reduced and modified fat diets (RR } 0.40,95 \% \mathrm{Cl} 0.08 \text { to } \\
2.03 \text { ). } \\
{ }^{4} \text { Fixed effects analysis produced similar results ( } R R 0.96,95 \% \mathrm{Cl} 0.91 \text { to } 1.01 \text { ). Almost all data were provided by studies of reduced fat diets (RR } 0.95,95 \% \mathrm{Cl} 0.88 \text { to } \\
1.02 \text { ). }\end{array}$} \\
\hline
\end{tabular}

\section{Overall completeness and applicability of evidence}

The review included adult participants at varying levels of risk of cardiovascular disease, men and women, in free-living and institutional settings, and across the past 50 years. Almost all the studies were conducted in industrialised countries, and almost no data were available from developing or transition countries. The effectiveness of reduced fat diets and modified fat diets have been well assessed (trials of at least 6 months included 62,000 and 13,000 participants respectively), but studies of the combined diet (reduced and modified fat) included fewer than 5000 participants. Over 4000 participants in the included trials died, 1400 died of a cardiovascular cause, and almost 5000 experienced at least one cardiovascular event.

Overall the external validity of the review in industrialised countries, men and women, people with low, moderate and high risk of cardiovascular disease was high, but it is not clear how this evidence relates to diets in developing and transition countries.

\section{Quality of the evidence}

All 48 trials and 60 comparisons included were randomised controlled trials, allocation concealment was judged well done in 26 comparisons, and 11 had adequate participant and study personnel blinding (difficult and expensive in dietary trials), see Figure 1. Ten comparisons ensured compete outcome data through checking of medical records or death registers, and we attempted to contact all trialists to ascertain deaths and cardiovascular events (that were often not important outcomes for the original studies). Just under half of the included comparisons (29) were free of systematic differences in care between study arms, and 39 were free of dietary changes other than those relating to dietary fat. 
The lack of blinding in most dietary trials is unlikely to alter outcome assessment when outcomes include death and cardiovascular events, but lack of blinding in the participants may have lead those in the control groups to alter their lifestyle and dietary practices (for example, feeling that they have not been helped to reduce their cardiovascular risk, they may act to reduce their own risk by altering other lifestyle behaviours such as smoking or exercise, leading to a potential lessening of the apparent effect of the dietary intervention). Systematic differences in care between arms may have lead to intervention groups receiving additional support in areas like self-efficacy and gaining support from new social circles, potentially beneficial to health regardless of dietary fat intake, or gaining additional health care professional time, possibly leading to earlier diagnosis and treatment of other risk factors such as raised blood pressure. Additional dietary messages (such as those around fruit and vegetable intake, fibre, alcohol and sugars), present in many studies, may have been protective, or may have diluted the effect and/or attainability of the fat goals.

This suggests that the evidence base for the dietary effects of changes in dietary fats on cardiovascular risk is not ideally valid, although it is large. In light of the uncertainty over allocation concealment, lack of blinding and presence of systematic differences in care and additional dietary differences between arms, the quality of evidence cannot be described as high (Figure 2). On the other hand, the scale and consistency of the evidence across studies and across decades, with very different designs and design flaws may make up for these deficits to some extent. For this reason, it is likely that the review provides robust conclusions on the effects of dietary fat modification in industrialised countries.

\section{Complex interventions}

With complex interventions, such as dietary ones, there are additional questions that need to be asked about included studies. Important issues to consider include defining the intervention, searching for and identifying all relevant studies, selecting studies for inclusion and data synthesis (Lenz 2007; Sheppherd 2009).

For this review we have worked to define the interventions clearly (see Characteristics of studies table), providing information on the type of intervention, stating the study aims and methods for each arm and the assessed total and saturated fat intakes attained within the study. However, while we have characterised the interventions (for example, sub-grouping studies into those that aimed to reduce, modify or reduce and modify dietary fat) no two studies that reduced dietary fat had exactly the same dietary goals for the intervention groups. Methods of attaining the dietary goals varied from providing a whole diet over several years (in studies based in institutions); to providing shops that sold either full or low fat versions of foods depending on the intervention or control status of the participant; to providing advice on diet alongside supplementary foods such as margarines or oils; to providing dietary advice with or without supplementary support in the way of group sessions, cooking classes, shopping tours, feedback, self-efficacy sessions and/or individual counselling. We aimed to use this variety in helping us to understand which dietary fat interventions are more useful, by using sub grouping (by reduced or modified fat aim, and by food provided or dietary advice for example) and meta-regression (on study duration and the difference between intervention and control arms in saturated or monounsaturated fat intake for example).
We addressed identifying all the relevant studies through use of a wide search strategy, that was time consuming. However, we believe that we have included most relevant trials. We also carefully defined acceptable interventions for each arm, so that decisions on inclusion were simpler, and the two independent assessors more often agreed. Data synthesis was augmented by sub-grouping and meta-regression to help us understand the effects of individual elements of dietary fat changes.

A study that sets out to assess the effect of a $30 \%$ reduction in saturated fat intake may attain this level of reduction in some participants, exceed it in some and not achieve it at all in others. The actual mean change attained in the intervention group may be less dramatic than that aimed for, and the participants in the control group may also reduce their saturated fat intake by a small amount, reducing the difference in saturated fat between the groups further and so reducing the scale of any outcome. This can be dealt with in the systematic review if we meta-regress the difference in saturated fat intake between the intervention and control group with the scale of the outcome (assuming a linear dose response), still allowing us to understand the effect of altering saturated fat intake. However, there is also a problem of measuring actual saturated fat intake achieved - some trials did not report this (whether because they did not assess it, or did assess it and were embarrassed by the results or simply didn't have space to report this relatively uninteresting outcome), and others did report the results of asking people what they are eating (using a food frequency questionnaire or several 24 hour food recalls). However, there is good evidence to believe that asking people how they are eating may produce somewhat biased information (Kristal 2005; Schatzkin 2003), and this may be a greater problem where the participant has been recently urged to eat in a particular way, as in a dietary trial.

A better solution to measuring the saturated fat intake achieved individually and as a mean in each study arm may be use of a biomarker of saturated fat intake. Our understanding of the relationship between dietary saturated fat intake and serum cholesterol has been quite clear since Hegsted's work in 1965 (Hegsted 1965). Meta-analysis by Mensink and Katan provided even more specific relationships between serum HDL and LDL cholesterol and triglycerides and changes in saturated, polyunsaturated and monounsaturated fats and carbohydrate from highly controlled short term studies (Mensink 1992). Unless long term dietary fat changes differ from these short term effects, understanding the changes in serum lipids in the trials included in this systematic review should allow us to understand the dietary fat changes. However, unfortunately few studies that experienced substantial numbers of deaths or cardiovascular events also report HDL, LDL or triglycerides (most older studies do not even report the composite serum total cholesterol). This has made it very difficult to understand whether lack of effect of dietary fat changes on total and cardiovascular mortality was due to minimal differences in fat intake between the control and intervention arms, or because such changes make no difference to mortality.

\section{Potential biases in the review process}

In compiling the included studies we worked hard to locate randomised studies that altered dietary fat for at least 6 months, even when cardiovascular events were not reported in study publications, or where such events were reported incidentally as reasons for participant drop outs. We attempted to contact all 
authors of potential studies to verify the presence, or not, of our outcomes. In many included studies no outcomes relevant to this review occurred within the participants, and the numbers of events occurring within single studies varied from none to over 2000 deaths, over 500 cardiovascular deaths, and over 3000 cardiovascular events (all within the WHI trial, the largest single study with almost 50,000 participants for many years). The addition of the WHI trial (WHI with CVD 2006, WHI without CVD 2006), despite its size, did not alter the results substantially from the previous review, and did not introduce heterogeneity to the review.

The number of cardiovascular deaths was relatively small, so while we can be quite confident in reporting a reduction in cardiovascular events with dietary fat intervention (especially fat modification), and a lack of effect on total mortality, the effect on cardiovascular mortality is less clear. The relative risk of 0.94 (95\% Cl 0.85 to 1.04$)$ may translate into a small protective effect, however this is unclear. The lack of effect on individual cardiovascular events is harder to explain - there were over 2000 myocardial infarctions, over 1000 strokes and non-fatal MI, almost 3000 cancer deaths, over 3000 diabetes diagnoses and 6000 cancer diagnoses. Lack of clear effects on any of these outcomes is surprising given the effects on total cardiovascular events.

The funnel plots are difficult to interpret (Figure 3, Figure 4) but there is some suggestion that there may be some trials of reduced fat interventions with higher relative risks missing, and some of modified fat interventions with lower relative risks missing (there are too few studies of reduced and modified interventions to tell). If additional studies of reduced fat diets with higher relative risks were found this would not alter the lack of effect of reduced fat diets on total mortality or cardiovascular events. If further modified fat studies were found these would be unlikely to alter the lack of effect of modified fat on mortality, but may strengthen its preventive effect on cardiovascular events.

\section{Agreements and disagreements with other studies or reviews}

All of the studies included in this review, whether they reduced or modified dietary fat, aimed to reduce saturated fat intake. Overall reduction and/or modification of dietary fat had no effect on total mortality or cardiovascular mortality but did appear to reduce cardiovascular events by $14 \%$ (although effects on myocardial infarction and stroke individually were not clear). This result was rather different from those of Siri-Tarino 2010 who systematically reviewed cohort studies that assessed relationships between saturated fat and cardiovascular events. They included 21 studies and did not find statistically significant associations between saturated fat intake and cardiovascular disease (RR 1.0, $95 \% \mathrm{Cl} 0.89$ to 1.11). As with our review they found no relationship between saturated fat intake and coronary heart disease (RR 1.07, $95 \% \mathrm{Cl} 0.96$ to 1.19 ) or stroke (RR $0.81,95 \% \mathrm{Cl} 0.62$ to 1.05 ).

In our review there was no effect of reducing total fat on total or cardiovascular mortality or cardiovascular events. This was also observed in a systematic review of observational studies. Skeaff 2009 included 28 US and European cohorts (including 6600 coronary heart disease deaths among 280000 participants) finding that total fat intake was not significantly associated with coronary heart disease mortality (RR $0.94,95 \% \mathrm{Cl} 0.74$ to 1.18 ) or coronary heart events (RR $1.02,95 \% \mathrm{Cl} 0.98$ to 1.05 ). Skeaff 2009 also partly updated the previous version of this review, adding in the Finnish Mental Hosp 1972 studies (excluded from both versions of this review as this was a cluster randomised study with only 2 clusters, and also because it carried out a crossover study, inappropriate in a progressive condition such as cardiovascular disease) and the Women's Health Initiative (WHI without CVD 2006; WHI with CVD 2006), the largest of the studies published between the versions of our review. They also found that studies reducing total fat had no effect on cardiovascular mortality or events. They also observed a marginally significant relationship between $\mathrm{P} / \mathrm{S}$ (polyunsaturated / saturated fat) ratio and cardiovascular events, but no relationship with cardiovascular mortality (similar to our suggestion that modifying dietary fat reduces cardiovascular events by $18 \%$, without an important effect on total or cardiovascular mortality).

While we found in this review that replacing saturated with unsaturated fats appeared to be beneficial in terms of cardiovascular events, it was not clear whether replacing saturated fats by polyunsaturated or monounsaturated fats, either or both, was beneficial. Meta-regression did not suggest any significant relationship between either polyunsaturated or monounsaturated fats and cardiovascular events in this review. A recent review by Mozaffarian 2010, which again included very similar studies to the last version of this review, with the Finnish Mental Hospital study and Women's Health Initiative data added, stated that their findings provided evidence that consuming polyunsaturated fat in place of saturated fat would reduce coronary heart disease. However, their evidence for this was limited and circumstantial, as they found that modifying fat reduced the risk of myocardial infarction or coronary heart disease death (combined) by 19\% (similar to our result). As the mean increase in polyunsaturated fat in these studies was $9.9 \%$ of energy they infer an effect of increasing polyunsaturated fat by $5 \%$ of energy of $10 \%$ reduction in risk of myocardial infarction or coronary heart disease death. They provided no suggestion or evidence of a relationship between degree of polyunsaturated fat increase and level of risk reduction.

Within the meta-regression we hoped to combine studies that effectively altered saturated fat by different degrees (so that studies that reduced saturated fat very little and studies that reduced it a great deal would all offer data points for the metaregression against mortality and morbidity endpoints, and similarly for total fat, polyunsaturated, monounsaturated and trans fats). Unfortunately many of the included studies did not report data on assessed dietary intake during the trial, reducing the quantity of data available to understand the relationships. Another limitation in understanding effects of individual classes of fatty acids on mortality and morbidity (in both trials and observational studies) was our ability to correctly assess participant's intake. We could overcome this by using biomarkers such as serum LDL cholesterol (differences between the LDL concentration in the intervention and control arms could be seen as a reasonable and independent approximation of saturated fat intake), however as many studies were carried out in the 1960s to 1990s few measured and reported LDL cholesterol. We tried using meta-regression with serum total cholesterol (although this is a composite marker and so less related to saturated fat intake), but although this was available for more studies that LDL it was still not available for all studies. It should be noted that the meta-regression with the smallest $P$ value (less than 0.1 ) was the effect of difference in serum LDL cholesterol between intervention and control arms and cardiovascular events. 
Pooled results of dietary fat trials indicate that reduction or modification of dietary fat intake does significantly reduce the incidence of combined cardiovascular events. The effect is consistent with a benefit as large as a $23 \%$ reduction, or as small as a $4 \%$ reduction, with a best estimate of $14 \%$ reduction in events (the first version of this review suggested an effect of $16 \%$ with slightly wider confidence intervals). This effect is seen almost exclusively in those who continue to modify their diet over at least two years. Duration of intervention does appear to be crucial. In the 4S trial (4S 1994) 4,444 participants were followed for roughly 19,339 person-years of observation, a mean of 4.35 years each. The Kaplan-Meier curve for all-cause mortality for the $4 \mathrm{~S}$ trial only showed a clear separation between the two randomisation groups at roughly two years. For this reason trials within the systematic review were grouped in the first version of this review into those with a mean follow-up of two years or less, and those with mean follow-up of more than two years. In our meta-analysis, studies of at least two years duration reduced the risk of cardiovascular events (by 22\%) while studies shorter than 2 years did not (best estimate of effect was a reduction in risk of $5 \%$ ), Analysis 7.17 . The trials with follow-up times from 6 months to 2 years may be diluting the effect of the trials with more than two years follow-up in the overall meta-analysis. Despite this meta-regression of study duration vs. cardiovascular events does not suggest a statically significant relationship. This may be due to confounding effect of the diet being less rigorously adhered to over longer durations, but we have no evidence of this.

Total mortality was examined as it is an important outcome, and there is little likelihood of ascertainment or diagnostic bias which may occur with cause-specific event outcomes. The data follow a similar trend, with no effect in the shorter trials and a suggestion of benefit in the trials of more than two years, but here the trend is not significant (the rate ratio for total mortality was 0.98 overall in the 2000 version of this review, and the relative risk is 0.98 in this version), with a relative risk of 1.05 in trials with mean followup of two years or less, 0.97 in trials with a mean follow-up of more than two years). This pattern suggests that the effects of dietary fat modification will take time to manifest themselves, and there is little evidence of immediate effects of fat reduction and/ or modification on factors such as thrombosis. The main effects of dietary fat reduction and modification are likely to be on the scale and type of atherosclerotic plaque, but other mechanisms may be operating.

\section{Participants level of risk}

As the rate of events is higher in high risk groups (by definition), it should require smaller sample sizes and shorter follow up to observe an effect of an intervention in a high risk group of participants (Davey Smith 1993). There have been suggestions that randomised controlled trials are unsuitable for assessing the effectiveness of interventions with very modest levels of effect in low risk populations, because of the huge numbers of person-years of observation needed to gain sufficient statistical power to avoid Type II errors (Ebrahim 1997). However, with the publication of the Women's Health Intervention trial (WHI with CVD 2006; WHI without CVD 2006) we now have data on more cardiovascular events in people at low risk of cardiovascular disease (3408 events) than in people with moderate (143 events) or high risk (1336 events). The same is true for cardiovascular deaths ( 879,23 and 505 respectively) and total mortality (3717, 47, and 528 deaths respectively). Given reasonable sized data sets for those at both high and low risk of cardiovascular disease, a similar level of risk reduction of combined cardiovascular events was seen in high and low risk groups (a 7\% reduction in risk, or RR 0.93), but this effect only reaches statistical significance in the high risk participants. The reason for this is unclear.

When endpoints such as total mortality are used the situation becomes more difficult as in low risk groups the proportion of deaths which are unrelated to cardiovascular disease (and perhaps unlikely to be influenced by dietary fat changes) rises, again diluting any differences in the numbers of deaths between intervention and control groups. It is more likely that significant changes in cardiovascular deaths will be seen than in total mortality. The trend is certainly in this direction (pooled relative risk for total mortality $0.98,95 \% \mathrm{Cl} 0.93$ to 1.04 , for cardiovascular mortality 0.94 , $95 \% \mathrm{Cl} 0.85$ to 1.04$)$. Our best estimate is that dietary fat reduction and modification result in a reduction of $6 \%$ in deaths due to cardiovascular disease, and a reduction of $2 \%$ in total deaths, but the confidence intervals are wide.

The high risk participants in the dietary fat trials all show evidence of cardiovascular disease at baseline. Under current guidelines most high risk participants with raised lipid levels should be on lipid lowering medication (ACC/AHA 2008; Fraker 2007; NICE 2006). This raises the question of whether there is any additional advantage of adherence to a low or modified fat diet in addition to statin therapy. Little evidence exists at present to answer this question. However, in all parts of the world where drug budgets are restricted and use of lipid lowering medication remains rationed even for those at high risk the use of modified fat diets would appear to be a cost-effective option leading to considerable reductions in cardiovascular events for populations (and so in health budgets) in only a few years.

Low risk participants are unlikely to be on lipid lowering medication under current guidelines. The suggestion of protection of this group from cardiovascular events, with a reduction of roughly $7 \%$ of events, by dietary fat modification (even though this does not reach statistical significance, but taking into account our best estimate) would appear to merit continued public health action.

\section{Low fat or modified fat diets}

An individuals dietary intake is a complex mixture of foods, each of which is a complex mixture of nutrients. Altering one dietary component leads to unintentional alterations in many others, each of which may have positive or negative effects on several risk factors and, eventually, health.

The fat interventions included in this review were low fat diets (where total fat is reduced, and energy is usually replaced by increasing carbohydrate intake), modified fat diets (where a proportion of saturated fat is replaced by unsaturated fats, and total fat intakes do not alter) and combinations of the two (with some fat reduction and some replacement with unsaturates). Modified fat diets appear to have rather better effects on major cardiovascular risk factors than reduced fat diets in indirect comparison, but not in direct comparisons (where the numbers of studies and participants are small). The indirect comparison data fits with data from Mensink 1992, suggesting that low fat, high carbohydrate diets are likely to result in higher triglyceride and lower HDL cholesterol levels than diets where saturated fats are wholly replaced by unsaturated fats, but direct comparisons will be important in confirming this. 
It is important that individuals and populations are receiving clear, evidence-based advice about the types of dietary fat changes which are most effective in reducing cardiovascular risk, as well as ways to achieve those changes. Further research comparing low fat and modified fat changes on cardiovascular disease risk factors would be feasible and helpful.

This review suggests that modified fat intake, or modified and reduced fat intake combined (but not reduced fat intake alone) are protective against combined cardiovascular events. No clear effects of these interventions on total or cardiovascular mortality were seen.

\section{AUTHORS' CONCLUSIONS}

\section{Implications for practice}

Dietary change to reduce saturated fat and partly replace it with unsaturated fats appears to reduce the incidence of cardiovascular events, but replacing the saturated fat with carbohydrate (creating a low fat diet) was not clearly protective of cardiovascular events (despite small improvements in weight, body mass index, total and LDL cholesterol). The protective effect was seen almost exclusively in those who continue to modify their diet over at least two years, and in studies of men (not those of women). Dietary advice to those at high risk of cardiovascular disease (particularly where lipid lowering medication may not be available), and probably also to lower risk population groups, should continue to include dietary fat modification, possibly as part of a Mediterranean dietary pattern, and it should be stressed that this is a permanent pattern of eating.

Effects on total and cardiovascular mortality are much less clear. No evidence was found on the long term health effects of altering trans fat intake.

\section{Implications for research}

Long term research to help us understand what types of unsaturated fats are most useful in the diet when replacing saturated fats (monounsaturated fats, polyunsaturated fats and the relevant specific fatty acids) are urgently needed.

The financial implications (costs and savings) of appropriate advice and legislation to modify fat intake in those at various levels of cardiovascular risk should be assessed and reflected in health policy. Whilst interventions to alter dietary fat intake in individuals at high cardiovascular risk have been fairly successful, such health promotion initiatives in the general population have been less successful. Further work is needed to help high and low risk individuals to make effective changes to dietary fat and to maintain these changes over their lifetimes. Research into the effects of legislation to alter fat contents of foods, improved labelling, pricing initiatives and improved availability of healthier foods, linking food production and processing into the health agenda may yield huge advances in this area.

It is not clear whether there is additional benefit of modifying dietary fat in those at high risk of cardiovascular disease who are on lipid lowering medication. Further research to examine the need for maintenance of dietary fat modification whilst on lipid lowering medication would be useful, but not as useful as understanding specific dietary fat replacements for saturated fat.
No trials assessing the long term health implications of reducing trans fat intake were found, and most trials did not report trans fat intake in intervention and control arms, so the evidence on long term impact of trans fat alteration rests on intermediate outcomes only. Long term trials of reductions in trans fats would be helpful to clarify effects on cardiovascular health and mortality.

\section{ACKNOWLEDGEMENTS}

The help of the following investigators in providing information about their own and others trials is gratefully acknowledged: H Arnesen (Ullevål University Hospital), AV Astrup (University of Copenhagen), K Aziz (NICVD, Karachi), F Azizi (Research Institute for Endocrine Sciences, Tehran), SAA Beresford (University of Washington), HS Black (Baylor College of Medicine), BPM Bloemberg (National Institute of Public Health and Environmental Protection, Netherlands), DJ Bowen (Fred Hutchinson Cancer Research Center, Seattle), NF Boyd (University of Toronto), GA Bray (Pennington Biomedical Research Center, Baton Rouge), ML Burr (University of Wales), G Carruba (University of Palermo), L Castagnetta (University of Palermo), JL Curzio (University of Glasgow), RF DeBusk (Stanford University), C Defoort (Méditerranée University), Z Djuric (Wayne State University), A Due (University of Copenhagen), S Druker (University of Massachusetts), RPF Dullaart (University Hospital, Groningen), GE Eyssen (University of Toronto), TM Hayes (University Hospital of Wales, Cardiff), JA Heady (retired, formerly of MRC Social Medicine Research Unit), J Hebert (University of South Carolina), RJ Heine (Free University Hospital, Amsterdam), M-L Hellenius (Karolinska Institute), RF Heller (University of Newcastle), TDR Hockaday (retired, formerly Radcliffe Infirmary), L-E Holm (Swedish Radiation Protection Institute), DJ Hyman (Baylor College of Medicine, Houston), AFM Kardinaal (University of Wageningen), F Khan (Ninewells Hospital and Medical School, Dundee), RH Knopp (University of Washington), D Lairon (Méditerranée University), MEJ Lean (University of Glasgow), B Leelarthaepin (University of Sydney), P Leren (University of Oslo), A Lindman (University of Oslo), S Mackey (Stanford University), R MacLennan (retired, formerly of Queensland Institute of Medical Research), F Macrae (Royal Melbourne Hospital), JI Mann (University of Otago), J Marniemi (Social Insurance Institute), K McManus (Harvard Medical School), RP Mensink (Maastricht University), PA Metcalf (University of Aukland), A Michalsen (University Duisburg-Essen), TF Moy (Johns Hopkins University), AR Ness (University of Bristol), I Okene (University of Massachusetts), GS Oostenbrug (Maastricht University), J Pierce (University of California, San Diego), SD Poppitt (University of Aukland), RJ Reber (University of Illinois), JE Reseland (University of Oslo), BM Retzlaff (University of Washington), AA Rivellese (Federico II University, Naples), P Roderick (University of Southampton), DP Rose (American Health Foundation), FM Sacks (Harvard School of Public Health), WHM Saris (University of Maastricht), ES Sarkkinen (University of Kuopio), A Schatzkin (National Cancer Institute), B Seppelt (German Institute of Human Nutrition), MS Simon (Wayne State University), B Smith (University of Kentucky), E Søndergaard (Svendborg Hospital, Svendborg), AS St. Leger (University of Manchester), VJ Stevens (Kaiser Permanente Centre for Health Research), A Stoddard (University of Massachusetts), LP Svetkey (Duke University Medical Center), LC Tapsell (University of Wollongong), BC Tilley (Medical University of South Carolina), H van den Berg (TNO Nutrition and Food Research Institute), W van Herpen (Unilever), $\mathrm{K}$ van het Hof (Unilever, Rotterdam), MW Verheijden 
(Wageningen University), GF Watts (University Hospital of Perth), AS Wierzbichi (St. Thomas's Hospital, London), PT Williams (Stanford University), RR Wing (University of Pittsburgh), PD Wood (Stanford University), I Zazpe (University of Navarra), PL Zock (Wageningen Centre for Food Studies).

The expertise and help of the following is also gratefully acknowledged: S Adams (Royal Free Hospital, London), B Anagnostelis (Royal Free Hospital, London), M Brand (Cochrane Hypertension Group), R Clarke (Universtiy of Oxford), D DarrahMorgan (Russian translation), A Donner (University of Western Ontario), D Fagard (University of East Anglia for duplication of inclusion and data extraction), Shweta Gidwani (University of Manchester), G Gubitz (Cochrane Stroke Group), M Haugh (Cochrane Renal Group), IU Haq (Northern General Hospital, Sheffield), J Hooper (Danish, Swedish and Norwegian translation), BK Hurley (Italian translation), L Jones (Systematic Reviews
Training Unit, London), SPH Keen (Cochrane Diabetes Group), S Logan (Systematic Reviews Training Unit, London), LI Mennen (INSERM), T Moore (Cochrane Heart Group), J Muscroft (German and French translation), HL Newmark (Rutgers), E Royle (Cochrane Peripheral Vascular Diseases Group), I Tumur (Pfizer Ltd), AS Truswell (University of Sydney), M Turner (Chinese translation), JM Walsh (University of California), A Wierzbicki (St. Thomas' Hospital, London), WC Willett (Harvard School of Public Health), AF Winder (University of London).

Many thanks to those people who were co-authors of the first version of this review: Julian PT Higgins, Shah Ebrahim, Rudolph Riemersma, Nigel E Capps and Gillian Clements, and to those reviewers who worked on study inclusion, data extraction and/or assessment of validity for the update but who are not authors (Indra Tumur and Dorotheé Fagard). 


\section{R E F E R E N C E S}

\section{References to studies included in this review}

Anderson 1990 \{published and unpublished data\}

* Anderson JW, Garrity TF, Smith BM, Whitis SE. Follow-up on a clinical trial comparing the effects of two lipid lowering diets. Arteriosclerosis 1990;10(5):882a.

Anderson JW, Garrity TF, Wood CL, Whitis SE, Smith BM, Oeltgen PR. Prospective, randomized, controlled comparison of the effects of low-fat and low-fat plus high-fiber diets on serum lipid concentrations. American Journal of Clinical Nutrition 1992;56(5):887-94.

\section{Azadbakht 2007 \{published and unpublished data\}}

Azadbakht L, Mirmiran P, Esmaillzadeh A, Azizi F. Better dietary adherence and weight maintenance achieved by a long-term moderate fat diet. British Journal of Nutrition 2007;97:399-404.

Ball 1965 \{published data only\}

Ball KP, Hanington E, McAllen PM, Pilkington TRE, Richards JM, Sharland DE, et al. Low-fat diet in myocardial infarction: A controlled trial. Lancet 1965;2(411):501-4.

\section{BDIT Pilot Studies 1996 \{published and unpublished data\}}

Boyd NF, Cousins M, Beaton M, Fishell E, Wright B, Fish E, et al. Clinical trial of low-fat, high-carbohydrate diet in subjects with mammographic dysplasia: report of early outcomes. Journal of the National Cancer Institute 1988;80:1244-8.

Boyd NF, Cousins M, Beaton M, Han L, McGuire V. Methodological issues in clinical trials of dietary fat reduction in patients with breast dysplasia. Progress in Clininical and Biological Research 1986;222:117-24.

Boyd NF, Cousins M, Beaton M, Kriukov V, Lockwood G, Tritchler D. Quantitative changes in dietary fat intake and serum cholesterol in women: results from a randomized, controlled trial. American Journal of Clinical Nutrition 1990;52(3):470-6.

Boyd NF, Cousins M, Kriukov V. A randomised controlled trial of dietary fat reduction: the retention of subjects and characteristics of drop outs. Journal of Clinical Epidemiology 1992;45(1):31-8.

Boyd NF, Cousins M, Lockwood G, Tritchler D. Dietary fat and breast cancer risk: the feasibility of a clinical trial of breast cancer prevention. Lipids 1992;27(10):821-6.

Boyd NF, Cousins M, Lockwood G, Tritchler D. The feasibility of testing experimentally the dietary fat-breast cancer hypothesis. Progress in Clinical and Biological Research 1990;346:231-41.

* Boyd NF, Martin LJ, Beaton M, Cousins M, Kriukov V. Longterm effects of participation in a randomized trial of a low-fat, high-carbohydrate diet. Cancer Epidemiology, Biomarkers \& Prevention 1996;5(3):217-22.

Lee-Han H, Cousins M, Beaton M, McGuire V, Kriukov V, Chipman $\mathrm{M}$, et al. Compliance in a randomized clinical trial of dietary fat reduction in patients with breast dysplasia. American Journal of Clinical Nutrition 1988;48(3):575-86.
beFIT 1997 \{published and unpublished data\}

Retzlaff BM, Walden CE, McNeney WB, Buck BL, McCann BS, Knopp RH. Nutritional intake of women and men on the NCEP Step I and Step II diets. Journal of the American College of Nutrition 1997;16(1):52-61.

* Walden CE, Retzlaff BM, Buck BL, McCann BS, Knopp RH. Lipoprotein lipid response to the National Cholesterol Education Program Step II diet by hypercholesterolemic and combined hyperlipidemic women and men. Arteriosclerosis, Thrombosis and Vascular Biology 1997;17:375-82.

Walden CE, Retzlaff BM, Buck BL, Wallick S, McCann BS, Knopp RH. Differential effect of National Cholesterol Education Program (NCEP) Step II Diet on HDL cholesterol, its subfractions, and apoprotein A-1 levels in hypercholesterolemic women and men after 1 year: the beFIT study. Arteriosclerosis, Thrombosis and Vascular Biology 2000;20(6):1580-7.

Black 1994 \{published and unpublished data\}

* Black HS, Herd JA, Goldberg LH, Wolf-JE J, Thornby JI, Rosen T, et al. Effect of a low-fat diet on the incidence of actinic keratosis. New England Journal of Medicine 1994;330(18):1272-5.

Black HS, Thornby JI, Wolf-JE J, Goldberg LH, Herd JA, Rosen T, et al. Evidence that a low-fat diet reduces the occurrence of non-melanoma skin cancer. International Journal of Cancer 1995;62(2):165-9.

Jaax S, Scott LW, Wolf-JE J, Thornby JI, Black HS. General guidelines for a low-fat diet effective in the management and prevention of nonmelanoma skin cancer. Nutrition and Cancer 1997;27(2):150-6.

Boyd 1988 \{published and unpublished data\}

Boyd NF, McGuire V, Shannon P, Cousins M, Kriukov V, Mahoney $L$, et al. Effect of a low-fat high-carbohydrate diet on symptoms of cyclical mastopathy. Lancet 1988;2(8603):128-32.

BRIDGES 2001 \{published and unpublished data\}

Hebert JR, Ebbeling CB, Olendzki BC, Hurley TG, Ma Y, Saal N, et al. Change in women's diet and body mass following intensive intervention for early-stage breast cancer. Journal of the American Dietetic Association 2001;101(4):421-31.

\section{CARMEN 2000 \{published and unpublished data\}}

Poppitt SD, Keogh GF, Prentice AM, Williams DEM, Sonnemans HMW, Valk EEJ, et al. Long-term effects of ad libitum low-fat, high-carbohydrate diets on body weight and serum lipids in overweight subjects with metabolic syndrome. American Journal of Clinical Nutrition 2002;75:11-20.

Raben A, Astrup A, Vasilaras TH, Prentice AM, Zunft H-JF, Formiguera $X$, et al. The CARMEN study [CARMEN-studiet]. Ugeskrift fur Laeger 2002;164(5):627-31.

Saris WHM, Astrup A, Prentice AM, Zunft FJF, Formiguera X. CARMEN Project: European multicentre study on the impact of dietary fat/CHO ratio and simple/complex $\mathrm{CHO}$ changes on 
long term weight control in overweight subjects. International Journal of Obesity 1997;21(Suppl 2):S71.

* Saris WHM, Astrup A, Prentice AM, Zunft HJF, Formiguera X, Verboeket-van de Venne WPHG, et al. Randomized controlled trial of changes in dietary carbohydrate/fat ratio and simple vs complex carbohydrates on body weight and blood lipids: the CARMEN study. International Journal of Obesity 2000;24:1310-8.

Vasilaras TH, Astrup A, Raben A. Micronutrient intake in overweight subjects is not deficient on and ad libitum fatreduced, high-simple carbohydrate diet. European Journal of Clinical Nutrition 2004;58:326-36.

\section{CARMEN MS sub-study 2002 \{published and unpublished data\}}

Poppitt SD, Keogh GF, Prentice AM, Williams DEM, Sonnemans HMW, Valk EEJ, et al. Long-term effects of ad libitum low-fat, high-carbohydrate diets on body weight and serum lipids in overweight subjects with metabolic syndrome. American Journal of Clinical Nutrition 2002;75:11-20.

\section{Curzio 1989 \{published and unpublished data\}}

Curzio JL, Kennedy SS, Elliott HL, Farish E, Barnes JF, Howie CA, et al. Hypercholesterolaemia in treated hypertensives: a controlled trial of intensive dietary advice. Journal of Hypertension. Supplement 1989;7(6):S254-5.

DART 1989 \{published and unpublished data\} Burr ML, Fehily AM. Fish and the heart. Lancet 1989;ii:1450-2.

* Burr ML, Fehily AM, Gilbert JF, Rogers S, Holliday RM, Sweetnam PM, et al. Effects of changes in fat, fish, and fibre intakes on death and myocardial reinfarction: diet and reinfarction trial (DART). Lancet 1989;2(8666):757-61.

Burr ML, Fehily AM, Rogers S, Welsby E, King S, Sandham S. Diet and reinfarction trial (DART): design, recruitment, and compliance. European Heart Journal 1989;10(6):558-67.

Burr ML, Holliday RM, Fehily AM, Whitehead PJ. Haematological prognostic indices after myocardial infarction: evidence from the diet and reinfarction trial (DART). European Heart Journal 1992;13(2):166-70.

Burr ML, Sweetham PM, Fehily AM. Diet and reinfarction [letter]. European Heart Journal 1994;15(8):1152-3.

Fehily AM, Vaughan-Williams E, Shiels K, Williams AH, Horner M, Bingham $G$, et al. The effect of dietary advice on nutrient intakes: evidence from the diet and reinfarction trial (DART). Journal of Human Nutrition \& Dietetics 1989;2(4):225-5.

\section{DO IT 2006 \{published and unpublished data\}}

Berstad P, Seljeflot I, Veierod MB, Hjerkinn EM, Arnesen H, Pedersen JI, et al. Supplementation with fish oil affects the association between very long-chain $n-3$ polyunsaturated fatty acids in serum non-esterified fatty acids and soluble vascular cell adhesion molecule-1. Clinical Science 2003;105(1):13-20.

Ellingsen I, Hjerkinn EM, Seljeflot I, Arnesen H, Tonstad S, Ellingsen I, et al. Consumption of fruit and berries is inversely associated with carotid atherosclerosis in elderly men.[Erratum appears in British Journal of Nutrition 2008;99(3):697]. British Journal of Nutrition 2008;99(3):674-81.

Ellingsen I, Seljeflot I, Arnesen H, Tonstad S, Ellingsen Ingrid, Seljeflot Ingebjorg, et al. Vitamin C consumption is associated with less progression in carotid intima media thickness in elderly men: A 3-year intervention study. Nutrition Metabolism \& Cardiovascular Diseases 2009;19(1):8-14.

Furenes EB, Seljeflot I, Solheim S, Hjerkinn EM, Arnesen H, Furenes EB, et al. Long-term influence of diet and/or omega-3 fatty acids on matrix metalloproteinase- 9 and pregnancyassociated plasma protein-A in men at high risk of coronary heart disease. Scandinavian Journal of Clinical \& Laboratory Investigation 2008;68(3):177-84.

* Hjerkinn EM, Abdelnoor M, Breivik L, Bergengen L, Ellingsen I, Seljeflot I, et al. Effect of diet or very long chain omega-3 fatty acids on progression of atherosclerosis, evaluated by carotid plaques, intima-media thickness and by pulse wave propagation in elderly men with hypercholesterolaemia. European Journal of Cardiovascular Prevention \& Rehabilitation 2006;13(3):325-33.

Hjerkinn EM, Seljeflot I, Ellingsen I, Berstad P, Hjermann I, Sandvik L, et al. Influence of long-term intervention with dietary counselling, long-chain n-3 fatty acid supplements, or both on circulating markers of endothelial activation in men with longstanding hyperlipidemia. American Journal of Clinical Nutrition 2005;81(3):583-9.

Lindman AS, Pedersen JI, Hjerkinn EM, Arnesen $\mathrm{H}$, Veierod MB, Ellingsen I, et al. The effects of long-term diet and omega-3 fatty acid supplementation on coagulation factor VII and serum phospholipids with special emphasis on the R353Q polymorphism of the FVII gene. Thrombosis \& Haemostasis 2004;91(6):1097-104.

Troseid M, Arnesen H, Hjerkinn EM, Seljeflot I. Serum levels of interleukin-18 are reduced by diet and n-3 fatty acid intervention in elderly high-risk men. Metabolism: Clinical \& Experimental 2009;58(11):1543-9.

Troseid M, Seljeflot I, Hjerkinn EM, Arnesen H. Interleukin-18 is a strong predictor of cardiovascular events in elderly men with the metabolic syndrome: synergistic effect of inflammation and hyperglycemia. Diabetes Care 2009;32(3):486-92.

\section{Due Low fat 2008 \{published and unpublished data\}}

Due A, Larsen TM, Hermansen K, Stender S, Holst JJ, Toubro S, et al. Comparison of the effects on insulin resistance and glucose tolerance of 6-mo high-monounsaturated-fat, lowfat, and control diets. American Journal of Clinical Nutrition 2008;87(4):855-62.

* Due A, Larsen TM, Mu H, Hermansen K, Stender S, Astrup A. Comparison of 3 ad libitum diets for weight-loss maintenance, risk of cardiovascular disease, and diabetes: a 6-mo randomized, controlled trial. American Journal of Clinical Nutrition 2008;88(5):1232-41.

Rasmussen LG, Larsen TM, Mortensen PK, Due A, Astrup A. Effect on 24-h energy expenditure of a moderate-fat diet high in monounsaturated fatty acids compared with that of a low-fat, 
carbohydrate-rich diet: a 6-mo controlled dietary intervention trial. American Journal of Clinical Nutrition 2007;85(4):1014-22.

Sloth B, Due A, Larsen TM, Holst JJ, Heding A, Astrup A, et al. The effect of a high-MUFA, low-glycaemic index diet and a low-fat diet on appetite and glucose metabolism during a 6month weight maintenance period. British Journal of Nutrition 2009;101(12):1846-58.

\section{Due Low vs Mod 2008 \{published and unpublished data\}}

Due A, Larsen TM, Hermansen K, Stender S, Holst JJ, Toubro S, et al. Comparison of the effects on insulin resistance and glucose tolerance of 6-mo high-monounsaturated-fat, lowfat, and control diets. American Journal of Clinical Nutrition 2008;87(4):855-62.

* Due A, Larsen TM, Mu H, Hermansen K, Stender S, Astrup A, et al. Comparison of 3 ad libitum diets for weight-loss maintenance, risk of cardiovascular disease, and diabetes: a 6 mo randomized, controlled trial. American Journal of Clinical Nutrition 2008;88(5):1232-41.

Rasmussen LG, Larsen TM, Mortensen PK, Due A, Astrup A. Effect on 24-h energy expenditure of a moderate-fat diet high in monounsaturated fatty acids compared with that of a low-fat, carbohydrate-rich diet: a 6-mo controlled dietary intervention trial. American Journal of Clinical Nutrition 2007;85(4):1014-22.

Sloth B, Due A, Larsen TM, Holst JJ, Heding A, Astrup A, et al. The effect of a high-MUFA, low-glycaemic index diet and a low-fat diet on appetite and glucose metabolism during a 6month weight maintenance period. British Journal of Nutrition 2009;101(12):1846-58

\section{Due Mod fat 2008 \{published and unpublished data\}}

Due A, Larsen TM, Hermansen K, Stender S, Holst JJ, Toubro S, et al. Comparison of the effects on insulin resistance and glucose tolerance of 6-mo high-monounsaturated-fat, lowfat, and control diets. American Journal of Clinical Nutrition 2008;87(4):855-62.

* Due A, Larsen TM, Mu H, Hermansen K, Stender S, Astrup A, et al. Comparison of 3 ad libitum diets for weight-loss maintenance, risk of cardiovascular disease, and diabetes: a 6mo randomized, controlled trial. American Journal of Clinical Nutrition 2008;88(5):1232-41.

Rasmussen LG, Larsen TM, Mortensen PK, Due A, Astrup A. Effect on 24-h energy expenditure of a moderate-fat diet high in monounsaturated fatty acids compared with that of a low-fat, carbohydrate-rich diet: a 6-mo controlled dietary intervention trial. American Journal of Clinical Nutrition 2007;85(4):1014-22.

Sloth B, Due A, Larsen TM, Holst JJ, Heding A, Astrup A, et al. The effect of a high-MUFA, low-glycaemic index diet and a low-fat diet on appetite and glucose metabolism during a 6month weight maintenance period. British Journal of Nutrition 2009;101(12):1846-58.

\section{Dullaart 1992 \{published and unpublished data\}}

Dullaart RP, Beusekamp BJ, Meijer S, Hoogenberg K, van DJ, Sluiter WJ. Long-term effects of linoleic-acid-enriched diet on albuminuria and lipid levels in type 1 (insulin-dependent) diabetic patients with elevated urinary albumin excretion. Diabetologia 1992;35(2):165-72.

Frenkiel 1986 \{published data only\}

Frenkiel PG, Lee DW, Cohen H, Gilmore CJ, Resser K, Bonorris GG, et al. The effect of diet on bile acid kinetics and biliary lipid secretion in gallstone patients treated with ursodeoxycholic acid. American Journal of Clinical Nutrition 1986;43(2):239-50.

Houtsmuller 1979 \{published data only\}

* Houtsmuller AJ, Zahn KJ, Henkes HE. Unsaturated fats and progression of diabetic retinopathy. Documenta Ophthalmologia 1979;48(2):363-71.

Houtsmuller AJ, van Hal-Ferwerda J, Zahn KJ, Henkes HE. Favourable influences of linoleic acid on the progression of diabetic micro- and macroangiopathy. Nutrition and Metabolism 1980;24(Suppl 1):105-18.

Houtsmuller AJ, van Hal-Ferwerda J, Zahn KJ, Henkes HE. Influence of different diets on the progression of diabetic retinopathy. Progress in Food Nutritition and Science 1980;4(5):41-6

\section{Lean 1997 \{published and unpublished data\}}

Han TS, Richmond P, Avenell A, Lean ME. Waist circumference reduction and cardiovascular benefits during weight loss in women. International Journal of Obesity and Related Metabolic Disorders 1997;21(2):127-34.

* Lean MEJ, Han TS, Prvan T, Richmond PR, Avenell A Weight loss with high and low carbohydrate $1200 \mathrm{kcal}$ diets in free living women. European Journal of Clinical Nutrition 1997;51(4):243-8

\section{Ley 2004 \{published and unpublished data\}}

* Ley SJ, Metcalf PA, Scragg RKR, Swinburn BA. Long-term effects of a reduced fat diet intervention on cardiovascular disease risk factors in individuals with glucose intolerance. Diabetes Research and Clinical Practice 2004;63:103-12.

Swinburn BA, Metcalf PA, Ley SJ. Long-term (5-year) effects of a reduced-fat diet intervention in individuals with glucose intolerance. Diabetes Care 2001;24(4):619-24.

Swinburn BA, Woollard GA, Chang EC, Wilson MR. Effects of reduced-fat diets consumed ad libitum on intake of nutrients particularly antioxidant vitamins. Journal of the American Dietetic Association 1999;99(11):1400-5.

McAuley 2005 \{published and unpublished data\}

* McAuley KA, Hopkins CM, Smith KJ, McLay RT, Williams SM, Taylor RW, et al. Comparison of a high-fat and high-protein diets with a high-carbohydrate diet in insulin-resistant obese women. Diabetologia 2005;48:8-16.

McAuley KA, Smith KJ, Taylor RW, McLay RT, Williams SM, Mann JI. Long-term effects of popular dietary approaches on weight loss and features of insulin resistance. International Journal of Obesity 2006;30:342-9. 
McKeown-Eyssen 1994 \{published and unpublished data\}

McKeown-Eyssen GE, Bright SE, Bruce WR, Jazmaji V. A randomized trial of a low fat high fibre diet in the recurrence of colorectal polyps. Journal of Clinical Epidemiology 1994;47(5):525-36.

\section{MeDiet 2002 \{published and unpublished data\}}

Carruba G, Granata OM, Pala V, Campisi I, Agostara B, Cusimano R, et al. A traditional Mediterranean diet decreases endogenous estrogens in healthy postmenopausal women. Nutrition and Cancer 2006;56(2):253-9.

* Castagnetta L, Granata OM, Cusimano R, Ravazzolo B, Liquori M, Polito L, et al. The Mediet Project. Annals of the New York Academy of Science 2002;963:282-9.

Granata OM, Traina A, Ramirez S, Campisi I, Zarcone M, Amodio R, et al. Dietary enterolactone affects androgen and estrogen levels in healthy postmenopausal women. Annals of the New York Academy of Science 2009;1155:232-6.

\section{Minnesota Coron men 1989 \{published data only\}}

Brewer ER, Ashman PL, Kuba K. The Minnesota Coronary Survey: composition of diets, adherence and serum lipid response [Abstract]. Circulation 1975;51 and 52(Suppl II):269.

Dawson EA, Gatewood LC. The Minnesota Coronary Survey: methodology and characteristics of the population [Abstract]. Circulation 1975;51 and 52(Suppl II):271.

* Frantz ID Jr, Dawson EA, Ashman PL, Gatewood LC, Bartsch GE, Kuba K, et al. Test of effect of lipid lowering by diet on cardiovascular risk. The Minnesota Coronary Survey. Arteriosclerosis 1989;9(1):129-35

Frantz ID, Dawson EA, Kuba K, et al. The Minnesota Coronary Survey: effect of diet on cardiovascular events and deaths [abstract]. Circulation 1975;51 and 52(Suppl II):4.

\section{Minnesota Coron women1989 \{published data only\}}

Brewer ER, Ashman PL, Kuba K. The Minnesota Coronary Survey: composition of diets, adherence and serum lipid response [Abstract]. Circulation 1975;51 and 52(Suppl II):269.

Dawson EA, Gatewood LC. The Minnesota Coronary Survey: methodology and characteristics of the population [Abstract]. Circulation 1975;51 and 52(Suppl II):271.

* Frantz ID Jr, Dawson EA, Ashman PL, Gatewood LC, Bartsch GE, Kuba K, et al. Test of effect of lipid lowering by diet on cardiovascular risk. The Minnesota Coronary Survey. Arteriosclerosis 1989;9(1):129-35.

Frantz ID, Dawson EA, Kuba K, et al. The Minnesota Coronary Survey: effect of diet on cardiovascular events and deaths [abstract]. Circulation 1975;51 and 52(Suppl II):4.

Moy 2001 \{published and unpublished data\} Moy TF, Yanek LR, Raqueno JV, Bezirdjian PJ, Blumenthal RS, Wilder LB, et al. Dietary counseling for high blood cholesterol in families at risk of coronary disease. Preventive Cardiology 2001;4(4):158-64.
MRC 1968 \{published and unpublished data\}

Ederer F, Leren P, Turpeinen O, Frantz ID Jr. Cancer among men on cholesterol lowering diets: experience of five clinical trials. Lancet 1971;2:203-6.

Heady JA. Are PUFA harmful?. British Medical Journal 1974;1(898):115-6.

* MRC. Controlled trial of soya-bean oil in myocardial infarction. Lancet 1968;2(570):693-9.

MSFAT 1997 \{published and unpublished data\}

Velthuis-te WE, van Leeuwen REW, Hendriks HF, Verhagen $\mathrm{H}$, Loft S, Poulsen $\mathrm{HE}$, et al. Short-term moderate energy restriction does not affect indicators of oxidative stress and genotoxicity in humans. Journal of Nutrition 1995;125:2631-9.

Velthuis-te WE, van-den BH, Weststrate JA, van het Hoff K, de Graaf $C$. Consumption of reduced-fat products: effects on parameters of anti-oxidative capacity. European Journal of Clinical Nutrition 1996;50(4):214-9.

Weststrate $\mathrm{JA}$, van het $\mathrm{Hof} \mathrm{KH}$, van den Berg $\mathrm{H}$, Velthuiste WE, de Graaf C, Zimmermanns NJ, et al. A comparison of the effect of free access to reduced fat products or their full fat equivalents on food intake, body weight, blood lipids and fat-soluble antioxidants levels and haemostasis variables. European Journal of Clinical Nutrition 1998;52:389-95.

* van het Hoff K, Weststrate JA, van-den BH, Velthuis-te WE, de Graaf C, Zimmermanns NJ, et al. A long-term study on the effect of spontaneous consumption of reduced fat products as part of a normal diet on indicators of health. International Journal of Food Sciences and Nutrition 1997;48(1):19-29.

NDHS Faribault 1968 \{published data only\}

The National Diet-Heart Study. Nutrition Reviews 1968;26(5):133-6

* The national diet-heart study final report. Circulation 1968;37(II):1-428.

Baker BM, Frantz ID Jr, Keys A, Kinsell LW, Page IH, Stamler J, et al. The National Diet-Heart Study: An initial report. JAMA 1963;185:105-6.

Brown HB. The National Diet Heart Study - implications for dietitians and nutritionists. Journal of the American Dietetic Association 1968;52:279-87.

Page IH, Brown HB. Some observations on the National DietHeart Study. Circulation 1968;37:313-5.

NDHS Open 1st L\&M 1968 \{published data only\}

The National Diet-Heart Study. Nutrition Reviews 1968;26(5):133-6.

* The national diet-heart study final report. Circulation 1968;37(II):1-428.

Baker BM, Frantz ID Jr, Keys A, Kinsell LW, Page IH, Stamler J, et al. The National Diet-Heart Study: An initial report. JAMA 1963:185:105-6. 
Brown HB. The National Diet Heart Study - implications for dietitians and nutritionists. Journal of the American Dietetic Association 1968;52:279-87.

Page IH, Brown HB. Some observations on the National DietHeart Study. Circulation 1968;37:313-5.

\section{NDHS Open 1st mod 1968 \{published data only\}}

The National Diet-Heart Study. Nutrition Reviews 1968;26(5):133-6.

* The national diet-heart study final report. Circulation 1968;37(II):1-428.

Baker BM, Frantz ID Jr, Keys A, Kinsell LW, Page IH, Stamler J, et al. The National Diet-Heart Study: An initial report. JAMA 1963;185:105-6.

Brown HB. The National Diet Heart Study - implications for dietitians and nutritionists. Journal of the American Dietetic Association 1968;52:279-87.

Page IH, Brown HB. Some observations on the National DietHeart Study. Circulation 1968;37:313-5.

\section{NDHS Open 2nd L\&M 1968 \{published data only\}}

The National Diet-Heart Study. Nutrition Reviews 1968;26(5):133-6.

* The national diet-heart study final report. Circulation 1968;37(II):1-428.

Baker BM, Frantz ID Jr, Keys A, Kinsell LW, Page IH, Stamler J, et al. The National Diet-Heart Study: An initial report. JAMA 1963;185:105-6.

Brown HB. The National Diet Heart Study - implications for dietitians and nutritionists. Journal of the American Dietetic Association 1968;52:279-87.

Page IH, Brown HB. Some observations on the National DietHeart Study. Circulation 1968;37:313-5.

\section{NDHS Open 2nd Mod 1968 \{published data only\}}

The National Diet-Heart Study. Nutrition Reviews 1968;26(5):133-6.

* The national diet-heart study final report. Circulation 1968;37(II):1-428.

Baker BM, Frantz ID Jr, Keys A, Kinsell LW, Page IH, Stamler J, et al. The National Diet-Heart Study: An initial report. JAMA 1963;185:105-6.

Brown HB. The National Diet Heart Study - implications for dietitians and nutritionists. Journal of the American Dietetic Association 1968;52:279-87.

Page IH, Brown HB. Some observations on the National DietHeart Study. Circulation 1968;37:313-5.

Nutrition \& Breast Health \{published and unpublished data\} Djuric Z, Poore KM, Depper JB, Uhley VE, Lababidi S, Covington $\mathrm{C}$, et al. Methods to increase fruit and vegetable intake with and without a decrease in fat intake: compliance and effects on body weight in the Nutrition and Breast Health Study. Nutrition and Cancer 2002;43(2):141-51.

Ole Study 2002 \{published and unpublished data\}

* Bray GA, Lovejoy JC, Most-Windhauser M, Smith SR, Volaufova J, Denkins Y, et al. A 9-mo randomized clinical trial comparing fat-substituted and fat-reduced diets in healthy obese men: the Ole Study. American Journal of Clinical Nutrition 2002;76:928-34.

Lovejoy JC, Bray GA, Lefevre M, Smith SR, Most MM, Denkins YM, et al. Consumption of a controlled low-fat diet containing olestra for 9 months improves health risk factors in conjunction with weight loss in obese men: the Ole Study. International Journal of Obesity 2003;27:1242-9.

Oslo Diet-Heart 1966 \{published and unpublished data\}

Leren P. Prevention of coronary heart disease, some results from the Oslo secondary and primary intervention studies. Journal of the American College of Nutrition 1989;8:407-10.

Leren P. The Oslo diet-heart study. Eleven year report. Circulation 1970;42:935-42.

* Leren P. The effect of plasma cholesterol lowering diet in male survivors of myocardial infarction. A controlled clinical trial. Acta Medica Scandinavia. Supplementum 1966;466:1-92.

Leren P. The effect of plasma-cholesterol-lowering diet in male survivors of myocardial infarction. A controlled clinical trial. Bulletin of the New York Academy of Medicine 1968;44:1012-20.

Leren $\mathrm{P}$. The effect of a cholesterol lowering diet in male survivors of myocardial infarction. (A controlled clinical trial) [Virkningen av cholesterolsenkende diett hos menn som har gjennomgatt hjerteinfarkt. Et kontrollert klinisk fors/ok]. Nordisk Medicin 1967;77(21):658-61.

Oxford Retinopathy 1978 \{published and unpublished data\} Coppack SW, Doll HA, Pim B, Hockaday TDR. Intravenous glucose tollerance and mortality in non-insulin-dependant diabetes mellitus. Quarterly Journal of Medicine 1990;75:451-60.

Hillson RM, Hockaday TDR, Mann JI, Newton DJ. Hyperinsulinaemia is associated with development of ECG abnormalities in diabetics. Diabetes Research 1984;1:143-9.

* Hockaday TD, Hockaday JM, Mann JI, Turner RC. Prospective comparison of modified fat-high-carbohydrate with standard low-carbohydrate dietary advice in the treatment of diabetes: one year follow-up study. British Journal of Nutrition 1978;39(2):357-62.

Howard-Williams J, Patel P, Jelfs R, Carter RD, Awdry P, Bron A, et al. Polyunsaturated fatty acids and diabetic retinopathy. British Journal of Ophthalmology 1985;69(1):15-8.

Lopez-Espinoza I, Howard WJ, Mann JI, Carter RD, Hockaday TD. Fatty acid composition of platelet phospholipids in non-insulindependent diabetics randomized for dietary advice. British Journal of Nutrition 1984;52(1):41-7. 
Polyp Prevention 1996 \{published and unpublished data\} Lanza E, Schatzkin A, Ballard BR, Clifford DC, Paskett E, Hayes D, et al. The polyp prevention trial II: dietary intervention program and participant baseline dietary characteristics. Cancer Epidemiology, Biomarkers and Prevention 1996;5(5):385-92.

* Schatzkin A, Lanza E, Freedman LS, Tangrea J, Cooper MR, Marshall JR, et al. The polyp prevention trial I: rationale, design, recruitment, and baseline participant characteristics. Cancer Epidemiology, Biomarkers and Prevention 1996;5(5):375-83.

PREMIER 2003 \{published and unpublished data\}

* Appel LJ, Champagne CM, Harsha DW, Cooper LS, Obarzanek E, Elmer PJ, et al. Effects of comprehensive lifestyle modification on blood pressure control: main results of the PREMIER clinical trial. JAMA 2003;289(16):2083-93.

Elmer PJ, Obarzanek E, Vollmer WM, Simons-Morton D, Stevens VJ, Young DR, et al. Effects of comprehensive lifestyle modification on diet, weight, physical fitness, and blood pressure control: 18-month results of a randomized trial. Annals of Internal Medicine 2006;144(7):485-95

Ledikwe JH, Rolls BJ, Smiciklas-Wright H, Mitchell DC, Ard JD, Champagne $\mathrm{C}$, et al. Reductions in dietary energy density are associated with weight loss in overweight and obese participants in the PREMIER trial. American Journal of Clinical Nutrition 2007;85(5):1212-21.

Lien LF, Brown AJ, Ard JD, Loria C, Erlinger TP, Feldstein AC, et al. Effects of PREMIER lifestyle modifications on participants with and without the metabolic syndrome. Hypertension 2007;50(4):609-16.

Lin PH, Appel LJ, Funk K, Craddick S, Chen C, Elmer P, et al. The PREMIER intervention helps participants follow the Dietary Approaches to Stop Hypertension dietary pattern and the current Dietary Reference Intakes recommendations. Journal of the American Dietetic Association 2007;107(9):1541-51.

Maruthur NM, Wang NY, Appel LJ, Maruthur Nisa M, Wang Nae Yuh, Appel Lawrence J. Lifestyle interventions reduce coronary heart disease risk: results from the PREMIER Trial. Circulation 2009;119(15):2026-31.

McGuire HL, Svetkey LP, Harsha DW, Elmer PJ, Appel LJ, Ard JD. Comprehensive lifestyle modification and blood pressure control: a review of the PREMIER trial. Journal of clinical hypertension (Greenwich., Conn.) 2004;6(7):383-90.

Obarzanek E, Vollmer WM, Lin PH, Cooper LS, Young DR, Ard JD, et al. Effects of individual components of multiple behavior changes: the PREMIER trial. American Journal of Health Behavior 2007;31(5):545-60.

Svetkey LP, Erlinger TP, Vollmer WM, Feldstein A, Cooper LS, Appel LJ, et al. Effect of lifestyle modifications on blood pressure by race, sex, hypertension status, and age. Journal of Human Hypertension 2005;19(1):21-31.

Svetkey LP, Harsha DW, Vollmer WM, Stevens VJ, Obarzanek E, Elmer PJ, et al. Premier: a clinical trial of comprehensive lifestyle modification for blood pressure control: rationale, design and baseline characteristics. Annals of Epidemiology 2003;13(6):462-71.

Rivellese 1994 \{published and unpublished data\}

Rivellese AA, Auletta P, Marotta G, Saldalamacchia G, Giacoo A, Mastrilli $V$, et al. Long term metabolic effects of two dietary methods of treating hyperlipidaemia. BMJ 1994;308:227-31.

Rose 1965 \{published data only\}

Rose GA, Thomson WB, Williams RT. Corn oil in treatment of ischaemic heart disease. British Medical Journal 1965;1:1531-3.

Sacks high protein 2009 \{published and unpublished data\} Sacks FM, Bray GA, Carey VJ, Smith SR, Ryan DH, Anton SD, et al. Comparison of weight-loss diets with different compositions of fat, protein, and carbohydrates. New England Journal of Medicine 2009;360(9):859-73.

Sacks low protein 2009 \{published and unpublished data\} Sacks FM, Bray GA, Carey VJ, Smith SR, Ryan DH, Anton SD, et al. Comparison of weight-loss diets with different compositions of fat, protein, and carbohydrates. New England Journal of Medicine 2009;360(9):859-73.

Sarkkinen Fat Mod 1995 \{published and unpublished data\} Makinen E, Uusitupa MI, Pietinen P, Aro A, Penttila I. Long term effects of three fat modified diets on serum lipids in free living hypercholesterolaemic subjects (abstract). European Heart Journal 1991;12:162.

Sarkkinen ES, Agren JJ, Ahola I, Ovaskainen ML, Uusitupa MI. Fatty acid composition of serum cholesterol esters, and erythrocyte and platelet membranes as indicators of long-term adherence to fat-modified diets. American Journal of Clinical Nutrition 1994;59(2):364-70.

Sarkkinen ES, Uusitupa MI, Nyyssonen K, Parviainen M, Penttila I, Salonen JT. Effects of two low-fat diets, high and low in polyunsaturated fatty acids, on plasma lipid peroxides and serum vitamin E levels in free-living hypercholesterolaemic men. European Journal of Clinical Nutrition 1993;47(9):623-30.

Sarkkinen ES, Uusitupa MI, Pietinen P, Aro A, Ahola I, Penttila I, et al. Long-term effects of three fat-modified diets in hypercholesterolemic subjects. Atherosclerosis 1994;105(1):9-23.

* Sarkkinen, E. Long-term feasibility and effects of three different fat-modified diets in free-living hypercholesterolemic subjects. Kuopio, Finland: Univ. of Kuopio, 1995.

Uusitupa MI, Sarkkinen ES, Torpstrom J, Pietinen P, Aro A. Long-term effects of four fat-modified diets on blood pressure. Journal of Human Hypertension 1994;8(3):209-18.

Sarkkinen Red \& Mod 1995 \{published and unpublished data\}

Makinen E, Uusitupa MI, Pietinen P, Aro A, Penttila I. Long term effects of three fat modified diets on serum lipids in free living hypercholesterolaemic subjects (abstract). European Heart Journal 1991;12:162.

Sarkkinen ES, Agren JJ, Ahola I, Ovaskainen ML, Uusitupa MI. Fatty acid composition of serum cholesterol esters, and 
erythrocyte and platelet membranes as indicators of long-term adherence to fat-modified diets. American Journal of Clinical Nutrition 1994;59(2):364-70.

Sarkkinen ES, Uusitupa MI, Nyyssonen K, Parviainen M, Penttila I, Salonen JT. Effects of two low-fat diets, high and low in polyunsaturated fatty acids, on plasma lipid peroxides and serum vitamin $E$ levels in free-living hypercholesterolaemic men. European Journal of Clinical Nutrition 1993;47(9):623-30.

Sarkkinen ES, Uusitupa MI, Pietinen P, Aro A, Ahola I, Penttila I, et al. Long-term effects of three fat-modified diets in hypercholesterolemic subjects. Atherosclerosis 1994;105(1):9-23.

* Sarkkinen, E. Long-term feasibility and effects of three different fat-modified diets in free-living hypercholesterolemic subjects. Kuopio, Finland: Univ. of Kuopio, 1995.

Uusitupa MI, Sarkkinen ES, Torpstrom J, Pietinen P, Aro A. Long-term effects of four fat-modified diets on blood pressure. Journal of Human Hypertension 1994;8(3):209-18.

\section{Sarkkinen Red Fat 1995 \{published and unpublished data\}}

Makinen E, Uusitupa MI, Pietinen P, Aro A, Penttila I. Long term effects of three fat modified diets on serum lipids in free living hypercholesterolaemic subjects (abstract). European Heart Journal 1991;12:162.

Sarkkinen ES, Agren JJ, Ahola I, Ovaskainen ML, Uusitupa MI. Fatty acid composition of serum cholesterol esters, and erythrocyte and platelet membranes as indicators of long-term adherence to fat-modified diets. American Journal of Clinical Nutrition 1994;59(2):364-70.

Sarkkinen ES, Uusitupa MI, Nyyssonen K, Parviainen M, Penttila I, Salonen JT. Effects of two low-fat diets, high and low in polyunsaturated fatty acids, on plasma lipid peroxides and serum vitamin E levels in free-living hypercholesterolaemic men. European Journal of Clinical Nutrition 1993;47(9):623-30.

Sarkkinen ES, Uusitupa MI, Pietinen P, Aro A, Ahola I, Penttila I, et al. Long-term effects of three fat-modified diets in hypercholesterolemic subjects. Atherosclerosis 1994;105(1):9-23.

* Sarkkinen, E. Long-term feasibility and effects of three different fat-modified diets in free-living hypercholesterolemic subjects. Kuopio, Finland: Univ. of Kuopio, 1995.

Uusitupa MI, Sarkkinen ES, Torpstrom J, Pietinen P, Aro A. Long-term effects of four fat-modified diets on blood pressure. Journal of Human Hypertension 1994;8(3):209-18.

Sarkkinen Red vs Mod1995 \{published and unpublished data\} Makinen E, Uusitupa MI, Pietinen P, Aro A, Penttila I. Long term effects of three fat modified diets on serum lipids in free living hypercholesterolaemic subjects (abstract). European Heart Journal 1991;12:162.

Sarkkinen ES, Agren JJ, Ahola I, Ovaskainen ML, Uusitupa MI. Fatty acid composition of serum cholesterol esters, and erythrocyte and platelet membranes as indicators of long-term adherence to fat-modified diets. American Journal of Clinical Nutrition 1994;59(2):364-70.

Sarkkinen ES, Uusitupa MI, Nyyssonen K, Parviainen M, Penttila I, Salonen JT. Effects of two low-fat diets, high and low in polyunsaturated fatty acids, on plasma lipid peroxides and serum vitamin $E$ levels in free-living hypercholesterolaemic men. European Journal of Clinical Nutrition 1993;47(9):623-30.

Sarkkinen ES, Uusitupa MI, Pietinen P, Aro A, Ahola I, Penttila I, et al. Long-term effects of three fat-modified diets in hypercholesterolemic subjects. Atherosclerosis 1994;105(1):9-23.

* Sarkkinen, E. Long-term feasibility and effects of three different fat-modified diets in free-living hypercholesterolemic subjects. Kuopio, Finland: Univ. of Kuopio, 1995.

Uusitupa MI, Sarkkinen ES, Torpstrom J, Pietinen P, Aro A. Long-term effects of four fat-modified diets on blood pressure. Journal of Human Hypertension 1994;8(3):209-18.

\section{Seppelt 1996 \{published and unpublished data\}}

Seppelt B, Weststrate JA, Reinert A, Johnson D, Luder W, Zunft HJ. Long-term effects of nutrition with fat-reduced foods on energy consumption and body weight [Langzeiteffekte einer Ernährung mit fettreduzierten Lebensmitteln auf die Energieaufnahme und das Körpergewicht]. Zeitschrift für Ernährungswissenschaft 1996;35(4):369-77.

Simon 1997 \{published and unpublished data\}

Djuric Z, Heilbrun LK, Reading BA, Boomer A, Valeriote FA, Martino S. Effects of a low fat diet on levels of oxidative damage to DNA in human peripheral nucleated blood cells. Journal of the National Cancer Institute 1991;83(11):766-9.

Djuric Z, Martino S, Heilbrun LK, Hart RW. Dietary modulation of oxidative DNA damage. Advances In Experimental Medicine and Biology 1994;354:71-83.

Kasim SE, Martino S, Kim P-N, Khilnani S, Boomer A, Depper J, et al. Dietary and anthropometric determinants of plasma lipoproteins during a long-term low-fat diet in healthy women. American Journal of Clinical Nutrition 1993;57:146-53.

* Simon MS, Heilbrun LK, Boomer A, Kresge C, Depper J, Kim PN, et al. A randomised trial of a low-fat dietary intervention in women at high risk for breast cancer. Nutrition and Cancer 1997;27(2):136-42.

\section{Sondergaard 2003 \{published and unpublished data\}}

Sondergaard E, Moller JE, Egstrup K. Effect of dietary intervention and lipid-lowering treatment on brachial vasoreactivity in patients with ischemic heart disease and hypercholesterolemia. American Heart Journal 2003;145(5):E19.

\section{STARS 1992 \{published and unpublished data\}}

Blann AD, Jackson P, Bath PM, Watts GF. von Willebrand factor, a possible indicator of endothelial cell damage, decreases during long-term compliance with a lipid-lowering diet. Journal of Internal Medicine 1995;237:557-61. 
Watts GF. Nutritional, metabolic, and genetic determinants of the progression of coronary heart disease. STARS Group. Journal of Cardiovascular Pharmacology 1995;25(Suppl 4):S11-9.

Watts GF, Brunt JNH, Coltart DJ, Lewis B. The St. Thomas Atherosclerosis Regression Study (STARS). Atherosclerosis 1992;97:231.

Watts GF, Jackson P, Burke V, Lewis B. Dietary fatty acids and progression of coronary artery disease in men. American Journal of Clinical Nutrition 1996;64:202-9.

Watts GF, Jackson P, Mandalia S, Brunt JN, Lewis ES, Coltart DJ, et al. Nutrient intake and progression of coronary artery disease. American Journal of Cardiology 1994;73(5):328-32.

* Watts GF, Lewis B, Brunt JN, Lewis ES, Coltart DJ, Smith LD, et al. Effects on coronary artery disease of lipid-lowering diet, or diet plus cholestyramine, in the St Thomas' Atherosclerosis Regression Study (STARS). Lancet 1992;339(8793):563-9.

Watts GF, Lewis B, Brunt JNH, Swan AV. Coronary Atheroma Regression Trials. Lancet 1992;339(i):1241-3.

Watts GF, Lewis B, Jackson P, Burke V, Lewis ES, Brunt JN, et al. Relationships between nutrient intake and progression/ regression of coronary atherosclerosis as assessed by serial quantitative angiography. Canadian Journal of Cardiology 1995;11(Suppl G):110G-4G.

Watts GF, Mandalia S, Brunt JN, Slavin BM, Coltart DJ, Lewis B. Independent associations between plasma lipoprotein subfraction levels and the course of coronary artery disease in the St. Thomas' Atherosclerosis Regression Study (STARS). Metabolism: Clinical and Experimental 1993;42:1461-7.

Watts GF, Mandalia S, Slavin BM, Brunt JN, Coltart DJ, Lewis B. Metabolic determinants of the course of coronary artery disease in men. Clinical Chemistry 1994;40(12):2240-6.

Strychar 2009 \{published and unpublished data\}

Strychar I, Cohn JS, Renier G, Rivard M, ris-Jilwan N, Beauregard $\mathrm{H}$, et al. Effects of a diet higher in carbohydrate/ lower in fat versus lower in carbohydrate/higher in monounsaturated fat on postmeal triglyceride concentrations and other cardiovascular risk factors in type 1 diabetes. Diabetes Care 2009;32(9):1597-9.

Sydney Diet-Heart 1978 \{published and unpublished data\} Blacket RB, Leelarthaepin B, McGilchrist C, Palmer AJ, Woodhill JM. The synergistic effect of weight loss and changes in dietary lipids on the serum cholesterol of obese men with hypercholesterolaemia: implications for prevention of coronary heart disease. Australian and New Zealand Journal of Medicine 1979;9:521-9.

* Woodhill JM, Palmer AJ, Leelarthaepin B, McGilchrist C, Blacket RB. Low fat, low cholesterol diet in secondary prevention of coronary heart disease. Advances in Experimental Medicine and Biology 1978;109:317-30.

\section{THIS DIET 2008 \{published data only\}}

Tuttle KR, Shuler LA, Packard DP, Milton JE, Daratha KB, Bibus DM, et al. Comparison of low-fat versus Mediterraneanstyle dietary intervention after first myocardial infarction (from The Heart Institute of Spokane Diet Intervention and Evaluation Trial). American Journal of Cardiology 2008;101(11):1523-30.

\section{Veterans Admin 1969 \{published data only\}}

Dayton S, Hashimoto S, Dixon W, Pearce ML. Composition of lipids in human serum and adipose tissue during prolonged feeding of a diet high in unsaturated fat. Journal of Lipid Research 1966;7:103-11.

Dayton S, Hashimoto S, Pearce ML. Adipose tissue linoleic acid as a criterion of adherence to a modified diet. Journal of Lipid Research 1967;8:508-10.

Dayton S, Hashimoto S, Pearce ML. Influence of a diet high in unsaturated fat upon composition of arterial tissue and atheromata in man. Circulation 1965;32:911-24.

Dayton S, Hashimoto S, Rosenblum D, Pearce M. Vitamin E status of humans during prolonged feeding of unsaturated fats. Journal of Laboratory and Clinical Medicine 1965;65(5):739-47.

Dayton S, Pearce ML. Diet and atherosclerosis. Lancet 1970;1(644):473-4.

Dayton S, Pearce ML. Diet and cardiovascular diseases. Lancet 1969;1(584):51-2.

Dayton S, Pearce ML. Diet high in unsaturated fat: a controlled clinical trial. Minnesota Medicine 1969;1969:1237-42.

Dayton S, Pearce ML. Prevention of coronary heart disease and other complications of atherosclerosis by modified diet. American Journal of Medicine 1969;46:751-62.

Dayton S, Pearce ML. Trial of unsaturated-fat diet. Lancet 1968;2(581):1296-7.

Dayton S, Pearce ML, Goldman H, Harnish A, Plotkin D, Shickman M, et al. Controlled trial of a diet high in unsaturated fat for prevention of atherosclerotic complications. Lancet 1968;2(577):1060-2.

* Dayton S, Pearce ML, Hashimoto S, Dixon WJ, Tomayasu U. A controlled clinical trial of a diet high in unsaturated fat in preventing complications of atherosclerosis. Circulation 1969;15(1, Suppl 2):II-1-63.

Dayton S, Pearce ML, Hashimoto S, Fakler LJ, Hiscock E, Dixon WJ. A controlled clinical trial of a diet high in unsaturated fat. New England Journal of Medicine 1962;266:1017-23.

Hiscock E, Dayton S, Pearce ML, Hashimoto S. A palatable diet high in unsaturated fat. Journal of the American Dietetic Association 1962;40:427-31.

Pearce ML, Dayton S. Incidence of cancer in men on a diet high in polyunsaturated fat. Lancet 1971;1(697):464-7. 
Sturdevant RA, Pearce ML, Dayton S. Increased prevalence of cholelithiasis in men ingesting a serum-cholesterol-lowering diet. New England Journal of Medicine 1973;288(1):24-7.

Tompkins MJ, Dayton S, Pearce ML. Effect of long-term feeding of various fats on whole blood clotting times in men. Journal of Laboratory and Clinical Medicine 1964;64(5):763-72.

\section{WHEL 2007 \{published data only\}}

Bardwell WA, Profant J, Casden DR, Dimsdale JE, ncoli-Israel S, Natarajan L, et al. The relative importance of specific risk factors for insomnia in women treated for early-stage breast cancer. Psycho-Oncology 2008;17(1):9-18.

Caan BJ, Flatt SW, Rock CL, Ritenbaugh C, Newman V, Pierce JP, et al. Low-energy reporting in women at risk for breast cancer recurrence. Women's Healthy Eating and Living Group. Cancer Epidemiology, Biomarkers \& Prevention 2000;9(10):1091-7.

Gold EB, Flatt SW, Pierce JP, Bardwell WA, Hajek RA, Newman VA, et al. Dietary factors and vasomotor symptoms in breast cancer survivors: the WHEL Study. Menopause 2006;13(3):423-33.

Gold EB, Pierce JP, Natarajan L, Stefanick ML, Laughlin GA, Caan BJ, et al. Dietary pattern influences breast cancer prognosis in women without hot flashes: the women's healthy eating and living trial. Journal of Clinical Oncology 2009;27(3):352-9.

Hernandez-Valero MA, Thomson CA, Hernandez M, Tran T, Detry MA, Theriault RL, et al. Comparison of baseline dietary intake of Hispanic and matched non-Hispanic white breast cancer survivors enrolled in the Women's Healthy Eating and Living study. Journal of the American Dietetic Association 2008;108(8):1323-9.

Hong S, Bardwell WA, Natarajan L, Flatt SW, Rock CL, Newman VA, et al. Correlates of physical activity level in breast cancer survivors participating in the Women's Healthy Eating and Living (WHEL) Study. Breast Cancer Research \& Treatment 2007;101(2):225-32.

Hyder JA, Thomson CA, Natarajan L, Madlensky L, Pu M, Emond J, et al. Adopting a plant-based diet minimally increased food costs in WHEL Study. American Journal of Health Behavior 2009;33(5):530-9.

Madlensky L, Natarajan L, Flatt SW, Faerber S, Newman VA, Pierce JP, et al. Timing of dietary change in response to a telephone counseling intervention: evidence from the WHEL study. Health Psychology 2008;27(5):539-47.

Mortimer JE, Flatt SW, Parker BA, Gold EB, Wasserman L, Natarajan L, et al. Tamoxifen, hot flashes and recurrence in breast cancer. Breast Cancer Research \& Treatment 2008;108(3):421-6.

Newman VA, Thomson CA, Rock CL, Flatt SW, Kealey S, Bardwell WA, et al. Achieving substantial changes in eating behavior among women previously treated for breast cancer: an overview of the intervention. Journal of the American Dietetic Association 2005;105(3):382-91.
Pierce JP. Diet and breast cancer prognosis: making sense of the Women's Healthy Eating and Living and Women's Intervention Nutrition Study trials. Current Opinion in Obstetrics \& Gynecology 2009;21(1):86-91.

Pierce JP, Faerber S, Wright FA, Rock CL, Newman V, Flatt SW, et al. A randomized trial of the effect of a plant-based dietary pattern on additional breast cancer events and survival: the Women's Healthy Eating and Living (WHEL) Study. Controlled Clinical Trials 2002;23(6):728-56.

Pierce JP, Natarajan L, Caan BJ, Flatt SW, Kealey S, Gold EB, et al. Dietary change and reduced breast cancer events among women without hot flashes after treatment of early-stage breast cancer: subgroup analysis of the Women's Healthy Eating and Living Study. American Journal of Clinical Nutrition 2009;89(5):1565S-71S.

* Pierce JP, Natarajan L, Caan BJ, Parker BA, Greenberg ER, Flatt SW, et al. Influence of a diet very high in vegetables, fruit, and fiber and low in fat on prognosis following treatment for breast cancer: the Women's Healthy Eating and Living (WHEL) randomized trial. JAMA 2007;298(3):289-98.

Pierce JP, Natarajan L, Sun S, Al-Delaimy W, Flatt SW, Kealey S, et al. Increases in plasma carotenoid concentrations in response to a major dietary change in the women's healthy eating and living study. Cancer Epidemiology, Biomarkers \& Prevention 2006;15(10):1886-92.

Pierce JP, Newman VA, Flatt SW, Faerber S, Rock CL, Natarajan L, et al. Telephone counseling intervention increases intakes of micronutrient- and phytochemical-rich vegetables, fruit and fiber in breast cancer survivors. Journal of Nutrition 2004;134(2):452-8.

Pierce JPF. A randomized trial of the effect of a plant-based dietary pattern on additional breast cancer events and survival: The Women's Healthy Eating and Living (WHEL) Study. Controlled Clinical Trials 2002;23(6):728-56.

Rock CL, Flatt SW, Laughlin GA, Gold EB, Thomson CA, Natarajan L, et al. Reproductive steroid hormones and recurrence-free survival in women with a history of breast cancer. Cancer Epidemiology, Biomarkers \& Prevention 2008;17(3):614-20.

Rock CL, Flatt SW, Newman V, Caan BJ, Haan MN, Stefanick ML, et al. Factors associated with weight gain in women after diagnosis of breast cancer. Women's Healthy Eating and Living Study Group. Journal of the American Dietetic Association 1999;99(10):1212-21.

Rock CL, Flatt SW, Thomson CA, Stefanick ML, Newman VA, Jones $L$, et al. Plasma triacylglycerol and HDL cholesterol concentrations confirm self-reported changes in carbohydrate and fat intakes in women in a diet intervention trial. Journal of Nutrition 2004;134(2):342-7.

Rock CL, Natarajan L, Pu M, Thomson CA, Flatt SW, Caan BJ, et al. Longitudinal biological exposure to carotenoids is associated with breast cancer-free survival in the Women's Healthy Eating and Living Study. Cancer Epidemiology, Biomarkers \& Prevention 2009;18(2):486-94. 
Saquib N, Flatt SW, Natarajan L, Thomson CA, Bardwell WA, Caan $B$, et al. Weight gain and recovery of pre-cancer weight after breast cancer treatments: evidence from the women's healthy eating and living (WHEL) study. Breast Cancer Research \& Treatment 2007;105(2):177-86.

Saxe GA, Madlensky L, Kealey S, Wu DP, Freeman KL, Pierce JP, et al. Disclosure to physicians of CAM use by breast cancer patients: findings from the Women's Healthy Eating and Living Study. Integrative Cancer Therapies 2008;7(3):122-9.

\section{WHI with CVD 2006 \{published data only\}}

Anderson G, Cummings S, Freedman LS, Furberg C, Henderson M, Johnson SR, et al. Design of the Women's Health Initiative clinical trial and observational study. Controlled Clinical Trials 1998;19(1):61-109.

* Howard BV, Van Horn L, Hsia J, Manson JE, Stefanick ML, Wassertheil-Smoller S, et al. Low-fat dietary pattern and risk of cardiovascular disease: the Women's Health Initiative Randomized Controlled Dietary Modification Trial. JAMA 2006;295(6):655-66.

Rossouw JE, Finnegan LP, Harlan WR, Pinn VW, Clifford C, McGowan JA. The evolution of the Women's Health Initiative: perspectives from the NIH. Journal of the American Medical Women's Association 1995;50(2):50-5.

\section{WHI without CVD 2006 \{published data only\}}

Anderson G, Cummings S, Freedman LS, Furberg C, Henderson M, Johnson SR, et al. Design of the Women's Health Initiative clinical trial and observational study. Controlled Clinical Trials 1998;19(1):61-109.

Anderson GL, Manson J, Wallace R, Lund B, Hall D, Davis S, et al. Implementation of the Women's Health Initiative study design. Annals of Epidemiology 2003;13(9 Suppl):S5-17.

Beresford SA, Johnson KC, Ritenbaugh C, Lasser NL, Snetselaar LG, Black HR, et al. Low-fat dietary pattern and risk of colorectal cancer: the Women's Health Initiative Randomized Controlled Dietary Modification Trial. JAMA 2006;295(6):643-54.

Bowen D, Ehret C, Pedersen M, Snetselaar L, Johnson M, Tinker $L$, et al. Results of an adjunct dietary intervention program in the Women's Health Initiative. Journal of the American Dietetic Association 2002;102(11):1631-7.

Curb JD, McTiernan A, Heckbert SR, Kooperberg C, Stanford J, Nevitt M, et al. Outcomes ascertainment and adjudication methods in the Women's Health Initiative. Annals of Epidemiology 2003;13(9 Suppl):S122-8.

Hays J, Hunt JR, Hubbell FA, Anderson GL, Limacher M, Allen C, et al. The Women's Health Initiative recruitment methods and results. Annals of Epidemiology 2003;13(9 Suppl):S18-77.

Hebert JR, Patterson RE, Gorfine M, Ebbeling CB, St Jeor ST, Chlebowski RT, et al. Differences between estimated caloric requirements and self-reported caloric intake in the women's health initiative. Annals of Epidemiology 2003;13(9):629-37.
Howard BV. Dietary fat and cardiovascular disease: putting the Women's Health Initiative in perspective. Nutrition Metabolism \& Cardiovascular Diseases 2007;17(3):171-4.

Howard BV, Manson JE, Stefanick ML, Beresford SA, Frank G, Jones B, et al. Low-fat dietary pattern and weight change over 7 years: the Women's Health Initiative Dietary Modification Trial. JAMA 2006;295(1):39-49.

* Howard BV, Van Horn L, Hsia J, Manson JE, Stefanick ML, Wassertheil-Smoller S, et al. Low-fat dietary pattern and risk of cardiovascular disease: the Women's Health Initiative Randomized Controlled Dietary Modification Trial. JAMA 2006;295(6):655-66.

Neuhouser ML, Tinker L, Shaw PA, Schoeller D, Bingham SA, Horn LV, et al. Use of recovery biomarkers to calibrate nutrient consumption self-reports in the Women's Health Initiative. American Journal of Epidemiology 2008;167(10):1247-59.

Patterson RE, Kristal A, Rodabough R, Caan B, Lillington L, Mossavar-Rahmani $Y$, et al. Changes in food sources of dietary fat in response to an intensive low-fat dietary intervention: early results from the Women's Health Initiative. Journal of the American Dietetic Association 2003;103(4):454-60.

Patterson RE, Kristal AR, Tinker LF, Carter RA, Bolton MP, gursCollins T, et al. Measurement characteristics of the Women's Health Initiative food frequency questionnaire. Annals of Epidemiology 1999;9(3):178-87.

Prentice RL, Caan B, Chlebowski RT, Patterson R, Kuller LH, Ockene JK, et al. Low-fat dietary pattern and risk of invasive breast cancer: the Women's Health Initiative Randomized Controlled Dietary Modification Trial. JAMA 2006;295(6):629-42.

Prentice RL, Thomson CA, Caan B, Hubbell FA, Anderson GL, Beresford SA, et al. Low-fat dietary pattern and cancer incidence in the Women's Health Initiative Dietary Modification Randomized Controlled Trial. Journal of the National Cancer Institute 2007;99(20):1534-43.

Ritenbaugh C, Patterson RE, Chlebowski RT, Caan B, FelsTinker L, Howard B, et al. The Women's Health Initiative Dietary Modification trial: overview and baseline characteristics of participants. Annals of Epidemiology 2003;13(9 Suppl):S87-97.

Rossouw JE, Finnegan LP, Harlan WR, Pinn VW, Clifford C, McGowan JA. The evolution of the Women's Health Initiative: perspectives from the NIH. Journal of the American Medical Women's Association 1995;50(2):50-5.

The Women's Health Initiative Study Group. Design of the Women's Health Initiative clinical trial and observational study. Controlled Clinical Trials 1998;19(1):61-109.

Tinker LF, Bonds DE, Margolis KL, Manson JE, Howard BV, Larson J, et al. Low-fat dietary pattern and risk of treated diabetes mellitus in postmenopausal women: the Women's Health Initiative randomized controlled dietary modification trial. Archives of Internal Medicine 2008;168(14):1500-11.

Tinker LF, Perri MG, Patterson RE, Bowen DJ, McIntosh M, Parker LM, et al. The effects of physical and emotional status 
on adherence to a low-fat dietary pattern in the Women's Health Initiative. Journal of the American Dietetic Association 2002;102(6):789-800.

Tinker LF, Rosal MC, Young AF, Perri MG, Patterson RE, Van Horn L, et al. Predictors of dietary change and maintenance in the Women's Health Initiative Dietary Modification Trial. Journal of the American Dietetic Association 2007;107(7):1155-66.

Women's Health Initiative Study Group. Dietary adherence in the Women's Health Initiative Dietary Modification Trial. Journal of the American Dietetic Association 2004;104(4):654-8.

WINS 2006 \{published and unpublished data\}

Chlebowski RT, Blackburn GL, Buzzard IM, Rose DP, Martino S, Khandekar JD, et al. Adherence to a dietary fat intake reduction program in postmenopausal women receiving therapy for early breast cancer. The Women's Intervention Nutrition Study. Journal of Clinical Oncology 1993;11(11):2072-80.

* Chlebowski RT, Blackburn GL, Thomson CA, Nixon DW, Shapiro A, Hoy MK, et al. Dietary fat reduction and breast cancer outcome: interim efficacy results from the women's intervention nutrition study. JNCI Journal of the National Cancer Institute 2006;98(24):1767-76.

Chlebowski RT, Rose DP, Buzzard IM, Blackburn GL, York M, Insull W, et al. Dietary fat reduction in adjuvant breast cancer therapy: current rationale and feasibility issues. Adjuvant. The Cancer Journal 1990;6:357-63.

Hoy MK, Winters BL, Chlebowski RT, Papoutsakis C, Shapiro A, Lubin MP, et al. Implementing a low-fat eating plan in the Women's Intervention Nutrition Study. Journal of the American Dietetic Association 2009;109(4):688-96.

Rose DP, Chlebowski RT, Connolly JM, Jones LA, Wynder EL. Effects of tamoxifen adjuvant therapy and a low-fat diet on serum binding proteins and estradiol bioavailability in postmenopausal breast cancer patients. Cancer Research 1992;52:5386-90.

Rose DP, Connolly JM, Chlebowski RT, Buzzard IM, Wynder EL. The effects of a low-fat dietary intervention and tamoxifen adjuvant therapy on the serum estrogen and sex hormonebinding globulin concentrations of postmenopausal breast cancer patients. Breast Cancer Research \& Treatment 1993;27(3):253-62.

Wynder EL, Cohen LA, Winters BL. The challenges of assessing fat intake in cancer research investigations. Journal of the American Dietetic Association 1997;97(7 Suppl):S5-8.

\section{References to studies excluded from this review}

\section{Agewall 2001 \{published data only\}}

Agewall S. Multiple risk intervention trial in high risk hypertensive men: comparison of ultrasound intima-media thickness and clinical outcome during 6 years of follow-up. Journal of Internal Medicine 2001;249(4):305-14.

\section{Ammerman 2003 \{published data only\}}

Ammerman AS, Keyserling TC, Atwood JR, Hosking JD, Zayed H, Krasny $\mathrm{C}$. A randomized controlled trial of a public health nurse directed treatment program for rural patients with high blood cholesterol. Preventive Medicine 2003;36(3):340-51.

\section{Anti-Coronary C 1966 \{published data only\}}

* Christakis G, Rinzler SH, Archer M, Maslansky E. Summary of the research activities of the Anti-Coronary Club. Public Health Report (Washington) 1966;81:64-70.

Christakis G, Rinzler SH, Archer M, et al. Effect of the AntiCoronary Club Program on coronary heart disease risk factor status. JAMA 1969;198:129-36.

Jolliffe N, Baumgarter L, Rinzler SH, Archer M, Stephenson JH, Christakis GJ. The Anti-Coronary Club: The first four years. New York State Journal of Medicine 1963;63:69-79.

Singman HS, Berman SN, Cowell C, Maslansky E, Archer M. The Anti-Coronary Club: 1957-1972. American Journal of Clinical Nutrition 1980;33(6):1183-91.

\section{Aquilani 2000 \{published data only\}}

Aquilani R, Tramarin R, Pedretti RFE, Bertolotti G, Sommaruga M, Mariani P, et al. Can a very-low-fat diet achieve cholesterol goals in CAD?. Cardiology Review 2000;17(10):36-40.

\section{Arntzenius 1985 \{published data only\}}

Arntzenius AC, Kromhout D, Bartn JE, Reiber JHC, Bruschke AVG, Buis Van Gent CM. Diet, lipoprotiens and progression of coronary atherosclerosis: The leiden intervention trial. New England Journal of Medicine 1985;312:805-8.

\section{Aro 1990 \{published data only\}}

Aro A, Ahola I, Jauhiainen M, et al. Effects of plasma phospholipid fatty acids of rapeseed oil and sunflower oil diets [Abstract]. Arteriosclerosis 1990;10:877a.

\section{ASSIST 2001 \{published data only\}}

Moher M, Yudkin P, Wright L, Turner R, Fuller A, Schofield T, et al. Cluster randomised controlled trial to compare three methods of promoting secondary prevention of coronary heart disease in primary care. BMJ 2001;322(7298):1338.

Australian Polyp Prev 95 \{published and unpublished data\} MacLennan R. Effect of fat, fibre and beta-carotene on colorectal adenomas after 24 months. Gastroenterology 1991;100:A382.

* MacLennan R, Macrae F, Bain C, Battistutta D, Chapuis P, Gratten $\mathrm{H}$, et al. Randomized trial of intake of fat, fiber, and beta carotene to prevent colorectal adenomas. The Australian Polyp Prevention Project. Journal of the National Cancer Institute 1995;87(23):1760-6.

Macrae FA, Hughes NR, Bhathal PS, Tay D, Selbie L, MacLennan R. Dietary suppression of rectal epthelial cell proliferation. Gastroenterology 1991;100:A383. 
Baer 1993 \{published data only\}

Baer JT. Improved plasma cholesterol levels in men after a nutrition education program at the worksite. Journal of the American Dietetic Association 1993;93(6):658-63.

\section{Bakx 1997 \{published data only\}}

Bakx JC, Stafleu A, van SW, van-den HH, van WC. Longterm effect of nutritional counseling: a study in family medicine. American Journal of Clinical Nutrition 1997;65(6 Suppl):1946S-50S.

\section{Barnard 2009 \{published data only\}}

Barnard ND, Cohen J, Jenkins DJ, Turner-McGrievy G, Gloede L, Green A, et al. A low-fat vegan diet and a conventional diabetes diet in the treatment of type 2 diabetes: a randomized, controlled, 74-wk clinical trial. American Journal of Clinical Nutrition 2009;89(5):1588S-96S.

\section{Barndt 1977 \{published data only\}}

Barndt R, Blankenhorn CH, Crawford DW, et al. Regression and progression of early femoral atherosclerosis in treated hyperlipidaemic patients. Annals of Internal Medicine 1977;86:139-46.

\section{Baron 1990 \{published data only\}}

Baron JA, Gleason R, Crowe B, Mann JI. Preliminary trial of the effect of general practice based nutritional advice. British Journal of General Practice 1990;40(333):137-41.

\section{Barr 1990 \{published data only\}}

Barr SL, Ramakrishnan R, Holleran S. A 30\% fat diet high in polyunsaturates and a $30 \%$ fat diet high in monounsaturates both lower total and low density lipoprotein cholesterol levels in normal males [Abstract]. Arteriosclerosis 1990;10:872a.

\section{Baumann 1982 \{published data only\}}

Baumann J, Martschick R. Therapy of hyperlipidemia with xanthinol nicotinate as opposed to low fat diet [Therapie der Hyperlipidämie mit Xantinolnicotinat gegenüber fettarmer Diät]. Die Medizinische Welt 1982;33(4):139-41.

\section{Beckmann 1988 \{published data only\}}

Beckmann SL, Os I, Kjeldsen SE, Mogensen B, Norum KR, Hjermann I. Non-pharmacological treatment of mild to moderate hypertension. A randomized, controlled study-results $11 / 2$ years later. Tidsskrift For Den Norske Laegeforening 1988;108:1593-7.

\section{Beckmann 1995 \{published data only\}}

Beckmann SL, Os I, Kjeldsen SE, Eide IK, Westheim AS, Hjermann I. Effect of dietary counselling on blood pressure and arterial plasma catecholamines in primary hypertension. American Journal of Hypertension 1995;8(7):704-11.

\section{Beresford 1992 \{published data only\}}

Beresford SAA, Farmer EMZ, Feingold L, Graves KL, Sumner SK, Baker RM. Evaluation of a self-help dietary intervention in a primary care setting. American Journal of Public Health 1992;82:79-84

\section{Bergstrom 1967 \{published data only\}}

Bergstrom G, Svanborg A. Dietary treatment of acute myocardial infarction. Acta Medica Scandinavica 1967;181(6):717-21.

\section{Bierenbaum 1963 \{published data only\}}

Bierenbaum ML, Fleischman AI, Raichelson RI, Hayton T, Watson P. Ten year experience of modified fat diets on younger men with coronary heart disease. Lancet 1973;i:1404-7.

Bierenbaum ML, Green DP, Florin A, Fleischman AI, Caldwell AB. Modified-fat dietary management of the young male with coronary disease. A five-year report. JAMA 1967;202(13):1119-23.

* Bierenbaum ML, Green DP, Gherman C, Florin A, Caldwell AB. The effects of two low fat dietary patterns on the blood cholesterol levels of young male coronary patients. Journal of Chronic Diseases 1963;16:1073-83.

\section{Bloemberg 1991 \{published and unpublished data\}}

Bloemberg BPM, Kromhout D, Goddijn HE, Jansen A, Obermann de Boer GL. The impact for the guidelines for a healthy diet of the Netherlands Nutrition Council on total and high density lipoprotein cholesterol in hypercholesterolemic free living men. American Journal of Epidemiology 1991;134:39-48.

\section{Bloomgarden 1987 \{published data only\}}

Bloomgarden ZT, Karmally W, Metzger MJ, Brothers M, Nechemias C, Bookman J, et al. Randomized, controlled trial of diabetic patient education: improved knowledge without improved metabolic status. Diabetes Care 1987;10:263-72.

\section{Bonnema 1995 \{published data only\}}

Bonnema SJ, Jespersen LT, Marving J, Gregersen G. Supplementation with olive oil rather than fish oil increases small arterial compliance in diabetic patients. Diabetes, Nutrition and Metabolism Clinical and Experimental 1995;8:81-7.

\section{Bosaeus 1992 \{published data only\}}

Bosaeus I, Belfrage L, Lindgren C, Andersson H. Olive oil instead of butter increases net cholesterol excretion from the small bowel. European Journal of Clinical Nutrition 1992;46(2):111-5.

Boyar 1988 \{published data only\}

Boyar AP, Rose DP, Loughridge JR, Engle A, Palge A, Laakso K, et al. Response to a diet low in total fat in women with postmenopausal breast cancer: a pilot study. Nutrition and Cancer 1988;11:93-9.

\section{Brensike 1982 \{published data only\}}

Brensike JF, Kelsey SF, Passamani ER, Fisher MR, Richardson JM, Loh IK, et al. National Heart, Lung, and Blood Institute type II Coronary Intervention Study: design, methods, and baseline characteristics. Controlled Clinical Trials 1982;3(2):91-111.

\section{Broekmans 2003 \{published and unpublished data\}}

Broekmans WMR, Klopping-Ketelaars IAA, Weststrate JA, Tijburg LBM, van Poppel G, Vink AA, et al. Decreased carotenoid concentrations due to dietary sucrose polyesters do not affect 
possible markers of disease risk in humans. Journal of Nutrition 2003;133:720-6.

\section{Brown 1984 \{published data only\}}

Brown GD, Whyte L, Gee MI, Crockford PM, Grace M, Oberle K, et al. Effects of two "lipid-lowering" diets on plasma lipid levels of patients with peripheral vascular disease. Journal of the American Dietetic Association 1984;84(5):546-50.

\section{Bruce 1994 \{published data only\}}

Bruce SL, Grove SK. The effect of a coronary artery risk evaluation program on serum lipid values and cardiovascular risk levels. Applied Nursing Research 1994;7(2):67-74.

\section{Bruno 1983 \{published data only\}}

Bruno R, Arnold C, Jacobson L, Winick M, Wynder E. Randomized controlled trial of a nonpharmacologic cholesterol reduction program at the worksite. Preventive Medicine 1983;12(4):523-32.

\section{Butcher 1990 \{published data only\}}

Butcher LA, O'Dea K, Sinclair AJ, Parkin JD, Smith IL, Blombery P. The effects of very low fat diets enriched with fish or kangaroo meat on cold-induced vasoconstriction and platelet function. Prostaglandins Leukot Essent. Fatty Acids 1990;39(3):221-6.

\section{Butowski 1998 \{published data only\}}

Butowski PF, Winder AF. Usual care dietary practice, achievement and implications for medication in the management of hypercholesterolaemia. European Heart Journal 1998;19:1328-33.

\section{Byers 1995 \{published data only\}}

Byers T, Mullis R, Anderson J, Dusenbury L, Gorsky R, Kimber C, et al. The costs and effects of a nutritional education program following work-site cholesterol screening. American Journal of Public Health 1995;85(5):650-5.

\section{Caggiula 1996 \{published data only\}}

Caggiula AW, Watson JE, Kuller LH, Olson MB, Milas NC, Berry M, et al. Cholesterol-lowering intervention program. Effect of the step I diet in community office practices. Archives of Internal Medicine 1996;156(11):1205-13.

\section{Cerin 1993 \{published data only\}}

Cerin A, Collins A, Landgren BM, Eneroth P. Hormonal and biochemical profiles of premenstrual syndrome. Treatment with essential fatty acids. Acta Obstetricia et Gynecologica Scandinavica 1993;72(5):337-43.

\section{Chan 1993 \{published data only\}}

Chan JK, McDonald BE, Gerrard JM, Bruce VM, Weaver BJ, Holub BJ. Effect of dietary alpha-linolenic acid and its ratio to linolenic acid on platelet and plasma fatty acids and thrombogenesis. Lipids 1993;28:811-7.

\section{Chapman 1950 \{published data only\}}

Chapman CB, Gibbons T, Henschel A. The effect of the rice-fruit diet on the composition of the body. New England Journal of Medicine 1950;243:899-905.

\section{Charbonnier 1975 \{published data only\}}

Charbonnier A, Nepveux P, Fluteau G, Fluteau D. Immediate effects of ingestion of olive oil on the principal lipid constituents of the plasma. Comparison with other edible fats. Medecine \& Chirurgie Digestives 1975;4:73-9.

\section{Cheng 2004 \{published data only\}}

Cheng C, Graziani C, Diamond JJ. Cholesterol-lowering effect of the Food for Heart Nutrition Education Program. Journal of the American Dietetic Association 2004;104(12):1868-72.

\section{Chicago CPEP 1977 \{published data only\}}

Farinaro E, Stamler J, Upton M, et al. Plasma glucose levels: long term effect of diet in the Chicago Coronary Prevention Evaluation Program. Annals of Internal Medicine 1977;86:147-54.

\section{Chiostri 1988 \{published data only\}}

Chiostri JE, Kwiterovich PO. Effect of American Heart Association Phase 2 diet versus eater's choice based diet on hypercholesterolaemia. Circulation 1988;78(4):II-385.

\section{Choudhury 1984 \{published data only\}}

Choudhury S, Jackson P, Katan MB, Marenah CB, Cortese C, Miller NE, et al. A multifactorial diet in the management of hyperlipidaemia. Atherosclerosis 1984;50:93-103.

\section{Clark 1997 \{published data only\}}

Clark M, Ghandour G, Miller NH, Taylor CB, Bandura A, DeBusk RF. Development and evaluation of a computer-based system for dietary management of hyperlipidemia. Journal of the American Dietetic Association 1997;97(2):146-50.

Clifton 1992 \{published data only\}

Clifton PM, Wight MB, Nestel PJ. Is fat restriction needed with HMGCoA reductase inhibitor treatment?. Atherosclerosis 1992;93(1-2):59-70.

\section{Cobb 1991 \{published data only\}}

Cobb MM, Teitelbaum HS, Breslow JL. Lovastatin efficacy in reducing low-density lipoprotein cholesterol levels on high-vs low-fat diets. JAMA 1991;265(8):997-1001.

\section{Cohen 1991 \{published data only\}}

Cohen MD, D'Amico FJ, Merenstein JH. Weight reduction in obese hypertensive patients. Family Medicine 1991;23(1):25-8.

\section{Cole 1988 \{published data only\}}

Cole TG, Schmeisser D, Prewitt TE. AHA phase 3 diet reduces cholesterol in moderately hypercholesterolemic premenopausal women [Abstract]. Circulation 1988;78(4):II-73.

Colquhoun 1990 \{published data only\}

Colquhoun DM, Moores D, Somerset SM. Comparison of the effects of an avocado enriched and american heart association diets on lipid levels [Abstract]. Arteriosclerosis 1990;10:875a.

\section{Consolazio 1946 \{published data only\}}

Consolazio FC, Forbes WH. The effects of high fat diet in a temperate environment. Journal of Nutrition 1946;32:195-204. 


\section{Cox 1996 \{published data only\}}

Cox RH, Gonzales-Vigilar MCRV, Novascone MA, Silva-Barbeau I. Impact of a cancer intervention on diet-related cardiovascular disease risks of white and african-american EFNEP clients. Journal of Nutrition Education 1996;28:209-18.

Croft 1986 \{published data only\}

Croft PR, Brigg D, Smith S, Harrison CB, Branthwaite A, Collins MF. How useful is weight reduction in the management of hypertension?. Journal of the Royal College of General Practitioners 1986;36(291):445-8.

\section{Crouch 1986 \{published data only\}}

Crouch M, Sallis JF, Farquar JW, et al. Personal and mediated health counselling for sustained dietary reduction of hypercholesterolaemia. Preventive Medicine 1986;15:282-91.

\section{Dalgard 2001 \{published data only\}}

Dalgard C, Thuroe A, Haastrup B, Haghfelt T, Stender S. Saturated fat intake is reduced in patients with ischemic heart disease 1 year after comprehensive counseling but not after brief counseling. Journal of the American Dietetic Association 2001;101(12):1420-9.

\section{Da Qing IGT 1997 \{published data only\}}

Pan XR, Li GW, Hu YH, Wang JX, Yang WY, An ZX, et al. Effects of diet and exercise in preventing NIDDM in people with impaired glucose tolerance. The Da Qing IGT and Diabetes Study. Diabetes Care 1997;20(4):537-44.

\section{DAS 2000 \{published data only\}}

Bovbjerg VE, McCann BS, Brief DJ, Follette WC, Retzlaff BM, Dowdy AA, et al. Spouse support and long-term adherence to lipid-lowering diets. American Journal of Epidemiology 1995;141(5):451-60.

* Knopp RH, Retzlaff B, Walden C, Fish B, Buck B, McCann B. One-year effects of increasingly fat-restricted, carbohydrateenriched diets on lipoprotein levels in free-living subjects. Proceedings of the Society for Experimental Biology \& Medicine 2000;225(3):191-9.

Knopp RH, Walden CE, McCann BS, Retzlaff B, Dowdy A Gey G, Cooper MN. Serial changes in lipoprotein cholesterol in hypercholesterolemic men treated with alternative diets [abstract]. Arteriosclerosis 1989;9:745A.

Knopp RH, Walden CE, Retzlaff BM, McCann BS, Dowdy AA, Albers JJ, et al. Long-term cholesterol-lowering effects of 4 fat-restricted diets in hypercholesterolaemic and combined hyperlipidaemic men: the Dietary Alternatives Study. JAMA 1997;278:1509-15.

Walden CE, McCann BS, Retzlaff B, Dowdy A, Hanson M, Fish B, et al. Alternative fat-restricted diets for hypercholesterolemia and combined hyperlipidemia: feasibility, design, subject recruitment, and baseline characteristics of the dietary alternatives study. Journal of the American College of Nutrition 1991;10(5):429-42.
DASH 1997 \{published data only\}

* Appel LJ, Moore TJ, Obarzanek E, Vollmer WM, Svetkey LP, Sacks FM, et al. A clinical trial of the effects of dietary patterns on blood pressure. DASH Collaborative Research Group. New England Journal of Medicine 1997;336(16):1117-24.

Blackburn GL. Functional foods in the prevention and treatment of disease: significance of the Dietary Approaches to Stop Hypertension Study. American Journal of Clinical Nutrition 1997;66(5):1067-71.

\section{Davey Smith 2005 \{published data only\}}

Davey Smith G, Bracha Y, Svendsen KH, Neaton JD, Haffner SM, Kuller $\mathrm{LH}$, et al. Incidence of type 2 diabetes in the randomized multiple risk factor intervention trial. Annals of Internal Medicine 2005;142(5):313-22.

\section{de Boer 1983 \{published data only\}}

de Boer AC, Turek JV, Pannebakker MA, den OG. The effect of diets high in polyunsaturated and high in saturated fatty acids on blood lipids and platelet tests in patients with coronary artery disease (CAD) [abstract]. Thrombosis and Haemostasis 1983;50:96

\section{de Bont 1981 \{published and unpublished data\}}

de Bont AJ, Baker IA, St, Sweetnam PM, Wragg KG, Stephens SM, Hayes TM. A randomised controlled trial of the effect of low fat diet advice on dietary response in insulin independent diabetic women. Diabetologia 1981;21(6):529-33.

\section{DeBusk 1994 \{published data only\}}

DeBusk RF, Miller NH, Superko HR, Dennis CA, Thomas RJ, Lew $\mathrm{HT}$, et al. A case-management system for coronary risk factor modification after acute myocardial infarction [see comments]. Annals of Internal Medicine 1994;120(9):721-9.

Delahanty 2001 \{published data only\}

Delahanty LM, Hayden D, Ammerman A, Nathan DM. Medical nutrition therapy for hypercholesterolemia positively affects patient satisfaction and quality of life outcomes. Annals of Behavioral Medicine 2002;24(4):269-78.

* Delahanty LM, Sonnenberg LM, Hayden D, Nathan DM. Clinical and cost outcomes of medical nutrition therapy for hypercholesterolemia: a controlled trial. Journal of the American Dietetic Association 2001;101(9):1012-23.

Delius 1969 \{published data only\}

Delius L. Treatment of hypotensive circulatory disorder [Die Behandlung der hypotonen Kreislaufregulationsstörung]. Deutsche Medizinische Wochenschrift 1969;94(42):2172-3.

\section{Demark 1990 \{published data only\}}

Demark WW, Bowering J, Cohen PS. Reduced serum cholesterol with dietary change using fat-modified and oat bran supplemented diets. Journal of the American Dietetic Association $1990 ; 90(2): 223-9$

\section{Dengel 1995 \{published data only\}}

Dengel JL, Katzel LI, Goldberg AP. Effect of an American Heart Association diet, with or without weight loss, on lipids in obese 
middle-aged and older men. American Journal of Clinical Nutrition 1995;62(4):715-21.

\section{Denke 1994 \{published data only\}}

Denke MA, Grundy SM. Individual responses to a cholesterol lowering diet in 50 men with moderate hypercholesterolaemia. Archives of Internal Medicine 1994;154:17-25.

\section{Diabetes CCT 1995 \{published data only\}}

Effect of intensive diabetes management on macrovascular events and risk factors in the Diabetes Control and Complications Trial. American Journal of Cardiology 1995;75:894-903.

\section{DIET 1998 \{published data only\}}

Dornelas EA, Wylie-Rosett J, Swencionis C. The DIET study: long term outcomes of a cognitive-behavioural weight control intervention in independent-living elders. Journal of the American Dietetic Association 1998;98(11):1276-81.

\section{Ding 1992 \{published data only\}}

Ding Q. Clinical study of qianxining in the treatment of 60 cases of yang hyperactivity due to yin deficiency type of hypertension. Chung Kuo Chung Hsi I Chieh Ho Tsa Chih 1992;12:409-11, 388.

\section{Dobs 1991 \{published data only\}}

Dobs AS, Sarma PS, Wilder L. Lipid-lowering diets in patients taking pravastatin, a new HMG-CoA reductase inhibitor: compliance and adequacy. American Journal of Clinical Nutrition 1991;54(4):696-700.

\section{Duffield 1982 \{published data only\}}

Duffield RG, Lewis B, Miller NE, Jamieson CW, Brunt JN, Colchester AC. Treatment of hyperlipidaemia retards progression of symptomatic femoral atherosclerosis. A randomised controlled trial. Lancet 1983;2(8351):639-42.

* Duffield RG, Miller NE, Jamieson CW, Lewis B. A controlled trial of plasma lipid reduction in peripheral atherosclerosis--an interim report. British Journal of Surgery 1982;69(Suppl):S3-S5.

\section{Dullaart 1997 \{published and unpublished data\}}

Dullaart RP, Hoogenberg K, Riemens SC, Groener JE, van TA, Sluiter WJ, et al. Cholesteryl ester transfer protein gene polymorphism is a determinant of HDL cholesterol and of the lipoprotein response to a lipid-lowering diet in type 1 diabetes. Diabetes 1997;46(12):2082-7.

\section{Eating Patterns 1997 \{published and unpublished data\}}

Beresford SA, Curry SJ, Kristal AR, Lazovich D, Feng Z, Wagner $\mathrm{EH}$. A dietary intervention in primary care practice: the Eating Patterns Study. American Journal of Public Health 1997;87(4):610-6.

\section{Ehnholm 1982 \{published data only\}}

Ehnholm C, Huttunen JK, Pietinen P, Leino U, Mutanen M, Kostiainen $\mathrm{E}$, et al. Effect of diet on serum lipoproteins in a population with a high risk of coronary heart disease. New England Journal of Medicine 1982;307:850-5.
Ehnholm 1984 \{published data only\}

Ehnholm C, Huttunen JK, Pietinen P, Leino U, Mutanen M, Kostiainen $\mathrm{E}$, et al. Effect of a diet low in saturated fatty acids on plasma lipids, lipoproteins, and HDL subfractions. Arteriosclerosis 1984;4(3):265-9.

Eisenberg 1990 \{published data only\}

Eisenberg S. The effect of dietary substitution of monounsaturated fatty acids with carbohydrates on lipoprotein levels, structure, and function in a free-living population [abstract]. Arteriosclerosis 1990;10:872A.

Elder 2000 \{published data only\}

Elder JP, Candelaria JI, Woodruff SI, Criqui MH, Talavera GA, Rupp JW. Results of language for health: cardiovascular disease nutrition education for Latino English-as-a-second-language students. Health Education \& Behavior 2000;27(1):50-63.

Ellegard 1991 \{published data only\}

Ellegard L, Bosaeus I. Sterol and nutrient excretion in ileostomists on prudent diets. European Journal of Clinical Nutrition 1991;45(9):451-7.

Esposito 2003 \{published data only\} Esposito K, Pontillo A, Di Palo C, Giugliano G, Masella M, Marfella R, et al. Effect of weight loss and lifestyle changes on vascular inflammatory markers in obese women: a randomized trial. JAMA 2003;289(14):1799-804.

\section{EUROACTION 2008 \{published data only\}}

Wood DA, Kotseva K, Connolly S, Jennings C, Mead A, Jones J, et al. Nurse-coordinated multidisciplinary, familybased cardiovascular disease prevention programme (EUROACTION) for patients with coronary heart disease and asymptomatic individuals at high risk of cardiovascular disease: a paired, cluster-randomised controlled trial. Lancet 2008;371(9629):1999-2012.

\section{FARIS 1997 \{published data only\}}

Goble A, Jackson B, Phillips P, Race E, Oliver RG, Worcester MC. The Family Atherosclerosis Risk Intervention Study (FARIS): risk factor profiles of patients and their relatives following an acute cardiac event. Australian and New Zealand Journal of Medicine 1997;27:568-77.

\section{Fasting HGS 1997 \{published data only\}}

Dyson PA, Hammersley MS, Morris RJ, Holman RR, Turner RC. The Fasting Hyperglycaemia Study: II. Randomized controlled trial of reinforced healthy-living advice in subjects with increased but not diabetic fasting plasma glucose. Metabolism 1997;46(12 Suppl 1):50-5.

\section{Ferrara 2000 \{published data only\}}

Ferrara LA, Raimondi AS, d'Episcopo L, Guida L, Dello Russo A, Marotta T. Olive oil and reduced need for antihypertensive medications. Archives of Internal Medicine 2000;160:837-42.

Fielding 1995 \{published data only\}

Fielding CJ, Havel RJ, Todd KM, Yeo KE, Schloetter MC Weinberg V, et al. Effects of dietary cholesterol and fat saturation on plasma lipoproteins in an ethnically diverse 
population of healthy young men. Journal of Clinical Investigation 1995;95(2):611-8.

\section{Finckenor 2000 \{published data only\}}

Finckenor M. Nutrition intervention group program based on preaction-stage-oriented change processes of the Transtheoretical Model promotes long-term reduction in dietary fat intake. Journal of the American Dietetic Association 2000;100(3):335-42.

\section{Finnish Diabet Prev 2000 \{published data only\}}

Uusitupa M, Louheranta A, Lindstrom J, Valle T, Sundvall J, Eriksson J, et al. The Finnish Diabetes Prevention Study. British Journal of Nutrition 2000;83(Suppl 1):S137-42.

\section{Finnish Mental Hosp 1972 \{published data only\}}

* Miettinen M, Turpeinen O, Karvonen MJ, Elosuo R, Paavilainen E. Effect of cholesterol-lowering diet on mortality from coronary heart-disease and other causes: a twelve-year clinical trial in men and women. Lancet 1972;2(782):835-8.

Miettinen M, Turpeinen O, Karvonen MJ, Pekkarinen M, Paavilainen E, Elosuo R. Dietary prevention of coronary heart disease in women: the Finnish mental hospital study. International Journal of Epidemiology 1983;12(1):17-25.

Turpeinen O, Miettinen M, Karvonen M, Roine P, Pekkarinen M, Lehtosuo EJ, et al. Dietary prevention of coronary heart disease: long-term experiment. I. Observations on male. American Journal of Clinical Nutrition 1968;21(4):255-76.

Fisher 1981 \{published data only\}

Fisher EA, Breslow JL, Zannis VI, Shen G, Blum CB. Dietary saturated fat, not cholesterol, affects plasma lipids and Apo E. Arteriosclerosis 1981;1(5):364a.

\section{Fleming 2002 \{published data only\}}

Fleming RM. The effect of high-, moderate-, and low-fat diets on weight loss and cardiovascular disease risk factors. Preventive Cardiology 2002;5:110-5.

\section{Fortmann 1988 \{published data only\}}

Fortmann SP, Haskell WL, Wood PD. Effects of weight loss on clinic and ambulatory blood pressure in normotensive men. American Journal of Cardiology 1988;62(1):89-93.

\section{Foster 2003 \{published data only\}}

Foster GD, Wyatt HR, Hill JO, McGuckin BG, Brill C, Mohammed BS, et al. A randomized trial of a lowcarbohydrate diet for obesity. New England Journal of Medicine 2003;348(21):2082-90.

\section{FRESH START 2007 \{published data only\}}

Denmark-Wahnefried W, Clipp EC, Lipkus IM, Lobach D, Snyder DC, Sloane R, et al. Main outcomes of the FRESH START trial: a sequentially tailored, diet and exercise mailed print intervention among breast and prostate cancer survivors. Journal of Clinical Oncology 2007;25(19):2709-18.

\section{Gambera 1995 \{published data only\}}

Gambera PJ, Schneeman BO, Davis PA. Use of the Food Guide Pyramid and US Dietary Guidelines to improve dietary intake and reduce cardiovascular risk in active-duty Air Force members. Journal of the American Dietetic Association 1995;95(11):1268-73.

\section{Gaullier 2007 \{published data only\}}

Gaullier J-M, Halse J, Hoivik HO, Hoye K, Syvertsen C, Nurminiemi M, et al. Six months supplementation with conjugated linoleic acid induces regional-specific fat mass decreases in overweight and obese. British Journal of Nutrition 2007;97:550-60.

\section{Ginsberg 1988 \{published data only\}}

Ginsberg H. Both a high monounsaturated fat diet and the step 1 AHA diet significantly reduce plasma cholesterol levels in healthy males [abstract]. Circulation 1988;78:I173.

Gjone 1972 \{published data only\}

Gjone E, Nordoy A, Blomhoff JP, Wiencke I. The effects of unsaturated and saturated dietary fats on plasma cholesterol, phospholipids and lecithin: cholesterol acyltransferase activity. Acta Medica Scandinavica 1972;191(6):481-4.

Glatzel 1966 \{published data only\}

Glatzel $H$. The relationship between postprandial triglyceridemia and the fat content of the basic diet [Die Abhängigkeit der postcenalen Triglyceridamie vom Fettgehalt der Grundkost]. Klinische Wochenschrift 1966;44(5):283-4.

\section{Goodpaster 1999 \{published data only\}}

Goodpaster BH, Kelley DE, Wing RR, Meier A, Thaete FL. Effects of weight loss on regional fat distribution and insulin sensitivity in obesity. Diabetes 1999;48:839-47.

\section{Grundy 1986 \{published data only\}}

Grundy SM, Nix D, Whelan MF, Franklin L. Comparison of three cholesterol-lowering diets in normolipidaemic men. JAMA 1986;256:2351-5.

\section{Hardcastle 2008 \{published data only\}}

Hardcastle S, Taylor A, Bailey M, Castle R, Hardcastle Sarah, Taylor Adrian, et al. A randomised controlled trial on the effectiveness of a primary health care based counselling intervention on physical activity, diet and CHD risk factors. Patient Education \& Counseling 2008;70(1):31-9.

\section{Harris 1990 \{published data only\}}

Harris WS, Feldman EB. Intensive dietary intervention in hypercholesterolemic patients. Observed versus predicted changes in cholesterol levels [abstract]. Arteriosclerosis 1990;10:853A.

\section{Hartman 1993 \{published data only\}}

Hartman T, McCarthy P, Himes J. Use of eating pattern messages to evaluate changes in eating behaviors in a worksite cholesterol education program. Journal of the American Dietetic Association 1993;93:1119-23.

Hartwell 1986 \{published data only\}

Hartwell SL, Kaplan RM, Wallace JP. Comparison of behavioral interventions for control of type II diabetes mellitus. Behavior Therapy 1986;17:447-61. 
Hashim 1960 \{published data only\}

Hashim SA, Arteaga A, van Itallie TB. Effect of saturated medium-chain triglyceride on serum-lipids in man. Lancet 1960;1:1105-7.

\section{Haynes 1984 \{published data only\}}

Haynes RB, Harper AC, Costley SR, Johnston M, Logan AG, Flanagan PT, et al. Failure of weight reduction to reduce mildly elevated blood pressure: a randomized trial. Journal of Hypertension 1984;2(5):535-9.

\section{Heber 1991 \{published data only\}}

Heber D, Ashley JM, Leaf DA, Barnard JA. Reduction of serum estradiol in postmenopausal women given free access to low-fat high carbohydrate diet. Nutrition 1991;7:137-41.

\section{Heine 1989 \{published and unpublished data\}}

Heine RJ, Mulder C, Popp-Snijders C, van der Meer J, van der Veen EA. Linoleic-acid-enriched diet: long-term effects on serum lipoprotein and apolipoprotein concentration and insulin sensitivity in noninsulin-dependent diabetic patients. American Journal of Clinical Nutrition 1989;49:448-56.

Hellenius Diet \& Ex 95 \{published and unpublished data\} Hellenius ML, Brismar KE, Berglund BH, de Faire U. Effects on glucose tolerance, insulin secretion, insulin-like growth factor 1 and its binding protein, IGFBP-1, in a randomized controlled diet and exercise study in healthy, middle-aged men. Journal of Internal Medicine 1995;238(2):121-30.

Hellenius ML, Dahlof C, Aberg H, Krakau I, de Faire U. Quality of life is not negatively affected by diet and exercise intervention in healthy men with cardiovascular risk factors. Quality of Life Research 1995;4(1):13-20.

Hellenius ML, Krakau I, De Faire U. Favourable long-term effects from advice on diet and exercise given to healthy men with raised cardiovascular risks. Nutrition, Metabolism and Cardiovascular Diseases 1997;7:293-300.

Hellenius ML, de Faire U, Berglund B, Hamsten A, Krakau I. Diet and exercise are equally effective in reducing risk for cardiovascular disease. Results of a randomized controlled study in men with slightly to moderately raised cardiovascular risk factors. Atherosclerosis 1993;103(1):81-91.

* Hellenius, ML. Prevention of cardiovascular disease: studies on the role of diet and exercise in the prevention of cardiovascular disease among middle-aged men. Huddinge, Sweden: Karolinska Intitute, 1995.

Naslund GK, Fredrikson M, Hellenius ML, de Faire U. Effect of diet and physical exercise intervention programmes on coronary heart disease risk in smoking and non-smoking men in Sweden. Journal of Epidemiology and Community Health 1996;50(2):131-6.

\section{Hellenius Diet 1995 \{published and unpublished data}

Hellenius ML, Brismar KE, Berglund BH, de Faire U. Effects on glucose tolerance, insulin secretion, insulin-like growth factor 1 and its binding protein, IGFBP-1, in a randomized controlled diet and exercise study in healthy, middle-aged men. Journal of Internal Medicine 1995;238(2):121-30.

Hellenius ML, Dahlof C, Aberg H, Krakau I, de Faire U. Quality of life is not negatively affected by diet and exercise intervention in healthy men with cardiovascular risk factors. Quality of Life Research 1995;4(1):13-20.

Hellenius ML, Krakau I, De Faire U. Favourable long-term effects from advice on diet and exercise given to healthy men with raised cardiovascular risks. Nutrition, Metabolism and Cardiovascular Diseases 1997;7:293-300.

Hellenius ML, de Faire U, Berglund B, Hamsten A, Krakau I. Diet and exercise are equally effective in reducing risk for cardiovascular disease. Results of a randomized controlled study in men with slightly to moderately raised cardiovascular risk factors. Atherosclerosis 1993;103(1):81-91.

* Hellenius, ML. Prevention of cardiovascular disease: studies on the role of diet and exercise in the prevention of cardiovascular disease among middle-aged men. Huddinge, Sweden: Karolinska Intitute, 1995.

Naslund GK, Fredrikson M, Hellenius ML, de FU. Effect of diet and physical exercise intervention programmes on coronary heart disease risk in smoking and non-smoking men in Sweden. J.Epidemiol.Community Health 1996;50(2):131-6.

Heller 1993 \{published and unpublished data\}

* Heller RF, Knapp JC, Valenti LA, Dobson AJ. Secondary prevention after acute myocardial infarction. American Journal of Cardiology 1993;72(11):759-62.

Heller RF, Walker RJ, Boyle CA, O'Connell DL, Rusakaniko S, Dobson AJ. A randomised controlled trial of a dietary advice program for relatives of heart attack victims. Medical Journal of Australia 1994;161(9):529-31.

Hildreth 1951 \{published data only\}

Hildreth EA, Mellinkoff SM, Blair GW, Hildreth DM. The effect of vegetable fat ingestion on human serum cholesterol concentration. Circulation 1951;3:641-6.

Holm 1990 \{published data only (unpublished sought but not used)\}

* Holm LE, Nordevang E, Ikkala E, Hallstrom L, Callmer E. Dietary intervention as adjuvant therapy in breast cancer patients--a feasibility study. Breast Cancer Research and Treatment 1990;16(2):103-9.

Nordevang E, Callmer E, Marmur A, Holm LE. Dietary intervention in breast cancer patients: effects on food choice. European Journal of Clinical Nutrition 1992;46(6):387-96.

Nordevang E, Ikkala E, Callmer E, Hallstrom L, Holm LE. Dietary intervention in breast cancer patients: effects on dietary habits and nutrient intake. European Journal of Clinical Nutrition 1990;44(9):681-7. 
Hood 1965 \{published data only\}

Hood B, Sanne H, Orndahl G, Ahlstrom M, Welin G. Long term prognosis in essential hypercholesterolaemia: the effect of a strict diet. Acta Medica Scandinavica 1965;178:161-73.

\section{Horlick 1957 \{published data only\}}

* Horlick L, Craig BM. Effect of long-chain polyunsaturated and saturated fatty acids on the serum-lipids of man. Lancet 1957;2:566-9.

\section{Horlick 1960 \{published data only\}}

Horlick L, O'Neil JB. Effect of modified egg-yolk fats on bloodcholesterol levels [letter]. Lancet 1960;1:438.

\section{Howard 1977 \{published data only\}}

Howard AN, Marks J. Hypocholesterolaemic effect of milk [letter]. Lancet 1977;2(8031):255-6.

\section{Hunninghake 1990 \{published data only\}}

Hunninghake DB, Laskarzewski PM. Gender difference in the response to lovastatin administration with and without a cholesterol lowering diet [abstract]. Arteriosclerosis 1990;10:786A.

\section{Hutchison 1983 \{published data only\}}

Hutchison K, Oberle K, Crockford P, Grace M, Whyte L, Gee M, et al. Effects of dietary manipulation on vascular status of patients with peripheral vascular disease. JAMA 1983;249(24):3326-30.

\section{Hyman 1998 \{published and unpublished data}

Hyman DJ, Ho KSI, Dunn K, Simons-Morton D. Dietary intervention for cholesterol reduction in public clinic patients. American Journal of Preventive Medicine 1998;15:139-45.

\section{Iacono 1981 \{published data only\}}

Iacono JM, Judd JT, Marshall MW, Canary JJ, Dougherty RM, Mackin JF, et al. The role of dietary essential fatty acids and prostaglandins in reducing blood pressure. Progress in Lipid Research 1981;20:349-64.

\section{IMPACT 1995 \{published data only\}}

Fielding JE, Mason T, Kinght K, Klesges R, Pelletier KR. A randomized trial of the IMPACT worksite cholesterol reduction program. American Journal of Preventive Medicine 1995;11:120-3.

\section{Ishikawa 1995 \{published data only\}}

Ishikawa H, Akedo I, Suzuki T, Otani T, Sobue T. Interventional trial for colorectal cancer prevention in Osaka: an introduction to the protocol. Japanese Journal of Cancer Research 1995;86(8):707-10.

\section{Iso 1991 \{published data only\}}

Iso H, Konishi M, Terao A, Kiyama M, Tanigaki M, Baba M, et al. A community-based education program for serum cholesterol reduction in urban hypercholesterolemic persons: comparison of intensive and usual education groups. Nippon.Koshu.Eisei.Zasshi [Japanese Journal of Public Health] 1991;38(9):751-61.

\section{Ives 1993 \{published data only\}}

Ives DG, Kuller LH, Traven ND. Use and outcomes of a cholesterol-lowering intervention for rural elderly subjects. American Journal of Preventive Medicine 1993;9(5):274-81.

\section{Jalkanen 1991 \{published data only\}}

Jalkanen $L$. The effect of a weight reduction program on cardiovascular risk factors among overweight hypertensives in primary health care. Scandinavian Journal of Social Medicine 1991;19(1):66-71.

\section{Jepson 1969 \{published data only\}}

Jepson EM, Fahmy MF, Torrens PE, Billimoria JD, Maclagan NF. Treatment of essential hyperlipidaemia. Lancet 1969;2(7634):1315-9.

\section{Jerusalem Nut 1992 \{published data only\}}

Berry EM, Eisenberg S, Friedlander Y, Harats D, Kaufmann NA, Norman Y, et al. Effects of diets rich in monounsaturated fatty acids on plasma lipoproteins: the Jerusalem Nutrition Study. II. Monounsaturated fatty acids vs carbohydrates. American Journal of Clinical Nutrition 1992;56(2):394-403.

Jula 1990 \{published data only\}

Jula A, Ronnemaa T, Rastas M, Karvetti RL, Maki J. Longterm nopharmacological treatment for mild to moderate hypertension. Journal of Internal Medicine 1990;227(6):413-21.

Junker 2001 \{published data only\}

Junker R, Pieke B, Schulte H, Nofer R, Neufeld M, Assmann G, et al. Changes in hemostasis during treatment of hypertriglyceridemia with a diet rich in monounsaturated and $\mathrm{n}-3$ polyunsaturated fatty acids in comparison with a low-fat diet. Thrombosis Research 2001;101(5):355-66.

\section{Karmally 1990 \{published data only\}}

Karmally W, Carpentiri C, Viscardi T, Cheverez V, Holleran S, Ramakrishnan $\mathrm{R}$, et al. Replacing monounsaturated by polyunsaturated fatty acids within an AHA step I diet does not affect the plasma levels or metabolism of low density and high density lipoproteins in normal men [abstract]. Arteriosclerosis 1990;10:877A.

\section{Karvetti 1992 \{published data only\}}

Karvetti RL, Hakala P. A seven-year follow-up of a weight reduction programme in Finnish primary health care. European Journal of Clinical Nutrition 1992;46:743-52.

Kastarinen 2002 \{published data only\}

Kastarinen MJ, Puska PM, Korhonen MH, Mustonen JN, Salomaa VV, Sundvall JE, et al. Non-pharmacological treatment of hypertension in primary health care: A 2-year open randomized controlled trial of lifestyle intervention against hypertension in eastern Finland. Journal of Hypertension 2002;20(12):01.

\section{Kather 1985 \{published data only\}}

Kather $\mathrm{H}$, Wildenberg $\mathrm{U}$, Wieland $\mathrm{E}$. Influence of different dietary conditions in ideal-weight subjects on serum levels of free fatty acids and of glycerol in vivo and on lipid mobilization in vitro [abstract]. European Journal of Clinical Investigation 1985;15:A. 


\section{Katzel 1995a \{published data only\}}

Katzel LI, Coon PJ, Dengel J, Goldberg AP. Effect of an American Heart Association Step I diet and weight loss on lipoprotein lipid levels in obese men with silent myocardial ischaemia and reduced high density lipoprotein cholesterol. Metabolism 1995;44:307-14.

\section{Katzel 1995b \{published data only\}}

Katzel LI, Bleecker ER, Colman EG, Rogus EM, Sorkin JD, Goldberg AP. Effects of weight loss vs aerobic exercise training on risk factors for coronary disease in healthy, obese, middleaged and older men. A randomized controlled trial [see comments]. JAMA 1995;274(24):1915-21.

\section{Kawamura 1993 \{published data only\}}

Kawamura M, Akasaka T, Kasatsuki T, Nakajima J, Onodera S, Fujiwara T, et al. Blood pressure is reduced by short-time calorie restriction in overweight hypertensive women with a constant intake of sodium and potassium. Journal of Hypertension. Supplement 1993;11(Suppl 5):S320-1.

Keidar 1988 \{published data only\} Keidar S, Krul ES, Goldberg AC, Bateman J, Schonfield G. Fatfree diet modulates epitope expression of LDL-apo [abstract]. Arteriosclerosis 1988;8:565A.

\section{Kempner 1948 \{published data only\}}

Kempner W. Treatment of hypertensive vascular disease with rice diet. American Journal of Medicine 1948;4:545-77.

Keys 1952 \{published data only\}

Keys A. Human atherosclerosis and the diet. Circulation 1952;5:115-8.

\section{Keys 1957a \{published data only\}}

Keys A, Anderson JT, Grande F. Serum-cholesterol response to dietary fat [letter]. Lancet 1957;1:787.

\section{Keys 1957b \{published data only\}}

Keys A, Anderson JT, Grande F. Essential fatty acids, degree of unsaturation, and effect of corn (maize) oil on the serumcholesterol level in man. Lancet 1957;1:66-8.

\section{Keys 1957c \{published data only\}}

Keys A. Prediction of serum-cholesterol responses of man to changes in fats in the diet. Lancet 1957;2:959-66.

\section{Khan 2003 \{published and unpublished data\}}

Khan F, Elherik K, Bolton-Smith C, Barr R, Hill A, Murrie I, et al. The effects of dietary fatty acid supplementation on endothelial function and vascular tone in healthy subjects. Cardiovascular Research 2003;59:955-62.

\section{King 2000 \{published data only\}}

King S, David S, Newton H, Hevey D, Rafferty F, Horgan JH. The effect of dietary modification on the training outcome and body composition in patients undergoing undergoing a cardiac rehabilitation programme. Coronary Health Care 2000;4(2):76-81.

\section{Kingsbury 1961 \{published data only\}}

Kingsbury KJ, Morgan DM, Aylott C, Emmerson R. Effects of ethyl arachidonate, cod-liver oil, and corn oil on the plasmacholesterol level: a comparison in normal volunteers. Lancet 1961;1:739-41.

\section{Kohler 1986 \{published data only\}}

Kohler VH, Voigt H, Reuter W, Peters H-J, Kuklinski B, Scheel $\mathrm{H}$, et al. Results of a long-term study of arteriosclerotic circulatory disorders with polyene fatty acid therapy [German]. Zeitschrift für die Gesamte Innere Medizin und ihre Grenzgebiete 1986;41:91-3.

\section{Koopman 1990 \{published data only\}}

Koopman H, Spreeuwenberg C, Westerman RF, Donker AJ. Dietary treatment of patients with mild to moderate hypertension in a general practice: a pilot intervention study (2). Beyond three months. Journal of Human Hypertension 1990;4(4):372-4.

\section{Korhonen 2003 \{published data only\}}

Korhonen M, Kastarinen M, Uusitupa M, Puska P, Nissinen A. The effect of intensified diet counselling on the diet of hypertensive subjects in primary health care: a 2-year open randomized controlled trial of lifestyle intervention against hypertension in eastern Finland. Preventive Medicine 2003;36(1):8-16.

\section{Kriketos 2001 \{published data only\}}

Kriketos AD, Robertson RM, Sharp TA, Drougas H, Reed GW, Storlien $\mathrm{LH}$, et al. Role of weight loss and polyunsaturated fatty acids in improving metabolic fitness in moderately obese, moderately hypertensive subjects. Journal of Hypertension 2001;19(10):1745-54.

\section{Kris 1994 \{published data only\}}

Kris EP, Mustad VA. Chocolate feeding studies: a novel approach for evaluating the plasma lipid effects of stearic acid. American Journal of Clinical Nutrition 1994;60(6 Suppl):1029S-36S.

\section{Kristal 1997 \{published data only\}}

Kristal AR, Shattuck AL, Bowen DJ, Sponzo RW, Nixon DW. Feasibility of using volunteer research staff to deliver and evaluate a low-fat dietary intervention: the American Cancer Society Breast Cancer Dietary Intervention Project. Cancer Epidemiology, Biomarkers and Prevention 1997;6(6):459-67.

\section{Kromhout 1987 \{published data only\}}

Kromhout D, Arntzenius AC, Kempen-Voogd N, Kempen HJ, Barth JD, van der Voort HA, et al. Long-term effects of linoleic-acid enriched diet, changes in body weight and alcohol consumption on serum total and HDL cholesterol. Atherosclerosis 1987;66:99-105.

\section{Kummel 2008 \{published data only\}}

Kummel M V. Effects of an intervention on health behaviors of older coronary artery bypass (CAB) patients. Archives of Gerontology and Geriatrics 2008;2(2):227-44.

Laitinen 1993 \{published data only\}

Laitinen JH, Ahola IE, Sarkkinen ES, Winberg RL, Harmaakorpi IP, Uusitupa MI. Impact of intensified dietary 
therapy on energy and nutrient intakes and fatty acid composition of serum lipids in patients with recently diagnosed non-insulin-dependent diabetes mellitus. Journal of the American Dietetic Association 1993;93(3):276-83.

\section{Laitinen 1994 \{published data only\}}

Laitinen J, Uusitupa M, Ahola I, Siitonen O. Metabolic and dietary determinants of serum lipids in obese patients with recently diagnosed non-insulin-dependent diabetes. Annals of Medicine 1994;26(2):119-24.

\section{Leduc 1994 \{published data only\}}

Leduc CP, Cherniak D, Faucher J. Effectiveness of a group dietary intervention on hypercholesterolaemia: a randomised controlled clinical trial [poster abstract]. Atherosclerosis 1994;19:149.

\section{Lewis 1958 \{published data only\}}

Lewis B. Effect of certain dietary oils on bile-acid secretion and serum-cholesterol. Lancet 1958;1:1090-2.

\section{Lewis 1981 \{published data only\}}

Lewis B, Hammett F, Katan M, Kay RM, Merkx I, Nobels A, et al. Towards an improved lipid-lowering diet: additive effects of changes in nutrient intake. Lancet 1981;2(8259):1310-3.

\section{Lewis 1985 \{published data only\}}

Lewis B. Randomised controlled trial of the treatment of hyperlipidaemia on progression of atherosclerosis. Acta Medica Scandinavica. Supplement 1985;701:53-7.

\section{Lichtenstein 2002 \{published data only\}}

Lichtenstein AH, Ausman LM, Jalbert SM, Vilella-Bach M, Jauhiainen M, McGladdery S, et al. Efficacy of a therapeutic lifestyle change/step 2 diet in moderately hypercholesterolemic middle-aged and elderly female and male subjects. Journal of Lipid Research 2002;43(2):264-73.

\section{Linko 1957 \{published data only\}}

Linko E. Vegetable oils and serum cholesterol: short-term experiments with rapeseed and sunflower oils. Acta Medica Scandinavica 1957;159:475-88.

\section{Lipid Res Clinic 1984 \{published data only\}}

* The Lipid Research Clinics Coronary Primary Prevention Trial results. I. Reduction in incidence of coronary heart disease. JAMA 1984;251(3):351-64.

The Lipid Research Clinics Coronary Primary Prevention Trial results. II. The relationship of reduction in incidence of coronary heart disease to cholesterol lowering. JAMA 1984;251(3):365-74.

Gordon DJ, Salz KM, Roggenkamp KJ. Dietary determinants of plasma cholesterol change in the recruitment phase of the Lipid Research Clinics Coronary Primary Prevention Trial. Arteriosclerosis 1982;2(6):537-48.

\section{Little 1990 \{published data only\}}

Little P, Girling G, Hasler A, Craven A, Trafford A. The effect of a combination low sodium, low fat, high fibre diet on serum lipids in treated hypertensive patients. European Journal of Clinical Nutrition 1990;44(4):293-300.

\section{Little 1991 \{published data only\}}

Little P, Girling G, Hasler A, Trafford A. A controlled trial of a low sodium, low fat, high fibre diet in treated hypertensive patients: effect on antihypertensive drug requirement in clinical practice. Journal of Human Hypertension 1991;5(3):175-81.

\section{Little 2004 \{published data only\}}

Little P, Kelly J, Barnett J, Dorward M, Margetts B, Warm D, et al. Randomised controlled factorial trial of dietary advice for patients with a single high blood pressure reading in primary care. BMJ 2004;328(7447):1054.

\section{Lottenberg 1996 \{published data only\}}

Lottenberg AM, Nunes VS, Lottenberg SA, Shimabukuro AF, Carrilho AJ, Malagutti S, et al. Plasma cholesteryl ester synthesis, cholesteryl ester transfer protein concentration and activity in hypercholesterolemic women: effects of the degree of saturation of dietary fatty acids in the fasting and postprandial states. Atherosclerosis 1996;126(2):265-75.

\section{Luszczynska 2007 \{published data only\}}

Luszczynska A, Scholz U, Sutton S. Planning to change diet: a controlled trial of an implementation intentions training intervention to reduce saturated fat intake among patients after myocardial infarction. Journal of Psychosomatic Research 2007;63(5):491-7.

\section{Lyon Diet Heart 1994 \{published data only\}}

* De Lorgeril M, Renaud S, Mamelle N, Salen P, Martin JL, Monjaud I, et al. Mediterranean alpha-linolenic acid-rich diet in secondary prevention of coronary heart disease. Lancet 1994;343(8911):1454-9.

De Lorgeril M, Salen P. Mediterranean diet in secondary prevention of coronary heart disease. Australian Journal of Nutrition and Dietetics 1998;55(Suppl):s16-s20.

De Lorgeril M, Salen P, Caillat-Vallet E, Hanauer M-T, Barthelemy JC, Mamelle N. Control of bias in dietary trial to prevent coronary recurrences: the Lyon Diet Heart study. European Journal of Clinical Nutrition 1997;51(2):116-22.

De Lorgeril M, Salen P, Martin J-L, Monjaud I, Delaye J, Mamelle N. Mediterranean diet, traditional risk factors, and the rate of cardiovascular complications after myocardial infarction: final report of the Lyon Diet Heart study. Circulation 1999;99:779-85.

De Lorgeril M, Salen P, Martin JL, Mamelle N, Monjaud I, Touboul P, et al. Effect of a Mediterranean type of diet on the rate of cardiovascular complications in patients with coronary artery disease. insights into the cardioprotective effect of certain nutriments. Journal of the American College of Cardiology 1996;28:1103-8.

De Lorgeril M, Salen P, Martin JL, Monjaud I, Boucher P, Mamelle N. Mediterranean dietary pattern in a randomised trial. Archives of Internal Medicine 1998;158:1181-7.

Renaud S, de Lorgeril M, Delaye J, Guidollet J, Jacquard F, Mamelle N, et al. Cretan Mediterranean diet for prevention of 
coronary heart disease. American Journal of Clinical Nutrition 1995;61(6, Suppl):1360S-7S.

\section{Lysikova 2003 \{published data only\}}

Lysikova SL, Pogozheva AV, Akol'zina SE, Vasil'ev AV, Vorob'eva LS. The study of the clinical potency of antiatherogenic diet containing flavonoids in cardiovascular patients [Russian]. Voprosy Pitaniia 2003;72(3):8-11.

\section{Macdonald 1972 \{published data only\}}

Macdonald I. Relationship between dietary carbohydrates and fats in their influence on serum lipid concentrations. Clinical Science 1972;43(2):265-74.

\section{Mansel 1990 \{published data only\}}

Mansel RE, Harrison BJ, Melhuish J, Sheridan W, Pye JK, Pritchard G, et al. A randomized trial of dietary intervention with essential fatty acids in patients with categorized cysts. Annals of the New York Academy of Sciences 1990;586:288-94.

\section{Marckmann 1993 \{published data only\}}

Marckmann P, Sandstrom B, Jespersen J. Favorable long-term effect of a low-fat/high fiber diet on human blood coagulation and fibrinolysis. Arteriosclerosis and Thrombosis 1993;13:505-11.

\section{MARGARIN 2002 \{published data only\}}

Bemelmans WJE, Broer J, Feskens EJM, Smit AJ, Muskiet FAJ, Lefrandt JD, et al. Effect of an increased intake of alphalinolenic acid and group nutritional education on cardiovascular risk factors: the Mediterranean Alpha-linolenic Enriched Groningen Dietary Intervention (MARGARIN) study. American Journal of Clinical Nutrition 2002;75:221-7.

\section{Marniemi 1990 \{published and unpublished data\}}

Hakala P, Karvetti RL. Weight reduction on lactovegetarian and mixed diets. European Journal of Clinical Nutrition 1989;43:421-30.

* Marniemi J, Seppanen A, Hakala P. Long-term effects on lipid metabolism of weight reduction on lactovegetarian and mixed diet. International Journal of Obesity 1990;14:113-25.

\section{Mattson 1985 \{published data only\}}

Mattson FH, Grundy SM. Comparison of effects of dietary saturated, monounsaturated and polyunsaturated fatty acids on plasma lipids and lipoproteins in man. Journal of Lipid Research 1985:26:194-202.

\section{McCarron 1997 \{published data only\}}

McCarron DA, Oparil S, Chait A, Haynes RB, Kris EP, Stern JS, et al. Nutritional management of cardiovascular risk factors. A randomized clinical trial. Archives of Internal Medicine 1997;157(2):169-77.

\section{McCarron 2001 \{published data only\}}

McCarron DA, Reusser ME. Reducing cardiovascular disease risk with diet. Obesity Research 2001;9(Suppl 4):335S-40S.

\section{McManus 2001 \{published and unpublished data\}}

McManus K, Antinoro L, Sacks F. Randomized controlled trial of a moderate-fat low-energy diet compared with a low fat, low- energy diet for weight loss in overweight adults. International Journal of Obesity 2001;25:1503-11.

McNamara 1981 \{published data only\}

McNamara DJ, Kolb R, Parker T, Batwin H, Brown C, Samuel P, et al. Diet and cholesterol homeostasis in men [abstract]. Arteriosclerosis 1981;1:369A.

\section{Medi-RIVAGE 2004 \{published and unpublished data\}}

Borel P, Moussa M, Reboul E, Lyan B, Defoort C, VincentBaudry $S$, et al. Human fasting plasma concentrations of vitamin $\mathrm{E}$ and carotenoids, and their association with genetic variants in apo C-III, cholesteryl ester transfer protein, hepatic lipase, intestinal fatty acid binding protein and microsomal triacylglycerol transfer protein. British Journal of Nutrition 2009;101(5):680-7.

Borel P, Moussa M, Reboul E, Lyan B, Defoort C, VincentBaudry $S$, et al. Human plasma levels of vitamin $E$ and carotenoids are associated with genetic polymorphisms in genes involved in lipid metabolism. Journal of Nutrition 2007;137(12):2653-9.

Gastaldi M, Diziere S, Defoort C, Portugal H, Lairon D, Darmon M, et al. Sex-specific association of fatty acid binding protein 2 and microsomal triacylglycerol transfer protein variants with response to dietary lipid changes in the 3-mo MediRIVAGE primary intervention study. American Journal of Clinical Nutrition 2007;86(6):1633-41.

* Vincent S, Gerber M, Bernard MC, Defoort C, Loundou A, Portugal $\mathrm{H}$, et al. The Medi-RIVAGE study (Mediterranean Diet, Cardiovascular Risks and Gene Polymorphisms): rationale, recruitment, design, dietary intervention and baseline characteristics of participants. Public Health Nutrition 2004;7(4):531-42.

Vincent-Baudry S, Defoort C, Gerber M, Bernard MC, Verger $\mathrm{P}$, Helal $\mathrm{O}$, et al. The Medi-RIVAGE study: reduction of cardiovascular disease risk factors after a 3-mo intervention with a Mediterranean-type diet or a low-fat diet. American Journal of Clinical Nutrition 2005;82(5):964-71.

\section{Mensink 1987 \{published data only\}}

Mensink RP, Katan MB. Effect of monounsaturated fatty acids versus complex carbohydrates on high-density lipoproteins in healthy men and women. Lancet 1987;1(8525):122-5.

\section{Mensink 1989 \{published data only\}}

Mensink RP, Katan MB. Effect of a diet enriched with monounsaturated or polyunsaturated fatty acids on levels of low density and high density lipoprotein cholesterol in healthy women and men. New England Journal of Medicine 1989;321:436-41.

\section{Mensink 1990a \{published data only\}}

Mensink RP, Katan MB. Effect of dietary trans fatty acids on high density and low density lipoprotein cholesterol levels in healthy subjects. New England Journal of Medicine 1990;323:439-45. 
Mensink 1990b \{published and unpublished data\}

Mensink RP. Effect of monounsaturated fatty acids on highdensity and low-density lipoprotein cholesterol levels and blood pressure in healthy men and women. Wageningen, Netherlands: Wageningen University and Research Centre, 1990.

Michalsen 2006 \{published and unpublished data\} Michalsen A, Lehmann N, Pithan C, Knoblauch NT, Moebus S, Kannenberg F, et al. Mediterranean diet has no effect on markers of inflammation and metabolic risk factors in patients with coronary artery disease. European Journal of Clinical Nutrition 2006;60(4):478-85.

\section{Miettinen 1994 \{published data only\}}

Miettinen TA, Vanhanen H. Dietary sitostanol related to absorption, synthesis and serum level of cholesterol in different apolipoprotein E phenotypes. Atherosclerosis 1994;105(2):217-26.

\section{Millar 1973 \{published data only\}}

Millar JH, Zilkha KJ, Langman MJS, Payling-Wright H, Smith AD, Belin J, et al. Double-blind trial of linoleate supplementation of the diet in multiple sclerosis. BMJ 1973;1(5856):765-8.

Miller 1998 \{published data only\}

Miller ER, Appel LJ, Risby TH. Effect of dietary patterns on measures of lipid peroxidation: results from a randomised clinical trial. Circulation 1998;98:2390-5.

Miller 2001 \{published and unpublished data\}

Miller SL, Reber RJ, Chapman-Novakofski K. Prevalence of CVD risk factors and impact of a two-year education program for premenopausal women. Women's Health Issues 2001;11(6):486-93.

\section{Milne 1994 \{published data only\}}

Milne RM, Mann JI, Chisholm AW, Williams SM. Long-term comparison of three dietary prescriptions in the treatment of NIDDM. Diabetes Care 1994;17(1):74-80.

\section{Minnesota HHP 1990 \{published data only\}}

Murray DM, Kurth C, Mullis R, Jeffery RW. Cholesterol reduction through low-intensity interventions: results from the Minnesota Heart Health Program. Preventive Medicine 1990;19(2):181-9.

\section{Mokuno 1988 \{published data only\}}

Mokuno H, Yamada N, Sugimoto T. Cholesterol free diet in heterozygous familial hypercholesterolaemia: significant lowering effect on plasma cholesterol (abstract). Arteriosclerosis 1988;8(5):590a.

\section{Moreno 1994 \{published data only\}}

Moreno VJ, Garcia AJ, Campillo AJ. Influence of diet and physical exercise on plasma lipid concentrations in an homogeneous sample of young Spanish Air Force pilots. European Journal of Applied Physiology and Occupational Physiology 1994;69(1):75-80.

\section{Morrison 1950 \{published data only\}}

Morrison LM, Awierlein M, Wolfson E. The effects of low fat low cholesterol diets on the serum lipids. Circulation 1950;2:475-6.

\section{Morrison 1951 \{published data only\}}

Morrison LM. Reduction of mortality rate in coronary atherosclerosis by a low cholesterol low fat diet. American Heart Journal 1951;42:538-45.

Morrison 1960 \{published data only\}

Morrison LM. Diet in coronary atherosclerosis. JAMA 1960;173:884-8.

\section{Mortensen 1983 \{published data only\}}

Mortensen JZ, Schmidt EB, Nielsen AH, Dyerberg J. The effect of $\mathrm{N}-6$ and $\mathrm{N}-3$ polyunsaturated fatty acids on hemostasis, blood lipids and blood pressure. Thrombosis and Haemostasis 1983;50(2):543-6.

\section{MRFIT substudy 1986 \{published data only\}}

Daniel GJ, Dolecek TA, Caggiula AW, Van HL, Epley L, Randall BL. Increasing the use of meatless meals: a nutrition intervention substudy in the Multiple Risk Factor Intervention Trial (MRFIT). Journal of the American Dietetic Association 1986;86(6):778-81.

\section{MSDELTA 1995 \{published data only\}}

Ginsberg HN. New directions in dietary studies and heart disease: the National Heart, Lung and Blood Institute sponsored Multicenter Study of Diet Effects on Lipoproteins and Thrombogenic Activity. Advances In Experimental Medicine and Biology 1995;369:241-7.

\section{Mujeres Felices 2003 \{published data only\}}

* Fitzgibbon ML, Gapstur SM, Knight SJ. Mujeres felices por ser saludables: a breast cancer risk reduction program for Latino women. Preventive Medicine 2003;36(5):536-46.

Fitzgibbon ML, Gapstur SM, Knight SJ. Results of Mujeres Felices por ser Saludables: a dietary/breast health randomized clinical trial for Latino women. Annals of Behavioral Medicine 2004;28(2):95-104.

\section{Mutanen 1997 \{published data only\}}

Mutanen M. Comparison between dietary monounsaturated and polyunsaturated fatty acids as regards diet-related diseases. Biomedicine and Pharmacotherapy 1997;51(8):314-7.

\section{Muzio 2007 \{published data only\}}

Muzio F, Mondazzi L, Harris WS, Sommariva D, Branchi A. Effects of moderate variations in the macronutrient content of the diet on cardiovascular disease risk factors in obese patients with the metabolic syndrome. American Journal of Clinical Nutrition 2007;86(4):946-51.

NAS 1987 \{published data only\}

Chlebowski RT, Nixon DW, Blackburn GL, Jochimsen P, Scanlon EF, Insull W, et al. A breast cancer Nutrition Adjuvant Study (NAS): protocol design and initial patient adherence. Breast Cancer Research and Treatment 1987;10(1):21-9.

\section{NCEP weight 1991 \{published and unpublished data\}}

Wood PD, Stefanick ML, Williams PT, Haskell WL. The effects on plasma lipoproteins of a prudent weight-reducing diet, with or without exercise, in overweight men and women. New England Journal of Medicine 1991;325(7):461-6. 
Neil 1995 \{published data only\}

Neil HA, Roe L, Godlee RJ, Moore JW, Clark GM, Brown J, et al. Randomised trial of lipid lowering dietary advice in general practice: the effects on serum lipids, lipoproteins, and antioxidants [see comments]. BMJ 1995;310(6979):569-73.

\section{Neverov 1997 \{published data only\}}

Neverov NI, Kaysen GA, Tareyeva IE. Effect of lipid-lowering therapy on the progression of renal disease in nondiabetic nephrotic patients. Contributions to Nephrology 1997;120:68-78.

\section{Next Step 1995 \{published and unpublished data\}}

Tilley BC, Vernon SW, Glanz K, Myers R, Sanders K, Lu M, et al. Worksite cancer screening and nutrition intervention for highrisk auto workers: design and baseline findings of the Next Step Trial. Preventive Medicine 1997;26(2):227-35.

* Tilley BC, Vernon SW, Myers R, Glanz K, Lu M, Sanders K, et al. Planning the next step. A screening promotion and nutrition intervention trial in the work site. Annals of the New York Academy of Sciences 1995;768:296-9.

\section{Nordoy 1971 \{published data only\}}

Nordoy A, Rodset JM. The influence of dietary fats on platelets in man. Acta Medica Scandinavica 1971;190(1-2):27-34.

\section{Norway Veg Oil 1968 \{published data only\}}

Natvig H, Borchgrevink CF, Dedichen J, Owren PA, Schiotz EH, Westlund K. A controlled trial of the effect of linolenic acid on incidence of coronary heart disease: the Norwegian Vegetable Oil Experiment of 1965-66. Scandinavian Journal of Clinical and Laboratory Investigation. Supplement 1968;105:1-20.

\section{O'Brien 1976 \{published data only\}}

O'Brien JR, Etherington MD, Jamieson S. Effect of a diet of polyunsaturated fats on some platelet-function tests. Lancet 1976;2(7993):995-6.

\section{ODES 2006 \{published data only\}}

Anderssen S, Holme I, Urdal P, Hjermann I. Diet and exercise intervention have favourable effects on blood pressure in mild hypertensives: the Oslo Diet and Exercise Study (ODES). Blood Pressure 1995;4(6):343-9.

Anderssen SA, Hjermann I, Urdal P, Torjesen PA, Holme I. Improved carbohydrate metabolism after physical training and dietary intervention in individuals with the "atherothrombogenic syndrome'. Oslo Diet and Exercise Study (ODES). A randomized trial. Journal of Internal Medicine 1996;240(4):203-9.

* Holme I, Haaheim LL, Tonstad S, Hjermann I, Holme I, Haaheim LL, et al. Effect of dietary and antismoking advice on the incidence of myocardial infarction: a 16-year follow-up of the Oslo Diet and Antismoking Study after its close. Nutrition Metabolism \& Cardiovascular Diseases 2006;16(5):330-8.

Rokling-Andersen MH, Reseland JE, Veierod MB, Anderssen SA, Jacobs DR Jr, Urdal P, et al. Effects of long-term exercise and diet intervention on plasma adipokine concentrations. American Journal of Clinical Nutrition 2007;86(5):1293-301.
The ODES Investigators. The Oslo Diet and Exercise Study (ODES): design and objectives. Controlled Clinical Trials 1993;14(3):229-43

Torjesen PA, Birkeland KI, Anderssen SA, Hjermann I, Holme I, Urdal P. Lifestyle changes may reverse development of the insulin resistance syndrome. The Oslo Diet and Exercise Study: a randomized trial. Diabetes Care 1997;20(1):26-31.

Oldroyd 2001 \{published data only\}

Oldroyd JC, Unwin NC, White M, Mathers JC, Alberti KG. Randomised controlled trial evaluating lifestyle interventions in people with impaired glucose tolerance. Diabetes Research \& Clinical Practice 2006;72(2):117-27.

* Oldroyd JCU. Randomised controlled trial evaluating the effectiveness of behavioural interventions to modify cardiovascular risk factors in men and women with impaired glucose tolerance: Outcomes at 6 months. Diabetes Research and Clinical Practice 2001;52(1):29-43.

\section{ORIGIN 2008 \{published data only\}}

Gerstein H, Yusuf S, Riddle MC, Ryden L, Bosch J. Rationale, design, and baseline characteristics for a large international trial of cardiovascular disease prevention in people with dysglycemia: the ORIGIN Trial (Outcome Reduction with an Initial Glargine Intervention). American Heart Journal 2008;155(1):26-32.

\section{Oslo Study 2004 \{published data only\}}

* Hjerkinn EM, Sandvik L, Hjermann I, Arnesen H. Effect of diet intervention on long-term mortality in healthy middleaged men with combined hyperlipidaemia. Journal of Internal Medicine 2004;255(1):68-73.

Hjermann I. Intervention of smoking and eating habits in healthy men carrying high risk for coronary heart disease. The Oslo Study. Acta Medica Scandinavica. Supplementum 1981;651:281-4.

Hjermann I. Smoking and diet intervention in healthy coronary high risk men. Methods and 5-year follow-up of risk factors in a randomized trial. The Oslo study. Journal of the Oslo City Hospitals 1980;30(1):3-17.

Hjermann I, Leren P, Norman N, Helgeland A, Holme I. Serum insulin response to oral glucose load during a dietary intervention trial in healthy coronary high risk men: the Oslo study. Scandinavian Journal of Clinical and Laboratory Investigation 1980;40(1):89-94.

Hjermann I, Velve BK, Holme I, Leren P. Effect of diet and smoking intervention on the incidence of coronary heart disease. Report from the Oslo Study Group of a randomised trial in healthy men. Lancet 1981;2(8259):1303-10.

\section{Pascale 1995 \{published data only\}}

Pascale RW, Wing RR, Butler BA, Mullen M, Bononi P. Effects of a behavioral weight loss program stressing calorie restriction versus calorie plus fat restriction in obese individuals with NIDDM or a family history of diabetes. Diabetes Care 1995;18(9):1241-8 
PEP 2001 \{published data only\}

Ohrig E, Geib HC, Haas G-M, Schwandt P. The prevention education program (PEP) Nuremberg: design and baseline data of a family oriented intervention study. International Journal of Obesity 2001;25(Suppl 1):S89-92.

PHYLLIS 1993 \{published data only\}

* Bond GM, Crepaldi G, Zanchetti A, Avogaro P, Marubini E, Maseri A, et al. Plaque hypertension lipid-lowering Italian study (PHYLLIS): A protocol for non-invasive evaluation of carotid atherosclerosis in hypercholesterolaemic hypertensive subjects. Journal of Hypertension 1993;11(Suppl 5):S314-5.

Pilkington 1960 \{published and unpublished data\}

Pilkington TRE, Stafford JL, Hankin VS, Simmonds FM, Koerselman HB. Practical diets for lowering serum lipids. British Medical Journal 1960;1(5165):23-5.

\section{Pritchard 2002 \{published data only\}}

Pritchard JE, Nowson CA, Billington T, Wark JD. Benefits of a year-long workplace weight loss program on cardiovascular risk factors. Nutrition and Dietetics 2002;59(2):87-96.

Puget Sound EP 2000 \{published and unpublished data\} Kristal AR, Curry SJ, Shattuck AL, Feng Z, Li S. A randomized trial of a tailored, self-help dietary intervention: the Puget Sound Eating Patterns Study. Preventive Medicine 2000;31:380-9.

\section{Rabast 1979 \{published data only\}}

Rabast U, Schonborn J, Kasper H. Dietetic treatment of obesity with low and high-carbohydrate diets: comparative studies and clinical results. International Journal of Obesity 1979;3(3):201-11.

\section{Rabkin 1981 \{published data only\}}

Rabkin SW, Boyko E, Streja DA. Relationship of weight loss and cigarette smoking to changes in high-density lipoprotein cholesterol. American Journal of Clinical Nutrition 1981;34:1764-8.

\section{Radack 1990 \{published data only\}}

Radack K, Deck C, Huster G. The comparative effects of $n-3$ and $\mathrm{n}-6$ polyunsaturated fatty acids on plasma fibrinogen levels: a controlled clinical trial in hypertriglyceridemic subjects. Journal of the American College of Nutrition 1990;9(4):352-7.

\section{Rasmussen 1995 \{published data only\}}

Rasmussen OW, Thomsen $\mathrm{CH}$, Hansen $\mathrm{KW}$, Vesterlund M, Winther E, Hermansen K. Favourable effect of olive oil in patients with non-insulin-dependent diabetes. The effect on blood pressure, blood glucose and lipid levels of a high-fat diet rich in monounsaturated fat compared with a carbohydrate-rich diet [Gunstig virkning af olivenolie hos ikkeinsulinkraevende diabetikere. Virkningen pa blodtryk, blodglukose og lipidniveauer af en dioet med et hojt indhold af monoumoettet fedt sammenlignet med en kulhydratrig dioet]. Ugeskrift for Laeger 1995;157(8):1028-32.

\section{Reaven 2001 \{published data only\}}

Reaven GM, Abbasi F, Bernhart S, Coulston A, Darnell B, Dashti N, et al. Insulin resistance, dietary cholesterol, and cholesterol concentration in postmenopausal women. Metabolism: Clinical \& Experimental 2001;50(5):594-7.

Reid 2002 \{published data only\}

Reid R, Fodor G, Lydon-Hassen K, D'Angelo MS, McCrea J, Bowlby $\mathrm{M}$, et al. Dietary counselling for dyslipidemia in primary care: results of a randomized trial. Canadian Journal of Dietetic Practice \& Research 2002;63(4):169-75.

Renaud 1986 \{published data only\}

Renaud S, Godsey F, Dumont E, Thevenon C, Ortchanian E, Martin JL. Influence of long-term diet modification on platelet function and composition in Moselle farmers. American Journal of Clinical Nutrition 1986;43:136-50.

Rivellese 2003 \{published data only\}

Rivellese AA, Maffettone A, Vessby B, Uusitupa M, Hermansen K, Berglund L, et al. Effects of dietary saturated, monounsaturated and $\mathrm{n}-3$ fatty acids on fasting lipoproteins, LDL size and postprandial lipid metabolism in healthy subjects. Atherosclerosis 2003;167(1):149-58.

\section{Roderick 1997 \{published and unpublished data\}}

Roderick $P$, Ruddock V, Hunt $P$, Miller $G$. A randomized trial to evaluate the effectiveness of dietary advice by practice nurses in lowering diet-related coronary heart disease risk. British Journal of General Practice 1997;47(414):7-12.

\section{Roman CHD prev 1986 \{published data only\}}

The Roman Coronary Disease Prevention Project: effectiveness of intervention and reduction of mortality over a 10-year period [II Progetto Romano di Prevenzione della Cardiopatia Coronarica: efficacia dell'intervento e riduzione della mortalita in 10 anni]. Giornale Italiano di Cardiologia 1986;16(3):196-202.

* Research Group of the Rome Project of Coronary Heart Disease Prevention. Eight-year follow-up results from the Rome Project of Coronary Heart Disease Prevention. Research Group of the Rome Project of Coronary Heart Disease Prevention. Preventive Medicine 1986;15(2):176-91.

Rose 1987 \{published data only\}

Rose DP, Boyar AP, Cohen C, Strong LE. Effect of a low fat diet on hormone levels in women with cystic breast disease I Serum steroids and gonadotropins. Journal of the National Cancer Institute 1987;78:623-6.

\section{Sandstrom 1992 \{published data only\}}

Sandstrom B, Marckmann P, Bindslev N. An eight-month controlled study of a low-fat high-fibre diet: effects on blood lipids and blood pressure in healthy young subjects. European Journal of Clinical Nutrition 1992;46(2):95-109.

\section{Sasaki 2000 \{published data only\}}

Sasaki S. Change and 1-year maintenance of nutrient and food group intakes at a 12-week worksite dietary intervention trial for men at high risk of coronary heart disease. Journal of Nutritional Science \& Vitaminology 2000;46(1):15-22. 


\section{Schaefer 1995a \{published data only\}}

Schaefer EJ, Lichtenstein AH, Lamon-Fava S, et al. Body weight and low density lipoprotein cholesterol changes after consumption of a low fat ad libitum diet. JAMA 1995;274:1450-5.

\section{Schaefer 1995b \{published data only\}}

Schaefer EJ, Lichtenstein AH, Lamon-Fava S, Contois JH, Li Z, Rasmussen H, et al. Efficacy of a National Cholesterol Education Program Step 2 diet in normolipidaemic and hypercholesterolaemic middle-aged and elderly men and women. Arteriosclerosis, Thrombosis, and Vascular Biology 1995; 15:1079-85.

\section{Schectman 1996 \{published data only\}}

Schectman G, Wolff N, Byrd JC, Hiatt JG, Hartz A. Physician extenders for cost-effective management of hypercholesterolemia. Journal of General Internal Medicine 1996;11(5):277-86.

\section{Schlierf 1995 \{published data only\}}

Schlierf G, Schuler G, Hambrecht R, Niebauer J, Hauer K, Vogel G, et al. Treatment of coronary heart disease by diet and exercise. Journal of Cardiovascular Pharmacology 1995;25(Suppl 4):S32-4.

\section{Seppanen-Laakso 1992 \{published data only\}}

Seppanen-Laakso T, Vanhanen H, Laakso I, Kohtamaki H, Viikari J. Replacement of butter on bread by rapeseed oil and rapeseed oil-containing margarine: effects on plasma fatty acid composition and serum cholesterol. British Journal of Nutrition 1992;68:639-54.

\section{Singh 1990 \{published data only\}}

Singh RB, Sircar AR, Rastogi SS, Singh R. Dietary modulators of blood pressure in hypertension. European Journal of Clinical Nutrition 1990;44(4):319-27.

\section{Singh 1991 \{published data only\}}

Singh RB, Rastogi SS, Sircar AR. Dietary strategies for risk-factor modification to prevent cardiovascular diseases. Nutrition 1991;7(3):210-4

\section{Singh 1992 \{published data only\}}

Singh RB, Niaz MA, Agarwal P, Begom R, Rastogi SS. Effect of antioxidant-rich foods on plasma ascorbic acid, cardiac enzyme, and lipid peroxide levels in patients hospitalized with acute myocardial infarction. Journal of the American Dietetic Association 1995;95(7):775-80.

Singh RB, Niaz MA, Ghosh S. Effect on central obesity and associated disturbances of low-energy, fruit- and vegetableenriched prudent diet in north Indians. Postgraduate Medical Journal 1994;70(830):895-900.

* Singh RB, Rastogi SS, Verma R, Bolaki L, Singh R. An Indian experiment with nutritional modulation in acute myocardial infarction. American Journal of Cardiology 1992;69(9):879-85.

Singh RB, Rastogi SS, Verma R, Laxmi B, Singh R, Ghosh S, et al. Randomised controlled trial of cardioprotective diet in patients with recent acute myocardial infarction: results of one year follow up. BMJ 1992;304(6833):1015-9.
Sirtori 1992 \{published data only\}

Sirtori CR, Gatti E, Tremoli E, Galli C, Gianfranceschi G, Franceschini $\mathrm{G}$, et al. Olive oil, corn oil, and n-3 fatty acids differently affect lipids, lipoproteins, platelets, and superoxide formation in type II hypercholesterolemia. American Journal of Clinical Nutrition 1992;56(1):113-22.

\section{SLIM 2008 \{published data only\}}

Roumen C, Corpeleijn E, Feskens EJ, Mensink M, Saris WH, Blaak EE, et al. Impact of 3-year lifestyle intervention on postprandial glucose metabolism: the SLIM study. Diabetic Medicine 2008;25(5):597-605.

\section{Sopotsinskaia 1992 \{published data only\}}

Sopotsinskaia EB, Balitskii KP, Tarutinov VI, Zhukova VM, Semenchuk DD, Kozlovskaia SG, et al. Experience with the use of a low-calorie diet in breast cancer patients to prevent metastasis [Opyt primeneniia nizkokaloriinoi diety u bol'nykh rakom molochnoi zhelezy s tsel'iu profilaktiki metastazi]. Voprosy Onkologii 1992;38(5):592-9.

\section{Staff HHP 1994 \{published data only\}}

Barratt A, Reznik R, Irwig L, Cuff A, Simpson JM, Oldenburg B, et al. Work-site cholesterol screening and dietary intervention: the Staff Healthy Heart Project. Steering Committee. American Journal of Public Health 1994;84(5):779-82.

\section{Stanford NAP 1997 \{published data only\}}

Howard PB, Winkleby MA, Albright CL, Bruce B, Fortmann SP. The Stanford Nutrition Action Program: a dietary fat intervention for low-literacy adults. American Journal of Public Health 1997;87(12):1971-6.

\section{Stanford Weight 1994 \{published and unpublished data\}}

Williams PT, Krauss RM, Stefanick ML, Vranizan KM, Wood PD. Effects of low-fat diet, calorie restriction, and running on lipoprotein subfraction concentrations in moderately overweight men. Metabolism 1994;43(5):655-63.

\section{Starmans 1995 \{published data only\}}

Starmans KM, Lustermans FT, Kragten HA, Struijker BH, Rilla H. Lowering cholesterol in patients with mild hypercholesterolaemia does not improve functional properties of large arteries [Abstract]. Netherlands Journal Of Medicine 1995; 46:A70

\section{Steinbach 1996 \{published data only\}}

Steinbach M. A Romanian contribution to the epidemiology and prevention of cardiovascular diseases. Romanian Journal of Internal Medicine 1996;34(1-2):137-48.

\section{Steptoe 2001 \{published data only\}}

Steptoe A, Kerry S, Rink E, Hilton S. The impact of behavioral counselling on stage of change in fat intake, physical activity, and cigarette smoking in adults at increased risk of coronary heart disease. American Journal of Public Health 2001;91(2):265-9.

\section{Stevens 2002 \{published and unpublished data\}}

Stevens VJ, Glasgow RE, Toobert DJ, Karanja N, Smith KS. Oneyear results from a brief, computer-assisted intervention to 
decrease consumption of fat and increase consumption of fruits and vegetables. Preventive Medicine 2003;36:594-600.

* Stevens VJ, Glasgow RE, Toobert DJ, Karanja N, Smith KS. Randomized trial of a brief dietary intervention to decrease consumption of fat and increase consumption of fruits and vegetables. American Journal of Health Promotion 2002;16(3):129-34.

\section{Stevenson 1988 \{published data only\}}

Stevenson DW, Darga LL, Spafford TR, Ahmad N, Lucas CP. Variable effects of weight loss on serum lipids and lipoproteins in obese patients. International Journal of Obesity 1988;12:495-502.

\section{Sweeney 2004 \{published data only\}}

Sweeney M, Sweeney Michael. Effects of very low-fat diets on anginal symptoms. Medical Hypotheses 2004;63(3):553.

\section{TAIM 1992 \{published data only\}}

Davis BR, Blaufox MD, Hawkins CM, Langford HG, Oberman A, Swencionis $C$, et al. Trial of antihypertensive interventions and management. Design, methods, and selected baseline results. Controlled Clinical Trials 1989;10(1):11-30.

Davis BR, Blaufox MD, Oberman A, Wassertheil SS, Zimbaldi N, Cutler JA, et al. Reduction in long-term antihypertensive medication requirements. Effects of weight reduction by dietary intervention in overweight persons with mild hypertension. Archives of Internal Medicine 1993;153(15):1773-82.

Davis BR, Oberman A, Blaufox MD, Wassertheil SS, Hawkins CM, Cutler JA, et al. Effect of antihypertensive therapy on weight loss. The Trial of Antihypertensive Interventions and Management Research Group. Hypertension 1992;19(4):393-9.

Langford HG, Davis BR, Blaufox D, Oberman A, Wassertheil Smoller $S$, et al. Effect of drug and diet treatment of mild hypertension on diastolic blood pressure. The TAIM Research. Hypertension 1991;17(2):210-7.

Oberman A, Wassertheil Smoller S, Langford HG, Blaufox MD, Davis BR, Blaszkowski T, et al. Pharmacologic and nutritional treatment of mild hypertension: changes in cardiovascular risk status. Annals of Internal Medicine 1990;112(2):89-95.

Wassertheil Smoller S, Davis BR, Breuer B, Chee JC, Oberman A, Blaufox MD. Differences in precision of dietary estimates among different population subgroups. Annals of Epidemiology 1993;3:619-28.

* Wassertheil Smoller S, Oberman A, Blaufox MD, Davis B, Langford $\mathrm{H}$. The Trial of Antihypertensive Interventions and Management (TAIM) Study. Final results with regard to blood pressure, cardiovascular risk, and quality of life. American Journal of Hypertension 1992;5(1):37-44.

Wylie Rosett J, Wassertheil Smoller S, Blaufox MD, Davis BR, Langford HG, Oberman A, et al. Trial of antihypertensive intervention and management: greater efficacy with weight reduction than with a sodium-potassium intervention. Journal of the American Dietetic Association 1993;93(4):408-15.

\section{Take Heart II 1997 \{published data only\}}

Glasgow RE, Terborg JR, Strycker LK, Boles SM, Hollis JF. Take Heart II: Replication of a worksite health promotion trial. Journal of Behavioral Medicine 1997;20:143-61.

\section{Taylor 1991 \{published data only\}}

Taylor CB, Fortmann SP, Flora J, Kayman S, Barrett DC, Jatulis D, et al. Effect of long-term community health education on body mass index. The Stanford Five-City Project. American Journal of Epidemiology 1991;134:235-49.

\section{TOHP I 1992 \{published data only\}}

* The effects of nonpharmacologic interventions on blood pressure of persons with high normal levels. Results of the Trials of Hypertension Prevention, Phase I. JAMA 1992;267(9):1213-20.

Kumanyika SK, Hebert PR, Cutler JA, Lasser VI, Sugars CP, Steffen Batey $L$, et al. Feasibility and efficacy of sodium reduction in the Trials of Hypertension Prevention, phase I. Hypertension 1993;22(4):502-12.

Satterfield S, Cutler JA, Langford HG, Applegate WB, Borhani NO, Brittain E, et al. Trials of hypertension prevention. Annals of Epidemiology 1991;1(5):455-71.

Stevens VJ, Corrigan SA, Obarzanek E, Bernauer E, Cook NR, Hebert $P$, et al. Weight loss intervention in phase I of the trials of hypertension prevention. The TOHP Collaborative Research Group. Archives of Internal Medicine 1993;153(7):849-58.

Whelton PK, Hebert PR, Cutler J, Applegate WB, Eberlein KA, Klag MJ, et al. Baseline characteristics of participants in phase I of the Trials of Hypertension Prevention. Annals of Epidemiology 1992;2(3):295-310

Whelton PK, Kumanyika SK, Cook NR, Cutler JA, Borhani NO, Hennekens $\mathrm{CH}$, et al. Efficacy of nonpharmacologic interventions in adults with high-normal blood pressure: results from phase 1 of the Trials of Hypertension Prevention. American Journal of Clinical Nutrition 1997;65(2 Suppl):652S-60S.

\section{TONE 1997 \{published data only\}}

Whelton PK, Appel LJ, Espeland MA, Applegate WB, Ettinger$\mathrm{WH} \mathrm{J}$, Kostis JB, et al. Sodium reduction and weight loss in the treatment of hypertension in older persons: a randomized controlled trial of nonpharmacologic interventions in the elderly (TONE). JAMA 1998;279(11):839-46.

* Whelton PK, Babnson J, Appel LJ, Charleston J, Cosgrove N, Espeland MA, et al. Recruitment in the Trial of Nonpharmacologic Intervention in the Elderly (TONE). Journal of the American Geriatrics Society 1997;45(2):185-93.

\section{Toobert 2003 \{published data only\}}

Toobert DJ, Glasgow RE, Strycker LA, Barrera M Jr, Radcliffe JL, Wander RC, et al. Biologic and quality-of-life outcomes from the Mediterranean Lifestyle Program: a randomized clinical trial. Diabetes Care 2003;26(8):2288-93. 
Towle 1994 \{published data only\}

Towle LA, Bergman EA, Joseph E. Low-fat bison-hybrid ground meat has no effects on serum lipid levels in a study of 12 men. Journal of the American Dietetic Association 1994;94(5):546-8.

\section{TRANSFACT 2006 \{published data only\}}

* Chardigny JM, Malpuech-Brugere C, Dionisi F, Bauman DE, German B, Mensink RP, et al. Rationale and design of the TRANSFACT project phase I: a study to assess the effect of the two different dietary sources of trans fatty acids on cardiovascular risk factors in humans. Contemporary Clinical Trials 2006;27(4):364-73.

Chardigny JMD. Do trans fatty acids from industrially produced sources and from natural sources have the same effect on cardiovascular disease risk factors in healthy subjects? Results of the trans Fatty Acids Collaboration (TRANSFACT) study. American Journal of Clinical Nutrition 2008;108(3):558-66.

Treatwell 1992 \{published and unpublished data\}

Sorensen G, Morris DM, Hunt MK, Hebert JR, Harris DR, Stoddard A, et al. Work-site nutrition intervention and employees' dietary habits: the Treatwell program. American Journal of Public Health 1992;82(6):877-80.

\section{Tromso Heart 1989 \{published data only\}}

Knutsen SF, Knutsen R. The Tromso Heart Study: family approach to intervention on CHD. Feasibility of risk factor reduction in high-risk persons--project description.

Scandinavian Journal of Social Medicine 1989;17:109-19.

\section{Turpeinen 1960 \{published data only\}}

Turpeinen O, Roine P, Pekkarinen M, Karvonen MJ, Rautanen Y, Runeberg J, et al. Effect on serum-cholesterol level of replacement of dietary milk fat by soybean oil. Lancet 1960;1:196-8.

\section{UK PDS 1996 \{published data only\}}

* Turner R, Cull C, Holman R. United Kingdom Prospective Diabetes Study 17: a 9-year update of a randomized, controlled trial on the effect of improved metabolic control on complications in non-insulin-dependent diabetes mellitus. Annals of Internal Medicine 1996;124(1 Pt 2):136-45.

Turner RC, Holman RR. Lessons from UK prospective diabetes study. Diabetes Research and Clinical Practice 1995;28(Suppl):S151-7.

\section{Urbach 1952 \{published data only\}}

Urbach R, Hildreth EA, Wackerman MT. The therapeutic uses of low fat, low cholesterol diets: I. Treatment of essential familial xanthomatosis. Journal of Clinical Nutrition 1952;1:52-6.

\section{Uusitupa 1993 \{published data only\}}

Uusitupa M, Laitinen J, Siitonen O, Vanninen E, Pyorala K. The maintenance of improved metabolic control after intensified diet therapy in recent type 2 diabetes. Diabetes Research and Clinical Practice 1993;19(3):227-38.

\section{Vavrikova 1958 \{published data only\}}

Vavrikova J. Essential fatty acids, lipid metabolism, and atherosclerosis [letter]. Lancet 1958;1:1337.

Wass 1981 \{published data only\}

Wass VJ, Jarrett RJ, Meilton V, Start MK, Mattock M, Ogg CS, et al. Effect of a long-term fat-modified diet on serum lipoprotein levels of cholesterol and triglyceride in patie. Clinical Science 1981;60(1):81-6.

\section{Wassertheil 1985 \{published data only\}}

Wassertheil SS, Blaufox MD, Langford HG, Oberman A, Cutter G, Pressel S. Prediction of response to sodium intervention for blood pressure control. Journal of Hypertension.Supplement 1986;4(5):S343-6.

* Wassertheil SS, Langford HG, Blaufox MD, Oberman A, Hawkins M, Levine B, et al. Effective dietary intervention in hypertensives: sodium restriction and weight reduction. Journal of the American Dietetic Association 1985;85(4):423-30.

\section{WATCH 1999 \{published and unpublished data\}}

Ockene IS, Hebert JR, Ockene JK, Saperia GM, Stanek E, Nicolosi R, et al. Effect of a physician-delivered nutrition counselling training and an office-support program on saturated fat intake, weight, and serum lipid measurements in a hyperlipidemic population: Worcester Area Trial for Counseling in Hyperlipidemia. Archives of Internal Medicine 1999;159:725-31.

\section{Watts 1988 \{published data only\}}

Watts GF, Ahmed W, Quiney J, Houlston R, Jackson P, Iles C, et al. Effective lipid lowering diets including lean meat. British Medical Journal (Clinical Research Ed.) 1988;296(6617):235-7.

\section{Weintraub 1992 \{published data only\}}

Weintraub M, Sundaresan PR, Schuster B. Long-term weight control study. VII (weeks 0 to 210). Serum lipid changes. Clinical Pharmacology and Therapeutics 1992;51(5):634-41.

\section{Westman 2006 \{published data only\}}

Westman EC, Yancy WS Jr, Olsen MK, Dudley T, Guyton JR. Effect of a low-carbohydrate, ketogenic diet program compared to a low-fat diet on fasting lipoprotein subclasses. International Journal of Cardiology 2006;110(2):212-6.

\section{Weststrate 1998 \{published data only\}}

Weststrate JA, Meijer GW. Plant sterol enriched margarines and reduction of plasma total-and LDL-cholesterol concentrations in normocholesterolaemic and mildly hypercholesterolaemic subjects. European Journal of Clinical Nutrition 1998;52:334-43.

WHO primary prev 1979 \{published data only\}

Primary prevention of ischaemic heart disease: WHO coordinated cooperative trial. A summary report. Bulletin Of The World Health Organization 1979;57:801-5.

WHT 1990 \{published and unpublished data\}

Bowen D. The role of participation in the women's health trial: feasibility study in minority populations. Preventive Medicine 2000;31(5):474-80. 
Bowen D, Clifford CK, Coates R, Evans M, Feng Z, Fouad M, et al. The Women's Health Trial feasibility study in minority populations: design and baseline descriptions. Annals of Epidemiology 1996;6(6):507-19.

Bowen DJ, Kestin M, McTiernan A, Carrell D, Green P. Effects of dietary fat intervention on mental health in women. Cancer Epidemiology, Biomarkers and Prevention 1995;4(5):555-9.

Gorbach SL, Morrill LA, Woods MN, Dwyer JT, Selles WD, Henderson $\mathrm{M}$, et al. Changes in food patterns during a low-fat dietary intervention in women. Journal of the American Dietetic Association 1990;90(6):802-9.

Henderson MM, Kushi LH, Thompson DJ, Gorbach SL, Clifford CK, Insull W, et al. Feasibility of a randomized trial of a low-fat diet for the prevention of breast cancer: dietary compliance in the Women's Health Trial Vanguard Study. Preventive Medicine 1990;19(2):115-33.

* Insull W, Henderson MM, Prentice RL, Thompson DJ, Clifford C, Goldman S, et al. Results of a randomized feasibility study of a low-fat diet. Archives of Internal Medicine 1990;150(2):421-7

Kristal AR, White E, Shattuck AL, Curry S, Anderson GL, Fowler A, et al. Long-term maintenance of a low-fat diet: durability of fatrelated dietary habits in the Women's Health Trial. Journal of the American Dietetic Association 1992;92(5):553-9.

Prentice RL, Kakar F, Hursting S, Sheppard L, Klein R, Kushi LH. Aspects of the rationale for the Women's Health Trial. Journal of the National Cancer Institute 1988;80(11):802-14.

Self S, Prentice R, Iverson D, Henderson M, Thompson D, Byar D, et al. Statistical design of the Women's Health Trial. Controlled Clininical Trials 1988;9(2):119-36.

Sheppard L, Kristal AR, Kushi LH. Weight loss in women participating in a randomised trial of low-fat diets. American Journal of Clinical Nutrition 1991;54:821-8.

Urban N, Baker M. The Women's Health Trial as an investment. Medical Decision Making 1989;9(1):59-64.

White E, Shattuck AL, Kristal AR, Urban N, Prentice RL, Henderson MM, et al. Maintenance of a low-fat diet: follow-up of the Women's Health Trial. Cancer Epidemiology, Biomarkers and Prevention 1992;1(4):315-23.

WHT Feasibility 2003 \{published and unpublished data\} Bowen D, Clifford CK, Coates R, Evans M, Feng Z, Fouad M, et al. The Women's Health Trial Feasibility Study in Minority Populations: design and baseline descriptions. Annals of Epidemiology 1996;6(6):507-19.

* Hall WD, Feng Z, George VA, Lewis CE, Oberman A, Huber M, et al. Low-fat diet: effect on anthropometrics, blood pressure, glucose, and insulin in older women. Ethnicity and Disease 2003;13:337-43. trans) [Verhalten der Serumlipide und des Körpergewichts unter Reduktionsdiät und medikamentöser Behandlung mit DTrijodthyronin]. Medizinische Klinik 1974;69(48):1986-9.

Williams 1990 \{published data only\}

Williams PT, Krauss RM, Vranizan KM, Wood PS. Changes in lipoprotein subfractions during diet-induced and exerciseinduced weight loss in moderately overweight men. Circulation 1990;81:1293-304.

Williams 1992 \{published data only\}

Williams PT, Krauss RM, Vranizan KM, Albers JJ, Wood PD. Effects of weight-loss by exercise and by diet on apolipoproteins AI and A-II and the particle-size distribution of high-density lipoproteins in men. Metabolism: Clinical and Experimental 1992;41:441-9.

\section{Williams 1994 \{published data only\}}

Williams PT, Stefanick ML, Vranizan KM, Wood PD. The effects of weight loss by exercise or by dieting on plasma high-density lipoprotein (HDL) levels in men with low, intermediate, and normal-to-high HDL at baseline. Metabolism 1994;43(7):917-24.

Wilmot 1952 \{published data only\}

Wilmot VA, Swank RL. The influence of low fat diet on blood lipid levels in health and in multiple sclerosis. American Journal of the Medical Sciences 1952;223:25-34.

Wing 1998 \{published data only\} Wing RR, Venditti E, Jakicic JM, Polley BA, Lang W. Lifestyle intervention in overweight individuals with a family history of diabetes. Diabetes Care 1998;21(3):350-9.

\section{WOMAN 2007 \{published data only\}}

Kuller LH, Kriska AM, Kinzel LS, Simkin-Silverman LR, SuttonTyrrell K, Johnson BD, et al. The clinical trial of Women On the Move through Activity and Nutrition (WOMAN) study. Contemporary Clinical Trials 2007;28(4):370-81.

\section{Wood 1988 \{published data only\}}

Wood PD, Stefanick ML, Dreon DM, Frey HB, Garay SC, Williams PT, et al. Changes in plasma lipids and lipoproteins in overweight men during weight loss through dieting as compared with exercise. New England Journal of Medicine 1988;319(18):1173-9.

\section{Woollard 2003 \{published data only\}}

Woollard J, Burke V, Beilin LJ, Verheijden M, Bulsara MK. Effects of a general practice-based intervention on diet, body mass index and blood lipids in patients at cardiovascular risk. Journal of Cardiovascular Risk 2003;10(1):31-40.

\section{Working Well 1996 \{published data only\}}

Sorensen G, Thompson B, Glanz K, Feng Z, Kinne S, DiClemente $C$, et al. Work site-based cancer prevention: primary results from the Working Well Trial. American Journal of Public Health 1996;86(7):939-47.

\section{Wilke 1974 \{published data only\}}

Wilke $\mathrm{H}$, Frahm H. Influence of low-caloric-diet and dtriiodothyronine on serum lipids and body weight (author's 
Zock 1995 \{published and unpublished data\}

* Zock PL. Dietary fatty acids and risk factors for coronary heart disease : controlled studies in healthy volunteers. Wageningen, Netherlands: Landbouwuniversiteit te Wageningen, 1995.

Zock PL, Mensink RP, Harryvan J, de VJ, Katan MB. Fatty acids in serum cholesteryl esters as quantitative biomarkers of dietary intake in humans. American Journal of Epidemiology 1997;145(12):1114-22.

\section{References to studies awaiting assessment}

Barsotti 1991 \{published data only\}

Barsotti A. Modern trends in the therapy of arteriosclerosis in the light of new physiopathological findings [Moderne tendenze della terapia dell'arteriosclerosi alla luce delle nuove acquisizioni fisiopatologiche]. Cardiologia 1991;36(12, Suppl 1):33-48.

\section{Bonk 1975 \{published data only\}}

Bonk S, Hubotter E, Nickel C, Stocksmeier U, Vahey P, Volk I, et al. Myocardial infarct patients with and without intensive nutrition consultation over several years-- comparison of physiological and social variables [Herzinfarktpatienten mit und ohne mehrjährige intensive Ernährungsberatung - Vergleich von physiologischen und sozialen Variablen]. Infusionstherapie und Klinische Ernährung 1975;2(4):290-6.

Brehm 2009 \{published data only (unpublished sought but not used)\}

Brehm BJ, Lattin BL, Summer SS, Boback JA, Gilchrist GM, Jandacek RJ, et al. One-year comparison of a highmonounsaturated fat diet with a high-carbohydrate diet in type 2 diabetes. Diabetes Care 2009;32(2):215-20.

\section{Canadian DBCP 1997 \{published data only (unpublished sought} but not used)\}

Boyd NF, Greenberg C, Lockwood G, Little L, Martin L, Byng J, et al. Effects at two years of a low-fat, high-carbohydrate diet on radiologic features of the breast: results from a randomized trial. Journal of the Nationl Cancer Institute 1997;89(7):488-96.

DEER 1998 \{published data only (unpublished sought but not used)\}

Stefanick ML, Mackey S, Sheehan RD, Ellsworth N, Haskell WL, Wood PD. Effects of diet and exercise in men and postmenopausal women with low levels of HDL cholesterol and high levels of LDL cholesterol. New England Journal of Medicine 1998;339(1):12-20

Diet \& Hormone Study 2003 \{published data only (unpublished sought but not used)\}

Gann PH, Chatterton RT, Gapstur SM, Liu K, Garside D, Giovanazzi S, et al. The effects of a low-fat/high-fiber diet on sex hormone levels and menstrual cycling in premenopausal women: a 12-month randomized trial (the Diet and Hormone Study). Cancer 2003;98:1870-9.
DIRECT 2009 \{published data only (unpublished sought but not used)\}

* Ben-Avraham S, Harman-Boehm I, Schwarzfuchs D, Shai I. Dietary strategies for patients with type 2 diabetes in the era of multi-approaches; review and results from the Dietary Intervention Randomized Controlled Trial (DIRECT). Diabetes Research and Clinical Practice 2009;86(Suppl 1):S41-8.

Shai I, Schwarzfuchs D, Henkin Y, Shahar DR, Witkow S, Greenberg I, et al. Weight loss with a low-carbohydrate, Mediterranean, or low-fat diet. New England Journal of Medicine 2008;359:229-41.

\section{Esposito 2004 \{published data only\}}

Esposito K, Marfella R, Ciotola M, Di Palo C, Giugliano F, Giugliano G, et al. Effect of a Mediterranean-style diet on endothelial dysfunction and markers of vascular inflammation in the metabolic syndrome: a randomized trial. JAMA 2004;292(12):1440-6.

\section{Koranyi 1963 \{published data only\}}

Koranyi A. Prophylaxis and treatment of the coronary syndrome. Therapia Hungarica 1963;11:17-20.

Metroville Health 2003 \{published data only (unpublished sought but not used)\}

Aziz KU, Dennis B, Davis CE, Sun K, Burke G, Manolio T, et al. Efficacy of CVD risk factor modification in a lower-middle class community in Pakistan: the Metroville Health Study. Asia Pacific Journal of Public Health 2003;15(1):30-6.

Mojonnier 1980 \{published data only\} Mojonnier ML, Hall Y, Berkson DM, Robinson E, Wethers B, Pannbacker B, et al. Experience in changing food habits of hyperlipidaemic men and women. Journal of the American Dietetic Association 1980;77:140-8.

Naglak 2000 \{published data only (unpublished sought but not used)\}

Naglak MC, Mitchell DC, Shannon BM, Pearson TA, Harkness WL, Kris-Etherton PM. Nutrient adequacy of diets of adults with hypercholesterolemia after a cholesterol-lowering intervention: long term assessment. Journal of the American Dietetic Association 2000;100(11):1385-91.

OLIVE 1997 \{published data only (unpublished sought but not used)\}

* Colquhoun DM. Rationale and design of the "OLIVE" study (Comparison of an Olive oil enriched to a low fat diet intervention study using vascular endpoints) [Abstract]. 11th International Symposium on Atherosclerosis, Paris. October 1997:326.

Colquhoun DM, Somerset S, Glasziou PP, Richards D, Weyers J. Comparison of an olive oil enriched diet to a low fat diet intervention study using vascular endpoints: assessed by repeat quantitative angiography (OLIVE study). Australian Journal of Nutrition and Dietetics 1998;55(Suppl 4):S24-9. 
Tapsell 2004 \{published data only (unpublished sought but not used)\}

Tapsell LC, Hokman A, Sebastiao A, Denmeade S, Martin G, Calvert GD, et al. The impact of usual dietary patterns, selection of significant foods and cuisine choices on changing dietary fat under 'free living' conditions. Asia Pacific Journal of Clinical Nutrition 2004;13(1):86-91.

Verheiden 2003 \{published data only (unpublished sought but not used)\}

Verheiden MW, van der Veen JE, van Zadelhoff WM, Bakx C, Koelen MA, van den Hoogen HJM, et al. Nutrition guidance in Dutch family practice: behavioural determinants of reduction in fat consumption. American Journal of Clinical Nutrition 2003;77(Suppl):1058S-64S.

\section{References to ongoing studies}

PREDIMED 2008 \{published data only (unpublished sought but not used)\}

Buil-Cosiales P, Irimia P, Ros E, Riverol M, Gilabert R, MartinezVila $E$, et al. Dietary fibre intake is inversely associated with carotid intima-media thickness: a cross-sectional assessment in the PREDIMED study. European Journal of Clinical Nutrition 2009;63(10):1213-9.

Estruch R, Martinez-Gonzalez MA, Corella D, Salas-Salvado J, Ruiz-Gutierrez V, Covas MI, et al. Effects of a Mediterranean-style diet on cardiovascular risk factors: a randomized trial. Annals of Internal Medicine 2006;145(1):1-11.

Razquin C, Martinez JA, Martinez-Gonzalez MA, Mitjavila MT, Estruch R, Marti A, et al. A 3 years follow-up of a Mediterranean diet rich in virgin olive oil is associated with high plasma antioxidant capacity and reduced body weight gain. European Journal of Clinical Nutrition 2009;63(12):1387-93.

Salas-Salvado J, Fernandez-Ballart J, Ros E, MartinezGonzalez MA, Fito M, Estruch R, et al. Effect of a Mediterranean diet supplemented with nuts on metabolic syndrome status: one-year results of the PREDIMED randomized trial. Archives of Internal Medicine 2008;168(22):2449-58.

Salas-Salvado J, Garcia-Arellano A, Estruch R, MarquezSandoval F, Corella D, Fiol M, et al. Components of the Mediterranean-type food pattern and serum inflammatory markers among patients at high risk for cardiovascular disease. European Journal of Clinical Nutrition 2008;62(5):651-9.

Sanchez-Tainta A, Estruch R, Bullo M, Corella D, Gomez-Gracia E, Fiol M, et al. Adherence to a Mediterranean-type diet and reduced prevalence of clustered cardiovascular risk factors in a cohort of 3,204 high-risk patients. European Journal of Cardiovascular Prevention \& Rehabilitation 2008;15(5):589-93.

Schroder H, de la Torre R, Estruch R, Corella D, MartinezGonzalez MA, Salas-Salvado J, et al. Alcohol consumption is associated with high concentrations of urinary hydroxytyrosol. American Journal of Clinical Nutrition 2009;90(5):1329-35.

Toledo E, Delgado-Rodriguez M, Estruch R, Salas-Salvado J, Corella D, Gomez-Gracia E, et al. Low-fat dairy products and blood pressure: follow-up of 2290 older persons at high cardiovascular risk participating in the PREDIMED study. British Journal of Nutrition 2009;101(1):59-67.

Waterhouse AL. Resveratrol metabolites in urine as biomarker of wine intake in free-living subjects: The PREDIMED Study. Free Radical Biology \& Medicine 2009;46(12):1561.

Zamora-Ros R, Urpi-Sarda M, Lamuela-Raventos RM, Estruch R, Martinez-Gonzalez MA, Bullo M, et al. Resveratrol metabolites in urine as a biomarker of wine intake in free-living subjects: The PREDIMED Study. Free Radical Biology \& Medicine 2009;46(12):1562-6.

Zazpe I, Estruch R, Toledo E, Sanchez-Tainta A, Corella D, Bullo $\mathrm{M}$, et al. Predictors of adherence to a Mediterraneantype diet in the PREDIMED trial. European Journal of Nutrition 2010;49(2):91-9.

* Zazpe I, Sanchez-Tainta A, Estruch R, Lamuela-Raventos RM, Schroder $\mathrm{H}$, Salas-Salvado J, et al. A large randomized individual and group intervention conducted by registered dietitians increased adherence to Mediterranean-type diets: the PREDIMED study. Journal of the American Dietetic Association 2008;108(7):1134-44.

\section{Additional references}

\section{S 1994}

Scandinavian Simvastatin Survival Study Group. Randomised trial of cholesterol lowering in $\mathbf{4 4 4 4}$ patients with coronary heart disease: the Scandinavian Simvastatin Survival Study (4S). Lancet 1994;344(8934):1383-9.

\section{ACC/AHA 2008}

ACC/AHA Writing group. ACC/AHA 2008 performance measures for adults with ST-elevation and non-ST-elevation myocardial infarction. Circulation 2008;118:2596-2648.

\section{Astrup 2000}

Astrup A, Ryan L, Grunwald GK, Storgaard M, Saris W, Melanson $\mathrm{E}$, et al. The role of dietary fat in body fatness: evidence from a preliminary meta-analysis of ad libitum lowfat dietary intervention studies. British Journal of Nutrition 2000;83(Suppl 1):S25-32.

\section{Berkley 1995}

Berkley CS, Hoaglin DC, Mosteller F, Colditz GA. A randomeffects regression model for meta-analysis. Statistics in Medicine 1995;14:395-411.

\section{Briel 2009}

Briel M, Ferreira-Gonzalez I, You JJ, Karanicolas PJ, Akl EA, Wu P, et al. Association between change in high density lipoprotein cholesterol and cardiovascular disease morbidity and mortality: systematic review and meta-regression analysis. BMJ 2009;338:b92. [DOI: 10.1136/bmj.b92]

\section{Brunner 1997}

Brunner E, White I, Thorogood M, Bristow A, Curle D, Marmot M. Can dietary interventions change diet and cardiovascular 
risk factors? A meta-analysis of randomized controlled trials. American Journal of Public Health 1997;87(9):1415-22.

\section{Brunner 2009}

Brunner E, Rees K, Ward K, Burke M, Thorogood M. Dietary advice for reducing cardiovascular risk. Cochrane Database of Systematic Reviews 2007, Issue 4. [DOI: 10.1002/14651858.CD002128.pub3]

\section{Bucher 1996}

Bucher HC, et al. Effects of dietary calcium supplementation on blood pressure: a meta-analysis of randomised controlled trials. JAMA 1996;275:1016-22.

\section{Chalmers 1990}

Chalmers I, Adams M, Dickersin K, Hetherington J, TarnowMordi W, Meinert C, et al. A cohort study of summary reports of controlled trials. JAMA 1990;263:1401-5.

\section{Clarke 1997}

Clarke R, Frost C, Collins R, Appleby P, Peto R. Dietary lipids and blood cholesterol: quantitative meta-analysis of metabolic ward studies. BMJ 1997;314:112-7.

\section{Davey Smith 1993}

Davey Smith G, Song F, Sheldon TA. Cholesterol lowering and mortality: the importance of considering initial level of risk. $B M J$ 1993;306:1367-73.

\section{De Caterina 2010}

De Caterina R, Scarano M, Marfisi RM, Lucisano G, Palma F, Tatasciore A, et al. Cholesterol-lowering interventions and stroke. Journal of the American College of Cardiology 2010;55:198-211.

\section{Denke 1995}

Denke MA. Cholesterol-lowering diets. A review of the evidence. Archives of Internal Medicine 1995;155:17-26.

\section{DerSimonian 1986}

DerSimonian R, Laird N. Meta-analysis in clinical trials. Controlled Clinical Trials 1986;7:177-88.

\section{Ebrahim 1997}

Ebrahim S, Davey Smith G. Systematic review of randomised controlled trials of multiple risk factor interventions for preventing coronary heart disease. BMJ 1997;314(7095):1666.

\section{Egger 1997}

Egger M, Davey Smith G, Schneider M, Minder C. Bias in meta-analysis detected by a simple graphical test. BMJ 1997;315:629-34.

\section{Fraker 2007}

Fraker TD, Fihn SD. 2007 Chronic angina: focused update of the ACC/AHA 2002 guidelines for the management of patients with chronic stable angina. Circulation 2007;116:2762-72.

\section{Gofman 1949}

Gofman JW, Lindgren FT, Elliott HA. Ultracentrifugal studies of lipoproteins of human serum. Journal of Biological Chemistry 1949;179:973-9.

\section{Hasselblad 1995}

Hasselblad V, McRory DC. Meta-analytic tools for medical decision making: a practical guide. Medical Decision Making 1995;15:81-96.

\section{Hauck 1991}

Hauck WW, Gilliss CL, Donner A, Gortner S. Randomisation by cluster. Nursing Research 1991;40(6):356-8.

\section{Hegsted 1965}

Hegsted DM, McGandy RB, Myers ML, Stare FJ. Quantitative effects of dietary fat on serum cholesterol in man. American Journal of Clinical Nutrition 1965;17(5):281-95.

\section{Hegsted 2000}

Hegsted DM. From chick nutrition to nutrition policy. Annual Review of Nutrition 2000;20:1-19.

\section{Hession 2009}

Hession M, Rolland C, Kulkarni U, Wise A, Broom J. Systematic review of randomized controlled trials of low-carbohydrate vs. low-fat/low-calorie diets in the management of obesity and its comorbidities. Obesity Reviews 2009;10:36-50.

\section{Higgins 1999}

Higgins JPT. ciplot: confidence interval plots using S-PLUS. Manual version 2, London: Institute of Child Health 1999.

\section{Higgins 2003}

Higgins JPT, Thompson SG, Deeks JJ, Altman DG. Measuring inconsistency in meta-analyses. BMJ 2003;327:557-60.

\section{Higgins 2011}

Higgins JPT, Green S (editors). Cochrane Handbook for Systematic Reviews of Interventions Version 5.1.0 [updated March 2011]. The Cochrane Collaboration, 2011. Available from www.cochrane-handbook.org. The Cochrane Collaboration.

\section{Kagan 1974}

Kagan A, Harris BR, Winkelstein W Jr, Johnson KG, Kato H, Syme SL, et al. Epidemiologic studies of coronary heart disease and stroke in Japanese men living in Japan, Hawaii and California: demographic, physical, dietary and biochemical characteristics. Journal of Chronic Disease 1974;27:345-64.

\section{Keys 1950}

Keys A, Mickelsen O, Miller EVO, Carleton B. The relation in man between cholesterol levels in the diet and in the blood. Science 1950;112:79-81.

\section{Keys 1986}

Keys A, Menotti A, Karvonen MJ, Aravanis C, Blackburn H, Buzina R, et al. The diet and 15-year death rate in the seven countries study. American Journal of Epidemiology 1986;124:903-15. 


\section{Kodama 2009}

Kodama S, Saito K, Tanaka S, Maki M, Yachi Y, Sato M, et al. Influence of fat and carbohydrate proportions on the metabolic profile in patients with type 2 diabetes: a meta-analysis. Diabetes Care 2009;32(5):959-65.

\section{Kristal 2005}

Kristal AR, Peters U, Potter JD. Is it time to abandon the food frequency questionnaire?. Cancer Epidemiology, Biomarkers and Prevention 2005;14(12):2826-8.

\section{Law 1991}

Law MR, Frost CD, Wald NJ. By how much does dietary salt restriction lower blood pressure?. BMJ 1991;302:811-24.

\section{Law 1994}

Law MR, Wald NJ, Thompson SG. By how much and how quickly does reduction in serum cholesterol concentration lower risk of ischaemic heart disease?. BMJ 1994;308:367-73.

\section{Lenz 2007}

Lenz M, Steckelberg A, Richter B, Mühlhauser I. Meta-analysis does not allow appraisal of complex interventions in diabetes and hypertension self-management:a methodological review. Diabetologia 2007;50:1375-83.

\section{Lichtenstein 2006}

Lichtenstein AH, Appel LJ, Brands M, Carnethon M, Daniels S, Franch HA, et al. Diet and lifestyle recommendations revision 2006: a scientific statement from the American Heart Association Nutrition Committee. Circulation 2006;114:82-96.

\section{Marchioli 1994}

Marchioli R, Prieto JC, Tognoni G. Surrogate end-points: the case of trials on coronary atherosclerotic plaque regression. Clinical Trials and Meta-Analyses 1994;29:139-76.

\section{Mensink 1992}

Mensink RP, Katan MB. Effect of dietary fatty acids on serum lipids and lipoproteins: a meta-analysis of 27 trials. Arteriosclerosis and Thrombosis 1992;12:911-9.

\section{Michels 2009}

Michels KB, Willett WC. The women's health initiative randomized controlled dietary modification trial: a postmortem. Breast Cancer Research and Treatment 2009;114:1-6.

\section{Mozaffarian 2010}

Mozaffarian D, Micha R, Wallace S. Effects of coronary heart disease of increasing polyunsaturated fat in place of saturated fat: a systematic review and meta-analysis of randomized controlled trials. PLOS Medicine 2010;7(3):e1000252.

\section{Ness 1997}

Ness AR, Powles JW. Fruit and vegetables, and cardiovascular disease: a review. International Journal of Epidemiology 1997;26:1-13

\section{NICE 2006}

National Institute for Health and Clinical Excellence. Technology Appraisal 94. Statins for the prevention of cardiovascular events. NICE at: www.nice.org.uk/TA094 2006.

\section{Oliver 1953}

Oliver MF, Boyd GS. The plasma lipids in coronary artery disease. British Heart Journal 1953;15:387-90.

\section{Oliver 2000}

Oliver MF. Pioneer research in Britain into atherosclerosis and coronary heart disease - an historical review. Atherosclerosis 2000;150:1-12.

\section{Prentice 2007}

Prentice RL. Observational studies, clinical trials, and the women's health initiative. Lifetime Data Analysis 2007;13:449-62.

\section{Rimm 1996}

Rimm EB, Klatsky A, Grobbee D, Stampfer MJ. Review of moderate alcohol consumption and reduced risk of coronary heart disease: is the effect due to beer, wine or spirits?. BMJ 1996;312:731-6.

\section{Robertson 1977}

Robertson TL, Kato H, Rhoads GG, Kagan A, Marmot M, Syme SL, et al. Epidemiologic studies of coronary heart disease and stroke in Japanese men living in Japan, Hawaii and California. Incidence of myocardial infarction and death from coronary heart disease. American Journal of Cardiology 1977;39:239-43.

\section{Robinson 2009}

Robinson JG, Wang S, Smith BJ, Jacobson TA. Meta-analysis of the relationship between non-high-density lipoprotein cholesterol reduction and coronary heart disease risk. Journal of the American College of Cardiology 2009;53:316-22.

\section{Rubins 1995}

Rubins HB. Cholesterol in patients with coronary heart disease: how low should we go?. Journal of General Internal Medicine 1995;10(8):464-71.

\section{Ryle 1949}

Ryle JA, Russell WT. The natural history of coronary disease. A clinical and epidemiological study. British Heart Journal 1949;11:370.

\section{Schatzkin 2003}

Schatzkin A, Kipnis V, Carroll RJ, et al. A comparison of a food frequency questionnaire with a 24-hour recall for use in an epidemiological cohort study: results from the biomarkerbased Observing Protein and EnergyNutrition (OPEN) Study. International Journal of Epidemiology 2003;32:1054-62.

\section{Shafiq 2010}

Shafiq N, Singh M, Kaur S, Khosla P, Malhotra S. Dietary treatment for familial hypercholesterolaemia. Cochrane Database of Systematic Reviews 2010, Issue 1. [DOI: 10.1002/14651858.CD001918.pub2] 


\section{Shah 2007}

Shah M, Adams-Huet B, Garg A. Effect of high-carbohydrate or high-cis-monounsaturated fat diets on blood pressure: a meta-analysis of intervention trials. American Journal of Clinical Nutrition 2007;85:1251-6.

\section{Sharp 1998}

Sharp S. Meta-analysis regression. Stats Technical Bulletin 1998;42:16-22.

\section{Sheppherd 2009}

Shepperd S, Lewin S, Straus S, Clarke M, Eccles MP, Fitzpatrick R, et al. Can we systematically review studies that evaluate complex interventions?. PLoS Medicine / Public Library of Science 2009;6(8):e1000086.

\section{SIGN 1996}

Scottish Intercollegiate Guidelines Network. Obesity in Scotland, integratingpPrevention with weight management. SIGN publication no. 8 1996:Edinburgh.

\section{Siri-Tarino 2010}

Siri-Tarino PW, Sun Q, Hu FB, Krauss RM. Meta-analysis of prospective cohort studies evaluating the association of saturated fat with cardiovascular disease. American Journal of Clinical Nutrition 2010;91:535-46.

\section{Skeaff 2009}

Skeaff CM, Miller J. Dietary fat and coronary heart disease: summary of evidence from prospective cohort and randomised controlled trials. Annals of Nutrition and Metabolism 2009;55:173-201.

\section{Stein 2006}

Stein K. After the media feeding frenzy: whither the Women's Health Initiative Dietary Modification Trial?. Journal of the American Dietetic Association 2006;106(6):794-800.

\section{Sterne 2009}

Sterne JAC. Meta-analysis in Stata: an updated collection from the Stata journal. Texas, USA: STATA Press, 2009.

\section{Sterne, Bradburn and Egger 2001}

Sterne JAC, Bradburn MJ, Egger M. Meta-analysis in STATA. In: Egger M, Davey Smith G, Altman DG editor(s). Systematic reviews in health care: meta-analysis in context. London: BMJ Books, 2001.

\section{CHARACTERISTICS OF STUDIES}

Characteristics of included studies [ordered by study ID]

\section{Thorogood 1996}

Thorogood M. Nutrition. In: Lawrence M, Neil A, Mant D, Fowler G editor(s). Prevention of Cardiovascular Disease: an evidence-based approach. Kings Lynn: Oxford University Press, 1996:54-66

\section{Walsh 1995}

Walsh JME, Grady D. Treatment of hyperlipidaemia in women. JAMA 1995;274:1152-58.

\section{Weggemans 2001}

Weggemans RM, Zock PL, Katan MB. Dietary cholesterol from eggs increases the ratio of total cholesterol to high-density lipoprotein cholesterol in humans: a meta-analysis. American Journal of Clinical Nutrition 2001;73:885-91.

\section{Yngve 2006}

Yngve A, Hambraeus L, Lissner L, Serra Majem L, Vaz de Almeida MD, Berg C, et al. Invited Commentary. The Women's Health Initiative. What is on trial: nutrition and chronic disease? Or misinterpreted science, media havoc and the sound of silence from peers. Public Health Nutrition 2006;9(2):269-72.

\section{Yu-Poth 1999}

Yu-Poth S, Zhao G, Etherton T, Naglak M, Jonnalagadda S, KrisEtherton PM. Effects of the National Cholesterol Education Program's Step I and Step II dietary intervention programs on cardiovascular disease risk factors: a meta-analysis. American Journal of Clinical Nutrition 1999;69:632-46.

\section{References to other published versions of this review Hooper 2000}

Hooper L, Summerbell CD, Higgins JPT, Thompson RL, Clements G, Capps N, et al. Reduced or modified dietary fat for preventing of cardiovascular disease. Cochrane Database of Systematic Reviews 2000, Issue 2. [DOI: 10.1002/14651858.CD002137]

\section{Hooper 2001}

Hooper L, Summerbell CD, Higgins JPT, Thompson RL, Capps N, Davey Smith G, et al. Dietary fat intake and prevention of cardiovascular disease: systematic review. BMJ 2001;322:757-63.

* Indicates the major publication for the study

\section{Anderson 1990}

\begin{tabular}{ll}
\hline Methods & RCT \\
\hline Participants & Moderately hypercholesterolaemic, non-obese Caucasian men and women aged 30-50 (USA) \\
& CVD risk: moderate \\
& Control: randomised 62, analysed 51 \\
Intervention: randomised 56, analysed 47
\end{tabular}


Anderson 1990 (Continued)

Mean years in trial: control 0.91, intervention 0.92

$\%$ male: control 61, intervention 66

Age: mean control 40.3 (sd 5.4), intervention 40.7 (sd 5.2) (all 30-50)

Reduced fat diet vs usual diet
Control aims: no diet intervention
Intervention aims: $25 \% \mathrm{E}$ from fats, $20 \% \mathrm{E}$ from protein, $55 \% \mathrm{E}$ from C
(Also an intervention arm with similar aims plus increased fibre intake)
Control methods: no intervention
Intervention methods: seminars and individual eating patterns taug
weeks maintenance
Total fat intake (at 1 year): low fat 30 (sd 7.5$)$, cont 31 (sd 5.7)\%E
Saturated fat intake (at 1 year): low fat 9 (sd 2.7), cont 10 (sd 2.9)\%E
Style: diet advice
Setting: community

\begin{tabular}{ll}
\hline Outcomes & Stated trial outcomes: diet composition, lipids \\
Data available on total mortality? yes (none) \\
Cardiovascular mortality? yes (none) \\
Events available for combined cardiovascular events: cardiovascular deaths, fatal and non-fatal MI, \\
stroke (none) \\
Secondary outcomes: total and non-fatal MI, stroke \\
Tertiary outcomes: total, LDL and HDL cholesterol \\
\hline
\end{tabular}

Notes

\section{Risk of bias}

\begin{tabular}{lll}
\hline Bias & Authors' judgement & Support for judgement \\
\hline $\begin{array}{l}\text { Random sequence genera- } \\
\text { tion (selection bias) }\end{array}$ & Low risk & $\begin{array}{l}\text { "matched on age, gender \& cholesterol level, randomly assigned to interven- } \\
\text { tion group using systematic random procedure" }\end{array}$ \\
\hline $\begin{array}{l}\text { Allocation concealment } \\
\text { (selection bias) }\end{array}$ & Unclear risk & Randomisation method not clearly described \\
\hline $\begin{array}{l}\text { Blinding (performance } \\
\text { bias and detection bias) } \\
\text { All outcomes }\end{array}$ & High risk & Participants were aware of their dietary advice, researchers were not \\
\hline $\begin{array}{l}\text { Incomplete outcome data } \\
\text { (attrition bias) }\end{array}$ & Unclear risk & Deaths, cancer and CV events are drop-outs, trialists asked for data \\
\hline $\begin{array}{l}\text { Sll outcomes } \\
\text { porting bias) }\end{array}$ & Low risk & Not relevant for primary and secondary outcomes as all trialists asked for data \\
\hline $\begin{array}{l}\text { Other bias } \\
\begin{array}{l}\text { Free of systematic differ- } \\
\text { ence in care? }\end{array}\end{array}$ High risk & $\begin{array}{l}\text { See Control and Intervention Methods in Interventions section of the Table of } \\
\text { Characteristics of Included Studies }\end{array}$ \\
\hline \hline
\end{tabular}


Anderson 1990 (Continued)

Free of dietary differences Low risk other than fat?
(As the high fibre arm has not been used in the data set). See Control and Intervention Aims in Interventions section of the Table of Characteristics of Included Studies

\section{Azadbakht 2007}

\begin{tabular}{|c|c|c|}
\hline Methods & \multicolumn{2}{|l|}{$\mathrm{RCT}$} \\
\hline Participants & \multicolumn{2}{|c|}{$\begin{array}{l}\text { Overweight and obese people (Iran) } \\
\text { CVD risk: low } \\
\text { Modified fat diet: } 50 \text { randomised, } 45 \text { analysed } \\
\text { Low fat diet: } 50 \text { randomised, } 44 \text { analysed } \\
\text { Mean years in trial: both groups } 1.1 \\
\% \text { male: modified fat diet } 32 \% \text {, low fat diet } 25 \% \\
\text { Age: mean modified fat } 45 \text { (sd 5), low fat diet } 46 \text { (sd 6) }\end{array}$} \\
\hline Interventions & $\begin{array}{l}\text { Reduced fat diet vs mc } \\
\text { Modified fat aims: } 30 \% \\
\text { terol <200mg/d, fibre } \\
\text { Low fat aims: } 20 \% \mathrm{E} \text { fro } \\
<200 \mathrm{mg} / \mathrm{d} \text {, fibre } 25 \mathrm{~g} / \mathrm{c} \\
\text { Modified fat methods: } \\
\text { dividual programmes } \\
\text { Low fat methods: mor } \\
\text { vidual programmes } \\
\text { Total fat intake (at } 14 \\
\text { Saturated fat intake (a } \\
\text { Style: diet advice } \\
\text { Setting: community }\end{array}$ & $\begin{array}{l}\text { lified fat diet } \\
\text { from fat, SFA } 5 \% \mathrm{E} \text {, MUFA } 15 \% \mathrm{E} \text {, PUFA } 10 \% \mathrm{E} \text {, protein } 15 \% \mathrm{E}, \mathrm{CHO} 55 \% \mathrm{E} \text {, choles- } \\
\text { n fat, SFA 5-6\%E, MUFA } 7 \% \mathrm{E} \text {, PUFA } 7 \% \mathrm{E} \text {, protein } 15 \% \mathrm{E}, \mathrm{CHO} 65 \% \mathrm{E} \text {, cholesterol } \\
500 \text { kcal below energy needs } \\
\text { nonthly appointments, oral and written information on healthy food choices, in- } \\
\text { hly appointments, oral and written information on healthy food choices, indi- } \\
\text { onths): low fat } 20 \text { (sd } 10) \% \mathrm{E}, \text { mod fat } 30 \text { (sd } 7.2) \% \mathrm{E} \\
14 \text { months): low fat } 6.8 \text { (sd 4.2)\%E, mod fat } 7.0 \text { (sd 3.6)\%E }\end{array}$ \\
\hline Outcomes & \multicolumn{2}{|c|}{$\begin{array}{l}\text { Stated trial outcomes: weight, metabolic risk } \\
\text { Data available on total mortality? yes (none) } \\
\text { Cardiovascular mortality? yes (none) } \\
\text { Events available for combined cardiovascular events: MI, stroke (no events) }\end{array}$} \\
\hline \multicolumn{3}{|l|}{ Notes } \\
\hline \multicolumn{3}{|l|}{ Risk of bias } \\
\hline Bias & Authors' judgement & Support for judgement \\
\hline $\begin{array}{l}\text { Random sequence genera- } \\
\text { tion (selection bias) }\end{array}$ & Low risk & Program generated by a random number table (?) \\
\hline $\begin{array}{l}\text { Allocation concealment } \\
\text { (selection bias) }\end{array}$ & Unclear risk & Randomisation method not clearly described \\
\hline
\end{tabular}


Azadbakht 2007 (Continued)

Blinding (performance High risk Participants and nutritionist were not blinded, lab staff were bias and detection bias)

All outcomes

\begin{tabular}{|c|c|c|}
\hline $\begin{array}{l}\text { Incomplete outcome data } \\
\text { (attrition bias) } \\
\text { All outcomes }\end{array}$ & Unclear risk & $\begin{array}{l}\text { Unclear, deaths, cancer and CV events are drop-outs - unclear if any data miss- } \\
\text { ing }\end{array}$ \\
\hline $\begin{array}{l}\text { Selective reporting (re- } \\
\text { porting bias) }\end{array}$ & Low risk & Not relevant for primary and secondary outcomes as all trialists asked for data \\
\hline Other bias & Low risk & \\
\hline $\begin{array}{l}\text { Free of systematic differ- } \\
\text { ence in care? }\end{array}$ & Low risk & $\begin{array}{l}\text { Both groups given equivalent intervention intensity and duration. See Control } \\
\text { and Intervention Methods in Interventions section of the Table of Characteris- } \\
\text { tics of Included Studies }\end{array}$ \\
\hline
\end{tabular}

Free of dietary differences Low risk other than fat?

Focus on fat and types of fat. See Control and Intervention Aims in Interventions section of the Table of Characteristics of Included Studies

\section{Ball 1965}

\begin{tabular}{ll}
\hline Methods & RCT \\
\hline Participants & Men who have recently recovered from their first MI (UK) \\
CVD risk: high \\
Control: unclear how many randomised, 129 analysed \\
Intervention: unclear how many randomised, 123 analysed \\
Mean years in trial: 3.0 \\
\% male: 100 \\
Age: unclear (all <65)
\end{tabular}

Interventions

Reduced fat intake vs. usual diet

Control aims: usual diet, overweight subjects given weight reduction advice (mainly $\mathrm{CHO}$ reduction) Intervention aims: reduce fat intake to $40 \mathrm{~g}$ daily, overweight subjects given weight reducing advice

Control methods: continued with normal diet, unclear if follow up for control group was as for intervention group.

Intervention methods: unclear who gave dietary advice or how often, patient and wife saw doctor and dietitian 2 weeks after hospital discharge, then every 2 weeks for 3 months, every 3 months for two years and six-monthly thereafter. Diet diaries checked by dietitian at each visit and problems discussed.

Total fat intake (at 4 years): low fat 21.8 (sd unclear), cont 44.3 (sd unclear)\%E

Saturated fat intake: unclear

Style: diet advice

Setting: community

$\begin{array}{ll}\text { Outcomes } & \text { Stated trial outcomes: reinfarction, death } \\ & \text { Data available on total mortality? yes } \\ & \text { Cardiovascular mortality? yes } \\ & \text { Events available for combined cardiovascular events: cardiovascular deaths, non-fatal MI }\end{array}$


Ball 1965 (Continued)

Secondary outcomes: total MI

Tertiary outcomes: none (weight and total cholesterol reported but no variance info)

\begin{tabular}{ll}
\hline Notes & At 3 years weight: control $-3.6 \mathrm{~kg} \mathrm{n}=67$, intervention $-5.4 \mathrm{~kg} \mathrm{n}=68$ \\
& total cholesterol: control $-0.85 \mathrm{mmol} / \mathrm{L} \mathrm{n}=52$, intervention $-1.14 \mathrm{mmol} / \mathrm{L} \mathrm{n}=54$
\end{tabular}

\section{Risk of bias}

\begin{tabular}{|c|c|c|}
\hline Bias & Authors' judgement & Support for judgement \\
\hline $\begin{array}{l}\text { Random sequence genera- } \\
\text { tion (selection bias) }\end{array}$ & Low risk & "allocated at random to one of two groups at each hospital" \\
\hline $\begin{array}{l}\text { Allocation concealment } \\
\text { (selection bias) }\end{array}$ & Unclear risk & Randomisation method not clearly described \\
\hline $\begin{array}{l}\text { Blinding (performance } \\
\text { bias and detection bias) } \\
\text { All outcomes }\end{array}$ & High risk & $\begin{array}{l}\text { Participants were not blinded, the researcher who assessed outcomes was } \\
\text { blinded to treatment arm }\end{array}$ \\
\hline $\begin{array}{l}\text { Incomplete outcome data } \\
\text { (attrition bias) } \\
\text { All outcomes }\end{array}$ & Unclear risk & Unclear if any lost to follow up \\
\hline $\begin{array}{l}\text { Selective reporting (re- } \\
\text { porting bias) }\end{array}$ & Low risk & Not relevant for primary and secondary outcomes as all trialists asked for data \\
\hline Other bias & Low risk & \\
\hline $\begin{array}{l}\text { Free of systematic differ- } \\
\text { ence in care? }\end{array}$ & Unclear risk & $\begin{array}{l}\text { See Control and Intervention Methods in Interventions section of the Table of } \\
\text { Characteristics of Included Studies }\end{array}$ \\
\hline $\begin{array}{l}\text { Free of dietary differences } \\
\text { other than fat? }\end{array}$ & Low risk & $\begin{array}{l}\text { See Control and Intervention Aims in Interventions section of the Table of } \\
\text { Characteristics of Included Studies }\end{array}$ \\
\hline
\end{tabular}

\section{BDIT Pilot Studies 1996}

\begin{tabular}{ll}
\hline Methods & RCT \\
\hline Participants & Women with mammographic dysplasia (Canada) \\
CVD risk: low \\
Control: 147 randomised, 110 analysed at over 8 years \\
Intervention: 148 randomised, 104 analysed at over 8 years \\
Mean years in trial: control 7.5, intervention 6.8 \\
\% male: 0 \\
Age: mean control 45, intervention 44 (all >30) \\
Reduced fat intake vs usual diet \\
Control aims: healthy diet advice, no alteration in dietary fat advised, aim to maintain weight \\
Intervention aims: total fat $15 \%$, replace fat by complex CHO, aim to maintain weight \\
Control methods: seen for advice once every 4 months for 12 months \\
Intervention methods: seen for advice once a month for 12 months
\end{tabular}


BDIT Pilot Studies 1996 (Continued)

Total fat intake (at 9.2 years): low fat 31.7 (sd 7.3)\%E, cont 35.3 (sd 5.6)\%E

Saturated fat intake (at 9.2 years): low fat 10.6 (sd 4.6), cont 12.3 (sd 4.6)\%E

Style: diet advice

Setting: community

Stated trial outcomes: dietary fat, serum cholesterol
Data available on total mortality? yes, but not clear from which groups
Cardiovascular mortality? no
Events available for combined cardiovascular events: none
Secondary outcomes: none
Tertiary outcomes: weight, BMI, total and HDL cholesterol

Notes

\section{Risk of bias}

\begin{tabular}{|c|c|c|}
\hline Bias & Authors' judgement & Support for judgement \\
\hline $\begin{array}{l}\text { Random sequence genera- } \\
\text { tion (selection bias) }\end{array}$ & Low risk & "randomly allocated" \\
\hline $\begin{array}{l}\text { Allocation concealment } \\
\text { (selection bias) }\end{array}$ & Unclear risk & Randomisation not described \\
\hline $\begin{array}{l}\text { Blinding (performance } \\
\text { bias and detection bias) } \\
\text { All outcomes }\end{array}$ & High risk & Participants not blinded, but outcome assessors blinded to intervention \\
\hline $\begin{array}{l}\text { Incomplete outcome data } \\
\text { (attrition bias) } \\
\text { All outcomes }\end{array}$ & Unclear risk & $\begin{array}{l}\text { Unclear, deaths, cancer and CV events are drop-outs, trialists asked for data - } \\
\text { unclear if any data missing }\end{array}$ \\
\hline $\begin{array}{l}\text { Selective reporting (re- } \\
\text { porting bias) }\end{array}$ & Low risk & Not relevant for primary and secondary outcomes as all trialists asked for data \\
\hline Other bias & Low risk & \\
\hline $\begin{array}{l}\text { Free of systematic differ- } \\
\text { ence in care? }\end{array}$ & High risk & $\begin{array}{l}\text { Minor, women in intervention group seen more frequently. See Control and In- } \\
\text { tervention Methods in Interventions section of the Table of Characteristics of } \\
\text { Included Studies }\end{array}$ \\
\hline $\begin{array}{l}\text { Free of dietary differences } \\
\text { other than fat? }\end{array}$ & Low risk & $\begin{array}{l}\text { See Control and Intervention Aims in Interventions section of the Table of } \\
\text { Characteristics of Included Studies }\end{array}$ \\
\hline
\end{tabular}

\section{beFIT 1997}

\begin{tabular}{ll}
\hline Methods & RCT \\
\hline Participants & Women and men with mild hypercholesterolemia (USA) \\
& CVD risk: moderate \\
& Control: unclear how many randomised, 192 analysed \\
Intervention: unclear how many randomised, 217 analysed \\
Mean years in trial: unclear (max duration 0.5 years)
\end{tabular}


beFIT 1997 (Continued)

$\%$ male: 52 (not divided by intervention group)

Age: mean 43.2 (not divided by intervention group) (all >30)

Interventions
Reduced and modified fat vs usual diet
Control aims:asked to delay dietary changes (provided intervention after the randomised trial)
Intervention aims: total fat $<30 \% \mathrm{E}, \mathrm{SFA}<7 \% \mathrm{E}$, dietary chol $<200 \mathrm{mg} / \mathrm{d}$
Control methods: usual intake
Intervention methods: 8 weekly classes with nutrition info and behaviour modification with spouses,
plus individual appointments at 3 and 6 months
Total fat intake (at 6 months): int 25.2 (sd unclear)\%E, cont unclear - no significant difference from
baseline 34 (sd unclear)\%E
Saturated fat intake (at 6 months): int $7.6 \%$ (sd unclear)\%E, cont unclear - no significant difference
from baseline 12 (sd unclear)\%
Style: diet advice
Setting: community

Stated trial outcomes: lipids
Data available on total mortality? yes (no events)
Cardiovascular mortality? no
Events available for combined cardiovascular events: unclear but authors stated that there were no
CVD events
Secondary outcomes: none
Tertiary outcomes: weight, total, LDL and HDL cholesterol, TGs (but variance data only provided for the
randomised comparison for LDL cholesterol)

Notes Weight: control 'no change', intervention $-2.7 \mathrm{~kg}$ at 6 months

\section{Risk of bias}

\begin{tabular}{|c|c|c|}
\hline Bias & Authors' judgement & Support for judgement \\
\hline $\begin{array}{l}\text { Random sequence genera- } \\
\text { tion (selection bias) }\end{array}$ & Low risk & Stratified random sampling scheme \\
\hline $\begin{array}{l}\text { Allocation concealment } \\
\text { (selection bias) }\end{array}$ & Unclear risk & Randomisation method not clearly described \\
\hline $\begin{array}{l}\text { Blinding (performance } \\
\text { bias and detection bias) } \\
\text { All outcomes }\end{array}$ & High risk & Participants knew their allocation, unclear for outcome assessors \\
\hline $\begin{array}{l}\text { Incomplete outcome data } \\
\text { (attrition bias) } \\
\text { All outcomes }\end{array}$ & Unclear risk & $\begin{array}{l}\text { Unclear, deaths, cancer and CV events are drop-outs, trialists asked for data - } \\
\text { unclear if any data missing }\end{array}$ \\
\hline $\begin{array}{l}\text { Selective reporting (re- } \\
\text { porting bias) }\end{array}$ & Low risk & Not relevant for primary and secondary outcomes as all trialists asked for data \\
\hline Other bias & Low risk & \\
\hline $\begin{array}{l}\text { Free of systematic differ- } \\
\text { ence in care? }\end{array}$ & High risk & $\begin{array}{l}\text { Intensive intervention for intervention group, but no intervention during the } 6 \\
\text { months of the randomised part of the study for the control group. See Control }\end{array}$ \\
\hline
\end{tabular}


beFIT 1997 (Continued)

and Intervention Methods in Interventions section of the Table of Characteristics of Included Studies

\begin{tabular}{ll}
\hline $\begin{array}{l}\text { Free of dietary differences } \\
\text { other than fat? }\end{array}$ & Low risk \\
& $\begin{array}{l}\text { See Control and Intervention Aims in Interventions section of the Table of } \\
\text { Chacteristics of Included Studies }\end{array}$ \\
\hline
\end{tabular}

Black 1994

\begin{tabular}{|c|c|}
\hline Methods & $\mathrm{RCT}$ \\
\hline Participants & $\begin{array}{l}\text { People with non-melanoma skin cancer (USA) } \\
\text { CVD risk: low } \\
\text { Control: randomised } 67 \text {, analysed } 58 \\
\text { Intervention: randomised } 66 \text {, analysed } 57 \\
\text { Mean years in trial: } 1.9 \\
\% \text { male: control } 67 \% \text {, intervention } 54 \% \\
\text { Age: mean control } 52.3 \text { (sd 13.2), intervention } 50.6 \text { (sd 9.7) }\end{array}$ \\
\hline Interventions & $\begin{array}{l}\text { Reduced fat vs. usual diet } \\
\text { Control aims: no dietary advice } \\
\text { Intervention aims: total fat } 20 \% \mathrm{E} \text {, protein } 15 \% \mathrm{E}, \mathrm{CHO} 65 \% \mathrm{E} \\
\text { Control methods: no dietary change, } 4 \text { monthly clinic visits } \\
\text { Intervention methods: } 8 \text { weekly classes, with behavioural techniques, plus } 4 \text { monthly clinic visits } \\
\text { Total fat intake ("during study" months 4-24): low fat } 20.7 \text { (sd 5.5), cont } 37.8 \text { (sd 4.1)\%E } \\
\text { Saturated fat intake ("during study, months 4-24): low fat } 6.6 \text { (sd 1.8), cont } 12.8 \text { (sd 2.0)\%E } \\
\text { Style: diet advice } \\
\text { Setting: community }\end{array}$ \\
\hline
\end{tabular}

Outcomes

Stated trial outcomes: incidence of actinic keratosis and non-melanoma skin cancer

Data available on total mortality? yes

Cardiovascular mortality? yes

Events available for combined cardiovascular events: cardiovascular deaths

Secondary outcomes: cancer deaths (none)

Tertiary outcomes: none (weight data provided, but no variance info)

Notes At 2 years control $-1.5 \mathrm{~kg} n=50$ ?, intervention $-1 \mathrm{~kg} n=51$ ?

\section{Risk of bias}

\begin{tabular}{lll}
\hline Bias & Authors' judgement & Support for judgement \\
\hline $\begin{array}{l}\text { Random sequence genera- } \\
\text { tion (selection bias) }\end{array}$ & Low risk & "list of randomly generated numbers" \\
\hline $\begin{array}{l}\text { Allocation concealment } \\
\text { (selection bias) }\end{array}$ & Unclear risk & Randomisation method not clearly described \\
\hline $\begin{array}{l}\text { Blinding (performance } \\
\text { bias and detection bias) }\end{array}$ & High risk & $\begin{array}{l}\text { Physician blinding: adequate } \\
\text { Participant blinding: inadequate }\end{array}$ \\
\hline
\end{tabular}


Black 1994 (Continued)

All outcomes

Incomplete outcome data Low risk $\quad$ For mortality. Unclear for other outcomes
(attrition bias)

(attrition bias)

All outcomes

\begin{tabular}{lll}
\hline $\begin{array}{l}\text { Selective reporting (re- } \\
\text { porting bias) }\end{array}$ & Low risk & Not relevant for primary and secondary outcomes as all trialists asked for data \\
\hline Other bias & Low risk \\
\hline
\end{tabular}

Free of systematic differ- High risk

Minor, all have 4 monthly clinic visits, the intervention group had 8 behaviourence in care? al technique classes that the control group did not have

Free of dietary differences Low risk other than fat?

See Control and Intervention Aims in Interventions section of the Table of Characteristics of Included Studies

Boyd 1988

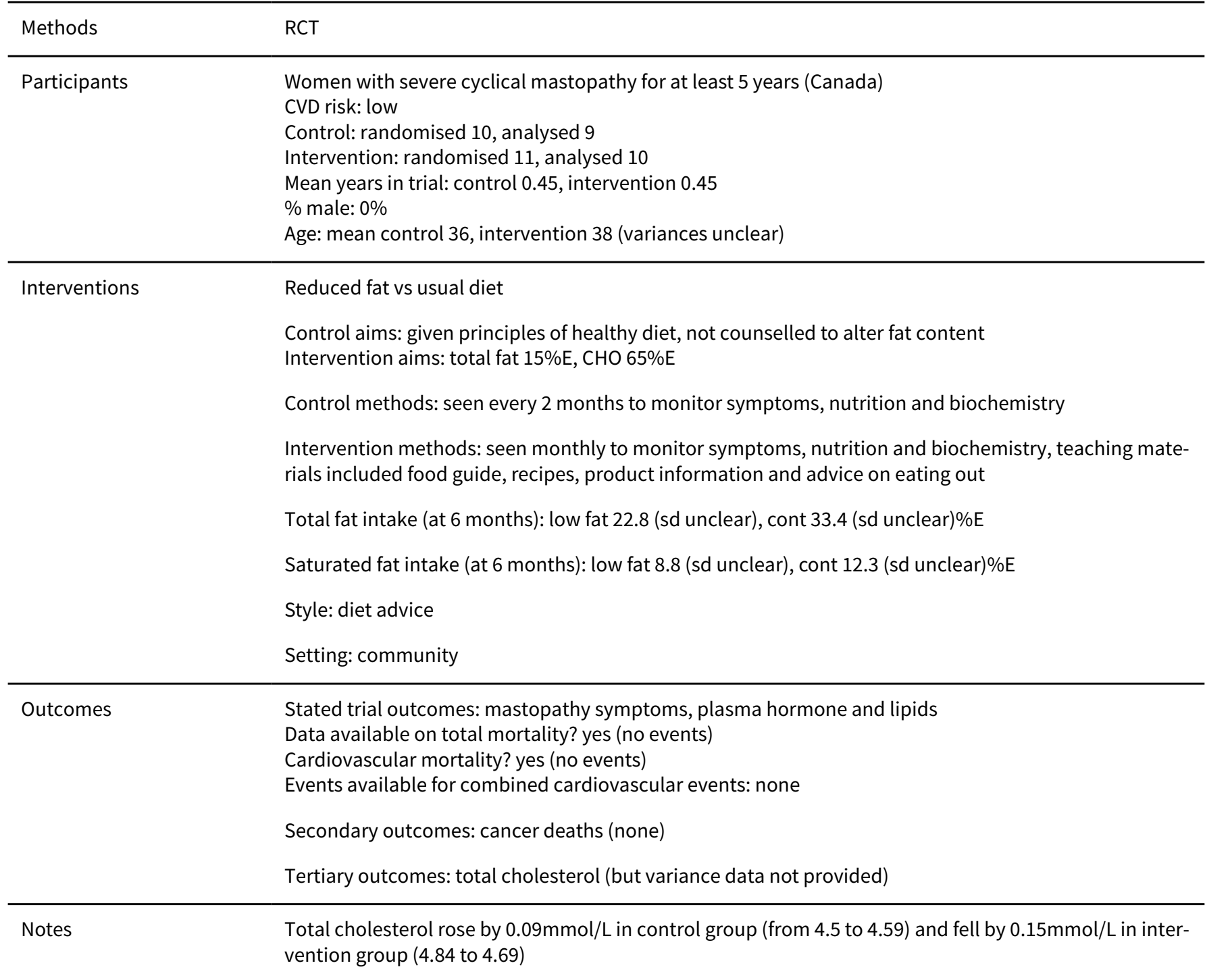


Boyd 1988 (Continued)

\section{Risk of bias}

\begin{tabular}{|c|c|c|}
\hline Bias & Authors' judgement & Support for judgement \\
\hline $\begin{array}{l}\text { Random sequence genera- } \\
\text { tion (selection bias) }\end{array}$ & Low risk & "randomly allocated" \\
\hline $\begin{array}{l}\text { Allocation concealment } \\
\text { (selection bias) }\end{array}$ & Unclear risk & Randomisation method not clearly described \\
\hline $\begin{array}{l}\text { Blinding (performance } \\
\text { bias and detection bias) } \\
\text { All outcomes }\end{array}$ & High risk & $\begin{array}{l}\text { Participants were not blinded, those assessing physical outcomes were blind- } \\
\text { ed, those assessing symptoms were not }\end{array}$ \\
\hline $\begin{array}{l}\text { Incomplete outcome data } \\
\text { (attrition bias) } \\
\text { All outcomes }\end{array}$ & Unclear risk & $\begin{array}{l}\text { Unclear, deaths, cancer and CV events are drop-outs, trialists asked for data - } \\
\text { unclear if any data missing }\end{array}$ \\
\hline $\begin{array}{l}\text { Selective reporting (re- } \\
\text { porting bias) }\end{array}$ & Low risk & Not relevant for primary and secondary outcomes as all trialists asked for data \\
\hline Other bias & Low risk & \\
\hline $\begin{array}{l}\text { Free of systematic differ- } \\
\text { ence in care? }\end{array}$ & High risk & $\begin{array}{l}\text { Minor differences in follow up frequency. See Control and Intervention Meth- } \\
\text { ods in Interventions section of the Table of Characteristics of Included Studies }\end{array}$ \\
\hline $\begin{array}{l}\text { Free of dietary differences } \\
\text { other than fat? }\end{array}$ & Low risk & $\begin{array}{l}\text { See Control and Intervention Aims in Interventions section of the Table of } \\
\text { Characteristics of Included Studies }\end{array}$ \\
\hline
\end{tabular}

\section{BRIDGES 2001}

\begin{tabular}{ll}
\hline Methods & RCT \\
\hline Participants & Women diagnosed with stage I or II breast cancer over the past 2 years (USA) \\
CVD risk: low \\
Control: randomised unclear (at least 56), analysed 56 \\
Intervention: randomised unclear (at least 50), analysed 50 \\
Mean years in trial: unclear (1 year max follow up) \\
\% male: 0 \\
Age: mean control unclear (71\% postmenopausal), intervention unclear (56\% postmenopausal) (all \\
20-65) \\
Reduced fat vs usual diet \\
Control aims: no formal intervention \\
Intervention diet aims: total fat 20\%E, high fibre, plant based micronutrients \\
Intervention stress: separate parallel arm, stress reduction programme (data not used here) \\
Control methods: no formal intervention \\
Intervention methods: nutrition intervention programme, 15 sessions (42 hours) over 15 weeks, group- \\
based, dietitian led, 2 individual sessions using social cognitive theory and patient centred counselling \\
to increase self efficacy and confidence \\
Total fat intake (at 12 months): low fat 29.9 (sd unclear), cont 33.6 (sd unclear)\%E \\
Saturated fat intake: unclear
\end{tabular}


BRIDGES 2001 (Continued)

Style: diet advice

Setting: community

\begin{tabular}{ll}
\hline Sutcomes & Stated trial outcomes: diet and BMI \\
Data available on total mortality? yes \\
Cardiovascular mortality? yes (no events) \\
Events available for combined cardiovascular events: cardiovascular deaths, non fatal MI, stroke (no \\
events) \\
Secondary outcomes: total and non-fatal MI, stroke, cancer deaths (events only for cancer deaths) \\
Tertiary outcomes: weight
\end{tabular}

Notes

Risk of bias

\begin{tabular}{|c|c|c|}
\hline Bias & Authors' judgement & Support for judgement \\
\hline $\begin{array}{l}\text { Random sequence genera- } \\
\text { tion (selection bias) }\end{array}$ & Low risk & $\begin{array}{l}\text { "randomised", stratified by medical centre, cancer stage and age, randomised } \\
\text { number/envelope method by project coordinator }\end{array}$ \\
\hline $\begin{array}{l}\text { Allocation concealment } \\
\text { (selection bias) }\end{array}$ & Low risk & $\begin{array}{l}\text { The project coordinator had contact with those from the University of Massa- } \\
\text { chussets, but not those from the other } 3 \text { centres, and allocation could not be } \\
\text { altered later }\end{array}$ \\
\hline $\begin{array}{l}\text { Blinding (performance } \\
\text { bias and detection bias) } \\
\text { All outcomes }\end{array}$ & High risk & Participants not blinded, unclear about researchers \\
\hline $\begin{array}{l}\text { Incomplete outcome data } \\
\text { (attrition bias) } \\
\text { All outcomes }\end{array}$ & Unclear risk & $\begin{array}{l}\text { Unclear, deaths, cancer and CV events are drop-outs, trialists asked for data - } \\
\text { unclear if any data missing }\end{array}$ \\
\hline $\begin{array}{l}\text { Selective reporting (re- } \\
\text { porting bias) }\end{array}$ & Low risk & Not relevant for primary and secondary outcomes as all trialists asked for data \\
\hline Other bias & Low risk & \\
\hline $\begin{array}{l}\text { Free of systematic differ- } \\
\text { ence in care? }\end{array}$ & High risk & $\begin{array}{l}\text { High intensity programme for intervention group, nothing for control group. } \\
\text { See Control and Intervention Methods in Interventions section of the Table of } \\
\text { Characteristics of Included Studies }\end{array}$ \\
\hline $\begin{array}{l}\text { Free of dietary differences } \\
\text { other than fat? }\end{array}$ & High risk & $\begin{array}{l}\text { Intervention also focused on fibre and plant based micronutrients. See Control } \\
\text { and Intervention Aims in Interventions section of the Table of Characteristics } \\
\text { of Included Studies }\end{array}$ \\
\hline
\end{tabular}

\section{CARMEN 2000}

\begin{tabular}{ll}
\hline Methods & RCT \\
\hline Participants & Healthy overweight people, BMI 26-34 (Europe, 5 centres) \\
CVD risk: low & \\
Control: unclear how many randomised, 77 analysed (290 randomised over all 3 arms) \\
Intervention with simple CHO: unclear how many randomised, 76 analysed
\end{tabular}


Intervention with complex $\mathrm{CHO}$ : unclear how many randomised, 83 analysed

Mean years in trial: unclear (max duration 0.5 years)

$\%$ male: control $48 \%$, simple $\mathrm{CHO}$ intervention $47 \%$, complex $\mathrm{CHO}$ intervention $52 \%$

Age: mean control 38 (sd 9), simple $\mathrm{CHO}$ intervention 41 (sd 9), complex $\mathrm{CHO}$ intervention 38 (sd 9)

Interventions

Reduced fat vs usual diet

Control aims: to attain national "normal" intake

Intervention aims: total fat reduced by $10 \%$ E with increases in simple or complex $\mathrm{CHO}$

Control methods: trial shop provided local selection of a specific set of national "normal" intake foods

Intervention methods: trial shop provided local selection of a specific set of low fat and high simple or complex $\mathrm{CHO}$ foods

Total fat intake (at 6 months): low fat complex $\mathrm{CHO} 27.8$ (sd unclear)\%E, low fat simple CHO 25.5 (sd unclear)\%E, cont 36.5 (sd unclear)\%E

Saturated fat intake (at 6 months): low fat complex CHO 9.9 (sd unclear)\%E, low fat simple CHO 8.6 (sd unclear)\%E, cont 12.7 (sd unclear)\%

Style: food provided

Setting: community

\section{Outcomes}

Stated trial outcomes: weight, body composition, lipids

Data available on total mortality? yes (no events)

Cardiovascular mortality? yes (no events)

Events available for combined cardiovascular events: CVD deaths, non-fatal MI, stroke, heart failure,

PVD (no events)

Secondary outcomes: cancer deaths and diagnoses (no events)

Tertiary outcomes: weight, total, LDL and HDL cholesterol, TGs

Notes

\section{Risk of bias}

\begin{tabular}{lll}
\hline Bias & Authors' judgement & Support for judgement \\
\hline $\begin{array}{l}\text { Random sequence genera- } \\
\text { tion (selection bias) }\end{array}$ & Low risk & Computer randomisation \\
\hline $\begin{array}{l}\text { Allocation concealment } \\
\text { (selection bias) }\end{array}$ & Low risk & $\begin{array}{l}\text { Randomisation by 3rd party, independent of research centres. Blind data were } \\
\text { sent to him for computer randomisation }\end{array}$ \\
\hline $\begin{array}{l}\text { Blinding (performance } \\
\text { bias and detection bias) } \\
\text { All outcomes }\end{array}$ & High risk & $\begin{array}{l}\text { Participants were clear about whether they were in the control or an interven- } \\
\text { tion group }\end{array}$ \\
\hline $\begin{array}{l}\text { Incomplete outcome data } \\
\text { (attrition bias) } \\
\text { All outcomes }\end{array}$ & Unclear risk & $\begin{array}{l}\text { Unclear, deaths, cancer and CV events are drop-outs, trialists asked for data - } \\
\text { unclear if any data missing }\end{array}$ \\
\hline $\begin{array}{l}\text { Selective reporting (re- } \\
\text { porting bias) }\end{array}$ & Low risk & Not relevant for primary and secondary outcomes as all trialists asked for data \\
\hline \begin{tabular}{l} 
Other bias \\
\hline
\end{tabular} & Low risk & \\
\hline
\end{tabular}




\section{CARMEN 2000 (Continued)}

Free of systematic differ- Low risk $\quad$ Trial shop used by both groups. See Control and Intervention Methods in Interence in care?

Free of dietary differences Low risk other than fat?

\author{
ventions section of the Table of Characteristics of Included Studies
}

See Control and Intervention Aims in Interventions section of the Table of Characteristics of Included Studies

CARMEN MS sub-study 2002

\begin{tabular}{|c|c|}
\hline Methods & RCT (data for this study excludes the 13 participants that were included in the main CARMEN data set) \\
\hline \multirow[t]{6}{*}{ Participants } & $\begin{array}{l}\text { People with at least } 3 \text { risk factors for metabolic syndrome (Europe, } 5 \text { centres) } \\
\text { CVD risk: moderate }\end{array}$ \\
\hline & $\begin{array}{l}\text { Control: } 12 \text { randomised, } 8 \text { analysed } \\
\text { Intervention with simple CHO: } 10 \text { randomised, } 9 \text { analysed }\end{array}$ \\
\hline & Intervention with complex CHO: 11 randomised, 9 analysed \\
\hline & Mean years in trial: control 0.4, simple $\mathrm{CHO} 0.5$, complex $\mathrm{CHO} 0.5$ \\
\hline & $\%$ male: control 0\%, simple $\mathrm{CHO} 33 \%$, complex $\mathrm{CHO} 22 \%$ \\
\hline & $\begin{array}{l}\text { Age: mean control } 47.5 \text { (sd 3.9), simple CHO intervention } 44.7 \text { (sd 4.7), complex CHO intervention } 43.4 \\
\text { (sd 4.5) }\end{array}$ \\
\hline \multirow[t]{10}{*}{ Interventions } & Reduced fat vs usual diet \\
\hline & Control aims: to attain national "normal" intake \\
\hline & Intervention aims: total fat reduced by $10 \% \mathrm{E}$ with increases in simple or complex $\mathrm{CHO}$ \\
\hline & Control methods: trial shop provided local selection of a specific set of national "normal" intake foods \\
\hline & $\begin{array}{l}\text { Intervention methods: trial shop provided local selection of a specific set of low fat and high simple or } \\
\text { complex } \mathrm{CHO} \text { foods }\end{array}$ \\
\hline & Total fat intake (at 6 months): low fat complex CHO 27.1 (sd 4.8), low fat simple CHO 20.6 (sd 6.6), cont \\
\hline & $30.4(\mathrm{sd} 2.3) \% \mathrm{E}$ \\
\hline & Saturated fat intake: unclear \\
\hline & Style: food provided \\
\hline & Setting: community \\
\hline
\end{tabular}

Outcomes

Stated trial outcomes: weight, body composition, lipids

Data available on total mortality? yes (no events)

Cardiovascular mortality? yes (no events)

Events available for combined cardiovascular events: CVD deaths, non-fatal MI, stroke, heart failure, PVD (no events)

Secondary outcomes: cancer deaths and diagnoses (no events)

Tertiary outcomes: BMI, total, LDL and HDL cholesterol, TGs, diastolic BP

Notes

\section{Risk of bias}

Bias Authors' judgement Support for judgement


CARMEN MS sub-study 2002 (Continued)

\begin{tabular}{lll}
$\begin{array}{l}\text { Random sequence genera- } \\
\text { tion (selection bias) }\end{array}$ & Low risk & Computer randomisation \\
\hline $\begin{array}{l}\text { Allocation concealment } \\
\text { (selection bias) }\end{array}$ & Low risk & $\begin{array}{l}\text { Randomisation by 3rd party, independent of research centres. Blind data were } \\
\text { sent to him for computer randomisation }\end{array}$
\end{tabular}

\begin{tabular}{|c|c|c|}
\hline $\begin{array}{l}\text { Blinding (performance } \\
\text { bias and detection bias) } \\
\text { All outcomes }\end{array}$ & High risk & $\begin{array}{l}\text { Participants were clear about whether they were in the control or an interven- } \\
\text { tion group }\end{array}$ \\
\hline
\end{tabular}

\begin{tabular}{|c|c|c|}
\hline $\begin{array}{l}\text { Incomplete outcome data } \\
\text { (attrition bias) } \\
\text { All outcomes }\end{array}$ & Unclear risk & $\begin{array}{l}\text { Unclear, deaths, cancer and CV events are drop-outs, trialists asked for data - } \\
\text { unclear if any data missing }\end{array}$ \\
\hline
\end{tabular}

\begin{tabular}{lll}
\hline $\begin{array}{l}\text { Selective reporting (re- } \\
\text { porting bias) }\end{array}$ & Low risk & Not relevant for primary and secondary outcomes as all trialists asked for data \\
\hline Other bias & Low risk & \\
\hline $\begin{array}{l}\text { Free of systematic differ- } \\
\text { ence in care? }\end{array}$ & Low risk & $\begin{array}{l}\text { Trial shop used by both groups. See Control and Intervention Methods in Inter- } \\
\text { ventions section of the Table of Characteristics of Included Studies }\end{array}$ \\
\hline $\begin{array}{l}\text { Free of dietary differences } \\
\text { other than fat? }\end{array}$ & Low risk & $\begin{array}{l}\text { See Control and Intervention Aims in Interventions section of the Table of } \\
\text { Characteristics of Included Studies }\end{array}$ \\
\hline
\end{tabular}

\section{Curzio 1989}

\begin{tabular}{|c|c|}
\hline Methods & $\mathrm{RCT}$ \\
\hline Participants & $\begin{array}{l}\text { Hypertensives with cholesterol }>6.5 \mathrm{mmol} / \mathrm{L} \text { (UK) } \\
\text { CVD risk: moderate } \\
\text { Control: randomised } 72 \text {, analysed } 63 \\
\text { Intervention: randomised } 72 \text {, analysed } 61 \\
\text { Mean years in trial: control } 0.47 \text {, intervention } 0.46 \\
\% \text { male: control } 54 \% \text {, intervention } 44 \% \\
\text { Age: mean control } 56 \text {, intervention } 57\end{array}$ \\
\hline \multirow[t]{8}{*}{ Interventions } & Unclear \\
\hline & $\begin{array}{l}\text { Control aims: no dietary advice } \\
\text { Intervention aims: advice to reduce serum cholesterol (?) }\end{array}$ \\
\hline & Control methods: no advice at any visit ( $0,1,3$ and 6 months) \\
\hline & $\begin{array}{l}\text { Intervention methods: intensive and specific dietary advice, by dietitian at each visit }(0,1,3 \text { and } 6 \\
\text { months) }\end{array}$ \\
\hline & Total fat intake: unclear \\
\hline & Saturated fat intake: unclear \\
\hline & Style: diet advice \\
\hline & Setting: community \\
\hline Outcomes & $\begin{array}{l}\text { Stated trial outcomes: blood pressure, weight, lipids } \\
\text { Data available on total mortality? yes (no events) } \\
\text { Cardiovascular mortality? yes (no events) }\end{array}$ \\
\hline
\end{tabular}


Secondary outcomes: cancer deaths (no events)

Tertiary outcomes: total, HDL and LDL cholesterol, TG (not used in analysis as unclear whether reduced and/or modified fat intervention)

Notes

Changes in lipid parameters from baseline to 6 months (control $n=63$, intervention $n=61$ ), all $\mathrm{mmol} / \mathrm{L}$

Total cholesterol control -0.3 (sd 0.7), intervention - 0.3 (sd 0.6)

LDL cholesterol control -0.1 (sd 0.7), intervention -0.3 (sd 0.7)

HDL cholesterol control -0.0 (sd 0.1), intervention 0.2 (sd 0.2)

TGs control -0.2 (sd 0.6), intervention -0.2 (sd 0.7)

\section{Risk of bias}

\begin{tabular}{|c|c|c|}
\hline Bias & Authors' judgement & Support for judgement \\
\hline $\begin{array}{l}\text { Random sequence genera- } \\
\text { tion (selection bias) }\end{array}$ & Low risk & "stratified by antihypertensive treatment, randomly allocated" \\
\hline $\begin{array}{l}\text { Allocation concealment } \\
\text { (selection bias) }\end{array}$ & Unclear risk & Randomisation method not clearly described \\
\hline $\begin{array}{l}\text { Blinding (performance } \\
\text { bias and detection bias) } \\
\text { All outcomes }\end{array}$ & High risk & Participants not blinded, researchers unclear \\
\hline $\begin{array}{l}\text { Incomplete outcome data } \\
\text { (attrition bias) } \\
\text { All outcomes }\end{array}$ & Unclear risk & $\begin{array}{l}\text { Unclear, deaths, cancer and CV events are drop-outs, trialists asked for data - } \\
\text { unclear if any data missing }\end{array}$ \\
\hline $\begin{array}{l}\text { Selective reporting (re- } \\
\text { porting bias) }\end{array}$ & Low risk & Not relevant for primary and secondary outcomes as all trialists asked for data \\
\hline Other bias & Low risk & \\
\hline $\begin{array}{l}\text { Free of systematic differ- } \\
\text { ence in care? }\end{array}$ & High risk & $\begin{array}{l}\text { Dietetic time for those on intervention only. See Control and Intervention } \\
\text { Methods in Interventions section of the Table of Characteristics of Included } \\
\text { Studies }\end{array}$ \\
\hline $\begin{array}{l}\text { Free of dietary differences } \\
\text { other than fat? }\end{array}$ & Unclear risk & Dietary goals unclear. \\
\hline
\end{tabular}

\section{DART 1989}

\begin{tabular}{ll}
\hline Methods & RCT \\
\hline Participants & Men recovering from an MI (UK) \\
& CVD risk: high \\
& Control: randomised 1015, analysed unclear \\
Intervention: randomised 1018, analysed unclear & Mean years in trial: control 1.9, randomised 1.9 \\
& $\%$ male: $100 \%$
\end{tabular}


DART 1989 (Continued)

Age: mean control 56.8, intervention 56.4 (all <70)

Interventions

Reduced and modified fat vs usual diet

Control aims: no dietary advice on fat, weight reducing advice if $\mathrm{BM} \mid>30$

Intervention aims: reduce fat intake to $30 \% \mathrm{E}$, increase $\mathrm{P} / \mathrm{S}$ to 1.0 , weight reducing advice if $\mathrm{BMI}>30$

Note: This was a factorial trial, and so some in each group were randomised to increased fatty fish and/ or increased cereal fibre.

Control methods: dietitians provided 'sensible eating' advice without specific information on fats

Intervention methods: dietitians provided the participants and their wives with initial individual advice and a diet information sheet, participants were revisited for further advice, recipes, encouragement at $1,3,6,9,12,15,18$ and 21 months.

Total fat intake (through study): int 31 (sd 7), cont 35 (sd 6)\%E

Saturated fat intake (through study): int 11 (sd 3), cont 15 (sd 3)\%E

Style: diet advice

Setting: community

Stated trial outcomes: mortality, reinfarction
Data available on total mortality? yes
Cardiovascular mortality? yes
Events available for combined cardiovascular events: cardiovascular deaths (including stroke deaths)
plus non-fatal MI
Secondary outcomes: cancer deaths, total MI, non-fatal MI
Tertiary outcomes: total and HDL cholesterol

Notes

\section{Risk of bias}

\begin{tabular}{|c|c|c|}
\hline Bias & Authors' judgement & Support for judgement \\
\hline $\begin{array}{l}\text { Random sequence genera- } \\
\text { tion (selection bias) }\end{array}$ & Low risk & randomised using sealed envelopes \\
\hline $\begin{array}{l}\text { Allocation concealment } \\
\text { (selection bias) }\end{array}$ & Unclear risk & Unclear if envelopes were opaque \\
\hline $\begin{array}{l}\text { Blinding (performance } \\
\text { bias and detection bias) } \\
\text { All outcomes }\end{array}$ & High risk & $\begin{array}{l}\text { Physician blinding: yes } \\
\text { Participant blinding: unclear }\end{array}$ \\
\hline $\begin{array}{l}\text { Incomplete outcome data } \\
\text { (attrition bias) } \\
\text { All outcomes }\end{array}$ & Low risk & $\begin{array}{l}\text { GPs contacted for information on mortality and morbidity when patients did } \\
\text { not attend }\end{array}$ \\
\hline $\begin{array}{l}\text { Selective reporting (re- } \\
\text { porting bias) }\end{array}$ & Low risk & Not relevant for primary and secondary outcomes as all trialists asked for data \\
\hline Other bias & Low risk & \\
\hline
\end{tabular}


DART 1989 (Continued)

Free of systematic differ- High risk ence in care?

Different levels of advice appear to have been provided. See Control and Intervention Methods in Interventions section of the Table of Characteristics of Included Studies

Free of dietary differences Low risk other than fat?

There were also other arms, testing fish and fibre interventions (in a factorial design). See Control and Intervention Aims in Interventions section of the Table of Characteristics of Included Studies

\section{DO IT 2006}

\begin{tabular}{|c|c|}
\hline Methods & $\mathrm{RCT}$ \\
\hline Participants & $\begin{array}{l}\text { Survivors of Oslo Diet Study who had hyperlipidaemia and high risk of CVD in the } 1970 \text { s (Norway) } \\
\text { CVD risk: moderate (although } 25 \% \text { have CVD) } \\
\text { control: No n-3 control, } 142 \text { randomised, } 117 \text { analysed } \\
\text { With n-3 control, } 140 \text { randomised, } 124 \text { analysed } \\
\text { intervention: No n-3 intervention, } 139 \text { randomised, } 122 \text { analysed } \\
\text { With n-3 intervention, } 142 \text { randomised, } 124 \text { analysed } \\
\text { Mean years in trial: } 3 \\
\% \text { male: } 100 \\
\text { Age: control groups median } 70 \text { years, intervention groups median } 70\end{array}$ \\
\hline Interventions & $\begin{array}{l}\text { Reduced fat intake vs usual diet } \\
\text { Control aims: usual care (with n-3 capsules or corn oil capsules) } \\
\text { Intervention aims: } 27-30 \% \mathrm{E} \text { from fat, protein } 15-18 \% \text {, CHO 50-55\%E, increase fruit, vegetables, fish, de- } \\
\text { crease meat, polyunsaturated margarine provided free (with } \mathrm{n}-3 \text { capsules or corn oil capsules) } \\
\text { Control methods: usual care, with either corn oil or omega-3 capsules } \\
\text { Intervention methods: dietary advice ( } 30-45 \text { mins initially plus } 30 \text { mins follow up at } 3 \text { months, then } 6 \\
\text { monthly), polyunsaturated margarines provided free, plus either corn oil or omega-3 capsules } \\
\text { Total fat intake (at } 36 \text { months): low fat } 27.6 \text { (sd 5.5), cont } 29.5 \text { (sd 5.4)\%E } \\
\text { Saturated fat intake (at } 36 \text { months): low fat } 9.2 \text { (sd 3.6), cont } 10.5 \text { (sd 3.7)\%E } \\
\text { Style: diet advice and supplement } \\
\text { Setting: community }\end{array}$ \\
\hline Outcomes & $\begin{array}{l}\text { Stated trial outcomes: CVD } \\
\text { Data available on total mortality? yes } \\
\text { Cardiovascular mortality? yes } \\
\text { Events available for combined cardiovascular events: total MI, verified cardiovascular events } \\
\text { Secondary outcomes: cancer deaths and diagnoses, diabetes, total MI } \\
\text { Tertiary outcomes: BMI, total, HDL, LDL cholesterol, TG, systolic BP }\end{array}$ \\
\hline
\end{tabular}

Notes

\section{Risk of bias}

\section{Bias \\ Authors' judgement Support for judgement}


DO IT 2006 (Continued)

Random sequence genera- Low risk $\quad$ 'randomly assigned'
tion (selection bias)

Allocation concealment Unclear risk
(selection bias)

\begin{tabular}{|c|c|c|}
\hline $\begin{array}{l}\text { Blinding (performance } \\
\text { bias and detection bias) } \\
\text { All outcomes }\end{array}$ & High risk & Not for dietary intervention. \\
\hline
\end{tabular}

\begin{tabular}{|c|c|c|}
\hline $\begin{array}{l}\text { Incomplete outcome data } \\
\text { (attrition bias) } \\
\text { All outcomes }\end{array}$ & Unclear risk & $\begin{array}{l}\text { Unclear, deaths, cancer and CV events are drop-outs, trialists asked for data - } \\
\text { unclear if any data missing }\end{array}$ \\
\hline
\end{tabular}

\begin{tabular}{lll}
\hline $\begin{array}{l}\text { Selective reporting (re- } \\
\text { porting bias) }\end{array}$ & Low risk & Not relevant for primary and secondary outcomes as all trialists asked for data \\
\hline Other bias & Low risk & $\begin{array}{l}\text { Additional dietary appointments in the intervention groups. See Control and } \\
\text { Intervention Methods in Interventions section of the Table of Characteristics of } \\
\text { Included Studies }\end{array}$ \\
\hline $\begin{array}{l}\text { Free of systematic differ- } \\
\text { ence in care? }\end{array}$ & High risk & $\begin{array}{l}\text { Also fruit, veg, fish and meat advice. See Control and Intervention Aims in In- } \\
\text { terventions section of the Table of Characteristics of Included Studies }\end{array}$ \\
\hline $\begin{array}{l}\text { Free of dietary differences } \\
\text { other than fat? }\end{array}$ & High risk & \\
\hline
\end{tabular}

Due Low fat 2008

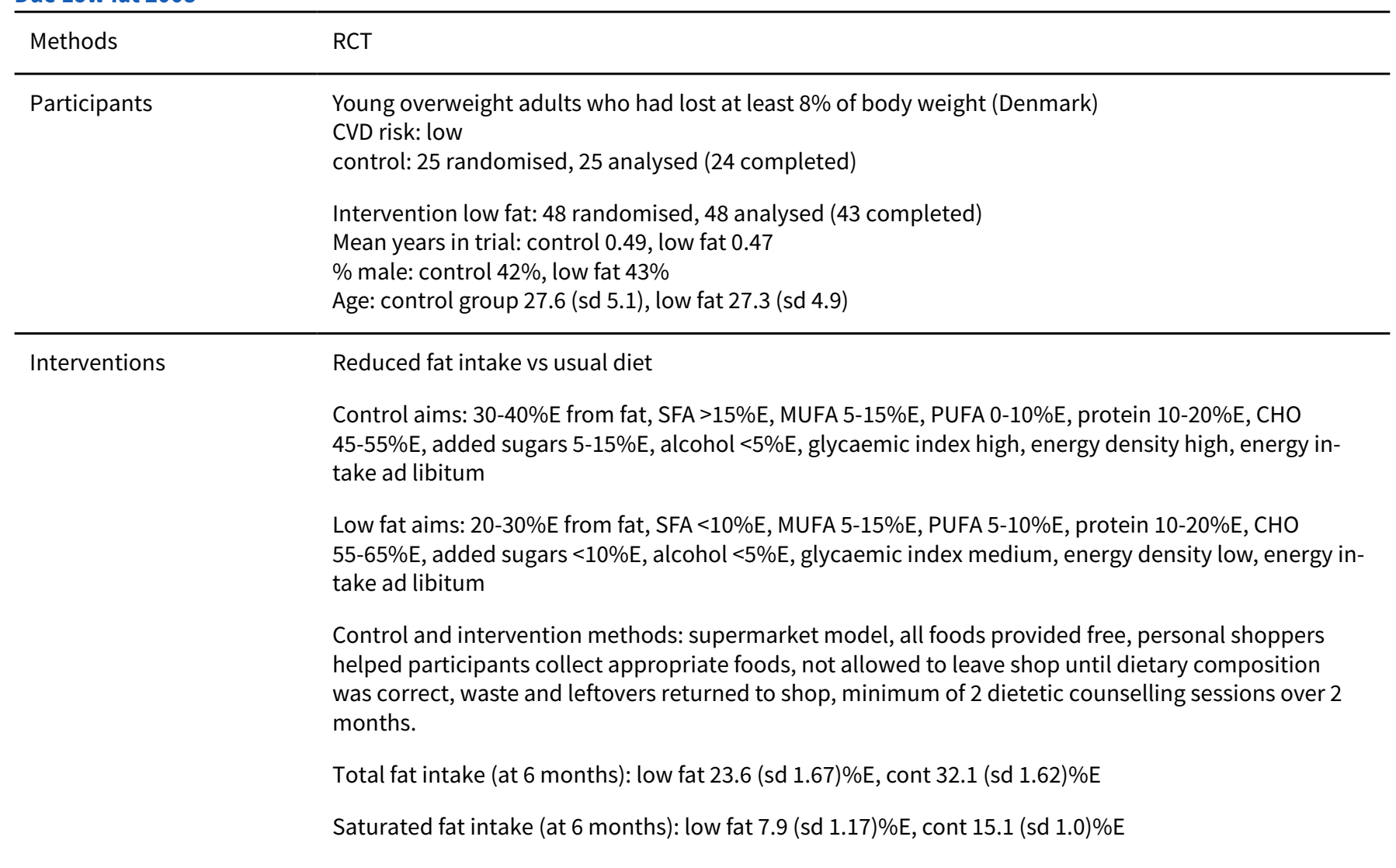


Due Low fat 2008 (Continued)

Style: food provided

Setting: community

Stated trial outcomes: CVD risk, diabetes risk, weight
Data available on total mortality? yes (no events)
Cardiovascular mortality? yes (no events)
Events available for combined cardiovascular events: total MI, stroke (no events)
Secondary outcomes: cancer deaths and diagnoses, total and non-fatal MI, stroke (no events in any
group)
Tertiary outcomes: weight, BMI, total, HDL, LDL cholesterol, TG

Notes

Risk of bias

\begin{tabular}{|c|c|c|}
\hline Bias & Authors' judgement & Support for judgement \\
\hline $\begin{array}{l}\text { Random sequence genera- } \\
\text { tion (selection bias) }\end{array}$ & Low risk & $\begin{array}{l}\text { Independently randomised by } 2 \text { study personnel, stratified by sex and initial } \\
\text { BMI }\end{array}$ \\
\hline $\begin{array}{l}\text { Allocation concealment } \\
\text { (selection bias) }\end{array}$ & Unclear risk & Randomisation method not clearly described \\
\hline $\begin{array}{l}\text { Blinding (performance } \\
\text { bias and detection bias) } \\
\text { All outcomes }\end{array}$ & High risk & $\begin{array}{l}\text { Participants were aware of their own allocated diet, those assessing outcomes } \\
\text { unclear }\end{array}$ \\
\hline $\begin{array}{l}\text { Incomplete outcome data } \\
\text { (attrition bias) } \\
\text { All outcomes }\end{array}$ & Unclear risk & Reasons for dropouts provided, ITT analysis used for continuous outcomes \\
\hline $\begin{array}{l}\text { Selective reporting (re- } \\
\text { porting bias) }\end{array}$ & Low risk & Not relevant for primary and secondary outcomes as all trialists asked for data \\
\hline Other bias & Low risk & \\
\hline $\begin{array}{l}\text { Free of systematic differ- } \\
\text { ence in care? }\end{array}$ & Low risk & Yes, advice and shop system was the same across all groups \\
\hline $\begin{array}{l}\text { Free of dietary differences } \\
\text { other than fat? }\end{array}$ & High risk & $\begin{array}{l}\text { No, there were also differences in sugary foods, legumes, dietary glycaemic in- } \\
\text { dex etc }\end{array}$ \\
\hline
\end{tabular}

\section{Due Low vs Mod 2008}

\begin{tabular}{|c|c|}
\hline Methods & $\mathrm{RCT}$ \\
\hline Participants & $\begin{array}{l}\text { Young overweight adults who had lost at least } 8 \% \text { of body weight (Denmark) } \\
\text { CVD risk: low } \\
\text { Intervention modified fat: } 52 \text { randomised, } 52 \text { analysed ( } 39 \text { completed) }\end{array}$ \\
\hline & $\begin{array}{l}\text { Intervention low fat: } 48 \text { randomised, } 48 \text { analysed ( } 43 \text { completed) } \\
\text { Mean years in trial: moderate fat } 0.44 \text {, low fat } 0.47 \\
\% \text { male: modified fat } 41 \% \text {, low fat } 43 \% \\
\text { Age: modified fat } 29.2 \text { (sd } 4.5 \text { ), low fat } 27.3 \text { (sd } 4.9)\end{array}$ \\
\hline
\end{tabular}


Due Low vs Mod 2008 (Continued)

Baseline total fat intake: int cont

Baseline saturated fat intake: int cont

Interventions

Reduced fat intake vs modified fat

Modified fat aims: $35-45 \% \mathrm{E}$ from fat, SFA $<10 \% \mathrm{E}$, MUFA $>20 \% \mathrm{E}$, PUFA $5-10 \% \mathrm{E}$, protein $10-20 \% \mathrm{E}$, CHO $40-50 \% \mathrm{E}$, added sugars $<10 \% \mathrm{E}$, alcohol $<5 \% \mathrm{E}$, glycaemic index low, energy density high, energy intake ad libitum

Low fat aims: $20-30 \% \mathrm{E}$ from fat, SFA $<10 \% \mathrm{E}$, MUFA 5-15\%E, PUFA 5-10\%E, protein $10-20 \% \mathrm{E}$, CHO $55-65 \% \mathrm{E}$, added sugars $<10 \% \mathrm{E}$, alcohol $<5 \% \mathrm{E}$, glycaemic index medium, energy density low, energy intake ad libitum

Control and intervention methods: supermarket model, all foods provided free, personal shoppers helped participants collect appropriate foods, not allowed to leave shop until dietary composition was correct, waste and leftovers returned to shop, minimum of 2 dietetic counselling sessions over 2 months.

Total fat intake (at 6 months): low fat 23.6 (sd 1.67)\%E, mod fat 38.4 (sd 1.75)\%E

Saturated fat intake (at 6 months): low fat 7.9 (sd 1.17)\%E, mod fat 7.1 (sd 0.80)\%E

Style: food provided

Setting: community

Outcomes

Stated trial outcomes: CVD risk, diabetes risk, weight

Data available on total mortality? yes (no events)

Cardiovascular mortality? yes (no events)

Events available for combined cardiovascular events: total MI, stroke (no events)

Secondary outcomes: cancer deaths and diagnoses, total and non-fatal MI, stroke (no events in any group)

Tertiary outcomes: weight, BMI, total, HDL, LDL cholesterol, TG

Notes

\section{Risk of bias}

\begin{tabular}{|c|c|c|}
\hline Bias & Authors' judgement & Support for judgement \\
\hline $\begin{array}{l}\text { Random sequence genera- } \\
\text { tion (selection bias) }\end{array}$ & Low risk & $\begin{array}{l}\text { Independently randomised by } 2 \text { study personnel, stratified by sex and initial } \\
\text { BMI }\end{array}$ \\
\hline $\begin{array}{l}\text { Allocation concealment } \\
\text { (selection bias) }\end{array}$ & Unclear risk & Randomisation method not clearly described \\
\hline $\begin{array}{l}\text { Blinding (performance } \\
\text { bias and detection bias) } \\
\text { All outcomes }\end{array}$ & High risk & $\begin{array}{l}\text { Participants were aware of their own allocated diet, those assessing outcomes } \\
\text { unclear }\end{array}$ \\
\hline $\begin{array}{l}\text { Incomplete outcome data } \\
\text { (attrition bias) } \\
\text { All outcomes }\end{array}$ & Unclear risk & Reasons for dropouts provided, ITT analysis used for continuous outcomes \\
\hline $\begin{array}{l}\text { Selective reporting (re- } \\
\text { porting bias) }\end{array}$ & Low risk & Not relevant for primary and secondary outcomes as all trialists asked for data \\
\hline Other bias & Low risk & \\
\hline
\end{tabular}


Due Low vs Mod 2008 (Continued)

Free of systematic differ- Low risk $\quad$ Yes, advice and shop system was the same across all groups ence in care?

Free of dietary differences High risk other than fat?

No, there were also differences in sugary foods, legumes, dietary glycaemic index etc

\section{Due Mod fat 2008}

\begin{tabular}{|c|c|}
\hline Methods & $\mathrm{RCT}$ \\
\hline \multirow[t]{4}{*}{ Participants } & $\begin{array}{l}\text { Young overweight adults who had lost at least } 8 \% \text { of body weight (Denmark) } \\
\text { CVD risk: low } \\
\text { control: } 25 \text { randomised, } 25 \text { analysed ( } 24 \text { completed) } \\
\text { Intervention modified fat: } 52 \text { randomised, } 52 \text { analysed ( } 39 \text { completed) }\end{array}$ \\
\hline & $\begin{array}{l}\text { Mean years in trial: control } 0.49 \text {, moderate fat } 0.44 \\
\% \text { male: control } 42 \% \text {, modified fat } 41 \% \\
\text { Age: control group } 27.6 \text { (sd 5.1), modified fat } 29.2(\mathrm{sd} 4.5)\end{array}$ \\
\hline & Baseline total fat intake: int cont \\
\hline & Baseline saturated fat intake: int cont \\
\hline
\end{tabular}

Control aims: $30-40 \%$ from fat, SFA $>15 \%$ E, MUFA 5-15\%E, PUFA 0-10\%E, protein $10-20 \%$ E, CHO $45-55 \% \mathrm{E}$, added sugars $5-15 \% \mathrm{E}$, alcohol $<5 \% \mathrm{E}$, glycaemic index high, energy density high, energy intake ad libitum

Modified fat aims: $35-45 \% \mathrm{E}$ from fat, SFA $<10 \% \mathrm{E}$, MUFA $>20 \% \mathrm{E}$, PUFA 5-10\%E, protein $10-20 \% \mathrm{E}$, CHO $40-50 \% \mathrm{E}$, added sugars $<10 \% \mathrm{E}$, alcohol $<5 \% \mathrm{E}$, glycaemic index low, energy density high, energy intake ad libitum

Control and intervention methods: supermarket model, all foods provided free, personal shoppers helped participants collect appropriate foods, not allowed to leave shop until dietary composition was correct, waste and leftovers returned to shop, minimum of 2 dietetic counselling sessions over 2 months.

Total fat intake (at 6 months): mod fat 38.4 (sd 1.75)\%E, cont 32.1 (sd 1.62)\%E

Saturated fat intake (at 6 months): mod fat 7.1 (sd 0.80)\%E, cont 15.1 (sd 1.0)\%E

Style: food provided

Setting: community

Outcomes

Stated trial outcomes: CVD risk, diabetes risk, weight

Data available on total mortality? yes (no events)

Cardiovascular mortality? yes (no events)

Events available for combined cardiovascular events: total MI, stroke (no events)

Secondary outcomes: cancer deaths and diagnoses, total and non-fatal MI, stroke (no events in any group)

Tertiary outcomes: weight, BMI, total, HDL, LDL cholesterol, TG

Notes

\section{Risk of bias}

Reduced or modified dietary fat for preventing cardiovascular disease (Review) 
Due Mod fat 2008 (Continued)

\begin{tabular}{|c|c|c|}
\hline Bias & Authors' judgement & Support for judgement \\
\hline $\begin{array}{l}\text { Random sequence genera- } \\
\text { tion (selection bias) }\end{array}$ & Low risk & $\begin{array}{l}\text { Independently randomised by } 2 \text { study personnel, stratified by sex and initial } \\
\text { BMI }\end{array}$ \\
\hline $\begin{array}{l}\text { Allocation concealment } \\
\text { (selection bias) }\end{array}$ & Unclear risk & Randomisation method not clearly described \\
\hline $\begin{array}{l}\text { Blinding (performance } \\
\text { bias and detection bias) } \\
\text { All outcomes }\end{array}$ & High risk & $\begin{array}{l}\text { Participants were aware of their own allocated diet, those assessing outcomes } \\
\text { unclear }\end{array}$ \\
\hline $\begin{array}{l}\text { Incomplete outcome data } \\
\text { (attrition bias) } \\
\text { All outcomes }\end{array}$ & Unclear risk & Reasons for dropouts provided, ITT analysis used for continuous outcomes \\
\hline $\begin{array}{l}\text { Selective reporting (re- } \\
\text { porting bias) }\end{array}$ & Low risk & Not relevant for primary and secondary outcomes as all trialists asked for data \\
\hline Other bias & Low risk & \\
\hline $\begin{array}{l}\text { Free of systematic differ- } \\
\text { ence in care? }\end{array}$ & Low risk & Yes, advice and shop system was the same across all groups \\
\hline $\begin{array}{l}\text { Free of dietary differences } \\
\text { other than fat? }\end{array}$ & High risk & $\begin{array}{l}\text { No, there were also differences in sugary foods, legumes, dietary glycaemic in- } \\
\text { dex etc }\end{array}$ \\
\hline
\end{tabular}

Dullaart 1992

\begin{tabular}{ll}
\hline Methods & RCT \\
\hline Participants & Type I diabetics with elevated urinary albumin (Netherlands) \\
& Risk: moderate \\
Control: randomised 20, analysed 20 \\
Intervention: randomised 18, analysed 16 \\
Mean years in trial: control 2.0, intervention 1.9 \\
\% male: control 75\%, intervention 81\% \\
Age: mean control 41 (sd 14), intervention 44 (sd 12) (all 21-65)
\end{tabular}

\section{Modified fat vs usual fat}

Control aims: usual diet (urged not to alter fat or protein intake)

Intervention aims: replace SFA by linoleic acid to achieve P/S of 1.0, total fat and protein intake to re-

main unchanged

Control methods: unclear

Intervention methods: counselling by a dietitian (unclear how often, but at least annually)

Total fat intake (at 2 years):mod fat 37 (sd 4), cont 40 (sd 7)\%E

Saturated fat intake (at 2 years): $\bmod$ fat 13 (sd 2), cont 16 (sd 3)\%E

Style: diet advice

Setting: community

Outcomes Stated trial outcomes: albuminuria and serum lipoproteins


Dullaart 1992 (Continued)

Data available on total mortality? yes (no events)

Cardiovascular mortality? yes (no events)

Events available for combined cardiovascular events: cardiovascular deaths, non-fatal MI, stroke (no events)

Secondary outcomes: stroke (no events), MI (no events), cancer deaths (no events)

Tertiary outcomes: weight, total, HDL and LDL cholesterol (data read off graph as data files no longer exist, total and LDL cholesterol data not used as very different in control and intervention arms at baseline)

Notes

\section{Risk of bias}

\begin{tabular}{lll}
\hline Bias & Authors' judgement & Support for judgement \\
\hline $\begin{array}{l}\text { Random sequence genera- } \\
\text { tion (selection bias) }\end{array}$ & Low risk & $\begin{array}{l}\text { "stratified according to gender, randomised in blocks of } 6 \text { using opaque sealed } \\
\text { envelopes" by independent statistical investigator with no contact with partic- } \\
\text { ipants }\end{array}$ \\
\hline
\end{tabular}

Allocation concealment Low risk
(selection bias)

Blinding (performance High risk

bias and detection bias)

All outcomes envelopes" by independent statistical investigator with no contact with partic-

\begin{tabular}{|c|c|c|}
\hline $\begin{array}{l}\text { Incomplete outcome data } \\
\text { (attrition bias) } \\
\text { All outcomes }\end{array}$ & Unclear risk & $\begin{array}{l}\text { Unclear, deaths, cancer and CV events are drop-outs, trialists asked for data - } \\
\text { unclear if any data missing }\end{array}$ \\
\hline
\end{tabular}

\begin{tabular}{|c|c|c|}
\hline $\begin{array}{l}\text { Selective reporting (re- } \\
\text { porting bias) }\end{array}$ & Low risk & Not relevant for primary and secondary outcomes as all trialists asked for data \\
\hline Other bias & Low risk & \\
\hline $\begin{array}{l}\text { Free of systematic differ- } \\
\text { ence in care? }\end{array}$ & Unclear risk & $\begin{array}{l}\text { Probably not, appears that the intervention group had more time with dietit- } \\
\text { ian. See Control and Intervention Methods in Interventions section of the Ta- } \\
\text { ble of Characteristics of Included Studies }\end{array}$ \\
\hline $\begin{array}{l}\text { Free of dietary differences } \\
\text { other than fat? }\end{array}$ & Low risk & $\begin{array}{l}\text { See Control and Intervention Aims in Interventions section of the Table of } \\
\text { Characteristics of Included Studies }\end{array}$ \\
\hline
\end{tabular}

\section{Frenkiel 1986}

\begin{tabular}{ll}
\hline Methods & RCT \\
\hline Participants & People with radiolucent gallstones taking ursodeoxycholic acid, UDCA (USA) \\
& CVD risk: low \\
& Control: randomised 17, analysed 16 \\
& Intervention: randomised 19, analysed 16 \\
& Mean years in trial: control 0.6, intervention 0.6 \\
& $\%$ male: control 35\%, intervention 58\% \\
& Age: mean control 52.4, intervention 53.1 \\
\hline Interventions & Modified fat vs average diet
\end{tabular}


Frenkiel 1986 (Continued)

Control aims: dietary advice for total fat $38-42 \% \mathrm{E}$, dietary cholesterol $500 \mathrm{mg} /$ day, protein $18-22 \% \mathrm{E}$,

CHO 38-42\%E, weight maintaining, low fibre

Intervention aims: as above but limit dietary cholesterol to $250 \mathrm{mg}$ /day, weight maintaining, low fibre

Control methods: dietary advice from dietitian every 3 months

Intervention methods: dietary advice from dietitian every 3 months

Total fat intake (at 6 or 9 months): mod fat 32.5 (sd 6.2), cont 36.0 (sd 5.1)\%E

Saturated fat intake: unclear

Style: diet advice

Setting: community

$\begin{array}{ll}\text { Outcomes } & \text { Stated trial outcomes: bile acid kinetics } \\ \text { Data available on total mortality? yes (no events) } \\ \text { Cardiovascular mortality? no } \\ \text { Events available for combined cardiovascular events: none } \\ \text { Secondary outcomes: none } \\ \text { Tertiary outcomes: none }\end{array}$

Notes

\section{Risk of bias}

\begin{tabular}{|c|c|c|}
\hline Bias & Authors' judgement & Support for judgement \\
\hline $\begin{array}{l}\text { Random sequence genera- } \\
\text { tion (selection bias) }\end{array}$ & Low risk & "randomly allocated" \\
\hline $\begin{array}{l}\text { Allocation concealment } \\
\text { (selection bias) }\end{array}$ & Unclear risk & Randomisation method not clearly described \\
\hline $\begin{array}{l}\text { Blinding (performance } \\
\text { bias and detection bias) } \\
\text { All outcomes }\end{array}$ & High risk & Participants knew their allocation, unclear for researchers \\
\hline $\begin{array}{l}\text { Incomplete outcome data } \\
\text { (attrition bias) } \\
\text { All outcomes }\end{array}$ & Unclear risk & $\begin{array}{l}\text { Unclear, deaths, cancer and CV events are drop-outs, trialists asked for data - } \\
\text { unclear if any data missing }\end{array}$ \\
\hline $\begin{array}{l}\text { Selective reporting (re- } \\
\text { porting bias) }\end{array}$ & Low risk & Not relevant for primary and secondary outcomes as all trialists asked for data \\
\hline Other bias & Low risk & \\
\hline $\begin{array}{l}\text { Free of systematic differ- } \\
\text { ence in care? }\end{array}$ & Low risk & $\begin{array}{l}\text { Similar level and duration of advice. See Control and Intervention Methods in } \\
\text { Interventions section of the Table of Characteristics of Included Studies }\end{array}$ \\
\hline $\begin{array}{l}\text { Free of dietary differences } \\
\text { other than fat? }\end{array}$ & Unclear risk & $\begin{array}{l}\text { Intervention unclear, aim was to maintain total fat intake in both groups but } \\
\text { reduce cholesterol intake - so fat modification must have occurred. See Con- } \\
\text { trol and Intervention Aims in Interventions section of the Table of Characteris- } \\
\text { tics of Included Studies }\end{array}$ \\
\hline
\end{tabular}


Houtsmuller 1979

\begin{tabular}{|c|c|}
\hline Methods & $\mathrm{RCT}$ \\
\hline \multirow[t]{5}{*}{ Participants } & $\begin{array}{l}\text { Adults with newly diagnosed diabetes (The Netherlands) } \\
\text { CVD risk: moderate }\end{array}$ \\
\hline & $\begin{array}{l}\text { Control: } 51 \text { randomised, unclear how many analysed (all analysed re deaths) } \\
\text { Intervention: } 51 \text { randomised, unclear how many analysed (all re deaths) }\end{array}$ \\
\hline & $\begin{array}{l}\text { Mean years in trial: unclear (max duration } 6 \text { years) } \\
\% \text { male: } 56 \% \text { overall } \\
\text { Age: mean unclear }\end{array}$ \\
\hline & Baseline total fat intake: int cont \\
\hline & Baseline saturated fat intake: int cont \\
\hline \multirow[t]{9}{*}{ Interventions } & Modified fat vs usual diet \\
\hline & Control aims: SFA 35\%E, CHO 50\%E, protein 15\%E \\
\hline & Intervention aims: total fat $40 \% \mathrm{E}, 1 / 3$ linoleic acid, $\mathrm{CHO} 45 \% \mathrm{E}$, protein $15 \% \mathrm{E}$ \\
\hline & Control methods: unclear, surveyed by dietitian \\
\hline & Intervention methods: unclear, surveyed by dietitian \\
\hline & Total fat intake: mod fat unclear, cont unclear \\
\hline & Saturated fat intake: mod fat unclear, cont unclear \\
\hline & Style: diet advice? \\
\hline & Setting: community \\
\hline \multirow[t]{6}{*}{ Outcomes } & Stated trial outcomes: progression of diabetic retinopathy \\
\hline & Data available on total mortality? no \\
\hline & Cardiovascular mortality? no \\
\hline & Events available for combined cardiovascular events: total MI and angina \\
\hline & Secondary outcomes: none \\
\hline & Tertiary outcomes: total cholesterol, TGs (data read off graph) \\
\hline
\end{tabular}

Notes

\section{Risk of bias}

\begin{tabular}{lll}
\hline Bias & Authors' judgement & Support for judgement \\
\hline $\begin{array}{l}\text { Random sequence genera- } \\
\text { tion (selection bias) }\end{array}$ & Low risk & Participants matched in pairs then randomised \\
\hline $\begin{array}{l}\text { Allocation concealment } \\
\text { (selection bias) }\end{array}$ & Unclear risk & Randomisation method not clearly described \\
\hline $\begin{array}{l}\text { Blinding (performance } \\
\text { bias and detection bias) }\end{array}$ & High risk & Neither participants nor physicians appear blinded \\
All outcomes & \\
\hline $\begin{array}{l}\text { Incomplete outcome data } \\
\text { (attrition bias) }\end{array}$ & Unclear risk & $\begin{array}{l}\text { Unclear, deaths, cancer and CV events are drop-outs, trialists asked for data - } \\
\text { All outcomes }\end{array}$ \\
\hline
\end{tabular}


Houtsmuller 1979 (Continued)

Selective reporting (re- Low risk Not relevant for primary and secondary outcomes as all trialists asked for data porting bias)

\begin{tabular}{lll}
\hline Other bias & Low risk & \\
\hline $\begin{array}{l}\text { Free of systematic differ- } \\
\text { ence in care? }\end{array}$ & Unclear risk & $\begin{array}{l}\text { Level and type of intervention unclear. See Control and Intervention Methods } \\
\text { in Interventions section of the Table of Characteristics of Included Studies }\end{array}$ \\
\hline $\begin{array}{l}\text { Free of dietary differences } \\
\text { other than fat? }\end{array}$ & Low risk & $\begin{array}{l}\text { State that fibre and dietary cholesterol were similar in control and interven- } \\
\text { tion. See Control and Intervention Aims in Interventions section of the Table of } \\
\text { Characteristics of Included Studies }\end{array}$ \\
\hline
\end{tabular}

Lean 1997

\begin{tabular}{|c|c|}
\hline Methods & RCT \\
\hline Participants & $\begin{array}{l}\text { Healthy women, } \mathrm{BMI}>25 \text { (UK) } \\
\text { CVD risk: low } \\
\text { Control: randomised } 53 \text {, analysed } 40 \\
\text { Intervention: randomised } 57 \text {, analysed } 42 \\
\text { Mean years in trial: control } 0.42 \text {, intervention } 0.43 \\
\% \text { male: } 0 \\
\text { Age: mean control } 50 \text {, intervention } 51\end{array}$ \\
\hline Interventions & $\begin{array}{l}\text { Reduced fat vs usual diet } \\
\text { Control aims: advice - total fat 35\%E, CHO 34.5\%E, 1200kcal per day encouraged but not prescribed } \\
\text { Intervention aims: total fat 20\%E, CHO 58\%E, 1200kcal/day encouraged but not prescribed } \\
\text { Control methods: dietary advice supported by exchanges and recipes } \\
\text { Intervention methods: dietary advice supported by exchanges and recipes } \\
\text { Total fat intake: unclear } \\
\text { Saturated fat intake: unclear } \\
\text { Style: diet advice } \\
\text { Setting: community }\end{array}$ \\
\hline Outcomes & $\begin{array}{l}\text { Stated trial outcomes: weight loss and cardiovascular risk factors } \\
\text { Data available on total mortality? yes (no events) } \\
\text { Cardiovascular mortality? yes (no events) } \\
\text { Events available for combined cardiovascular events: cardiovascular deaths, non fatal MI, stroke (no } \\
\text { events) } \\
\text { Secondary outcomes: total and non-fatal MI, stroke, cancer deaths (no events) } \\
\text { Tertiary outcomes: BMI, total, LDL and HDL cholesterol, TG, BP }\end{array}$ \\
\hline
\end{tabular}

Notes

\section{Risk of bias}

Bias

Authors' judgement Support for judgement 
Lean 1997 (Continued)

Random sequence genera- Low risk " "medical officer drew coloured straws from a box" tion (selection bias)

Allocation concealment $\quad$ Unclear risk
(selection bias)

(selection bias)

Blinding (performance High risk Participants were not blinded, unclear about researchers
bias and detection bias)
All outcomes

\begin{tabular}{lll}
\hline $\begin{array}{l}\text { Incomplete outcome data } \\
\text { (attrition bias) } \\
\text { All outcomes }\end{array}$ & Unclear risk & Deaths, cancer and CV events are drop-outs, trialists asked for data \\
\hline $\begin{array}{l}\text { Selective reporting (re- } \\
\text { porting bias) }\end{array}$ & Low risk & Not relevant for primary and secondary outcomes as all trialists asked for data \\
\hline Other bias & Low risk & Low risk \\
\hline $\begin{array}{l}\text { Free of systematic differ- } \\
\text { ence in care? }\end{array}$ & $\begin{array}{l}\text { Similar intervention in both groups. See Control and Intervention Methods in } \\
\text { Interventions section of the Table of Characteristics of Included Studies }\end{array}$ \\
\hline $\begin{array}{l}\text { Free of dietary differences } \\
\text { other than fat? }\end{array}$ & Low risk & $\begin{array}{l}\text { See Control and Intervention Aims in Interventions section of the Table of } \\
\text { Characteristics of Included Studies }\end{array}$ \\
\hline
\end{tabular}

Ley 2004

\begin{tabular}{|c|c|}
\hline Methods & $\mathrm{RCT}$ \\
\hline Participants & $\begin{array}{l}\text { People with impaired glucose intolerance or high normal blood glucose (New Zealand) } \\
\text { CVD risk: moderate } \\
\text { Control: unclear how many randomised ( } 176 \text { between both groups), unclear how many analysed (112 } \\
\text { between both groups at } 5 \text { years) } \\
\text { Intervention: as above } \\
\text { Mean years in trial: } 4.1 \text { over whole trial } \\
\% \text { male: control } 80 \% \text {, intervention } 68 \% \\
\text { Age: mean control } 52.0 \text { (SE } 0.8 \text { ), intervention } 52.5 \text { (SE } 0.8 \text { ) }\end{array}$ \\
\hline \multirow[t]{8}{*}{ Interventions } & Reduced fat vs usual diet \\
\hline & $\begin{array}{l}\text { Control aims: usual diet } \\
\text { Intervention aims: reduced fat diet (no specific goal stated) }\end{array}$ \\
\hline & Control methods: usual intake \\
\hline & $\begin{array}{l}\text { Intervention methods: monthly meetings to follow a } 1 \text { year structured programme aimed at reducing } \\
\text { fat in the diet, includes education, personal goal setting, self-monitoring }\end{array}$ \\
\hline & Total fat intake (at 1 year): low fat 26.1 (sd 7.7), cont 33.6 (sd 7.8)\%E \\
\hline & Saturated fat intake (at 1 year): low fat 10.0 (sd 4.2), cont 13.4 (sd 4.7)\%E \\
\hline & Style: diet advice \\
\hline & Setting: community \\
\hline Outcomes & $\begin{array}{l}\text { Stated trial outcomes: lipids, glucose, blood pressure } \\
\text { Data available on total mortality? yes }\end{array}$ \\
\hline
\end{tabular}


Ley 2004 (Continued)

Cardiovascular mortality? yes

Events available for combined cardiovascular events: MI, angina, stroke, heart failure

Secondary outcomes: total MI, stroke, cancer diagnoses, cancer deaths

Tertiary outcomes: weight, total, LDL and HDL cholesterol, TGs, BP

Notes

\section{Risk of bias}

\begin{tabular}{lll}
\hline Bias & Authors' judgement & Support for judgement \\
\hline $\begin{array}{l}\text { Random sequence genera- } \\
\text { tion (selection bias) }\end{array}$ & Low risk & $\begin{array}{l}\text { Unmarked opaque envelopes were opened by the person recruiting, unable to } \\
\text { alter allocation later }\end{array}$ \\
\hline
\end{tabular}

\begin{tabular}{|c|c|c|}
\hline $\begin{array}{l}\text { Allocation concealment } \\
\text { (selection bias) }\end{array}$ & Low risk & \\
\hline $\begin{array}{l}\text { Blinding (performance } \\
\text { bias and detection bias) } \\
\text { All outcomes }\end{array}$ & High risk & Participants were not blinded, outcome assessors were \\
\hline $\begin{array}{l}\text { Incomplete outcome data } \\
\text { (attrition bias) } \\
\text { All outcomes }\end{array}$ & Unclear risk & $\begin{array}{l}\text { Unclear, deaths, cancer and CV events are drop-outs, trialists asked for data - } \\
\text { unclear if any data missing }\end{array}$ \\
\hline $\begin{array}{l}\text { Selective reporting (re- } \\
\text { porting bias) }\end{array}$ & Low risk & Not relevant for primary and secondary outcomes as all trialists asked for data \\
\hline Other bias & Low risk & \\
\hline $\begin{array}{l}\text { Free of systematic differ- } \\
\text { ence in care? }\end{array}$ & High risk & $\begin{array}{l}\text { See Control and Intervention Methods in Interventions section of the Table of } \\
\text { Characteristics of Included Studies }\end{array}$ \\
\hline $\begin{array}{l}\text { Free of dietary differences } \\
\text { other than fat? }\end{array}$ & Low risk & $\begin{array}{l}\text { See Control and Intervention Aims in Interventions section of the Table of } \\
\text { Characteristics of Included Studies }\end{array}$ \\
\hline
\end{tabular}

McAuley 2005

\begin{tabular}{|c|c|}
\hline Methods & $\mathrm{RCT}$ \\
\hline Participants & $\begin{array}{l}\text { Overweight and insulin-resistant women (New Zealand) } \\
\text { CVD risk: low } \\
\text { Low fat: randomised } 32 \text {, analysed } 24 \text { (at } 1 \text { year) } \\
\text { Modified fat: randomised } 30 \text {, analysed } 28 \text { (at } 1 \text { year) } \\
\text { Mean years in trial: low fat } 0.88, \text { modified fat } 0.97 \\
\% \text { male: } 0 \\
\text { Age: mean low fat } 45 \text { (sd } 7.5 \text { ), modified fat } 47 \text { (sd } 7.9)\end{array}$ \\
\hline Interventions & $\begin{array}{l}\text { Reduced fat vs Modified fat diet } \\
\text { Modified fat aims: } 30 \% \mathrm{E} \text { from fat, predominantly MUFA, protein } 30 \% \mathrm{E}, \mathrm{CHO} \text { low glycaemic index } 40 \% \mathrm{E} \text {, } \\
5 \text { meals/d, less than } 5 \text { hours between meals, ad libitum consumption, } 30 \text { mins activity } 5 \text { days/week ad- } \\
\text { vised } \\
\text { Low fat aims: total fat }<30 \% \mathrm{E} \text {, SFA }<8 \% \text {, protein } 15 \% \mathrm{E}, \mathrm{CHO}>55 \% \mathrm{E} \text {, advised to reduce dietary fat, salt } \\
\text { and sugar, national healthy eating guidelines plus at least } 6 \text { servings of bread and whole-grains } / \mathrm{d} \text {, at }\end{array}$ \\
\hline
\end{tabular}


McAuley 2005 (Continued)

least 3 of vegetables, 2 of fruit, 2 of low fat dairy/d, ad libitum consumption, 30mins activity 5 days/ week advised

(Also a high fat Atkins-type arm)

Low fat methods: unclear how much dietary advice, or who delivered

Modified fat methods: unclear how much dietary advice or who delivered it

Total fat intake (at 6 months): low fat 28 (sd 7)\%E, mod fat 35 (sd 7)\%E

Saturated fat intake (at 6 months): low fat $10(\mathrm{sd} 4) \% \mathrm{E}$, mod fat 11 (sd 3)\%E

Style: diet advice

Setting: community

Stated trial outcomes: weight loss, lipids
Data available on total mortality? yes (none)
Cardiovascular mortality? yes (none)
Events available for combined cardiovascular events: total MI, stroke (no CVD events)
Secondary outcomes: non-fatal and total MI, stroke, cancer deaths and diagnoses (no events for any
outcome)
Tertiary outcomes: weight, BMI, total, LDL and HDL cholesterol, TG, systolic and diastolic BP

Notes

\section{Risk of bias}

\begin{tabular}{|c|c|c|}
\hline Bias & Authors' judgement & Support for judgement \\
\hline $\begin{array}{l}\text { Random sequence genera- } \\
\text { tion (selection bias) }\end{array}$ & Low risk & 'randomised' \\
\hline $\begin{array}{l}\text { Allocation concealment } \\
\text { (selection bias) }\end{array}$ & Unclear risk & Randomisation method not clearly described \\
\hline $\begin{array}{l}\text { Blinding (performance } \\
\text { bias and detection bias) } \\
\text { All outcomes }\end{array}$ & High risk & Participants knew allocation, unclear whether researchers did also \\
\hline $\begin{array}{l}\text { Incomplete outcome data } \\
\text { (attrition bias) } \\
\text { All outcomes }\end{array}$ & Unclear risk & $\begin{array}{l}\text { Unclear, deaths, cancer and CV events are drop-outs, trialists asked for data - } \\
\text { unclear if any data missing }\end{array}$ \\
\hline $\begin{array}{l}\text { Selective reporting (re- } \\
\text { porting bias) }\end{array}$ & Low risk & Not relevant for primary and secondary outcomes as all trialists asked for data \\
\hline Other bias & Low risk & \\
\hline $\begin{array}{l}\text { Free of systematic differ- } \\
\text { ence in care? }\end{array}$ & Unclear risk & $\begin{array}{l}\text { Probably, as both groups were taught their diets. See Control and Intervention } \\
\text { Methods in Interventions section of the Table of Characteristics of Included } \\
\text { Studies }\end{array}$ \\
\hline $\begin{array}{l}\text { Free of dietary differences } \\
\text { other than fat? }\end{array}$ & High risk & Differences in meal frequency, salt and sugar advice. \\
\hline
\end{tabular}


McKeown-Eyssen 1994

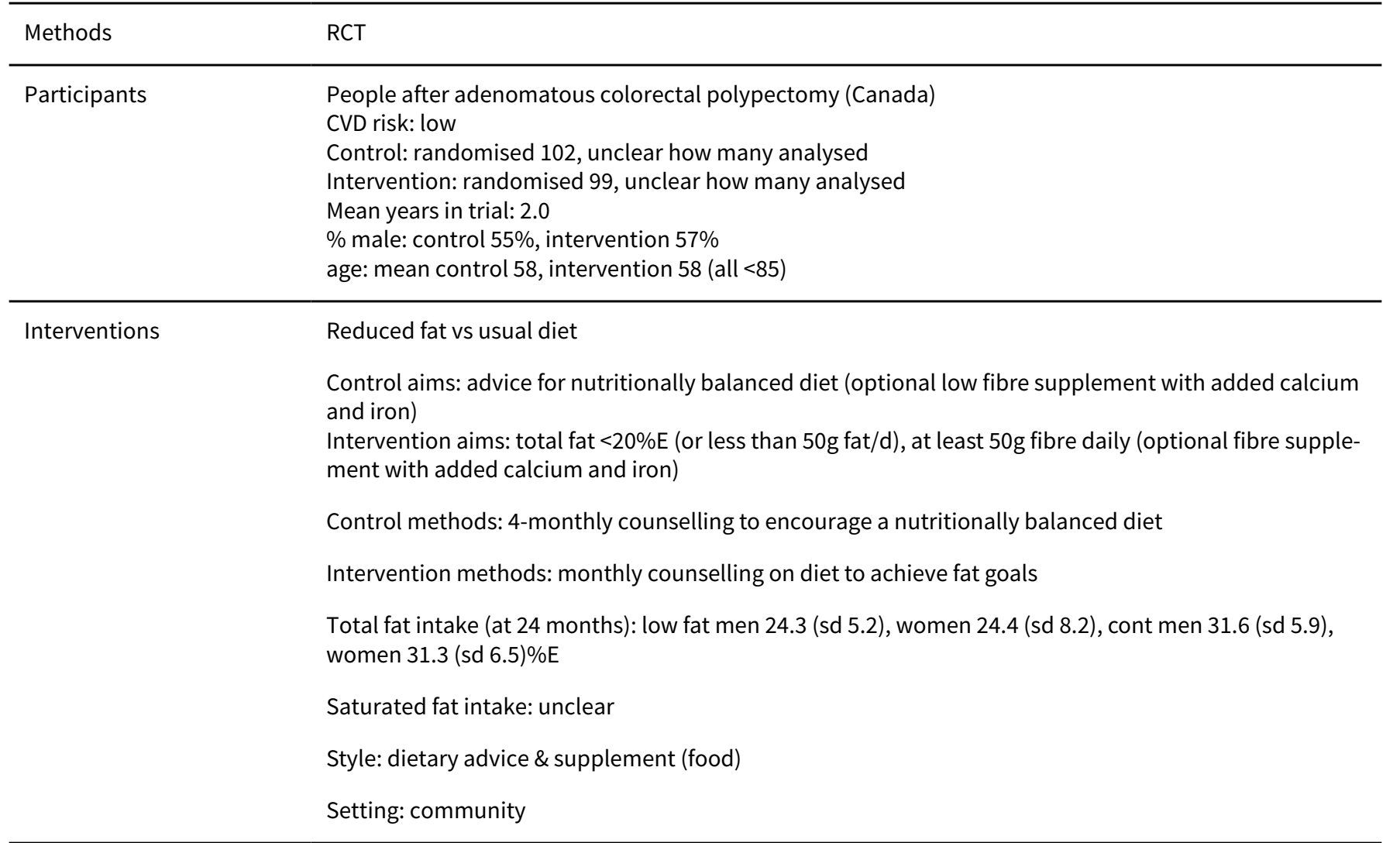

\section{Outcomes}

Stated trial outcomes: recurrence of neoplastic polyps

Data available on total mortality? yes (no events)

Cardiovascular mortality? yes (no events)

Events available for combined cardiovascular events: none

Secondary outcomes: cancer diagnoses, cancer deaths (no deaths)

Tertiary outcomes: total cholesterol (but no variance data presented and graph too small to read)

\section{Notes}

\section{Risk of bias}

\begin{tabular}{|c|c|c|}
\hline Bias & Authors' judgement & Support for judgement \\
\hline $\begin{array}{l}\text { Random sequence genera- } \\
\text { tion (selection bias) }\end{array}$ & Low risk & \\
\hline $\begin{array}{l}\text { Allocation concealment } \\
\text { (selection bias) }\end{array}$ & Low risk & $\begin{array}{l}\text { "stratification by physician, gender, age, randomisation by research associate, } \\
\text { centrally, using random numbers generated by computer" }\end{array}$ \\
\hline $\begin{array}{l}\text { Blinding (performance } \\
\text { bias and detection bias) } \\
\text { All outcomes }\end{array}$ & High risk & Participants not blinded, physicians were \\
\hline $\begin{array}{l}\text { Incomplete outcome data } \\
\text { (attrition bias) } \\
\text { All outcomes }\end{array}$ & Unclear risk & $\begin{array}{l}\text { Unclear, deaths, cancer and CV events are drop-outs - unclear if any data miss- } \\
\text { ing. ITT analysis performed. }\end{array}$ \\
\hline
\end{tabular}


McKeown-Eyssen 1994 (Continued)

Selective reporting (re- Low risk Not relevant for primary and secondary outcomes as all trialists asked for data
porting bias)

\begin{tabular}{lll}
\hline Other bias & Low risk & \\
\hline $\begin{array}{l}\text { Free of systematic differ- } \\
\text { ence in care? }\end{array}$ & High risk & $\begin{array}{l}\text { More frequent visits for intervention group. See Control and Intervention } \\
\text { Methods in Interventions section of the Table of Characteristics of Included } \\
\text { Studies }\end{array}$ \\
\hline $\begin{array}{l}\text { Free of dietary differences } \\
\text { other than fat? }\end{array}$ & High risk & Dietary fibre advice also in intervention group \\
\hline
\end{tabular}

\section{MeDiet 2002}

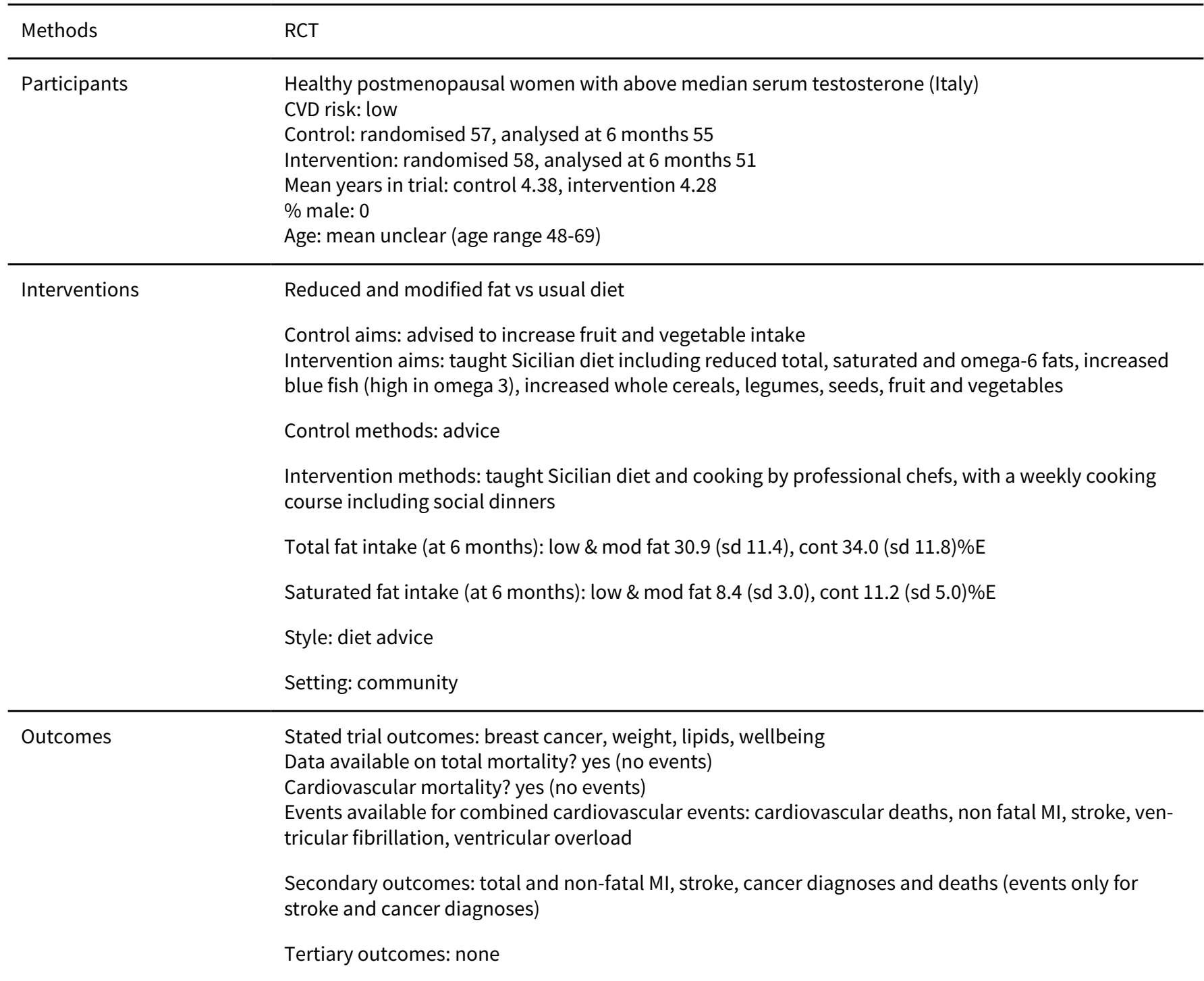

Notes

\section{Risk of bias}


MeDiet 2002 (Continued)

\begin{tabular}{|c|c|c|}
\hline Bias & Authors' judgement & Support for judgement \\
\hline $\begin{array}{l}\text { Random sequence genera- } \\
\text { tion (selection bias) }\end{array}$ & Low risk & "individually randomised" \\
\hline $\begin{array}{l}\text { Allocation concealment } \\
\text { (selection bias) }\end{array}$ & Unclear risk & Randomisation method not clearly described \\
\hline $\begin{array}{l}\text { Blinding (performance } \\
\text { bias and detection bias) } \\
\text { All outcomes }\end{array}$ & High risk & Participants were aware of assignment, researchers unclear \\
\hline $\begin{array}{l}\text { Incomplete outcome data } \\
\text { (attrition bias) } \\
\text { All outcomes }\end{array}$ & Unclear risk & $\begin{array}{l}\text { Unclear, deaths, cancer and CV events are drop-outs, trialists asked for data - } \\
\text { unclear if any data missing }\end{array}$ \\
\hline $\begin{array}{l}\text { Selective reporting (re- } \\
\text { porting bias) }\end{array}$ & Low risk & Not relevant for primary and secondary outcomes as all trialists asked for data \\
\hline Other bias & Low risk & \\
\hline $\begin{array}{l}\text { Free of systematic differ- } \\
\text { ence in care? }\end{array}$ & High risk & $\begin{array}{l}\text { Intensive cookery course with social element compared with brief advice. } \\
\text { See Control and Intervention Methods in Interventions section of the Table of } \\
\text { Characteristics of Included Studies }\end{array}$ \\
\hline $\begin{array}{l}\text { Free of dietary differences } \\
\text { other than fat? }\end{array}$ & High risk & $\begin{array}{l}\text { Both groups encouraged to increase fruit and vegetables, but intervention } \\
\text { group also encouraged to increase fish, pulses, seeds, whole grains. }\end{array}$ \\
\hline
\end{tabular}

Minnesota Coron men 1989

\begin{tabular}{ll}
\hline Methods & RCT \\
\hline Participants & Institutionalised men living in a mental hospital (USA) \\
CVD risk: low & Control: randomised 2196, analysed unclear \\
Intervention: randomised 2197, analysed unclear & Mean years in trial: control 1.0, intervention 1.1 \\
& \% male: 100 \\
& Age: unclear, ranges from <30 to >70 \\
& Modified fat diet vs. usual diet \\
& Control aims: usual institutional diet provided \\
Intervention aims: institutional diet modified to total fat 45\%E, PUFA 18-20\%E, P/S 2.5, less than & 150mg/day dietary chol \\
Interventions & Control methods: whole diet provided \\
& Intervention methods: whole diet provided \\
& Total fat intake (over 4 years): mod fat 37.8 (sd unclear)\%E, cont 39.1 (sd unclear)\%E \\
& Saturated fat intake (over 4 years): mod fat 9.2 (sd unclear)\%E, cont 18.3 (sd unclear)\%E \\
& Style: diet provided \\
& Setting: residential institution \\
\hline
\end{tabular}


Minnesota Coron men 1989 (Continued)

Outcomes Stated trial outcomes: MI, mortality, sudden deaths

Data available on total mortality? yes

Cardiovascular mortality? yes

Events available for combined cardiovascular events: total MI plus sudden death plus stroke

Secondary outcomes: stroke, cancer deaths, total MI

Tertiary outcomes: none (data provided on total cholesterol and TGs but no variance info)

\begin{tabular}{ll}
\hline Notes & This was a 4.5 year institutional study, but as turnover of participants was very high average time in tri- \\
al per participant was actually around one year. Participants were replaced as they left, and often left \\
the institution and later returned.
\end{tabular}

Risk of bias

\begin{tabular}{|c|c|c|}
\hline Bias & Authors' judgement & Support for judgement \\
\hline $\begin{array}{l}\text { Random sequence genera- } \\
\text { tion (selection bias) }\end{array}$ & Low risk & "stratified randomisation" \\
\hline $\begin{array}{l}\text { Allocation concealment } \\
\text { (selection bias) }\end{array}$ & Unclear risk & Randomisation method not clearly described \\
\hline $\begin{array}{l}\text { Blinding (performance } \\
\text { bias and detection bias) } \\
\text { All outcomes }\end{array}$ & Low risk & $\begin{array}{l}\text { Physician blinding: adequate } \\
\text { Participant blinding: adequate }\end{array}$ \\
\hline $\begin{array}{l}\text { Incomplete outcome data } \\
\text { (attrition bias) } \\
\text { All outcomes }\end{array}$ & High risk & No, participants appear to have been lost on leaving the institution \\
\hline $\begin{array}{l}\text { Selective reporting (re- } \\
\text { porting bias) }\end{array}$ & Low risk & Not relevant for primary and secondary outcomes as all trialists asked for data \\
\hline Other bias & Low risk & \\
\hline $\begin{array}{l}\text { Free of systematic differ- } \\
\text { ence in care? }\end{array}$ & Low risk & $\begin{array}{l}\text { Whole diet provided for both groups. See Control and Intervention Methods in } \\
\text { Interventions section of the Table of Characteristics of Included Studies }\end{array}$ \\
\hline $\begin{array}{l}\text { Free of dietary differences } \\
\text { other than fat? }\end{array}$ & Low risk & $\begin{array}{l}\text { See Control and Intervention Aims in Interventions section of the Table of } \\
\text { Characteristics of Included Studies }\end{array}$ \\
\hline
\end{tabular}

Minnesota Coron women1989

\begin{tabular}{ll}
\hline Methods & \\
\hline Participants & Institutionalised women living in a mental hospit \\
& CVD risk: low \\
& Control: randomised 2320, analysed unclear \\
& Intervention: randomised 2344, analysed unclear \\
& Mean years in trial: control 1.0, intervention 1.1 \\
& \% male: 0 \\
& Age: unclear, ranges from $<30$ to $>70$ \\
Interventions & Modified fat diet vs. usual diet \\
& Control aims: usual institutional diet provided
\end{tabular}


Minnesota Coron women1989 (Continued)

Intervention aims: institutional diet modified to total fat 45\%E, PUFA $18-20 \% \mathrm{E}, \mathrm{P} / \mathrm{S} 2.5$, less than

$150 \mathrm{mg} /$ day dietary chol

Control methods: whole diet provided

Intervention methods: whole diet provided

Total fat intake (over 4 years): mod fat 37.8 (sd unclear)\%E, cont 39.1 (sd unclear)\%E

Saturated fat intake (over 4 years): mod fat 9.2 (sd unclear)\%E, cont 18.3 (sd unclear)\%E

Style: diet provided

Setting: residential institution

\begin{tabular}{ll}
\hline Outcomes & Stated trial outcomes: MI, mortality, sudden deaths \\
Data available on total mortality? yes \\
Cardiovascular mortality? yes \\
Events available for combined cardiovascular events: total MI plus sudden death plus stroke \\
Secondary outcomes: stroke, cancer deaths, total MI \\
Tertiary outcomes: none (data provided on total cholesterol and TGs but no variance info) \\
\hline Notes \\
$\begin{array}{l}\text { This was a } 4.5 \text { year institutional study, but as turnover of participants was very high average time in tri- } \\
\text { al per participant was actually around one year. Participants were replaced as they left, and often left }\end{array}$ \\
\hline
\end{tabular}

\section{Risk of bias}

\begin{tabular}{|c|c|c|}
\hline Bias & Authors' judgement & Support for judgement \\
\hline $\begin{array}{l}\text { Random sequence genera- } \\
\text { tion (selection bias) }\end{array}$ & Low risk & "stratified randomisation" \\
\hline $\begin{array}{l}\text { Allocation concealment } \\
\text { (selection bias) }\end{array}$ & Unclear risk & Randomisation method not clearly described \\
\hline $\begin{array}{l}\text { Blinding (performance } \\
\text { bias and detection bias) } \\
\text { All outcomes }\end{array}$ & Low risk & $\begin{array}{l}\text { Physician blinding: adequate } \\
\text { Participant blinding: adequate }\end{array}$ \\
\hline $\begin{array}{l}\text { Incomplete outcome data } \\
\text { (attrition bias) } \\
\text { All outcomes }\end{array}$ & High risk & No, participants appear to have been lost on leaving the institution \\
\hline $\begin{array}{l}\text { Selective reporting (re- } \\
\text { porting bias) }\end{array}$ & Low risk & Not relevant for primary and secondary outcomes as all trialists asked for data \\
\hline Other bias & Low risk & \\
\hline $\begin{array}{l}\text { Free of systematic differ- } \\
\text { ence in care? }\end{array}$ & Low risk & $\begin{array}{l}\text { Whole diet provided for both groups. See Control and Intervention Methods in } \\
\text { Interventions section of the Table of Characteristics of Included Studies }\end{array}$ \\
\hline $\begin{array}{l}\text { Free of dietary differences } \\
\text { other than fat? }\end{array}$ & Low risk & $\begin{array}{l}\text { See Control and Intervention Aims in Interventions section of the Table of } \\
\text { Characteristics of Included Studies }\end{array}$ \\
\hline
\end{tabular}


Moy 2001

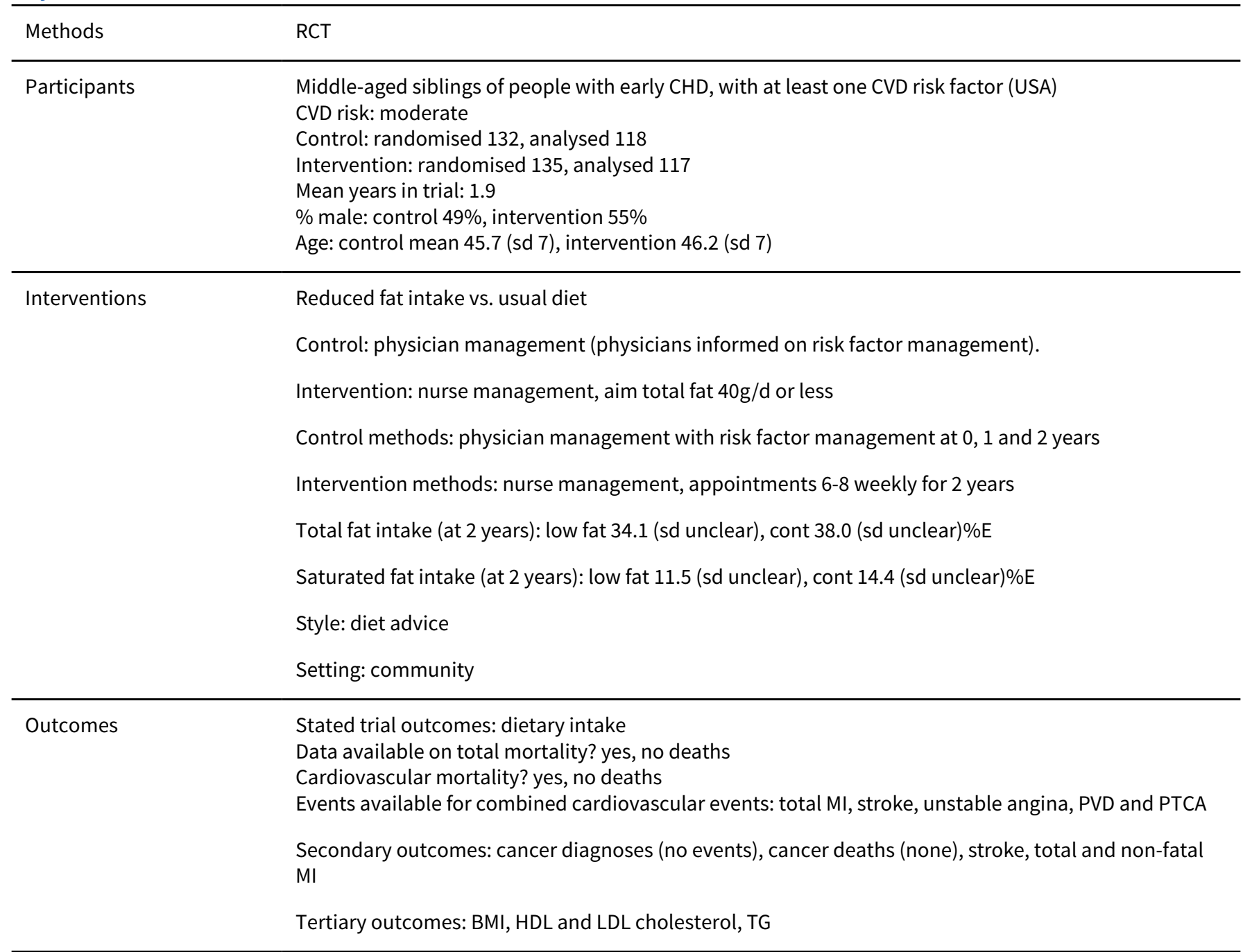

Notes

\section{Risk of bias}

\begin{tabular}{lll}
\hline Bias & Authors' judgement & Support for judgement \\
\hline $\begin{array}{l}\text { Random sequence genera- } \\
\text { tion (selection bias) }\end{array}$ & Low risk & $\begin{array}{l}\text { Randomly assigned via computerised schema after all eligible siblings from a } \\
\text { family had been screened }\end{array}$ \\
\hline $\begin{array}{l}\text { Allocation concealment } \\
\text { (selection bias) }\end{array}$ & Unclear risk & Randomisation method not clearly described \\
\hline
\end{tabular}

\begin{tabular}{lll}
$\begin{array}{l}\text { Blinding (performance } \\
\text { bias and detection bias) } \\
\text { All outcomes }\end{array}$ & High risk & Participants and trialists clear about their allocation \\
\hline $\begin{array}{l}\text { Incomplete outcome data } \\
\begin{array}{l}\text { (attrition bias) } \\
\text { All outcomes }\end{array}\end{array}$ & Unclear risk & $\begin{array}{l}\text { Unclear, deaths, cancer and CV events are drop-outs, trialists asked for data - } \\
\text { unclear if any data missing }\end{array}$ \\
\hline $\begin{array}{l}\text { Selective reporting (re- } \\
\text { porting bias) }\end{array}$ & Low risk & Not relevant for primary and secondary outcomes as all trialists asked for data
\end{tabular}


Moy 2001 (Continued)

\begin{tabular}{lll} 
Other bias & Low risk & \\
\hline $\begin{array}{l}\text { Free of systematic differ- } \\
\text { ence in care? }\end{array}$ & High risk & $\begin{array}{l}\text { Differences in frequency of follow up, but unclear what differences in care } \\
\text { curred between the physician and nurse-led care. See Control and Interven- } \\
\text { tion Methods in Interventions section of the Table of Characteristics of Includ } \\
\text { ed Studies }\end{array}$ \\
\hline $\begin{array}{l}\text { Free of dietary differences } \\
\text { other than fat? }\end{array}$ & Unclear risk & $\begin{array}{l}\text { See Control and Intervention Aims in Interventions section of the Table of } \\
\text { Characteristics of Included Studies }\end{array}$ \\
\hline
\end{tabular}

\section{MRC 1968}

\begin{tabular}{ll}
\hline Methods & RCT \\
\hline Participants & Free-living men who have survived a first MI (UK) \\
CVD risk: high \\
Control: randomised 194, analysed 181 at 2 years \\
Intervention: randomised 199, analysed 172 at 2 years \\
Mean years in trial: control 3.7, intervention 3.8 \\
\% male: 100 \\
Age: unclear (all <60)
\end{tabular}

Interventions

Modified fat vs usual diet

Control aims: usual diet

Intervention aims: reduce dietary fat to $35 \mathrm{~g}$ fat per day, add $84 \mathrm{~g}$ soya oil per day

Control methods: usual diet

Intervention methods: unclear who gave dietary advice or how often

Total fat intake (at 3.5 years): mod fat 46 (sd unclear), cont 43 (sd unclear)\%E

Saturated fat intake: unclear

Style: diet advice \& supplement (soy oil)

Setting: community

$\begin{array}{ll}\text { Outcomes } & \text { Stated trial outcomes: MI or sudden death } \\ \text { Data available on total mortality? yes } \\ \text { Cardiovascular mortality? yes } \\ \text { Events available for combined cardiovascular events: cardiovascular deaths and fatal or non-fatal MI } \\ \text { Secondary outcomes: total and non-fatal MI } \\ \text { Tertiary outcomes: none (data for weight, total cholesterol and BP, but no variance info) } \\ \text { For all, data at } 4 \text { years, control } \mathrm{n}=89 \text {, intervention } \mathrm{n}=88 \\ \text { Weight change: control - } 3 \mathrm{~kg}, \text { intervention 0kg } \\ \text { Total cholesterol change: control -0.47mmol/L, intervention }-1.11 \mathrm{mmol} / \mathrm{L} \\ \text { Systolic BP change: control 0mmHg, intervention }+2 \mathrm{mmHg} \\ \text { Diastolic BP change: control }+3 \mathrm{mmHg}, \text { intervention }-1 \mathrm{mmHg}\end{array}$

\section{Risk of bias}

Reduced or modified dietary fat for preventing cardiovascular disease (Review) 
MRC 1968 (Continued)

\begin{tabular}{|c|c|c|}
\hline Bias & Authors' judgement & Support for judgement \\
\hline $\begin{array}{l}\text { Random sequence genera- } \\
\text { tion (selection bias) }\end{array}$ & Unclear risk & "using random numbers, by blocks within hospitals" \\
\hline $\begin{array}{l}\text { Allocation concealment } \\
\text { (selection bias) }\end{array}$ & Unclear risk & Randomisation method not clearly described \\
\hline $\begin{array}{l}\text { Blinding (performance } \\
\text { bias and detection bias) } \\
\text { All outcomes }\end{array}$ & High risk & $\begin{array}{l}\text { Physician blinding: adequate } \\
\text { Participant blinding: inadequate }\end{array}$ \\
\hline $\begin{array}{l}\text { Incomplete outcome data } \\
\text { (attrition bias) } \\
\text { All outcomes }\end{array}$ & High risk & $\begin{array}{l}\text { Data collection was thorough, but some participants dropped out and contact } \\
\text { was lost }\end{array}$ \\
\hline $\begin{array}{l}\text { Selective reporting (re- } \\
\text { porting bias) }\end{array}$ & Low risk & Not relevant for primary and secondary outcomes as all trialists asked for data \\
\hline Other bias & Low risk & \\
\hline $\begin{array}{l}\text { Free of systematic differ- } \\
\text { ence in care? }\end{array}$ & High risk & $\begin{array}{l}\text { Unlikely as control group continued diet as usual, intervention group were } \\
\text { likely to have had additional contact. See Control and Intervention Methods in } \\
\text { Interventions section of the Table of Characteristics of Included Studies }\end{array}$ \\
\hline $\begin{array}{l}\text { Free of dietary differences } \\
\text { other than fat? }\end{array}$ & Low risk & $\begin{array}{l}\text { See Control and Intervention Aims in Interventions section of the Table of } \\
\text { Characteristics of Included Studies }\end{array}$ \\
\hline
\end{tabular}

\section{MSFAT 1997}

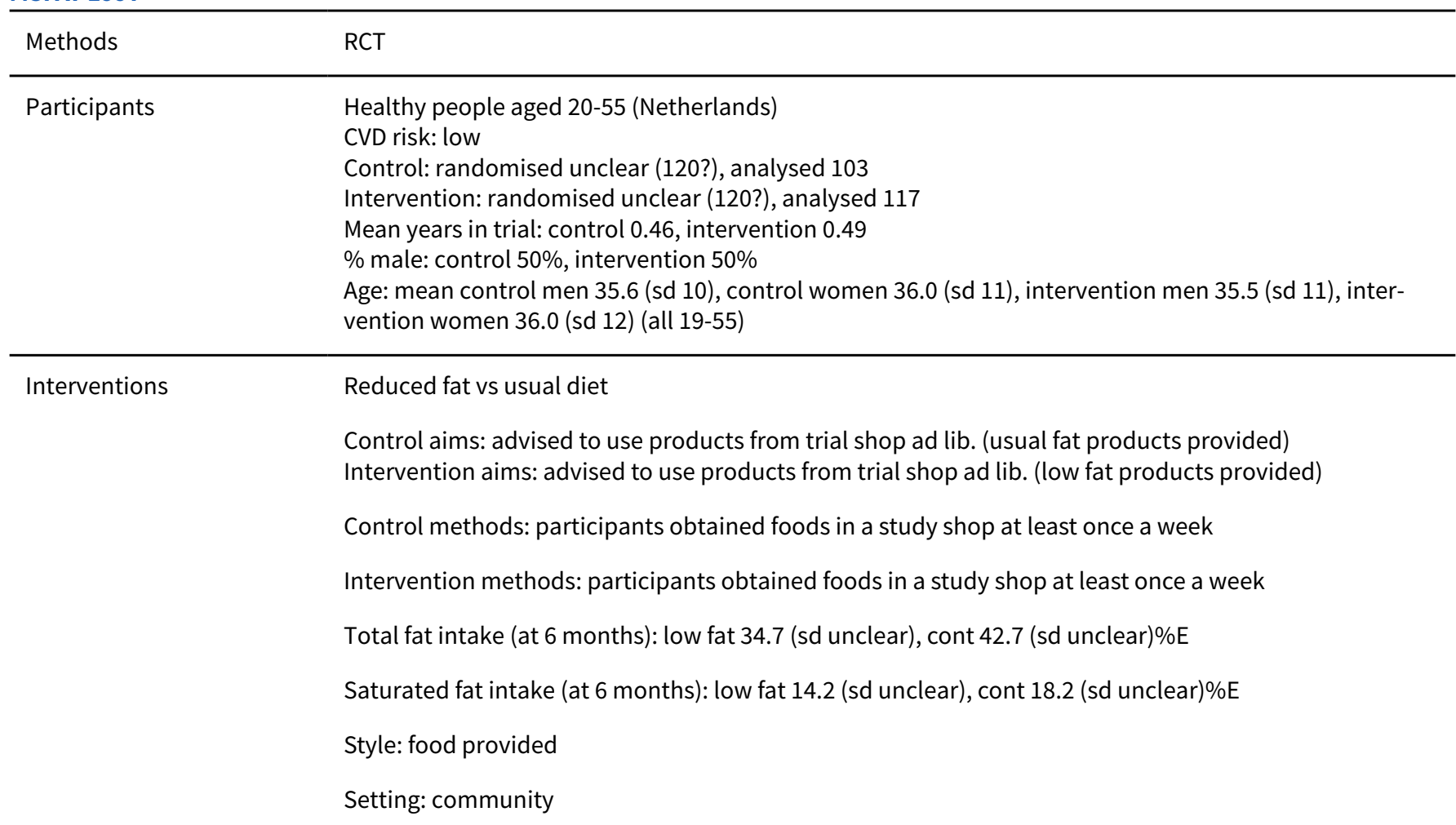


MSFAT 1997 (Continued)

Outcomes
Stated trial outcomes: weight, vitamin and fatty acid intake, anti-oxidative capacity

Data available on total mortality? yes (no events)

Cardiovascular mortality? yes (no events)

Events available for combined cardiovascular events: MI, stroke and CVD deaths (no events)

Secondary outcomes: stroke, MI, cancer diagnoses and deaths (no events for any outcome)

Tertiary outcomes: weight (for subgroup), weight and lipids provided for larger group, but without variance data

Notes

Weight, kg: 1.1, 0.4

total cholesterol, $\mathrm{mmol} / \mathrm{L}: 0.07,-0.09$

HDL cholesterol, mmol/L: -0.03, -0.06

LDL cholesterol, $\mathrm{mmol} / \mathrm{L}:$ : 0.15, 0.16

TGs, $\mathrm{mmol} / \mathrm{L}: 0.04,-0.04$

\section{Risk of bias}

\begin{tabular}{lll}
\hline Bias & Authors' judgement & Support for judgement \\
\hline $\begin{array}{ll}\text { Random sequence genera- } \\
\text { tion (selection bias) }\end{array}$ & Low risk & $\begin{array}{l}\text { "stratified randomisation (according to sex, age, QI index and eating behav- } \\
\text { iour) by co-ordinating centre", a statistician at Unilever Research, SAS soft- } \\
\text { ware, and allocation could not be altered later }\end{array}$ \\
\end{tabular}

Allocation concealment Low risk
(selection bias)

Blinding (performance High risk Participants aware of allocation, those analysing biochemistry were not
bias and detection bias) All outcomes

\begin{tabular}{|c|c|c|}
\hline $\begin{array}{l}\text { Incomplete outcome data } \\
\text { (attrition bias) }\end{array}$ & Unclear risk & $\begin{array}{l}\text { Unclear, deaths, cancer and CV events are drop-outs, trialists asked for data - } \\
\text { unclear if any data missing }\end{array}$ \\
\hline
\end{tabular}

All outcomes

Low risk

Not relevant for primary and secondary outcomes as all trialists asked for data

Selective reporting (re- Low risk
porting bias)

\begin{tabular}{ll}
\hline Other bias $\quad$ Low risk \\
\hline
\end{tabular}

Free of systematic differ- Low risk ence in care?

Both groups used study shop. See Control and Intervention Methods in Interventions section of the Table of Characteristics of Included Studies

\begin{tabular}{|c|c|c|}
\hline $\begin{array}{l}\text { Free of dietary differences } \\
\text { other than fat? }\end{array}$ & Low risk & $\begin{array}{l}\text { See Control and Intervention Aims in Interventions section of the Table of } \\
\text { Characteristics of Included Studies }\end{array}$ \\
\hline
\end{tabular}

NDHS Faribault 1968

\begin{tabular}{ll}
\hline Methods & RCT \\
\hline Participants & Men living in a mental health institute (USA) \\
\hline
\end{tabular}


NDHS Faribault 1968 (Continued)

CVD risk: low

Control: randomised 57, analysed 52

Interventions B, C, E combined: randomised 167, analysed 143

Mean years in trial: control 1.0, Interventions 0.9

$\%$ male: 100

Age: unclear (all 45-54)

\begin{tabular}{|c|c|}
\hline \multirow[t]{7}{*}{ Interventions } & $\begin{array}{l}\text { Modified fat vs. usual diet } \\
\text { Control aims: total fat } 40 \% E \text {, SFA } 16-18 \% E \text {, dietary chol } 650-750 \mathrm{mg} / \mathrm{d}, \mathrm{P} / \mathrm{S} 0.4 \\
\text { Intervention aims: B (C, E) total fat } 30 \% \mathrm{E}(40 \% \mathrm{E}, 40 \% \mathrm{E}) \text {, SFA }<9 \% \mathrm{E}(<9 \% \mathrm{E} \text {, not stated), dietary chol } \\
350-450 \mathrm{mg} / \mathrm{d}(350-450 \mathrm{mg} / \mathrm{d} \text {, not stated), PUFA } 15 \% \mathrm{E}(18-20 \% \mathrm{E} \text {, not stated), P/S } 1.5(2.0,4.4)\end{array}$ \\
\hline & Control methods: whole diet provided \\
\hline & Intervention methods: whole diet provided \\
\hline & $\begin{array}{l}\text { Total fat intake (at } 28 \& 44 \text { weeks combined): B (C, E) mod fat } 29.2(38.5,37.1) \text { (sds unclear)\%E, cont } \\
39.4 \text { (sd unclear)\%E }\end{array}$ \\
\hline & $\begin{array}{l}\text { Saturated fat intake (at } 28 \& 44 \text { weeks combined): B (C, E) mod fat } 6.6(7.4,4.9) \text { (sds unclear)\%E, cont } \\
15.6 \text { (sd unclear)\%E }\end{array}$ \\
\hline & Style: diet provided \\
\hline & Setting: residential institution \\
\hline
\end{tabular}

Stated trial outcomes: lipid levels and dietary assessment
Data available on total mortality? yes
Cardiovascular mortality? no
Events available for combined cardiovascular events: none
Secondary outcomes: no cancer deaths or diagnoses occurred
Tertiary outcomes: total cholesterol

Notes

\section{Risk of bias}

\begin{tabular}{lll}
\hline Bias & Authors' judgement & Support for judgement \\
\hline $\begin{array}{l}\text { Random sequence genera- } \\
\text { tion (selection bias) }\end{array}$ & Low risk & Stratified randomisation by the statistical centre \\
\hline $\begin{array}{l}\text { Allocation concealment } \\
\text { (selection bias) }\end{array}$ & Low risk & \\
\hline $\begin{array}{l}\text { Blinding (performance } \\
\text { bias and detection bias) } \\
\text { All outcomes }\end{array}$ & Low risk & Double blind, facilitated by provision of the whole diet \\
\hline $\begin{array}{l}\text { Incomplete outcome data } \\
\text { (attrition bias) } \\
\text { All outcomes }\end{array}$ & Low risk & Institution so able to follow up all participants through study. \\
\hline $\begin{array}{l}\text { Selective reporting (re- } \\
\text { porting bias) }\end{array}$ & Low risk & Not relevant for primary and secondary outcomes as all trialists asked for data \\
\hline \begin{tabular}{l} 
Other bias \\
\hline
\end{tabular} & Low risk & \\
\hline
\end{tabular}


NDHS Faribault 1968 (Continued)

Free of systematic differ- Low risk Whole diet provided for both groups. See Control and Intervention Methods in ence in care? Interventions section of the Table of Characteristics of Included Studies
Free of dietary differences Low risk other than fat?
See Control and Intervention Aims in Interventions section of the Table of Characteristics of Included Studies

\section{NDHS Open 1st L\&M 1968}

\begin{tabular}{ll}
\hline Methods & RCT \\
\hline Participants & Free living men (USA) \\
& CVD risk: low \\
& Control: randomised 382, analysed 341 \\
Intervention B: randomised 385, analysed 332 \\
Intervention X: randomised 54, analysed 46 \\
Mean years in trial: control 1.0, B 0.9, C 0.9, X 0.9 \\
\% male: 100 \\
Age: unclear (all 45-54)
\end{tabular}

Interventions

Reduced and modified fat diet vs. usual diet

Control aims: total fat 40\%E, SFA 16-18\%E, dietary chol 650-750mg/d, P/S 0.4

Intervention B: total fat 30\%E, SFA <9\%E, dietary chol 350-450mg/d, PUFA 15\%E, P/S 1.5

Intervention X: total fat 30\%E, SFA $<9 \%$ E, dietary chol 350-450mg/d, PUFA 15\%E, P/S 1.5

Control methods: dietary advice to reduce saturated fat and cholesterol (plus 10 follow up visits with nutritionist), purchase of 'usual fat' items from a trial shop

Intervention B methods: dietary advice to reduce saturated fat and cholesterol (plus 10 follow up visits with nutritionist), plus purchase of appropriately reduced and modified fat items from a trial shop

Intervention X methods: dietary advice but no trial shop

Total fat intake (through study): B 29.7 (sd unclear)\%E, X 31.7 (sd unclear), cont 34.9 (sd unclear)\%E

Saturated fat intake (through study): B 7.1 (sd unclear)\%E, X 8.9 (sd unclear), cont 11.6 (sd unclear)\%E

Style: B diet provided, $\mathrm{X}$ - diet advice

Setting: community

Outcomes Stated trial outcomes: lipid levels and dietary assessment

Data available on total mortality? no

Cardiovascular mortality? yes (none occurred)

Events available for combined cardiovascular events: fatal and non-fatal MI, peripheral vascular events

Secondary outcomes: no cancer diagnoses, total or non-fatal MI occurred

Tertiary outcomes: total cholesterol (some weight and BP data presented but no variance info)

Notes

At 52 weeks weight change in the control was not presented, weight change in B was $-2.4 \mathrm{~kg}$.

At 52 weeks diastolic BP change from baseline was $-2.2 \mathrm{~kg}$ in control, -1.9 in B and -5.8 in X.

\section{Risk of bias}


NDHS Open 1st L\&M 1968 (Continued)
Random sequence genera- Low risk
Stratified randomisation by the statistical centre tion (selection bias)

Allocation concealment Low risk
(selection bias)

\begin{tabular}{|c|c|c|}
\hline $\begin{array}{l}\text { Blinding (performance } \\
\text { bias and detection bias) } \\
\text { All outcomes }\end{array}$ & Low risk & $\begin{array}{l}\text { Intervention B: All reduced saturated fat and purchased blinded foods from a } \\
\text { trial shop, double blind. } \\
\text { Intervention X: No trial shop, so participants not blinded, though those } \\
\text { analysing blood samples etc were. }\end{array}$ \\
\hline $\begin{array}{l}\text { Incomplete outcome data } \\
\text { (attrition bias) } \\
\text { All outcomes }\end{array}$ & High risk & $\begin{array}{l}\text { Dropouts do not appear to have been followed for death or CV events. Deaths, } \\
\text { cancer and CV events for participants otherwise still included in the study were } \\
\text { collated as a reason for study exclusion }\end{array}$ \\
\hline
\end{tabular}

Selective reporting (re- Low risk Not relevant for primary and secondary outcomes as all trialists asked for data
porting bias)

\begin{tabular}{lll}
\hline Other bias & Low risk & \\
\hline $\begin{array}{l}\text { Free of systematic differ- } \\
\text { ence in care? }\end{array}$ & Low risk & $\begin{array}{l}\text { Yes for intervention B (as both intervention and control received dietary advice } \\
\text { and purchased food from trial shop). No for intervention X (as it did not include } \\
\text { a trial shop as in the control group). See Control and Intervention Methods in } \\
\text { Interventions section of the Table of Characteristics of Included Studies }\end{array}$ \\
\hline $\begin{array}{l}\text { Free of dietary differences } \\
\text { other than fat? }\end{array}$ & Low risk & $\begin{array}{l}\text { See Control and Intervention Aims in Interventions section of the Table of } \\
\text { Characteristics of Included Studies }\end{array}$ \\
\hline
\end{tabular}

\section{NDHS Open 1st mod 1968}

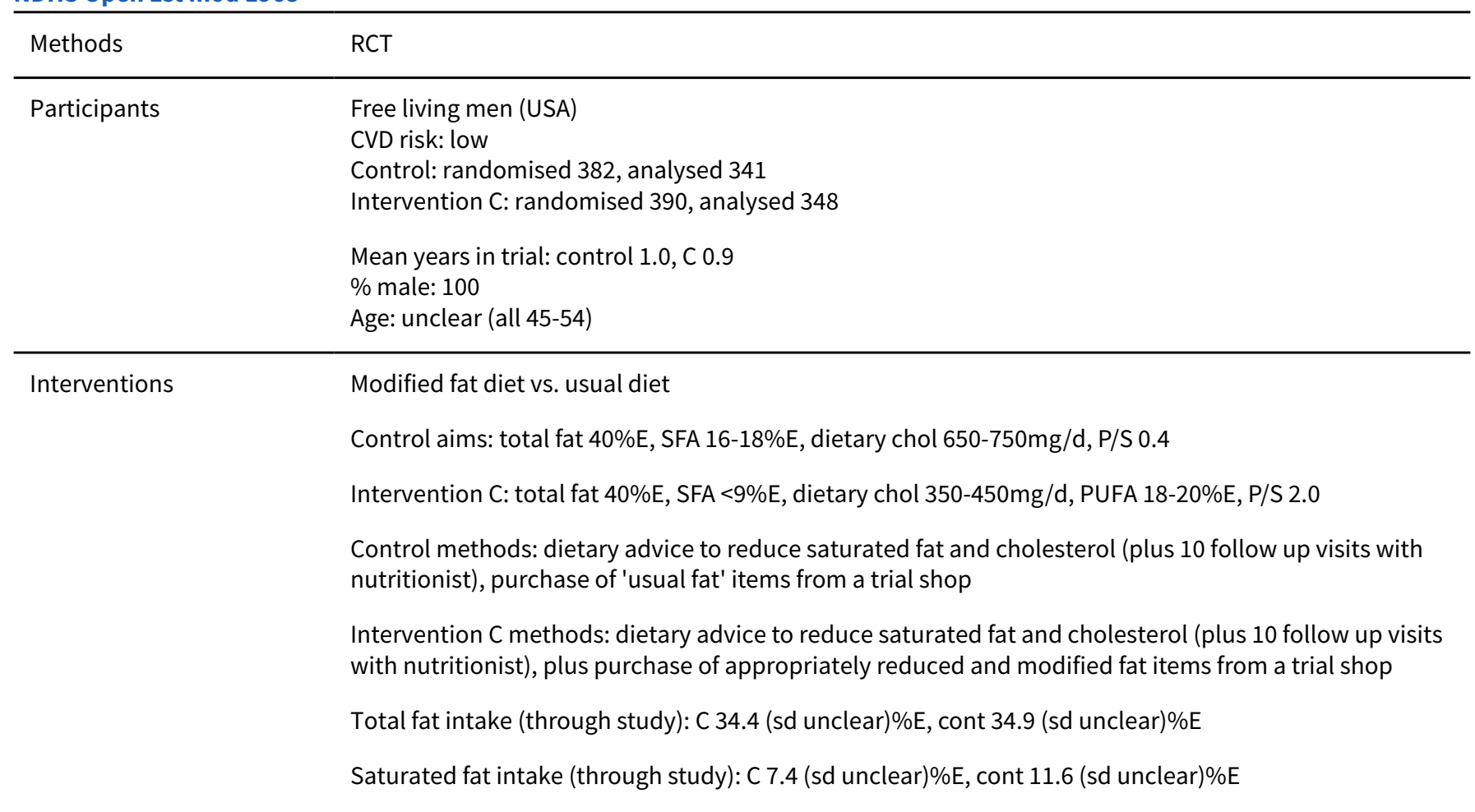


NDHS Open 1st mod 1968 (Continued)

Style: food provided

Setting: community

Stated trial outcomes: lipid levels and dietary assessment
Data available on total mortality? no
Cardiovascular mortality? yes (no events)
Events available for combined cardiovascular events: fatal and non-fatal MI, peripheral vascular events
Secondary outcomes: cancer diagnoses, total and non-fatal MI
Tertiary outcomes: none (total cholesterol and BP data presented but no variance info)

Notes

\section{Risk of bias}

\begin{tabular}{|c|c|c|}
\hline Bias & Authors' judgement & Support for judgement \\
\hline $\begin{array}{l}\text { Random sequence genera- } \\
\text { tion (selection bias) }\end{array}$ & Low risk & Stratified randomisation by the statistical centre \\
\hline $\begin{array}{l}\text { Allocation concealment } \\
\text { (selection bias) }\end{array}$ & Low risk & \\
\hline $\begin{array}{l}\text { Blinding (performance } \\
\text { bias and detection bias) } \\
\text { All outcomes }\end{array}$ & Low risk & $\begin{array}{l}\text { All reduced saturated fat and purchased blinded foods from a trial shop, dou- } \\
\text { ble blind. }\end{array}$ \\
\hline $\begin{array}{l}\text { Incomplete outcome data } \\
\text { (attrition bias) } \\
\text { All outcomes }\end{array}$ & High risk & $\begin{array}{l}\text { Dropouts do not appear to have been followed for death or CV events. Deaths, } \\
\text { cancer and CV events for participants otherwise still included in the study were } \\
\text { collated as a reason for study exclusion }\end{array}$ \\
\hline $\begin{array}{l}\text { Selective reporting (re- } \\
\text { porting bias) }\end{array}$ & Low risk & Not relevant for primary and secondary outcomes as all trialists asked for data \\
\hline Other bias & Low risk & \\
\hline $\begin{array}{l}\text { Free of systematic differ- } \\
\text { ence in care? }\end{array}$ & Low risk & $\begin{array}{l}\text { Trial shop used by both groups, plus dietary advice. See Control and Interven- } \\
\text { tion Methods in Interventions section of the Table of Characteristics of Includ- } \\
\text { ed Studies }\end{array}$ \\
\hline $\begin{array}{l}\text { Free of dietary differences } \\
\text { other than fat? }\end{array}$ & Low risk & $\begin{array}{l}\text { See Control and Intervention Aims in Interventions section of the Table of } \\
\text { Characteristics of Included Studies }\end{array}$ \\
\hline
\end{tabular}

\section{NDHS Open 2nd L\&M 1968}

\begin{tabular}{ll}
\hline Methods & RCT \\
\hline Participants & Free living men who had participated in NDHS 1st studies (USA) \\
& CVD risk: low \\
& Control: randomised 304, analysed 280 \\
Intervention BC: randomised 194, analysed 179 & Mean years in trial: control 0.6, intervention BC 0.6 \\
& $\%$ male: 100 \\
& Age: unclear (all 45-54) \\
\hline
\end{tabular}


NDHS Open 2nd L\&M 1968 (Continued)

Interventions $\quad$ Reduced and modified fat vs usual diet

Control aims: total fat 40\%E, SFA $16-18 \% \mathrm{E}$, dietary chol $650-750 \mathrm{mg} / \mathrm{d}, \mathrm{P} / \mathrm{S} 0.4, \mathrm{X}$ - advice to continue usual diet

Intervention aims: BC total fat $30-40 \% \mathrm{E}$, SFA reduced, dietary chol $350-450 \mathrm{mg} / \mathrm{d}$, increased PUFA, P/S $1.5-2.0$

Control methods: dietary advice to reduce saturated fat and cholesterol (plus 10 follow up visits with nutritionist), purchase of 'usual fat' items from a trial shop

Intervention $\mathrm{BC}$ methods: dietary advice to reduce saturated fat and cholesterol (plus 10 follow up visits with nutritionist), plus purchase of appropriately reduced and modified fat items from a trial shop

Total fat intake (through study): BC 32.5 (sd unclear)\%E, cont 35.5 (sd unclear)\%E

Saturated fat intake (through study): BC 7.4 (sd unclear)\%E, cont 12.0 (sd unclear)\%E

Style: food provided

Setting: community

Stated trial outcomes: lipid levels and dietary assessment
Data available on total mortality? no
Cardiovascular mortality? yes (no events)
Events available for combined cardiovascular events: fatal and non-fatal MI, peripheral vascular events
Secondary outcomes: cancer diagnoses (no events), total and non-fatal MI
Tertiary outcomes: none

\section{Notes}

\section{Risk of bias}

\begin{tabular}{|c|c|c|}
\hline Bias & Authors' judgement & Support for judgement \\
\hline $\begin{array}{l}\text { Random sequence genera- } \\
\text { tion (selection bias) }\end{array}$ & Low risk & Stratified randomisation by the statistical centre \\
\hline $\begin{array}{l}\text { Allocation concealment } \\
\text { (selection bias) }\end{array}$ & Low risk & \\
\hline $\begin{array}{l}\text { Blinding (performance } \\
\text { bias and detection bias) } \\
\text { All outcomes }\end{array}$ & Low risk & $\begin{array}{l}\text { Some participants continued with advice to reduce saturated fat and pur- } \\
\text { chased blinded foods from a trial shop, but half of the participants were in- } \\
\text { structed in their own purchase of appropriate foods from normal shops to } \\
\text { compile their own dietary regimen. }\end{array}$ \\
\hline $\begin{array}{l}\text { Incomplete outcome data } \\
\text { (attrition bias) } \\
\text { All outcomes }\end{array}$ & High risk & $\begin{array}{l}\text { Dropouts do not appear to have been followed for death or CV events. Deaths, } \\
\text { cancer and CV events for participants otherwise still included in the study were } \\
\text { collated as a reason for study exclusion }\end{array}$ \\
\hline $\begin{array}{l}\text { Selective reporting (re- } \\
\text { porting bias) }\end{array}$ & Low risk & Not relevant for primary and secondary outcomes as all trialists asked for data \\
\hline Other bias & Low risk & \\
\hline $\begin{array}{l}\text { Free of systematic differ- } \\
\text { ence in care? }\end{array}$ & Low risk & $\begin{array}{l}\text { Trial shop used by both groups, plus dietary advice. See Control and Interven- } \\
\text { tion Methods in Interventions section of the Table of Characteristics of Includ- } \\
\text { ed Studies }\end{array}$ \\
\hline
\end{tabular}


NDHS Open 2nd L\&M 1968 (Continued)

Free of dietary differences Low risk other than fat?
See Control and Intervention Aims in Interventions section of the Table of Characteristics of Included Studies

\section{NDHS Open 2nd Mod 1968}

\begin{tabular}{ll}
\hline Methods & RCT \\
\hline Participants & Free living men who had participated in NDHS 1st studies (USA) \\
& CVD risk: low \\
& Control: randomised 304, analysed 280 \\
& Intervention F: randomised 127, analysed 112 \\
& Mean years in trial: control 0.6, intervention 0.6 \\
& \% male: 100 \\
& Age: unclear (all 45-54)
\end{tabular}

Interventions

Modified fat vs usual diet

Control aims: total fat $40 \% \mathrm{E}$, SFA $16-18 \% \mathrm{E}$, dietary chol $650-750 \mathrm{mg} / \mathrm{d}, \mathrm{P} / \mathrm{S} 0.4$, Intervention aims: $F$ total fat 40\%E, SFA no data, dietary chol $350-450 \mathrm{mg} / \mathrm{d}$, increased PUFA, P/S 3.0

Control methods: dietary advice to reduce saturated fat and cholesterol (plus 10 follow up visits with nutritionist), purchase of 'usual fat' items from a trial shop

Intervention $\mathrm{F}$ methods: dietary advice to reduce saturated fat and cholesterol (plus 10 follow up visits with nutritionist), plus purchase of appropriately reduced and modified fat items from a trial shop

Total fat intake (through study): F 35.1 (sd unclear)\%E, cont 35.5 (sd unclear)\%E

Saturated fat intake (through study): F 7.8 (sd unclear)\%E, cont 12.0 (sd unclear)\%E

Style: food provided

Setting: community

Outcomes

Stated trial outcomes: lipid levels and dietary assessment

Data available on total mortality? no

Cardiovascular mortality? yes (none)

Events available for combined cardiovascular events: fatal and non-fatal MI, peripheral vascular events

Secondary outcomes: cancer diagnoses (none occurred), total and non-fatal MI

Tertiary outcomes: none

\section{Notes}

\section{Risk of bias}

\begin{tabular}{lll}
\hline Bias & Authors' judgement & Support for judgement \\
\hline $\begin{array}{l}\text { Random sequence genera- } \\
\text { tion (selection bias) }\end{array}$ & Low risk & Stratified randomisation by the statistical centre \\
\hline $\begin{array}{l}\text { Allocation concealment } \\
\text { (selection bias) }\end{array}$ & Low risk & \\
\hline $\begin{array}{l}\text { Blinding (performance } \\
\text { bias and detection bias) } \\
\text { All outcomes }\end{array}$ & Low risk & $\begin{array}{l}\text { Some participants continued with advice to reduce saturated fat and pur- } \\
\text { chased blinded foods from a trial shop, but half of the participants were in- }\end{array}$ \\
\hline
\end{tabular}


NDHS Open 2nd Mod 1968 (Continued)

structed in their own purchase of appropriate foods from normal shops to compile their own dietary regimen

\begin{tabular}{ll}
\hline $\begin{array}{l}\text { Incomplete outcome data } \\
\text { (attrition bias) }\end{array}$ & High risk \\
All outcomes & $\begin{array}{l}\text { Dropouts do not appear to have been followed for death or CV events. Deaths, } \\
\text { cancer and CV events for participants otherwise still included in the study were } \\
\text { collated as a reason for study exclusion }\end{array}$
\end{tabular}

\begin{tabular}{lll}
\hline $\begin{array}{l}\text { Selective reporting (re- } \\
\text { porting bias) }\end{array}$ & Low risk & Not relevant for primary and secondary outcomes as all trialists asked for data \\
\hline Other bias & Low risk & \\
\hline $\begin{array}{l}\text { Free of systematic differ- } \\
\text { ence in care? }\end{array}$ & Low risk & $\begin{array}{l}\text { Trial shop used by both groups, plus dietary advice. See Control and Interven- } \\
\text { tion Methods in Interventions section of the Table of Characteristics of Includ- } \\
\text { ed Studies }\end{array}$ \\
\hline $\begin{array}{l}\text { Free of dietary differences } \\
\text { other than fat? }\end{array}$ & Low risk & $\begin{array}{l}\text { See Control and Intervention Aims in Interventions section of the Table of } \\
\text { Characteristics of Included Studies }\end{array}$ \\
\hline
\end{tabular}

Nutrition \& Breast Health

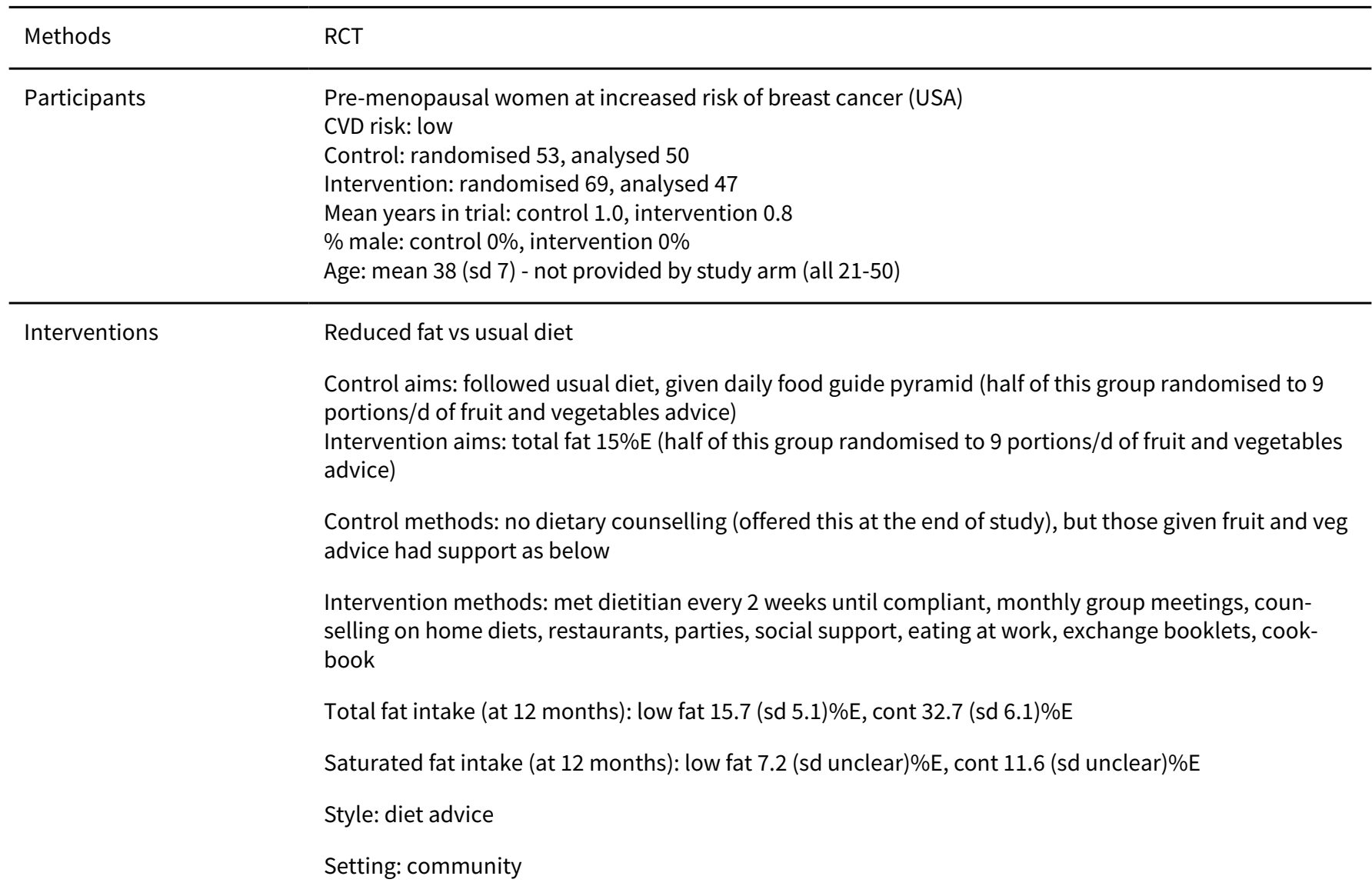

\section{Outcomes}

Stated trial outcomes: body weight, dietary compliance

Data available on total mortality? yes (no events)

Cardiovascular mortality? yes (no events)

Events available for combined cardiovascular events: total MI, stroke (no events) 
Nutrition \& Breast Health (Continued)

Secondary outcomes: non-fatal and total MI, stroke, cancer diagnoses and deaths (no events for any outcome)

Tertiary outcomes: weight, total, LDL and HDL cholesterol, TG, BMI (but variance data not provided for any but weight)

Change from baseline to 12 months for the control $(n=23)$, control plus fruit \& veg $(n=25)$, low fat $(n=24)$,
low fat plus fruit \& veg $(n=23)$ :
Total cholesterol $\mathrm{mg} / \mathrm{dl}: 9,2,-8,0$
TGs $\mathrm{mg} / \mathrm{dl}:-7,1,5,8$
HDL chol $\mathrm{mg} / \mathrm{dl}: 0,0,-4,0$
LDL chol mg/dl: $11,2,-6,-2$
BMI kg/m2: $0,4,-13,0$

\section{Risk of bias}

\begin{tabular}{|c|c|c|}
\hline Bias & Authors' judgement & Support for judgement \\
\hline $\begin{array}{l}\text { Random sequence genera- } \\
\text { tion (selection bias) }\end{array}$ & Low risk & $\begin{array}{l}\text { The statistician made envelopes ahead of time, dietitians handed out en- } \\
\text { velopes at first visit }\end{array}$ \\
\hline $\begin{array}{l}\text { Allocation concealment } \\
\text { (selection bias) }\end{array}$ & Low risk & Allocation could not be altered once made \\
\hline $\begin{array}{l}\text { Blinding (performance } \\
\text { bias and detection bias) } \\
\text { All outcomes }\end{array}$ & High risk & $\begin{array}{l}\text { Paricipants were aware of allocation, researchers and those assessing lipids } \\
\text { were not }\end{array}$ \\
\hline $\begin{array}{l}\text { Incomplete outcome data } \\
\text { (attrition bias) } \\
\text { All outcomes }\end{array}$ & Unclear risk & $\begin{array}{l}\text { Unclear, deaths, cancer and CV events are drop-outs, trialists asked for data - } \\
\text { unclear if any data missing }\end{array}$ \\
\hline $\begin{array}{l}\text { Selective reporting (re- } \\
\text { porting bias) }\end{array}$ & Low risk & Not relevant for primary and secondary outcomes as all trialists asked for data \\
\hline Other bias & Low risk & \\
\hline $\begin{array}{l}\text { Free of systematic differ- } \\
\text { ence in care? }\end{array}$ & High risk & $\begin{array}{l}\text { High levels of intervention for those on low fat or high fruit and vegetable di- } \\
\text { ets. See Control and Intervention Methods in Interventions section of the Ta- } \\
\text { ble of Characteristics of Included Studies }\end{array}$ \\
\hline $\begin{array}{l}\text { Free of dietary differences } \\
\text { other than fat? }\end{array}$ & Low risk & $\begin{array}{l}\text { Randomisation to fruit and vegetable intervention was independent of low fat } \\
\text { allocation }\end{array}$ \\
\hline
\end{tabular}

\section{Ole Study 2002}

\begin{tabular}{ll}
\hline Methods & RCT \\
\hline Participants & Moderately obese healthy men (USA) \\
& CVD risk: low \\
& Control: randomised 15, analysed 12 \\
& Intervention: randomised 15, analysed 13 \\
& Mean years in trial: control 0.68, intervention 0.70 \\
\hline
\end{tabular}


Ole Study 2002 (Continued)

$\%$ male: control $100 \%$, intervention $100 \%$

Age: mean control 37.0 (SE 2.54), intervention 36.1 (SE 2.49)

\begin{tabular}{|c|c|}
\hline \multirow[t]{10}{*}{ Interventions } & Reduced fat vs usual diet \\
\hline & Control aims: total fat 33\%E, CHO 52\%E, protein $15 \% \mathrm{E}$ \\
\hline & $\begin{array}{l}\text { Intervention aims: total fat } 25 \% \mathrm{E}, \mathrm{CHO} 58 \% \mathrm{E} \text {, protein } 17 \% \mathrm{E} \text {, provided with } 11 \% \text { less energy than con- } \\
\text { trols, but were allowed to ask for more }\end{array}$ \\
\hline & Also second intervention arm with Olestra added to \\
\hline & $\begin{array}{l}\text { Control methods: } 5 \text { meals/week eaten in centre, other meals in takeout containers, asked to return un- } \\
\text { eaten food, allowed to ask for more }\end{array}$ \\
\hline & $\begin{array}{l}\text { Intervention methods: } 5 \text { meals/week eaten in centre, other meals in takeout containers, asked to re- } \\
\text { turn uneaten food, allowed to ask for more }\end{array}$ \\
\hline & Total fat intake (at 6 months): low fat 26.2 (sd 2.8)\%E, cont 34.1 (sd 2.7)\%E \\
\hline & Saturated fat intake (at 6 months): low fat 6.2 (sd 0.7)\%E, cont 7.6 (sd 0.9)\%E \\
\hline & Style: diet provided \\
\hline & Setting: community \\
\hline
\end{tabular}

Outcomes Stated trial outcomes: body weight, body fat, lipids, glucose, insulin

Data available on total mortality? yes (no events)

Cardiovascular mortality? yes (no events)

Events available for combined cardiovascular events: total MI, stroke, angina, CABG, angioplasty, peripheral vascular events (no events)

Secondary outcomes: non-fatal and total MI, stroke, cancer diagnoses and deaths (no events for any outcome), Quality of Life

Tertiary outcomes: weight, total, LDL and HDL cholesterol, TG, BP

Notes

\section{Risk of bias}

\begin{tabular}{lll}
\hline Bias & Authors' judgement & Support for judgement \\
\hline $\begin{array}{l}\text { Random sequence genera- } \\
\text { tion (selection bias) }\end{array}$ & Low risk & $\begin{array}{l}\text { Computer stratified and randomised by personnel not involved with partici- } \\
\text { pants }\end{array}$ \\
\hline $\begin{array}{l}\text { Allocation concealment } \\
\text { (selection bias) }\end{array}$ & Low risk & \\
\hline $\begin{array}{l}\text { Blinding (performance } \\
\text { bias and detection bias) }\end{array}$ & Low risk & $\begin{array}{l}\text { Workers in the dietary kitchen, who provided the meals, were the only ones } \\
\text { who knew the allocations }\end{array}$ \\
\hline $\begin{array}{l}\text { Incomplete outcome data } \\
\text { (attrition bias) } \\
\text { All outcomes }\end{array}$ & Unclear risk & $\begin{array}{l}\text { Unclear, deaths, cancer and CV events are drop-outs, trialists asked for data - } \\
\text { unclear if any data missing }\end{array}$ \\
\hline $\begin{array}{l}\text { Selective reporting (re- } \\
\text { porting bias) }\end{array}$ & Low risk & Not relevant for primary and secondary outcomes as all trialists asked for data \\
\hline \begin{tabular}{l} 
Other bias \\
\hline
\end{tabular} & Low risk & \\
\hline
\end{tabular}


Ole Study 2002 (Continued)

Free of systematic differ- Low risk Most food provided for both groups. See Control and Intervention Methods in ence in care? Interventions section of the Table of Characteristics of Included Studies

Free of dietary differences High risk

Potential difference in energy intake, but unclear what effect this had other than fat?

\section{Oslo Diet-Heart 1966}

\begin{tabular}{ll}
\hline Methods & RCT \\
\hline Participants & Men with previous MI (Norway) \\
CVD risk: high \\
Control: randomised 206, analysed 148 (at 5 years) \\
Intervention: randomised 206, analysed 152 (at 5 years) \\
Mean years in trial: control 4.3, intervention 4.3 \\
\% male: 100 \\
age: mean control 56.3, intervention 56.2 (all 30-67)
\end{tabular}

Control aims: no dietary advice but direct questions answered, supplement = 1 vitamin tablet daily Intervention aims: reduce meat \& dairy fats, increase fish, vegetables, supplement - 1 vitamin tablet daily, $0.5 \mathrm{~L}$ soy bean oil per week (free to $25 \%$ of participants), sardines in cod liver oil (free at certain times to encourage compliance)

Control methods: usual diet

Intervention methods: continuous instruction and supervision by dietitian, including home visits, letters and phone calls

Total fat intake: mod fat unclear, cont unclear

Saturated fat intake: mod fat unclear, cont unclear

Style: diet advice \& supplement (food)

Setting: community

Outcomes Stated trial outcomes: coronary heart disease morbidity and mortality

Data available on total mortality? yes

Cardiovascular mortality? yes

Events available for combined cardiovascular events: total MI, sudden death, stroke, angina

Secondary outcomes: non-fatal and total MI, stroke

Tertiary outcomes: weight, total cholesterol, systolic and diastolic BP (but no variance information is provided)

Total cholesterol change from baseline was $-0.46 \mathrm{mmol} / \mathrm{L}$ in the control group and $-1.53 \mathrm{mmol} / \mathrm{L}$ in the intervention group at 51 months.

Systolic BP at baseline was $153.8 \mathrm{mmHg}$ in control and 159.0 in intervention, and mean SBP through trial was $154.3 \mathrm{mmHg}$ in control and $158.2 \mathrm{mmHg}$ in the intervention group.

Diastolic BP at baseline was $93.5 \mathrm{mmHg}$ in control and $97.1 \mathrm{mmHg}$ in intervention, through trial mean $\mathrm{dBP}$ was $95.5 \mathrm{mmHg}$ in control and $98.6 \mathrm{~mm} \mathrm{Hg}$ in intervention participants. 
Oslo Diet-Heart 1966 (Continued)

\section{Risk of bias}

\begin{tabular}{|c|c|c|}
\hline Bias & Authors' judgement & Support for judgement \\
\hline $\begin{array}{l}\text { Random sequence genera- } \\
\text { tion (selection bias) }\end{array}$ & Low risk & "table of random numbers used", by Prof Knut Westlund \\
\hline $\begin{array}{l}\text { Allocation concealment } \\
\text { (selection bias) }\end{array}$ & Low risk & $\begin{array}{l}\text { Randomisation appears to have occurred before medical examination within } \\
\text { the study }\end{array}$ \\
\hline $\begin{array}{l}\text { Blinding (performance } \\
\text { bias and detection bias) } \\
\text { All outcomes }\end{array}$ & High risk & $\begin{array}{l}\text { Participants were aware of their allocation as was the main trialist. Outcomes } \\
\text { were categorised by a diagnostic board, but their blinded status was unclear. }\end{array}$ \\
\hline $\begin{array}{l}\text { Incomplete outcome data } \\
\text { (attrition bias) } \\
\text { All outcomes }\end{array}$ & Low risk & $\begin{array}{l}\text { The participants who could not be directly followed up for the } 5 \text { years were fol- } \\
\text { lowed until death or study end through personal interviews, or contact with } \\
\text { their physicians or relatives. }\end{array}$ \\
\hline $\begin{array}{l}\text { Selective reporting (re- } \\
\text { porting bias) }\end{array}$ & Low risk & Not relevant for primary and secondary outcomes as all trialists asked for data \\
\hline Other bias & Low risk & \\
\hline $\begin{array}{l}\text { Free of systematic differ- } \\
\text { ence in care? }\end{array}$ & High risk & $\begin{array}{l}\text { Dietetic input level very different, although medical care appeared similar. } \\
\text { See Control and Intervention Methods in Interventions section of the Table of } \\
\text { Characteristics of Included Studies }\end{array}$ \\
\hline $\begin{array}{l}\text { Free of dietary differences } \\
\text { other than fat? }\end{array}$ & High risk & Differences in fruit and vegetables, fish etc. as above. \\
\hline
\end{tabular}

Oxford Retinopathy 1978

\begin{tabular}{ll}
\hline Methods & RCT \\
\hline Participants & Newly diagnosed non-insulin dependant diabetics (UK) \\
CVD risk: moderate \\
Control: randomised unclear (249 split between the 2 groups, 125?), analysed for mortality unclear (all \\
but 2 overall at 16 years) \\
Intervention: randomised unclear (249 split between the 2 groups, 125?), analysed as above \\
Mean years in trial: overall 9.3? \\
\% male: overall 49 \\
Age: mean overall 47.1 (all <65)
\end{tabular}

Interventions

Reduced and modified dietary fat vs average diet

Control aims: total fat 40\%E, PUFA $12 \% \mathrm{E}$, protein $20 \% \mathrm{E}, \mathrm{CHO} 40 \% \mathrm{E}$ (reducing simple sugars), 1500kcal/ day

Intervention aims: total fat 26\%E, PUFA $16 \%$ E, protein 20\%E, CHO 54\%E (reducing simple sugars), $1500 \mathrm{kcal} /$ day

Control methods: dietary advice from diabetes dietitian

Intervention methods: dietary advice from diabetes dietitian

Total fat intake (at 7-9 years): low \& mod fat 32 (sd unclear), cont 41 (sd unclear)\%E

Saturated fat intake (at 7-9 years): low \& mod fat 10.7 (sd unclear), cont 20.4 (sd unclear)\%E 
Oxford Retinopathy 1978 (Continued)

Style: diet advice

Setting: community (outpatients clinic)

Stated trial outcomes: retinopathy
Data available on total mortality? yes, but unable to ascertain from which intervention groups (34
deaths at 10 years)
Cardiovascular mortality? no
Events available for combined cardiovascular events: none
Secondary outcomes: none
Tertiary outcomes: BMI, total cholesterol

Notes

Risk of bias

\begin{tabular}{|c|c|c|}
\hline Bias & Authors' judgement & Support for judgement \\
\hline $\begin{array}{l}\text { Random sequence genera- } \\
\text { tion (selection bias) }\end{array}$ & Low risk & $\begin{array}{l}\text { "random number sequence, provided and allotted by a separate agency" (Prof } \\
\text { Richard Peto) }\end{array}$ \\
\hline $\begin{array}{l}\text { Allocation concealment } \\
\text { (selection bias) }\end{array}$ & Low risk & \\
\hline $\begin{array}{l}\text { Blinding (performance } \\
\text { bias and detection bias) } \\
\text { All outcomes }\end{array}$ & High risk & Participants not blinded, physicians unclear \\
\hline $\begin{array}{l}\text { Incomplete outcome data } \\
\text { (attrition bias) } \\
\text { All outcomes }\end{array}$ & Unclear risk & $\begin{array}{l}\text { Unclear, deaths, cancer and CV events are drop-outs - unclear if any data miss- } \\
\text { ing }\end{array}$ \\
\hline $\begin{array}{l}\text { Selective reporting (re- } \\
\text { porting bias) }\end{array}$ & Low risk & Not relevant for primary and secondary outcomes as all trialists asked for data \\
\hline Other bias & Low risk & \\
\hline $\begin{array}{l}\text { Free of systematic differ- } \\
\text { ence in care? }\end{array}$ & Low risk & $\begin{array}{l}\text { Dietetic advice for both groups. See Control and Intervention Methods in Inter- } \\
\text { ventions section of the Table of Characteristics of Included Studies }\end{array}$ \\
\hline $\begin{array}{l}\text { Free of dietary differences } \\
\text { other than fat? }\end{array}$ & Low risk & $\begin{array}{l}\text { See Control and Intervention Aims in Interventions section of the Table of } \\
\text { Characteristics of Included Studies }\end{array}$ \\
\hline
\end{tabular}

\section{Polyp Prevention 1996}

\begin{tabular}{|c|c|}
\hline Methods & $\mathrm{RCT}$ \\
\hline \multirow[t]{4}{*}{ Participants } & $\begin{array}{l}\text { People with at least one adenomatous polyp of the large bowel removed (USA) } \\
\text { CVD risk: low }\end{array}$ \\
\hline & Control: 1042 randomised, 947 analysed \\
\hline & Intervention: 1037 randomised, 958 analysed \\
\hline & Mean years in trial: control 3.05, intervention 3.05 \\
\hline
\end{tabular}


Polyp Prevention 1996 (Continued)

$\%$ male: control $64 \%$, intervention $66 \%$

Age: mean control 61.5, intervention 61.4 (all at least 35 )

Low fat vs usual diet
Control: general dietary guidelines
Intervention: total fat $20 \% \mathrm{E}, 18 \mathrm{~g}$ fibre $1000 \mathrm{kcal}, 5-8$ servings fruit and veg daily
Control methods: leaflet, no additional information or behaviour modification
Intervention methods: $>50$ hours of counselling over 4 years, included skill building, behaviour modifi-
cation, self monitoring and nutritional materials
Total fat intake (at 4 years): low fat 23.8 (sd 6.0$)$, cont 33.9 (sd 5.9)\%E
Saturated fat intake: unclear
Style: diet advice
Setting: community
Stated trial outcomes: recurrence of polyps, prostate cancer
Data available on total mortality? yes
Cardiovascular mortality? no
Events available for combined cardiovascular events: none
Secondary outcomes: cancer diagnoses
Tertiary outcomes: weight, total cholesterol

Notes

\section{Risk of bias}

Bias Authors' judgement Support for judgement

Random sequence genera- Low risk "randomly assigned"

tion (selection bias)

Allocation concealment Low risk Phone call to computer randomisation centre, stratified according to centre
(selection bias)

\begin{tabular}{|c|c|c|}
\hline $\begin{array}{l}\text { Blinding (performance } \\
\text { bias and detection bias) } \\
\text { All outcomes }\end{array}$ & High risk & Outcome assessors blinded, participants not. \\
\hline $\begin{array}{l}\text { Incomplete outcome data } \\
\text { (attrition bias) } \\
\text { All outcomes }\end{array}$ & Unclear risk & $\begin{array}{l}\text { Unclear, deaths, cancer and CV events are drop-outs - unclear if any data miss- } \\
\text { ing }\end{array}$ \\
\hline $\begin{array}{l}\text { Selective reporting (re- } \\
\text { porting bias) }\end{array}$ & Low risk & Not relevant for primary and secondary outcomes as all trialists asked for data \\
\hline Other bias & Low risk & \\
\hline $\begin{array}{l}\text { Free of systematic differ- } \\
\text { ence in care? }\end{array}$ & High risk & $\begin{array}{l}50 \text { hours behaviour modification in intervention group, not in control. See } \\
\text { Control and Intervention Methods in Interventions section of the Table of } \\
\text { Characteristics of Included Studies }\end{array}$ \\
\hline
\end{tabular}


Polyp Prevention 1996 (Continued)

Free of dietary differences High risk

Fibre, fruit and vegetable goals in intervention group other than fat?

\section{PREMIER 2003}

\begin{tabular}{ll}
\hline Methods & RCT \\
\hline Participants & Adults with above optimal BP or stage 1 hypertension (USA) \\
CVD risk: moderate \\
Control: 269 randomised, 269 analysed \\
Intervention: 268 randomised, 268 analysed \\
Mean years in trial: 1.5 \\
\% male: control $35.3 \%$, intervention $42.8 \%$ \\
Age, years: control 50.2 mean (sd 8.6), intervention 50.2 (sd 9.3)
\end{tabular}

Interventions

Reduced fat vs usual diet

Control: 'established' goals weight loss of $6.8 \mathrm{~kg}$ at $6 \mathrm{months}, 180 \mathrm{mins} /$ week of moderate physical activity, limited sodium intake, limited alcohol.

Intervention: 'established plus DASH' goals as 'established' plus saturated fat intake 7\%E or less, total fat intake $25 \%$ E or less, 2-3 portions low fat dairy foods/d, 9-12 portions fruit and veg/d.

Other arms: Advice only standard care arm, data not used.

Control methods: 18 face to face contacts (14 group and 4 individual), food diaries, physical activity records, calorie and sodium intake records

Intervention methods: 18 face to face contacts (14 group and 4 individual), food diaries, physical activity records, calorie and sodium intake records

Total fat intake (at 6 months): low fat 23.8 (sd 8.6), cont 29.4 (sd 8.4)\%E

Saturated fat intake (at 6 months): low fat 7.7 (sd 3.2), cont 9.4 (sd 3.5)\%E

Style: diet advice

Setting: community

Stated trial outcomes: blood pressure
Data available on total mortality? yes
Cardiovascular mortality? yes, none by 18 months
Events available for combined cardiovascular events: total MI, stroke.
Secondary outcomes: cancer deaths (none), cancer diagnoses, diabetes, stroke, total and non-fatal MI
Tertiary outcomes: weight, BP

Notes

\section{Risk of bias}

\begin{tabular}{lll}
\hline Bias & Authors' judgement & Support for judgement \\
\hline $\begin{array}{l}\text { Random sequence genera- } \\
\text { tion (selection bias) }\end{array}$ & Low risk & $\begin{array}{l}\text { Randomisation was carried out centrally by computer programme, stratified } \\
\text { by clinic and baseline BP, blocked }\end{array}$ \\
\hline
\end{tabular}


PREMIER 2003 (Continued)

Allocation concealment Low risk (selection bias)

Blinding (performance

bias and detection bias)

All outcomes
High risk

Participants were not blinded to the intervention, though efforts were made to mask centre staff involved in outcome assessments to allocation

\begin{tabular}{lll}
\hline $\begin{array}{l}\text { Incomplete outcome data } \\
\text { (attrition bias) } \\
\text { All outcomes }\end{array}$ & Low risk & $\begin{array}{l}\text { All those randomised were included in the analysis of both events and blood } \\
\text { pressure }\end{array}$ \\
\hline $\begin{array}{l}\text { Selective reporting (re- } \\
\text { porting bias) }\end{array}$ & Low risk & Not relevant for primary and secondary outcomes as all trialists asked for data \\
\hline $\begin{array}{l}\text { Other bias } \\
\text { Free of systematic differ- } \\
\text { ence in care? }\end{array}$ & Low risk & $\begin{array}{l}\text { Similar style and duration of interventions. See Control and Intervention Meth- } \\
\text { ods in Interventions section of the Table of Characteristics of Included Studies }\end{array}$ \\
\hline $\begin{array}{l}\text { Free of dietary differences } \\
\text { other than fat? }\end{array}$ & High risk & Differences in fruit and vegetable advice \\
\hline
\end{tabular}

Rivellese 1994

\begin{tabular}{|c|c|}
\hline Methods & $\mathrm{RCT}$ \\
\hline Participants & $\begin{array}{l}\text { Adults with primary hyperlipoproteinaemia (Italy) } \\
\text { CVD risk: moderate } \\
\text { Intervention reduced fat: } 33 \text { randomised, } 27 \text { analysed } \\
\text { Intervention modified fat: } 30 \text { randomised, } 17 \text { analysed } \\
\text { Mean years in trial: reduced fat } 0.4 \text {, modified fat } 0.4 \\
\% \text { male: reduced fat } 82 \% \text {, modified fat } 63 \% \\
\text { Age, years: reduced fat } 47.4 \text { mean (sd } 10.3 \text { ), modified fat } 48.6 \text { (sd } 8.1 \text { ) }\end{array}$ \\
\hline Interventions & $\begin{array}{l}\text { Reduced fat vs Modified fat diet } \\
\text { Reduced fat aims: total fat } 25 \% \mathrm{E}, \text { SFA } 8 \% \mathrm{E} \text {, MUFA } 15 \% \text {, PUFA } 2 \% \text {, dietary chol }<300 \mathrm{mg} / \mathrm{d}, \mathrm{CHO} 58 \% \text {, } \\
\text { protein } 17 \% \mathrm{E} \text {, soluble fibre } 41 \mathrm{~g} / \mathrm{d} \\
\text { Modified fat aims: total fat } 38 \% \mathrm{E}, \mathrm{SFA}<10 \% \mathrm{E}, \text { MUFA } 20 \% \mathrm{E}, \text { PUFA } 10 \% \mathrm{E} \text {, dietary chol<300mg/d, CHO } \\
47 \% \mathrm{E} \text {, protein } 15 \% \mathrm{E} \text {, soluble fibre } 19 \mathrm{~g} / \mathrm{d} \\
\text { Reduced fat methods:seen monthly by dietitian and doctor, feedback based on } 7 \text { day food diary each } \\
\text { time } \\
\text { Modified fat methods: seen monthly by dietitian and doctor, feedback based on } 7 \text { day food diary each } \\
\text { time } \\
\text { Total fat intake (at 5-6 months): low fat } 27 \text { (sd unclear) } \% \mathrm{E}, \text { mod fat } 36 \text { (sd unclear) } \% \mathrm{E} \\
\text { Saturated fat intake (at 5-6 months): low fat } 6 \text { (sd unclear) } \% \mathrm{E} \text {, mod fat } 7 \text { (sd unclear) } \% \mathrm{E} \\
\text { Style: diet advice } \\
\text { Setting: community }\end{array}$ \\
\hline Outcomes & $\begin{array}{l}\text { Stated trial outcomes: metabolic effects } \\
\text { Data available on total mortality? yes (no events) } \\
\text { Cardiovascular mortality? yes (no events) }\end{array}$ \\
\hline
\end{tabular}


Rivellese 1994 (Continued)

Events available for combined cardiovascular events: total MI, cardiovascular deaths, stroke (no events)

Secondary outcomes: stroke, total and non-fatal MI (no events for any outcomes)

Tertiary outcomes: total, LDL and HDL cholesterol, TGs

Notes

\section{Risk of bias}

\begin{tabular}{|c|c|c|}
\hline Bias & Authors' judgement & Support for judgement \\
\hline $\begin{array}{l}\text { Random sequence genera- } \\
\text { tion (selection bias) }\end{array}$ & Low risk & $\begin{array}{l}\text { Following } 3 \text { or } 6 \text { weeks compliance with control diet run-in, stratified block } \\
\text { randomisation with tables of random numbers }\end{array}$ \\
\hline $\begin{array}{l}\text { Allocation concealment } \\
\text { (selection bias) }\end{array}$ & Unclear risk & Randomisation method not clearly described \\
\hline $\begin{array}{l}\text { Blinding (performance } \\
\text { bias and detection bias) } \\
\text { All outcomes }\end{array}$ & High risk & None \\
\hline $\begin{array}{l}\text { Incomplete outcome data } \\
\text { (attrition bias) } \\
\text { All outcomes }\end{array}$ & Unclear risk & $\begin{array}{l}\text { Unclear, deaths, cancer and CV events are drop-outs - unclear if any data miss- } \\
\text { ing }\end{array}$ \\
\hline $\begin{array}{l}\text { Selective reporting (re- } \\
\text { porting bias) }\end{array}$ & Low risk & Not relevant for primary and secondary outcomes as all trialists asked for data \\
\hline Other bias & Low risk & \\
\hline $\begin{array}{l}\text { Free of systematic differ- } \\
\text { ence in care? }\end{array}$ & Low risk & $\begin{array}{l}\text { Identical follow up. See Control and Intervention Methods in Interventions sec- } \\
\text { tion of the Table of Characteristics of Included Studies }\end{array}$ \\
\hline $\begin{array}{l}\text { Free of dietary differences } \\
\text { other than fat? }\end{array}$ & High risk & Some differences in soluble fibre intake \\
\hline
\end{tabular}

Rose 1965

\begin{tabular}{ll}
\hline Methods & RCT \\
\hline Participants & Men (?) with angina or following MI (UK) \\
CVD risk: high & Control: randomised 26, analysed 18 \\
Intervention - olive: randomised 28, analysed 12 \\
Intervention - corn: randomised 26, analysed 13 \\
Mean years in trial: control 1.7, olive 1.5, corn 1.5 \\
\% male: unclear (100\%?) \\
Age: mean control 58.8, olive 55.0, corn 52.6 (all <70) \\
Modified fat vs. usual diet \\
Control aims: usual diet \\
Intervention aims -olive: restrict dietary fat, plus $80 \mathrm{~g} /$ day olive oil provided \\
Interventions
\end{tabular}


Rose 1965 (Continued)

Control methods: usual physician care plus follow up clinic monthly, then every 2 months, no dietary fat advice or oil provided

Intervention methods: usual physician care plus follow up clinic monthly, then every 2 months, dietary fat advice plus oil provided

Total fat intake (at 18 months): corn 50.5 (sd unclear), olive 46.2 (sd unclear), cont 32.6 (sd unclear)\%E

Saturated fat intake: unclear

Style: diet advice \& supplement (oil)

Setting: community

Stated trial outcomes: cardiac events
Data available on total mortality? yes
Cardiovascular mortality? yes
Events available for combined cardiovascular events: cardiovascular deaths, non-fatal MI, angina,
stroke
Secondary outcomes: stroke (none), non-fatal and total MI
Tertiary outcomes: total cholesterol

\section{Notes}

\section{Risk of bias}

\begin{tabular}{|c|c|c|}
\hline Bias & Authors' judgement & Support for judgement \\
\hline $\begin{array}{l}\text { Random sequence genera- } \\
\text { tion (selection bias) }\end{array}$ & Low risk & "sealed envelopes" \\
\hline $\begin{array}{l}\text { Allocation concealment } \\
\text { (selection bias) }\end{array}$ & Unclear risk & Unclear if envelopes were opaque \\
\hline $\begin{array}{l}\text { Blinding (performance } \\
\text { bias and detection bias) } \\
\text { All outcomes }\end{array}$ & High risk & $\begin{array}{l}\text { Physician blinding: inadequate } \\
\text { Participant blinding: inadequate }\end{array}$ \\
\hline $\begin{array}{l}\text { Incomplete outcome data } \\
\text { (attrition bias) } \\
\text { All outcomes }\end{array}$ & High risk & Some lost to follow up by 2 years \\
\hline $\begin{array}{l}\text { Selective reporting (re- } \\
\text { porting bias) }\end{array}$ & Low risk & Not relevant for primary and secondary outcomes as all trialists asked for data \\
\hline Other bias & Low risk & \\
\hline $\begin{array}{l}\text { Free of systematic differ- } \\
\text { ence in care? }\end{array}$ & Low risk & $\begin{array}{l}\text { All received conventional treatments at the discretion of the physicians, all at- } \\
\text { tended a special follow up clinic. See Control and Intervention Methods in In- } \\
\text { terventions section of the Table of Characteristics of Included Studies }\end{array}$ \\
\hline $\begin{array}{l}\text { Free of dietary differences } \\
\text { other than fat? }\end{array}$ & Low risk & $\begin{array}{l}\text { See Control and Intervention Aims in Interventions section of the Table of } \\
\text { Characteristics of Included Studies }\end{array}$ \\
\hline
\end{tabular}


Sacks high protein 2009

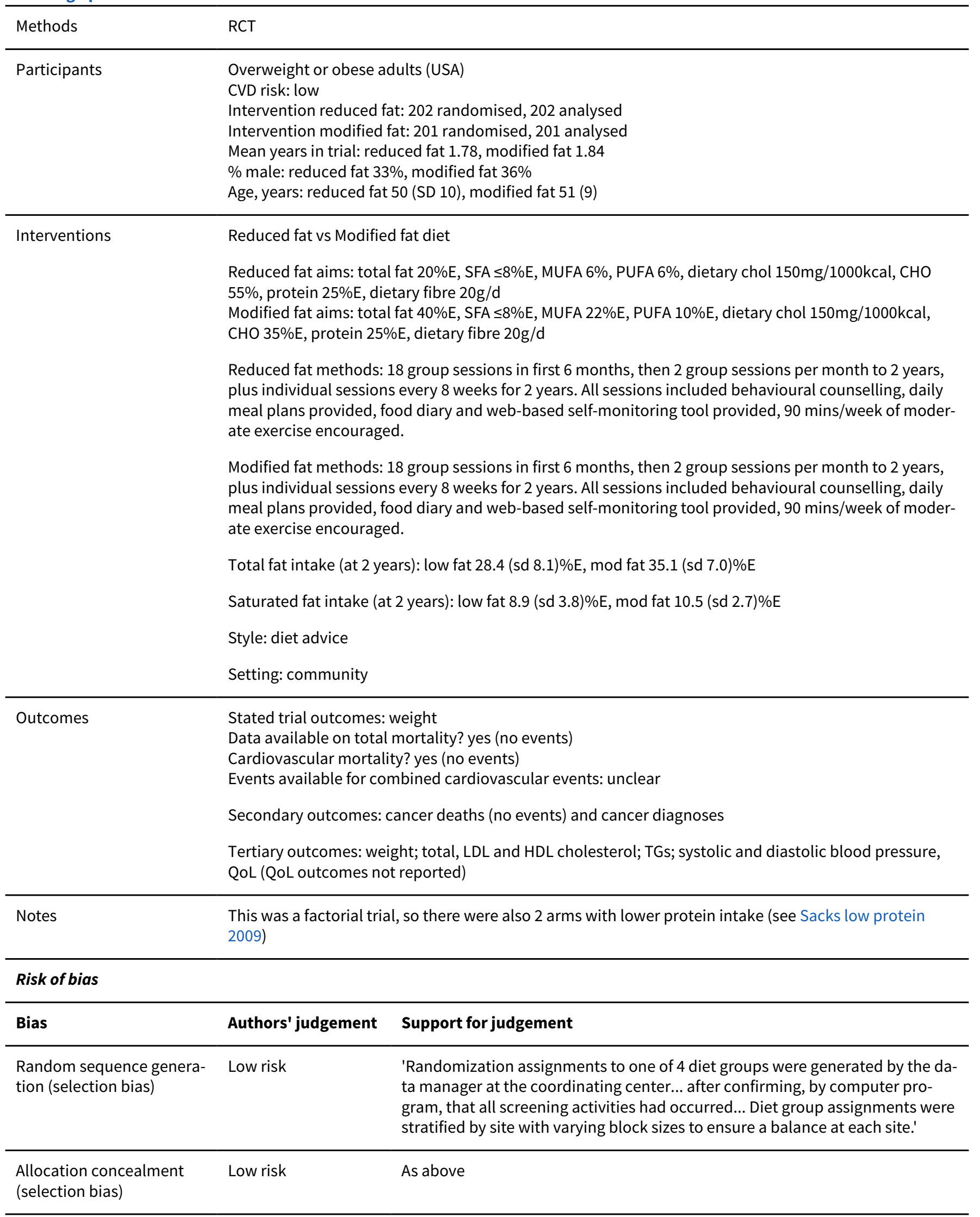


Sacks high protein 2009 (Continued)

Blinding (performance Low risk bias and detection bias)

All outcomes
'Blinding was established by naming each diet with colors, and using the same foods for each diet. Blinding and equipoise were strictly maintained by emphasizing to intervention staff and participants that each diet adheres to healthy principles, and each is advocated by certain experts to be superior for longterm weight-loss. Except for interventionists ... investigators and staff were kept blind to diet assignment of the participants. The trial adhered to established procedures to maintain separation between staff that take outcome measurements and staff that deliver the intervention. Staff members who obtained outcome measurements were not informed of the diet group assignment.... All investigators, staff and participants were kept masked to outcome measurements and trial results.'

\begin{tabular}{|c|c|c|}
\hline $\begin{array}{l}\text { Incomplete outcome data } \\
\text { (attrition bias) } \\
\text { All outcomes }\end{array}$ & Unclear risk & $\begin{array}{l}\text { Unclear for cardiovascular events. Yes for tertiary outcomes - intention to } \\
\text { treat analysis, imputing zero change from baseline for missing data (except } \\
\text { for weight which was more complex, assuming weight regain for missing da- } \\
\text { ta following weight loss, and zero change for those who had previously gained } \\
\text { weight). }\end{array}$ \\
\hline
\end{tabular}

\begin{tabular}{|c|c|c|}
\hline $\begin{array}{l}\text { Selective reporting (re- } \\
\text { porting bias) }\end{array}$ & Low risk & $\begin{array}{l}\text { ClinicalTrials.gov number NCT00072995. Protocol secondary outcomes (he- } \\
\text { patic and skeletal muscle, visceral fat, and quality of life) not yet reported in } \\
\text { full }\end{array}$ \\
\hline
\end{tabular}
full

\begin{tabular}{lll}
\hline Other bias & Low risk & \\
\hline $\begin{array}{l}\text { Free of systematic differ- } \\
\text { ence in care? }\end{array}$ & Low risk & Identical follow up for all groups \\
\hline $\begin{array}{l}\text { Free of dietary differences } \\
\text { other than fat? }\end{array}$ & Low risk & Clear dietary goal similarities across saturated fat, fibre, cholesterol etc. \\
\hline
\end{tabular}

Sacks low protein 2009

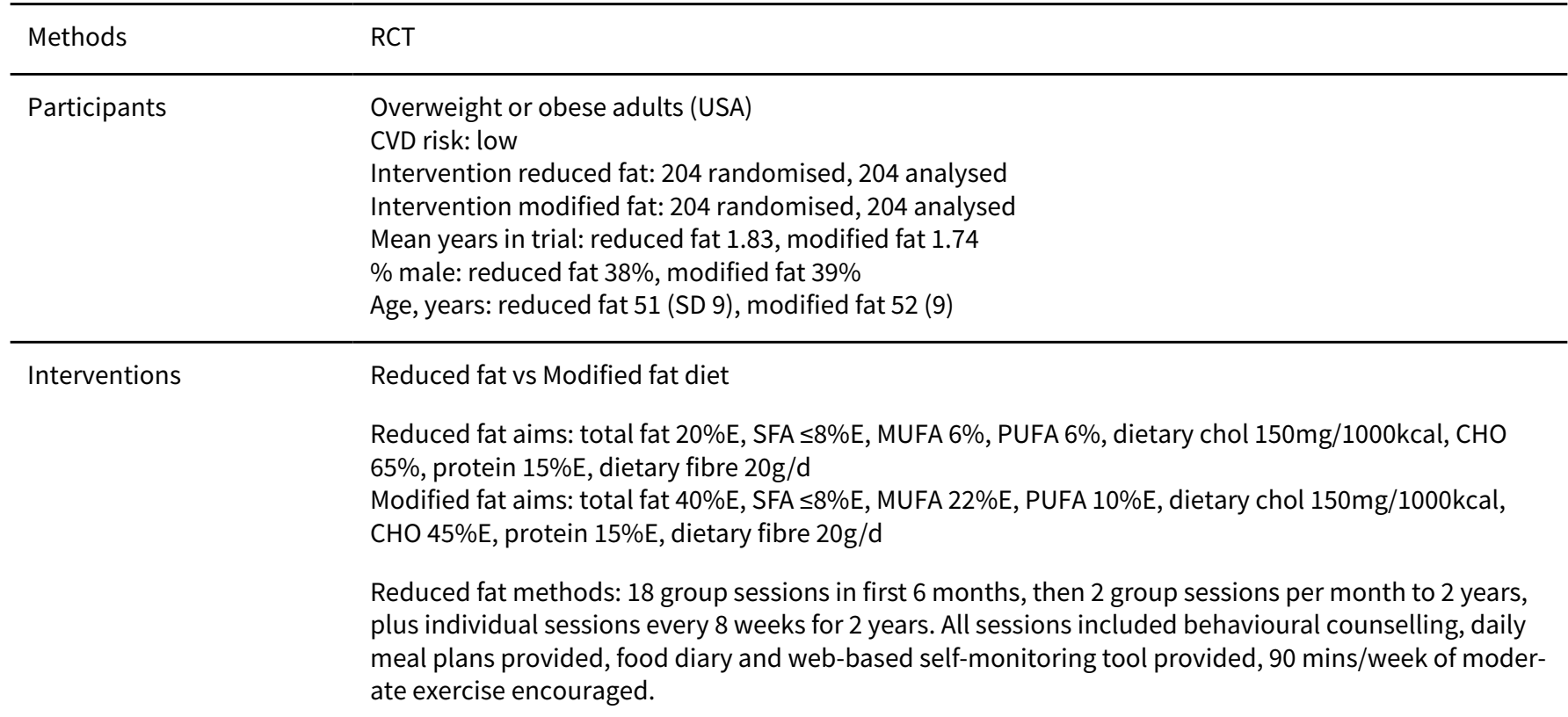

Modified fat methods: 18 group sessions in first 6 months, then 2 group sessions per month to 2 years, plus individual sessions every 8 weeks for 2 years. All sessions included behavioural counselling, daily 
Sacks low protein 2009 (Continued)

meal plans provided, food diary and web-based self-monitoring tool provided, 90 mins/week of moderate exercise encouraged.

Total fat intake (at 2 years): low fat 26.5 (sd 8.0)\%E, mod fat 33.3 (sd 8.2)\%E

Saturated fat intake (at 2 years): low fat 8.0 (sd 3.1)\%E, mod fat 9.8 (sd 3.3)\%E

Style: diet advice

Setting: community

\begin{tabular}{ll}
\hline Outcomes & Stated trial outcomes: weight \\
Data available on total mortality? yes (no events) \\
Cardiovascular mortality? yes (no events) \\
Events available for combined cardiovascular events: unclear \\
Secondary outcomes: cancer deaths (no events) and cancer diagnoses \\
Tertiary outcomes: weight; total, LDL and HDL cholesterol; TGs; systolic and diastolic blood pressure, \\
QoL (QoL outcomes not reported) \\
\hline Notes \\
This was a factorial trial, so there were also 2 arms with higher protein intake (see Sacks high protein \\
2009)
\end{tabular}

\section{Risk of bias}

Bias Authors' judgement Support for judgement

Random sequence genera- Low risk tion (selection bias)
'Randomization assignments to one of 4 diet groups were generated by the data manager at the coordinating center... after confirming, by computer program, that all screening activities had occurred... Diet group assignments were stratified by site with varying block sizes to ensure a balance at each site.'

Allocation concealment Low risk As above.

Blinding (performance Low risk
bias and detection bias) All outcomes

\begin{abstract}
'Blinding was established by naming each diet with colors, and using the same foods for each diet. Blinding and equipoise were strictly maintained by emphasizing to intervention staff and participants that each diet adheres to healthy principles, and each is advocated by certain experts to be superior for longterm weight-loss. Except for interventionists ... investigators and staff were kept blind to diet assignment of the participants. The trial adhered to established procedures to maintain separation between staff that take outcome measurements and staff that deliver the intervention. Staff members who obtained outcome measurements were not informed of the diet group assignment.... All investigators, staff and participants were kept masked to outcome measurements and trial results.'
\end{abstract}

Unclear for cardiovascular events. Yes for tertiary outcomes - intention to treat analysis, imputing zero change from baseline for missing data (except for weight which was more complex, assuming weight regain for missing data following weight loss, and zero change for those who had previously gained weight).

ClinicalTrials.gov number NCT00072995. Protocol secondary outcomes (hepatic and skeletal muscle, visceral fat, and quality of life) not yet reported in full
Selective reporting (re- Low risk porting bias)
Incomplete outcome data Unclear risk

(attrition bias)

All outcomes

Other bias Low risk


Sacks low protein 2009 (Continued)

Free of systematic differ- Low risk Identical follow up for all groups ence in care?

Free of dietary differences Low risk other than fat?

Clear dietary goal similarities across saturated fat, fibre, cholesterol etc.

Sarkkinen Fat Mod 1995

\begin{tabular}{ll}
\hline Methods & RCT (the 3 Kuopio trials share a common control group) \\
\hline Participants & Free-living people aged 30-60 with serum total cholesterol levels 6.5-8.0mmol/L (Finland) \\
CVD risk: moderate \\
Control: randomised 37, analysed 37 \\
Intervention Mono: randomised 41, analysed 41 \\
Mean years in trial: for both groups 0.5 \\
\% male: control 46, Mono 46 \\
Age: mean control 43.2, Mono 46.4 (all 30-60) \\
Modified fat vs usual diet \\
Control aims: advised total fat 38\%E, SFA <18\%E, MUFA 15\%E, PUFA <5\%E, rapeseed oil, butter and se- \\
mi-skimmed milk provided \\
Intervention aims Mono: total fat 38\%E, SFA <14\%E, MUFA 18\%E, PUFA <6\%E, rapeseed oil, rapeseed \\
spread and skimmed milk provided \\
Control and intervention methods: given written dietary instructions and a diet plan with checking and \\
reinforcement for 3 visits, then at 2, 6, 12, 18 and 26 weeks \\
Total fat intake (weeks 14-28): mod fat 35 (sd 5), cont 36 (sd 5)\%E \\
Saturated fat intake (weeks 14-28): mod fat 11 (sd 2), cont 15 (sd 2)\%E \\
Style: dietary advice \& supplement (food) \\
Setting: community
\end{tabular}

Outcomes Stated trial outcomes: lipids and blood pressure

Data available on total mortality? yes (no events)

Cardiovascular mortality? no

Events available for combined cardiovascular events: none

Secondary outcomes: none

Tertiary outcomes: BMI, total, LDL and HDL cholesterol, TG, BP

Notes

\section{Risk of bias}

\begin{tabular}{lll}
\hline Bias & Authors' judgement & Support for judgement \\
\hline $\begin{array}{l}\text { Random sequence genera- } \\
\text { tion (selection bias) }\end{array}$ & Low risk & $\begin{array}{l}\text { "randomisation stratified for men and women, singles and couples, random } \\
\text { number tables" }\end{array}$ \\
\hline $\begin{array}{l}\text { Allocation concealment } \\
\text { (selection bias) }\end{array}$ & Unclear risk & Randomisation method not clearly described \\
\hline
\end{tabular}


Sarkkinen Fat Mod 1995 (Continued)
Blinding (performance
High risk
Participants and researchers knew allocation
bias and detection bias)
All outcomes

\begin{tabular}{lll}
\hline $\begin{array}{l}\text { Incomplete outcome data } \\
\text { (attrition bias) } \\
\text { All outcomes }\end{array}$ & Unclear risk & $\begin{array}{l}\text { Unclear, deaths, cancer and CV events are drop-outs, trialists asked for data - } \\
\text { unclear if any data missing }\end{array}$ \\
\hline $\begin{array}{l}\text { Selective reporting (re- } \\
\text { porting bias) }\end{array}$ & Low risk & Not relevant for primary and secondary outcomes as all trialists asked for data \\
\hline Other bias & Low risk & Low risk \\
\hline $\begin{array}{l}\text { Free of systematic differ- } \\
\text { ence in care? }\end{array}$ & $\begin{array}{l}\text { Similar intensity and duration for both groups. See Control and Intervention } \\
\text { Methods in Interventions section of the Table of Characteristics of Included } \\
\text { Studies. }\end{array}$ \\
\hline $\begin{array}{l}\text { Free of dietary differences } \\
\text { other than fat? }\end{array}$ & Low risk & $\begin{array}{l}\text { See Control and Intervention Aims in Interventions section of the Table of } \\
\text { Characteristics of Included Studies }\end{array}$ \\
\hline
\end{tabular}

\section{Sarkkinen Red \& Mod 1995}

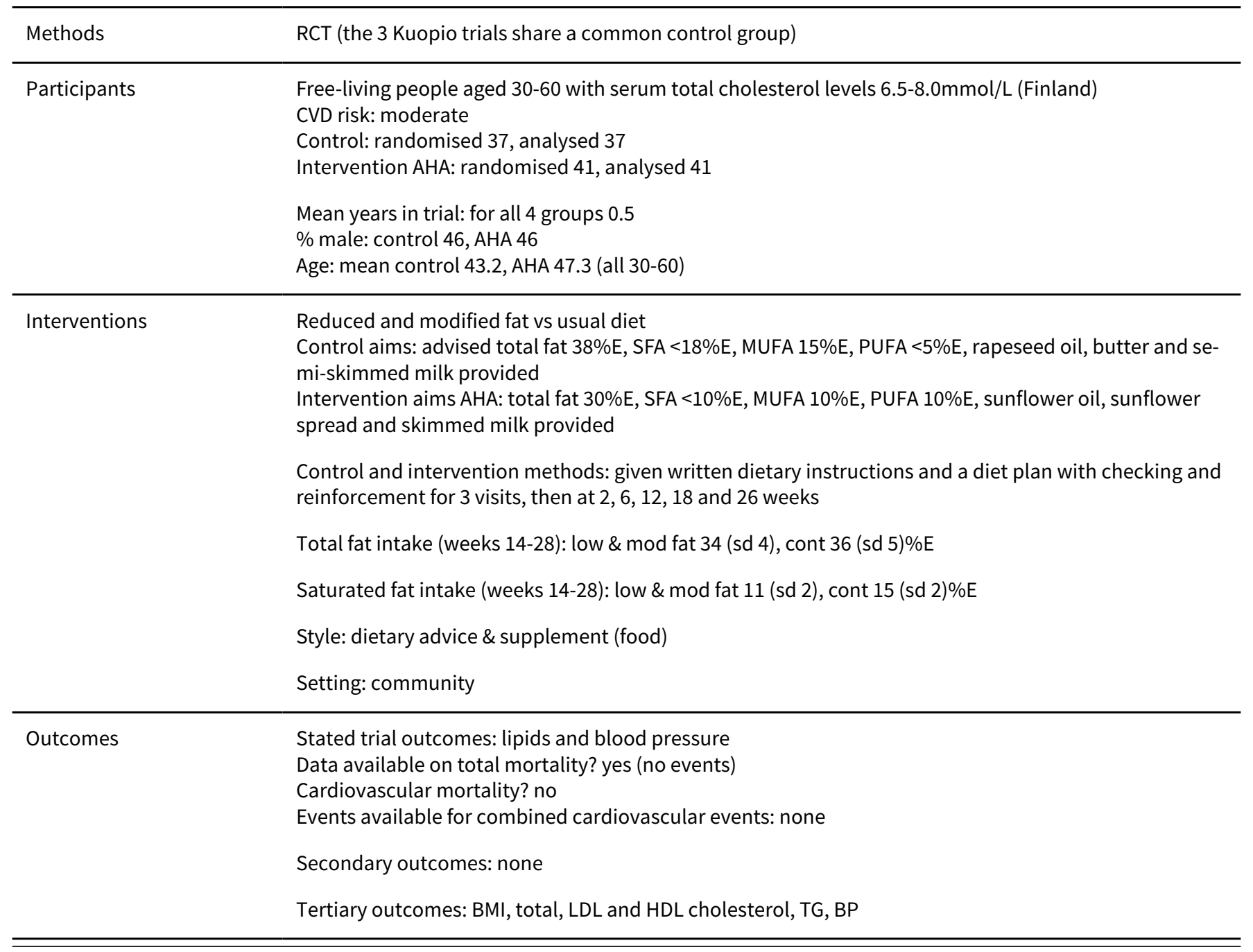


Sarkkinen Red \& Mod 1995 (Continued)

Notes

\section{Risk of bias}

\begin{tabular}{|c|c|c|}
\hline Bias & Authors' judgement & Support for judgement \\
\hline $\begin{array}{l}\text { Random sequence genera- } \\
\text { tion (selection bias) }\end{array}$ & Low risk & $\begin{array}{l}\text { "randomisation stratified for men and women, singles and couples, random } \\
\text { number tables" }\end{array}$ \\
\hline $\begin{array}{l}\text { Allocation concealment } \\
\text { (selection bias) }\end{array}$ & Unclear risk & Randomisation method not clearly described \\
\hline $\begin{array}{l}\text { Blinding (performance } \\
\text { bias and detection bias) } \\
\text { All outcomes }\end{array}$ & High risk & Participants and researchers knew allocation \\
\hline $\begin{array}{l}\text { Incomplete outcome data } \\
\text { (attrition bias) } \\
\text { All outcomes }\end{array}$ & Unclear risk & $\begin{array}{l}\text { Unclear, deaths, cancer and CV events are drop-outs, trialists asked for data - } \\
\text { unclear if any data missing }\end{array}$ \\
\hline $\begin{array}{l}\text { Selective reporting (re- } \\
\text { porting bias) }\end{array}$ & Low risk & Not relevant for primary and secondary outcomes as all trialists asked for data \\
\hline Other bias & Low risk & \\
\hline $\begin{array}{l}\text { Free of systematic differ- } \\
\text { ence in care? }\end{array}$ & Low risk & $\begin{array}{l}\text { Similar intensity and duration in both groups. See Control and Intervention } \\
\text { Methods in Interventions section of the Table of Characteristics of Included } \\
\text { Studies }\end{array}$ \\
\hline $\begin{array}{l}\text { Free of dietary differences } \\
\text { other than fat? }\end{array}$ & Low risk & $\begin{array}{l}\text { See Control and Intervention Aims in Interventions section of the Table of } \\
\text { Characteristics of Included Studies }\end{array}$ \\
\hline
\end{tabular}

\section{Sarkkinen Red Fat 1995}

\begin{tabular}{ll}
\hline Methods & RCT (the 3 Kuopio trials share a common control group) \\
\hline Participants & Free-living people aged 30-60 with serum total cholesterol levels 6.5-8.0mmol/L (Finland) \\
CVD risk: moderate \\
Control: randomised 37, analysed 37 \\
Intervention low fat: randomised 40, analysed 40 \\
Mean years in trial: for both groups 0.5 \\
\% male: control 46, low fat 48 \\
Age: mean control 43.2, low fat 45.8 (all 30-60) \\
Reduced fat vs usual diet (low fat vs control) \\
Control aims: advised total fat 38\%E, SFA <18\%E, MUFA 15\%E, PUFA <5\%E, rapeseed oil, butter and se- \\
mi-skimmed milk provided \\
Intervention aims low fat: total fat 28-30\%E, SFA <14\%E, MUFA 10\%E, PUFA 4\%E, butter and rapeseed \\
spread and skimmed milk provided \\
Control and intervention methods: given written dietary instructions and a diet plan with checking and \\
reinforcement for 3 visits, then at 2, 6, 12, 18 and 26 weeks \\
Total fat intake (weeks 14-28): low fat 31 (sd 5), cont 36 (sd 5)\%E \\
Saturated fat intake (weeks 14-28): low fat 12 (sd 2), cont 15 (sd 2)\%E
\end{tabular}


Sarkkinen Red Fat 1995 (Continued)

Style: dietary advice \& supplement (food)

Setting: community

$\begin{array}{ll}\text { Sutcomes } & \text { Stated trial outcomes: lipids and blood pressure } \\ \text { Data available on total mortality? yes (no events) } \\ \text { Cardiovascular mortality? no } \\ \text { Events available for combined cardiovascular events: none } \\ \text { Secondary outcomes: none } \\ \text { Tertiary outcomes: BMI, total, LDL and HDL cholesterol, TG, BP }\end{array}$

Notes

\section{Risk of bias}

\begin{tabular}{|c|c|c|}
\hline Bias & Authors' judgement & Support for judgement \\
\hline $\begin{array}{l}\text { Random sequence genera- } \\
\text { tion (selection bias) }\end{array}$ & Low risk & $\begin{array}{l}\text { "randomisation stratified for men and women, singles and couples, random } \\
\text { number tables" }\end{array}$ \\
\hline $\begin{array}{l}\text { Allocation concealment } \\
\text { (selection bias) }\end{array}$ & Unclear risk & Randomisation method not clearly described \\
\hline $\begin{array}{l}\text { Blinding (performance } \\
\text { bias and detection bias) } \\
\text { All outcomes }\end{array}$ & High risk & Participants and researchers knew allocation \\
\hline $\begin{array}{l}\text { Incomplete outcome data } \\
\text { (attrition bias) } \\
\text { All outcomes }\end{array}$ & Unclear risk & $\begin{array}{l}\text { Unclear, deaths, cancer and CV events are drop-outs, trialists asked for data - } \\
\text { unclear if any data missing }\end{array}$ \\
\hline $\begin{array}{l}\text { Selective reporting (re- } \\
\text { porting bias) }\end{array}$ & Low risk & Not relevant for primary and secondary outcomes as all trialists asked for data \\
\hline Other bias & Low risk & \\
\hline $\begin{array}{l}\text { Free of systematic differ- } \\
\text { ence in care? }\end{array}$ & Low risk & $\begin{array}{l}\text { Similar intensity and duration in both groups. See Control and Intervention } \\
\text { Methods in Interventions section of the Table of Characteristics of Included } \\
\text { Studies }\end{array}$ \\
\hline $\begin{array}{l}\text { Free of dietary differences } \\
\text { other than fat? }\end{array}$ & Low risk & $\begin{array}{l}\text { See Control and Intervention Aims in Interventions section of the Table of } \\
\text { Characteristics of Included Studies }\end{array}$ \\
\hline
\end{tabular}

\section{Sarkkinen Red vs Mod1995}

\begin{tabular}{|c|c|}
\hline Methods & RCT \\
\hline Participants & $\begin{array}{l}\text { Free-living people aged } 30-60 \text { with serum total cholesterol levels } 6.5-8.0 \mathrm{mmol} / \mathrm{L} \text { (Finland) } \\
\text { CVD risk: moderate } \\
\text { Intervention Mono: randomised } 41 \text {, analysed } 41\end{array}$ \\
\hline & $\begin{array}{l}\text { Intervention low fat: randomised } 40 \text {, analysed } 40 \\
\text { Mean years in trial: for both groups } 0.5 \\
\% \text { male: mono } 46 \text {, low fat } 48 \\
\text { Age: mean Mono } 46.4 \text { (all } 30-60 \text { ), low fat } 45.8 \text { (all 30-60) }\end{array}$ \\
\hline
\end{tabular}


Sarkkinen Red vs Mod1995 (Continued)

Reduced fat vs modified fat (low fat vs Mono)
Intervention aims Mono: total fat 38\%E, SFA <14\%E, MUFA 18\%E, PUFA
spread and skimmed milk provided
Intervention aims low fat: total fat 28-30\%E, SFA <14\%E, MUFA 10\%E, PUFA
spread and skimmed milk provided
Both intervention methods: given written dietary instructions and a diet
forcement for 3 visits, then at 2, 6, 12, 18 and 26 weeks
Total fat intake (weeks 14-28): mod fat 35 (sd 5), low fat 31 (sd 5)\%E
Saturated fat intake (weeks 14-28): mod fat 11 (sd 2), low fat 12 (sd 2)\%E
Style: dietary advice \& supplement (food)
Setting: community

$\begin{array}{ll}\text { Outcomes } & \text { Stated trial outcomes: lipids and blood pressure } \\ \text { Data available on total mortality? yes (no events) } \\ \text { Cardiovascular mortality? no } \\ \text { Events available for combined cardiovascular events: none } \\ \text { Secondary outcomes: none } \\ \text { Tertiary outcomes: BMI, total, LDL and HDL cholesterol, TG, BP }\end{array}$

Notes

\section{Risk of bias}

\begin{tabular}{|c|c|c|}
\hline Bias & Authors' judgement & Support for judgement \\
\hline $\begin{array}{l}\text { Random sequence genera- } \\
\text { tion (selection bias) }\end{array}$ & Low risk & $\begin{array}{l}\text { "randomisation stratified for men and women, singles and couples, random } \\
\text { number tables" }\end{array}$ \\
\hline $\begin{array}{l}\text { Allocation concealment } \\
\text { (selection bias) }\end{array}$ & Unclear risk & Randomisation method not clearly described \\
\hline $\begin{array}{l}\text { Blinding (performance } \\
\text { bias and detection bias) } \\
\text { All outcomes }\end{array}$ & High risk & Participants and researchers knew allocation \\
\hline $\begin{array}{l}\text { Incomplete outcome data } \\
\text { (attrition bias) } \\
\text { All outcomes }\end{array}$ & Unclear risk & $\begin{array}{l}\text { Unclear, deaths, cancer and CV events are drop-outs, trialists asked for data - } \\
\text { unclear if any data missing }\end{array}$ \\
\hline $\begin{array}{l}\text { Selective reporting (re- } \\
\text { porting bias) }\end{array}$ & Low risk & Not relevant for primary and secondary outcomes as all trialists asked for data \\
\hline Other bias & Low risk & \\
\hline $\begin{array}{l}\text { Free of systematic differ- } \\
\text { ence in care? }\end{array}$ & Low risk & $\begin{array}{l}\text { Similar intensity and duration in both groups. See Control and Intervention } \\
\text { Methods in Interventions section of the Table of Characteristics of Included } \\
\text { Studies }\end{array}$ \\
\hline $\begin{array}{l}\text { Free of dietary differences } \\
\text { other than fat? }\end{array}$ & Low risk & $\begin{array}{l}\text { See Control and Intervention Aims in Interventions section of the Table of } \\
\text { Characteristics of Included Studies }\end{array}$ \\
\hline
\end{tabular}


Seppelt 1996

\begin{tabular}{ll}
\hline Methods & RCT \\
\hline Participants & Women with BMI 24-29 (Germany) \\
CVD risk: low & Control: randomised 35, analysed 32 \\
Intervention: randomised 35, analysed 35 & Mean years in trial: control 0.7, intervention 0.8 \\
\% male: 0 & Age: mean control 46, intervention 48 (all 40-60) \\
\hline Interventions & Reduced fat vs usual diet \\
& Control aims: advice to buy foods from trial shop, usual fat foods supplied \\
Intervention aims: advice to buy foods from trial shop, low fat foods supplied \\
Control methods: trial shop provided ad libitum usual fat foods \\
Intervention methods: trial shop provided ad libitum low fat foods \\
Total fat intake (at 9 months): low fat 35.1 (sd unclear), cont 35.5 (sd unclear)\%E \\
Saturated fat intake: unclear \\
Style: food provided \\
Setting: community \\
\hline
\end{tabular}

\begin{tabular}{ll}
\hline Outcomes & Stated trial outcomes: weight \\
Data available on total mortality? yes (no events) \\
Cardiovascular mortality? yes (no events) \\
Events available for combined cardiovascular events: cardiovascular deaths, non-fatal MI, stroke (no \\
events) \\
Secondary outcomes: total and non-fatal MI, stroke, cancer deaths (no events for any outcomes) \\
Tertiary outcomes: weight, total, LDL and HDL cholesterol, TG
\end{tabular}

Notes

\section{Risk of bias}

\begin{tabular}{lll}
\hline Bias & Authors' judgement & Support for judgement \\
\hline $\begin{array}{l}\text { Random sequence genera- } \\
\text { tion (selection bias) }\end{array}$ & Low risk & "participants assigned to a random number, later numbers sorted \& assigned" \\
\hline $\begin{array}{l}\text { Allocation concealment } \\
\text { (selection bias) }\end{array}$ & Unclear risk & Randomisation method not clearly described \\
\hline $\begin{array}{l}\text { Blinding (performance } \\
\text { bias and detection bias) } \\
\text { All outcomes }\end{array}$ & Unclear risk & Blinding unclear for participants and researchers \\
\hline $\begin{array}{l}\text { Incomplete outcome data } \\
\text { (attrition bias) } \\
\text { All outcomes }\end{array}$ & Unclear risk & $\begin{array}{l}\text { Unclear, deaths, cancer and CV events are drop-outs, trialists asked for data - } \\
\text { unclear if any data missing }\end{array}$ \\
\hline
\end{tabular}


Seppelt 1996 (Continued)

Selective reporting (reporting bias)

Other bias Low risk

Free of systematic differ- Low risk ence in care?

Low risk

Not relevant for primary and secondary outcomes as all trialists asked for data
Trial shop for both groups. See Control and Intervention Methods in Interventions section of the Table of Characteristics of Included Studies
Free of dietary differences Low risk other than fat?

\section{Simon 1997}

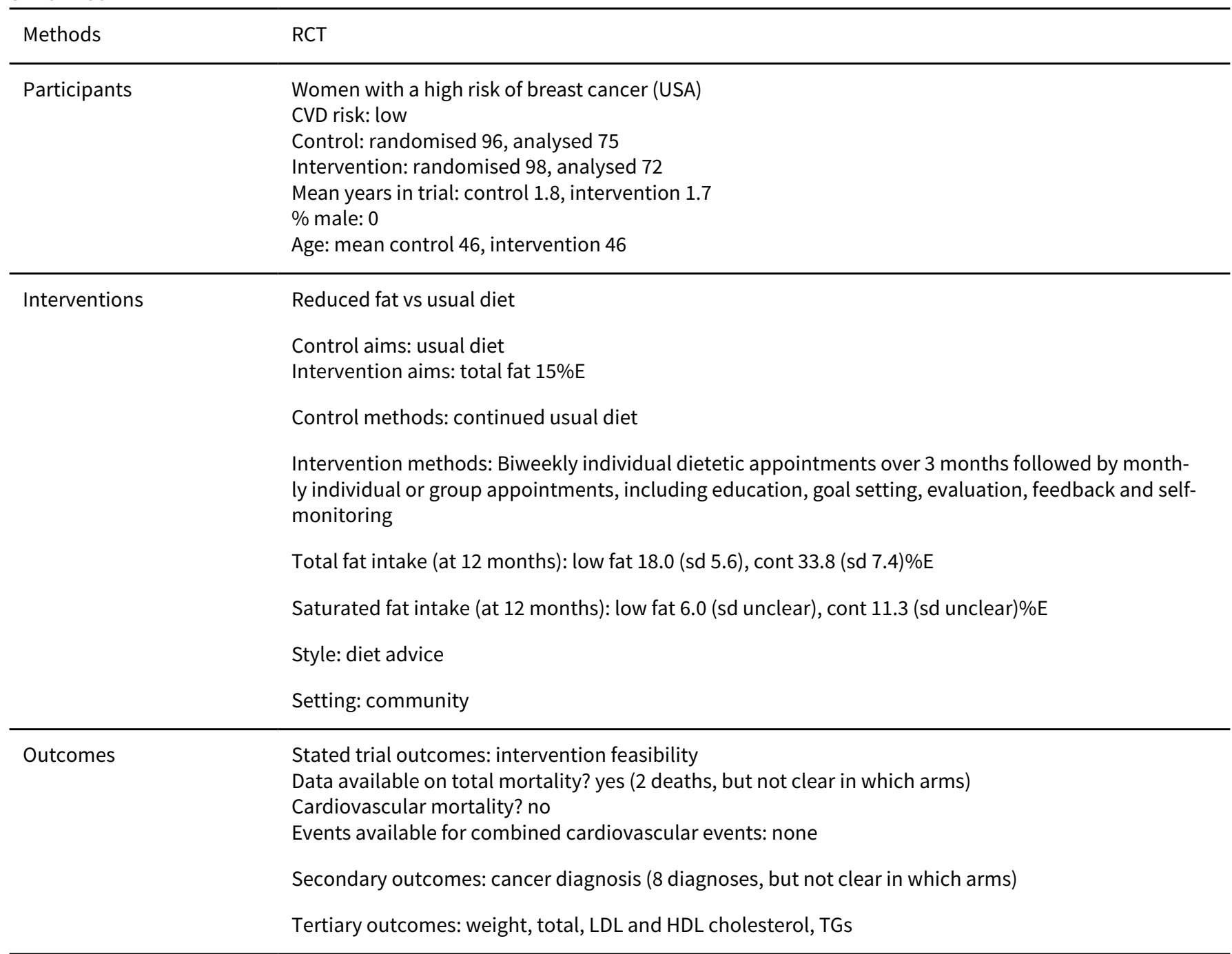

\footnotetext{
Notes
}

\section{Risk of bias}

Bias

Authors' judgement Support for judgement 
Simon 1997 (Continued)

Random sequence genera- Low risk Stratified by age and randomised (block size 2)
tion (selection bias)

Allocation concealment Unclear risk
(selection bias)

\begin{tabular}{lll}
\hline $\begin{array}{l}\text { Blinding (performance } \\
\text { bias and detection bias) } \\
\text { All outcomes }\end{array}$ & High risk & Participants knew their allocation, unclear whether physicians did \\
\hline $\begin{array}{l}\text { Incomplete outcome data } \\
\text { (attrition bias) }\end{array}$ & Unclear risk & $\begin{array}{l}\text { Unclear, deaths, cancer and CV events are drop-outs - unclear if any data miss- } \\
\text { All outcomes }\end{array}$ \\
\hline
\end{tabular}

\begin{tabular}{lll}
\hline $\begin{array}{l}\text { Selective reporting (re- } \\
\text { porting bias) }\end{array}$ & Low risk & Not relevant for primary and secondary outcomes as all trialists asked for data \\
\hline Other bias & Low risk & \\
\hline $\begin{array}{l}\text { Free of systematic differ- } \\
\text { ence in care? }\end{array}$ & High risk & $\begin{array}{l}\text { Very different contact time with dietitian, but medical appointments same in } \\
\text { both groups. See Control and Intervention Methods in Interventions section of } \\
\text { the Table of Characteristics of Included Studies }\end{array}$ \\
\hline
\end{tabular}

Free of dietary differences Low risk other than fat?

See Control and Intervention Aims in Interventions section of the Table of Characteristics of Included Studies

Sondergaard 2003

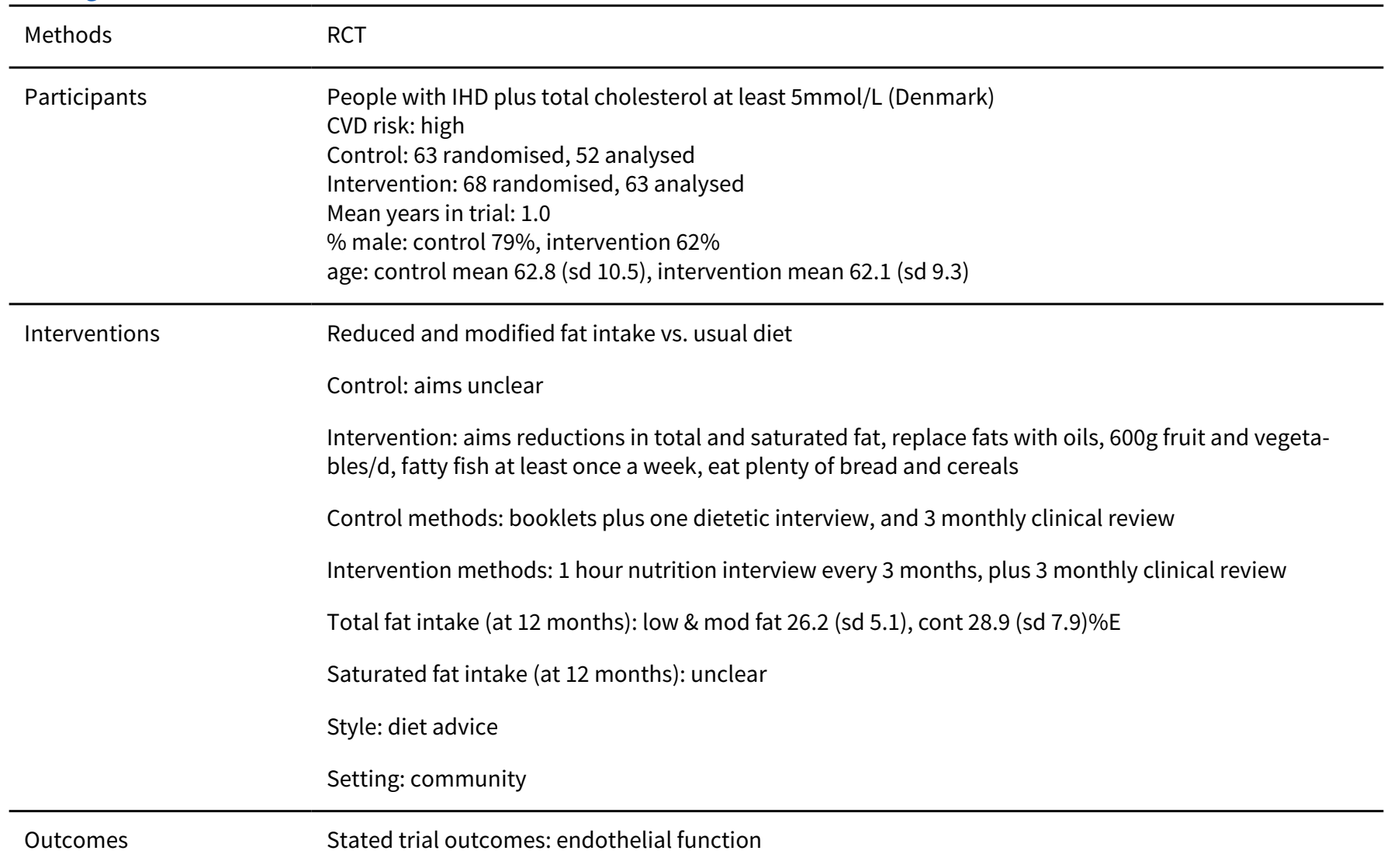


Sondergaard 2003 (Continued)

Data available on total mortality? yes

Cardiovascular mortality? yes

Events available for combined cardiovascular events: total MI, cardiovascular deaths, stroke, angina, heart failure, $\mathrm{CABG}, \mathrm{PCl}$ and atrial fibrillation.

Secondary outcomes: cancer diagnoses and deaths, stroke, total MI

Tertiary outcomes: total, LDL and HDL cholesterol, TG

\section{Notes}

\section{Risk of bias}

\begin{tabular}{|c|c|c|}
\hline Bias & Authors' judgement & Support for judgement \\
\hline $\begin{array}{l}\text { Random sequence genera- } \\
\text { tion (selection bias) }\end{array}$ & High risk & "randomised in unblinded 1:1 fashion" \\
\hline $\begin{array}{l}\text { Allocation concealment } \\
\text { (selection bias) }\end{array}$ & High risk & \\
\hline $\begin{array}{l}\text { Blinding (performance } \\
\text { bias and detection bias) } \\
\text { All outcomes }\end{array}$ & High risk & Participants aware of allocation, unclear about others \\
\hline $\begin{array}{l}\text { Incomplete outcome data } \\
\text { (attrition bias) } \\
\text { All outcomes }\end{array}$ & Unclear risk & $\begin{array}{l}\text { Unclear, deaths, cancer and CV events are drop-outs - unclear if any data miss- } \\
\text { ing }\end{array}$ \\
\hline $\begin{array}{l}\text { Selective reporting (re- } \\
\text { porting bias) }\end{array}$ & Low risk & Not relevant for primary and secondary outcomes as all trialists asked for data \\
\hline Other bias & Low risk & \\
\hline $\begin{array}{l}\text { Free of systematic differ- } \\
\text { ence in care? }\end{array}$ & High risk & $\begin{array}{l}\text { Additional dietetic time for intervention group. See Control and Intervention } \\
\text { Methods in Interventions section of the Table of Characteristics of Included } \\
\text { Studies }\end{array}$ \\
\hline $\begin{array}{l}\text { Free of dietary differences } \\
\text { other than fat? }\end{array}$ & High risk & $\begin{array}{l}\text { Additional dietary advice for intervention group (fruit, vegetables, fish, cere- } \\
\text { als). }\end{array}$ \\
\hline
\end{tabular}

STARS 1992

\begin{tabular}{ll}
\hline Methods & RCT \\
\hline Participants & Men with angina referred for angiography (UK) \\
CVD risk: high & Control: unclear randomised (30?), analysed 24 \\
& Intervention: unclear how many randomised (30?), analysed 26 \\
& Mean years in trial: control 2.9, intervention 3.0 \\
& $\%$ male: 100 \\
& age: mean control 53.9, intervention 48.9 (all <66)
\end{tabular}

Interventions

Reduced and modified fat diet vs usual diet

Control aims: no diet intervention but advised to lose weight if $\mathrm{BMI}>25$ 
Intervention aims: total fat 27\%E, SFA 8-10\%E, omega-3 and omega-6 PUFA 8\%E, increase in plant-derived soluble fibre, dietary cholesterol $100 \mathrm{mg} / 1000 \mathrm{kcal}$, advised to lose weight if $\mathrm{BMI}>25$

Control methods: usual care but no formal dietetic counselling

Intervention methods: Usual care plus dietetic assessment of diet and advice

Total fat intake (through study): int 27 (sd unclear), cont 37 (sd unclear)\%E

Saturated fat intake (through study): int 9 (sd unclear), cont 17 (sd unclear)\%E

Style: diet advice

Setting: community

Stated trial outcomes: angiography
Data available on total mortality? yes
Cardiovascular mortality? yes
Events available for combined cardiovascular events: cardiovascular deaths, non-fatal MI, angina,
stroke, CABG, angioplasty
Secondary outcomes: cancer deaths (none), stroke, total MI
Tertiary outcomes: total, HDL, LDL cholesterol, TGs (weight and BP "remained similar" but were not re-
ported)

\section{Notes}

\section{Risk of bias}

\begin{tabular}{|c|c|c|}
\hline Bias & Authors' judgement & Support for judgement \\
\hline $\begin{array}{l}\text { Random sequence genera- } \\
\text { tion (selection bias) }\end{array}$ & Low risk & "blinded random cards issued centrally by statistician advisor" \\
\hline $\begin{array}{l}\text { Allocation concealment } \\
\text { (selection bias) }\end{array}$ & Low risk & \\
\hline $\begin{array}{l}\text { Blinding (performance } \\
\text { bias and detection bias) } \\
\text { All outcomes }\end{array}$ & High risk & $\begin{array}{l}\text { Physician blinding: unclear } \\
\text { Participant blinding: inadequate }\end{array}$ \\
\hline $\begin{array}{l}\text { Incomplete outcome data } \\
\text { (attrition bias) } \\
\text { All outcomes }\end{array}$ & Unclear risk & $\begin{array}{l}\text { Unclear, deaths, cancer and CV events are drop-outs - unclear if any data miss- } \\
\text { ing }\end{array}$ \\
\hline $\begin{array}{l}\text { Selective reporting (re- } \\
\text { porting bias) }\end{array}$ & Low risk & Not relevant for primary and secondary outcomes as all trialists asked for data \\
\hline Other bias & Low risk & \\
\hline $\begin{array}{l}\text { Free of systematic differ- } \\
\text { ence in care? }\end{array}$ & High risk & $\begin{array}{l}\text { Usual care in both groups, dietetic counselling only in the intervention group. } \\
\text { See Control and Intervention Methods in Interventions section of the Table of } \\
\text { Characteristics of Included Studies }\end{array}$ \\
\hline $\begin{array}{l}\text { Free of dietary differences } \\
\text { other than fat? }\end{array}$ & High risk & Intervention group also encouraged to increase plant derived soluble fibre \\
\hline
\end{tabular}


Strychar 2009

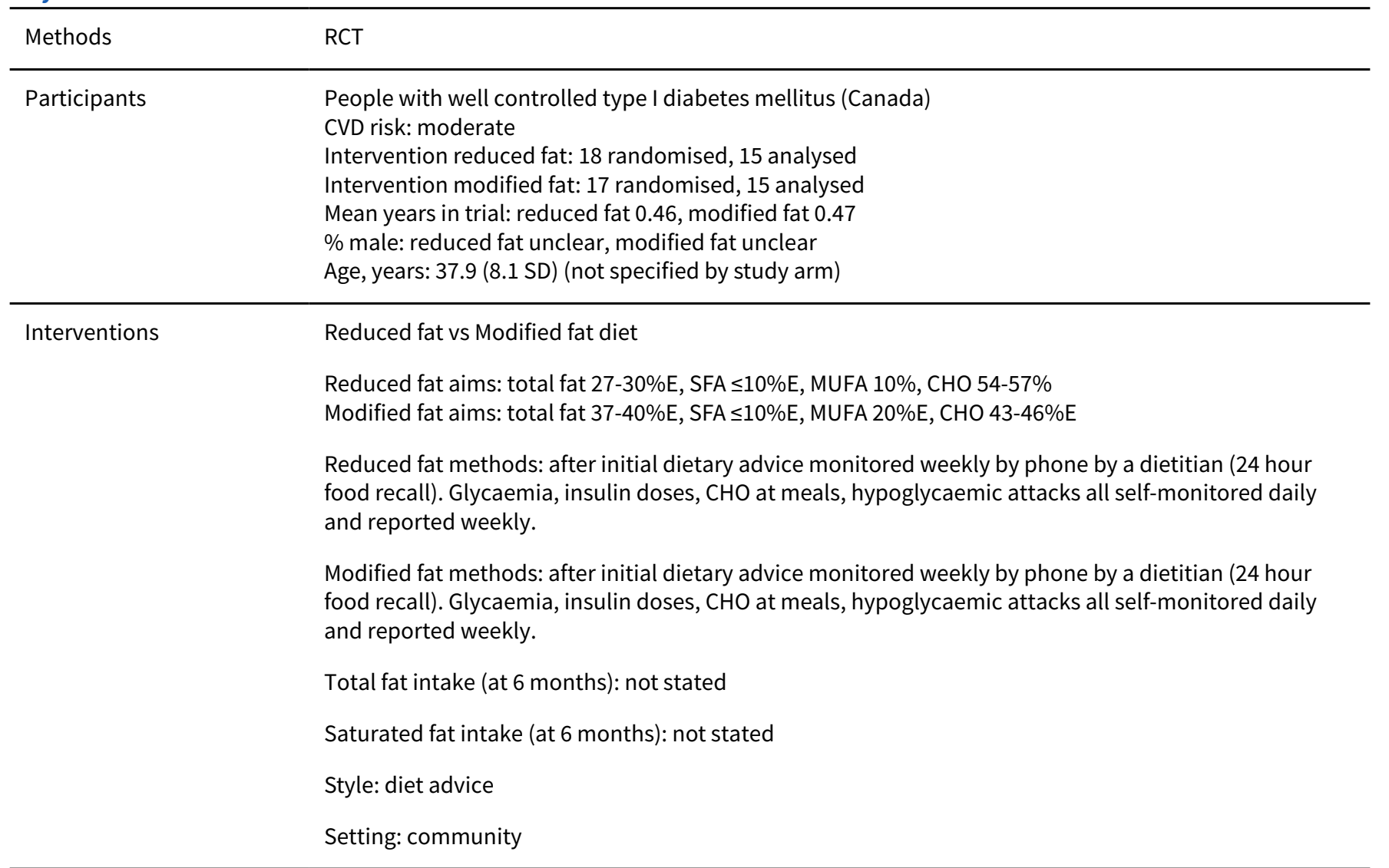

Stated trial outcomes: Triglycerides and other CVD risk factors
Data available on total mortality? yes (no events)
Cardiovascular mortality? yes (no events)
Events available for combined cardiovascular events: none
Secondary outcomes: cancer deaths (no events) and cancer diagnoses (no events)
Tertiary outcomes: weight; BMI; total, LDL and HDL cholesterol; TGs; systolic and diastolic blood pres-
sure

Notes

\section{Risk of bias}

\begin{tabular}{lll}
\hline Bias & Authors' judgement & Support for judgement \\
\hline $\begin{array}{l}\text { Random sequence genera- } \\
\text { tion (selection bias) }\end{array}$ & Low risk & "randomly assigned" \\
\hline $\begin{array}{l}\text { Allocation concealment } \\
\text { (selection bias) }\end{array}$ & Unclear risk & No details provided \\
\hline $\begin{array}{l}\text { Blinding (performance } \\
\text { bias and detection bias) } \\
\text { All outcomes }\end{array}$ & High risk & $\begin{array}{l}\text { No details provided, but participants had to make decisions about what they } \\
\text { ate }\end{array}$ \\
\hline
\end{tabular}

Incomplete outcome data High risk Data reported for those who completed
(attrition bias)

All outcomes 
Strychar 2009 (Continued)

Selective reporting (re- Unclear risk Unclear porting bias)

\begin{tabular}{lll}
\hline Other bias & Low risk & \\
\hline $\begin{array}{l}\text { Free of systematic differ- } \\
\text { ence in care? }\end{array}$ & Low risk & Similar intervention in both groups \\
\hline
\end{tabular}

Free of dietary differences Low risk Focus on fat and $\mathrm{CHO}$ intake

other than fat?

Sydney Diet-Heart 1978

\begin{tabular}{ll}
\hline Methods & RCT \\
\hline Participants & Men with previous MI (Australia) \\
CVD risk: high \\
Control: randomised 237, analysed 221 at 2 years \\
Intervention: randomised 221, analysed 205 at 2 years \\
Mean years in trial: control 4.3, intervention 4.3 \\
\% male: 100 \\
Age: mean control 49.1 (sd 6.5), intervention 48.7 (sd 6.8) \\
\hline
\end{tabular}

Interventions

Modified fat diet vs usual diet

Control aims: reduction in energy if overweight, no other specific dietary advice, allowed to use PUFA margarine instead of butter

Intervention aims: SFA $10 \% \mathrm{E}$, PUFA $15 \% \mathrm{E}$, reduction in energy if overweight, dietary chol $<300 \mathrm{mg} /$ day

Control methods: no specific dietary instruction (except re weight)

Intervention methods: advised and tutored individually, diet assessed 3 times in first year and twice annually thereafter

Total fat intake ("during follow up"): mod fat 38.3 (sd 5.9), cont 38.1 (sd 5.4)\%E

Saturated fat intake ("during follow up"): mod fat 9.8 (sd 2.6), cont 13.5 (sd 3.2)\%E

Style: diet advice

Setting: community

Outcomes Stated trial outcomes: cardiovascular mortality and morbidity

Data available on total mortality? yes

Cardiovascular mortality? no (overall number given, but not by intervention group)

Events available for combined cardiovascular events: none

Secondary outcomes: none

Tertiary outcomes: total cholesterol, TG

Notes

\section{Risk of bias}

Bias

Authors' judgement Support for judgement 
Sydney Diet-Heart 1978 (Continued)

\begin{tabular}{lll}
$\begin{array}{l}\text { Random sequence genera- } \\
\text { tion (selection bias) }\end{array}$ & Low risk & "random numbers" \\
\hline $\begin{array}{l}\text { Allocation concealment } \\
\text { (selection bias) }\end{array}$ & Unclear risk & Randomisation method not clearly described \\
\hline
\end{tabular}

\begin{tabular}{|c|c|c|}
\hline $\begin{array}{l}\text { Blinding (performance } \\
\text { bias and detection bias) }\end{array}$ & High risk & $\begin{array}{l}\text { Physician blinding: adequate } \\
\text { participant blinding: inadequate }\end{array}$ \\
\hline
\end{tabular}

\begin{tabular}{|c|c|c|}
\hline $\begin{array}{l}\text { Incomplete outcome data } \\
\text { (attrition bias) } \\
\text { All outcomes }\end{array}$ & Low risk & Survival analysis used \\
\hline $\begin{array}{l}\text { Selective reporting (re- } \\
\text { porting bias) }\end{array}$ & Low risk & Not relevant for primary and secondary outcomes as all trialists asked for data \\
\hline Other bias & Low risk & \\
\hline $\begin{array}{l}\text { Free of systematic differ- } \\
\text { ence in care? }\end{array}$ & High risk & $\begin{array}{l}\text { Advice and follow up in intervention group, not in control. See Control and In- } \\
\text { tervention Methods in Interventions section of the Table of Characteristics of } \\
\text { Included Studies }\end{array}$ \\
\hline
\end{tabular}

$\begin{array}{ll}\begin{array}{l}\text { Free of dietary differences } \\ \text { other than fat? }\end{array} & \text { Low risk }\end{array}$

THIS DIET 2008

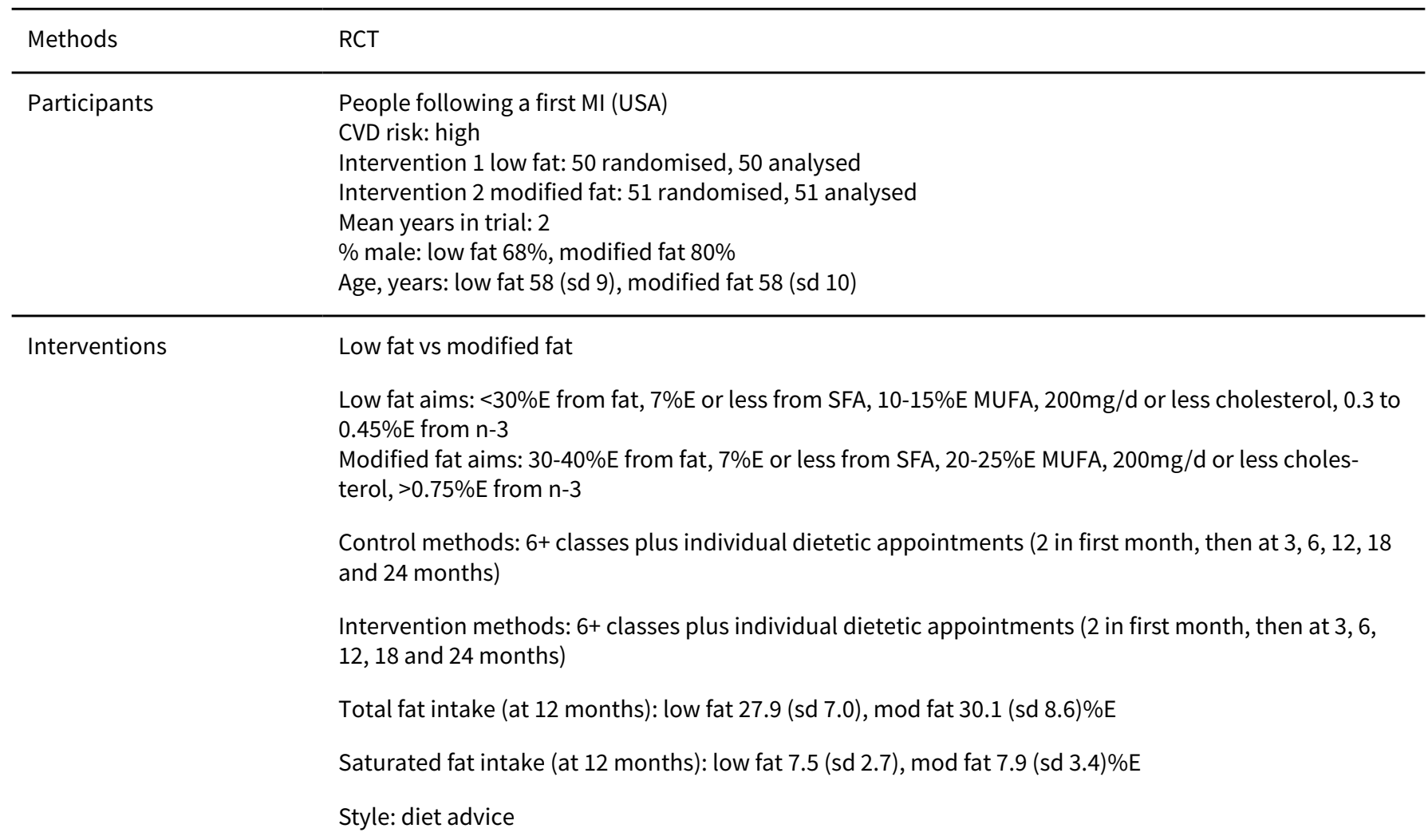


THIS DIET 2008 (Continued)

\section{Setting: community}

Outcomes

Stated trial outcomes: mortality and morbidity

Data available on total mortality? yes, none

Cardiovascular mortality? yes, none

Events available for combined cardiovascular events: MI, unstable angina, stroke

Secondary outcomes: cancer deaths (none), stroke, total and non-fatal MI

Tertiary outcomes: HDL and LDL cholesterol, TG, BMI, BP

\section{Notes}

\section{Risk of bias}

\begin{tabular}{lll}
\hline Bias & Authors' judgement & Support for judgement \\
\hline $\begin{array}{l}\text { Random sequence genera- } \\
\text { tion (selection bias) }\end{array}$ & Low risk & $\begin{array}{l}\text { Sealed envelopes, stratified by diabetes, 10 envelope blocks, selected in pre- } \\
\text { pared order from locked drawer by study dietitian. }\end{array}$ \\
\hline $\begin{array}{l}\text { Allocation concealment } \\
\text { (selection bias) }\end{array}$ & Low risk & \\
\hline
\end{tabular}

\begin{tabular}{|c|c|c|}
\hline $\begin{array}{l}\text { Blinding (performance } \\
\text { bias and detection bias) } \\
\text { All outcomes }\end{array}$ & High risk & Participants were aware of allocation as taught the diet. \\
\hline $\begin{array}{l}\text { Incomplete outcome data } \\
\text { (attrition bias) } \\
\text { All outcomes }\end{array}$ & Low risk & For primary outcomes \\
\hline $\begin{array}{l}\text { Selective reporting (re- } \\
\text { porting bias) }\end{array}$ & Low risk & Not relevant for primary and secondary outcomes as all trialists asked for data \\
\hline Other bias & Low risk & \\
\hline $\begin{array}{l}\text { Free of systematic differ- } \\
\text { ence in care? }\end{array}$ & Low risk & $\begin{array}{l}\text { Same number of individual dietetic appointments and group sessions in both } \\
\text { arms. See Control and Intervention Methods in Interventions section of the Ta- } \\
\text { ble of Characteristics of Included Studies }\end{array}$ \\
\hline
\end{tabular}

Free of dietary differences Low risk Smoking cessation, weight loss and exercise encouraged in both arms.
other than fat?

\section{Veterans Admin 1969}

\begin{tabular}{ll}
\hline Methods & RCT \\
\hline Participants & Men living at the Veterans Administration Centre (USA) \\
& CVD risk: low \\
& Control: randomised 422, analysed 422 \\
& Intervention: randomised 424, analysed 424 \\
& Mean years in trial: control 3.7, intervention 3.7 \\
& \% male: 100 \\
& age: mean control 65.6, intervention 65.4 (all 54-88) \\
\hline
\end{tabular}


Veterans Admin 1969 (Continued)

Control aims: provided, total fat $40 \% \mathrm{E}$

Intervention aims: total fat $40 \% \mathrm{E}, 2 / 3$ of SFA replaced by unsaturated fats, dietary chol reduced

Control methods: whole diet provided

Intervention methods: whole diet provided

Total fat intake (during trial): mod fat 38.9 (sd unclear), cont 40 (sd unclear)\%E

Saturated fat intake (during trial): mod fat 8.3 (sd unclear), cont 18.5 (sd unclear)\%E

Style: diet provided

Setting: residential institution

$\begin{array}{ll}\text { Outcomes } & \text { Stated trial outcomes: mortality, heart disease } \\ & \text { Data available on total mortality? yes } \\ \text { Cardiovascular mortality? yes } \\ \text { Events available for combined cardiovascular events: sudden death, definite MI, definite stroke, angi- } \\ \text { na, PV events } \\ \text { Secondary outcomes: cancer deaths, cancer diagnoses, stroke, non-fatal MI, total MI } \\ \text { Tertiary outcomes: none (some data on total cholesterol, but no variance info) }\end{array}$

Notes

\section{Risk of bias}

\begin{tabular}{|c|c|c|}
\hline Bias & Authors' judgement & Support for judgement \\
\hline $\begin{array}{l}\text { Random sequence genera- } \\
\text { tion (selection bias) }\end{array}$ & Low risk & "table of random numbers used" \\
\hline $\begin{array}{l}\text { Allocation concealment } \\
\text { (selection bias) }\end{array}$ & Unclear risk & Randomisation method not clearly described \\
\hline $\begin{array}{l}\text { Blinding (performance } \\
\text { bias and detection bias) } \\
\text { All outcomes }\end{array}$ & Low risk & $\begin{array}{l}\text { physician blinding: adequate } \\
\text { participant blinding: adequate }\end{array}$ \\
\hline $\begin{array}{l}\text { Incomplete outcome data } \\
\text { (attrition bias) } \\
\text { All outcomes }\end{array}$ & Low risk & All followed up via Veterans Admin system \\
\hline $\begin{array}{l}\text { Selective reporting (re- } \\
\text { porting bias) }\end{array}$ & Low risk & Not relevant for primary and secondary outcomes as all trialists asked for data \\
\hline Other bias & Low risk & \\
\hline $\begin{array}{l}\text { Free of systematic differ- } \\
\text { ence in care? }\end{array}$ & Low risk & $\begin{array}{l}\text { All ate centre food as usual. See Control and Intervention Methods in Interven- } \\
\text { tions section of the Table of Characteristics of Included Studies }\end{array}$ \\
\hline $\begin{array}{l}\text { Free of dietary differences } \\
\text { other than fat? }\end{array}$ & Low risk & $\begin{array}{l}\text { See Control and Intervention Aims in Interventions section of the Table of } \\
\text { Characteristics of Included Studies }\end{array}$ \\
\hline
\end{tabular}


WHEL 2007

\begin{tabular}{|c|c|c|}
\hline Methods & \multicolumn{2}{|l|}{$\mathrm{RCT}$} \\
\hline Participants & \multicolumn{2}{|c|}{$\begin{array}{l}\text { Women with previously treated early breast cancer (USA) } \\
\text { CVD risk: low } \\
\text { Control: randomised } 1561 \text {, analysed } 1551 \\
\text { Intervention: randomised } 1546 \text {, analysed } 1537 \\
\text { Mean years in trial: unclear, } 11 \text { years max, around } 11 \text { years mean? } \\
\% \text { male: } 0 \\
\text { Age: control mean } 53.0 \text { (sd 9.0), intervention mean } 53.3 \text { (sd 8.9) }\end{array}$} \\
\hline Interventions & $\begin{array}{l}\text { Reduced fat intake vs } \\
\text { Control: aim 30\%E fro } \\
\text { Intervention: aim } 15-2 \\
\text { Control methods: give } \\
\text { Intervention methods } \\
\text { offered in first year, } 4 \\
\text { self-efficacy, self-mon } \\
\text { Total fat intake (at } 72 \\
\text { Saturated fat intake (a } \\
\text { Style: diet advice } \\
\text { Setting: community }\end{array}$ & $\begin{array}{l}\text { fat } \\
\% \text { E from fat, } 5 \mathrm{veg} / \mathrm{d}, 3 \text { fruit/d, } 16 \mathrm{oz} \text { veg juice and } 30 \mathrm{~g} / \mathrm{d} \text { fibre } \\
\text { print materials only } \\
\text { elephone counselling programme ( } 31 \text { calls by study end), cooking classes (12 } \\
\text { tended on average) and monthly newsletters ( } 48 \text { by study end), all focused on } \\
\text { oring and barriers, retaining motivation } \\
\text { onths): low fat } 28.9 \text { (sd 9.0), cont } 32.4 \text { (sd } 8.0 \text { )\%E } \\
72 \text { months): low fat } 7.2 \text { (sd unclear), cont } 8.9 \text { (sd unclear)\%E }\end{array}$ \\
\hline Outcomes & \multicolumn{2}{|c|}{$\begin{array}{l}\text { Stated trial outcomes: mortality, invasive breast cancer } \\
\text { Data available on total mortality? yes } \\
\text { Cardiovascular mortality? yes } \\
\text { Events available for combined cardiovascular events: none } \\
\text { Secondary outcomes: cancer diagnoses and deaths } \\
\text { Tertiary outcomes: weight, total, LDL and HDL cholesterol, TG }\end{array}$} \\
\hline \multicolumn{3}{|l|}{ Notes } \\
\hline \multicolumn{3}{|l|}{ Risk of bias } \\
\hline Bias & Authors' judgement & Support for judgement \\
\hline $\begin{array}{l}\text { Random sequence genera- } \\
\text { tion (selection bias) }\end{array}$ & Low risk & Randomisation via computer programme \\
\hline $\begin{array}{l}\text { Allocation concealment } \\
\text { (selection bias) }\end{array}$ & Low risk & \\
\hline $\begin{array}{l}\text { Blinding (performance } \\
\text { bias and detection bias) } \\
\text { All outcomes }\end{array}$ & High risk & Participants aware of allocation \\
\hline $\begin{array}{l}\text { Incomplete outcome data } \\
\text { (attrition bias) } \\
\text { All outcomes }\end{array}$ & Low risk & Monitoring of national death register and medical records \\
\hline
\end{tabular}


WHEL 2007 (Continued)

\begin{tabular}{lll}
$\begin{array}{l}\text { Selective reporting (re- } \\
\text { porting bias) }\end{array}$ & Low risk & Not relevant for primary and secondary outcomes as all trialists asked for data \\
\hline Other bias & Low risk & \\
\hline $\begin{array}{l}\text { Free of systematic differ- } \\
\text { ence in care? }\end{array}$ & High risk & $\begin{array}{l}\text { High intensity intervention compared with leaflets. See Control and Interven- } \\
\text { tion Methods in Interventions section of the Table of Characteristics of Includ- } \\
\text { ed Studies }\end{array}$
\end{tabular}

Free of dietary differences High risk Fruit and veg intervention in low fat arm, not in control.

other than fat?

WHI with CVD 2006

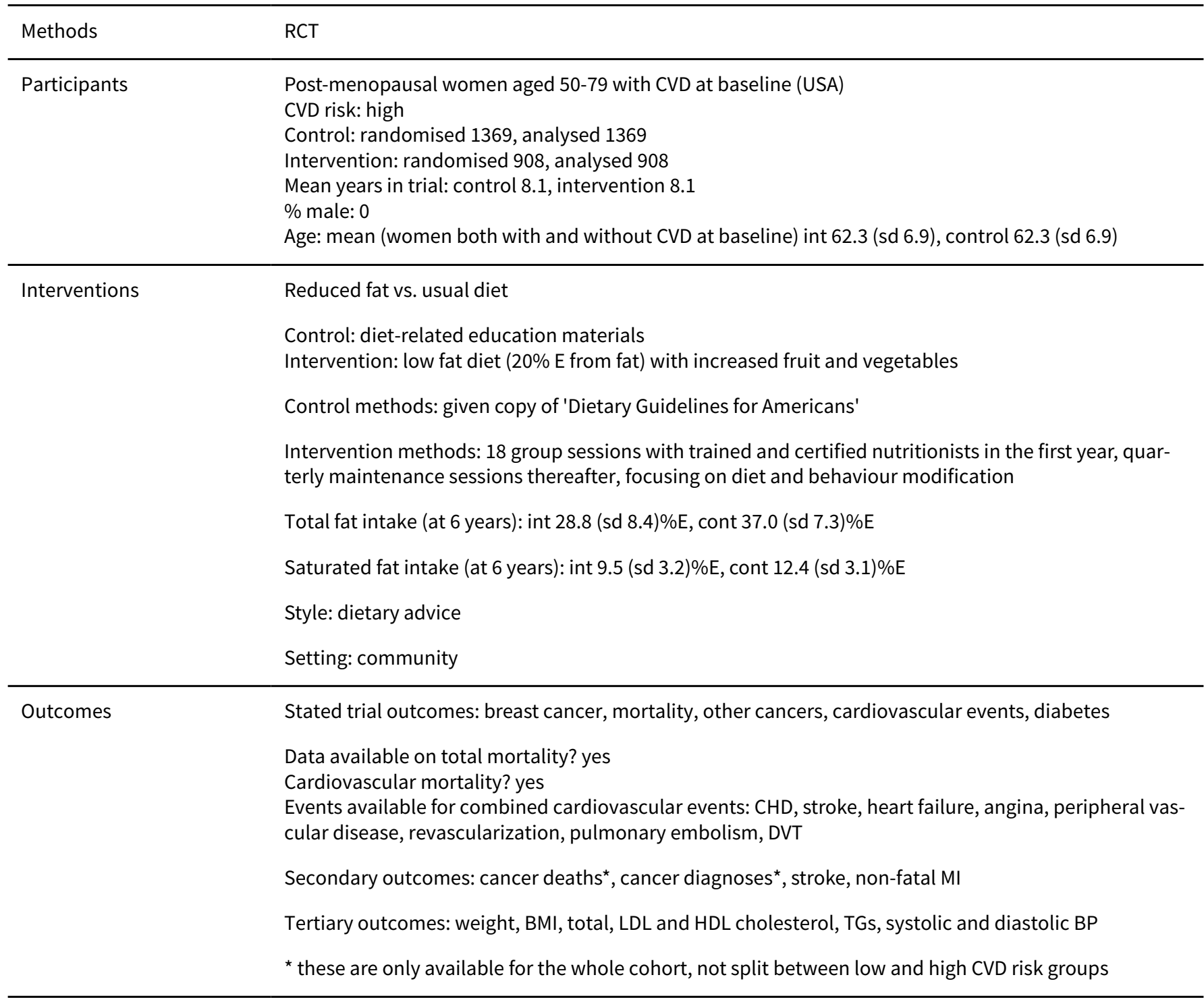

Notes

\section{Risk of bias}


WHI with CVD 2006 (Continued)

\begin{tabular}{|c|c|c|}
\hline Bias & Authors' judgement & Support for judgement \\
\hline $\begin{array}{l}\text { Random sequence genera- } \\
\text { tion (selection bias) }\end{array}$ & Low risk & Computer algorithm \\
\hline $\begin{array}{l}\text { Allocation concealment } \\
\text { (selection bias) }\end{array}$ & Low risk & \\
\hline $\begin{array}{l}\text { Blinding (performance } \\
\text { bias and detection bias) } \\
\text { All outcomes }\end{array}$ & High risk & Participants aware of allocation \\
\hline $\begin{array}{l}\text { Incomplete outcome data } \\
\text { (attrition bias) } \\
\text { All outcomes }\end{array}$ & Low risk & ITT analysis \\
\hline $\begin{array}{l}\text { Selective reporting (re- } \\
\text { porting bias) }\end{array}$ & Low risk & Not relevant for primary and secondary outcomes as all trialists asked for data \\
\hline Other bias & Low risk & \\
\hline $\begin{array}{l}\text { Free of systematic differ- } \\
\text { ence in care? }\end{array}$ & High risk & $\begin{array}{l}\text { Intervention participants received } 18 \text { group sessions with behavioural mod- } \\
\text { ification plus quarterly maintenance sessions thereafter, control groups re- } \\
\text { ceived a leaflet. See Control and Intervention Methods in Interventions section } \\
\text { of the Table of Characteristics of Included Studies }\end{array}$ \\
\hline $\begin{array}{l}\text { Free of dietary differences } \\
\text { other than fat? }\end{array}$ & High risk & $\begin{array}{l}\text { Also fruit and vegetable intervention. See Control and Intervention Aims in In- } \\
\text { terventions section of the Table of Characteristics of Included Studies }\end{array}$ \\
\hline
\end{tabular}

WHI without CVD 2006

\begin{tabular}{ll}
\hline Methods & RCT \\
\hline
\end{tabular}

\section{Participants}

Post-menopausal women aged 50-79 without CVD at baseline (USA)

CVD risk: low

Control: randomised 29294, analysed 29294

Intervention: randomised 19541, analysed 19541

Mean years in trial: control 8.1, intervention 8.1

$\%$ male: 0

Age: mean (both with and without CVD at baseline) int 62.3 (sd 6.9), control 62.3 (sd 6.9)

Control: diet-related education materials

Intervention: low fat diet (20\% E from fat) with increased fruit and vegetables

Control methods: given copy of 'Dietary Guidelines for Americans'

Intervention methods: 18 group sessions with trained and certified nutritionists in the first year, quarterly maintenance sessions thereafter, focusing on diet and behaviour modification

Total fat intake (at 6 years): int 28.8 (sd 8.4)\%E, cont 37.0 (sd 7.3)\%E

Saturated fat intake (at 6 years): int 9.5 (sd 3.2)\%E, cont 12.4 (sd 3.1)\%E

Style: dietary advice 
WHI without CVD 2006 (Continued)

$$
\text { Setting: community }
$$

Outcomes Stated trial outcomes: breast cancer, mortality, other cancers, cardiovascular events, diabetes

Data available on total mortality? yes ${ }^{\star}$

Cardiovascular mortality? yes

Events available for combined cardiovascular events: CHD, stroke, heart failure, angina, peripheral vas-

cular disease, revascularization, pulmonary embolism, DVT

Secondary outcomes: cancer deaths ${ }^{\star}$, cancer diagnoses*, stroke, non-fatal MI, diabetes diagnosis ${ }^{\star}$

Tertiary outcomes: weight, BMI, total, LDL and HDL cholesterol, TGs, systolic and diastolic BP

* these are only available for the whole cohort, not split between low and high CVD risk groups

\section{Notes}

\section{Risk of bias}

\begin{tabular}{|c|c|c|}
\hline Bias & Authors' judgement & Support for judgement \\
\hline $\begin{array}{l}\text { Random sequence genera- } \\
\text { tion (selection bias) }\end{array}$ & Low risk & Computer algorithm \\
\hline $\begin{array}{l}\text { Allocation concealment } \\
\text { (selection bias) }\end{array}$ & Low risk & \\
\hline $\begin{array}{l}\text { Blinding (performance } \\
\text { bias and detection bias) } \\
\text { All outcomes }\end{array}$ & High risk & Participants aware of allocation \\
\hline $\begin{array}{l}\text { Incomplete outcome data } \\
\text { (attrition bias) } \\
\text { All outcomes }\end{array}$ & Low risk & ITT analysis \\
\hline $\begin{array}{l}\text { Selective reporting (re- } \\
\text { porting bias) }\end{array}$ & Low risk & Not relevant for primary and secondary outcomes as all trialists asked for data \\
\hline Other bias & Low risk & \\
\hline $\begin{array}{l}\text { Free of systematic differ- } \\
\text { ence in care? }\end{array}$ & High risk & $\begin{array}{l}\text { Intervention participants received } 18 \text { group sessions with behavioural modifi- } \\
\text { cation plus quarterly maintenance sessions thereafter. See Control and Inter- } \\
\text { vention Methods in Interventions section of the Table of Characteristics of In- } \\
\text { cluded Studies }\end{array}$ \\
\hline $\begin{array}{l}\text { Free of dietary differences } \\
\text { other than fat? }\end{array}$ & High risk & $\begin{array}{l}\text { Also fruit and vegetable intervention. See Control and Intervention Aims in In- } \\
\text { terventions section of the Table of Characteristics of Included Studies }\end{array}$ \\
\hline
\end{tabular}

WINS 2006

\begin{tabular}{ll}
\hline Methods & RCT \\
\hline Participants & Women with localised re-sected breast cancer (USA) \\
CVD risk: low \\
Control: 1462 randomised, 1462 analysed \\
Intervention: 975 randomised, 975 analysed \\
\hline
\end{tabular}


WINS 2006 (Continued)

Mean years in trial: overall 5.0

$\%$ men: 0

Age: control mean 58.5 ( $95 \% \mathrm{Cl} 43.6$ to 73.4$)$, intervention mean 58.6 ( $95 \% \mathrm{Cl} 44.4$ to 72.8 ) (all postmenopausal)

Reduced fat intake vs. usual diet
Control aims: minimal nutritional counselling focused on nutritional adequa
Intervention aims: total fat 15-20\%E
Control methods: 1 baseline dietetic session plus 3-monthly sessions
Intervention methods: 8 bi-weekly individual dietetic sessions, then optio
incorporating individual fat gram goals, social cognitive theory, self-monito
ling, social support and relapse prevention and management
Total fat intake (at 1 year): low fat 20.3 (sd 8.1), cont 29.2 (sd 7.4)\%E
Saturated fat intake (at 1 year): low fat 10.4 (sd 6.7), cont 16.6 (sd 9.3)\%E
Style: dietary advice
Setting: community

Stated trial outcomes: dietary fat intake, total cholesterol, weight and waist
Data available on total mortality? yes
Cardiovascular mortality? no
Events available for combined cardiovascular events: none
Secondary outcomes: cancer diagnoses
Tertiary outcomes: weight, BMI

Notes

\section{Risk of bias}

\begin{tabular}{lll}
\hline Bias & Authors' judgement & Support for judgement \\
\hline $\begin{array}{l}\text { Random sequence genera- } \\
\text { tion (selection bias) }\end{array}$ & Low risk & $\begin{array}{l}\text { random stratified permuted block design, carried out at the statistical co-ordi- } \\
\text { nating centre of WINS }\end{array}$ \\
\hline $\begin{array}{l}\text { Allocation concealment } \\
\text { (selection bias) }\end{array}$ & Low risk & \\
\hline $\begin{array}{l}\text { Blinding (performance } \\
\text { bias and detection bias) } \\
\text { All outcomes }\end{array}$ & High risk & $\begin{array}{l}\text { Participants not blinded, not relevant for assessment of mortality by re- } \\
\text { searchers }\end{array}$ \\
\hline $\begin{array}{l}\text { Incomplete outcome data } \\
\begin{array}{l}\text { (attrition bias) } \\
\text { All outcomes }\end{array}\end{array}$ & Low risk & All assessed. \\
\hline $\begin{array}{l}\text { Selective reporting (re- } \\
\text { porting bias) }\end{array}$ & Low risk & Not relevant for primary and secondary outcomes as all trialists asked for data \\
\hline \begin{tabular}{l} 
Other bias \\
\hline
\end{tabular} & Low risk & \\
\hline
\end{tabular}


WINS 2006 (Continued)

Free of systematic differ- High risk Differences in attention - more time for those in intervention group. See Conence in care? trol and Intervention Methods in Interventions section of the Table of Characteristics of Included Studies

Free of dietary differences Low risk other than fat?

See Control and Intervention Aims in Interventions section of the Table of Characteristics of Included Studies

Abreviations:

$\mathrm{CHO}=$ carbohydrates,

$\% \mathrm{E}=$ percent of total energy intake,

$\mathrm{P} / \mathrm{S}=$ polyunsaturated / saturated fat ratio,

chol = cholesterol,

CVD = cardiovascular disease,

$\mathrm{MI}=$ myocardial infarction

Characteristics of excluded studies [ordered by study ID]

\begin{tabular}{|c|c|}
\hline Study & Reason for exclusion \\
\hline Agewall 2001 & Multifactorial intervention \\
\hline Ammerman 2003 & No appropriate control group (and not low fat vs modified fat) \\
\hline Anti-Coronary C 1966 & Not randomised \\
\hline Aquilani 2000 & No appropriate control group (and not low fat vs modified fat) \\
\hline Arntzenius 1985 & No appropriate control group (and not low fat vs modified fat) \\
\hline Aro 1990 & Intervention and randomised follow up less than 6 months \\
\hline ASSIST 2001 & Intervention is not dietary fat modification or low fat diet \\
\hline Australian Polyp Prev 95 & $\begin{array}{l}\text { Neither mortality nor cardiovascular morbidity data available (only decided after contact with at } \\
\text { least one author) }\end{array}$ \\
\hline Baer 1993 & Not randomised \\
\hline Bakx 1997 & Multifactorial intervention \\
\hline Barnard 2009 & Weight reduction encouraged in the conventional diet, but not in the vegan diet arm \\
\hline Barndt 1977 & No appropriate control group (and not low fat vs modified fat) \\
\hline Baron 1990 & Multifactorial intervention \\
\hline Barr 1990 & Intervention and randomised follow up less than 6 months \\
\hline Baumann 1982 & Intervention and randomised follow up less than 6 months \\
\hline Beckmann 1988 & Not randomised \\
\hline Beckmann 1995 & Intervention is not dietary fat modification or low fat diet \\
\hline Beresford 1992 & Intervention and randomised follow up less than 6 months \\
\hline
\end{tabular}




\begin{tabular}{|c|c|}
\hline Study & Reason for exclusion \\
\hline Bergstrom 1967 & Intervention and randomised follow up less than 6 months \\
\hline Bierenbaum 1963 & No appropriate control group (and not low fat vs modified fat) \\
\hline Bloemberg 1991 & $\begin{array}{l}\text { Neither mortality nor cardiovascular morbidity data available (only decided after contact with at } \\
\text { least one author) }\end{array}$ \\
\hline Bloomgarden 1987 & Multifactorial intervention \\
\hline Bonnema 1995 & No appropriate control group (and not low fat vs modified fat) \\
\hline Bosaeus 1992 & Intervention and randomised follow up less than 6 months \\
\hline Boyar 1988 & Not randomised \\
\hline Brensike 1982 & No appropriate control group (and not low fat vs modified fat) \\
\hline Broekmans 2003 & Intervention is not dietary fat modification or low fat diet \\
\hline Brown 1984 & No appropriate control group (and not low fat vs modified fat) \\
\hline Bruce 1994 & No appropriate control group (and not low fat vs modified fat) \\
\hline Bruno 1983 & Multifactorial intervention \\
\hline Butcher 1990 & Intervention and randomised follow up less than 6 months \\
\hline Butowski 1998 & Not randomised \\
\hline Byers 1995 & No appropriate control group (and not low fat vs modified fat) \\
\hline Caggiula 1996 & No appropriate control group (and not low fat vs modified fat) \\
\hline Cerin 1993 & Intervention and randomised follow up less than 6 months \\
\hline Chan 1993 & Intervention and randomised follow up less than 6 months \\
\hline Chapman 1950 & Intervention and randomised follow up less than 6 months \\
\hline Charbonnier 1975 & Intervention and randomised follow up less than 6 months \\
\hline Cheng 2004 & Intervention and randomised follow up less than 6 months \\
\hline Chicago CPEP 1977 & Not randomised \\
\hline Chiostri 1988 & Intervention and randomised follow up less than 6 months \\
\hline Choudhury 1984 & Intervention and randomised follow up less than 6 months \\
\hline Clark 1997 & Multifactorial intervention \\
\hline Clifton 1992 & Intervention and randomised follow up less than 6 months \\
\hline Cobb 1991 & Intervention and randomised follow up less than 6 months \\
\hline
\end{tabular}




\begin{tabular}{|c|c|}
\hline Study & Reason for exclusion \\
\hline Cohen 1991 & Intervention is not dietary fat modification or low fat diet \\
\hline Cole 1988 & Intervention and randomised follow up less than 6 months \\
\hline Colquhoun 1990 & Intervention and randomised follow up less than 6 months \\
\hline Consolazio 1946 & Intervention and randomised follow up less than 6 months \\
\hline Cox 1996 & Multifactorial intervention \\
\hline Croft 1986 & Intervention is not dietary fat modification or low fat diet \\
\hline Crouch 1986 & Not randomised \\
\hline Da Qing IGT 1997 & Intervention is not dietary fat modification or low fat diet \\
\hline Dalgard 2001 & No appropriate control group (and not low fat vs modified fat) \\
\hline DAS 2000 & No appropriate control group (and not low fat vs modified fat) \\
\hline DASH 1997 & Intervention and randomised follow up less than 6 months \\
\hline Davey Smith 2005 & Multifactorial intervention \\
\hline de Boer 1983 & Intervention and randomised follow up less than 6 months \\
\hline de Bont 1981 & Neither mortality nor cardiovascular morbidity data available as study data have been lost \\
\hline DeBusk 1994 & Multifactorial intervention \\
\hline Delahanty 2001 & No appropriate control group (and not low fat vs modified fat) \\
\hline Delius 1969 & Intervention is not dietary fat modification or low fat diet \\
\hline Demark 1990 & Intervention and randomised follow up less than 6 months \\
\hline Dengel 1995 & No appropriate control group (and not low fat vs modified fat) \\
\hline Denke 1994 & Intervention and randomised follow up less than 6 months \\
\hline Diabetes CCT 1995 & Intervention is not dietary fat modification or low fat diet \\
\hline DIET 1998 & Multifactorial intervention \\
\hline Ding 1992 & Intervention and randomised follow up less than 6 months \\
\hline Dobs 1991 & No appropriate control group (and not low fat vs modified fat) \\
\hline Duffield 1982 & Multifactorial intervention \\
\hline Dullaart 1997 & Not randomised \\
\hline Eating Patterns 1997 & $\begin{array}{l}\text { Neither mortality nor cardiovascular morbidity data available (only decided after contact with at } \\
\text { least one author) }\end{array}$ \\
\hline
\end{tabular}




\begin{tabular}{|c|c|}
\hline Study & Reason for exclusion \\
\hline Ehnholm 1982 & Intervention and randomised follow up less than 6 months \\
\hline Ehnholm 1984 & Intervention and randomised follow up less than 6 months \\
\hline Eisenberg 1990 & Intervention and randomised follow up less than 6 months \\
\hline Elder 2000 & No appropriate control group (and not low fat vs modified fat) \\
\hline Ellegard 1991 & Intervention and randomised follow up less than 6 months \\
\hline Esposito 2003 & No appropriate control group (and not low fat vs modified fat) \\
\hline EUROACTION 2008 & Multifactorial intervention \\
\hline FARIS 1997 & Multifactorial intervention \\
\hline Fasting HGS 1997 & No appropriate control group (and not low fat vs modified fat) \\
\hline Ferrara 2000 & No appropriate control group (and not low fat vs modified fat) \\
\hline Fielding 1995 & Intervention and randomised follow up less than 6 months \\
\hline Finckenor 2000 & Not randomised \\
\hline Finnish Diabet Prev 2000 & Multifactorial intervention \\
\hline Finnish Mental Hosp 1972 & Not randomised (cluster randomised, but $<6$ clusters) \\
\hline Fisher 1981 & Intervention and randomised follow up less than 6 months \\
\hline Fleming 2002 & No appropriate control group (and not low fat vs modified fat) \\
\hline Fortmann 1988 & Intervention is not dietary fat modification or low fat diet \\
\hline Foster 2003 & Weight reduction in one arm but not the other \\
\hline FRESH START 2007 & Participants were newly diagnosed with cancer \\
\hline Gambera 1995 & Intervention and randomised follow up less than 6 months \\
\hline Gaullier 2007 & No appropriate control group (and not low fat vs modified fat) \\
\hline Ginsberg 1988 & Intervention and randomised follow up less than 6 months \\
\hline Gjone 1972 & Intervention and randomised follow up less than 6 months \\
\hline Glatzel 1966 & No appropriate control group (and not low fat vs modified fat) \\
\hline Goodpaster 1999 & No appropriate control group (and not low fat vs modified fat) \\
\hline Grundy 1986 & Intervention and randomised follow up less than 6 months \\
\hline Hardcastle 2008 & Multifactorial intervention \\
\hline Harris 1990 & Intervention and randomised follow up less than 6 months \\
\hline
\end{tabular}




\begin{tabular}{|c|c|}
\hline Study & Reason for exclusion \\
\hline Hartman 1993 & No appropriate control group (and not low fat vs modified fat) \\
\hline Hartwell 1986 & No appropriate control group (and not low fat vs modified fat) \\
\hline Hashim 1960 & Intervention and randomised follow up less than 6 months \\
\hline Haynes 1984 & Intervention is not dietary fat modification or low fat diet \\
\hline Heber 1991 & Intervention and randomised follow up less than 6 months \\
\hline Heine 1989 & $\begin{array}{l}\text { Neither mortality nor cardiovascular morbidity data available (only decided after contact with at } \\
\text { least one author) }\end{array}$ \\
\hline Hellenius Diet \& Ex 95 & The study aimed for weight loss in one arm and not in the comparison arm \\
\hline Hellenius Diet 1995 & The study aimed for weight loss in one arm and not in the comparison arm \\
\hline Heller 1993 & $\begin{array}{l}\text { Neither mortality nor cardiovascular morbidity data available (only decided after contact with at } \\
\text { least one author) }\end{array}$ \\
\hline Hildreth 1951 & No appropriate control group (and not low fat vs modified fat) \\
\hline Holm 1990 & $\begin{array}{l}\text { Neither mortality nor cardiovascular morbidity data available (only decided after contact with at } \\
\text { least one author) }\end{array}$ \\
\hline Hood 1965 & Not randomised \\
\hline Horlick 1957 & Intervention and randomised follow up less than 6 months \\
\hline Horlick 1960 & Intervention and randomised follow up less than 6 months \\
\hline Howard 1977 & Intervention and randomised follow up less than 6 months \\
\hline Hunninghake 1990 & Intervention and randomised follow up less than 6 months \\
\hline Hutchison 1983 & No appropriate control group (and not low fat vs modified fat) \\
\hline Hyman 1998 & $\begin{array}{l}\text { Neither mortality nor cardiovascular morbidity data available (only decided after contact with at } \\
\text { least one author) }\end{array}$ \\
\hline lacono 1981 & Not randomised, Intervention and randomised follow up less than 6 months \\
\hline IMPACT 1995 & Multifactorial intervention \\
\hline Ishikawa 1995 & Not randomised \\
\hline Iso 1991 & No appropriate control group (and not low fat vs modified fat) \\
\hline Ives 1993 & Multifactorial intervention \\
\hline Jalkanen 1991 & Multifactorial intervention \\
\hline Jepson 1969 & Not randomised \\
\hline Jerusalem Nut 1992 & Intervention and randomised follow up less than 6 months \\
\hline
\end{tabular}




\begin{tabular}{|c|c|}
\hline Study & Reason for exclusion \\
\hline Jula 1990 & Multifactorial intervention \\
\hline Junker 2001 & Intervention and randomised follow up less than 6 months \\
\hline Karmally 1990 & Intervention and randomised follow up less than 6 months \\
\hline Karvetti 1992 & Multifactorial intervention \\
\hline Kastarinen 2002 & Multifactorial intervention \\
\hline Kather 1985 & Intervention and randomised follow up less than 6 months \\
\hline Katzel 1995a & Not randomised \\
\hline Katzel 1995b & Intervention is not dietary fat modification or low fat diet \\
\hline Kawamura 1993 & Intervention and randomised follow up less than 6 months \\
\hline Keidar 1988 & Intervention and randomised follow up less than 6 months \\
\hline Kempner 1948 & No appropriate control group (and not low fat vs modified fat) \\
\hline Keys 1952 & Not randomised \\
\hline Keys 1957a & Intervention and randomised follow up less than 6 months \\
\hline Keys 1957b & Intervention and randomised follow up less than 6 months \\
\hline Keys 1957c & Intervention and randomised follow up less than 6 months \\
\hline Khan 2003 & $\begin{array}{l}\text { Neither mortality nor cardiovascular morbidity data available (only decided after contact with at } \\
\text { least one author) }\end{array}$ \\
\hline King 2000 & Intervention and randomised follow up less than 6 months \\
\hline Kingsbury 1961 & Intervention and randomised follow up less than 6 months \\
\hline Kohler 1986 & Not randomised \\
\hline Koopman 1990 & Intervention and randomised follow up less than 6 months \\
\hline Korhonen 2003 & Multifactorial intervention \\
\hline Kriketos 2001 & Intervention and randomised follow up less than 6 months \\
\hline Kris 1994 & Intervention and randomised follow up less than 6 months \\
\hline Kristal 1997 & Multifactorial intervention \\
\hline Kromhout 1987 & No appropriate control group (and not low fat vs modified fat) \\
\hline Kummel 2008 & Intervention is not dietary fat modification or low fat diet \\
\hline Laitinen 1993 & Multifactorial intervention \\
\hline
\end{tabular}




\begin{tabular}{|c|c|}
\hline Study & Reason for exclusion \\
\hline Laitinen 1994 & Multifactorial intervention \\
\hline Leduc 1994 & Multifactorial intervention \\
\hline Lewis 1958 & Intervention and randomised follow up less than 6 months \\
\hline Lewis 1981 & Intervention and randomised follow up less than 6 months \\
\hline Lewis 1985 & Multifactorial intervention \\
\hline Lichtenstein 2002 & Intervention and randomised follow up less than 6 months \\
\hline Linko 1957 & Intervention and randomised follow up less than 6 months \\
\hline Lipid Res Clinic 1984 & No appropriate control group (and not low fat vs modified fat) \\
\hline Little 1990 & Intervention and randomised follow up less than 6 months \\
\hline Little 1991 & Not randomised \\
\hline Little 2004 & Intervention is not dietary fat modification or low fat diet \\
\hline Lottenberg 1996 & Intervention and randomised follow up less than 6 months \\
\hline Luszczynska 2007 & No appropriate control group (and not low fat vs modified fat) \\
\hline Lyon Diet Heart 1994 & Intervention is not dietary fat modification or low fat diet \\
\hline Lysikova 2003 & Intervention and randomised follow up less than 6 months \\
\hline Macdonald 1972 & Intervention and randomised follow up less than 6 months \\
\hline Mansel 1990 & Intervention is not dietary fat modification or low fat diet \\
\hline Marckmann 1993 & Not randomised \\
\hline MARGARIN 2002 & No appropriate control group (and not low fat vs modified fat) \\
\hline Marniemi 1990 & Both intervention groups aimed to lose weight, while the control group did not. \\
\hline Mattson 1985 & Intervention and randomised follow up less than 6 months \\
\hline McCarron 1997 & Intervention and randomised follow up less than 6 months \\
\hline McCarron 2001 & Intervention is not dietary fat modification or low fat diet \\
\hline McManus 2001 & $\begin{array}{l}\text { Neither mortality nor cardiovascular morbidity data available (only decided after contact with at } \\
\text { least one author) }\end{array}$ \\
\hline McNamara 1981 & Intervention and randomised follow up less than 6 months \\
\hline Medi-RIVAGE 2004 & $\begin{array}{l}\text { Weight reduction for some low fat diet participants (those with } \mathrm{BMI}>25 \text { ) but not in Mediterranean } \\
\text { group }\end{array}$ \\
\hline Mensink 1987 & Intervention and randomised follow up less than 6 months \\
\hline
\end{tabular}




\begin{tabular}{|c|c|}
\hline Study & Reason for exclusion \\
\hline Mensink 1989 & Intervention and randomised follow up less than 6 months \\
\hline Mensink 1990a & Intervention and randomised follow up less than 6 months \\
\hline Mensink 1990b & Intervention and randomised follow up less than 6 months \\
\hline Michalsen 2006 & Diet plus stress management vs no intervention \\
\hline Miettinen 1994 & Intervention and randomised follow up less than 6 months \\
\hline Millar 1973 & No appropriate control group (and not low fat vs modified fat) \\
\hline Miller 1998 & Intervention and randomised follow up less than 6 months \\
\hline Miller 2001 & $\begin{array}{l}\text { Neither mortality nor cardiovascular morbidity data available (only decided after contact with at } \\
\text { least one author) }\end{array}$ \\
\hline Milne 1994 & $\begin{array}{l}\text { No appropriate control group (and not low fat vs modified fat) - the high CHO diet is neither 'usual' } \\
\text { or 'low fat' to compare with the modified fat diet }\end{array}$ \\
\hline Minnesota HHP 1990 & No appropriate control group (and not low fat vs modified fat) \\
\hline Mokuno 1988 & Intervention and randomised follow up less than 6 months \\
\hline Moreno 1994 & Not randomised \\
\hline Morrison 1950 & Not randomised \\
\hline Morrison 1951 & Not randomised \\
\hline Morrison 1960 & Not randomised \\
\hline Mortensen 1983 & Intervention and randomised follow up less than 6 months \\
\hline MRFIT substudy 1986 & Intervention and randomised follow up less than 6 months \\
\hline MSDELTA 1995 & Intervention and randomised follow up less than 6 months \\
\hline Mujeres Felices 2003 & Diet and breast self examination vs no intervention \\
\hline Mutanen 1997 & Intervention and randomised follow up less than 6 months \\
\hline Muzio 2007 & Intervention and randomised follow up less than 6 months \\
\hline NAS 1987 & Intervention and randomised follow up less than 6 months \\
\hline NCEP weight 1991 & $\begin{array}{l}\text { Neither mortality nor cardiovascular morbidity data available (only decided after contact with at } \\
\text { least one author) }\end{array}$ \\
\hline Neil 1995 & No appropriate control group (and not low fat vs modified fat) \\
\hline Neverov 1997 & Multifactorial intervention \\
\hline Next Step 1995 & $\begin{array}{l}\text { Neither mortality nor cardiovascular morbidity data available (only decided after contact with at } \\
\text { least one author) }\end{array}$ \\
\hline
\end{tabular}




\begin{tabular}{|c|c|}
\hline Study & Reason for exclusion \\
\hline Nordoy 1971 & Intervention and randomised follow up less than 6 months \\
\hline Norway Veg Oil 1968 & No appropriate control group (and not low fat vs modified fat) \\
\hline O'Brien 1976 & Intervention and randomised follow up less than 6 months \\
\hline ODES 2006 & The study aimed for weight loss in one arm and not in the other arm \\
\hline Oldroyd 2001 & Multifactorial intervention \\
\hline ORIGIN 2008 & Intervention is not dietary fat modification or low fat diet \\
\hline Oslo Study 2004 & Multifactorial intervention \\
\hline Pascale 1995 & Multifactorial intervention \\
\hline PEP 2001 & Multifactorial intervention \\
\hline PHYLLIS 1993 & No appropriate control group (and not low fat vs modified fat) \\
\hline Pilkington 1960 & $\begin{array}{l}\text { Neither mortality nor cardiovascular morbidity data available (only decided after contact with at } \\
\text { least one author) }\end{array}$ \\
\hline Pritchard 2002 & The study aimed for weight loss in one arm and not in the comparison arm \\
\hline Puget Sound EP 2000 & $\begin{array}{l}\text { Neither mortality nor cardiovascular morbidity data available (only decided after contact with at } \\
\text { least one author) }\end{array}$ \\
\hline Rabast 1979 & Intervention and randomised follow up less than 6 months \\
\hline Rabkin 1981 & Intervention and randomised follow up less than 6 months \\
\hline Radack 1990 & Intervention and randomised follow up less than 6 months \\
\hline Rasmussen 1995 & Intervention and randomised follow up less than 6 months \\
\hline Reaven 2001 & Intervention and randomised follow up less than 6 months \\
\hline Reid 2002 & No appropriate control group (and not low fat vs modified fat) \\
\hline Renaud 1986 & Not randomised \\
\hline Rivellese 2003 & Intervention and randomised follow up less than 6 months \\
\hline Roderick 1997 & $\begin{array}{l}\text { Neither mortality nor cardiovascular morbidity data available (only decided after contact with at } \\
\text { least one author) }\end{array}$ \\
\hline Roman CHD prev 1986 & Multifactorial intervention \\
\hline Rose 1987 & No appropriate control group (and not low fat vs modified fat) \\
\hline Sandstrom 1992 & Not randomised \\
\hline Sasaki 2000 & Not randomised \\
\hline
\end{tabular}




\begin{tabular}{|c|c|}
\hline Study & Reason for exclusion \\
\hline Schaefer 1995a & Intervention and randomised follow up less than 6 months \\
\hline Schaefer 1995b & Intervention and randomised follow up less than 6 months \\
\hline Schectman 1996 & Multifactorial intervention \\
\hline Schlierf 1995 & Multifactorial intervention \\
\hline Seppanen-Laakso 1992 & Intervention and randomised follow up less than 6 months \\
\hline Singh 1990 & Not randomised \\
\hline Singh 1991 & Multifactorial intervention \\
\hline Singh 1992 & No appropriate control group (and not low fat vs modified fat) \\
\hline Sirtori 1992 & Intervention and randomised follow up less than 6 months \\
\hline SLIM 2008 & Multifactorial intervention \\
\hline Sopotsinskaia 1992 & The study aimed for weight loss in one arm and not in the comparison arm \\
\hline Staff HHP 1994 & Not randomised \\
\hline Stanford NAP 1997 & Intervention and randomised follow up less than 6 months \\
\hline Stanford Weight 1994 & The study aimed for weight loss in one arm and not in the comparison arm \\
\hline Starmans 1995 & Intervention and randomised follow up less than 6 months \\
\hline Steinbach 1996 & Multifactorial intervention \\
\hline Steptoe 2001 & No appropriate control group (and not low fat vs modified fat) \\
\hline Stevens 2002 & Diet plus breast self-examination vs no intervention \\
\hline Stevenson 1988 & No appropriate control group (and not low fat vs modified fat) \\
\hline Sweeney 2004 & Intervention is not dietary fat modification or low fat diet \\
\hline TAIM 1992 & Intervention is not dietary fat modification or low fat diet \\
\hline Take Heart II 1997 & Not randomised \\
\hline Taylor 1991 & Not randomised \\
\hline TOHP I 1992 & Multifactorial intervention \\
\hline TONE 1997 & Intervention is not dietary fat modification or low fat diet \\
\hline Toobert 2003 & Multifactorial intervention \\
\hline Towle 1994 & Intervention and randomised follow up less than 6 months \\
\hline TRANSFACT 2006 & Intervention and randomised follow up less than 6 months \\
\hline
\end{tabular}




\begin{tabular}{|c|c|}
\hline Study & Reason for exclusion \\
\hline Treatwell 1992 & $\begin{array}{l}\text { Neither mortality nor cardiovascular morbidity data available (only decided after contact with at } \\
\text { least one author) }\end{array}$ \\
\hline Tromso Heart 1989 & Multifactorial intervention \\
\hline Turpeinen 1960 & Not randomised \\
\hline UK PDS 1996 & No appropriate control group (and not low fat vs modified fat) \\
\hline Urbach 1952 & No appropriate control group (and not low fat vs modified fat) \\
\hline Uusitupa 1993 & Multifactorial intervention \\
\hline Vavrikova 1958 & Intervention and randomised follow up less than 6 months \\
\hline Wass 1981 & Intervention and randomised follow up less than 6 months \\
\hline Wassertheil 1985 & Intervention is not dietary fat modification or low fat diet \\
\hline WATCH 1999 & $\begin{array}{l}\text { Neither mortality nor cardiovascular morbidity data available (only decided after contact with at } \\
\text { least one author) }\end{array}$ \\
\hline Watts 1988 & Intervention and randomised follow up less than 6 months \\
\hline Weintraub 1992 & No appropriate control group (and not low fat vs modified fat) \\
\hline Westman 2006 & Intervention is not dietary fat modification or low fat diet \\
\hline Weststrate 1998 & Intervention and randomised follow up less than 6 months \\
\hline WHO primary prev 1979 & Multifactorial intervention \\
\hline WHT 1990 & $\begin{array}{l}\text { Neither mortality nor cardiovascular morbidity data available as such data were not collected in } \\
\text { the study }\end{array}$ \\
\hline WHT Feasibility 2003 & $\begin{array}{l}\text { Neither mortality nor cardiovascular morbidity data available (only decided after contact with at } \\
\text { least one author) }\end{array}$ \\
\hline Wilke 1974 & Intervention and randomised follow up less than 6 months \\
\hline Williams 1990 & Intervention is not dietary fat modification or low fat diet \\
\hline Williams 1992 & Intervention is not dietary fat modification or low fat diet \\
\hline Williams 1994 & Intervention is not dietary fat modification or low fat diet \\
\hline Wilmot 1952 & No appropriate control group (and not low fat vs modified fat) \\
\hline Wing 1998 & No appropriate control group (and not low fat vs modified fat) \\
\hline WOMAN 2007 & Lifestyle intervention includes exercise and weight as well as diet \\
\hline Wood 1988 & Intervention is not dietary fat modification or low fat diet \\
\hline Woollard 2003 & Multifactorial intervention including smoking, weight, exercise and alcohol components \\
\hline
\end{tabular}




\begin{tabular}{ll}
\hline Study & Reason for exclusion \\
\hline Working Well 1996 & Multifactorial intervention \\
\hline Zock 1995 & Intervention and randomised follow up less than 6 months \\
\hline
\end{tabular}

The figure in brackets following the Study ID of each reference is a code for "reason for exclusion". The code is given in full in "Description of Studies section of the text.

Characteristics of studies awaiting assessment [ordered by study ID]

\section{Barsotti 1991}

\begin{tabular}{ll}
\hline Methods & RCT \\
\hline Participants & 356 people with hyperlipidaemia \\
\hline Interventions & Allocated to bezafibrate, not bezafibrate, simvastatin or low fat low cholesterol diet for 4 years \\
\hline Outcomes & Plaque progression \\
\hline Notes & Complex paper in Italian, unclear whether cardiovascular events occurred \\
\hline
\end{tabular}

Bonk 1975

\begin{tabular}{ll}
\hline Methods & Trial, unclear if randomised \\
\hline Participants & 300 people who have had a myocardial infarction (Germany) \\
\hline Interventions & Intensive change of nutrition vs. usual diet (both with comprehensive medical care) \\
\hline Outcomes & Return to work \\
\hline Notes & No answer to requests to clarify the way that participants were allocated to intervention or control \\
\hline
\end{tabular}

Brehm 2009

\begin{tabular}{ll}
\hline Methods & RCT \\
\hline Participants & 124 overweight or obese people with type 2 diabetes \\
\hline Interventions & Low fat vs modified fat diet \\
\hline Outcomes & Weight and glycaemic control \\
\hline Notes & LH has recently contacted the authors about whether any deaths or CV events occurred \\
\hline
\end{tabular}

Methods RCT


Canadian DBCP 1997 (Continued)

Participants 4690 women with mammographic densities $>50 \%$ breast area (Canada)

Interventions

Control: self-selected diet (no advice) vs intervention: total fat $15 \% \mathrm{E}$, protein $20 \% \mathrm{E}, \mathrm{CHO} 65 \% \mathrm{E}$, followed up for 10 years

style: diet advice

\begin{tabular}{ll}
\hline Outcomes & Stated trial outcomes: incidence of breast cancer \\
\hline Notes & No answer to requests for data on deaths or health events \\
\hline
\end{tabular}

\section{DEER 1998}

\begin{tabular}{ll}
\hline Methods & RCT \\
\hline Participants & 180 Postmenopausal women and 197 men with low HDL and raised LDL \\
\hline Interventions & NCEP step 2 diet vs aerobic exercise vs diet and exercise vs no intervention, all for 1 year \\
\hline Outcomes & lipids and diet \\
\hline Notes & No answer to requests for data on deaths or health events \\
\hline
\end{tabular}

Diet \& Hormone Study 2003

\begin{tabular}{ll}
\hline Methods & RCT \\
\hline Participants & 213 healthy women aged 20-40 years (USA) \\
\hline Interventions & Low fat, high fruit, vegetable and fibre diet vs usual diet, for 1 year \\
\hline Outcomes & Hormone levels \\
\hline Notes & No answer to requests for data on deaths or health events \\
\hline
\end{tabular}

DIRECT 2009

\begin{tabular}{ll}
\hline Methods & RCT \\
\hline Participants & 322 moderately obese people \\
\hline Interventions & Low fat restricted-calorie diet vs modified fat restricted-calorie diet, for 24 months \\
\hline Outcomes & Weight and safety \\
\hline Notes & LH has recently contacted the authors about whether any deaths or CV events occurred \\
\hline
\end{tabular}


Esposito 2004

\begin{tabular}{ll}
\hline Methods & RCT \\
\hline Participants & 180 men and women with metabolic syndrome (Italy) \\
\hline Interventions & Mediterranean style diet vs low fat diet, for 2 years \\
\hline Outcomes & Remaining metabolic syndrome, inflammatory markers \\
\hline Notes & No answer to requests for data on deaths or health events \\
\hline
\end{tabular}

Koranyi 1963

\begin{tabular}{ll}
\hline Methods & Intervention study, unclear if randomised \\
\hline Participants & Nearly 250 people with severe coronary stenosis (mostly post MI) acrued since 1957 (Hungary) \\
\hline Interventions & $\begin{array}{l}\text { Low fat diet (35-40g fat/d) vs Modified fat (50g edible oil but no butter, lard etc/d) vs Modified fat } 2 \\
(50 \mathrm{~g} \text { fat/d made up of both edible oil and butter, lard etc) vs control (no intervention) }\end{array}$ \\
\hline Outcomes & $\begin{array}{l}\text { Mortality rates (8.6\% in low fat diet, } 19.7 \% \text { controls, other groups not reported, nor numbers of par- } \\
\text { ticipants in each group) }\end{array}$ \\
\hline
\end{tabular}

Notes

Metroville Health 2003

\begin{tabular}{ll}
\hline Methods & RCT \\
\hline Participants & 403 middle class urban households (Pakistan) \\
\hline Interventions & Counselling on changing cooking fats and reducing salt vs. usual diet \\
\hline Outcomes & Dietary intake \\
\hline Notes & No answer to requests for data on deaths or health events \\
\hline
\end{tabular}

Mojonnier 1980

\begin{tabular}{ll}
\hline Methods & RCT \\
\hline Participants & 418 people with hypercholesterolaemia (USA) \\
\hline Interventions & $\begin{array}{l}\text { Self-teaching vs group-teaching vs individual teaching vs mixed teaching (all teaching eating with } \\
\text { reduced and modified dietary fat) vs usual care, for } 9 \text { months }\end{array}$ \\
\hline Outcomes & Dietary intake and total cholesterol \\
\hline Notes & No answer to requests for data on deaths or health events \\
\hline
\end{tabular}


Naglak 2000

\begin{tabular}{ll}
\hline Methods & RCT \\
\hline Participants & 153 people within 16 primary care practices - cluster randomised by practice (USA) \\
\hline Interventions & Home based healthy heart programme vs usual care \\
\hline Outcomes & Dietary intake \\
\hline Notes & Have written to ask whether any deaths or CV events occurred, but no reply \\
\hline
\end{tabular}

\section{OLIVE 1997}

\begin{tabular}{ll}
\hline Methods & RCT \\
\hline Participants & 180 people with CHD documented by angiography \\
\hline Interventions & Mediterranean diet vs low fat diet, for 2.5 years \\
\hline Outcomes & Angiographic endpoints \\
\hline Notes & No answer to requests for data on deaths or health events \\
\hline
\end{tabular}

\section{Tapsell 2004}

\begin{tabular}{ll}
\hline Methods & RCT \\
\hline Participants & 86 people with type II diabetes (Australia) \\
\hline Interventions & Low fat diet vs modified fat diet, for 1 year \\
\hline Outcomes & Uptake of advice \\
\hline Notes & No answer to questions about whether any deaths or CV events occurred. \\
\hline
\end{tabular}

Verheiden 2003

\begin{tabular}{ll}
\hline Methods & RCT \\
\hline Participants & 143 patients at risk of CVD in Dutch general practices (Netherlands) \\
\hline Interventions & $\begin{array}{l}9 \text { family practices were randomised to dietary advice according to stages of change vs. usual care } \\
\text { for } 12 \text { months }\end{array}$ \\
\hline Outcomes & Fat intake and lipids \\
\hline Notes & No answer to requests for data on deaths or health events \\
\hline
\end{tabular}


Characteristics of ongoing studies [ordered by study ID]

PREDIMED 2008

\begin{tabular}{ll}
\hline Trial name or title & PREDIMED \\
\hline Methods & RCT \\
\hline Participants & 372 people at high cardiovascular risk \\
\hline Interventions & Low fat diet vs. modified fat diets (2), for 4 years \\
\hline Outcomes & Various CVD risk factors \\
\hline Starting date & 2003 \\
\hline Contact information & Professor Ramon Estruch, Department of Internal Medicine, University of Barcelona \\
\hline Notes & Authors replied that mortality and morbidity data will not be analysed until 2011 \\
\hline
\end{tabular}

\section{DATA AND ANALYSES}

Comparison 1. fat modification or reduction vs usual diet - primary outcomes

\begin{tabular}{|c|c|c|c|c|}
\hline Outcome or subgroup title & No. of studies & $\begin{array}{l}\text { No. of partici- } \\
\text { pants }\end{array}$ & Statistical method & Effect size \\
\hline 1 Total mortality & 21 & 71790 & Risk Ratio (M-H, Random, 95\% Cl) & $0.98[0.93,1.04]$ \\
\hline 1.1 Modified fat intake & 8 & 11441 & Risk Ratio (M-H, Random, 95\% Cl) & $1.02[0.88,1.18]$ \\
\hline 1.2 Reduced fat intake & 10 & 58130 & Risk Ratio (M-H, Random, 95\% Cl) & $0.97[0.90,1.04]$ \\
\hline $\begin{array}{l}1.3 \text { Reduced and modified fat } \\
\text { intake }\end{array}$ & 3 & 2219 & Risk Ratio (M-H, Random, 95\% Cl) & $0.97[0.76,1.23]$ \\
\hline 2 Cardiovascular mortality & 16 & 65978 & Risk Ratio (M-H, Random, 95\% Cl) & $0.94[0.85,1.04]$ \\
\hline 2.1 Modified fat intake & 6 & 10788 & Risk Ratio (M-H, Random, 95\% Cl) & $0.92[0.73,1.15]$ \\
\hline 2.2 Reduced fat intake & 7 & 52971 & Risk Ratio (M-H, Random, 95\% Cl) & $0.96[0.82,1.13]$ \\
\hline $\begin{array}{l}2.3 \text { Reduced and modified fat } \\
\text { intake }\end{array}$ & 3 & 2219 & Risk Ratio (M-H, Random, 95\% Cl) & $0.98[0.76,1.27]$ \\
\hline $\begin{array}{l}3 \text { Combined cardiovascular } \\
\text { events }\end{array}$ & 23 & 65508 & Risk Ratio (M-H, Random, 95\% Cl) & $0.86[0.77,0.96]$ \\
\hline 3.1 Modified fat intake & 9 & 11660 & Risk Ratio (M-H, Random, 95\% Cl) & $0.82[0.66,1.02]$ \\
\hline 3.2 Reduced fat intake & 8 & 50655 & Risk Ratio (M-H, Random, 95\% Cl) & $0.97[0.87,1.08]$ \\
\hline $\begin{array}{l}3.3 \text { Reduced and modified fat } \\
\text { intake }\end{array}$ & 6 & 3193 & Risk Ratio (M-H, Random, 95\% Cl) & $0.77[0.57,1.03]$ \\
\hline
\end{tabular}




\section{Analysis 1.1. Comparison 1 fat modification or reduction vs usual diet - primary outcomes, Outcome 1 Total mortality.}

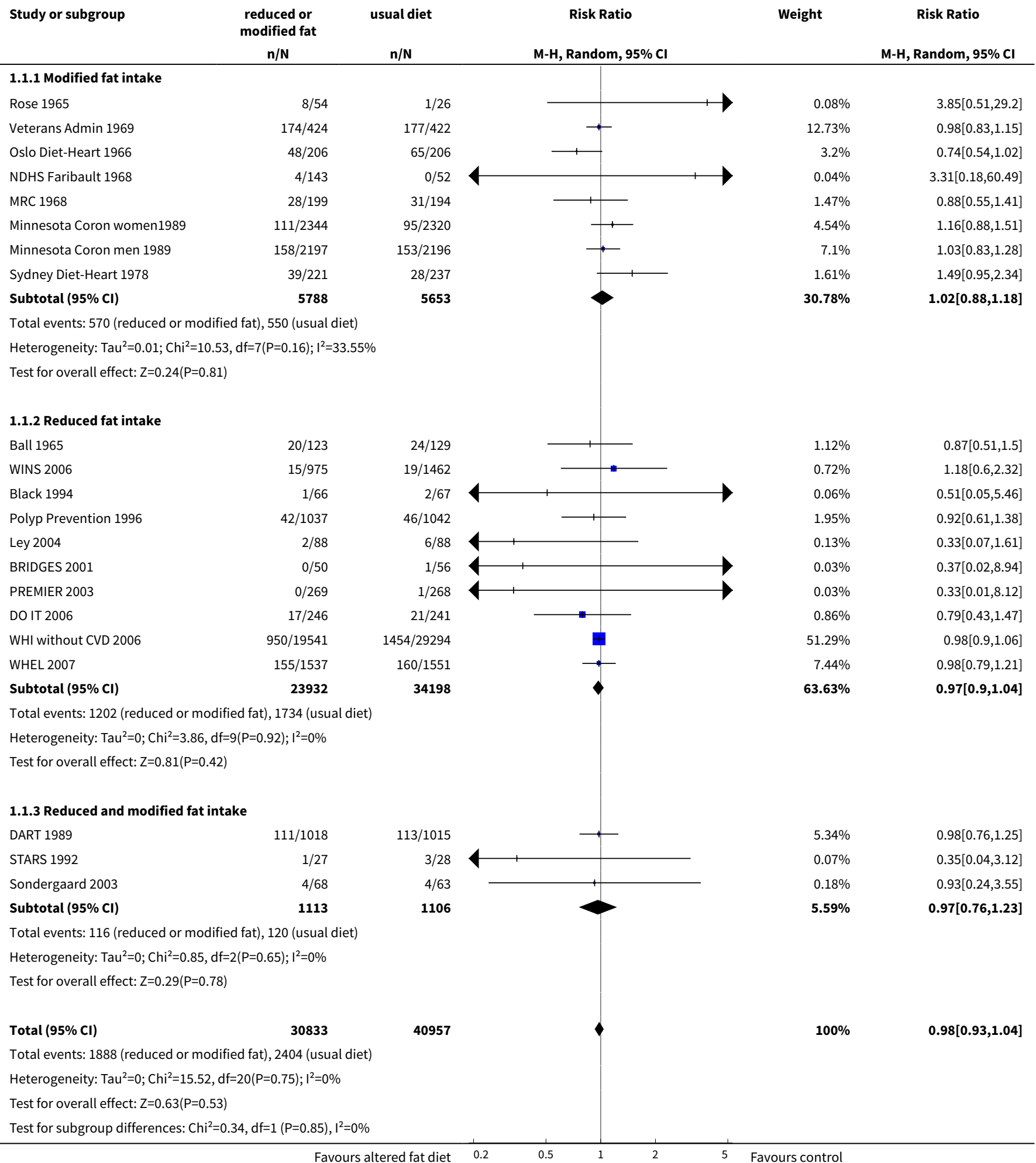




\section{Analysis 1.2. Comparison 1 fat modification or reduction vs usual diet - primary outcomes, Outcome $\mathbf{2}$ Cardiovascular mortality.}

\section{Study or subgroup}

\section{reduced or modified fat}

$\mathbf{n} / \mathbf{N}$

usual diet

$\mathbf{n} / \mathbf{N}$

Risk Ratio

Weight

M-H, Random, 95\% CI
Risk Ratio

M-H, Random, $95 \% \mathrm{Cl}$

\subsubsection{Modified fat intake}

Rose 1965

$8 / 54$

Veterans Admin 1969

$57 / 424$

$38 / 206$

Oslo Diet-Heart 1966

$$
27 / 199
$$

Minnesota Coron men 1989

$92 / 2197$

$65 / 2344$

5424

Subtotal $(95 \% \mathrm{Cl})$

Total events: 287 (reduced or modified fat), 306 (usual diet)

Heterogeneity: $\operatorname{Tau}^{2}=0.03 ; \mathrm{Chi}^{2}=9.13, \mathrm{df}=5(\mathrm{P}=0.1) ; \mathrm{I}^{2}=45.22 \%$

Test for overall effect: $Z=0.74(P=0.46)$

\subsubsection{Reduced fat intake}

Ball 1965

Black 1994

Ley 2004

DO IT 2006

WHI without CVD 2006

WHI with CVD 2006

WHEL 2007

Subtotal $(95 \% \mathrm{Cl})$

Total events: 241 (reduced or modified fat), 361 (usual diet) Heterogeneity: $\mathrm{Tau}^{2}=0 ; \mathrm{Chi}^{2}=4.1, \mathrm{df}=6(\mathrm{P}=0.66) ; \mathrm{I}^{2}=0 \%$

Test for overall effect: $\mathrm{Z}=0.46(\mathrm{P}=0.65)$

\subsubsection{Reduced and modified fat intake}

DART 1989

STARS 1992

$101 / 1018$

Sondergaard 2003

$1 / 27$

$3 / 68$

Subtotal $(95 \% \mathrm{Cl})$

1113

Total events: 105 (reduced or modified fat), 107 (usual diet) Heterogeneity: $\mathrm{Tau}^{2}=0 ; \mathrm{Chi}^{2}=1.12, \mathrm{df}=2(\mathrm{P}=0.57) ; \mathrm{I}^{2}=0 \%$

Test for overall effect: $\mathrm{Z}=0.15(\mathrm{P}=0.88)$

Total $(95 \% \mathrm{Cl})$

28138

Total events: 633 (reduced or modified fat), 774 (usual diet) Heterogeneity: $\mathrm{Tau}^{2}=0 ; \mathrm{Chi}^{2}=14.72, \mathrm{df}=15(\mathrm{P}=0.47) ; \mathrm{I}^{2}=0 \%$

Test for overall effect: $Z=1.19(P=0.23)$

Test for subgroup differences: $\mathrm{Chi}^{2}=0.17, \mathrm{df}=1(\mathrm{P}=0.92), \mathrm{I}^{2}=0 \%$

$1 / 2$

$1 / 26$
$81 / 422$
$52 / 206$
$25 / 194$
$94 / 2196$
$53 / 2320$
$\mathbf{5 3 6 4}$

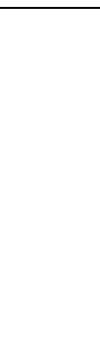

$3.85[0.51,29.2]$
$0.7[0.51,0.96]$
$0.73[0.5,1.06]$
$1.05[0.63,1.75]$
$0.98[0.74,1.3]$
$1.21[0.85,1.74]$
$\mathbf{0 . 9 2}[\mathbf{0 . 7 3 , 1 . 1 5}]$
$0.25 \%$

$10.79 \%$

$7.57 \%$

$4.06 \%$

$13.18 \%$

$8.13 \%$

$43.97 \%$
$0.89[0.49,1.62]$

$0.2[0.01,4.15]$

$0.25[0.03,2.19]$

$0.78[0.31,1.95]$

$0.99[0.81,1.2]$

$1.05[0.72,1.53]$

$0.4[0.08,2.08]$

$0.96[0.82,1.13]$
$1.01[0.77,1.31]$

$0.35[0.04,3.12]$

$0.69[0.16,2.98]$

$0.98[0.76,1.27]$ 
Analysis 1.3. Comparison 1 fat modification or reduction vs usual diet - primary outcomes, Outcome 3 Combined cardiovascular events.

\section{Study or subgroup}

\begin{abstract}
reduced or
modified fat
$\mathbf{n} / \mathbf{N}$
\end{abstract}

Risk Ratio

$\mathbf{n} / \mathbf{N}$
Weight

M-H, Random, $95 \% \mathrm{CI}$

\begin{abstract}
1.3.1 Modified fat intake
\end{abstract}
Veterans Admin 1969

Rose 1965

Oslo Diet-Heart 1966

NDHS Open 1st mod 1968

NDHS Open 2nd Mod 1968

MRC 1968

Minnesota Coron men 1989

Minnesota Coron women1989

Houtsmuller 1979

Subtotal $(95 \% \mathrm{CI})$

\section{$97 / 424$}

$26 / 54$

$64 / 206$

$122 / 422$

$11 / 26$

$90 / 206$

$4 / 348$

$1 / 170$

$1 / 112$

$2 / 140$

$62 / 199$

$74 / 194$

$67 / 2197$

$78 / 2196$

$67 / 2344$

$8 / 51$

5935

Total events: 396 (reduced or modified fat), 459 (usual diet)

Heterogeneity: $\mathrm{Tau}^{2}=0.06 ; \mathrm{Chi}^{2}=20.43, \mathrm{df}=8(\mathrm{P}=0.01) ; \mathrm{I}^{2}=60.85 \%$

Test for overall effect: $Z=1.79(P=0.07)$

\subsubsection{Reduced fat intake}

Ball 1965

Black 1994

Ley 2004

Moy 2001

PREMIER 2003

DO IT 2006

WHI without CVD 2006

WHI with CVD 2006

Subtotal $(95 \% \mathrm{Cl})$

Total events: 1439 (reduced or modified fat), 2193 (usual diet) Heterogeneity: $\mathrm{Tau}^{2}=0 ; \mathrm{Chi}^{2}=8.44, \mathrm{df}=7(\mathrm{P}=0.3) ; \mathrm{I}^{2}=17.06 \%$

Test for overall effect: $Z=0.6(P=0.55)$

\subsubsection{Reduced and modified fat intake}

NDHS Open 2nd L\&M 1968

NDHS Open 1st L\&M 1968

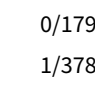

DART 1989

STARS 1992

Sondergaard 2003

MeDiet 2002

Subtotal $(95 \% \mathrm{CI})$
$38 / 123$

$0 / 66$

$11 / 88$

$5 / 117$

$0 / 269$

$28 / 246$

$1132 / 18633$

$225 / 908$

20450
$51 / 2320$

$30 / 51$

5725
$8.76 \%$

$3.39 \%$

$8.06 \%$

$0.26 \%$

$0.22 \%$

$7.6 \%$

$6.48 \%$

$5.7 \%$

$2.28 \%$

42.74\%
Risk Ratio

M-H, Random, 95\% CI
$5.64 \%$

$0.14 \%$

$2.11 \%$

$0.6 \%$

$0.13 \%$

$4.28 \%$

$13.18 \%$

$11.16 \%$

$37.25 \%$
$0.79[0.63,1]$

$1.14[0.67,1.93]$

$0.71[0.55,0.92]$

$1.95[0.22,17.35]$

$0.63[0.06,6.8]$

$0.82[0.62,1.07]$

$0.86[0.62,1.18]$

$1.3[0.91,1.86]$

$0.27[0.14,0.52]$

$0.82[0.66,1.02]$
$0.95[0.66,1.36]$

$0.2[0.01,4.15]$

$0.69[0.34,1.4]$

$1.68[0.41,6.87]$

$0.2[0.01,4.13]$

$0.69[0.44,1.07]$

$0.95[0.89,1.03]$

$1.09[0.94,1.27]$

$0.97[0.87,1.08]$
Total events: 185 (reduced or modified fat), 215 (usual diet)

Heterogeneity: $\mathrm{Tau}^{2}=0.04 ; \mathrm{Chi}^{2}=8.36, \mathrm{df}=5(\mathrm{P}=0.14) ; \mathrm{I}^{2}=40.21 \%$

Test for overall effect: $\mathrm{Z}=1.77(\mathrm{P}=0.08)$

Total $(95 \% \mathrm{Cl})$

28106

37402

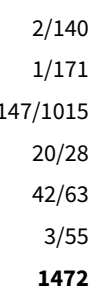

$0.13 \%$

$0.16[0.01,3.24]$

$0.16 \%$

$0.45[0.03,7.19]$

$9.16 \%$

$0.92[0.74,1.15]$

$0.41[0.22,0.78]$

$0.88[0.68,1.15]$

$7.82 \%$

$0.15[0.01,2.91]$

$20 \%$

$0.77[0.57,1.03]$

$100 \%$

$0.86[0.77,0.96]$

Total events: 2020 (reduced or modified fat), 2867 (usual diet) Heterogeneity: $\mathrm{Tau}^{2}=0.02 ; \mathrm{Chi}^{2}=44.3, \mathrm{df}=22(\mathrm{P}=0) ; \mathrm{I}^{2}=50.33 \%$

Test for overall effect: $Z=2.71(P=0.01)$

Test for subgroup differences: $\mathrm{Chi}^{2}=3.51, \mathrm{df}=1(\mathrm{P}=0.17), \mathrm{I}^{2}=42.97 \%$ 


\section{Comparison 2. fat modification or reduction vs usual diet - secondary outcomes}

\begin{tabular}{|c|c|c|c|c|}
\hline $\begin{array}{l}\text { Outcome or subgroup ti- } \\
\text { tle }\end{array}$ & No. of studies & $\begin{array}{l}\text { No. of partici- } \\
\text { pants }\end{array}$ & Statistical method & Effect size \\
\hline 1 Myocardial infarctions & 19 & 64891 & Risk Ratio (M-H, Random, 95\% Cl) & $0.93[0.84,1.02]$ \\
\hline 1.1 Modified fat intake & 9 & 11831 & Risk Ratio (M-H, Random, 95\% Cl) & $0.91[0.72,1.16]$ \\
\hline 1.2 Reduced fat intake & 6 & 50522 & Risk Ratio (M-H, Random, 95\% Cl) & $0.97[0.86,1.08]$ \\
\hline $\begin{array}{l}\text { 1.3 Reduced and modified } \\
\text { fat intake }\end{array}$ & 4 & 2538 & Risk Ratio (M-H, Random, 95\% Cl) & $0.90[0.72,1.11]$ \\
\hline 2 Stroke & 11 & 59853 & Risk Ratio (M-H, Random, 95\% Cl) & $0.99[0.89,1.11]$ \\
\hline 2.1 Modified fat intake & 4 & 10315 & Risk Ratio (M-H, Random, 95\% Cl) & $0.70[0.36,1.34]$ \\
\hline 2.2 Reduced fat intake & 4 & 49246 & Risk Ratio (M-H, Random, 95\% Cl) & $1.01[0.90,1.13]$ \\
\hline $\begin{array}{l}2.3 \text { Reduced and modified } \\
\text { fat intake }\end{array}$ & 3 & 292 & Risk Ratio (M-H, Random, 95\% Cl) & $0.40[0.08,2.04]$ \\
\hline 3 Cancer deaths & 10 & 64759 & Risk Ratio (M-H, Random, 95\% Cl) & $0.98[0.91,1.06]$ \\
\hline 3.1 Modified fat intake & 3 & 9903 & Risk Ratio (M-H, Random, 95\% Cl) & $1.46[0.96,2.21]$ \\
\hline 3.2 Reduced fat intake & 5 & 52692 & Risk Ratio (M-H, Random, 95\% Cl) & $0.97[0.90,1.05]$ \\
\hline $\begin{array}{l}\text { 3.3 Reduced and modified } \\
\text { fat intake }\end{array}$ & 2 & 2164 & Risk Ratio (M-H, Random, 95\% Cl) & $0.81[0.25,2.61]$ \\
\hline 4 Cancer diagnoses & 12 & 58847 & Risk Ratio (M-H, Random, 95\% Cl) & $0.96[0.91,1.01]$ \\
\hline 4.1 Modified fat intake & 2 & 1535 & Risk Ratio (M-H, Random, 95\% Cl) & $1.17[0.85,1.60]$ \\
\hline 4.2 Reduced fat intake & 8 & 57075 & Risk Ratio (M-H, Random, 95\% Cl) & $0.95[0.88,1.02]$ \\
\hline $\begin{array}{l}4.3 \text { Reduced and modified } \\
\text { intake }\end{array}$ & 2 & 237 & Risk Ratio (M-H, Random, 95\% Cl) & $0.73[0.06,9.03]$ \\
\hline 5 Diabetes diagnoses & 3 & 49859 & Risk Ratio (M-H, Random, 95\% Cl) & $0.96[0.90,1.02]$ \\
\hline 5.1 Modified fat intake & 0 & 0 & Risk Ratio (M-H, Random, 95\% Cl) & $0.0[0.0,0.0]$ \\
\hline 5.2 Reduced fat intake & 3 & 49859 & Risk Ratio (M-H, Random, 95\% Cl) & $0.96[0.90,1.02]$ \\
\hline $\begin{array}{l}5.3 \text { Reduced and modified } \\
\text { intake }\end{array}$ & 0 & 0 & Risk Ratio (M-H, Random, 95\% Cl) & $0.0[0.0,0.0]$ \\
\hline 6 Non-fatal MI & 13 & 54883 & Risk Ratio (M-H, Random, 95\% Cl) & $0.95[0.81,1.12]$ \\
\hline 6.1 Modified fat intake & 6 & 2672 & Risk Ratio (M-H, Random, 95\% Cl) & $0.86[0.64,1.16]$ \\
\hline 6.2 Reduced fat intake & 5 & 49859 & Risk Ratio (M-H, Random, 95\% Cl) & $1.06[0.80,1.40]$ \\
\hline
\end{tabular}




\begin{tabular}{lllll}
\hline $\begin{array}{l}\text { Outcome or subgroup ti- } \\
\text { tle }\end{array}$ & No. of studies & $\begin{array}{l}\text { No. of partici- } \\
\text { pants }\end{array}$ & Statistical method & Effect size \\
\hline $\begin{array}{l}\text { 6.3 Reduced and modified } \\
\text { intake }\end{array}$ & 2 & 2352 & Risk Ratio (M-H, Random, 95\% Cl) & $0.72[0.47,1.10]$ \\
\hline
\end{tabular}

\section{Analysis 2.1. Comparison 2 fat modification or reduction vs usual diet - secondary outcomes, Outcome 1 Myocardial infarctions.}

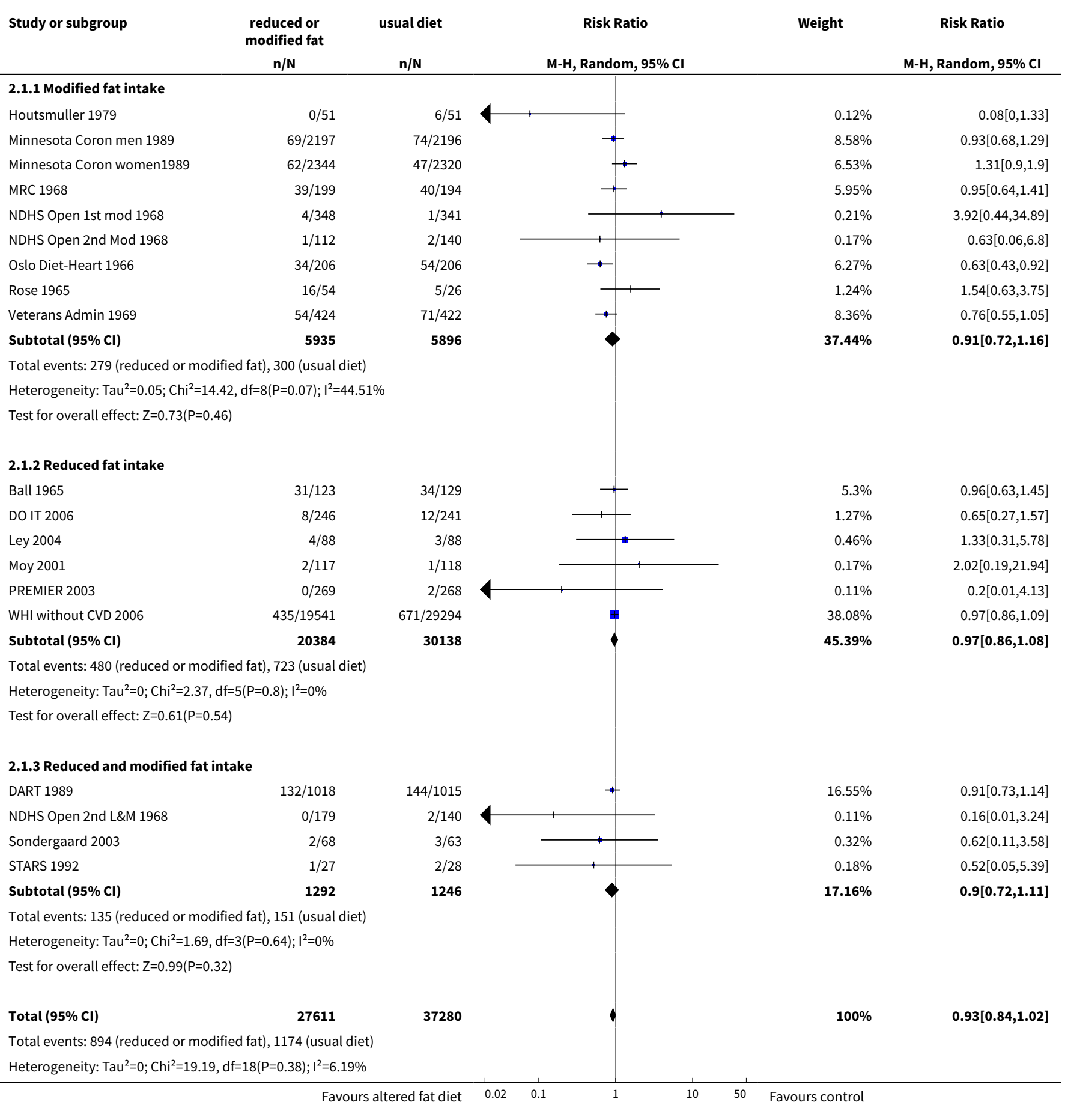




\begin{tabular}{|c|c|c|c|c|c|c|c|c|}
\hline Study or subgroup & $\begin{array}{c}\text { reduced or } \\
\text { modified fat } \\
n / N\end{array}$ & $\begin{array}{l}\text { usual diet } \\
\qquad \mathrm{n} / \mathrm{N}\end{array}$ & \multicolumn{3}{|c|}{ Risk Ratio } & \multicolumn{2}{|r|}{ Weight } & $\begin{array}{c}\text { Risk Ratio } \\
\text { M-H, Random, } 95 \% \mathrm{Cl}\end{array}$ \\
\hline \multicolumn{9}{|c|}{ Test for overall effect: $Z=1.52(P=0.13)$} \\
\hline \multicolumn{9}{|c|}{ Test for subgroup differences: $\mathrm{Chi}^{2}=0.45, \mathrm{df}=1(\mathrm{P}=0.8), \mathrm{I}^{2}=0 \%$} \\
\hline
\end{tabular}

Analysis 2.2. Comparison 2 fat modification or reduction vs usual diet - secondary outcomes, Outcome 2 Stroke.

\begin{tabular}{ccccc} 
Study or subgroup & $\begin{array}{c}\text { reduced or } \\
\text { modified fat } \\
\mathrm{n} / \mathrm{N}\end{array}$ & $\mathrm{n} / \mathrm{N}$ & Risk Ratio & Weight \\
& & $\mathrm{M}-\mathrm{H}, \mathrm{Random}, 95 \% \mathrm{Cl}$ & $\mathrm{M}-\mathrm{H}, \mathrm{Random}, 95 \% \mathrm{C}$ \\
\hline
\end{tabular}

\subsubsection{Modified fat intake}

Minnesota Coron men 1989

Minnesota Coron women1989

Oslo Diet-Heart 1966

Veterans Admin 1969

Subtotal $(95 \% \mathrm{Cl})$

$\mathrm{n} / \mathrm{N}$

M-H, Random, 95\% Cl

$\begin{array}{rr}0.14 \% & 0.11[0.01,2.06] \\ 0.72 \% & 1.24[0.33,4.6] \\ 0.22 \% & 2[0.18,21.89] \\ 2.73 \% & 0.59[0.3,1.15] \\ \mathbf{3 . 8 1} \% & \mathbf{0 . 7}[\mathbf{0 . 3 6 , 1 . 3 4}]\end{array}$

Total events: 20 (reduced or modified fat), 31 (usual diet)

Heterogeneity: $\mathrm{Tau}^{2}=0.05 ; \mathrm{Chi}^{2}=3.26, \mathrm{df}=3(\mathrm{P}=0.35) ; \mathrm{I}^{2}=8.11 \%$

Test for overall effect: $\mathrm{Z}=1.08(\mathrm{P}=0.28)$

\subsubsection{Reduced fat intake}

$\begin{array}{lrr}\text { Ley } 2004 & 1 / 88 & 5 / 88 \\ \text { Moy 2001 } & 1 / 117 & 1 / 118 \\ \text { WHI with CVD 2006 } & 206 / 908 & 308 / 1369 \\ \text { WHI without CVD 2006 } & 228 / 18633 & 334 / 27925 \\ \text { Subtotal (95\% Cl) } & \mathbf{1 9 7 4 6} & \mathbf{2 9 5 0 0}\end{array}$

Total events: 436 (reduced or modified fat), 648 (usual diet)

29500

Heterogeneity: $\mathrm{Tau}^{2}=0 ; \mathrm{Chi}^{2}=2.25, \mathrm{df}=3(\mathrm{P}=0.52) ; \mathrm{I}^{2}=0 \%$

Test for overall effect: $Z=0.18(P=0.86)$

\subsubsection{Reduced and modified fat intake}

MeDiet 2002

Sondergaard 2003

$1 / 68$

STARS 1992

Total events: 1 (reduced or modified fat), 4 (usual diet) Heterogeneity: $\mathrm{Tau}^{2}=0 ; \mathrm{Chi}^{2}=0.03, \mathrm{df}=2(\mathrm{P}=0.99) ; \mathrm{I}^{2}=0 \%$ Test for overall effect: $\mathrm{Z}=1.1(\mathrm{P}=0.27)$

\begin{tabular}{l|l}
\hline \\
\hline+1 \\
\hline
\end{tabular}

$0.7[0.36,1.34]$

Total events: 457 (reduced or modified fat), 683 (usual diet) 
Analysis 2.3. Comparison 2 fat modification or reduction vs usual diet - secondary outcomes, Outcome 3 Cancer deaths.

\begin{tabular}{|c|c|c|c|c|c|}
\hline Study or subgroup & $\begin{array}{c}\text { reduced or } \\
\text { modified fat } \\
n / N\end{array}$ & usual diet & M-H, Random, 95\% Cl & Weight & $\begin{array}{c}\text { Risk Ratio } \\
\text { M-H, Random, } 95 \% \mathrm{CI} \\
\end{array}$ \\
\hline \multicolumn{6}{|l|}{ 2.3.1 Modified fat intake } \\
\hline Minnesota Coron men 1989 & $16 / 2197$ & $12 / 2196$ & - & $0.97 \%$ & $1.33[0.63,2.81]$ \\
\hline Minnesota Coron women1989 & $7 / 2344$ & $8 / 2320$ & $\longrightarrow+$ & $0.52 \%$ & $0.87[0.31,2.38]$ \\
\hline Veterans Admin 1969 & $31 / 424$ & $17 / 422$ & 1 & $1.62 \%$ & $1.81[1.02,3.23]$ \\
\hline Subtotal $(95 \% \mathrm{Cl})$ & 4965 & 4938 & & $3.11 \%$ & $1.46[0.96,2.21]$ \\
\hline \multicolumn{6}{|c|}{ Total events: 54 (reduced or modified fat), 37 (usual diet) } \\
\hline \multicolumn{6}{|c|}{ Heterogeneity: $\operatorname{Tau}^{2}=0 ; \mathrm{Chi}^{2}=1.63, \mathrm{df}=2(\mathrm{P}=0.44) ; \mathrm{I}^{2}=0 \%$} \\
\hline \multicolumn{6}{|c|}{ Test for overall effect: $Z=1.77(P=0.08)$} \\
\hline \multicolumn{6}{|l|}{ 2.3.2 Reduced fat intake } \\
\hline BRIDGES 2001 & $0 / 50$ & $1 / 56$ & & $0.05 \%$ & $0.37[0.02,8.94]$ \\
\hline Ley 2004 & $1 / 88$ & $2 / 88$ & & $0.09 \%$ & $0.5[0.05,5.41]$ \\
\hline WHEL 2007 & $139 / 1537$ & $150 / 1551$ & + & $11.13 \%$ & $0.94[0.75,1.16]$ \\
\hline WHI without CVD 2006 & $950 / 19541$ & $1454 / 29294$ & & $84.53 \%$ & $0.98[0.9,1.06]$ \\
\hline Subtotal $(95 \% \mathrm{Cl})$ & 21462 & 31230 & 1 & $96.5 \%$ & $0.97[0.9,1.05]$ \\
\hline \multicolumn{6}{|c|}{ Total events: 1099 (reduced or modified fat), 1617 (usual diet) } \\
\hline \multicolumn{6}{|c|}{ Heterogeneity: $\mathrm{Tau}^{2}=0 ; \mathrm{Chi}^{2}=0.85, \mathrm{df}=4(\mathrm{P}=0.93) ; \mathrm{I}^{2}=0 \%$} \\
\hline \multicolumn{6}{|c|}{ Test for overall effect: $Z=0.74(P=0.46)$} \\
\hline \multicolumn{6}{|c|}{ 2.3.3 Reduced and modified fat intake } \\
\hline DART 1989 & $4 / 1018$ & $6 / 1015$ & $\longrightarrow$ & $0.34 \%$ & $0.66[0.19,2.35]$ \\
\hline Sondergaard 2003 & $1 / 68$ & $0 / 63$ & & $0.05 \%$ & $2.78[0.12,67.08]$ \\
\hline Subtotal $(95 \% \mathrm{CI})$ & 1086 & 1078 & & $0.39 \%$ & $0.81[0.25,2.61]$ \\
\hline \multicolumn{6}{|c|}{ Total events: 5 (reduced or modified fat), 6 (usual diet) } \\
\hline \multicolumn{6}{|c|}{ Test for overall effect: $Z=0.36(P=0.72)$} \\
\hline Total $(95 \% \mathrm{Cl})$ & 27513 & 37246 & 1 & $100 \%$ & $0.98[0.91,1.06]$ \\
\hline \multicolumn{6}{|c|}{ Total events: 1158 (reduced or modified fat), 1660 (usual diet) } \\
\hline \multicolumn{6}{|c|}{ Heterogeneity: $\mathrm{Tau}^{2}=0 ; \mathrm{Chi}^{2}=6.77, \mathrm{df}=9(\mathrm{P}=0.66) ; \mathrm{I}^{2}=0 \%$} \\
\hline \multicolumn{6}{|c|}{ Test for overall effect: $Z=0.43(P=0.66)$} \\
\hline Test for subgroup differences: & 52, $d f=1(P=0.16)$, & $68 \%$ & & & \\
\hline
\end{tabular}

Analysis 2.4. Comparison 2 fat modification or reduction vs usual diet - secondary outcomes, Outcome 4 Cancer diagnoses.

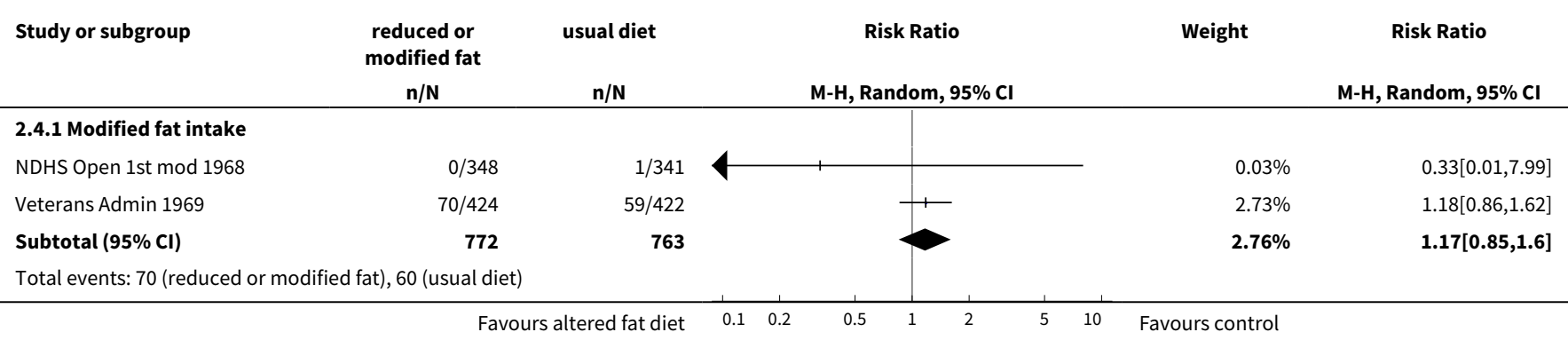




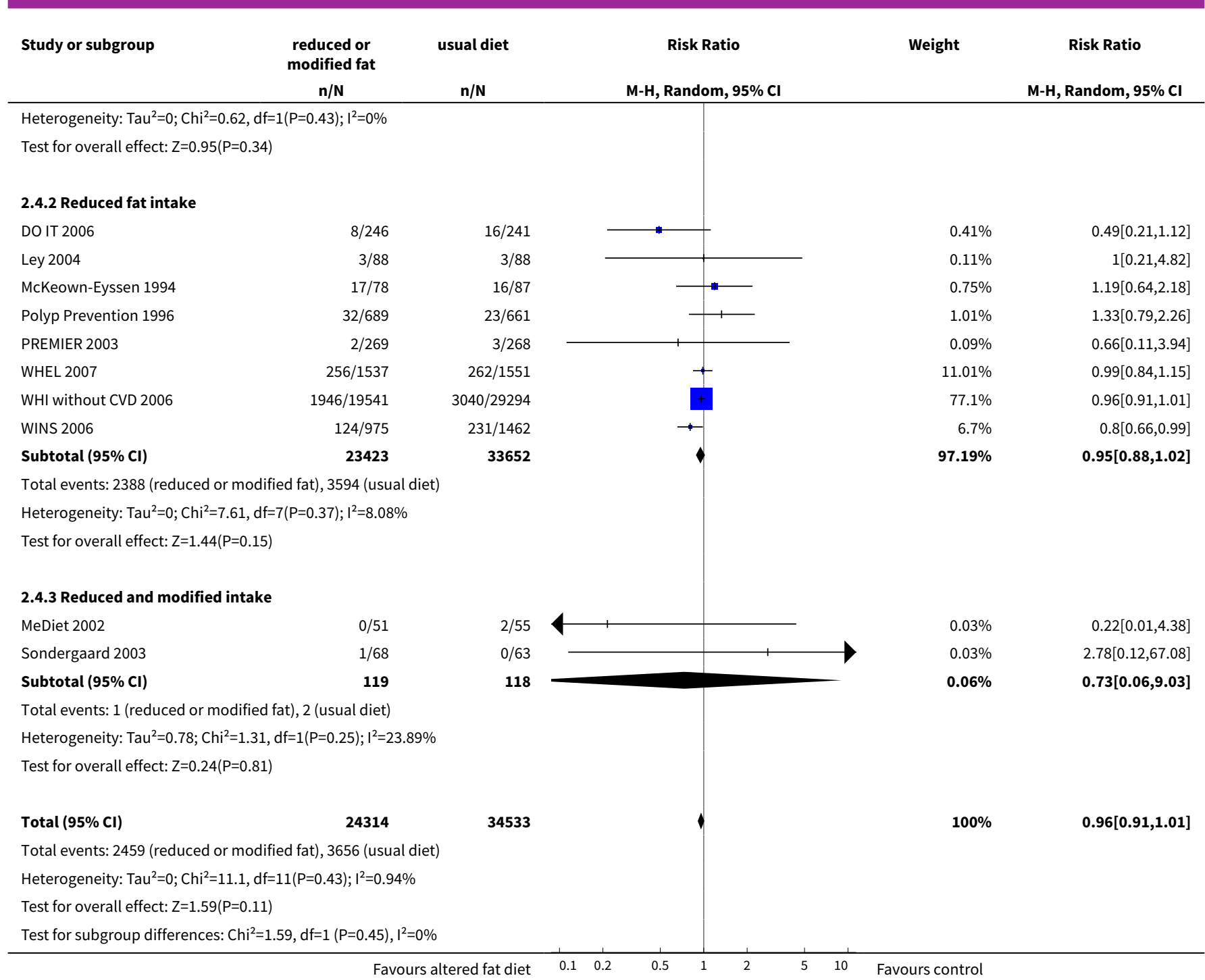

Analysis 2.5. Comparison 2 fat modification or reduction vs usual diet - secondary outcomes, Outcome 5 Diabetes diagnoses.

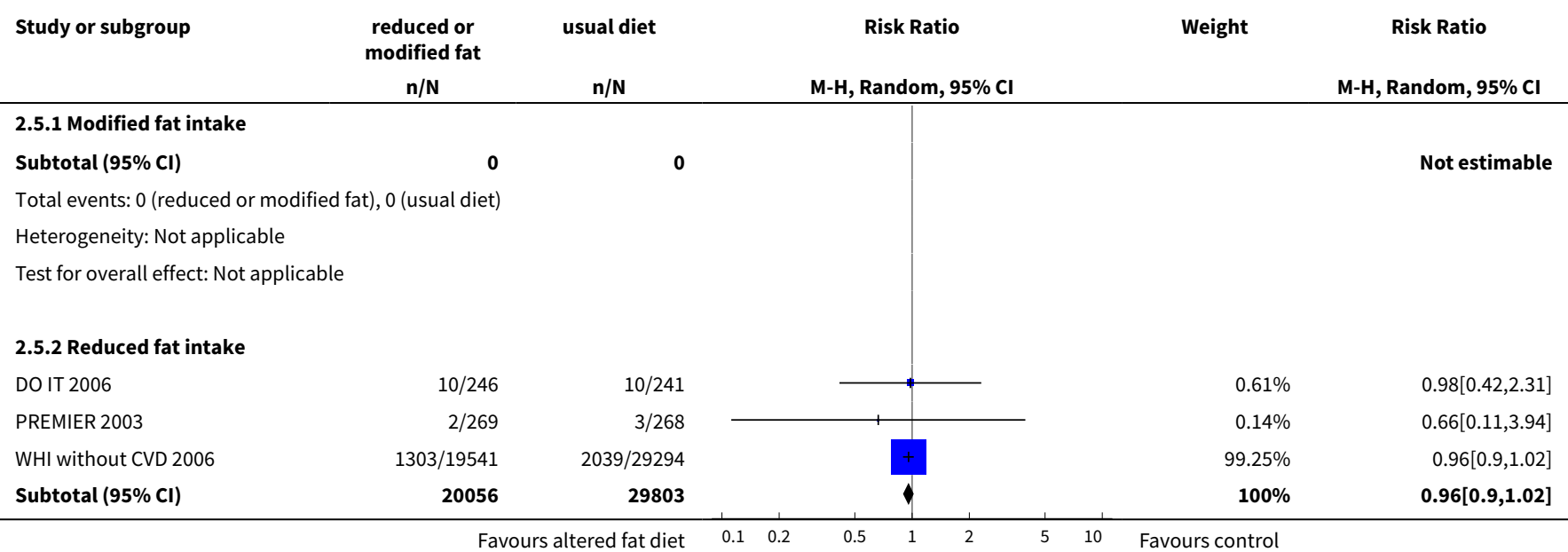




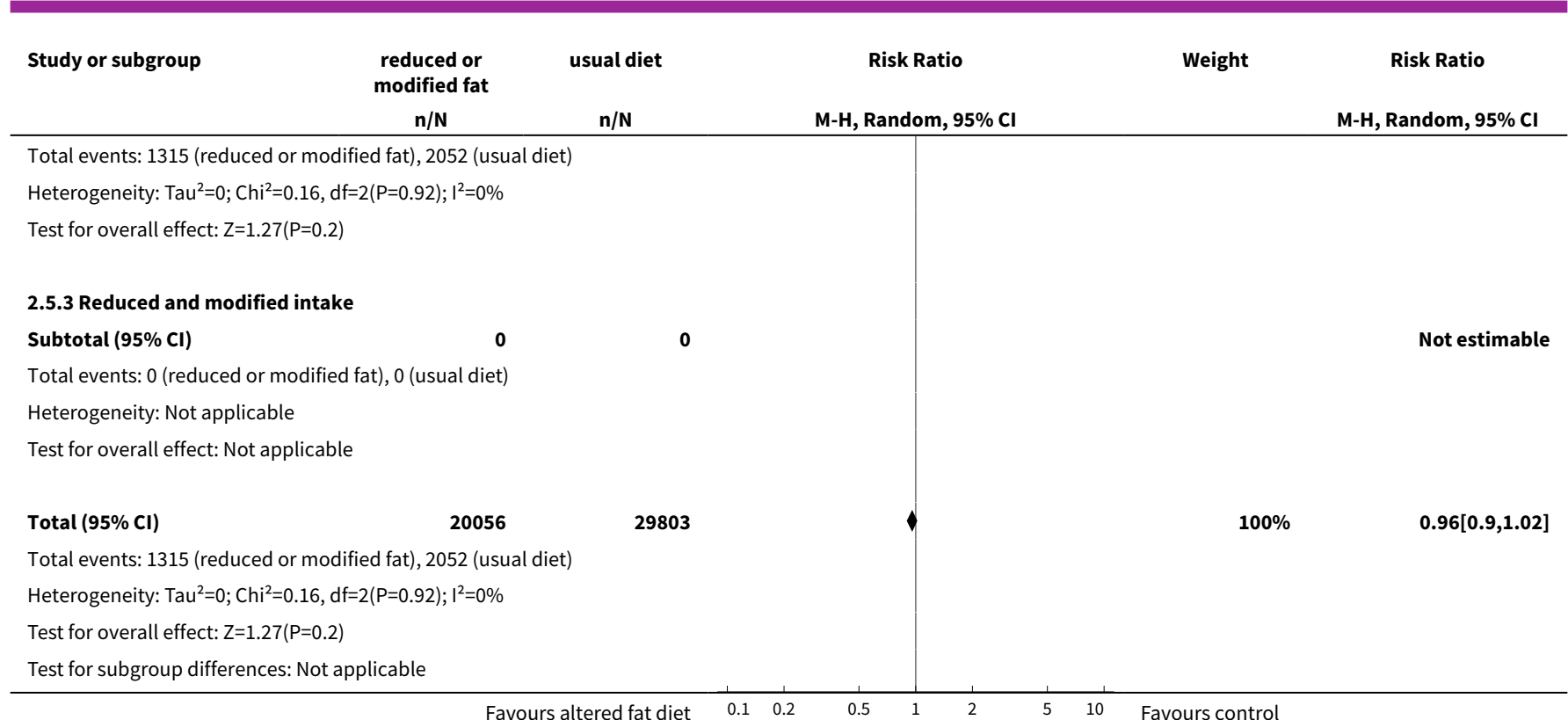

\section{Analysis 2.6. Comparison $\mathbf{2}$ fat modification or reduction vs usual diet - secondary outcomes, Outcome 6 Non-fatal MI.}

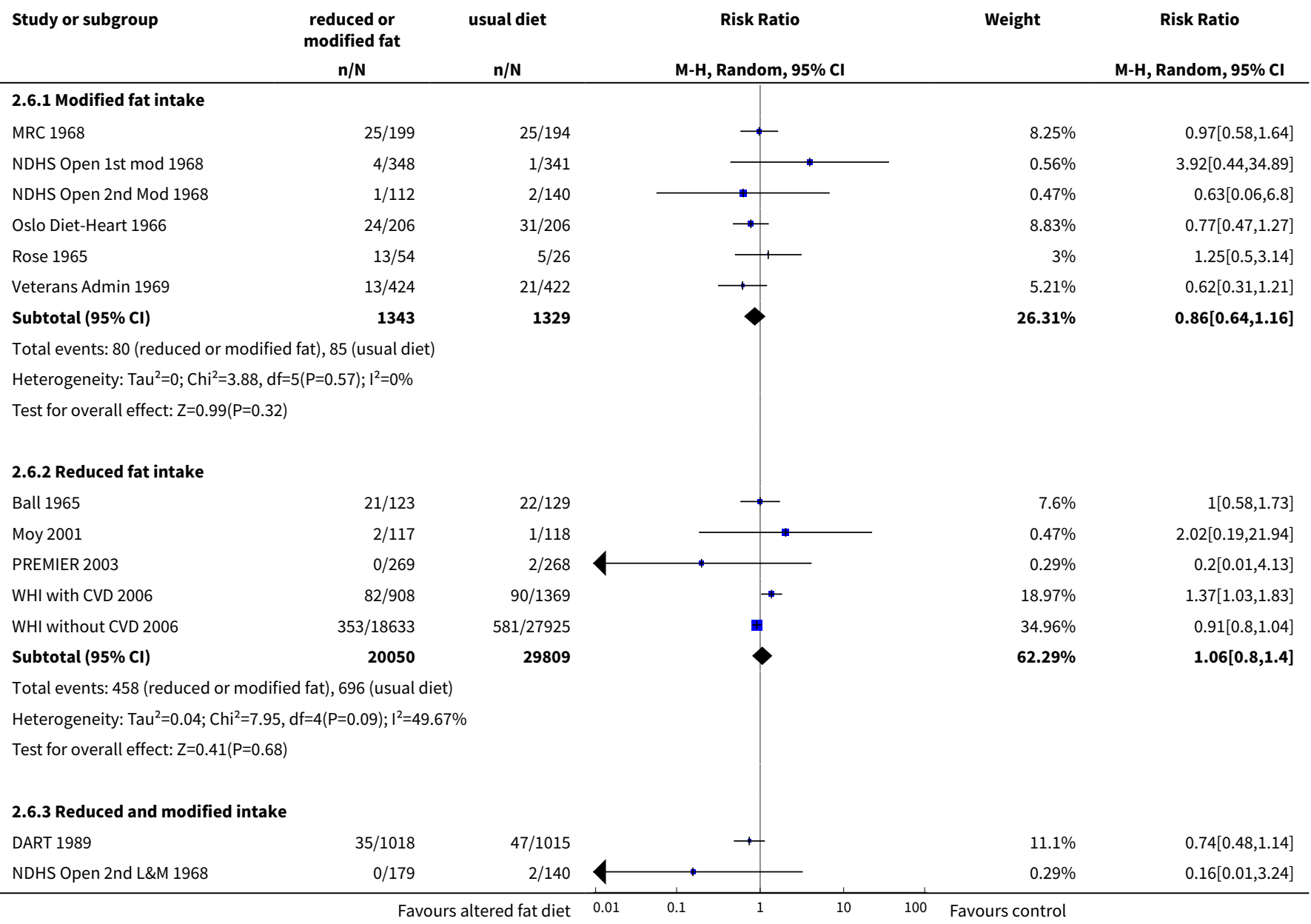




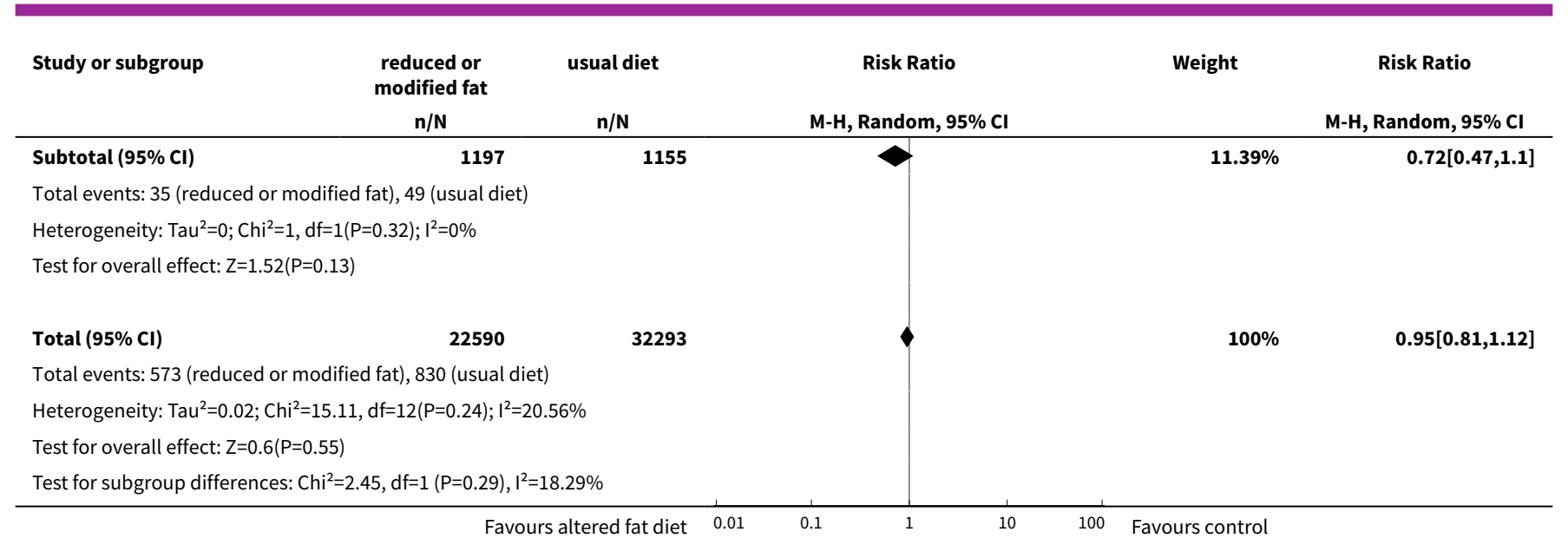

\section{Comparison 3. fat modification or reduction vs usual diet - tertiary outcomes}

\begin{tabular}{|c|c|c|c|c|}
\hline Outcome or subgroup title & No. of studies & $\begin{array}{l}\text { No. of partici- } \\
\text { pants }\end{array}$ & Statistical method & Effect size \\
\hline 1 Weight, kg & 18 & & $\begin{array}{l}\text { Mean Difference (IV, Random, 95\% } \\
\mathrm{Cl} \text { ) }\end{array}$ & Subtotals only \\
\hline 1.1 Modified fat intake & 2 & 99 & $\begin{array}{l}\text { Mean Difference (IV, Random, 95\% } \\
\mathrm{CI})\end{array}$ & $-1.10[-3.14,0.93]$ \\
\hline 1.2 Reduced fat intake & 16 & 11058 & $\begin{array}{l}\text { Mean Difference (IV, Random, 95\% } \\
\mathrm{CI})\end{array}$ & $-0.83[-1.37,-0.30]$ \\
\hline $\begin{array}{l}1.3 \text { Reduced and modified } \\
\text { fat intake }\end{array}$ & 0 & 0 & $\begin{array}{l}\text { Mean Difference (IV, Random, 95\% } \\
\mathrm{CI})\end{array}$ & $0.0[0.0,0.0]$ \\
\hline $2 \mathrm{BMI}, \mathrm{kg} / \mathrm{m} 2$ & 14 & & $\begin{array}{l}\text { Mean Difference (IV, Random, 95\% } \\
\mathrm{CI})\end{array}$ & Subtotals only \\
\hline 2.1 Modified fat intake & 2 & 116 & $\begin{array}{l}\text { Mean Difference (IV, Random, 95\% } \\
\mathrm{CI})\end{array}$ & $-0.34[-0.99,0.31]$ \\
\hline 2.2 Reduced fat intake & 10 & 5972 & $\begin{array}{l}\text { Mean Difference (IV, Random, 95\% } \\
\mathrm{CI})\end{array}$ & $-0.47[-0.72,-0.23]$ \\
\hline $\begin{array}{l}2.3 \text { Reduced and modified } \\
\text { fat intake }\end{array}$ & 2 & 111 & $\begin{array}{l}\text { Mean Difference (IV, Random, 95\% } \\
\mathrm{CI})\end{array}$ & $-0.20[-1.30,0.91]$ \\
\hline 3 LDL cholesterol, mmol/L & 20 & & $\begin{array}{l}\text { Mean Difference (IV, Random, 95\% } \\
\mathrm{CI})\end{array}$ & Subtotals only \\
\hline 3.1 Modified fat intake & 2 & 116 & $\begin{array}{l}\text { Mean Difference (IV, Random, 95\% } \\
\mathrm{CI})\end{array}$ & $-0.20[-0.47,0.07]$ \\
\hline 3.2 Reduced fat intake & 14 & 6971 & $\begin{array}{l}\text { Mean Difference (IV, Random, 95\% } \\
\mathrm{CI})\end{array}$ & $-0.10[-0.14,-0.05]$ \\
\hline $\begin{array}{l}3.3 \text { Reduced and modified } \\
\text { fat intake }\end{array}$ & 4 & 627 & $\begin{array}{l}\text { Mean Difference (IV, Random, 95\% } \\
\mathrm{Cl} \text { ) }\end{array}$ & $-0.21[-0.35,-0.08]$ \\
\hline
\end{tabular}




\begin{tabular}{|c|c|c|c|c|}
\hline Outcome or subgroup title & No. of studies & $\begin{array}{l}\text { No. of partici- } \\
\text { pants }\end{array}$ & Statistical method & Effect size \\
\hline $\begin{array}{l}4 \mathrm{HDL} \text { cholesterol, mmol/ } \\
\text { Lkg }\end{array}$ & 22 & & $\begin{array}{l}\text { Mean Difference (IV, Random, 95\% } \\
\mathrm{Cl} \text { ) }\end{array}$ & Subtotals only \\
\hline 4.1 Modified fat intake & 3 & 152 & $\begin{array}{l}\text { Mean Difference (IV, Random, 95\% } \\
\mathrm{CI})\end{array}$ & $-0.04[-0.18,0.09]$ \\
\hline 4.2 Reduced fat intake & 15 & 7082 & $\begin{array}{l}\text { Mean Difference (IV, Random, 95\% } \\
\mathrm{CI} \text { ) }\end{array}$ & $-0.01[-0.02,0.01]$ \\
\hline $\begin{array}{l}4.3 \text { Reduced and modified } \\
\text { fat intake }\end{array}$ & 4 & 2073 & $\begin{array}{l}\text { Mean Difference (IV, Random, 95\% } \\
\mathrm{CI})\end{array}$ & $-0.01[-0.04,0.01]$ \\
\hline 5 Total cholesterol, $\mathrm{mmol} / \mathrm{L}$ & 28 & & $\begin{array}{l}\text { Mean Difference (IV, Random, 95\% } \\
\mathrm{Cl} \text { ) }\end{array}$ & Subtotals only \\
\hline 5.1 Modified fat intake & 8 & 2280 & $\begin{array}{l}\text { Mean Difference (IV, Random, 95\% } \\
\mathrm{Cl} \text { ) }\end{array}$ & $-0.44[-0.60,-0.28]$ \\
\hline 5.2 Reduced fat intake & 15 & 7602 & $\begin{array}{l}\text { Mean Difference (IV, Random, 95\% } \\
\mathrm{CI} \text { ) }\end{array}$ & $-0.10[-0.14,-0.05]$ \\
\hline $\begin{array}{l}5.3 \text { Reduced and modified } \\
\text { fat intake }\end{array}$ & 5 & 2131 & $\begin{array}{l}\text { Mean Difference (IV, Random, 95\% } \\
\mathrm{CI})\end{array}$ & $-0.26[-0.47,-0.04]$ \\
\hline 6 Triglycerides, $\mathrm{mmol} / \mathrm{L}$ & 21 & & $\begin{array}{l}\text { Mean Difference (IV, Random, 95\% } \\
\mathrm{CI})\end{array}$ & Subtotals only \\
\hline 6.1 Modified fat intake & 5 & 706 & $\begin{array}{l}\text { Mean Difference (IV, Random, 95\% } \\
\mathrm{CI})\end{array}$ & $-0.11[-0.22,-0.00]$ \\
\hline 6.2 Reduced fat intake & 13 & 6875 & $\begin{array}{l}\text { Mean Difference (IV, Random, 95\% } \\
\mathrm{CI} \text { ) }\end{array}$ & $-0.00[-0.00,0.00]$ \\
\hline $\begin{array}{l}6.3 \text { Reduced and modified } \\
\text { fat intake }\end{array}$ & 3 & 218 & $\begin{array}{l}\text { Mean Difference (IV, Random, 95\% } \\
\mathrm{CI} \text { ) }\end{array}$ & $-0.27[-0.53,-0.00]$ \\
\hline $\begin{array}{l}7 \text { Systolic Blood Pressure, } \\
\mathrm{mmHg}\end{array}$ & 7 & 4059 & $\begin{array}{l}\text { Mean Difference (IV, Random, 95\% } \\
\mathrm{Cl} \text { ) }\end{array}$ & $-0.67[-1.61,0.28]$ \\
\hline 7.1 Modified fat intake & 0 & 0 & $\begin{array}{l}\text { Mean Difference (IV, Random, 95\% } \\
\mathrm{Cl} \text { ) }\end{array}$ & $0.0[0.0,0.0]$ \\
\hline 7.2 Reduced fat intake & 6 & 3981 & $\begin{array}{l}\text { Mean Difference (IV, Random, 95\% } \\
\mathrm{CI} \text { ) }\end{array}$ & $-0.56[-1.52,0.40]$ \\
\hline $\begin{array}{l}7.3 \text { Reduced and modified } \\
\text { fat intake }\end{array}$ & 1 & 78 & $\begin{array}{l}\text { Mean Difference (IV, Random, 95\% } \\
\mathrm{CI})\end{array}$ & $-5.08[-11.22,1.06]$ \\
\hline $\begin{array}{l}8 \text { Diastolic Blood Pressure, } \\
\mathrm{mmHg}\end{array}$ & 7 & 3621 & $\begin{array}{l}\text { Mean Difference (IV, Random, 95\% } \\
\mathrm{CI} \text { ) }\end{array}$ & $-0.40[1.00,0.20]$ \\
\hline 8.1 Modified fat intake & 0 & 0 & $\begin{array}{l}\text { Mean Difference (IV, Random, 95\% } \\
\mathrm{CI} \text { ) }\end{array}$ & $0.0[0.0,0.0]$ \\
\hline
\end{tabular}




\begin{tabular}{lllll}
\hline Outcome or subgroup title & No. of studies & $\begin{array}{l}\text { No. of partici- } \\
\text { pants }\end{array}$ & Statistical method & Effect size \\
\hline 8.2 Reduced fat intake & 6 & 3543 & $\begin{array}{l}\text { Mean Difference (IV, Random, 95\% } \\
\text { Cl) }\end{array}$ & $-0.35[-0.96,0.26]$ \\
\hline $\begin{array}{l}8.3 \text { Reduced and modified } \\
\text { fat intake }\end{array}$ & 1 & 78 & $\begin{array}{l}\text { Mean Difference (IV, Random, 95\% } \\
\text { Cl) }\end{array}$ & $-2.31[-6.20,1.58]$ \\
\hline
\end{tabular}

Analysis 3.1. Comparison 3 fat modification or reduction vs usual diet - tertiary outcomes, Outcome 1 Weight, $\mathrm{kg}$.

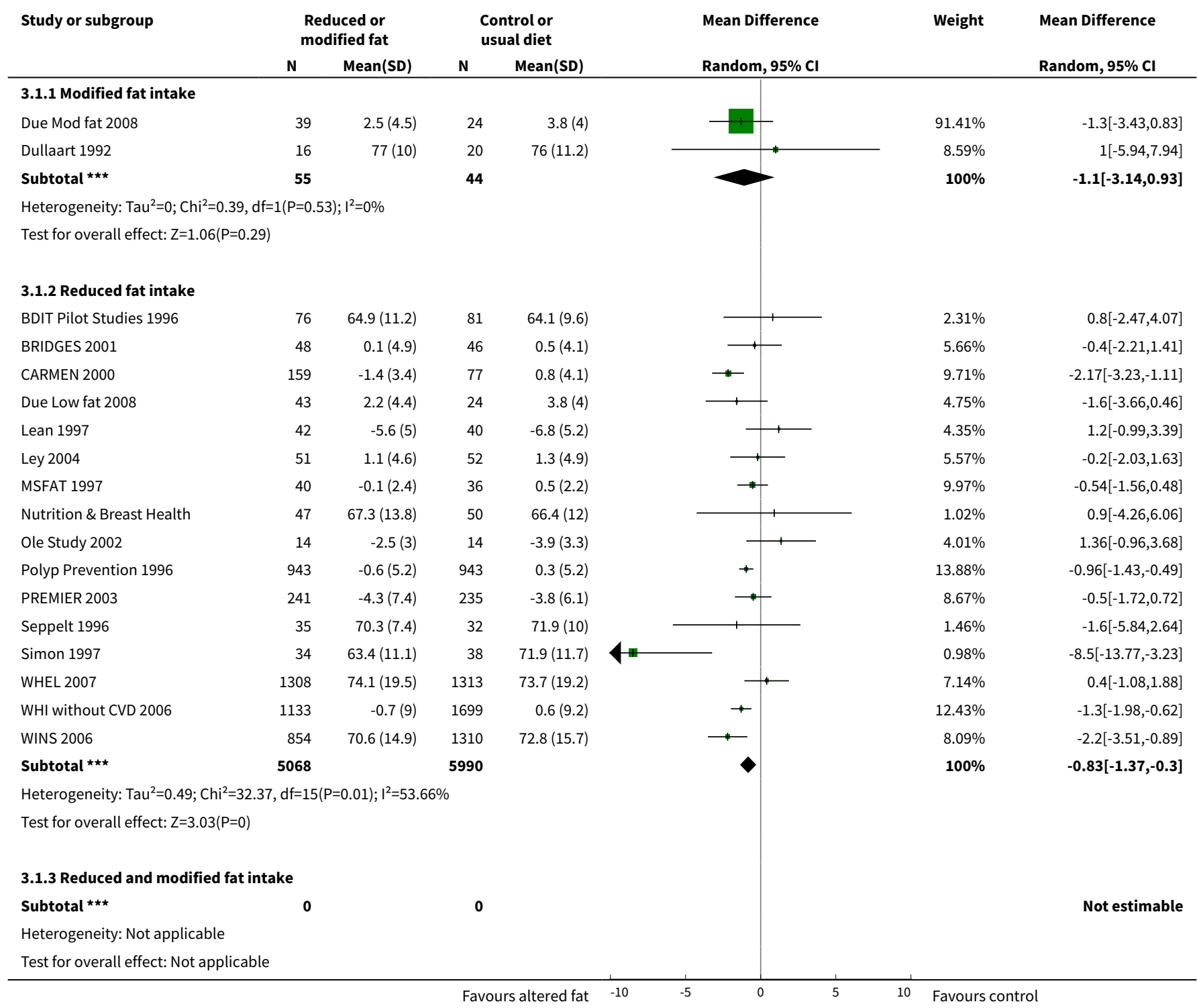


Analysis 3.2. Comparison 3 fat modification or reduction vs usual diet - tertiary outcomes, Outcome 2 BMI, $\mathrm{kg} / \mathrm{m} 2$.

\begin{tabular}{|c|c|c|c|c|c|c|c|}
\hline \multirow[t]{2}{*}{ Study or subgroup } & \multicolumn{2}{|c|}{$\begin{array}{l}\text { Reduced or } \\
\text { modified fat }\end{array}$} & \multicolumn{2}{|c|}{$\begin{array}{l}\text { Control or } \\
\text { usual diet }\end{array}$} & \multirow{2}{*}{$\begin{array}{l}\text { Mean Difference } \\
\text { Random, } 95 \% \mathrm{Cl}\end{array}$} & \multirow[t]{2}{*}{ Weight } & \multirow{2}{*}{$\begin{array}{l}\text { Mean Difference } \\
\text { Random, } 95 \% \mathrm{Cl}\end{array}$} \\
\hline & $\mathbf{N}$ & $\operatorname{Mean}(S D)$ & $\mathbf{N}$ & Mean(SD) & & & \\
\hline \multicolumn{8}{|l|}{ 3.2.1 Modified fat intake } \\
\hline Due Mod fat 2008 & 39 & $0.8(1.4)$ & 24 & $1.2(1.3)$ & & $93.83 \%$ & $-0.4[-1.07,0.27]$ \\
\hline Sarkkinen Fat Mod 1995 & 41 & $26.3(3.6)$ & 12 & $25.7(4.2)$ & & $6.17 \%$ & $0.6[-2.02,3.22]$ \\
\hline Subtotal $* \star \star$ & 80 & & 36 & & & $100 \%$ & $-0.34[-0.99,0.31]$ \\
\hline \multicolumn{8}{|c|}{ Heterogeneity: $\operatorname{Tau}^{2}=0 ; \mathrm{Chi}^{2}=0.53, \mathrm{df}=1(\mathrm{P}=0.47) ;\left.\right|^{2}=0 \%$} \\
\hline \multicolumn{8}{|c|}{ Test for overall effect: $Z=1.02(P=0.31)$} \\
\hline \multicolumn{8}{|l|}{ 3.2.2 Reduced fat intake } \\
\hline BDIT Pilot Studies 1996 & 76 & $24.3(3.8)$ & 81 & $24.3(3.6)$ & & $3.81 \%$ & $0[-1.16,1.16]$ \\
\hline CARMEN MS sub-study 2002 & 18 & $-0.8(1.4)$ & 8 & $0.4(0.9)$ & & $5.83 \%$ & $-1.17[-2.07,-0.28]$ \\
\hline DO IT 2006 & 233 & $-0.2(1.3)$ & 231 & $0.2(1.3)$ & $*$ & $21.57 \%$ & $-0.37[-0.61,-0.13]$ \\
\hline Lean 1997 & 42 & $-2.2(1.8)$ & 40 & $-2.6(2.1)$ & -1 & $6.3 \%$ & $0.4[-0.45,1.25]$ \\
\hline Moy 2001 & 117 & $-0.1(1)$ & 118 & $0.2(2)$ & $\rightarrow$ & $15.55 \%$ & $-0.31[-0.71,0.09]$ \\
\hline Sarkkinen Red Fat 1995 & 40 & $26.2(3.2)$ & 12 & $25.7(4.2)$ & & $0.87 \%$ & $0.5[-2.07,3.07]$ \\
\hline Simon 1997 & 34 & $23.8(4.7)$ & 38 & $27.4(4.9)$ & & $1.16 \%$ & $-3.6[-5.82,-1.38]$ \\
\hline WHI without CVD 2006 & 1133 & $-0.2(2.7)$ & 1699 & $0.3(2.7)$ & + & $22.79 \%$ & $-0.5[-0.7,-0.3]$ \\
\hline WINS 2006 & 755 & $26.8(5.6)$ & 1230 & $27.6(5.4)$ & $\longrightarrow$ & $12.67 \%$ & $-0.8[-1.3,-0.3]$ \\
\hline Subtotal $\star \star \star$ & 2491 & & 3481 & & $\diamond$ & $100 \%$ & $-0.47[-0.72,-0.23]$ \\
\hline \multicolumn{8}{|c|}{ Heterogeneity: $\operatorname{Tau}^{2}=0.06 ; \mathrm{Chi}^{2}=18.21, \mathrm{df}=9(\mathrm{P}=0.03) ; \mathrm{I}^{2}=50.59 \%$} \\
\hline \multicolumn{8}{|c|}{ Test for overall effect: $Z=3.78(P=0)$} \\
\hline \multicolumn{8}{|c|}{ 3.2.3 Reduced and modified fat intake } \\
\hline Oxford Retinopathy 1978 & 29 & $-1(2.8)$ & 29 & $-0.7(1.8)$ & & $82.96 \%$ & $-0.3[-1.51,0.91]$ \\
\hline Sarkkinen Red \& Mod 1995 & 41 & $26(4)$ & 12 & $25.7(4.2)$ & & $17.04 \%$ & $0.3[-2.37,2.97]$ \\
\hline Test for overall effect: $Z=0.35$ & & & & & & & \\
\hline
\end{tabular}

\section{Analysis 3.3. Comparison 3 fat modification or reduction vs usual diet - tertiary outcomes, Outcome 3 LDL cholesterol, mmol/L.}

\begin{tabular}{|c|c|c|c|c|c|c|c|}
\hline \multirow[t]{2}{*}{ Study or subgroup } & \multicolumn{2}{|c|}{$\begin{array}{l}\text { Reduced or } \\
\text { modified fat }\end{array}$} & \multicolumn{2}{|c|}{$\begin{array}{l}\text { Control or } \\
\text { usual diet }\end{array}$} & \multirow{2}{*}{$\begin{array}{l}\text { Mean Difference } \\
\text { Random, } 95 \% \mathrm{Cl}\end{array}$} & \multirow[t]{2}{*}{ Weight } & \multirow{2}{*}{$\begin{array}{l}\text { Mean Difference } \\
\text { Random, } 95 \% \mathrm{Cl}\end{array}$} \\
\hline & $\mathbf{N}$ & Mean(SD) & $\mathbf{N}$ & Mean(SD) & & & \\
\hline \multicolumn{8}{|l|}{ 3.3.1 Modified fat intake } \\
\hline Due Mod fat 2008 & 39 & $-0.1(0.5)$ & 24 & $0.1(0.6)$ & - & $81.73 \%$ & $-0.22[-0.51,0.07]$ \\
\hline Sarkkinen Fat Mod 1995 & 41 & $4.3(1)$ & 12 & $4.4(1)$ & $\rightarrow$ & $18.27 \%$ & $-0.11[-0.73,0.51]$ \\
\hline 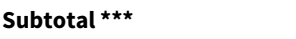 & 80 & & 36 & & & $100 \%$ & $-0.2[-0.47,0.07]$ \\
\hline \multicolumn{8}{|c|}{ Test for overall effect: $Z=1.48(P=0.14)$} \\
\hline \multicolumn{8}{|l|}{ 3.3.2 Reduced fat intake } \\
\hline Anderson 1990 & 47 & $-0.6(0.6)$ & 51 & $-0.4(0.4)$ & & $5.31 \%$ & $-0.16[-0.36,0.04]$ \\
\hline CARMEN 2000 & 159 & $-0.1(0.5)$ & 77 & $-0(0.7)$ & $\longrightarrow$ & $7.26 \%$ & $-0.02[-0.19,0.15]$ \\
\hline CARMEN MS sub-study 2002 & 18 & $-0.1(0.5)$ & 8 & $0.2(0.9)$ & - & $0.5 \%$ & $-0.3[-0.94,0.33]$ \\
\hline
\end{tabular}




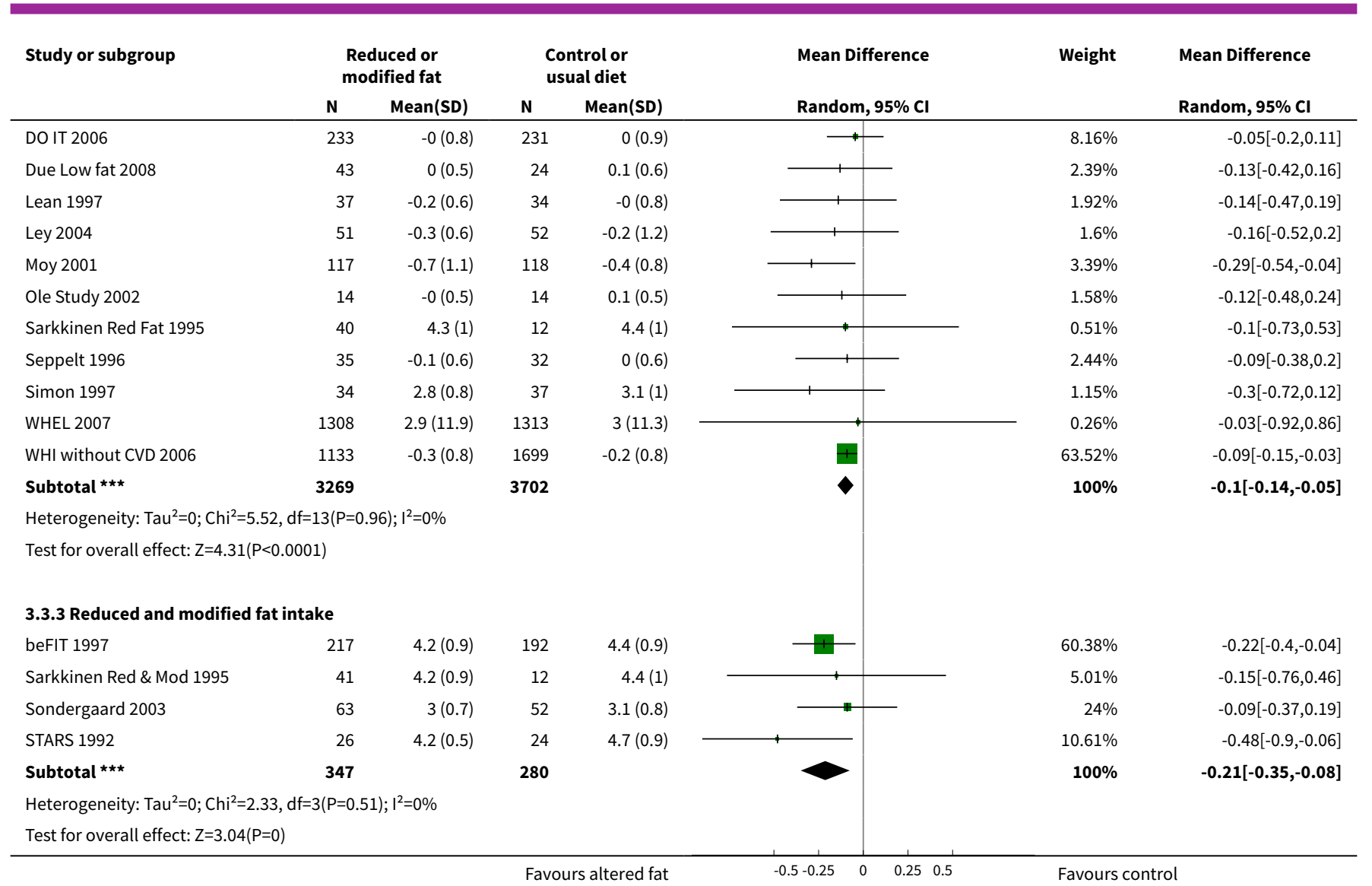

\section{Analysis 3.4. Comparison $\mathbf{3}$ fat modification or reduction vs usual diet - tertiary outcomes, Outcome $4 \mathrm{HDL}$ cholesterol, mmol/Lkg.}

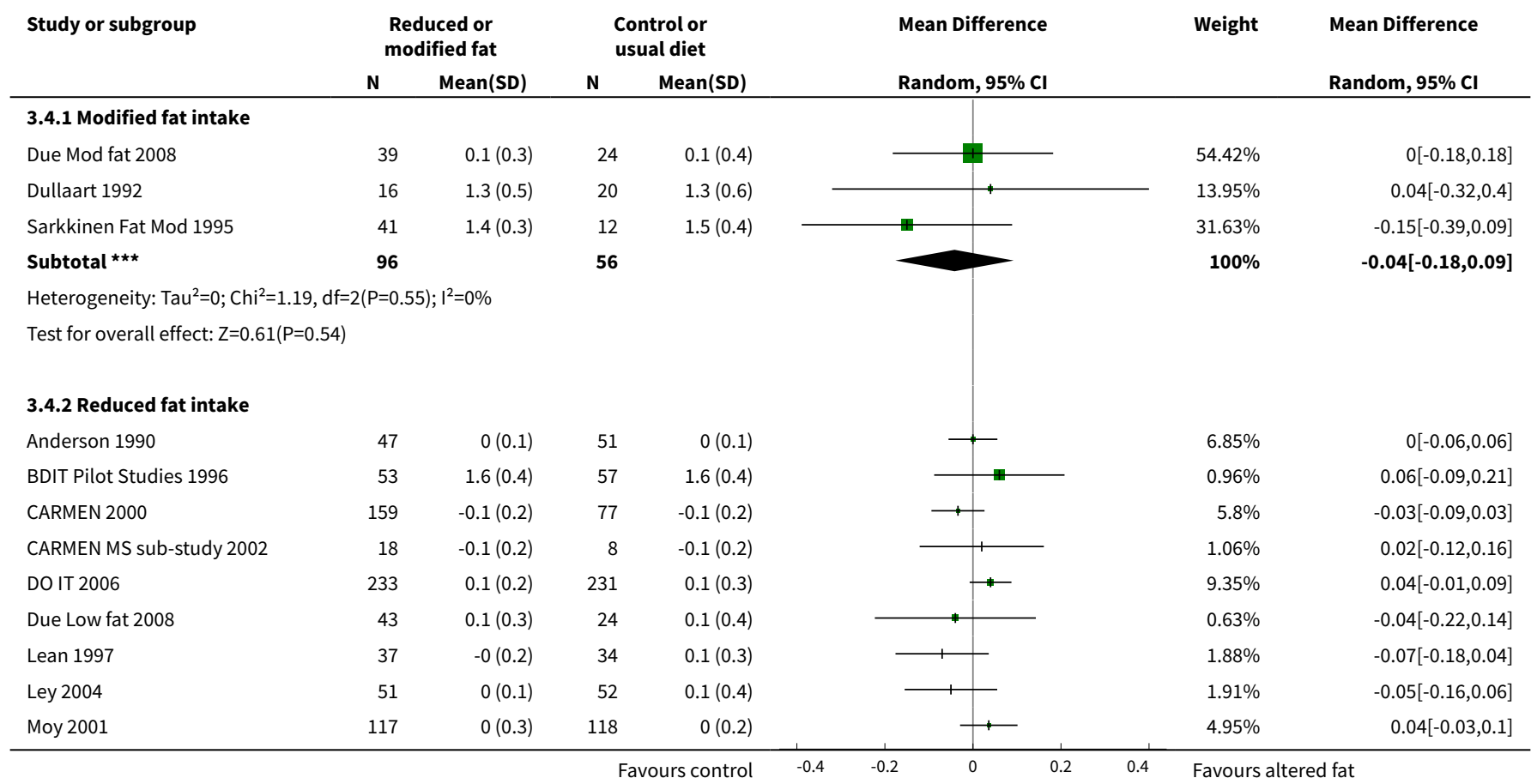




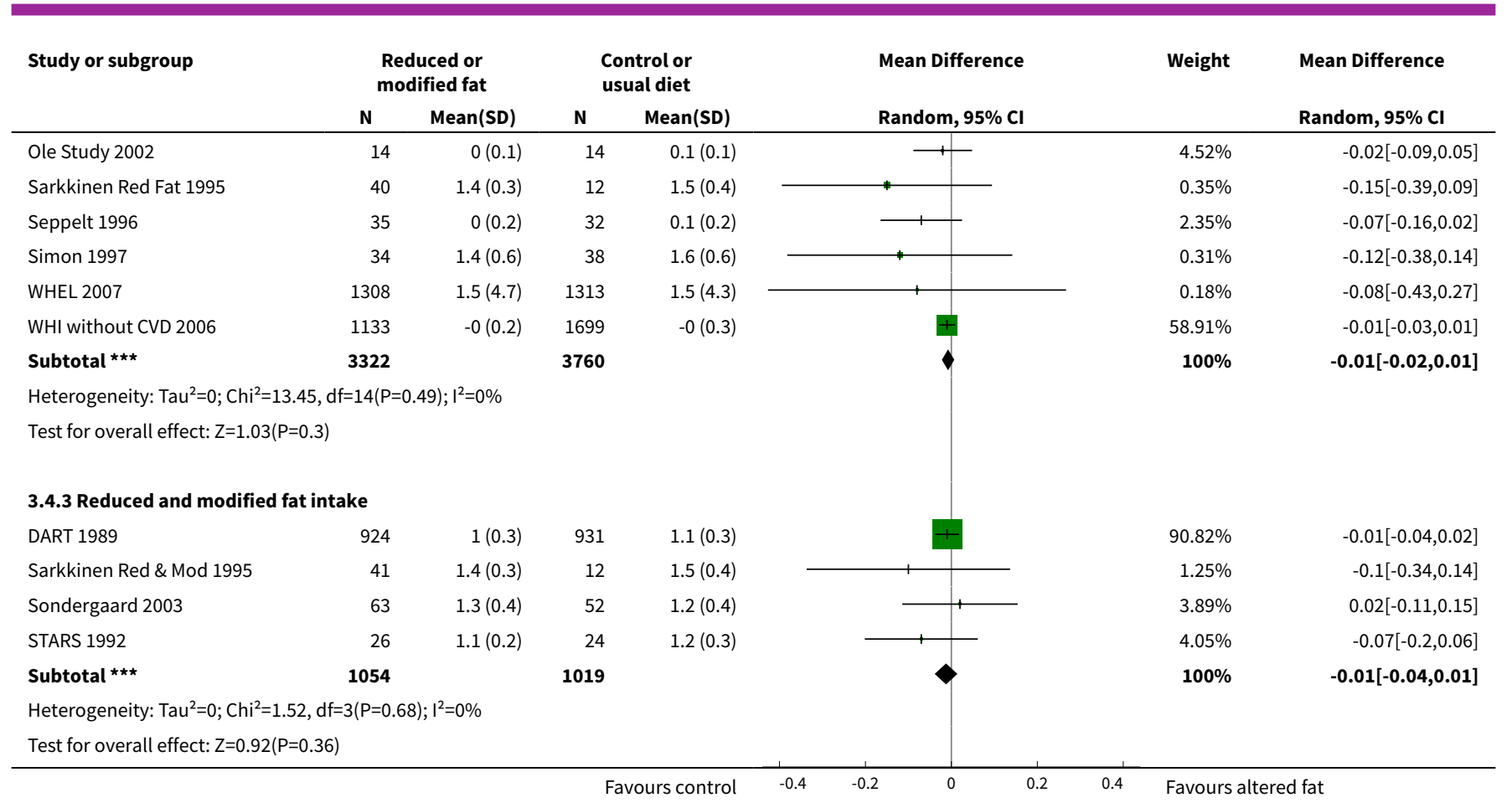

\section{Analysis 3.5. Comparison 3 fat modification or reduction vs usual diet - tertiary outcomes, Outcome 5 Total cholesterol, mmol/L.}

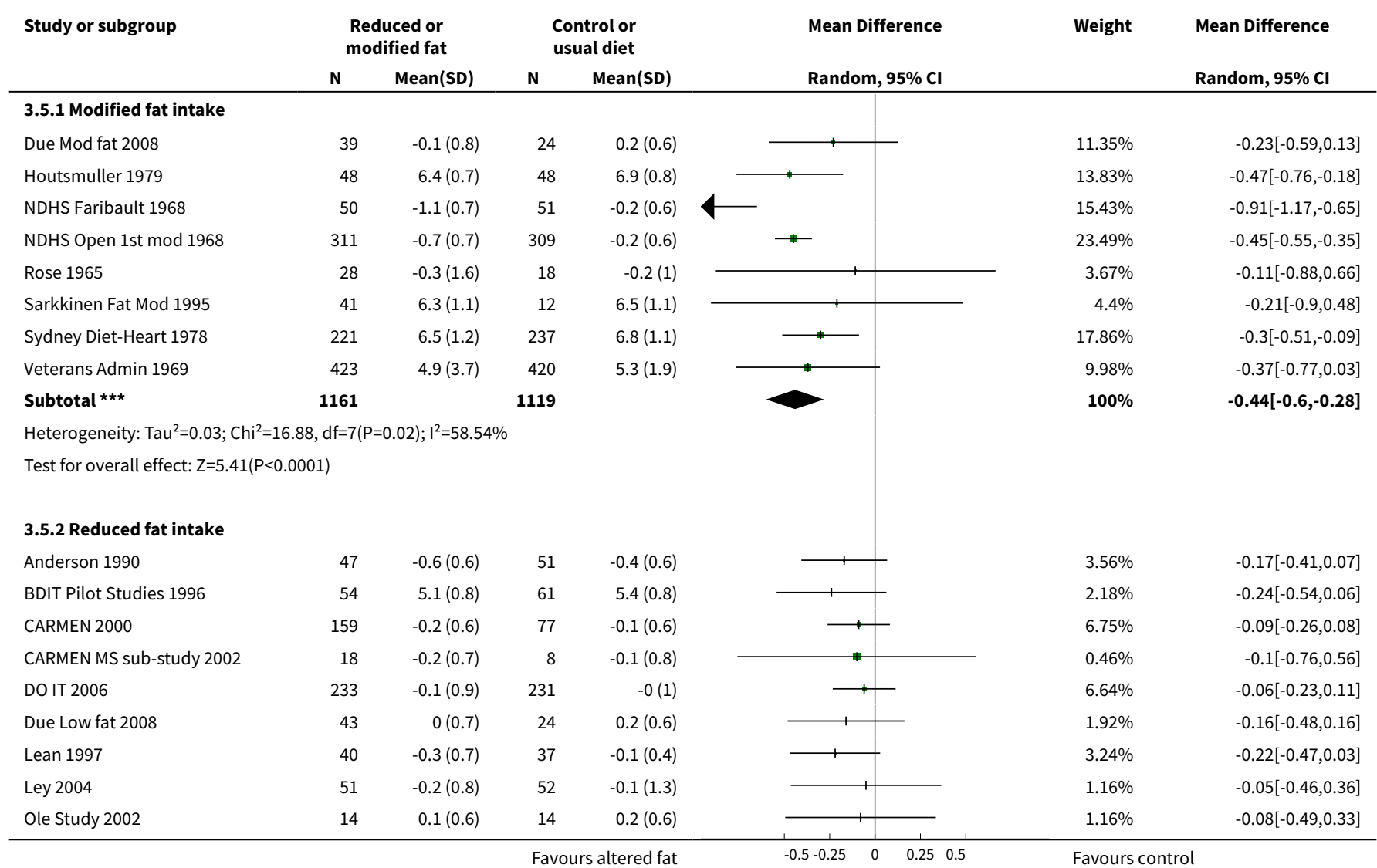




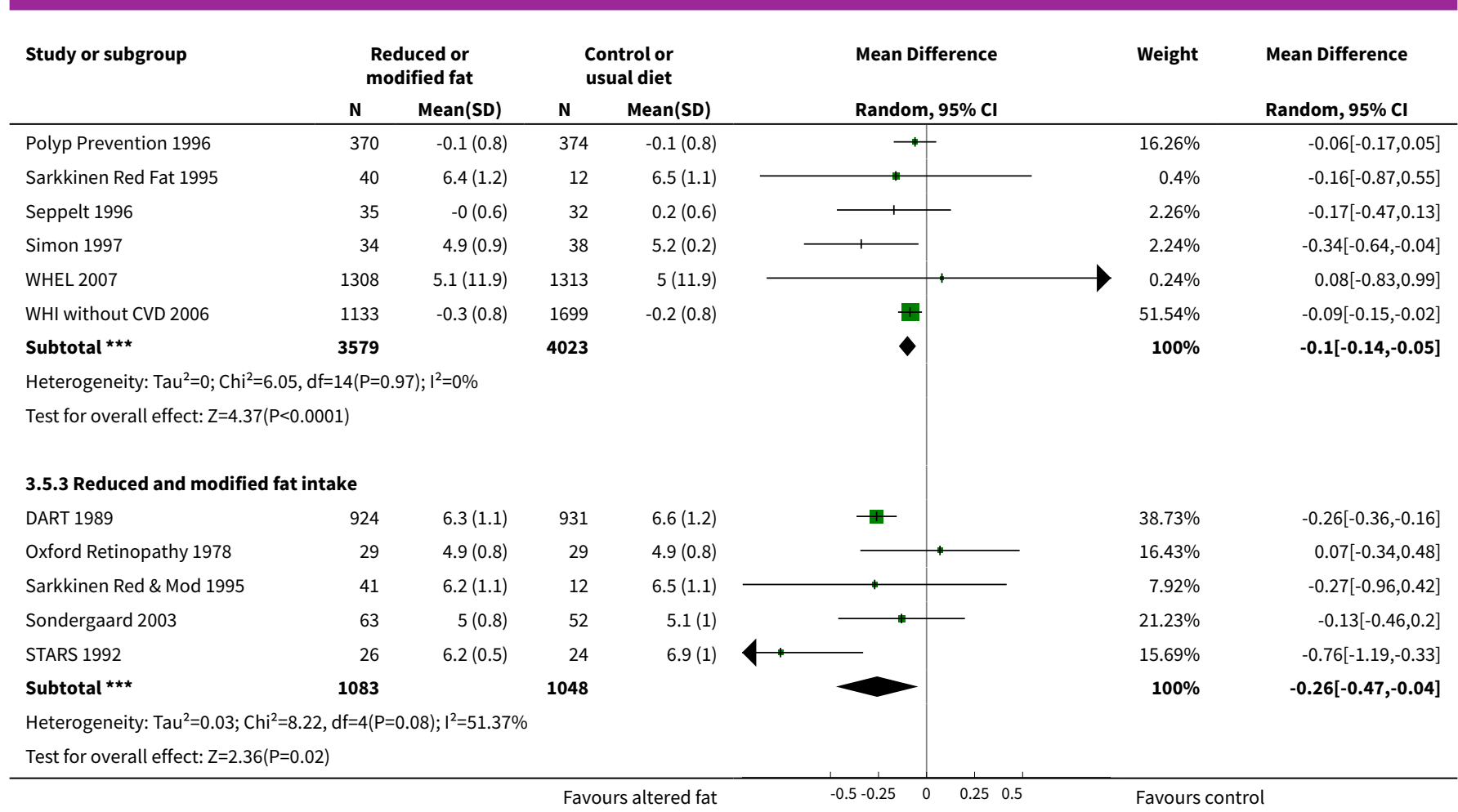

Analysis 3.6. Comparison 3 fat modification or reduction vs usual diet - tertiary outcomes, Outcome 6 Triglycerides, mmol/L.

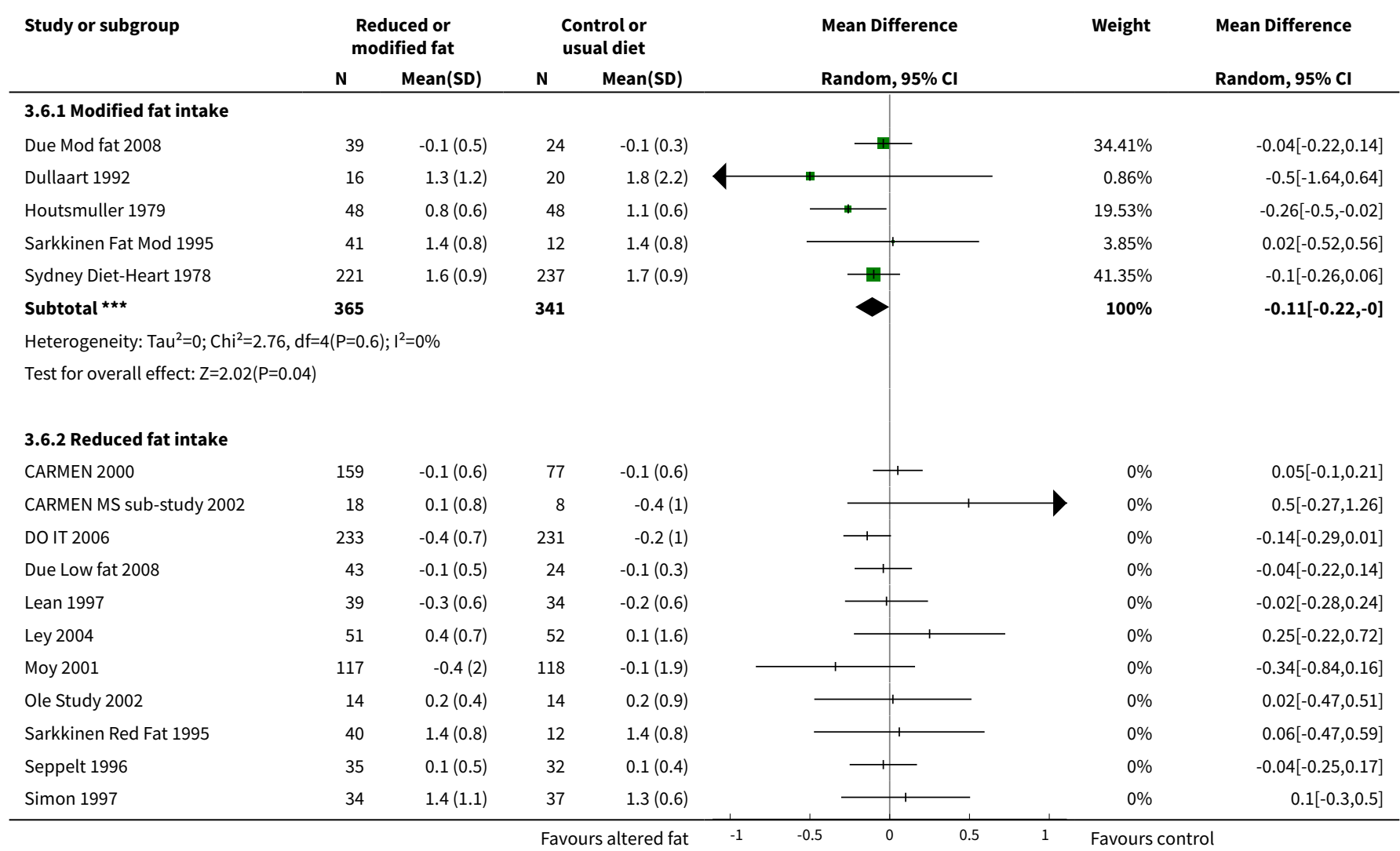




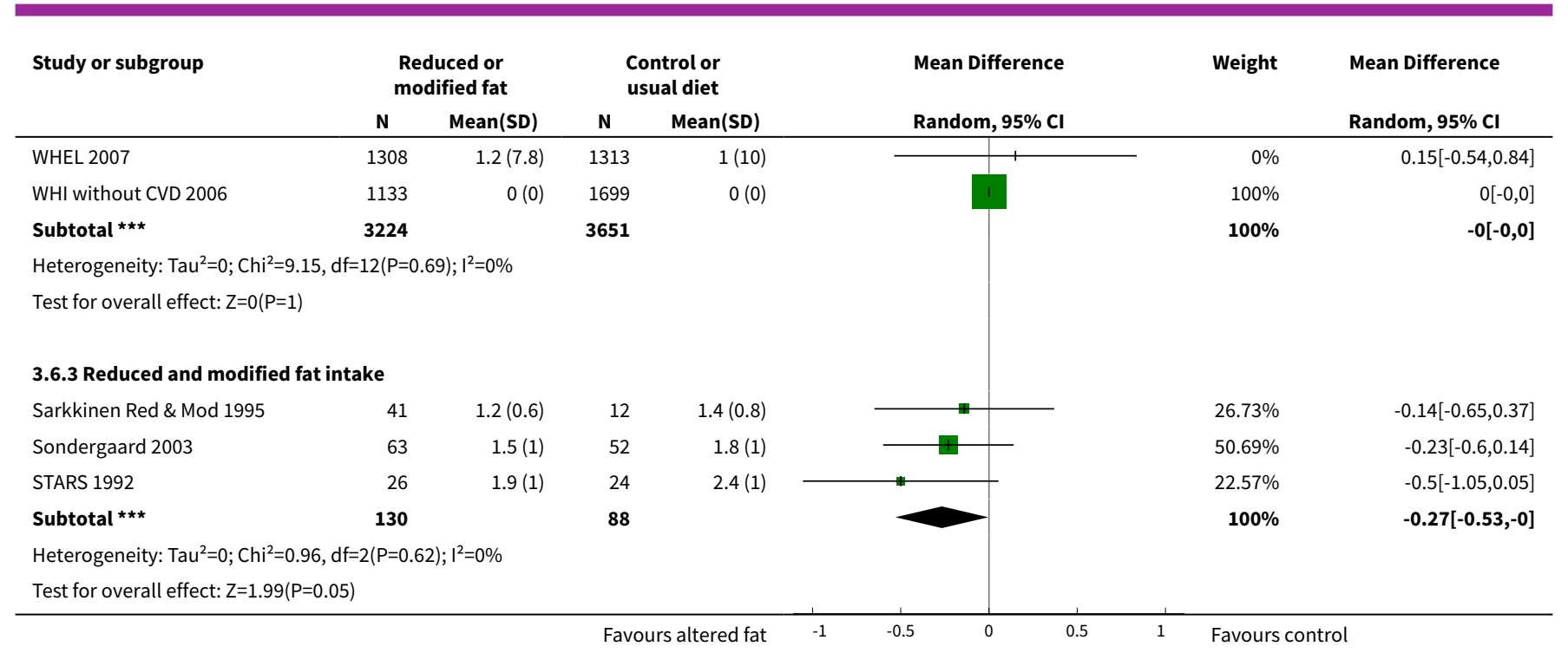

Analysis 3.7. Comparison 3 fat modification or reduction vs usual diet - tertiary outcomes, Outcome 7 Systolic Blood Pressure, $\mathbf{m m H g}$.

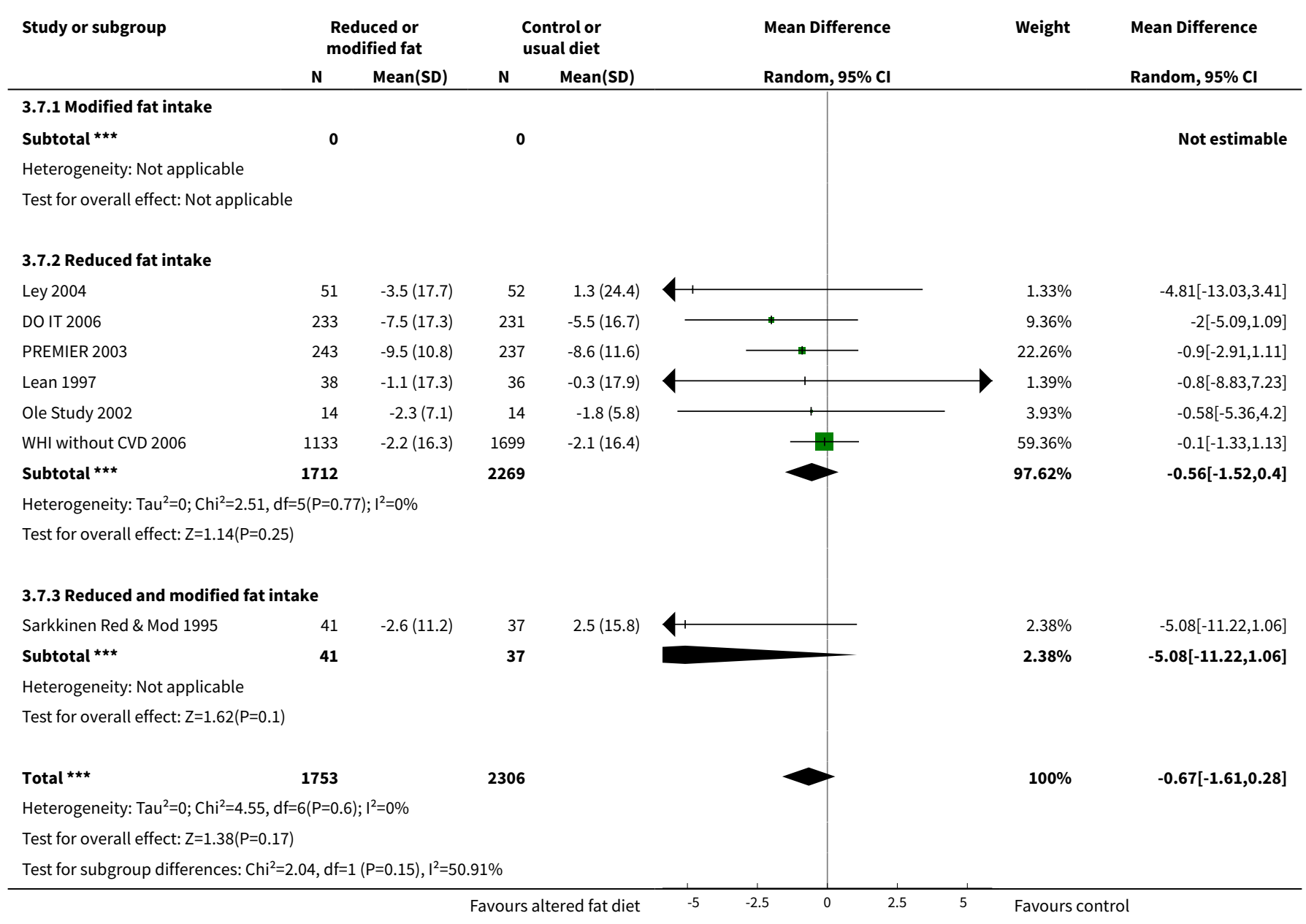


Analysis 3.8. Comparison 3 fat modification or reduction vs usual diet - tertiary outcomes, Outcome 8 Diastolic Blood Pressure, mmHg.

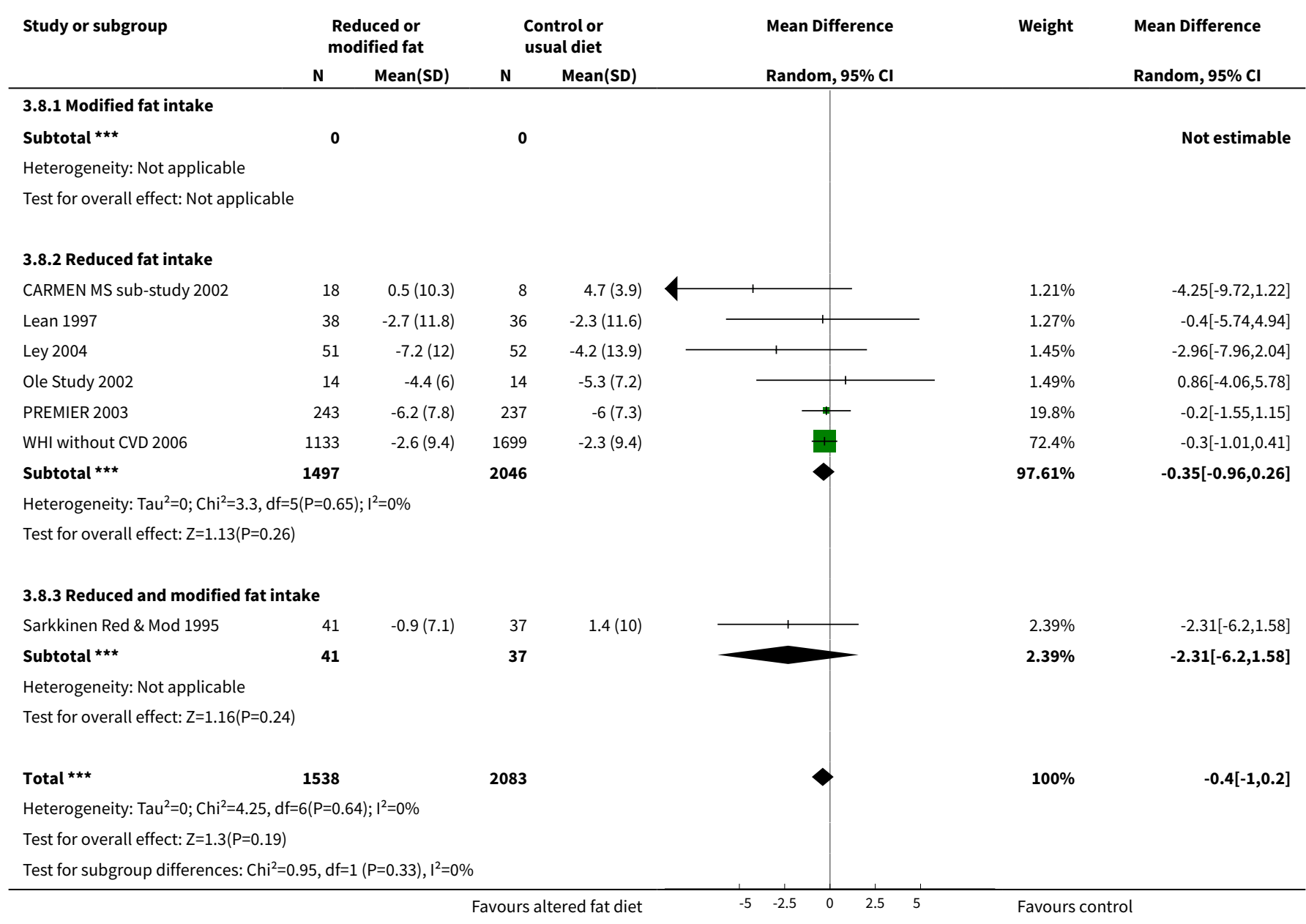

Comparison 4. fat reduction vs fat modification - primary outcomes

\begin{tabular}{llllll}
\hline Outcome or subgroup title & No. of studies & $\begin{array}{l}\text { No. of partici- } \\
\text { pants }\end{array}$ & Statistical method & Effect size \\
\hline 1 Total mortality & 0 & 0 & Risk Ratio (M-H, Random, 95\% Cl) & $0.0[0.0,0.0]$ \\
\hline $\begin{array}{lllll}\text { 2 Cardiovascular mortality } \\
\text { 3 Combined cardiovascular }\end{array}$ & 0 & 0 & Risk Ratio (M-H, Random, 95\% Cl) & $0.0[0.0,0.0]$ \\
\hline \begin{tabular}{l} 
events \\
\hline
\end{tabular}
\end{tabular}


Analysis 4.3. Comparison 4 fat reduction vs fat modification primary outcomes, Outcome 3 Combined cardiovascular events.

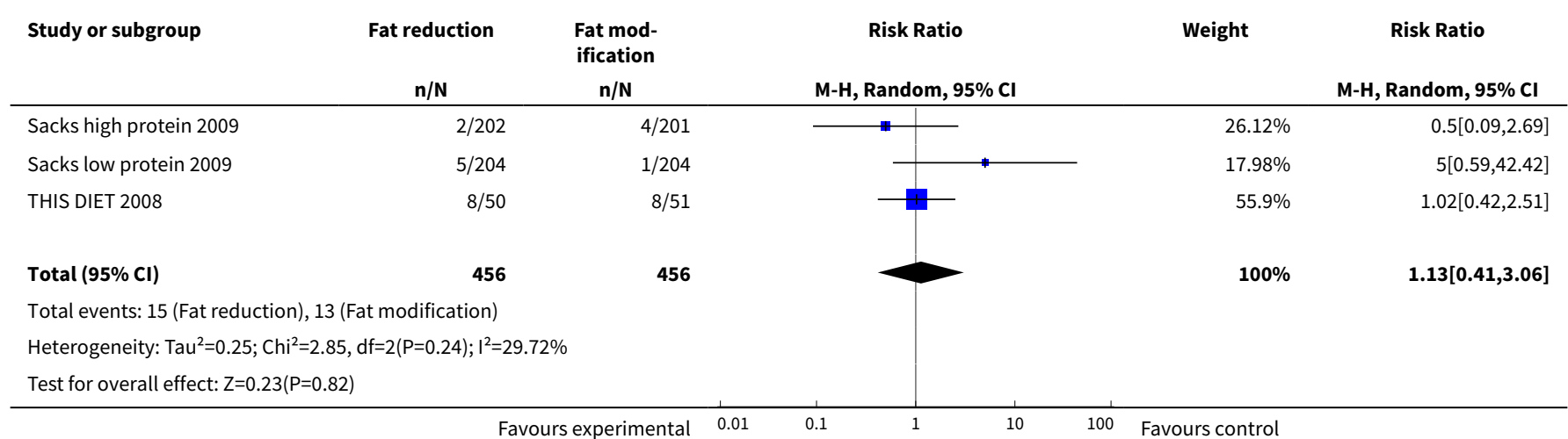

\section{Comparison 5. fat reduction vs fat modification - secondary outcomes}

\begin{tabular}{lllll}
\hline $\begin{array}{l}\text { Outcome or subgroup } \\
\text { title }\end{array}$ & No. of studies & $\begin{array}{l}\text { No. of partici- } \\
\text { pants }\end{array}$ & Statistical method & Effect size \\
\hline 1 Myocardial infarction & 1 & 101 & Risk Ratio (M-H, Random, 95\% Cl) & $3.06[0.33,28.44]$ \\
\hline 2 Stroke & 1 & 101 & Risk Ratio (M-H, Random, 95\% Cl) & $0.34[0.04,3.16]$ \\
\hline 3 Cancer deaths & 0 & 0 & Risk Ratio (M-H, Random, 95\% Cl) & $0.0[0.0,0.0]$ \\
\hline 4 Cancer diagnoses & 2 & Risk Ratio (M-H, Random, 95\% Cl) & $2.07[0.27,15.98]$ \\
\hline 5 Diabetes diagnoses & 0 & 811 & Risk Ratio (M-H, Random, 95\% Cl) & $0.0[0.0,0.0]$ \\
\hline 6 Non-fatal MI & 1 & 0 & Risk Ratio (M-H, Random, 95\% Cl) & $3.06[0.33,28.44]$ \\
\hline
\end{tabular}

Analysis 5.1. Comparison 5 fat reduction vs fat modification - secondary outcomes, Outcome 1 Myocardial infarction.

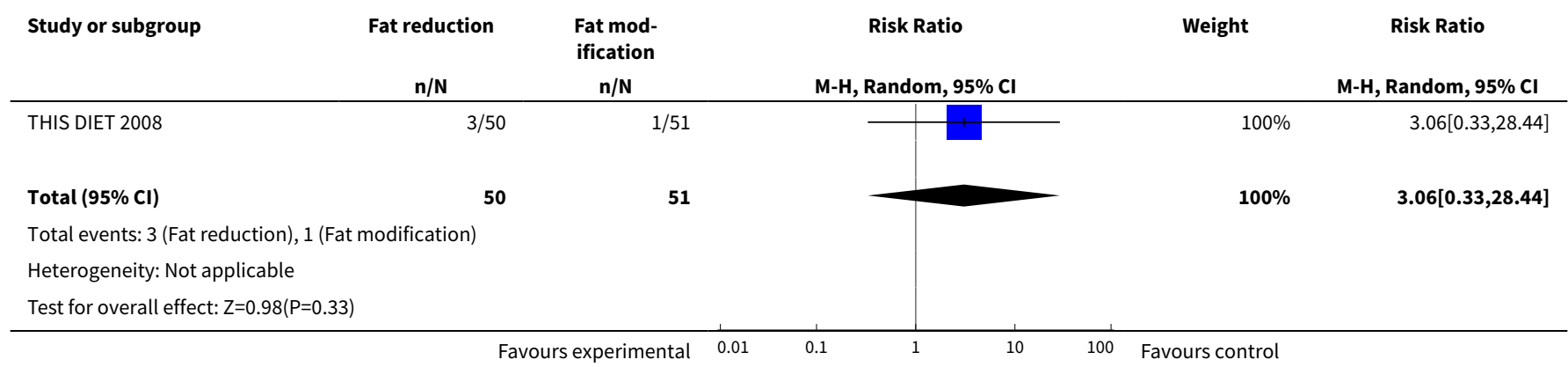


Analysis 5.2. Comparison 5 fat reduction vs fat modification - secondary outcomes, Outcome 2 Stroke.

\begin{tabular}{|c|c|c|c|c|c|}
\hline \multirow[t]{2}{*}{ Study or subgroup } & \multirow{2}{*}{$\begin{array}{l}\text { Fat reduction } \\
\qquad n / N \\
\end{array}$} & \multirow{2}{*}{$\begin{array}{c}\text { Fat mod- } \\
\text { ification } \\
n / N \\
\end{array}$} & Risk Ratio & \multirow[t]{2}{*}{ Weight } & \multirow{2}{*}{$\begin{array}{c}\text { Risk Ratio } \\
\text { M-H, Random, 95\% CI }\end{array}$} \\
\hline & & & M-H, Random, $95 \% \mathrm{Cl}$ & & \\
\hline THIS DIET 2008 & $1 / 50$ & $3 / 51$ & \begin{tabular}{l|l|l}
+1 & & \\
\end{tabular} & $100 \%$ & $0.34[0.04,3.16]$ \\
\hline Total $(95 \% \mathrm{Cl})$ & 50 & 51 & & $100 \%$ & $0.34[0.04,3.16]$ \\
\hline \multicolumn{6}{|c|}{ Total events: 1 (Fat reduction), 3 (Fat modification) } \\
\hline \multicolumn{6}{|c|}{ Test for overall effect: $Z=0.95(P=0.34)$} \\
\hline
\end{tabular}

Analysis 5.4. Comparison 5 fat reduction vs fat modification - secondary outcomes, Outcome 4 Cancer diagnoses.

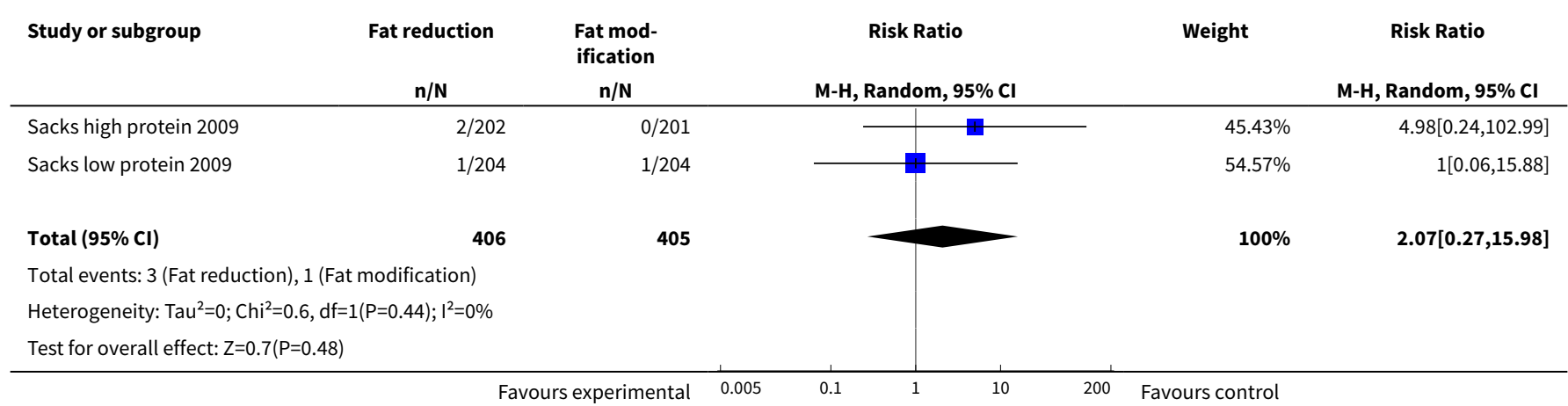

Analysis 5.6. Comparison 5 fat reduction vs fat modification - secondary outcomes, Outcome 6 Non-fatal MI.

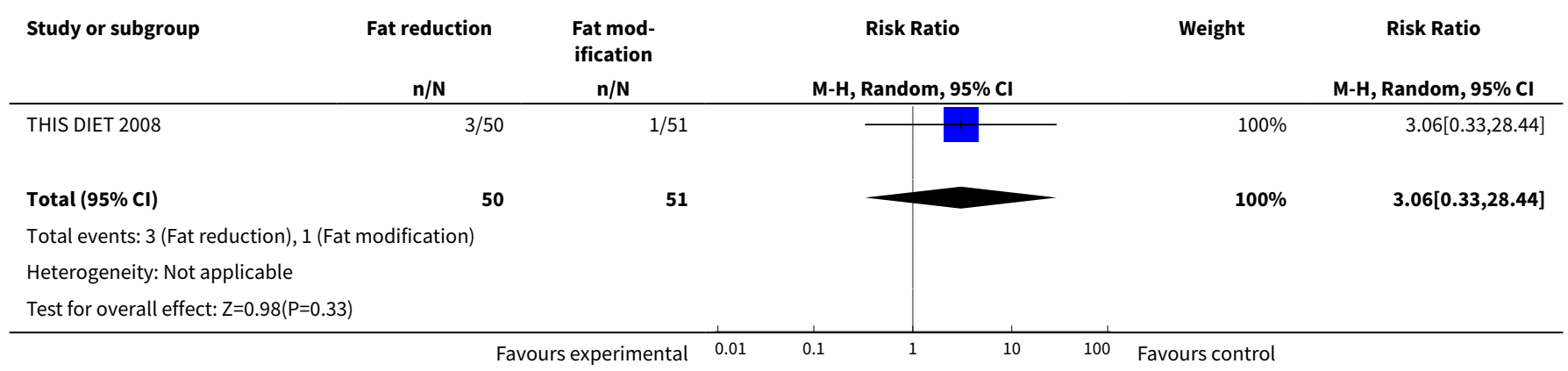

\section{Comparison 6. fat reduction vs fat modification - tertiary outcomes}

\begin{tabular}{lllll}
\hline $\begin{array}{l}\text { Outcome or subgroup } \\
\text { title }\end{array}$ & No. of studies & $\begin{array}{l}\text { No. of partici- } \\
\text { pants }\end{array}$ & Statistical method & Effect size \\
\hline 1 Weight, kg & 6 & 1057 & Mean Difference (IV, Random, 95\% Cl) & $-0.25[-1.88,1.39]$ \\
\hline $2 \mathrm{BMl}, \mathrm{kg} / \mathrm{m} 2$ & 5 & 345 & Mean Difference (IV, Random, 95\% Cl) & $0.06[-0.79,0.92]$ \\
\hline
\end{tabular}




\begin{tabular}{|c|c|c|c|c|}
\hline $\begin{array}{l}\text { Outcome or subgroup } \\
\text { title }\end{array}$ & No. of studies & $\begin{array}{l}\text { No. of partici- } \\
\text { pants }\end{array}$ & Statistical method & Effect size \\
\hline $\begin{array}{l}3 \mathrm{LDL} \text { cholesterol, mmol/ } \\
\mathrm{L}\end{array}$ & 9 & 1275 & Mean Difference (IV, Random, 95\% CI) & $-0.01[-0.13,0.12]$ \\
\hline $\begin{array}{l}4 \mathrm{HDL} \text { cholesterol, mmol/ } \\
\text { Lkg }\end{array}$ & 9 & 1275 & Mean Difference (IV, Random, 95\% CI) & $-0.02[-0.06,0.02]$ \\
\hline $\begin{array}{l}5 \text { Total cholesterol, } \\
\mathrm{mmol} / \mathrm{L}\end{array}$ & 7 & 1130 & Mean Difference (IV, Random, 95\% CI) & $-0.04[-0.18,0.09]$ \\
\hline 6 Triglycerides, $\mathrm{mmol} / \mathrm{L}$ & 9 & 1275 & Mean Difference (IV, Random, 95\% CI) & $0.05[-0.02,0.12]$ \\
\hline $\begin{array}{l}7 \text { Systolic Blood Pres- } \\
\text { sure, } \mathrm{mmHg}\end{array}$ & 6 & 1068 & Mean Difference (IV, Random, 95\% CI) & $-0.13[-2.16,1.90]$ \\
\hline $\begin{array}{l}8 \text { Diastolic Blood Pres- } \\
\text { sure, } \mathrm{mmHg}\end{array}$ & 6 & 1068 & Mean Difference (IV, Random, 95\% CI) & $-0.23[-1.90,1.43]$ \\
\hline 9 Dropouts & 9 & 1353 & Risk Ratio (M-H, Random, 95\% Cl) & $0.84[0.58,1.22]$ \\
\hline
\end{tabular}

Analysis 6.1. Comparison 6 fat reduction vs fat modification - tertiary outcomes, Outcome 1 Weight, kg.

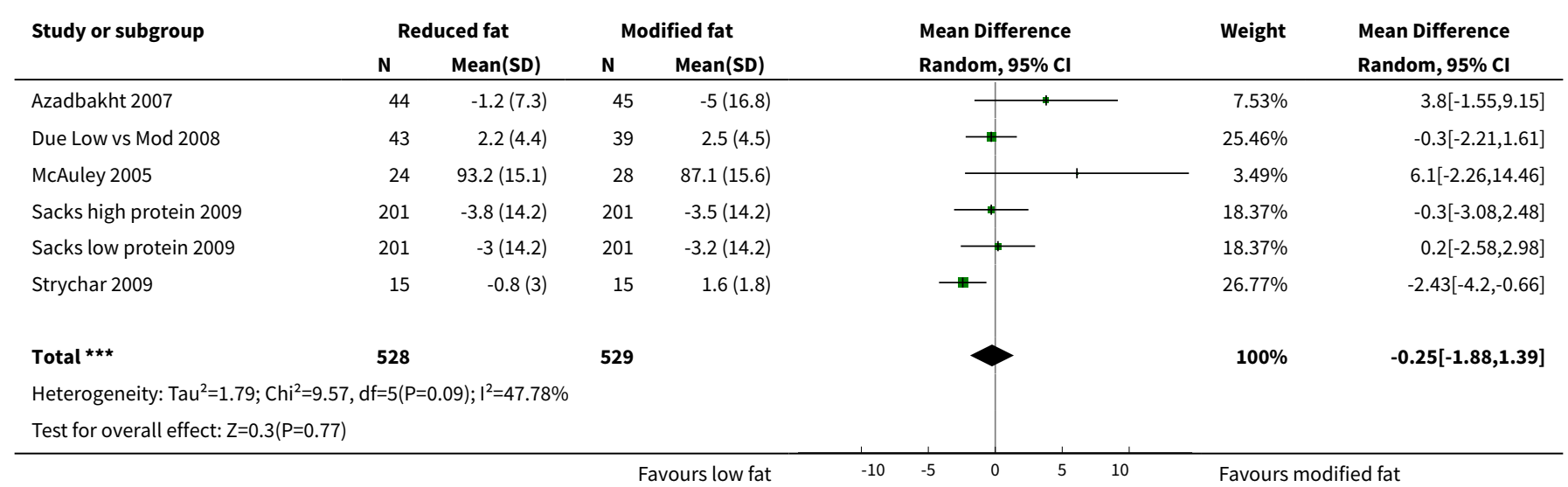

\section{Analysis 6.2. Comparison 6 fat reduction vs fat modification - tertiary outcomes, Outcome 2 BMI, kg/m2.}

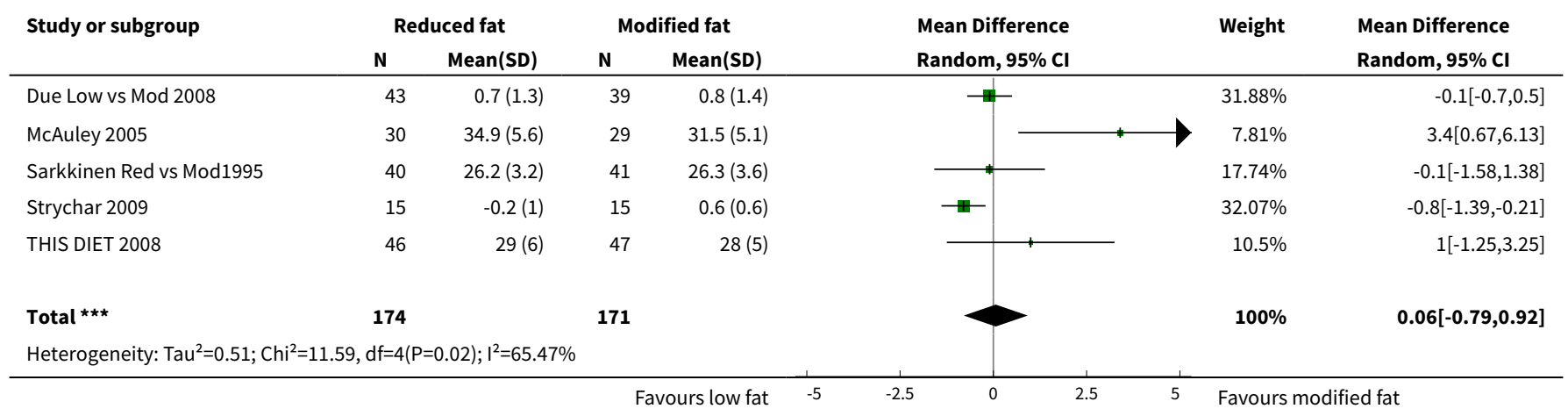




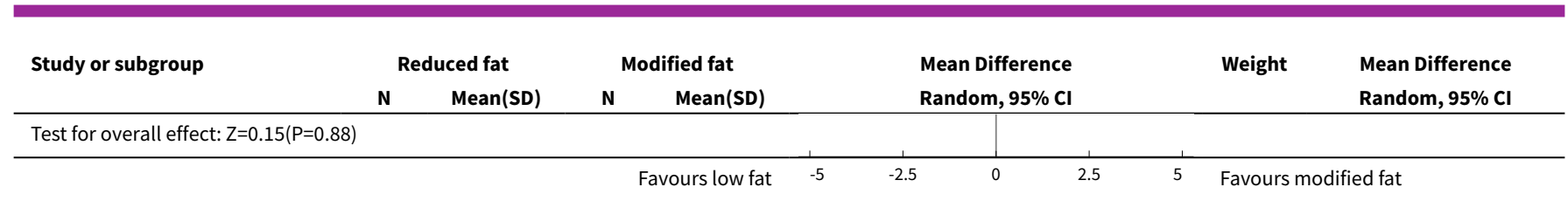

Analysis 6.3. Comparison 6 fat reduction vs fat modification - tertiary outcomes, Outcome 3 LDL cholesterol, $\mathrm{mmol} / \mathrm{L}$.

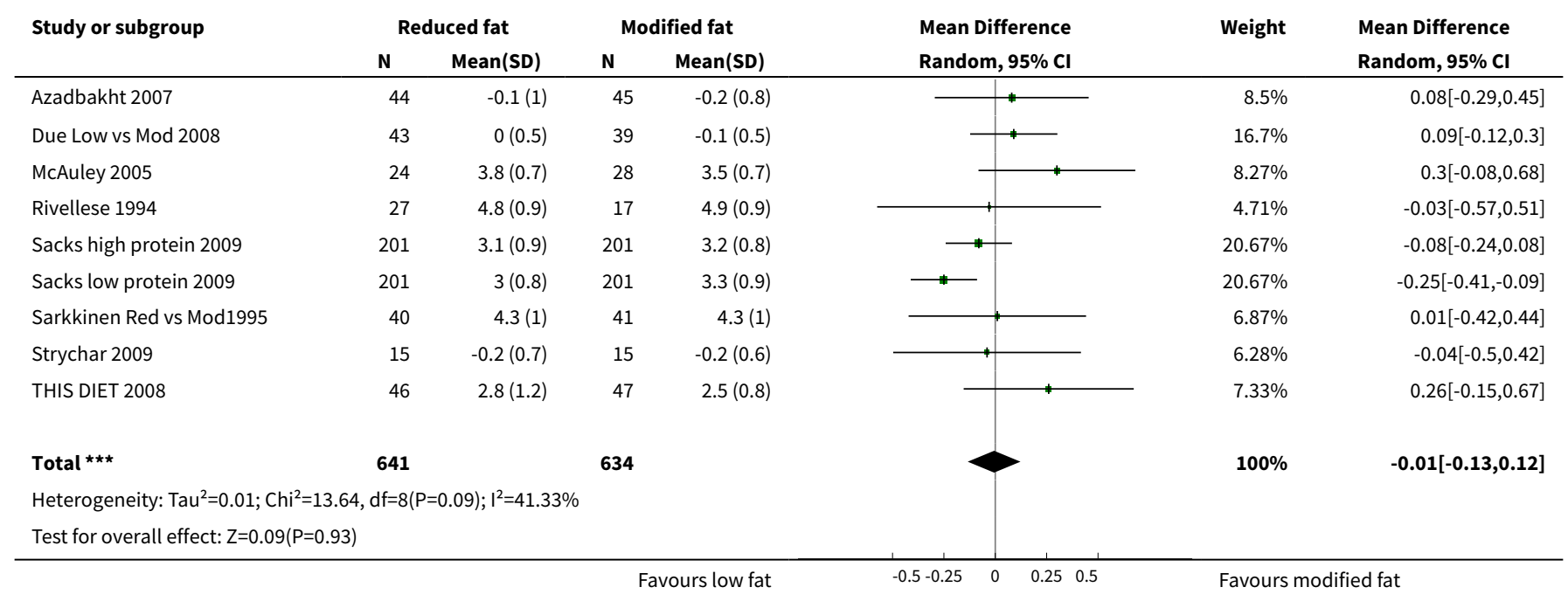

Analysis 6.4. Comparison 6 fat reduction vs fat modification - tertiary outcomes, Outcome $4 \mathrm{HDL}$ cholesterol, mmol/Lkg.

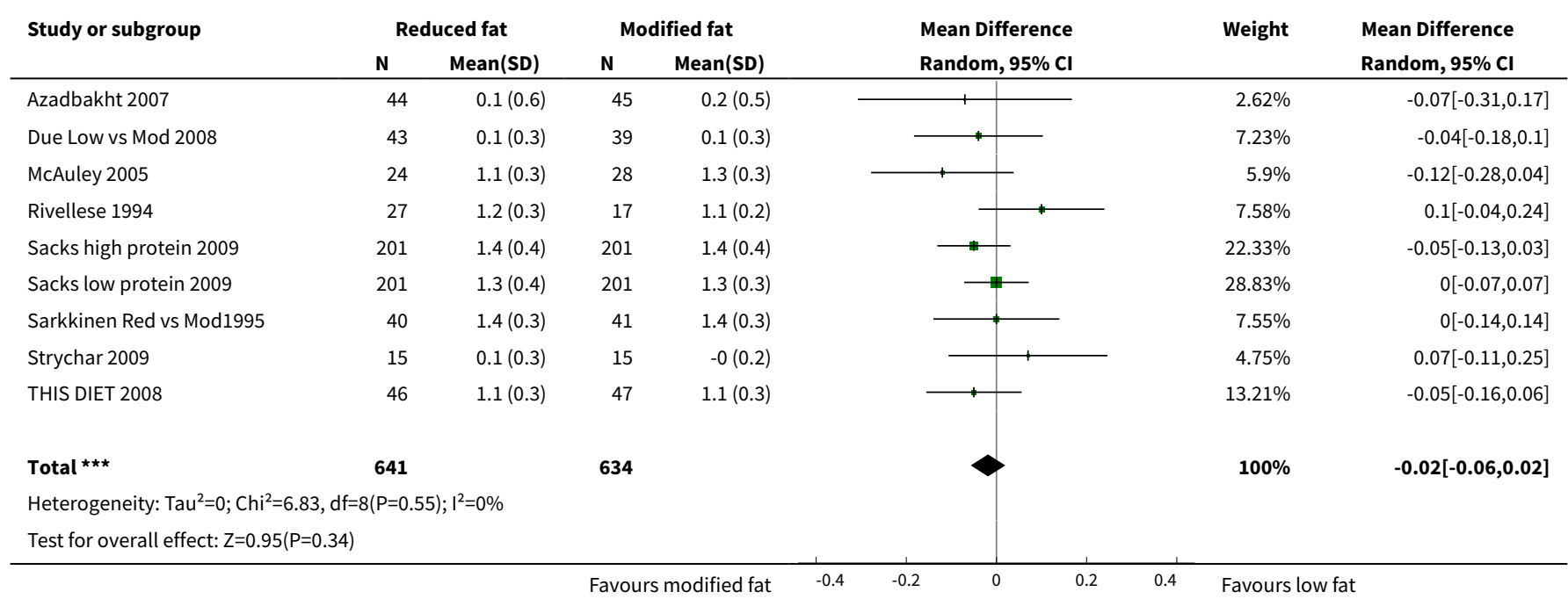


Analysis 6.5. Comparison 6 fat reduction vs fat modification - tertiary outcomes, Outcome 5 Total cholesterol, mmol/L.

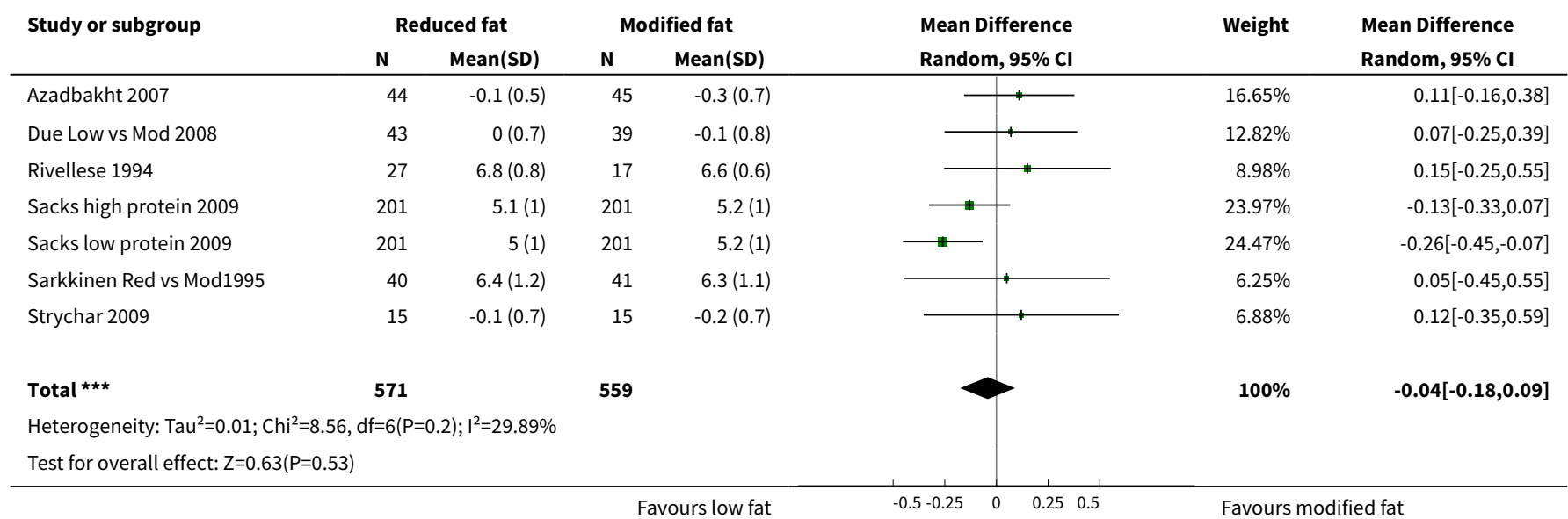

Analysis 6.6. Comparison 6 fat reduction vs fat modification - tertiary outcomes, Outcome 6 Triglycerides, $\mathrm{mmol} / \mathrm{L}$.

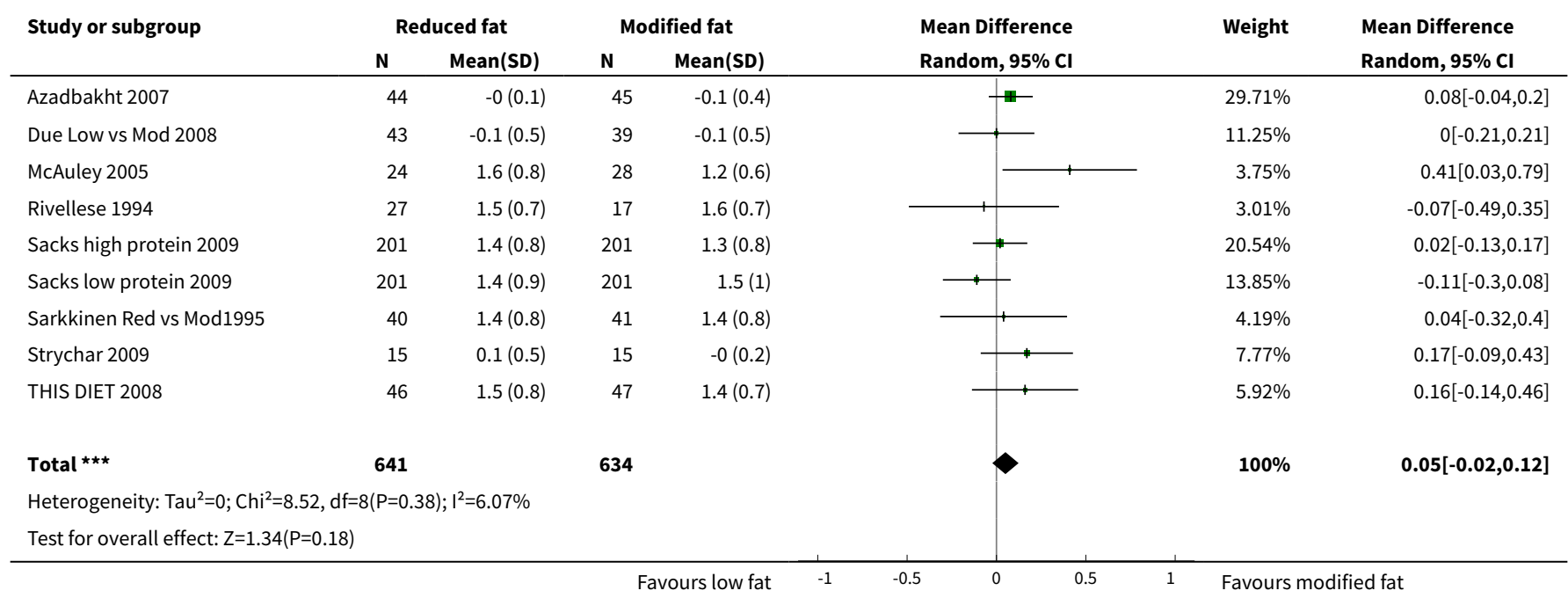

Analysis 6.7. Comparison 6 fat reduction vs fat modification tertiary outcomes, Outcome 7 Systolic Blood Pressure, $\mathrm{mmHg}$.

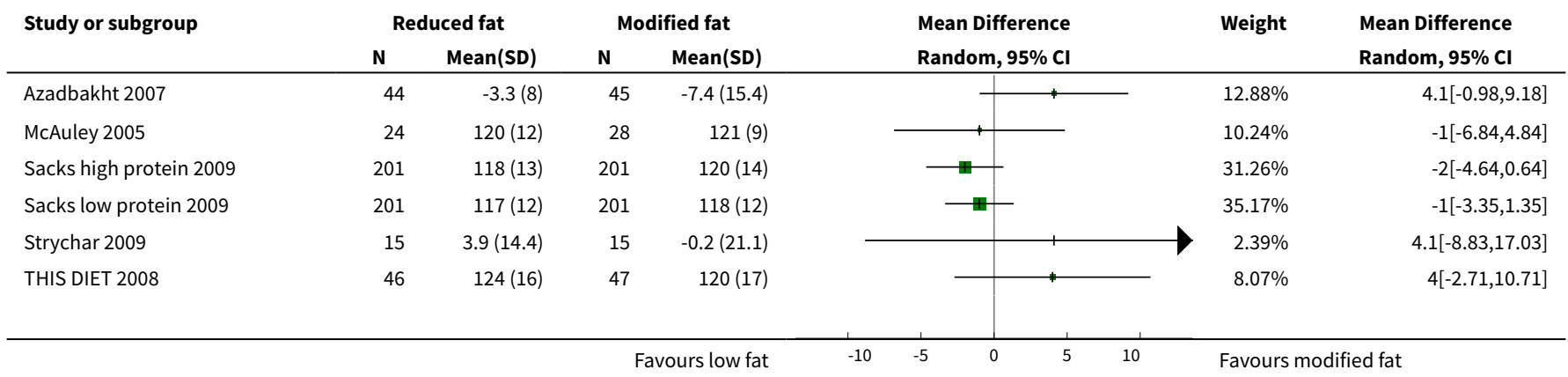




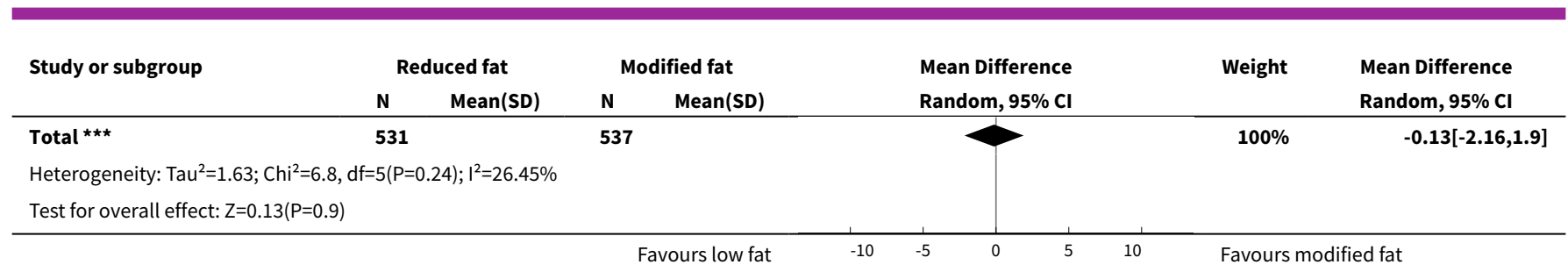

Favours low fat

Analysis 6.8. Comparison 6 fat reduction vs fat modification tertiary outcomes, Outcome 8 Diastolic Blood Pressure, $\mathrm{mmHg}$.

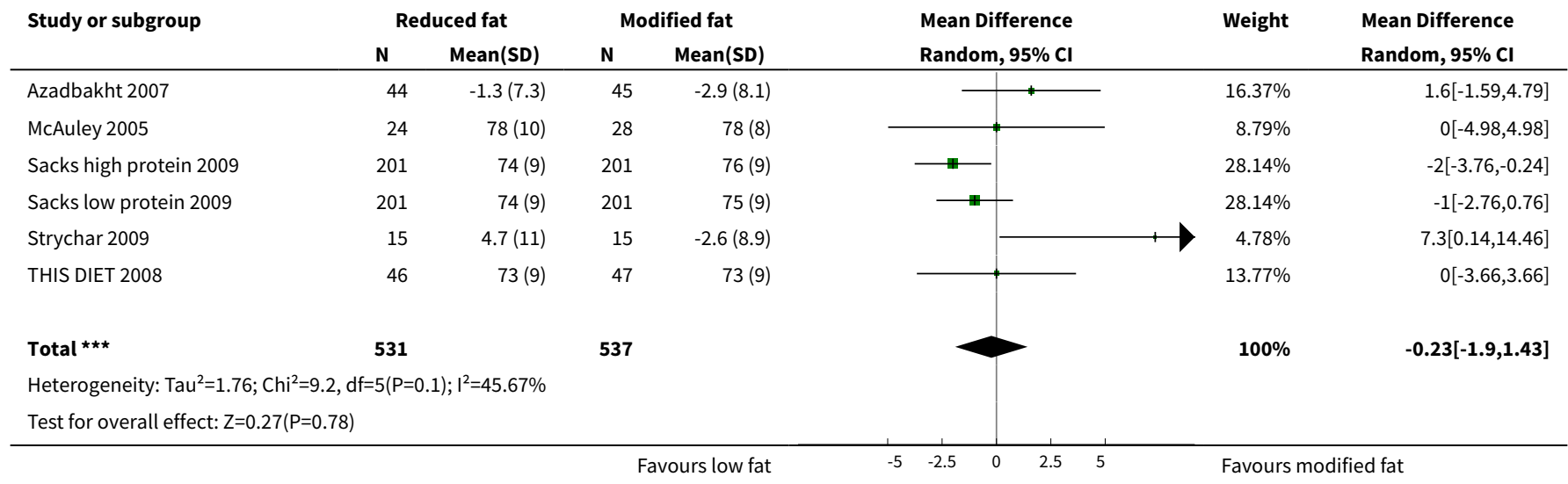

Analysis 6.9. Comparison 6 fat reduction vs fat modification - tertiary outcomes, Outcome 9 Dropouts.

\begin{tabular}{|c|c|c|c|c|c|}
\hline Study or subgroup & $\begin{array}{c}\text { Reduced fat } \\
n / N\end{array}$ & $\begin{array}{c}\text { Modified fat } \\
n / \mathbf{N}\end{array}$ & $\begin{array}{c}\text { Risk Ratio } \\
\text { M-H, Random, } 95 \% \text { Cl }\end{array}$ & Weight & $\begin{array}{c}\text { Risk Ratio } \\
\text { M-H, Random, 95\% Cl }\end{array}$ \\
\hline Azadbakht 2007 & $6 / 50$ & $5 / 50$ & + & $8.45 \%$ & $1.2[0.39,3.68]$ \\
\hline Due Low vs Mod 2008 & $8 / 48$ & $15 / 52$ & $\longrightarrow$ & $14.17 \%$ & $0.58[0.27,1.24]$ \\
\hline McAuley 2005 & $2 / 32$ & $3 / 30$ & & $4.18 \%$ & $0.63[0.11,3.48]$ \\
\hline Rivellese 1994 & $6 / 33$ & $13 / 30$ & $\longrightarrow$ & $12.77 \%$ & $0.42[0.18,0.96]$ \\
\hline Sacks high protein 2009 & $45 / 202$ & $33 / 201$ & $\rightarrow$ & $24.53 \%$ & $1.36[0.91,2.03]$ \\
\hline Sacks low protein 2009 & $35 / 204$ & $53 / 204$ & $\rightarrow-$ & $25.37 \%$ & $0.66[0.45,0.97]$ \\
\hline Sarkkinen Red vs Mod1995 & $0 / 40$ & $0 / 41$ & & & Not estimable \\
\hline Strychar 2009 & $3 / 18$ & $2 / 17$ & + & $4.43 \%$ & $1.42[0.27,7.46]$ \\
\hline THIS DIET 2008 & $5 / 50$ & $3 / 51$ & $\longrightarrow$ & $6.1 \%$ & $1.7[0.43,6.74]$ \\
\hline Total $(95 \% \mathrm{Cl})$ & 677 & 676 & & $100 \%$ & $0.84[0.58,1.22]$ \\
\hline \multicolumn{6}{|c|}{ Total events: 110 (Reduced fat), 127 (Modified fat) } \\
\hline \multicolumn{6}{|c|}{ Test for overall effect: $Z=0.92(P=0.36)$} \\
\hline
\end{tabular}

\section{ADDITIONAL TABLES}


Table 1. Sensitivity analyses of primary outcomes

\begin{tabular}{|c|c|c|c|c|c|}
\hline Outcome & Sensitivity Analysis & Subgroup & $\begin{array}{l}\text { Risk ratio }(95 \% \\
\mathrm{CI})\end{array}$ & $\begin{array}{l}\text { Number of } \\
\text { studies / par- } \\
\text { ticipants/ } \\
\text { events }\end{array}$ & $\mathbf{1}^{2}$ \\
\hline Total mortality & Mantel-Haenszel, Fixed effects & Overall analysis & $\begin{array}{l}0.98(0.93 \text { to } \\
1.04)\end{array}$ & $\begin{array}{l}21 / 71795 / \\
4292\end{array}$ & $0 \%$ \\
\hline Total mortality & Mantel-Haenszel, Fixed effects & $\begin{array}{l}\text { Modified fat in- } \\
\text { take }\end{array}$ & $\begin{array}{l}1.03(0.92 \text { to } \\
1.14)\end{array}$ & $8 / 11441 / 1120$ & $34 \%$ \\
\hline Total mortality & Mantel-Haenszel, Fixed effects & $\begin{array}{l}\text { Reduced fat in- } \\
\text { take }\end{array}$ & $\begin{array}{l}0.97(0.90 \text { to } \\
1.04)\end{array}$ & $\begin{array}{l}10 / 58130 / \\
2936\end{array}$ & $0 \%$ \\
\hline Total mortality & Mantel-Haenszel, Fixed effects & $\begin{array}{l}\text { Reduced \& modi- } \\
\text { fied fat intake }\end{array}$ & $\begin{array}{l}0.96 \text { ( } 0.76 \text { to } \\
1.22)\end{array}$ & $2 / 2224 / 236$ & $0 \%$ \\
\hline Total mortality & Peto Odds Ratio (fixed effects) & Overall analysis & $\begin{array}{l}\text { OR } 0.98(0.92 \text { to } \\
1.05)\end{array}$ & $\begin{array}{l}21 / 71795 / \\
4292\end{array}$ & $0 \%$ \\
\hline Total mortality & Peto Odds Ratio (fixed effects) & $\begin{array}{l}\text { Modified fat in- } \\
\text { take }\end{array}$ & $\begin{array}{l}\text { OR } 1.03 \text { (0.91 to } \\
1.18)\end{array}$ & $8 / 11441 / 1120$ & $39 \%$ \\
\hline Total mortality & Peto Odds Ratio (fixed effects) & $\begin{array}{l}\text { Reduced fat in- } \\
\text { take }\end{array}$ & $\begin{array}{l}\text { OR } 0.97 \text { ( } 0.90 \text { to } \\
1.04)\end{array}$ & $\begin{array}{l}10 / 58130 / \\
2936\end{array}$ & $0 \%$ \\
\hline Total mortality & Peto Odds Ratio (fixed effects) & $\begin{array}{l}\text { Reduced and } \\
\text { modified fat in- } \\
\text { take }\end{array}$ & $\begin{array}{l}\text { OR } 0.96 \text { ( } 0.73 \text { to } \\
1.25)\end{array}$ & $2 / 2224 / 236$ & $0 \%$ \\
\hline Total mortality & Excluding WHI & Overall analysis & $\begin{array}{l}0.98(0.91 \text { to } \\
1.07)\end{array}$ & $\begin{array}{l}20 / 22960 / \\
1888\end{array}$ & $0 \%$ \\
\hline Total mortality & Excluding WHI & $\begin{array}{l}\text { Reduced fat in- } \\
\text { take }\end{array}$ & $\begin{array}{l}0.94(0.80 \text { to } \\
1.10)\end{array}$ & 9/9295/532 & $0 \%$ \\
\hline Total mortality & $\begin{array}{l}\text { Excluding studies with systematic } \\
\text { difference in care }\end{array}$ & Overall analysis & $\begin{array}{l}1.03(0.92 \text { to } \\
1.16)\end{array}$ & $6 / 10715 / 882$ & $0 \%$ \\
\hline Total mortality & $\begin{array}{l}\text { Excluding studies with systematic } \\
\text { difference in care }\end{array}$ & $\begin{array}{l}\text { Modified fat in- } \\
\text { take }\end{array}$ & $\begin{array}{l}1.03(0.92 \text { to } \\
1.16)\end{array}$ & $5 / 10178 / 881$ & $0 \%$ \\
\hline Total mortality & $\begin{array}{l}\text { Excluding studies with systematic } \\
\text { difference in care }\end{array}$ & $\begin{array}{l}\text { Reduced fat in- } \\
\text { take }\end{array}$ & $\begin{array}{l}0.33(0.01 \text { to } \\
8.12)\end{array}$ & $1 / 537 / 1$ & NR \\
\hline Total mortality & $\begin{array}{l}\text { Excluding studies with systematic } \\
\text { difference in care }\end{array}$ & $\begin{array}{l}\text { Reduced \& modi- } \\
\text { fied fat intake }\end{array}$ & NR & $0 / 0 / 0$ & NR \\
\hline Total mortality & $\begin{array}{l}\text { Excluding studies with systematic } \\
\text { differences in diet other than fat }\end{array}$ & Overall analysis & $\begin{array}{l}1.03(0.93 \text { to } \\
1.13)\end{array}$ & $\begin{array}{l}12 / 16060 / \\
1320\end{array}$ & $0 \%$ \\
\hline Total mortality & $\begin{array}{l}\text { Excluding studies with systematic } \\
\text { differences in diet other than fat }\end{array}$ & $\begin{array}{l}\text { Modified fat in- } \\
\text { take }\end{array}$ & $\begin{array}{l}1.05 \text { (0.93 to } \\
1.18)\end{array}$ & $7 / 11029 / 1005$ & $7 \%$ \\
\hline Total mortality & $\begin{array}{l}\text { Excluding studies with systematic } \\
\text { differences in diet other than fat }\end{array}$ & $\begin{array}{l}\text { Reduced fat in- } \\
\text { take }\end{array}$ & $\begin{array}{l}0.90(0.60 \text { to } \\
1.34)\end{array}$ & 4/ 2998/ 89 & $0 \%$ \\
\hline
\end{tabular}


Table 1. Sensitivity analyses of primary outcomes (Continued)

\begin{tabular}{|c|c|c|c|c|c|}
\hline Total mortality & $\begin{array}{l}\text { Excluding studies with systematic } \\
\text { differences in diet other than fat }\end{array}$ & $\begin{array}{l}\text { Reduced \& modi- } \\
\text { fied fat intake }\end{array}$ & $\begin{array}{l}0.98(0.76 \text { to } \\
1.25)\end{array}$ & $1 / 2033 / 224$ & NR \\
\hline $\begin{array}{l}\text { Cardiovascular } \\
\text { mortality }\end{array}$ & Mantel-Haenszel, Fixed effects & Overall analysis & $\begin{array}{l}0.95(0.85 \text { to } \\
1.05)\end{array}$ & $\begin{array}{l}16 / 65983 / \\
1407\end{array}$ & $0 \%$ \\
\hline $\begin{array}{l}\text { Cardiovascular } \\
\text { mortality }\end{array}$ & Mantel-Haenszel, Fixed effects & $\begin{array}{l}\text { Modified fat in- } \\
\text { take }\end{array}$ & $\begin{array}{l}0.92(0.79 \text { to } \\
1.08)\end{array}$ & 6/10788/ 593 & $45 \%$ \\
\hline $\begin{array}{l}\text { Cardiovascular } \\
\text { mortality }\end{array}$ & Mantel-Haenszel, Fixed effects & $\begin{array}{l}\text { Reduced fat in- } \\
\text { take }\end{array}$ & $\begin{array}{l}0.96(0.82 \text { to } \\
1.12)\end{array}$ & $7 / 52971 / 602$ & $0 \%$ \\
\hline $\begin{array}{l}\text { Cardiovascular } \\
\text { mortality }\end{array}$ & Mantel-Haenszel, Fixed effects & $\begin{array}{l}\text { Reduced \& modi- } \\
\text { fied fat intake }\end{array}$ & $\begin{array}{l}0.98(0.76 \text { to } \\
1.26)\end{array}$ & $3 / 2224 / 212$ & $0 \%$ \\
\hline $\begin{array}{l}\text { Cardiovascular } \\
\text { mortality }\end{array}$ & Peto Odds Ratio (fixed effects) & Overall analysis & $\begin{array}{l}\text { OR } 0.94(0.84 \text { to } \\
1.05)\end{array}$ & $\begin{array}{l}16 / 65983 / \\
1407\end{array}$ & $11 \%$ \\
\hline $\begin{array}{l}\text { Cardiovascular } \\
\text { mortality }\end{array}$ & Peto Odds Ratio (fixed effects) & $\begin{array}{l}\text { Modified fat in- } \\
\text { take }\end{array}$ & $\begin{array}{l}\text { OR } 0.91 \text { ( } 0.77 \text { to } \\
1.08)\end{array}$ & $6 / 10788 / 593$ & $51 \%$ \\
\hline $\begin{array}{l}\text { Cardiovascular } \\
\text { mortality }\end{array}$ & Peto Odds Ratio (fixed effects) & $\begin{array}{l}\text { Reduced fat in- } \\
\text { take }\end{array}$ & $\begin{array}{l}\text { OR } 0.96 \text { ( } 0.81 \text { to } \\
1.13)\end{array}$ & $7 / 52971 / 602$ & $0 \%$ \\
\hline $\begin{array}{l}\text { Cardiovascular } \\
\text { mortality }\end{array}$ & Peto Odds Ratio (fixed effects) & $\begin{array}{l}\text { Reduced \& Modi- } \\
\text { fied fat intake }\end{array}$ & $\begin{array}{l}\text { OR } 0.97(0.73 \text { to } \\
1.29)\end{array}$ & $3 / 2224 / 212$ & $0 \%$ \\
\hline $\begin{array}{l}\text { Cardiovascular } \\
\text { mortality }\end{array}$ & Excluding WHI & 50verall analysis & $\begin{array}{l}0.91 \text { ( } 0.79 \text { to } \\
1.04)\end{array}$ & $14 / 17148 / 874$ & $7 \%$ \\
\hline $\begin{array}{l}\text { Cardiovascular } \\
\text { mortality }\end{array}$ & Excluding WHI & $\begin{array}{l}\text { Reduced fat in- } \\
\text { take }\end{array}$ & $\begin{array}{l}0.74(0.47 \text { to } \\
1.17)\end{array}$ & $5 / 4136 / 69$ & $0 \%$ \\
\hline $\begin{array}{l}\text { Cardiovascular } \\
\text { mortality }\end{array}$ & $\begin{array}{l}\text { Excluding studies with systematic } \\
\text { difference in care }\end{array}$ & Overall analysis & $\begin{array}{l}0.97(0.70 \text { to } \\
1.33)\end{array}$ & 4/9983/451 & $59 \%$ \\
\hline $\begin{array}{l}\text { Cardiovascular } \\
\text { mortality }\end{array}$ & $\begin{array}{l}\text { Excluding studies with systematic } \\
\text { difference in care }\end{array}$ & $\begin{array}{l}\text { Modified fat in- } \\
\text { take }\end{array}$ & $\begin{array}{l}0.97(0.70 \text { to } \\
1.33)\end{array}$ & 4/ 9983/ 451 & $59 \%$ \\
\hline $\begin{array}{l}\text { Cardiovascular } \\
\text { mortality }\end{array}$ & $\begin{array}{l}\text { Excluding studies with systematic } \\
\text { difference in care }\end{array}$ & $\begin{array}{l}\text { Reduced fat in- } \\
\text { take }\end{array}$ & NR & $0 / 0 / 0$ & NR \\
\hline $\begin{array}{l}\text { Cardiovascular } \\
\text { mortality }\end{array}$ & $\begin{array}{l}\text { Excluding studies with systematic } \\
\text { difference in care }\end{array}$ & $\begin{array}{l}\text { Reduced \& modi- } \\
\text { fied fat intake }\end{array}$ & NR & $0 / 0 / 0$ & NR \\
\hline $\begin{array}{l}\text { Cardiovascular } \\
\text { mortality }\end{array}$ & $\begin{array}{l}\text { Excluding studies with systematic } \\
\text { differences in diet other than fat }\end{array}$ & Overall analysis & $\begin{array}{l}0.95(0.80 \text { to } \\
1.13)\end{array}$ & 9/12970/ 748 & $22 \%$ \\
\hline $\begin{array}{l}\text { Cardiovascular } \\
\text { mortality }\end{array}$ & $\begin{array}{l}\text { Excluding studies with systematic } \\
\text { differences in diet other than fat }\end{array}$ & $\begin{array}{l}\text { Modified fat in- } \\
\text { take }\end{array}$ & $\begin{array}{l}0.97(0.75 \text { to } \\
1.26)\end{array}$ & $5 / 10376 / 503$ & $47 \%$ \\
\hline $\begin{array}{l}\text { Cardiovascular } \\
\text { mortality }\end{array}$ & $\begin{array}{l}\text { Excluding studies with systematic } \\
\text { differences in diet other than fat }\end{array}$ & $\begin{array}{l}\text { Reduced fat in- } \\
\text { take }\end{array}$ & $\begin{array}{l}0.75(0.40 \text { to } \\
1.42)\end{array}$ & $3 / 561 / 44$ & $3 \%$ \\
\hline $\begin{array}{l}\text { Cardiovascular } \\
\text { mortality }\end{array}$ & $\begin{array}{l}\text { Excluding studies with systematic } \\
\text { differences in diet other than fat }\end{array}$ & $\begin{array}{l}\text { Reduced \& modi- } \\
\text { fied fat intake }\end{array}$ & $\begin{array}{l}1.01 \text { ( } 0.77 \text { to } \\
1.31)\end{array}$ & $1 / 2033 / 201$ & NR \\
\hline
\end{tabular}


Table 1. Sensitivity analyses of primary outcomes (Continued)

\begin{tabular}{|c|c|c|c|c|c|}
\hline $\begin{array}{l}\text { Combined cardio- } \\
\text { vascular events }\end{array}$ & Mantel-Haenszel, Fixed effects & Overall analysis & $\begin{array}{l}0.93 \text { (0.88 to } \\
0.98)\end{array}$ & $\begin{array}{l}23 / 65508 / \\
4887\end{array}$ & $50 \%$ \\
\hline $\begin{array}{l}\text { Combined cardio- } \\
\text { vascular events }\end{array}$ & Mantel-Haenszel, Fixed effects & $\begin{array}{l}\text { Modified fat in- } \\
\text { take }\end{array}$ & $\begin{array}{l}0.83(0.73 \text { to } \\
0.93)\end{array}$ & $9 / 11660 / 855$ & $61 \%$ \\
\hline $\begin{array}{l}\text { Combined cardio- } \\
\text { vascular events }\end{array}$ & Mantel-Haenszel, Fixed effects & $\begin{array}{l}\text { Reduced fat in- } \\
\text { take }\end{array}$ & $\begin{array}{l}0.96(0.91 \text { to } \\
1.03)\end{array}$ & $8 / 50655 / 3632$ & $17 \%$ \\
\hline $\begin{array}{l}\text { Combined cardio- } \\
\text { vascular events }\end{array}$ & Mantel-Haenszel, Fixed effects & $\begin{array}{l}\text { Reduced \& modi- } \\
\text { fied fat intake }\end{array}$ & $\begin{array}{l}0.84(0.71 \text { to } \\
1.00)\end{array}$ & $6 / 3193 / 400$ & $40 \%$ \\
\hline $\begin{array}{l}\text { Combined cardio- } \\
\text { vascular events }\end{array}$ & Peto Odds Ratio (fixed effects) & Overall analysis & $\begin{array}{l}\text { OR } 0.92 \text { ( } 0.86 \text { to } \\
0.97)\end{array}$ & $\begin{array}{l}23 / 65508 / \\
4887\end{array}$ & $61 \%$ \\
\hline $\begin{array}{l}\text { Combined cardio- } \\
\text { vascular events }\end{array}$ & Peto Odds Ratio (fixed effects) & $\begin{array}{l}\text { Modified fat in- } \\
\text { take }\end{array}$ & $\begin{array}{l}\text { OR } 0.78 \text { (0.67 to } \\
0.91)\end{array}$ & $9 / 11660 / 855$ & $70 \%$ \\
\hline $\begin{array}{l}\text { Combined cardio- } \\
\text { vascular events }\end{array}$ & Peto Odds Ratio (fixed effects) & $\begin{array}{l}\text { Reduced fat in- } \\
\text { take }\end{array}$ & $\begin{array}{l}\text { OR } 0.96 \text { (0.90 to } \\
1.03)\end{array}$ & $8 / 50655 / 3632$ & $30 \%$ \\
\hline $\begin{array}{l}\text { Combined cardio- } \\
\text { vascular events }\end{array}$ & Peto Odds Ratio (fixed effects) & $\begin{array}{l}\text { Reduced \& modi- } \\
\text { fied fat intake }\end{array}$ & $\begin{array}{l}\text { OR } 0.79 \text { (0.63 to } \\
1.00)\end{array}$ & $6 / 3193 / 400$ & $60 \%$ \\
\hline $\begin{array}{l}\text { Combined cardio- } \\
\text { vascular events }\end{array}$ & Excluding WHI & Overall analysis & $\begin{array}{l}0.81(0.70 \text { to } \\
0.93)\end{array}$ & $\begin{array}{l}21 / 16673 / \\
1442\end{array}$ & $39 \%$ \\
\hline $\begin{array}{l}\text { Combined cardio- } \\
\text { vascular events }\end{array}$ & Excluding WHI & $\begin{array}{l}\text { Reduced fat in- } \\
\text { take }\end{array}$ & $\begin{array}{l}0.82(0.63 \text { to } \\
1.05)\end{array}$ & $6 / 1820 / 187$ & $0 \%$ \\
\hline $\begin{array}{l}\text { Combined cardio- } \\
\text { vascular events }\end{array}$ & $\begin{array}{l}\text { Excluding studies with systematic } \\
\text { difference in care }\end{array}$ & Overall analysis & $\begin{array}{l}0.93(0.77 \text { to } \\
1.12)\end{array}$ & $9 / 12158 / 533$ & $12 \%$ \\
\hline $\begin{array}{l}\text { Combined cardio- } \\
\text { vascular events }\end{array}$ & $\begin{array}{l}\text { Excluding studies with systematic } \\
\text { difference in care }\end{array}$ & $\begin{array}{l}\text { Modified fat in- } \\
\text { take }\end{array}$ & $\begin{array}{l}0.95(0.77 \text { to } \\
1.17)\end{array}$ & $6 / 10753 / 527$ & $24 \%$ \\
\hline $\begin{array}{l}\text { Combined cardio- } \\
\text { vascular events }\end{array}$ & $\begin{array}{l}\text { Excluding studies with systematic } \\
\text { difference in care }\end{array}$ & $\begin{array}{l}\text { Reduced fat in- } \\
\text { take }\end{array}$ & $\begin{array}{l}0.20(0.01 \text { to } \\
4.13)\end{array}$ & $1 / 537 / 2$ & NR \\
\hline $\begin{array}{l}\text { Combined cardio- } \\
\text { vascular events }\end{array}$ & $\begin{array}{l}\text { Excluding studies with systematic } \\
\text { difference in care }\end{array}$ & $\begin{array}{l}\text { Reduced \& modi- } \\
\text { fied fat intake }\end{array}$ & $\begin{array}{l}0.28(0.04 \text { to } \\
2.15)\end{array}$ & $2 / 868 / 4$ & $0 \%$ \\
\hline $\begin{array}{l}\text { Combined cardio- } \\
\text { vascular events }\end{array}$ & $\begin{array}{l}\text { Excluding studies with systematic } \\
\text { differences in diet other than fat }\end{array}$ & Overall analysis & $\begin{array}{l}0.85(0.72 \text { to } \\
1.01)\end{array}$ & $\begin{array}{l}14 / 14710 / \\
1097\end{array}$ & $42 \%$ \\
\hline $\begin{array}{l}\text { Combined cardio- } \\
\text { vascular events }\end{array}$ & $\begin{array}{l}\text { Excluding studies with systematic } \\
\text { differences in diet other than fat }\end{array}$ & $\begin{array}{l}\text { Modified fat in- } \\
\text { take }\end{array}$ & $\begin{array}{l}0.84(0.65 \text { to } \\
1.09)\end{array}$ & $8 / 11248 / 701$ & $63 \%$ \\
\hline $\begin{array}{l}\text { Combined cardio- } \\
\text { vascular events }\end{array}$ & $\begin{array}{l}\text { Excluding studies with systematic } \\
\text { differences in diet other than fat }\end{array}$ & $\begin{array}{l}\text { Reduced fat in- } \\
\text { take }\end{array}$ & $\begin{array}{l}0.87(0.63 \text { to } \\
1.20)\end{array}$ & $3 / 561 / 109$ & $0 \%$ \\
\hline $\begin{array}{l}\text { Combined cardio- } \\
\text { vascular events }\end{array}$ & $\begin{array}{l}\text { Excluding studies with systematic } \\
\text { differences in diet other than fat }\end{array}$ & $\begin{array}{l}\text { Reduced \& modi- } \\
\text { fied fat intake }\end{array}$ & $\begin{array}{l}0.91 \text { ( } 0.73 \text { to } \\
1.13)\end{array}$ & $3 / 2901 / 287$ & $0 \%$ \\
\hline
\end{tabular}


Table 2. Subgrouping data for primary outcomes

\begin{tabular}{|c|c|c|c|c|c|c|}
\hline Analysis described & $\begin{array}{l}\text { Total mor- } \\
\text { tality: } \\
\text { Number } \\
\text { of stud- } \\
\text { ies, par- } \\
\text { ticipants, } \\
\text { events }\end{array}$ & $\begin{array}{l}\text { Total mortality: } \\
\text { Relative Risk } \\
(95 \% \mathrm{CI}) \text {, Het- } \\
\text { erogeneity - } \mathrm{I}^{2} \text {, } \\
\%\end{array}$ & $\begin{array}{l}\text { CVD mor- } \\
\text { tality: } \\
\text { Number } \\
\text { of stud- } \\
\text { ies, par- } \\
\text { ticipants, } \\
\text { events }\end{array}$ & $\begin{array}{l}\text { CVD mortality: } \\
\text { Relative Risk } \\
(95 \% \mathrm{CI}) \text {, Het- } \\
\text { erogeneity - } \text { I2, }^{2} \\
\%\end{array}$ & $\begin{array}{l}\text { CVD } \\
\text { events: } \\
\text { Number } \\
\text { of stud- } \\
\text { ies, par- } \\
\text { ticipants, } \\
\text { events }\end{array}$ & $\begin{array}{l}\text { CVD events: } \\
\text { Relative Risk } \\
(95 \% \mathrm{Cl}) \text {, Het- } \\
\text { erogeneity - }{ }^{2} \text {, } \\
\%\end{array}$ \\
\hline Main meta-analysis & $\begin{array}{l}21,71790 \\
4292\end{array}$ & $\begin{array}{l}0.98(0.93 \text { to } \\
1.04), 0\end{array}$ & $\begin{array}{l}16,65978 \\
1407\end{array}$ & $\begin{array}{l}0.94(0.85 \text { to } \\
1.04), 0\end{array}$ & $\begin{array}{l}23,65508 \\
4887\end{array}$ & $\begin{array}{l}0.86(0.77 \text { to } \\
0.96), 50\end{array}$ \\
\hline $\begin{array}{l}\text { Sub-group 'mean fol- } \\
\text { low-up } \leq 2 \text { years' }\end{array}$ & $\begin{array}{l}9,12272 \\
767\end{array}$ & $\begin{array}{l}1.05(0.91 \text { to } \\
1.20), 0\end{array}$ & $\begin{array}{l}6,11434 \\
523\end{array}$ & $\begin{array}{l}1.04(0.88 \text { to } \\
1.23), 0\end{array}$ & $\begin{array}{l}12,13844 \\
689\end{array}$ & $\begin{array}{l}0.95(0.84 \text { to } \\
1.09), 0\end{array}$ \\
\hline $\begin{array}{l}\text { Sub-group 'mean fol- } \\
\text { low-up >2 years' }\end{array}$ & $\begin{array}{l}12,59518 \\
3525\end{array}$ & $\begin{array}{l}0.97(0.91 \text { to } \\
1.03), 0\end{array}$ & $\begin{array}{l}10,54544 \\
884\end{array}$ & $\begin{array}{l}0.89(0.78 \text { to } \\
1.01), 0\end{array}$ & $\begin{array}{l}11,51664 \\
4198\end{array}$ & $\begin{array}{l}0.78(0.67 \text { to } \\
0.92), 72\end{array}$ \\
\hline Sub-group 'low CVD risk' & $\begin{array}{l}10,66776 \\
3717\end{array}$ & $\begin{array}{l}0.99(0.93 \text { to } \\
1.05), 0\end{array}$ & $\begin{array}{l}6,59682 \\
879\end{array}$ & $\begin{array}{l}0.93(0.77 \text { to } \\
1.13), 35\end{array}$ & $\begin{array}{l}10,58338 \\
3408\end{array}$ & $\begin{array}{l}0.93(0.82 \text { to } \\
1.05), 13\end{array}$ \\
\hline $\begin{array}{l}\text { Sub-group 'moderate CVD } \\
\text { risk' }\end{array}$ & $3,1200,47$ & $\begin{array}{l}0.69(0.39 \text { to } \\
1.21), 0\end{array}$ & $2,663,23$ & $\begin{array}{l}0.66(0.28 \text { to } \\
1.53), 0\end{array}$ & $5,1537,143$ & $\begin{array}{l}0.57(0.33 \text { to } \\
0.99), 53\end{array}$ \\
\hline Sub-group 'high CVD risk' & $8,3814,528$ & $\begin{array}{l}0.95(0.78 \text { to } \\
1.17), 24\end{array}$ & $8,5633,505$ & $\begin{array}{l}0.95(0.80 \text { to } \\
1.12), 0\end{array}$ & $\begin{array}{l}8,5633 \\
1336\end{array}$ & $\begin{array}{l}0.88(0.75 \text { to } \\
1.02), 58\end{array}$ \\
\hline Sub-group 'Dietary advice' & $\begin{array}{l}13,60320 \\
3201\end{array}$ & $\begin{array}{l}0.98(0.92 \text { to } \\
1.05), 0\end{array}$ & $\begin{array}{l}9,54703 \\
796\end{array}$ & $\begin{array}{l}0.97(0.85 \text { to } \\
1.12), 0\end{array}$ & $\begin{array}{l}12,52595 \\
3998\end{array}$ & $\begin{array}{l}0.85(0.72 \text { to } \\
1.00), 62\end{array}$ \\
\hline $\begin{array}{l}\text { Sub-group 'dietary advice } \\
\text { plus supplementation' }\end{array}$ & 4, 1372, 219 & $\begin{array}{l}0.80(0.63 \text { to } \\
1.02), 0\end{array}$ & 4, 1372, 169 & $\begin{array}{l}0.87(0.62 \text { to } \\
1.21), 15\end{array}$ & 4, 1372, 395 & $\begin{array}{l}0.78(0.66 \text { to } \\
0.92), 0\end{array}$ \\
\hline Sub-group 'diet provided' & $\begin{array}{l}4,10098 \\
872\end{array}$ & $\begin{array}{l}1.03(0.92 \text { to } \\
1.15), 0\end{array}$ & 3, 9903, 442 & $\begin{array}{l}0.93(0.69 \text { to } \\
1.26), 63\end{array}$ & $\begin{array}{l}7,11541 \\
494\end{array}$ & $\begin{array}{l}0.91(0.73 \text { to } \\
1.14), 19\end{array}$ \\
\hline $\begin{array}{l}\text { Sub-group 'total fat in } \\
\text { control }<30 \% \mathrm{E}^{\prime}\end{array}$ & $4,3592,81$ & $\begin{array}{l}0.93(0.61 \text { to } \\
1.43), 0\end{array}$ & $2,618,25$ & $\begin{array}{l}0.76(0.35 \text { to } \\
1.64), 0\end{array}$ & $3,1155,152$ & $\begin{array}{l}0.82(0.65 \text { to } \\
1.03), 1\end{array}$ \\
\hline $\begin{array}{l}\text { Sub-group 'total fat in } \\
\text { control } 30-34.9 \% \mathrm{E}^{\prime}\end{array}$ & $4,3450,333$ & $\begin{array}{l}0.91(0.46 \text { to } \\
1.81), 23\end{array}$ & $3,3344,21$ & $\begin{array}{l}0.72(0.15 \text { to } \\
3.53), 51\end{array}$ & $5,1429,74$ & $\begin{array}{l}0.93(0.62 \text { to } \\
1.39), 0\end{array}$ \\
\hline $\begin{array}{l}\text { Sub-group 'total fat in } \\
\text { control } 35-39.9 \% \mathrm{E}^{\prime}\end{array}$ & $\begin{array}{l}8,62820 \\
3311\end{array}$ & $\begin{array}{l}1.00(0.94 \text { to } \\
1.07), 0\end{array}$ & $\begin{array}{l}6,60088 \\
1054\end{array}$ & $\begin{array}{l}1.00(0.88 \text { to } \\
1.12), 0\end{array}$ & $\begin{array}{l}10,60919 \\
4034\end{array}$ & $\begin{array}{l}0.96(0.84 \text { to } \\
1.10), 44\end{array}$ \\
\hline $\begin{array}{l}\text { Sub-group 'total fat in } \\
\text { control } 40+\% \mathrm{E}^{\prime}\end{array}$ & $3,1491,454$ & $\begin{array}{l}0.96(0.83 \text { to } \\
1.11), 0\end{array}$ & $3,1491,227$ & $\begin{array}{l}0.80(0.63 \text { to } \\
1.02), 0\end{array}$ & $3,1491,435$ & $\begin{array}{l}0.83(0.71 \text { to } \\
0.97), 0\end{array}$ \\
\hline $\begin{array}{l}\text { Sub-group 'saturated fat } \\
\text { in control }<10 \% \mathrm{E}^{\prime}\end{array}$ & $2,3625,316$ & $\begin{array}{l}0.97(0.79 \text { to } \\
1.20), 0\end{array}$ & $1,3088,7$ & $\begin{array}{l}0.40(0.08 \text { to } \\
2.08),-\end{array}$ & $1,537,2$ & $\begin{array}{l}0.20(0.01 \text { to } \\
4.13),-\end{array}$ \\
\hline $\begin{array}{l}\text { Sub-group 'saturated fat } \\
\text { in control } 10-14.9 \% \mathrm{E}^{\prime}\end{array}$ & $\begin{array}{l}5,50089 \\
2520\end{array}$ & $\begin{array}{l}1.01(0.77 \text { to } \\
1.32), 32\end{array}$ & $\begin{array}{l}5,49631 \\
558\end{array}$ & $\begin{array}{l}0.98(0.83 \text { to } \\
1.16), 0\end{array}$ & $\begin{array}{l}11,51610 \\
3565\end{array}$ & $\begin{array}{l}0.97(0.87 \text { to } \\
1.07), 10\end{array}$ \\
\hline $\begin{array}{l}\text { Sub-group 'saturated fat } \\
\text { in control } 15-19.9 \% \mathrm{E}^{\prime}\end{array}$ & $\begin{array}{l}6,14598 \\
1134\end{array}$ & $\begin{array}{l}1.02(0.92 \text { to } \\
1.13), 0\end{array}$ & $\begin{array}{l}4,11966 \\
657\end{array}$ & $\begin{array}{l}0.90 \text { ( } 0.74 \text { to } \\
1.11), 37\end{array}$ & $\begin{array}{l}5,11991 \\
793\end{array}$ & $\begin{array}{l}0.86 \text { ( } 0.68 \text { to } \\
1.09), 64\end{array}$ \\
\hline
\end{tabular}


Table 2. Subgrouping data for primary outcomes (Continued)

\begin{tabular}{|c|c|c|c|c|c|c|}
\hline $\begin{array}{l}\text { Sub-group 'saturated fat } \\
\text { in control } 20+\% E^{\prime}\end{array}$ & $0,0,0$ & - & $0,0,0$ & - & $0,0,0$ & - \\
\hline $\begin{array}{l}\text { Sub-group 'studies of } \\
\text { men' }\end{array}$ & $\begin{array}{l}11,9604 \\
1224\end{array}$ & $\begin{array}{l}0.97(0.87 \text { to } \\
1.08), 7\end{array}$ & $9,8951,735$ & $\begin{array}{l}0.89(0.77 \text { to } \\
1.02), 0\end{array}$ & $\begin{array}{l}13,10589 \\
1162\end{array}$ & $\begin{array}{l}0.82(0.74 \text { to } \\
0.90), 0\end{array}$ \\
\hline $\begin{array}{l}\text { Sub-group 'studies of } \\
\text { women' }\end{array}$ & $\begin{array}{l}5,59130 \\
2960\end{array}$ & $\begin{array}{l}0.99(0.92 \text { to } \\
1.07), 0\end{array}$ & $\begin{array}{l}4,56587 \\
658\end{array}$ & $\begin{array}{l}1.03(0.88 \text { to } \\
1.20), 0\end{array}$ & $\begin{array}{l}4,53605 \\
3566\end{array}$ & $\begin{array}{l}1.04(0.89 \text { to } \\
1.21), 53\end{array}$ \\
\hline $\begin{array}{l}\text { Sub-group 'studies of men } \\
\text { and women' }\end{array}$ & $5,3056,108$ & $\begin{array}{l}0.84(0.58 \text { to } \\
1.22), 0\end{array}$ & $3,440,14$ & $\begin{array}{l}0.45(0.15 \text { to } \\
1.37), 0\end{array}$ & $6,1314,159$ & $\begin{array}{l}0.60(0.32 \text { to } \\
1.13), 66\end{array}$ \\
\hline $\begin{array}{l}\text { Sub-group 'community } \\
\text { setting' }\end{array}$ & $\begin{array}{l}17,61692 \\
3420\end{array}$ & $\begin{array}{l}0.97(0.91 \text { to } \\
1.03), 0\end{array}$ & $\begin{array}{l}13,56077 \\
965\end{array}$ & $\begin{array}{l}0.95(0.84 \text { to } \\
1.07), 0\end{array}$ & $\begin{array}{l}20,55605 \\
4405\end{array}$ & $\begin{array}{l}0.84(0.73 \text { to } \\
0.95), 51\end{array}$ \\
\hline $\begin{array}{l}\text { Sub-group 'residential set- } \\
\text { ting' }\end{array}$ & $\begin{array}{l}4,10098 \\
872\end{array}$ & $\begin{array}{l}1.03(0.92 \text { to } \\
1.15), 0\end{array}$ & $3,9903,442$ & $\begin{array}{l}0.93(0.69 \text { to } \\
1.26), 63\end{array}$ & $3,9903,482$ & $\begin{array}{l}0.94(0.71 \text { to } \\
1.24), 63\end{array}$ \\
\hline $\begin{array}{l}\text { Sub-group 'published in } \\
\text { 1960s' }\end{array}$ & $6,2178,580$ & $\begin{array}{l}0.92(0.80 \text { to } \\
1.06), 2\end{array}$ & $5,1983,326$ & $\begin{array}{l}0.80,0.64 \text { to } \\
1.00), 11\end{array}$ & $9,3621,638$ & $\begin{array}{l}0.81(0.71 \text { to } \\
0.92), 0\end{array}$ \\
\hline $\begin{array}{l}\text { Sub-group 'published in } \\
\text { 1970s' }\end{array}$ & $3,9515,584$ & $\begin{array}{l}1.13(0.95 \text { to } \\
1.33), 9\end{array}$ & $2,9057,304$ & $\begin{array}{l}1.06(0.85 \text { to } \\
1.33), 0\end{array}$ & 3, 9159, 301 & $\begin{array}{l}0.71(0.35 \text { to } \\
1.43), 88\end{array}$ \\
\hline $\begin{array}{l}\text { Sub-group 'published in } \\
\text { 1980s' }\end{array}$ & $1,2033,224$ & $\begin{array}{l}0.98(0.76 \text { to } \\
1.25),-\end{array}$ & 1, 2033, 201 & $\begin{array}{l}1.01(0.77 \text { to } \\
1.31),-\end{array}$ & $1,2033,283$ & $\begin{array}{l}0.92(0.74 \text { to } \\
1.15),-\end{array}$ \\
\hline $\begin{array}{l}\text { Sub-group 'published in } \\
\text { 1990s' }\end{array}$ & $5,4880,137$ & $\begin{array}{l}0.90(0.65 \text { to } \\
1.26), 0\end{array}$ & $3,364,11$ & $\begin{array}{l}0.27(0.07 \text { to } \\
1.08), 0\end{array}$ & $3,364,57$ & $\begin{array}{l}0.51(0.32 \text { to } \\
0.81), 0\end{array}$ \\
\hline $\begin{array}{l}\text { Sub-group 'published } \\
\text { since } 2000 \text { ' }\end{array}$ & $\begin{array}{l}6,53184 \\
2767\end{array}$ & $\begin{array}{l}0.98(0.91 \text { to } \\
1.05), 0\end{array}$ & $\begin{array}{l}5,52541 \\
565\end{array}$ & $\begin{array}{l}0.98(0.83 \text { to } \\
1.15), 0\end{array}$ & $\begin{array}{l}7,50331 \\
3608\end{array}$ & $\begin{array}{l}0.96(0.85 \text { to } \\
1.08), 29\end{array}$ \\
\hline
\end{tabular}

Table 3. Meta-regression, exploring effects of dietary changes on the primary outcomes

\begin{tabular}{|c|c|c|c|c|}
\hline Outcome / Variable & $\begin{array}{l}\text { Total mortality, coeffi- } \\
\text { cient }(95 \% \mathrm{Cl}) \text {, number } \\
\text { of studies, } 1^{2}\end{array}$ & $\begin{array}{l}\text { Cardiovascular mortali- } \\
\text { ty, coefficient }(95 \% \mathrm{Cl}) \text {, } \\
\text { number of studies, } \mathrm{I}^{2}\end{array}$ & $\begin{array}{l}\text { Cardiovascular events, } \\
\text { coefficient }(95 \% \mathrm{Cl}) \text {, } \\
\text { number of studies, } \mathrm{I}^{2}\end{array}$ & $\begin{array}{l}\text { CVD events (co-regres- } \\
\text { sion with duration) co- } \\
\text { efficient ( } 95 \% \mathrm{CI}) \text {, num- } \\
\text { ber of studies, } \mathrm{I}^{2} \text {, }\end{array}$ \\
\hline $\begin{array}{l}\text { Difference in total fat } \\
\text { intake, } \% \mathrm{E}\end{array}$ & $\begin{array}{l}-0.010(-0.030 \text { to } 0.010) \\
20,0 \%{ }^{*}\end{array}$ & $\begin{array}{l}-0.023(-0.074 \text { to } 0.029) \\
14,4 \%\end{array}$ & $\begin{array}{l}-0.012(-0.037 \text { to } 0.012) \\
21,25 \%\end{array}$ & $\begin{array}{l}-0.016(-0.045 \text { to } 0.013) \text {, } \\
21,23 \%\end{array}$ \\
\hline $\begin{array}{l}\text { Difference in SFA in- } \\
\text { take, \%E }\end{array}$ & $\begin{array}{l}0.005(-0.016 \text { to } 0.026) \\
18,0 \%\end{array}$ & $\begin{array}{l}-0.006(-0.050 \text { to } 0.037) \\
14,3 \%\end{array}$ & $\begin{array}{l}-.013(-0.043 \text { to } 0.017), 21, \\
19 \%\end{array}$ & $\begin{array}{l}-0.008(-0.048 \text { to } 0.032), \\
21,22 \%\end{array}$ \\
\hline $\begin{array}{l}\text { Difference in MUFA } \\
\text { intake, } \% \mathrm{E}\end{array}$ & $\begin{array}{l}-0.036(-0.112 \text { to } 0.040) \\
15,0 \%\end{array}$ & $\begin{array}{l}-0.073(-0.203 \text { to } 0.056) \\
13,3 \%\end{array}$ & $\begin{array}{l}-0.023(-0.067 \text { to } 0.021) \\
14,41 \%\end{array}$ & $\begin{array}{l}-0.024(-0.071 \text { to } 0.022) \text {, } \\
14,36 \%\end{array}$ \\
\hline $\begin{array}{l}\text { Difference in PUFA } \\
\text { intake, } \% \mathrm{E}\end{array}$ & $\begin{array}{l}-0.004 \text { (-0.016 to } 0.008) \\
17,0 \%\end{array}$ & $\begin{array}{l}-0.000(-0.027 \text { to } 0.026) \\
14,4 \%\end{array}$ & $\begin{array}{l}-0.002(-0.022 \text { to } 0.019) \\
19,24 \%\end{array}$ & $\begin{array}{l}-0.006(-0.032 \text { to } 0.021) \text {, } \\
19,27 \%\end{array}$ \\
\hline $\begin{array}{l}\text { Difference in trans fat } \\
\text { intake, } \% \mathrm{E}\end{array}$ & $\begin{array}{l}\text { Insufficient data, } 2 \\
\text { studies }\end{array}$ & $\begin{array}{l}\text { Insufficient data, } 2 \text { stud- } \\
\text { ies }\end{array}$ & $\begin{array}{l}\text { Insufficient data, } 2 \text { stud- } \\
\text { ies }\end{array}$ & $\begin{array}{l}\text { Insufficient data, } 2 \\
\text { studies }\end{array}$ \\
\hline
\end{tabular}


Table 3. Meta-regression, exploring effects of dietary changes on the primary outcomes (Continued)

\begin{tabular}{lllll}
$\begin{array}{l}\text { Difference in weight, } \\
\mathrm{kg}\end{array}$ & $\begin{array}{l}-0.035(-0.138 \text { to } 0.068), \\
14,0 \%\end{array}$ & $\begin{array}{l}-0.067(-0.240 \text { to } 0.106), 9, \\
17 \%\end{array}$ & $\begin{array}{l}-0.038(-0.154 \text { to } 0.078), \\
14,47 \%\end{array}$ & $\begin{array}{l}-0.033(-0.118 \text { to } 0.052), \\
14,15 \% * *\end{array}$ \\
\hline $\begin{array}{l}\text { Difference in LDL } \\
\text { cholesterol, } \mathrm{mmol} / \mathrm{L}\end{array}$ & $\begin{array}{l}-0.948(-5.752 \text { to } 3.856), \\
6,0 \%\end{array}$ & $\begin{array}{l}-2.909(-10.529 \text { to } \\
4.710), 6,0 \%\end{array}$ & $\begin{array}{l}-1.600(-3.815 \text { to } 0.616), 7, \\
28 \%\end{array}$ & $\begin{array}{l}-1.465(-4.094 \text { to } 1.164), \\
7,25 \%\end{array}$ \\
\hline $\begin{array}{l}\text { Difference in serum } \\
\text { total cholesterol, }\end{array}$ & $\begin{array}{l}0.024(-0.168 \text { to } 0.217), \\
\text { mmol/L }\end{array}$ & $\begin{array}{l}-0.187(-0.505 \text { to } 0.131), \\
15,0 \%\end{array}$ & $\begin{array}{l}-0.155(-0.554 \text { to } 0.244), \\
17,49 \%\end{array}$ & $-0.155(-0.635$ to 0.325$)$, \\
\hline Study duration, years & $-0.004(-0.024$ to 0.016$)$, & $\begin{array}{l}-0.014(-0.072 \text { to } 0.044), \\
16,4 \%\end{array}$ & $\begin{array}{l}-0.037(-0.106 \text { to } 0.032), \\
24,45 \%\end{array}$ &
\end{tabular}

\section{APPENDICES}

\section{Appendix 1. MEDLINE search strategies 1998}

\section{MEDLINE on SilverPlatter - diet and cardiovascular disease or mortality - from 1966 to May 1998}

explode "NUTRITION"/adverse-effects , classification , contraindications , drug-effects , education , mortality , methods , nursing , physiology, utilization

explode "DIET"/ adverse-effects, blood, contraindications, drug-effects , metabolism , mortality , methods , nursing , physiology , utilization

explode "DIET-THERAPY"/ all subheadings

explode "LIPIDS"/ administration-and-dosage, adverse-effects, therapeutic-use

explode "FOOD"/administration-and-dosage, adverse-effects, drug-effects , therapeutic-use

explode "VITAMINS"/administration-and-dosage, adverse-effects, therapeutic-use

"SELENIUM"/administration-and-dosage, adverse-effects, therapeutic-use

"CALCIUM"/administration-and-dosage, adverse-effects, therapeutic-use

explode "CHLORIDES"/administration-and-dosage, adverse-effects, therapeutic-use

"MAGNESIUM"/administration-and-dosage, adverse-effects, therapeutic-use

"PHOSPHORUS,-DIETARY"/ all subheadings

"POTASSIUM,-DIETARY"/ all subheadings

explode "SODIUM-CHLORIDE"/ all subheadings

explode "TRACE-ELEMENTS"/administration-and-dosage, adverse-effects, therapeutic-use

explode "FLUORIDES"/administration-and-dosage, adverse-effects, therapeutic-use

MEDITERRAN* in TI,AB

explode "ANTIOXIDANTS"/administration-and-dosage, adverse-effects, therapeutic-use

$\# 1$ or \#2 or \#3 or \#4 or \#5 or \#6 or \#7

$\# 8$ or \#9 or \#10 or \#11 or \#12 or \#13 or \#14 or \#15 or \#16 or \#17

\#18 or \#19

LIPID* near $\left(\right.$ LOW $^{\star}$ or REDUC* or MODIFI*)

$\mathrm{DIET}^{*}$ in $\mathrm{TI}, \mathrm{AB}$

FAT $^{\star}$ near $\left(\right.$ LOW $^{\star}$ or MODIFI* or ANIMAL ${ }^{\star}$ or VEGETABLE* or ACID* or MONO?UNSAT* or POLY?UNSAT* or SATURAT* or UNSATUR*)

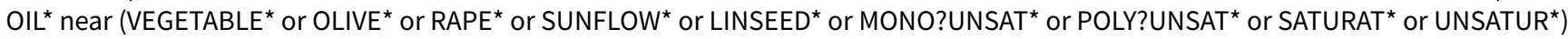

$\mathrm{MEAT}^{\star}$ in $\mathrm{TI}, \mathrm{AB}$

WEIGHT* near (REDUC* in TI,AB)

$\mathrm{SLIMM}^{*}$ in TI,AB

FISH in TI,AB

ANTI?OXIDA* in TI,AB

VITAMIN* in TI,AB

MINERAL* in TI,AB

$\mathrm{SALT}^{\star}$ in $\mathrm{TI}, \mathrm{AB}$

SODIUM* near (ORAL ${ }^{*}$ or SUPPLEMEN ${ }^{\star}$ or $\mathrm{ADD}^{\star}$ or CAPSUL ${ }^{\star}$ or TABLET ${ }^{*}$ or $\mathrm{HIGH}^{\star}$ or $\mathrm{LOW}^{\star}$ or ENRICH${ }^{\star}$ )

VEGETABLE* in TI,AB

FRUIT* in TI,AB

POTASSIUM $^{\star}$ near $\left(\mathrm{ORAL}^{\star}\right.$ or SUPPLEMEN ${ }^{\star}$ or $\mathrm{ADD}^{\star}$ or CAPSUL ${ }^{\star}$ or TABLET ${ }^{\star}$ or $\mathrm{HIGH}^{\star}$ or $\mathrm{LOW}^{\star}$ or ENRICH ${ }^{\star}$ ) 


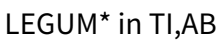

$\mathrm{SOY}^{*}$ in $\mathrm{TI}, \mathrm{AB}$

$\# 21$ or \#22 or \#23 or \#24 or \#25 or \#26 or \#27 or \#28 or \#29 or \#30

$\# 31$ or \#32 or \#33 or \#34 or \#35 or \#36 or \#37 or \#38

\#39 or \#40

$\mathrm{OAT}^{*}$ in $\mathrm{TI}, \mathrm{AB}$

FOLIC $^{*}$ in TI,AB

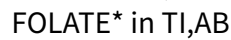

IRON $^{\star}$ near $\left(\mathrm{ORAL}^{\star}\right.$ or SUPPLEMEN ${ }^{\star}$ or $\mathrm{ADD}^{\star}$ or CAPSUL ${ }^{\star}$ or TABLET ${ }^{\star}$ or $\mathrm{HIGH}^{\star}$ or $\mathrm{LOW}^{\star}$ or ENRICH${ }^{\star}$ )

FERROUS* in TI,AB

FERRIC* in TI,AB

MARG?RINE* in TI,AB

BUTTER $^{\star}$ in TI,AB

$\mathrm{STARCH}^{*}$ in TI,AB

GRAIN* in TI,AB

NUT in TI,AB

NUTS in TI,AB

CAFFEIN* in TI,AB

COFFEE $^{*}$ in $T I, A B$

MULTI?VITAMIN ${ }^{*}$ in TI,AB

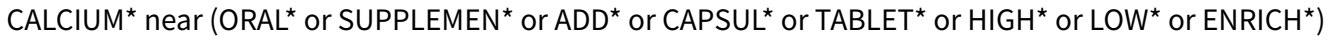

SELENIUM ${ }^{\star}$ near $\left(\mathrm{ORAL}^{\star}\right.$ or SUPPLEMEN ${ }^{\star}$ or $\mathrm{ADD}^{\star}$ or CAPSUL ${ }^{\star}$ or TABLET ${ }^{\star}$ or $\mathrm{HIGH}^{\star}$ or $\mathrm{LOW}^{\star}$ or ENRICH ${ }^{\star}$ )

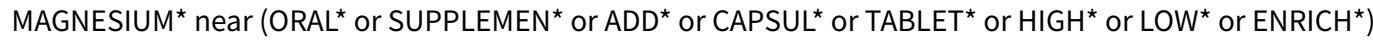

MANGANESE* near (ORAL ${ }^{\star}$ or SUPPLEMEN ${ }^{\star}$ or $\mathrm{ADD}^{\star}$ or CAPSUL ${ }^{\star}$ or TABLET ${ }^{\star}$ or $\mathrm{HIGH}^{\star}$ or $\mathrm{LOW}^{\star}$ or ENRICH ${ }^{\star}$ )

RETINOL* in TI,AB

CAROTENE* in TI,AB

BETA?CAROTENE* in TI,AB

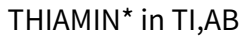

RIBOFLAV* in TI,AB

PYRIDOXIN* in TI,AB

ASCORB $^{*}$ in TI,AB

TOCOPHEROL* in TI,AB

ALPHA?TOCOPHER* in TI,AB

MOLYBDENUM* in TI,AB

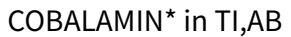

$\mathrm{BIOTIN}^{*}$ in TI,AB

FOLACIN* in TI,AB

$N I A C I N^{*}$ in $T I, A B$

NICOTINIC* in TI,AB

PANTOTHEN* in TI,AB

PHOSPHORUS $^{\star}$ near (ORAL ${ }^{\star}$ or SUPPLEMEN ${ }^{\star}$ or $\mathrm{ADD}^{\star}$ or CAPSUL ${ }^{\star}$ or TABLET ${ }^{\star}$ or $\mathrm{HIGH}^{\star}$ or $\mathrm{LOW}^{\star}$ or ENRICH${ }^{\star}$ )

CHROMIUM $^{\star}$ near (ORAL* or SUPPLEMEN ${ }^{\star}$ or ADD* or CAPSUL ${ }^{\star}$ or TABLET ${ }^{\star}$ or $\mathrm{HIGH}^{\star}$ or LOW* or ENRICH ${ }^{\star}$ )

COBALT $^{*}$ in TI,AB

IODINE* near (ORAL ${ }^{\star}$ or SUPPLEMEN ${ }^{\star}$ or $\mathrm{ADD}^{\star}$ or $\mathrm{CAPSUL}^{\star}$ or TABLET ${ }^{\star}$ or $\mathrm{HIGH}^{\star}$ or $\mathrm{LOW}^{\star}$ or ENRICH${ }^{\star}$ )

GARLIC* in TI, AB

ZINC* near (ORAL* or SUPPLEMEN ${ }^{*}$ or ADD* or CAPSUL ${ }^{*}$ or TABLET ${ }^{\star}$ or $\mathrm{HIGH}^{\star}$ or $\mathrm{LOW}^{\star}$ or ENRICH ${ }^{\star}$ )

$\# 42$ or \#43 or \#44 or \#45 or \#46 or \#47

$\# 48$ or \#49 or \#50 or \#51 or \#52 or \#53 or \#54 or \#55 or \#56 or \#57

$\# 58$ or \#59 or \#60 or \#61 or \#62 or \#63 or \#64 or \#65 or \#66 or \#67

$\# 68$ or \#69 or \#70 or \#71 or \#72 or \#73 or \#74 or \#75 or \#76 or \#77

$\# 78$ or \#79 or \#80 or \#81 or \#82

$\# 20$ or \#41 or \#83 or \#84 or \#85 or \#86 or \#87

explode "CARDIOVASCULAR-DISEASES"/ complications , diet-therapy , epidemiology , etiology , mortality , prevention-and-control , rehabilitation, secondary, therapy

explode "CEREBROVASCULAR-DISORDERS"/ complications , diet-therapy , epidemiology , etiology , mortality , prevention-and-control , rehabilitation, therapy

explode "NEOPLASMS"/ diet-therapy, mortality, prevention-and-control

CORONARY* near (BYPAS* or GRAFT* ${ }^{\star}$ or DISEASE* or EVENT*)

CEREBRO?VASCULA*

CARDIO?VASC*

MYOCARDIAL* near (INFARCT* or RE?VASCULAR* ${ }^{*}$ or ISCH?EMI*)

MORTAL* 
MORBID* near (HEART* or CORONARY* or ISCH?EM ${ }^{\star}$ or MYOCARD*)

VASCULAR $^{\star}$ near (PERIPHERAL ${ }^{\star}$ or DISEASE* or COMPLICATION ${ }^{*}$ )

ANGINA*

STROKE*

HEART $^{\star}$ near (DISEASE* ${ }^{*}$ or ATTACK ${ }^{\star}$ or BYPASS*)

$\# 91$ or \#92 or \#93 or \#94 or \#95 or \#96 or \#97 or \#98 or \#99 or \#100 or \#101 or \#89 or \#90

$\# 102$ and \#88

(TG=ANIMAL) not ((TG=HUMAN) and (TG=ANIMAL))

RANDOMIZED-CONTROLLED-TRIAL in PT

CONTROLLED-CLINICAL-TRIAL in PT

RANDOMIZED-CONTROLLED-TRIALS

RANDOM-ALLOCATION

DOUBLE-BLIND-METHOD

SINGLE-BLIND-METHOD

\#107 or \#108 or \#109 or \#110 or \#105 or \#106

\#103 not \#104

explode "CHILD"/ all subheadings

explode "ADULT"/ all subheadings

$\# 113$ and \#114

\#113 not \#115

111 and \#112

\#117 not \#116

\section{MEDLINE on SilverPlatter - lipid outcomes - 1966 to June 1998}

diet $^{\star}$ in $\mathrm{TI}, \mathrm{AB}$

fib? $r^{\star}$ in $T I, A B$

"Diet,-Atherogenic"

"Diet,-Fat-Restricted"/ all subheadings

explode "Fats"/ all subheadings

explode "Fatty-Acids"/ all subheadings

explode "Oils"/ all subheadings

explode "Dairy-Products"/ all subheadings

explode "Dietary-Fats"/ all subheadings

"Dietary-Fiber"/ all subheadings

"Food,-Fortified"/ all subheadings

explode "Nuts"/ all subheadings

lipid* near (low* or reduc ${ }^{\star}$ or modif*)

fat $^{\star}$ near (diet ${ }^{\star}$ or low ${ }^{\star}$ or modifi* or animal $^{\star}$ or vegetable* or acid $^{\star}$ or mono? unsat ${ }^{\star}$ or poly? unsat ${ }^{\star}$ or saturat ${ }^{\star}$ or unsatur ${ }^{\star}$ )

oil $^{\star}$ near (vegetable ${ }^{\star}$ or olive or rape $^{\star}$ or sunflow ${ }^{\star}$ or linseed ${ }^{\star}$ or mono? unsat* ${ }^{\star}$ or poly? unsat ${ }^{\star}$ or saturat ${ }^{\star}$ or unsatur $^{\star}$ )

lard $^{*}$ in $\mathrm{TI}, \mathrm{AB}$

meat* in $T I, A B$

garlic* in $T I, A B$

legum* in $\mathrm{TI}, \mathrm{AB}$

marg?rine ${ }^{\star}$ in $\mathrm{TI}, \mathrm{AB}$

butter* in $\mathrm{TI}, \mathrm{AB}$

bean $^{*}$ in $\mathrm{TI}, \mathrm{AB}$

$\# 1$ or \#2 or \#3 or \#4 or \#5 or \#6 or \#7 or \#8 or \#9 or \#10 or \#11 or \#12 or \#13 or \#14 or \#15 or \#16 or \#17 or \#18 or \#19 or \#20 or \#21 or \#22 explode "Lipoproteins"/ all subheadings

explode "Triglycerides"/ all subheadings

lipid $^{*}$

cholesterol* $^{*}$

lipoprotein*

triglyceride*

$\mathrm{HDL}^{*}$

$\mathrm{LDL}^{*}$

$\# 24$ or \#25 or \#26 or \#27 or \#28 or \#29 or \#30 or \#31

\#23 and \#32

(TG=ANIMAL) not ((TG=HUMAN) and (TG=ANIMAL))

RANDOMIZED-CONTROLLED-TRIAL in PT

CONTROLLED-CLINICAL-TRIAL in PT

RANDOMIZED-CONTROLLED-TRIALS 
RANDOM-ALLOCATION

DOUBLE-BLIND-METHOD

SINGLE-BLIND-METHOD

\#35 or \#36 or \#37 or \#38 or \#39 or \#40

\#33 not \#34

explode "Child"/ all subheadings

explode "Adult"/ all subheadings

\#43 and \#44

\#43 not \#45

$\# 41$ and \#42

\#47 not \#46

\section{Appendix 2. Search strategies June 2010}

\section{CENTRAL}

\#1lipid near (low ${ }^{\star}$ or reduc ${ }^{\star}$ or modifi ${ }^{\star}$ )

$\# 2$ cholesterol $^{\star}$ near (low* or modifi* or reduc ${ }^{\star}$ )

\#3(\#1 OR \#2)

\#4MeSH descriptor Nutrition Therapy explode all trees

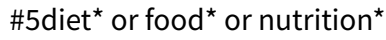

\#6(\#4 OR \#5)

\#7(\#3 AND \#6)

\#8fat ${ }^{\star}$ near (low ${ }^{\star}$ or reduc ${ }^{\star}$ or modifi ${ }^{\star}$ or animal ${ }^{\star}$ or saturat ${ }^{\star}$ or unsaturat ${ }^{\star}$ )

\#9MeSH descriptor Diet, Atherogenic explode all trees

$\# 10 \mathrm{MeSH}$ descriptor Diet Therapy explode all trees

\#11(\#7 OR \#8 OR \#9 OR \#10)

\#12MeSH descriptor Cardiovascular Diseases, this term only

\#13MeSH descriptor Heart Diseases explode all trees

\#14MeSH descriptor Vascular Diseases explode all trees

\#15MeSH descriptor Cerebrovascular Disorders, this term only

\#16MeSH descriptor Brain Ischemia explode all trees

\#17MeSH descriptor Carotid Artery Diseases explode all trees

\#18MeSH descriptor Dementia, Vascular explode all trees

\#19MeSH descriptor Intracranial Arterial Diseases explode all trees

\#20MeSH descriptor Intracranial Embolism and Thrombosis explode all trees

\#21MeSH descriptor Intracranial Hemorrhages explode all trees

\#22MeSH descriptor Stroke explode all trees

\#23 coronar* near (bypas* or graft $^{\star}$ or disease ${ }^{\star}$ or event ${ }^{\star}$ )

$\# 24$ cerebrovasc $^{\star}$ or cardiovasc ${ }^{\star}$ or mortal ${ }^{\star}$ or angina* or stroke or strokes or tia or ischaem ${ }^{\star}$ or ischem ${ }^{*}$

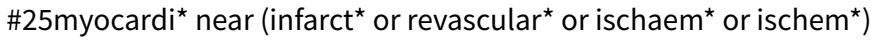

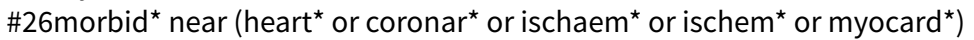

\#27vascular* near (peripheral ${ }^{\star}$ or disease ${ }^{\star}$ or complication $^{\star}$ )

$\# 28$ heart* near (disease ${ }^{\star}$ or attack* or bypas ${ }^{\star}$ )

\#29(\#12 OR \#13 OR \#14 OR \#15 OR \#16 OR \#17 OR \#18 OR \#19 OR \#20 OR \#21 OR \#22 OR \#23 OR \#24 OR \#25 OR \#26 OR \#27 OR \#28)

\#30(\#11 AND \#29)

\section{Ovid MEDLINE}

1 randomized controlled trial.pt.

2 controlled clinical trial.pt.

3 Randomized Controlled Trials/

4 Random Allocation/

5 Double-Blind Method/

6 Single-Blind Method/

7 or/1-6

8 Animal/ not Human/

97 not 8 (419534)

10 (lipid\$ adj5 (low\$ or reduc\$ or modifi\$)).mp.

11 (cholesterol\$ adj5 (low\$ or modific\$ or reduc\$)).mp.

1211 or 10

13 exp Nutrition Therapy/

14 (diet\$ or food\$ or nutrition\$).mp. 
1514 or 13

1612 and 15

17 (fat adj5 (low\$ or reduc\$ or modifi or animal\$ or saturat\$ or unsatur\$)).mp.

18 exp Diet, Atherogenic/

19 exp Diet Therapy/

2017 or 18 or 19 or 16

21 cardiovascular diseases/ or exp heart diseases/ or exp vascular diseases/

22 cerebrovascular disorders/ or exp brain ischemia/ or exp carotid artery diseases/or exp dementia, vascular/ or exp intracranial arterial diseases/ or exp "intracranial embolism and thrombosis"/ or exp intracranial hemorrhages/ or exp stroke/

23 (coronar\$ adj5 (bypas\$ or graft\$ or disease\$ or event\$)).mp.

24 (cerebrovasc $\$$ or cardiovasc $\$$ or mortal\$ or angina $\$$ or stroke or strokes).mp.

25 (myocardi\$ adj5 (infarct\$ or revascular\$ or ischaemi\$ or ischemi\$)).mp. (190649)

26 (morbid\$ adj5 (heart\$ or coronar\$ or ischaem\$ or ischem\$ or myocard\$)).mp.

27 (vascular\$ adj5 (peripheral\$ or disease or complication\$)).mp.

28 (heart\$ adj5 (disease $\$$ or attack\$ or bypass $\$$ )).mp.

2927 or 26 or 21 or 25 or 28 or 24 or 22 or 23

309 and 29 and 20

31 limit 30 to $y r=" 1998$ - current"

\section{EMBASE <1980 to 2010 Week 23>}

1 cardiovascular diseases/ or exp heart diseases/ or exp vascular diseases/ (1304100)

2 cerebrovascular disorders/ or exp brain ischemia/ or exp carotid artery diseases/or exp dementia, vascular/ or exp intracranial arterial diseases/ or exp "intracranial embolism and thrombosis"/ or exp intracranial hemorrhages/ or exp stroke/ (320121)

3 (coronar\$ adj5 (bypas\$ or graft\$ or disease\$ or event\$)).mp. [mp=title, abstract, subject headings, heading word, drug trade name, original title, device manufacturer, drug manufacturer name] (149449)

4 (cerebrovasc $\$$ or cardiovasc $\$$ or mortal\$ or angina $\$$ or stroke or strokes).mp. [mp=title, abstract, subject headings, heading word, drug trade name, original title, device manufacturer, drug manufacturer name] (838125)

5 (myocardi\$ adj5 (infarct\$ or revascular\$ or ischaemi or ischemi\$)).mp. [mp=title, abstract, subject headings, heading word, drug trade name, original title, device manufacturer, drug manufacturer name] (119244)

6 (morbid\$ adj5 (heart\$ or coronar\$ or ischaem \$ or ischem\$ or myocard\$)).mp. [mp=title, abstract, subject headings, heading word, drug trade name, original title, device manufacturer, drug manufacturer name] (20403)

7 (vascular\$ adj5 (peripheral\$ or disease $\$$ or complication\$)).mp. [mp=title, abstract, subject headings, heading word, drug trade name, original title, device manufacturer, drug manufacturer name] (160186)

8 (heart\$ adj5 (disease or attack\$ or bypass\$)). mp. [mp=title, abstract, subject headings, heading word, drug trade name, original title, device manufacturer, drug manufacturer name] (217597)

9 random\$.ti,ab. (442747)

10 factorial\$.ti,ab. (9473)

11 placebo\$.ti,ab. (119397)

12 (doubl\$ adj blind\$).ti,ab. (90667)

13 (singl\$ adj blind\$).ti,ab. (8183)

14 double-blind procedure.sh. (78190)

15 randomized controlled trial.sh. (189654)

16 single blind procedure.sh. (9526)

179 or 10 or 11 or 12 or 13 or 14 or 15 or $16(553493)$

$18 \mathrm{animal} /$ or nonhuman/ or animal experiment/ (3698016)

19 human/ (7063453)

2019 and 18 (617530)

2118 not 20 (3080486)

2217 not $21(485728)$

23 (lipid\$ adj5 (low\$ or reduc\$ or modifi\$)).mp. [mp=title, abstract, subject headings, heading word, drug trade name, original title, device manufacturer, drug manufacturer name] (46393)

24 (cholesterol\$ adj5 (low\$ or modific\$ or reduc\$)).mp. [mp=title, abstract, subject headings, heading word, drug trade name, original title, device manufacturer, drug manufacturer name] (64458)

2524 or $23(89868)$

26 (diet\$ or food\$ or eat\$ or nutrition\$).mp. [mp=title, abstract, subject headings, heading word, drug trade name, original title, device manufacturer, drug manufacturer name] (596443)

27 exp nutrition/ (977243)

2826 or $27(1182970)$

2928 and $25(34840)$

30 (fat adj5 (low\$ or reduc\$ or modifi or animal\$ or saturat\$ or unsatur\$)).mp. [mp=title, abstract, subject headings, heading word, drug trade name, original title, device manufacturer, drug manufacturer name] (24569) 
31 exp lipid diet/ or exp fat intake/ or exp low fat diet/ (31724)

3231 or 29 or $30(71041)$

336 or 3 or 7 or 2 or 8 or 1 or 4 or 5 (1779345)

3422 and 33 and 32 (3615)

35 limit 34 to $y r=" 2008$-Current" (626)*

*Previous updates were from 1998, so the updated EMBASE searches covered the period January 1998 to June 2010.

\section{FEE D B A C K}

\section{Question}

\section{Summary}

Name: Jos Verbeek

Email Address: jos.verbeek@ttl.fi

Personal Description: Occupation Occupational Physician, Cochrane Review Author

Feedback: Dear Authors,

Many compliments for the enormous amount of work in putting together the evidence on reducing or modifying fat intake as a dietary change to prevent CVD. However, I am of the opinion that you overstated your conclusions and that the recommendation to change to a non-saturated fat diet is not based on sufficient evidence. My arguments are as follows. First of all you have picked one positive outcome out of a dozen of negative outcomes, in fact all other outcomes, to underpin your recommendation. You do not even discuss how come that only this one is supportive and the rest not. I believe that this does not make a strong case for a preventive effect. You do not explain why a much better defined and also plausible outcome as mortality does not show any effect whereas a quite ill-defined effect such as all cardiovascular events does show an effect. In addition, it is difficult to understand why none of the other cardiovascular events, such as myocardial infarctions, shows an effect. I believe that this points much more strongly to no effect than to the overall positive effect that you have formulated.For a person who should decide to change diet, the figures are not very convincing either. Even though you say that this reduces the risk with $14 \%$, in practice, this means that my CVD risk would change from $7 \%$ to $6 \%$. Being totally healthy otherwise, it is probably even much lower than that. That is not a big incentive to start changing something important as your diet.

I believe that taking these items into account would further improve the review.

Best wishes

Jos Verbe

\section{Reply}

Thank you so much for your thoughts, it is great to get feedback!

We don't agree that we have over-stated our conclusions - we have stated clearly in the abstract that we found no effect on mortality or cardiovascular mortality, but that we did find a small but statistically significant effect on cardiovascular events.

In the abstract we quote the overall effect of reducing or modifying dietary fat on cardiovascular events, but within the review we show that the effect is only seen in longer studies, and in studies that modified fat rather than reducing it. This means that the effect of modifying fat for at least 2 years is considerably greater than the quoted $14 \%$, as the $14 \%$ is diluted by reduced fat studies and short studies. The actual effect (of the subgroup of studies that were at least 2 years long and modified fat intake) was around $27 \%$ (RR $0.73,95 \% \mathrm{Cl} 0.56$ to $0.95, \mathrm{p}$ $0.02,1267 \%, 584$ events). However, this was a subgroup analysis and so did not feature in the abstract, and if we had quoted it we would correctly have been challenged for overstating our case.

You worry that there is no supportive evidence of effects on individual cardiovascular events - but there were very few people who experienced myocardial infarction in the studies of modified fat (only 579 , with a RR of $0.91,95 \% \mathrm{Cl} 0.72$ to 1.16 ) and even fewer people with stroke (51 people, with a RR of $0.70,95 \% \mathrm{Cl} 0.36$ to 1.34). It is quite feasible that reductions in both stroke and MI lead to the reduction in cardiovascular events, but that short term studies diluted the effect seen, and low numbers led to low power, obscuring any effect.

It is not surprising at all that while we saw reductions in cardiovascular events we did not see similar reductions in mortality - fortunately most cardiovascular events are not fatal, and many deaths are not cardiovascular in origin, so if modifying fat intake reduces cardiovascular events the effect on cardiovascular mortality and all-cause mortality will be much less clear.

It is great to hear that your cardiovascular risk is so low - well done! Given your risk is only $7 \%$ (or less) even a $50 \%$ risk reduction would make only a small difference to you. However, to a person with a larger baseline risk, or for a whole population, a reduction of $14 \%$ (or $27 \%$ from a long term modified fat diet) is a great help.

With best wishes, Lee and the review team 
WHAT'S NEW

\begin{tabular}{lll}
\hline Date & Event & Description \\
\hline 21 November 2017 & Review declared as stable & This review was split into seven smaller reviews. \\
\hline
\end{tabular}

\section{HISTORY}

Protocol first published: Issue 2, 1999

Review first published: Issue 2, 2000

\begin{tabular}{|c|c|c|}
\hline Date & Event & Description \\
\hline 27 March 2012 & Amended & New feedback. \\
\hline 27 May 2011 & $\begin{array}{l}\text { New citation required and conclusions } \\
\text { have changed }\end{array}$ & $\begin{array}{l}\text { - The objectives have been clarified and extended } \\
\text { - Studies divided by major intervention type: reduced fat, mod- } \\
\text { ified fat, reduced and modified fat intervention. Studies com- } \\
\text { paring reduced with modified fat diets also included. } \\
\text { - Analyses run in RevMan as relative risks (rather than as rate ra- } \\
\text { tios) } \\
\text { - All meta-analyses, sub-grouping, sensitivity analyses and } \\
\text { meta-regressions re-run } \\
\text { - Validity assessment updated in all included studies } \\
\text { - The first version of the review included } 27 \text { studies, } 40 \text { interven- } \\
\text { tion arms, } 30901 \text { person years. } \\
\text { - This update includes } 48 \text { studies, including } 60 \text { comparisons and } \\
80760 \text { individual participants, published between } 1965 \text { and } \\
2009 \\
\text { - Of these } 25 \text { comparisons, including } 61,958 \text { participants com- } \\
\text { pared a reduced fat diet with usual or control diet, while } 15 \\
\text { comparisons, including } 13,004 \text { participants compared a modi- } \\
\text { fied fat diet with control or usual diet } \\
\text { - In this update } 10 \text { interventions, including } 4,931 \text { participants } \\
\text { compared a reduced and modified fat diet with usual or con- } \\
\text { trol diet, while nine interventions, including } 1290 \text { participants } \\
\text { compared a low fat diet with a modified fat diet. The final com- } \\
\text { parison could not be classified. }\end{array}$ \\
\hline
\end{tabular}

27 May $2011 \quad$ New search has been performed The searches were updated by the authors to June 2010. New au-
thors have been added.

\begin{tabular}{lll}
\hline 9 September 2008 & Amended & Converted to new review format. \\
\hline 1 February 2000 & $\begin{array}{l}\text { New citation required and conclusions } \\
\text { have changed }\end{array}$ & Substantive amendment \\
\hline
\end{tabular}

\section{CONTRIBUTIONS OF AUTHORS}

All authors were active in the design of the review and in providing critical revisions of the manuscript. Julian Higgins also performed the statistical analyses for the first version of this review, Lee Hooper carried out the statistical analyses for the update; Rachel Thompson, Helen Moore, Diredre Sills and Felicia Roberts (with Indra Tumur and Dorothee Fagard) duplicated the inclusion / exclusion and data extraction of all studies; and Rudolph Riemersma arbitrated on study inclusion where necessary. Shah Ebrahim and Carolyn Summerbell 
were primary advisors to the initial review. Lee Hooper originated and was primarily responsible for planning and carrying out the review and was the principal author of the first and update versions.

\section{DECLARATIONS OF INTEREST}

LH was employed as a dietitian working in the area of cardiac rehabilitation for much of the duration of the first version of this review. RLT and CDS are also dietitians.

\section{SOURCES OF SUPPORT}

\section{Internal sources}

- University of East Anglia, UK.

Help with acquiring papers for the review, time for Lee Hooper to work on the review

- University of Manchester, UK.

Support with collection of papers for the review.

\section{External sources}

- Studentship, Systematic Reviews Training Unit, Institute of Child Health, University of London, UK.

Funding to support Lee Hooper to carry out the first version of the systematic review

\section{NOTES}

This review was split into seven reviews with more specific questions:

Reduction in saturated fat intake for cardiovascular disease

Effects of a long term reduced fat diet on prevention of cardiovascular disease

Effects of a long term modified fat diet on prevention of cardiovascular disease

Effects of a long term reduced and modified fat diet on prevention of cardiovascular disease

Effects of a reduced fat versus a modified fat diet on prevention of cardiovascular disease

Effects of total fat intake on body weight in adults

Effects of total fat intake on body weight in children

\section{INDEX TERMS}

\section{Medical Subject Headings (MeSH)}

Cardiovascular Diseases [epidemiology] [ ${ }^{*}$ prevention \& control]; Cholesterol [blood]; Diet, Fat-Restricted [ ${ }^{*}$ methods]; Dietary Fats [*administration \& dosage]; Fats, Unsaturated [administration \& dosage]; Randomized Controlled Trials as Topic; Risk Factors; Triglycerides [blood]

\section{MeSH check words}

Adult; Aged; Humans; Middle Aged 